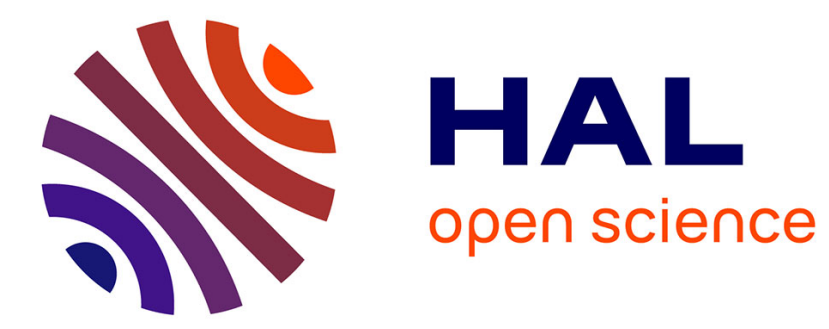

\title{
Cryogenic AFM-STM for mesoscopic physics
}

Hélène Le Sueur

\section{To cite this version:}

Hélène Le Sueur. Cryogenic AFM-STM for mesoscopic physics. Condensed Matter [cond-mat]. Université Pierre et Marie Curie - Paris VI, 2007. English. NNT ： . tel-00261434v3

\section{HAL Id: tel-00261434 https://theses.hal.science/tel-00261434v3}

Submitted on 10 Oct 2008

HAL is a multi-disciplinary open access archive for the deposit and dissemination of scientific research documents, whether they are published or not. The documents may come from teaching and research institutions in France or abroad, or from public or private research centers.
L'archive ouverte pluridisciplinaire HAL, est destinée au dépôt et à la diffusion de documents scientifiques de niveau recherche, publiés ou non, émanant des établissements d'enseignement et de recherche français ou étrangers, des laboratoires publics ou privés. 
Electronic spectroscopy based on electron tunneling gives access to the electronic Density of States (DoS) in conductive materials, and thus provides detailed information about their electronic properties.

During this thesis work, we have developed a microscope in order to perform spatially resolved $(10 \mathrm{~nm})$ tunneling spectroscopy, with an unprecedented energy resolution $(10 \mu \mathrm{eV})$, on individual nanocircuits. This machine combines an Atomic Force Microscope (AFM mode) together with a Scanning Tunneling Spectroscope (STS mode), and functions at very low temperatures $(30 \mathrm{mK})$. In the AFM mode, the sample topography is recorded using a piezoelectric quartz tuning fork, which allows locating and imaging nanocircuits. Tunneling can then be performed on conductive areas of the circuit.

With this microscope, we have measured the local DoS in a hybrid Superconductor-Normal metal-Superconductor (S-N-S) structure. In such circuit, the electronic properties of $\mathrm{N}$ and $\mathrm{S}$ are modified by the superconducting proximity effect. In particular, for short $\mathrm{N}$ wires, we have observed a minigap in the DoS of the $\mathrm{N}$ wire, independent of position. Moreover, when varying the superconducting phase difference between the $S$ electrodes, we have measured the modification of the minigap, and its disappearance when the phase difference equals $\pi$.

Our experimental results for the DoS, and its dependences (with phase, position, $\mathrm{N}$ length) are quantitatively accounted for by the quasiclassical theory of superconductivity. Some predictions of this theory are observed for the first time.

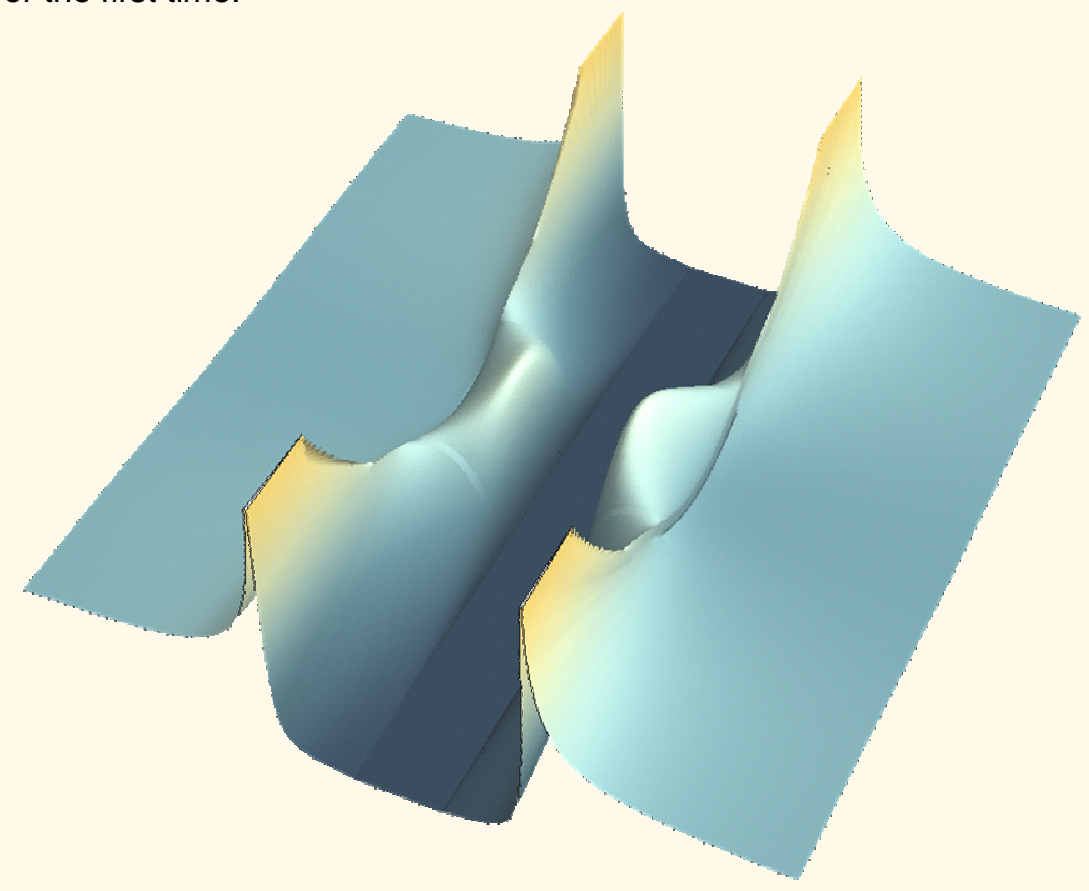

\section{Cryogenic AFM-STM for} mesoscopic physics

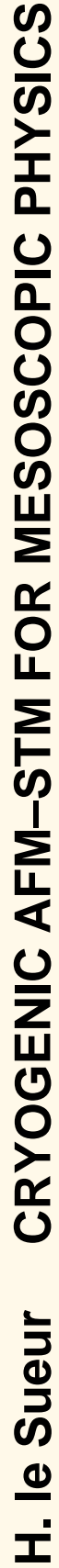

\section{Hélène le Sueur}

\author{
Quantronics Group \\ SPEC \\ CEA - Saclay
}


THESE DE DOCTORAT DE

L'UNIVERSITÉ PARIS 6 - PIERRE ET MARIE CURIE

\author{
Spécialité
}

\title{
Physique des Solides
}

Présentée par Hélène le Sueur

pour obtenir le grade de

\section{DOCTEUR de l'UNIVERSITÉ PIERRE ET MARIE CURIE}

Sujet de la thèse:

Développement d'un AFM-STM pour la spectroscopie électronique haute résolution de nanostructures et application à la supraconductivité de proximité

soutenue le 21 septembre 2007

devant un jury composé de

M Marco APRILI

M Claude CHAPELIER rapporteur

M Juan Carlos CUEVAS rapporteur

M Daniel ESTEVE directeur de thèse

M William SACKS

M Christian SCHÖNENBERGER 
This thesis can be downloaded as a pdf file at:

http://www-drecam.cea.fr/drecam/spec/pres/quantro/qsite/index.htm

or

http://tel.archives-ouvertes.fr/

\section{COVER:}

The front cover shows an AFM image of a small circuit fabricated by electronic lithography and double angle evaporation, comprising a $350 \mathrm{~nm}$ long, $30 \mathrm{~nm}$ thick silver wire (colorized in orange) and a $60 \mathrm{~nm}$ thick aluminium half loop (dark grey). This structure has been imaged using an atomic force sensor, and tunneling spectroscopy was subsequently performed at several positions. Curves on the background are tunneling spectra measured for different values of the superconducting phase difference across the normal wire (at the location of the tip represented on the AFM image).

The graph on the backside of the cover is a 3D plot of the predicted local density of states along a 1D S-N-S structure, with a normal wire length $\mathrm{L}=1.4 \xi$ (corresponding to $350 \mathrm{~nm}$ ), and perfect interfaces. 


\section{REMERCIEMENTS}

Ce fût un réel plaisir pour moi de travailler au Service de Physique de l'Etat Condensé. Je ne parle pas ici du seul groupe Quantronique, car ce sont réellement le rassemblement en un lieu unique d'autant de compétences diverses et leur prompte disponibilité qui m’ont donné la chance inouïe de mener à bon terme ce projet.

Tout d'abord, un grand merci à Eric Vincent qui m'a soutenue dans toutes mes demandes auprès du CEA, et m'a ainsi permis de rester parmi vous le temps nécessaire à la réalisation de notre première expérience de physique mésoscopique : quel bonheur ! Merci à ceux qui ont rendu cela possible, par leur intervention à divers niveaux : Louis Laurent, Philis Livanos, lors de mon entrée au SPEC, Didier Normand lors de la prolongation de mon contrat CFR, Sandrine Thunin et Nathalie Royer dans mes diverses démarches administratives.

Merci infiniment à Daniel Esteve, mon directeur de thèse, pour avoir appuyé sans réserve ma candidature, et tout ce qui s'en est suivi : Travailler avec un «chef»comme toi est un enrichissement extrême, tant pour ton recul sur la compréhension des phénomènes envisagés, que pour ton esprit pratique qui nous permet de parvenir plus vite (et mieux bien sûr!) au résultat souhaité... Et puis tu n'es pas chef pour rien : ce don d'ubiquité qui te permet de consacrer à tous les projets simultanément la même attention exclusive force sincèrement l'admiration !

J'espère avoir mérité toute la confiance que m'a accordée Philippe Joyez en me laissant la responsabilité de porter avec lui son projet de recherche, à ses débuts lors de mon arrivée dans le groupe. Ma seule gratitude ne peut exprimer justement tout ce que tu as fait pour moi car tu m'as fait vivre durant cette thèse une expérience de recherche inoubliable : construire une manip' entière depuis la racine, l'élaborer pas par pas, et la faire marcher pour en tirer des mesures de premier ordre! Quel thésard peut rêver mieux ?

Bien entendu une telle entreprise n'aurait pu aboutir sans le soutien technique de nombreuses personnes. Sans les compétences de Pascal Senat, les pièces du microscope seraient probablement usinées de travers, et cette mécanique si délicate n'aurait jamais fonctionné. Pief Orfila, non content de gérer la logistique du groupe ainsi que l'installation et le bon fonctionnement de la majorité des machines de nano-fabrication au SPEC, a de plus aménagé notre labo de manière astucieuse et confortable. Jean-Claude Tack a été d'une grande disponibilité pour l'usinage d'un bon nombre de pièces de câblage. Grâce à Michel Juignet, qui m'a initiée aux machines d'atelier, j'ai pu usiner moi-même -avec les conseils de Pascal- 
quelques pièces du microscope. Je remercie chaleureusement toutes ces personnes pour leur aide précieuse.

Le réfrigérateur que nous utilisons pour faire l'expérience présentée dans ce manuscrit est un modèle construit par Air-Liquide, qui a présenté dès son achat de nombreux dysfonctionnements. Ce réfrigérateur a été envoyé en réparation avant le début de ma thèse, et n'est revenu qu'au milieu de la 2è année, en raison des difficultés rencontrées pour le réparer. Je tiens à remercier chaudement le personnel du CRTBT qui s'est attaché à ce travail difficile et peu gratifiant, en sus de leur taches personnelles. Le travail bénévole de Thomas Prouve, un étudiant en thèse, et Lionel Germani, un technicien cryogéniste m'aura permis -malgré le retard pris- d'utiliser le microscope que j'avais construit à des fins de recherche. Patrick Pari a aussi contribué à cette réparation puisqu'il a levé le tout dernier problème de cryogénie, nous permettant de récupérer ce réfrigérateur tant attendu pour commencer à le câbler. Patrick Pari continue, avec Mathieu de Combarieu et Philippe Forget, le groupe de cryogénie du SPEC, de nous aider à améliorer le fonctionnement du réfrigérateur. Merci à eux !

Faire de la physique dans le groupe Quantronique est en soi une chance, tant pour l'éclectisme que pour l'effervescence scientifique qui y règnent. Difficile de passer quatre années au contact de ses membres sans être contaminé par la passion qui les anime...

Denis Vion d'un enthousiasme constant pour mes divers balbutiements en montage de manip', et d'un dévouement exceptionnel pour améliorer mon discours de soutenance : merci de ton soutien dans ces moments difficiles, et merci d'avoir pris sur toi tout mon stress le jour J, comme en témoignaient tes grimaces pendant mon exposé!

Hugues Pothier sans qui la salle café serait jonchée de miettes de gâteau. Cette seule description est un peu réductrice je l'avoue : il faut aussi noter la finesse de ses explications physiques qui savent aborder la problématique de manière simple et limpide en utilisant les bonnes images. Merci à toi de m'avoir introduite aux subtilités de l'effet de proximité, et m'avoir évité de tourner en rond trop longtemps pour choisir les bons paramètres d'expérience.

J'ai beaucoup apprécié les compétences et l'exigence de Cristiàn Urbina, dans de multiples domaines et notamment en matière d'écriture anglaise. Merci pour ta relecture attentive de mon manuscrit et tes corrections qui sont sûrement responsables des compliments que j'ai reçus en ce sens.

Merci à Patrice Bertet, nouveau membre permanent du groupe, pour ses idées originales en provenance du grand nord, sa grande capacité d'écoute et de conseil, et sa contenance qui contraste avec l'impertinence du groupe ! A bientôt à La tuile à loup... 
Je souhaite une bonne continuation aux thésards et post-docs que j'ai côtoyés durant mon séjour : Martin Chauvin et Peter Vom Stein, le premier duo P08, responsable de nombreuses soirées pizza / vidéo-projecteur qui m’ont souvent remonté le moral ! Anne Anthore et Ronald Cron, qui ont partagé durant quelques mois ces soirées avec nous, Benjamin Huard et MariaLuisa Della-Rocca dignes successeurs de Peter et Martin, et Quentin Le Masne, qui prend maintenant la relève avec autant de punch. Bon vent aux manipulateurs de Q-Bit : Eddy Collin, Grégoire Ithier, Nicolas Boulant, François Nguyen, François Mallet et Agustin Palacios : puissent les suivants animer la P4 team aussi bien que vous !

J'ai apprécié de passer ce temps en compagnie des divers groupes du SPEC: les magnétiseurs, le groupe bruitonique (le sous-sol humide est-il toujours propice à la culture des blagues graveleuses ?), les LEMuriens, les turbulents... thésards, post-doc ou permanents avec qui il était toujours agréable d'échanger.

Je remercie également les différentes personnes qui m'ont accordé leur disponibilité et m’ont fait partager leurs connaissances de manière toujours très sympathique: merci tout particulièrement à Juan Carlos Cuevas, Wolfgang Belzig, Elke Sheer, Terö Heikkilä, Mathias Eschrig, Takis Kontos, Audrey Cottet, Anne Anthore, Sophie Guéron, Hélène Bouchiat, Francesca Chiodi pour nos échanges très instructifs sur l'effet de proximité, à Hervé Courtois, Gabino Rubio-Bollinger, Nicolas Agrait, Herman Suderow et Olivier Klein pour avoir partagé avec moi leur expérience sur la microscopie, à Marcelo Goffman et Roland Lefebvre pour m'avoir fourni des échantillons lors des tests initiaux du microscope.

Je suis très reconnaissante envers Juan Carlos Cuevas, Claude Chapelier, Marco Aprili, William Sacks, et Christian Schönenberger d'avoir accepté de faire partie de mon jury.

Enfin, mes pensées vont vers tous ceux qui m'ont épaulé durant cette thèse et ont rendu ces années plus agréables. Je pense tout particulièrement à mon père Henri, ma mère MarieClaude, et à Florence et Benjamin, ma famille au sens large, qui accompagnent mes réussites et mes échecs depuis le début.

Je regrette déjà cette dualité d'une grande profondeur d'esprit avec des discours irrévérencieux, qui caractérise le groupe Quantronique, et souhaite sincèrement pouvoir prolonger dans l'avenir nos discussions passionnantes -de tout ordre- et notamment celles sur les macarons ! 


\section{TABLE OF CONTENTS}

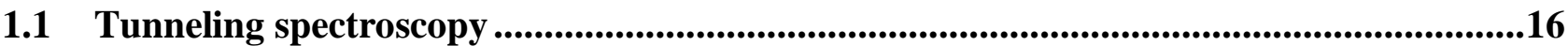

1.2 Spatially resolved measurement of the LDOS with a combined AFM-STM....................19

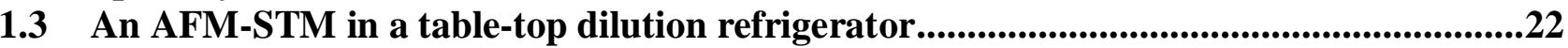

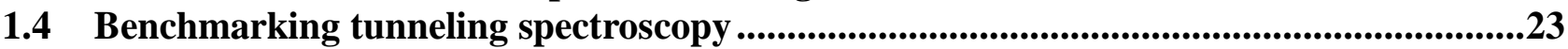

1.5 An experiment on the proximity effect in $\mathrm{S}-\mathrm{N}-\mathrm{S}$ structures .............................................25

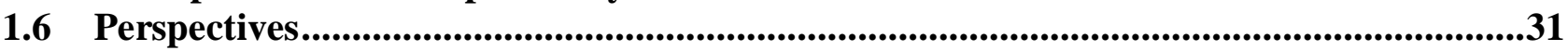

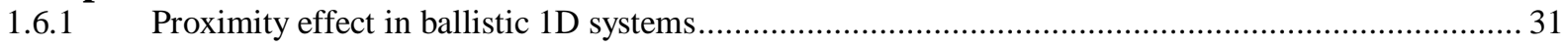

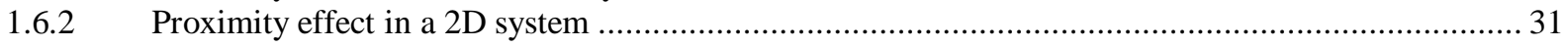

1.6.3 Spin injection and relaxation in superconductors ........................................................................... 32

1.6.4 Energy relaxation in quasi-ballistic ("Superdiffusive”) structures...................................................... 32

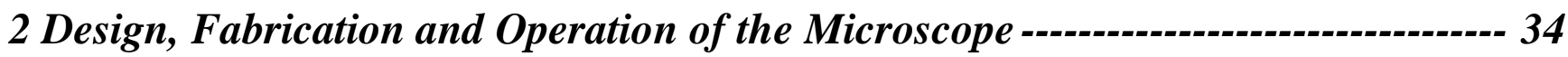

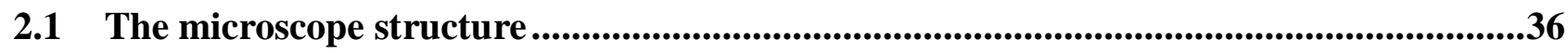

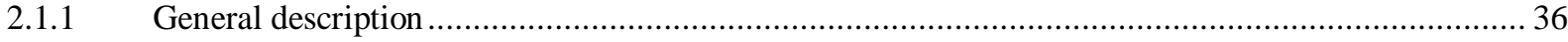

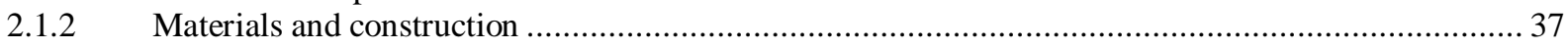

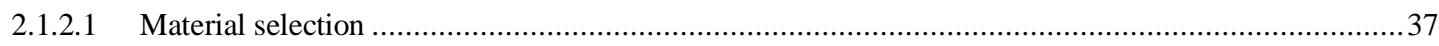

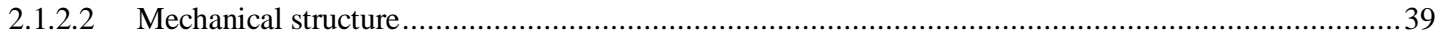

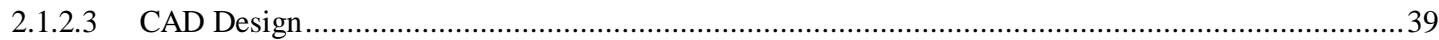

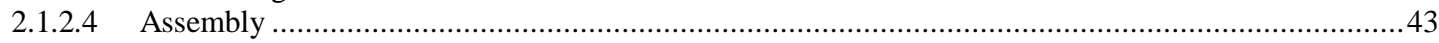

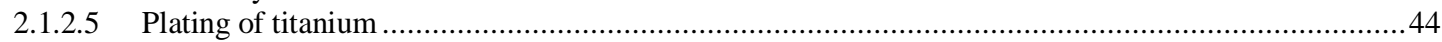

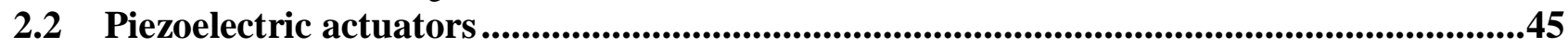

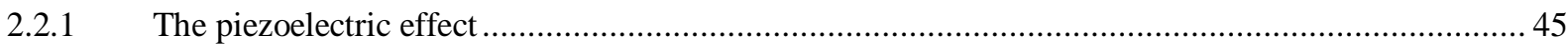

2.2.1.1 General mathematical description of piezoelectricity ...........................................................45

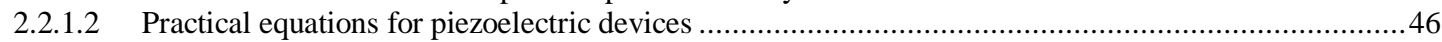

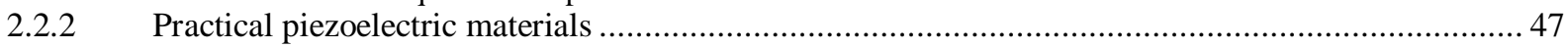

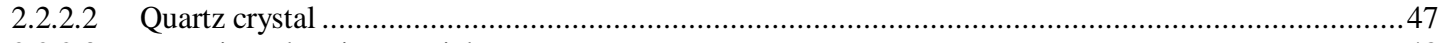

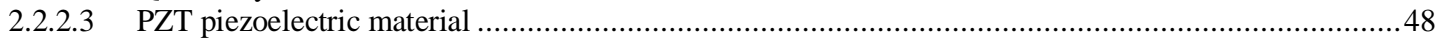

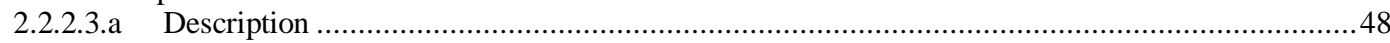

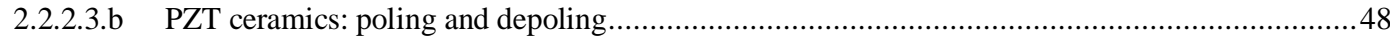

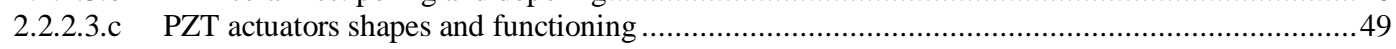

2.2.2.3.d Imperfections of PZT ceramics: drift and hysteresis .......................................................52

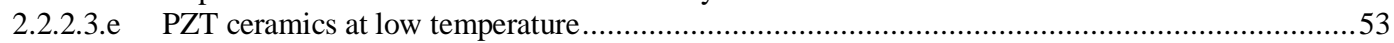

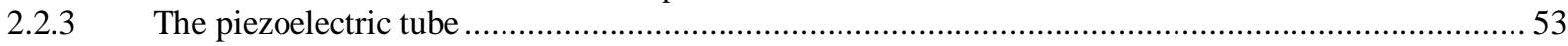

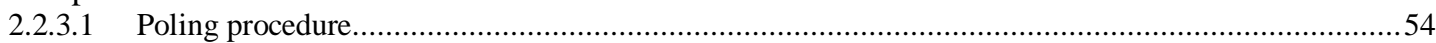

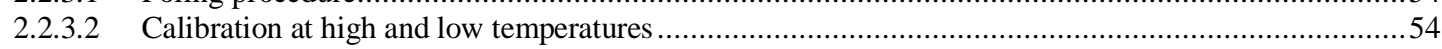

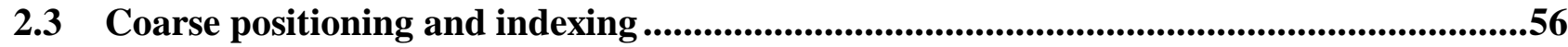

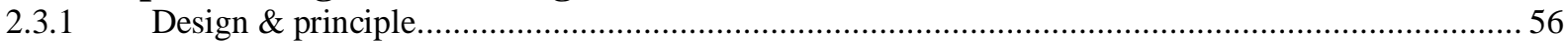

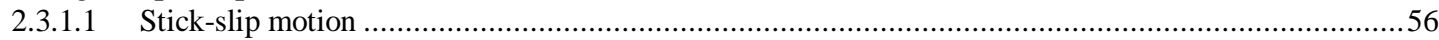

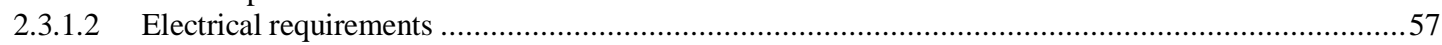

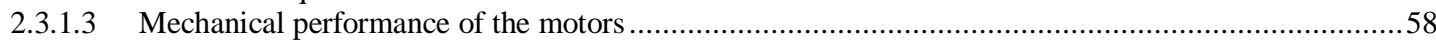

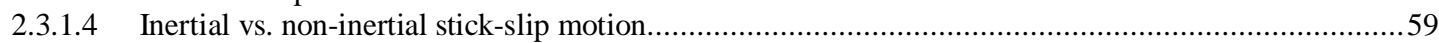

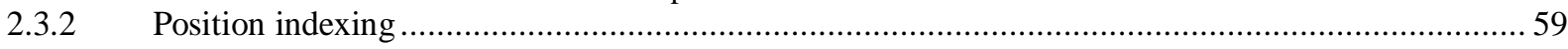

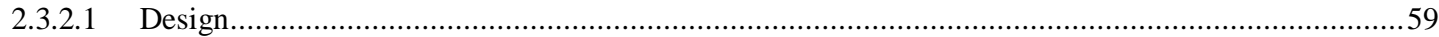

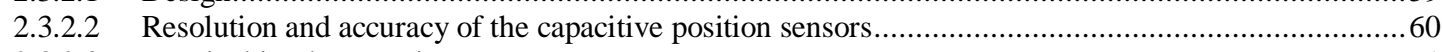

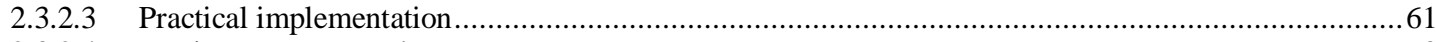

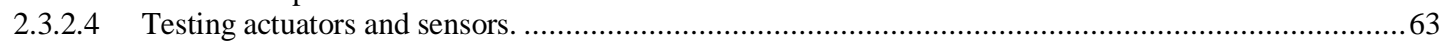

2.4 The atomic force sensor .............................................................................................66

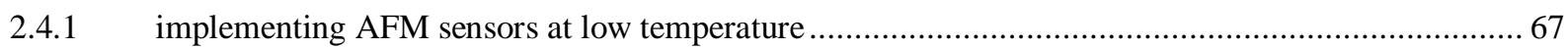

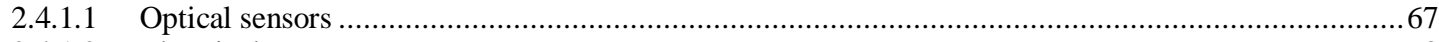

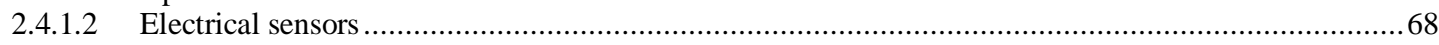

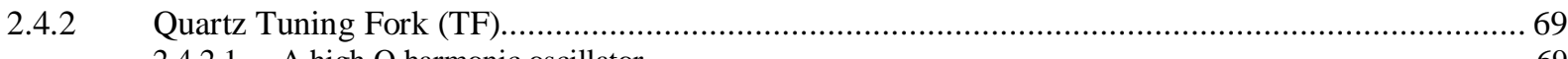

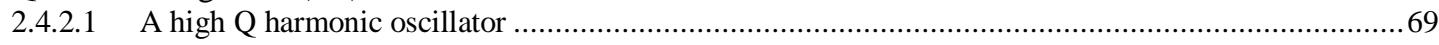




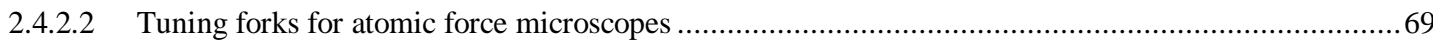

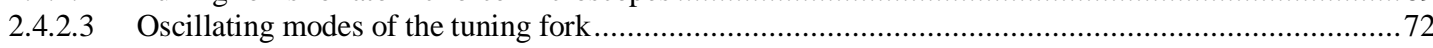

2.4.2.4 Basic analysis of a tuning fork: 1 Degree-of-Freedom harmonic oscillator model...........................73

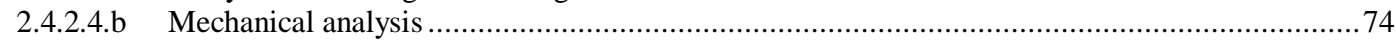

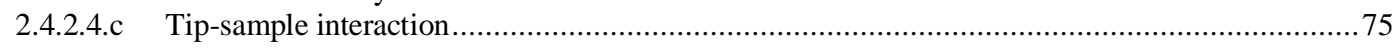

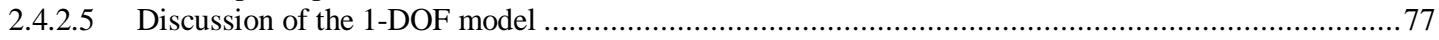

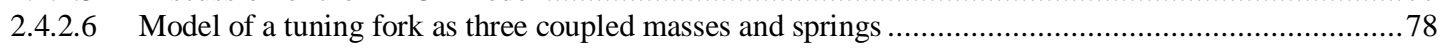

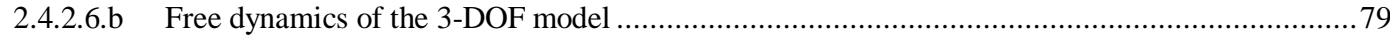

2.4.2.6.c Balanced tuning fork: the unperturbed modes................................................................ 81

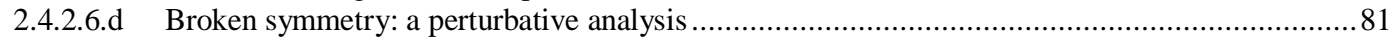

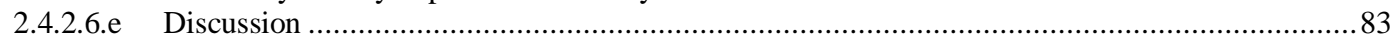

2.4.2.7 Estimating characteristics of the connecting wire for preserving the tuning fork balance ......................8

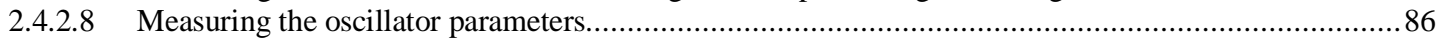

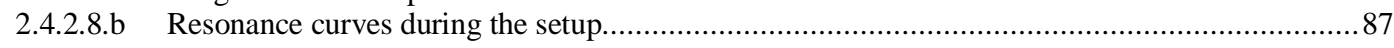

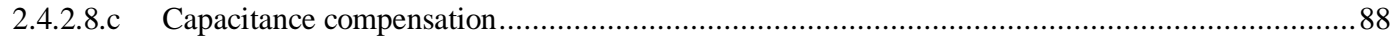

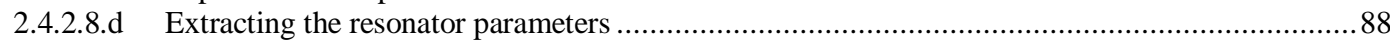

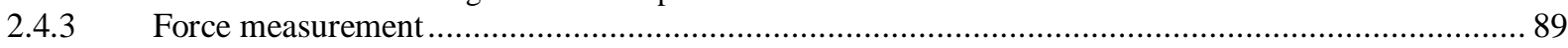

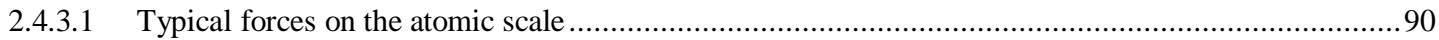

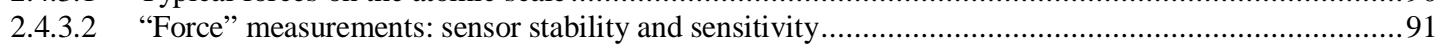

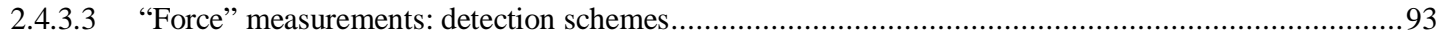

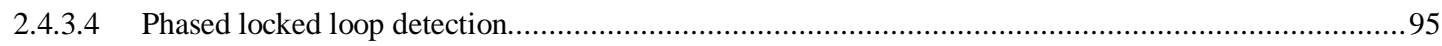

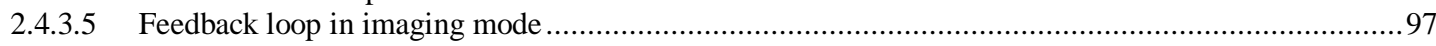

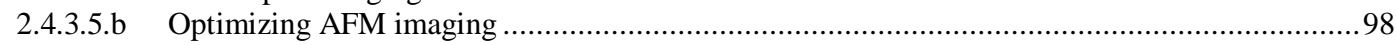

3.1 A dilution refrigerator well adapted for a local probe microscope................................102

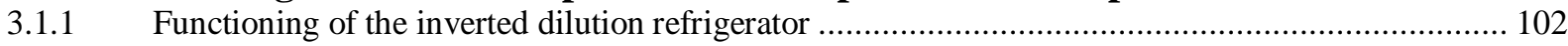

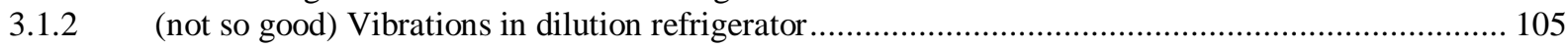

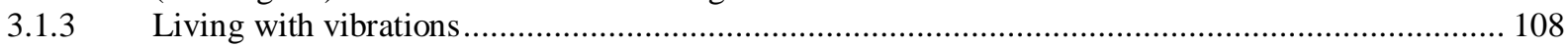

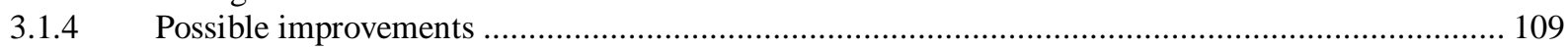

3.2 Wiring a microscope for very low temperature experiments...........................................110

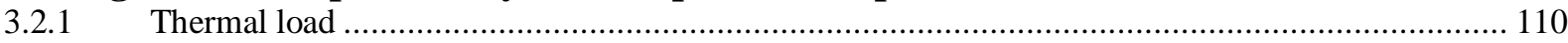

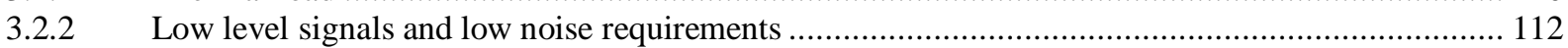

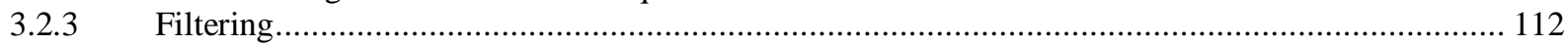

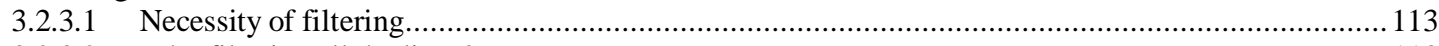

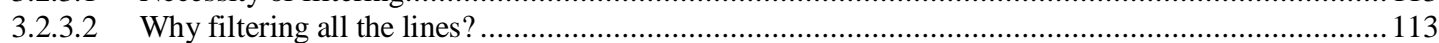

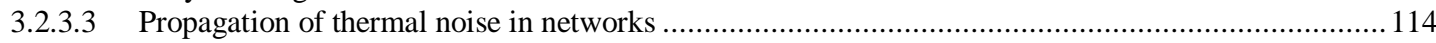

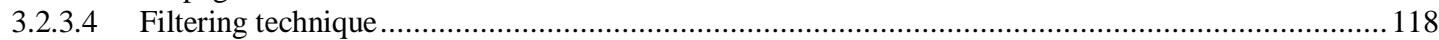

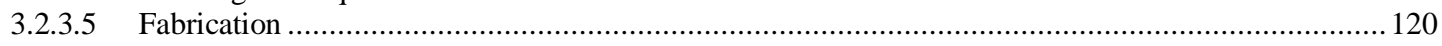

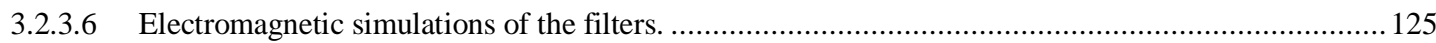

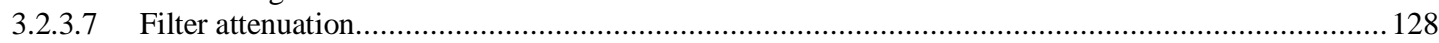

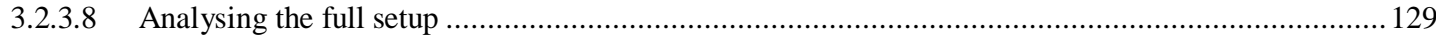

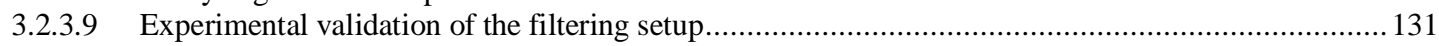

3.2.3.10 Article reprint: microfabricated filters ....…………………………………………………. 131

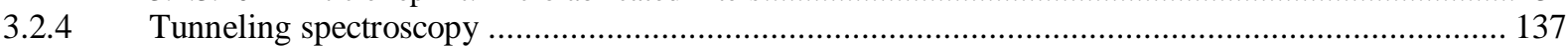

3.2.4.1 Tunneling spectroscopy measurements ………………………………………………………..... 137

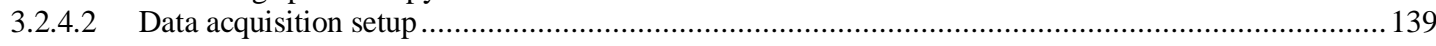

3.2.4.3 Article reprint : Tunnel current pre-amplifier ...…………………………………………...... 143

3.3 Fabrication techniques .............................................................................................149

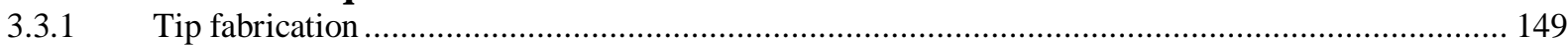

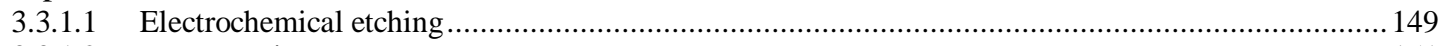

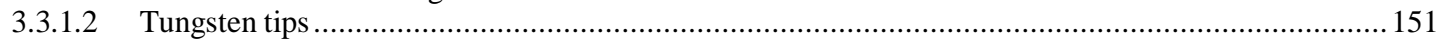

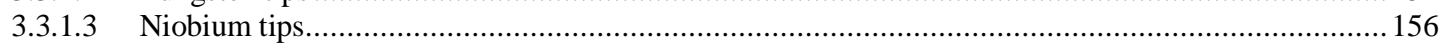

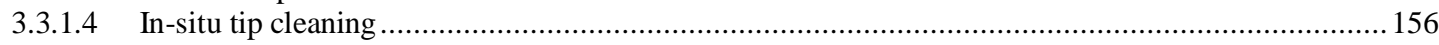

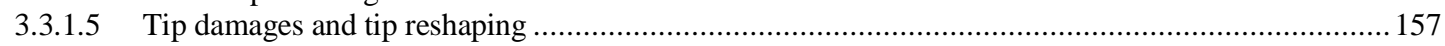

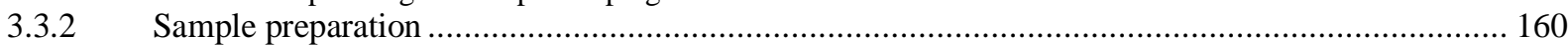

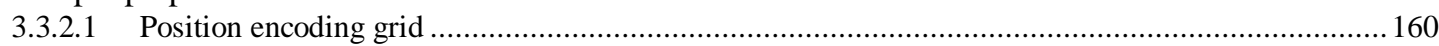

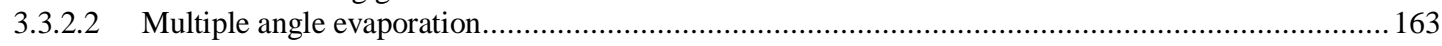




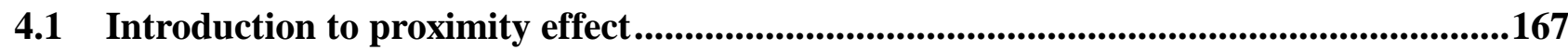

4.2 Theoretical description of Proximity Effect...........................................................168

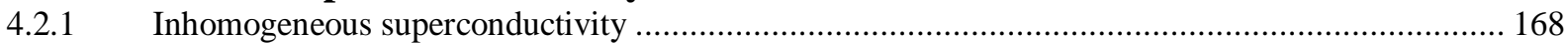

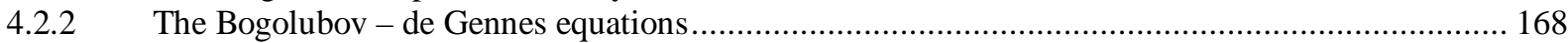

4.2.3 Theoretical description of the proximity effect in diffusive systems at equilibrium ......................... 169

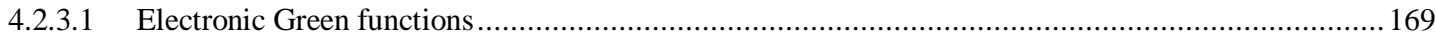

4.2.3.2 Green functions in the Nambu space..................................................................................... 169

4.2.3.3 Quasiclassical Green functions in the dirty limit - Usadel equation .................................................. 170

4.2.3.4 Properties of the Green function elements - Physical quantities .................................................... 170

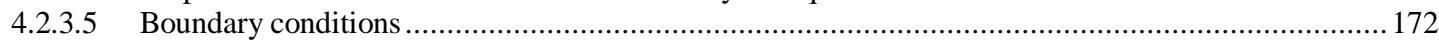

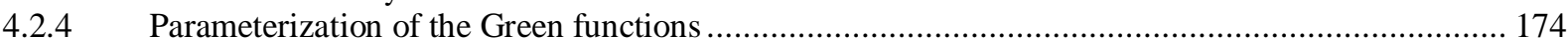

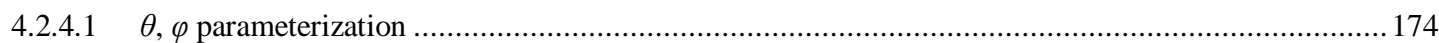

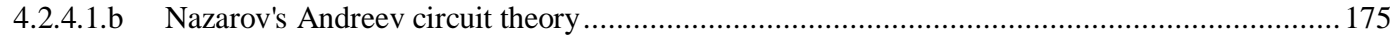

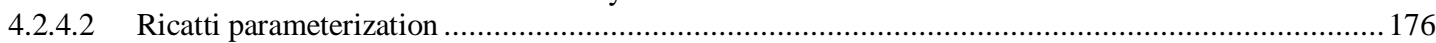

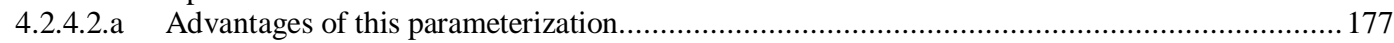

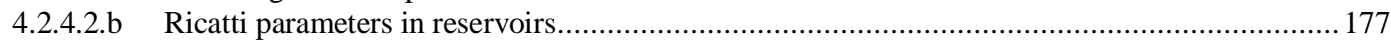

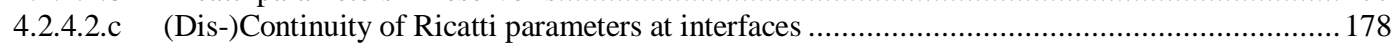

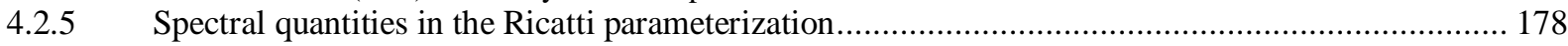

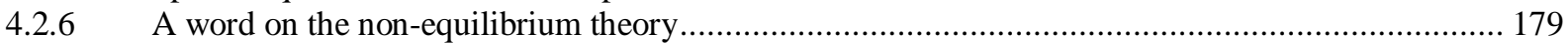

4.3 Solving the Usadel equations numerically ............................................................180

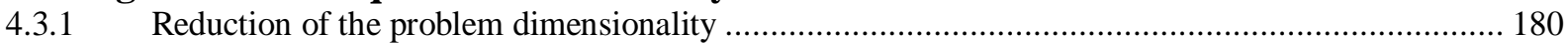

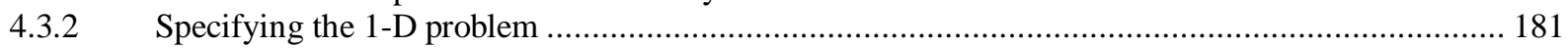

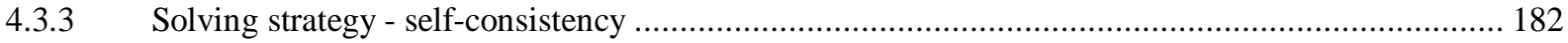

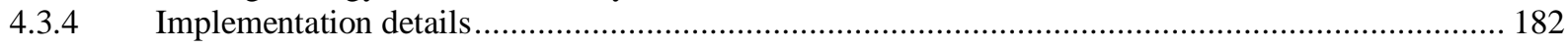

4.4 General results on proximity effect in SNS structures ............................................186

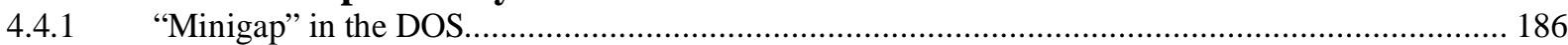

4.4.2 Inverse proximity effect - Pairing amplitude and pairing potential.............................................. 187

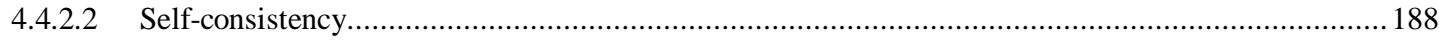

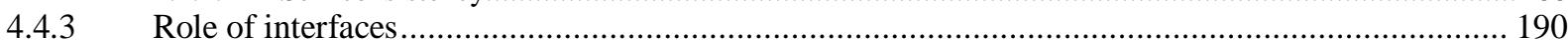

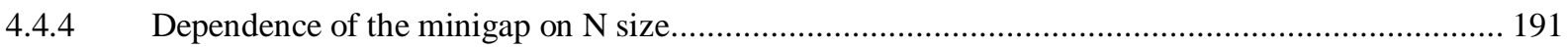

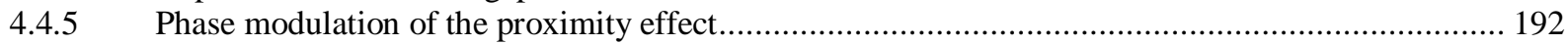

4.5 SNS systems viewed as scattering structures ........................................................193

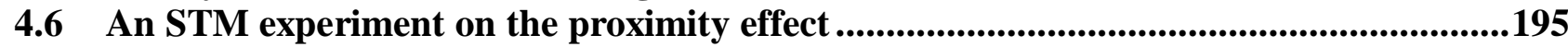

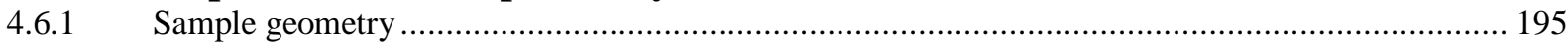

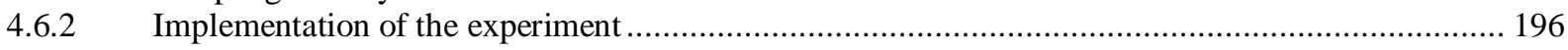

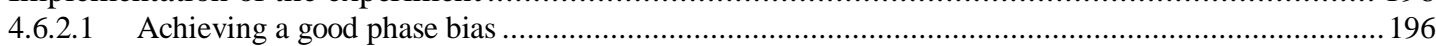

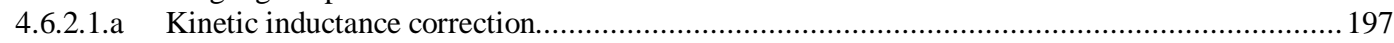

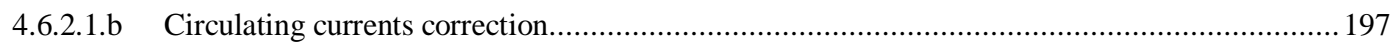

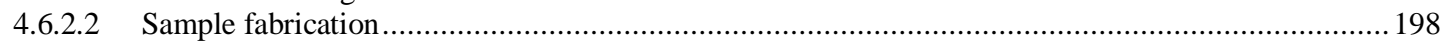

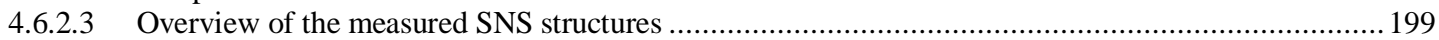

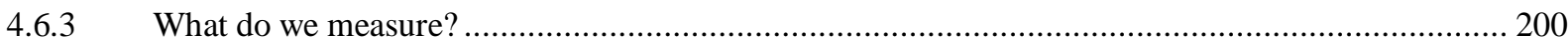

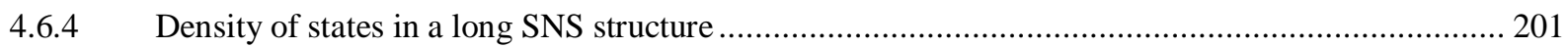

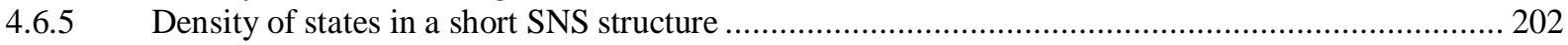

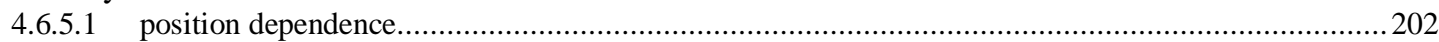

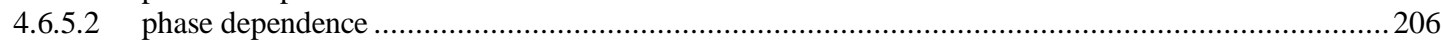

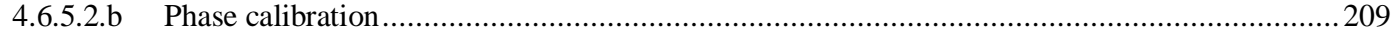

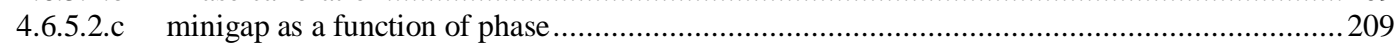

4.6.5.3 Is the Al electrode affected by the applied field?................................................................... 210

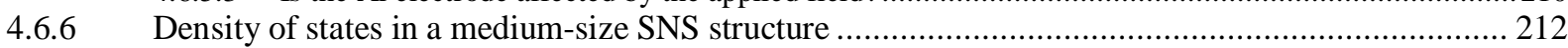

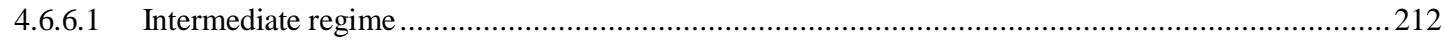

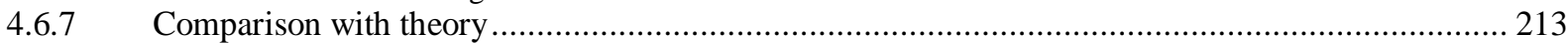

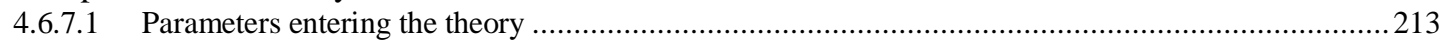

4.6.7.2 Comparison with theoretical predictions ...................................................................................22 216

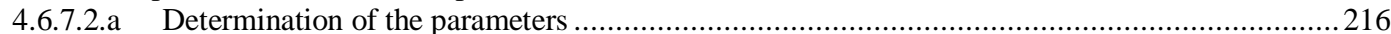

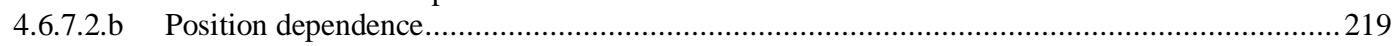

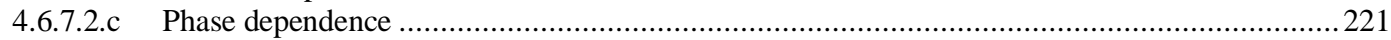

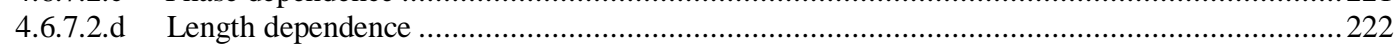

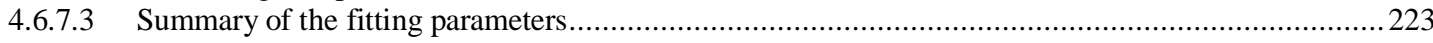

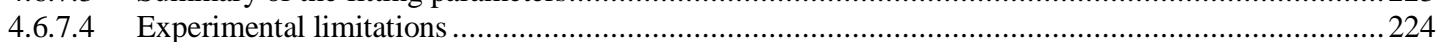




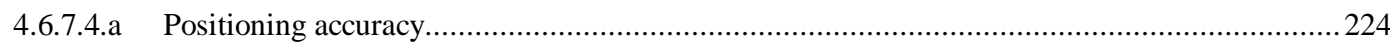

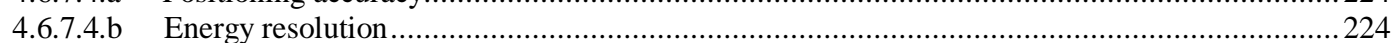

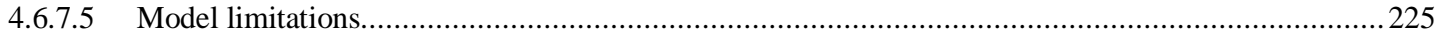

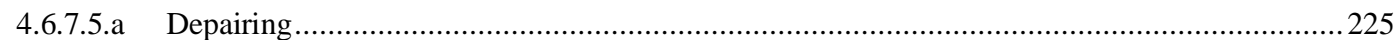

4.6.7.5.b Self consistent treatment of the proximity effect..........................................................225

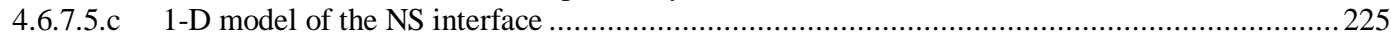

4.6.7.5.d Transverse extension, sample geometry …................................................................22

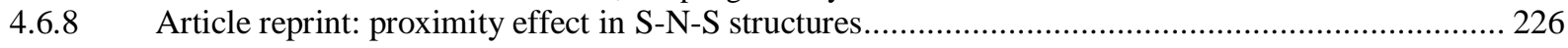

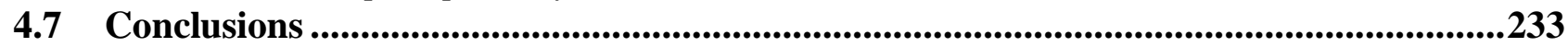

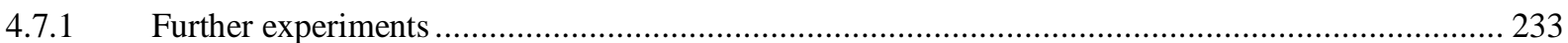

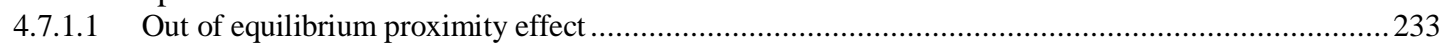

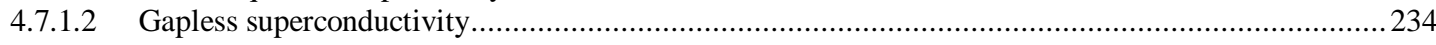


Chapter 1

GENERAL

INTRODUCTION 
The ability to combine in a given nanostructure materials with different electronic properties such as normal metal conductors, magnetic or superconducting electrodes, conductors of reduced dimensionality, single molecules,... has opened new perspectives in mesoscopic physics and nanophysics. The operation of field-effect transistors based on single gated carbon nanotubes [1], and the production of spintronic devices in which the orientation of a single nano-size magnetic domain controls (or is controlled by) an electrical current [2], exemplify the progress achieved during the recent years. A wide range of electronic phenomena have been found in the numerous combinations of materials explored: superconductivity in nanostructures made of both superconducting and non-superconducting materials, Kondo effect in quantum dots connected to bulk Fermi reservoirs [3], spin and charge transport in spintronic devices... Understanding the behaviour of such heterogeneous nanostructures with competing electronic orders remains a major challenge in mesoscopic physics.

At an interface between two different materials, variations of the electronic properties often occur on short length-scales. Local probing of electronic spectroscopy, with a good spatial resolution, is therefore necessary. Besides, such local spectroscopic measurements are often more direct tests of microscopic models of the system than transport measurements are.

Moreover, the energy range over which these effects appear is often quite small (typically $\sim 1 \mathrm{meV}$ and down to a few $10 \mu \mathrm{eV}$ ), compared to, for instance, the thermal energy at room temperature $\left(\mathrm{k}_{\mathrm{B}} \mathrm{T} \sim 30 \mathrm{meV}\right)$. It is thus mandatory to cool the experiments down to very low temperatures, in the millikelvin range, first to make these phenomena observable, as well as to measure them without thermal blurring.

Many different kinds of spectroscopies using electron, ion or photon beams, such as Electron Energy Loss Spectroscopy or photo-emission, can be used to perform a local electronic characterisation. However, their spatial resolution is often poor, and the probed energy range ( $\mathrm{eV}$ or higher) is larger than the relevant one for mesoscopic physics. In order to obtain spatially resolved information, and to probe small energies, various types of scanning probe microscopies have been developed. Two main operation modes, electronic or optical, can be used to probe either electronic or electro-magnetic spectroscopies. In the electronic mode, a very sharp conducting tip is scanned above the sample surface, while the conductance between the tip and the sample is recorded. This method used in the low conductance regime measures the ability to inject electrons from the tip to the sample. It is called "tunneling spectroscopy". In the optical mode, an optical fiber with a very narrow apex is used to collect optical photons from the sample surface, to probe for instance the electromagnetic density of 
states [4]. Combined electronic-optical systems operating both in excitation and detection modes are also feasible $[5,6]$.

The aim of this thesis work was precisely to design, fabricate and operate an apparatus able to probe the electronic ordering in isolated conducting nanostructures using tunneling spectroscopy, in the low energy range suitable for mesoscopic physics.

For this purpose, a microscope with both imaging and local electron spectroscopy abilities was built and mounted in a dilution refrigerator. 


\subsection{Tunneling spectroscopy}

Tunneling spectroscopy was experimentally introduced in 1960 by Giaever in the particular case of tunneling of quasiparticles between a superconductor and a normal metal, using planar junctions [7]. In 1981, Binnig and Rohrer developed the Scanning Tunneling Microscope, providing condensed matter physics with a tool able to perform spatially resolved electronic spectroscopy [8].

Although the basic theory of tunneling dates back to the early days of quantum mechanics [9], the interpretation of most tunneling measurements relies on the tunneling-Hamiltonian formalism introduced in the early 1960s, in particular by Bardeen [10, 11], and by Cohen, Falicov and Philips [12]. In this framework, two independent systems are linked by a Hamiltonian of the form

$$
H_{T}=\sum_{a, b} T_{a, b} c_{a}^{\dagger} c_{b}+\text { h.c. }
$$

which transfers single electrons between states in electrode $a$ and states in electrode $b$, and where $c^{+}$and $c$ are the creation and annihilation operators of single particle states in the electrodes, and $T_{a, b}=\frac{\hbar^{2}}{2 m} \int \Psi_{b}(r) \Psi_{a}^{*}(r) d r$ is the tunneling matrix element between $a$ states and $b$ states $^{1}$.

In the case of tunneling from a sharp STM tip, one expects the tunneling process to be essentially local, limited to the close vicinity of the tip apex. Indeed, Tersoff and Hammann [13] have shown that, assuming the tip apex can be modelled by a sphere, the tunneling matrix elements $T_{a, b}$ are such that

$$
\left|T_{a, b}\right|^{2} \propto\left|\Psi_{b}\left(r_{c}\right)\right|^{2}
$$

where $\Psi_{b}\left(r_{c}\right)$ is the value of the sample single-particle wave function $\mathrm{b}$ at the center $\left(r_{c}\right)$ of the tip apex. For the sake of simplicity, the contact point between tip and sample is assimilated to $r_{c}$.

From this result, one easily derives a perturbative expression for the tunneling current within the linear response theory, involving only quantities in the electrodes at $r_{\mathrm{c}}$ [13]:

\footnotetext{
${ }^{1}$ We neglect here the possible coupling of tunneling electrons to collective electromagnetic or phonon modes which would give rise to inelastic processes that modify the tunneling current. This is generally valid when dealing with "good" metallic electrodes having low electromagnetic impedance, and at voltages below a few $\mathrm{mV}$.
} 


$$
I(V)=\frac{4 e^{2}|\tau|^{2}}{\hbar} \int d \varepsilon\left[f_{\text {tip }}(\varepsilon-e V)-f_{\text {sample }}\left(r_{c}, \varepsilon\right)\right] \rho_{\text {tip }}(\varepsilon-e V) \rho_{\text {sample }}\left(r_{c}, \varepsilon\right),
$$

where $\rho_{\text {tip,sample }}$ denote the local electronic densities of states (LDOS) in the tip, and in the sample at the contact point $r_{c}$ and $f_{\text {tip,sample }}(\varepsilon)$ are the local energy distribution functions - or occupation number of electronic states - in the electrodes (which reduce to Fermi functions at thermal equilibrium). $|\tau|$ is the average value of the tunneling matrix elements $\left|T_{a, b}\right|$.

Expression (1.3) can be easily understood in terms of a balance of tunneling events in both directions from occupied to empty states. It is the usual basis for analysing local tunneling spectroscopy experiments and it is worth noticing and stressing that, to date, most STM spectroscopic measurements have been successfully and often accurately analysed within this rather simple Tersoff and Hammann picture. Thus, deviations from this description due to tip shape effects or tip-sample interactions are generally negligible.

As we will now show, under favourable conditions, one can disentangle the factors contributing in (1.3), thereby gaining knowledge of local spectral functions in the electrodes. The case when both tip and sample are Fermi liquids with arbitrary densities of states is depicted in Fig. 1.1, where it is further assumed that both electrodes are at thermal equilibrium.

When one wants to probe the LDOS in the sample, one uses a tip where the density of states can be assumed as constant over the probed relevant energy range (see Fig. 1.2). This is the case for normal-metal tips, in the energy range suitable for mesoscopic physics (meV). The LDOS plays a major role in the knowledge of physical properties of the system. It is formally amenable to calculation once the Hamiltonian of the system is known; for instance it can be expressed as a retarded Green function for electrons.

In order to determine the local energy distribution function in a sample, one rather uses a superconducting tip ${ }^{1}$. In this case the sharp BCS singularity [14] of the tip DOS probes the energy distribution discontinuities. In this mode, out-of-equilibrium situations can be probed, giving access to fast dynamics inside the structure. Out-of-equilibrium distribution functions can in principle be calculated from Green functions, but here within the Keldysh formalism.

\footnotetext{
${ }^{1}$ It is possible to access energy distribution of electrons using a normal tip in a high impedance environment, using the Zero Bias Anomaly (ZBA) induced in the density of states of the tip by dynamical coulomb blockade [15]. However, the ZBA has a less sharp singularity, compared to the BCS singularity (which is nearly a delta peak) and is thus a less efficient probe.
} 


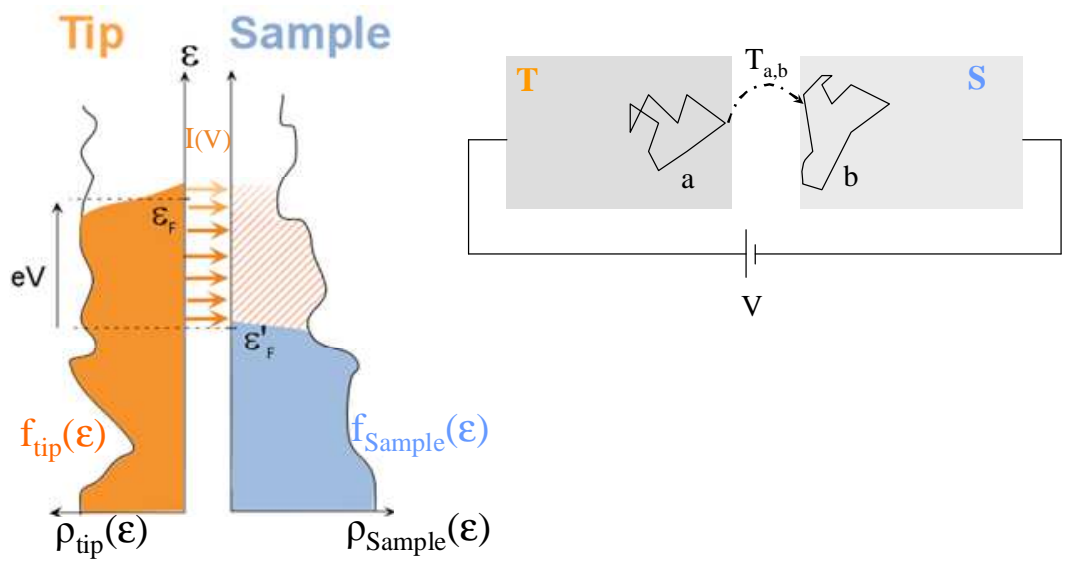

Fig. 1.1 On the left are shown side to side the densities of states of arbitrary probe $\left(\rho_{\text {tip }}\right)$ and sample $\left(\rho_{\text {sample }}\right)$ as a function of energy (vertical axis). The filling of states (f between 0 and 1) is represented in plain color (orange for the tip, blue for the sample). Fermi levels are shifted by the voltage bias applied to the junction. The current flowing through a tunnel junction between the tip and the sample involves the convolution product of spectral functions $\rho$ and $\mathrm{f}$ of the electrodes (see expression (1.3)). The right panel exemplifies tunneling from a state $\mathrm{a}$ in the tip into a state $\mathrm{b}$ of the sample with a coupling $\mathrm{T}_{\mathrm{a}, \mathrm{b}}$.

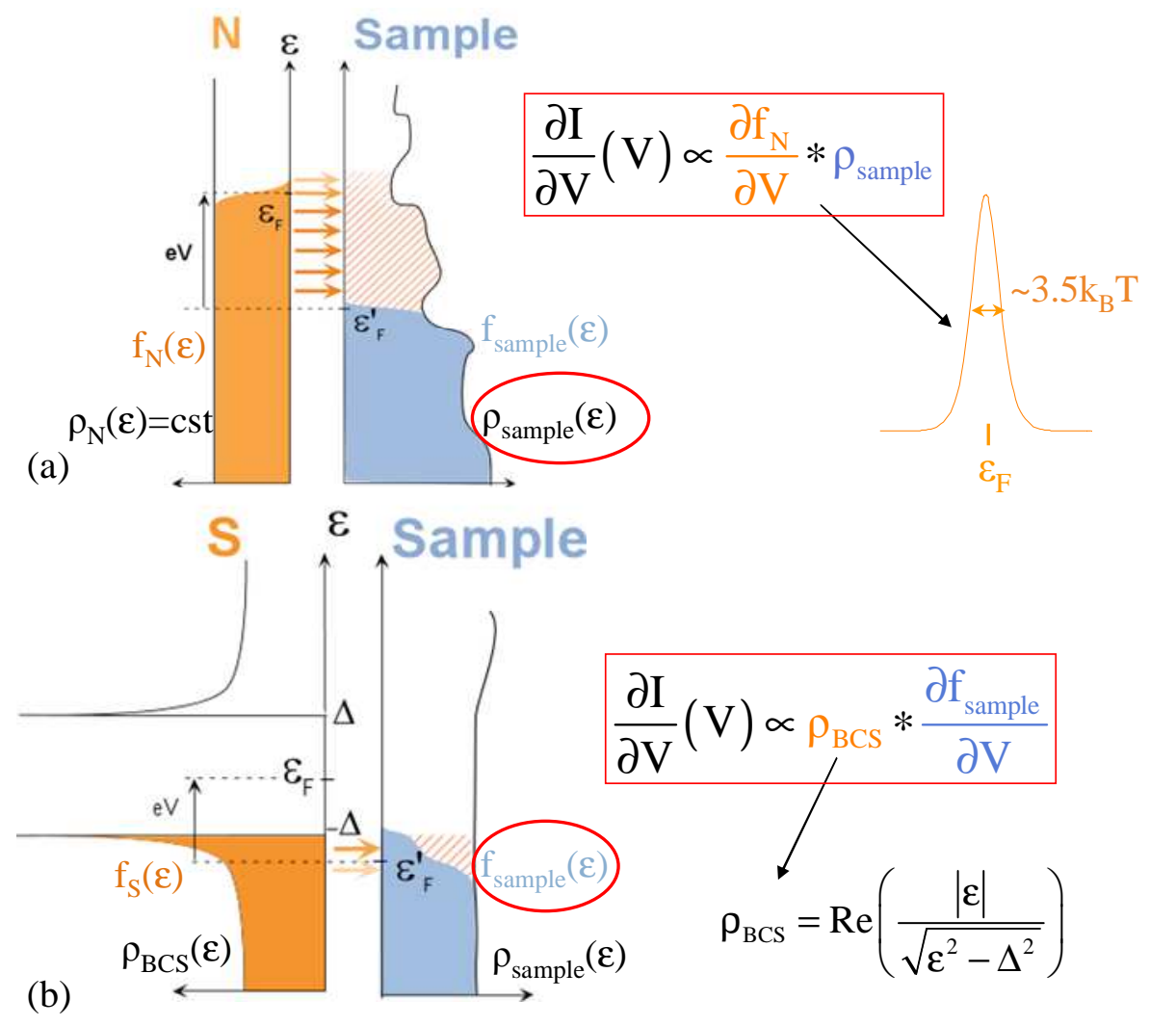

Fig. 1.2 Tunneling spectroscopy with normal metal (a), and with superconducting tips (b). (a) When $\rho_{\text {tip }}(\varepsilon)$ is featureless (normal metal), the differential conductance, which is the derivative of current with respect to applied voltage, gives direct access to the local density of states in the sample, with a maximal resolution given by the sharpness of the Fermi-Dirac function, involving the temperature $\mathrm{T}$ in the tip (note that temperatures are not necessarily equal). (b) With a superconducting tip, tunneling gives access to the energy distribution function in the sample, provided $\rho_{\text {sample }}(\varepsilon)$ is constant at Fermi level, using a deconvolution procedure. Experimental techniques about the DOS measurement are discussed in chapter 3. 


\subsection{Spatially resolved measurement of the LDOS with a combined AFM-STM}

Scanning Tunneling Microscopes have been exploited for spectroscopy purposes since the very beginning. The visualization by $\mathrm{D}$. Eigler et al. of a quantum corral for electronic surface states delimited by atoms added on a crystalline surface is one famous example [16, 17]. Despite its obvious interest, scanning tunneling spectroscopy has however not been much used to probe nanostructures. There are two main reasons for this:

- $\quad$ Circuits are designed on insulator:

Most of nanostructures are fabricated on an insulating substrate, on which an STM cannot be operated: as soon as the tip hovers on an insulating part, tunneling current drops to zero, just as if it were far from the sample surface. Therefore, the feedback mechanism lowers the tip to reach the target tunnel current, unavoidably resulting in a "tip crash" most dreaded by all STM users. In order to perform local probe measurements on such samples, one needs to resort to another type of local probe. Atomic Force Microscopy (AFM) is the most obvious choice since it has topographic imaging capability with high spatial resolution, quite independently of the sample properties, in particular of its conductivity.

\section{- $\quad$ Circuits are small compared to the chip:}

On a given sample of macroscopic size, there is often one or a few nanostructures connected to the outside by large bonding pads. Fig. 1.3 shows as an example one of the samples measured during this thesis work. The area of interest has a characteristic size of a few microns, located at the center of a $5 \times 5 \mathrm{~mm}^{2}$ chip. Reliably bringing the local probe of a microscope on top of the nanostructure requires precise mechanical alignment capabilities of the local probe with respect to the sample in all three dimensions.

In order to perform spatially resolved tunneling spectroscopy on nanostructures, and to cope with the first point above, we have built a near-field microscope having a combined Atomic Force and Tunneling probe, which can be used in either mode. In AFM mode, topographic images of the sample can be obtained whatever its electrical properties. The STM mode is primarily used to measure LDOS at desired locations. This dual mode sensor is shown in Fig. 1.4, and will be described in chapter 2. It is based on a tuning fork normally used as the time-base resonator in wristwatches. Such a tuning fork resonator is made of quartz, a 
piezoelectric material, which allows easy, low power, electrical excitation and detection of its mechanical resonance $(\sim 32 \mathrm{kHz})$ and is thus well adapted for very low temperature experiments. This tuning fork is equipped with a metallic probe tip attached to one arm. The attractive or repulsive interaction between the tip and the sample surface affects the tuning fork resonance. A feedback system regulating the tip-to-sample distance is used to maintain a constant target resonance frequency while the tip scans the sample surface. This way, one can form topographic images of the sample surface. Once the area of interest is located, switching to the STM mode, and performing tunneling spectroscopy at precise locations is then possible without endangering the tip. The small wire connected to the tip carries the tunnel current, and allows tip biasing with respect to sample.

To cope with the second point, we incorporated in our microscope means of precisely displace the sensor with respect to the sample in all three directions of space over large distances (several $\mathrm{mm}$ ). This motion is performed by piezoelectric actuators based on stick-slip motion. To compensate for the lack of reproducibility of these actuators, and since operation at very low temperatures does not allow visual access, we fitted our microscope with position sensors. We further designed the samples so as to help us rapidly locate the nanostructure of interest. All these features are described in details in chapters 2 and 3. 


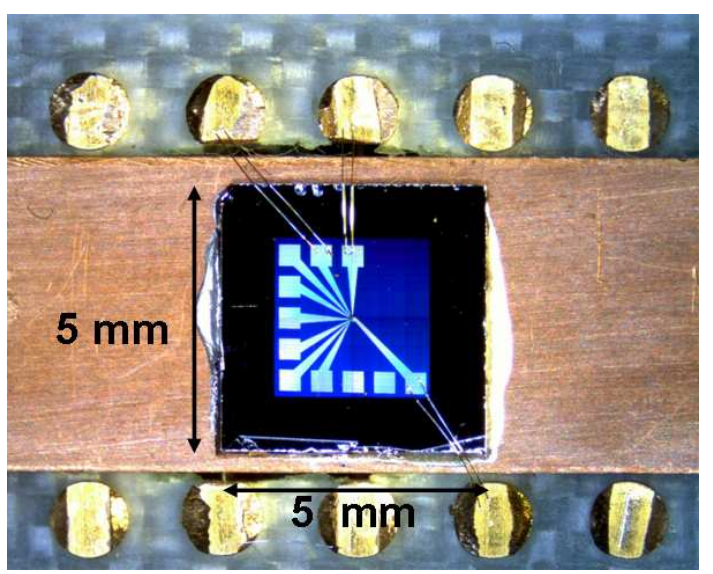

Fig. 1.3 A typical sample made on a $5 \mathrm{~mm}$ chip by standard e-beam lithography and metal deposition, is glued by silver paint on a sample holder, and bonded to contacts for transport measurements. The Moiré patterns that can be seen on a $3 \mathrm{~mm}$ field in the center of the sample are position encoding marks for the microscope. For the description of the encoding see chapter 3.

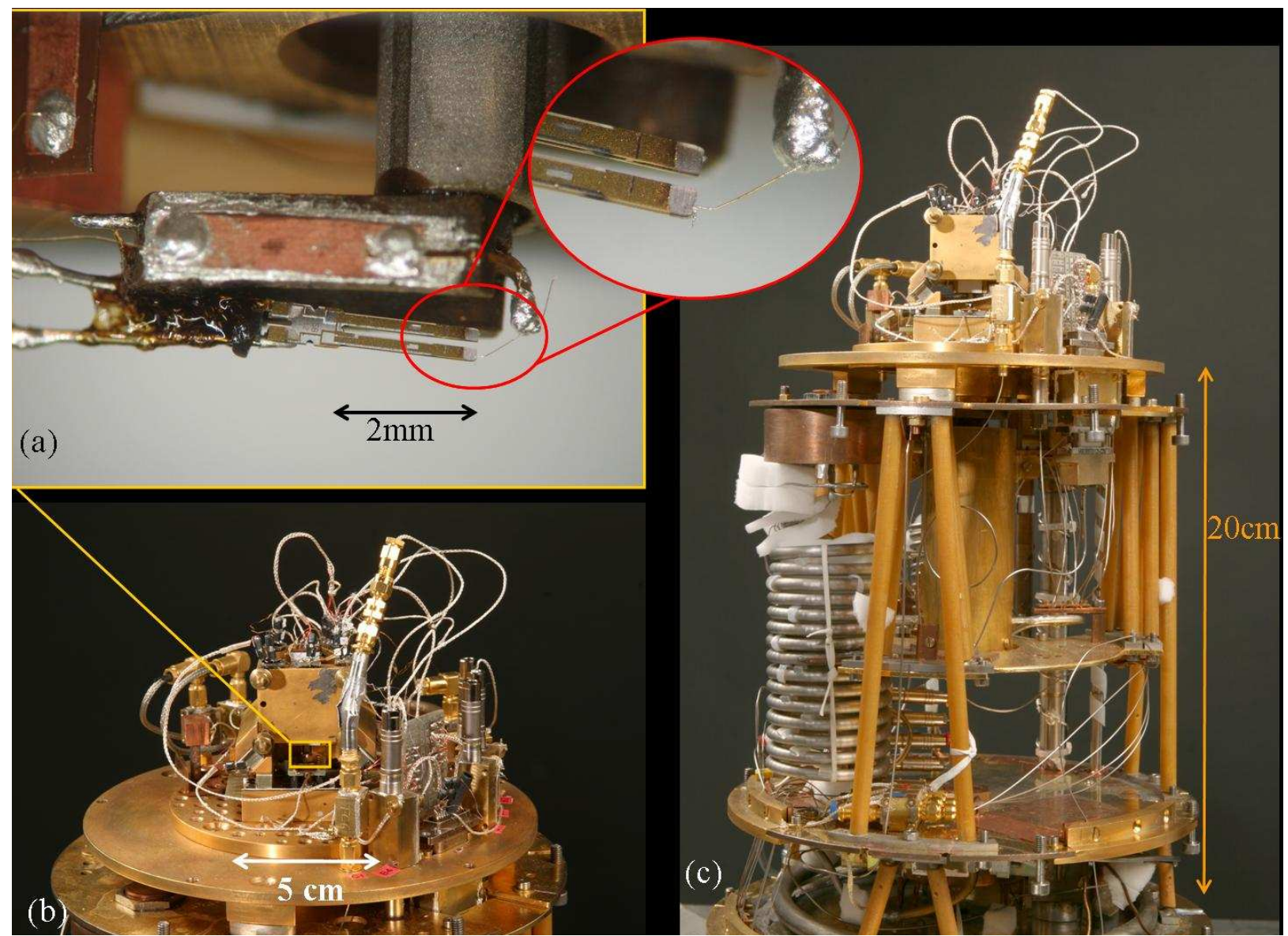

Fig. 1.4 (a) Picture of the combined AFM-STM probe, attached to the end of a piezoelectric scanning tube. A sharp metallic tip (inset) is glued at the end of the lower arm of the tuning fork. The fork senses the interaction between tip and sample, which allows to image topography with a precision mainly limited by the shape of the tip apex. The tip is electrically connected by a thin wire to a filtered measuring line for performing tunneling spectroscopy. (b) Picture of the microscope mounted and wired on the top plate of an inverted dilution refrigerator. (c) Ensemble view of the dilution refrigerator, model SIONLUDI designed by A. Benoit from the CNRS (Grenoble). This fridge cools the experiment down to $30 \mathrm{mK}$ in about 8 hours. 


\subsection{An AFM-STM in a table-top dilution refrigerator}

Mesoscopic physics experiments are often performed at low temperature since the characteristic energy scales of the investigated phenomena are generally small: for instance, for pure metals superconductivity occurs only below a critical temperature of the order of $1-10 \mathrm{~K}$. Moreover, electron coherence effects tend to disappear as temperature rises, due to incoherent scattering by phonons... In general, a temperature higher than the relevant energy scale blurs the measurement: as explained in Fig. 1.2, the energy resolution of tunneling spectroscopy is ultimately limited by the temperature at which the experiment is performed.

However, to reach this ultimate limit, or even approach it, it is not enough to cool the sample and the microscope. It is also necessary to carefully thermalize and filter all the lines connected to the sample so that not only the external noise (either noise from roomtemperature apparatus, or ambient noise), but also the Johnson-Nyquist noise arising from all dissipative elements placed in these lines at temperatures higher than the sample temperature, are suitably attenuated.

For these reasons, the microscope was placed in thermal contact with the mixing chamber of a dilution refrigerator with a base temperature of about $30 \mathrm{mK}$ and the wiring of the experiment was carefully designed so as to benefit from the low operating temperature. In particular, special filters were designed, fabricated and installed on every line, and a new type of tunnel current preamplifier was designed. The fabrication and setup of the full apparatus is described in chapter 3 .

We use a table-top dilution refrigerator, developed by Alain Benoit from the Centre de Recherche sur les Très Basses Températures (CRTBT), and which has the advantage of being of comfortable use and suitable for a microscope such as ours. All leak-tight seals are situated at room temperature, and the whole fridge and the experiment are placed inside the same vacuum chamber. This fridge is not surrounded by a cryostat filled with liquid helium, but it operates with circulating liquid helium fed from a slightly pressurized storage Dewar placed underneath. Although a couple of groups are successfully using this type of dilution refrigerator with STMs, we found our own fridge, which was actually fabricated by Air Liquide, to be plagued by internal vibrations, presently forbidding normal tunnel vacuum STM operation. We could nevertheless perform very stable tunneling spectroscopy by bringing the tip in mechanical contact with the sample, through a thin insulating layer grown on the sample surface. 


\subsection{Benchmarking tunneling spectroscopy}

A convenient benchmark for tunneling spectroscopy is the measurement of the DOS of a BCS superconductor with a normal metal tip, as shown in Fig. 1.2. Ideally, the measured conductance of a N-S tunnel junction should be the convolution of the BCS DOS [14]

$$
\rho_{B C S}(\varepsilon)=\operatorname{Re} \frac{|\varepsilon|}{\sqrt{\varepsilon^{2}-\Delta^{2}}},
$$

where $\Delta$ is the superconducting gap, with the derivative of the Fermi function in the tip at a temperature given by the thermometer.

In practice, it turns out that in most very low temperature experiments one can only account for the measurements by assuming that the energy distribution function in the tip is at an effective temperature higher than that of the refrigerator. Such a discrepancy is usually attributed to an imperfect filtering of the electric signals. The practical energy resolution achieved in such experiments is then determined by this effective temperature.

In order to test the performance of our setup, we measured the DOS of a thin aluminum film (thickness: $25 \mathrm{~nm}$ ), with a chemically etched tungsten tip (see fabrication process in chapter 3). The differential conductance curve, shown in Fig. 1.5 was taken at a fridge temperature of $40 \mathrm{mK}$. The data are best fitted using the BCS density of states for aluminum (with a gap energy $187 \mu \mathrm{eV}$ ), and a Fermi distribution function of $50 \mathrm{mK}$ for the electrons in the tip. This result already demonstrates that the electrons in the tip are fairly well thermalized, and that not much extra noise is present.

This result presently displays the best effective temperature ever obtained in STM tunneling spectroscopy. Although the effective temperature is still higher than the thermometer temperature, this result validates our work on wiring, filtering and instrumentation (Chap. 3). 


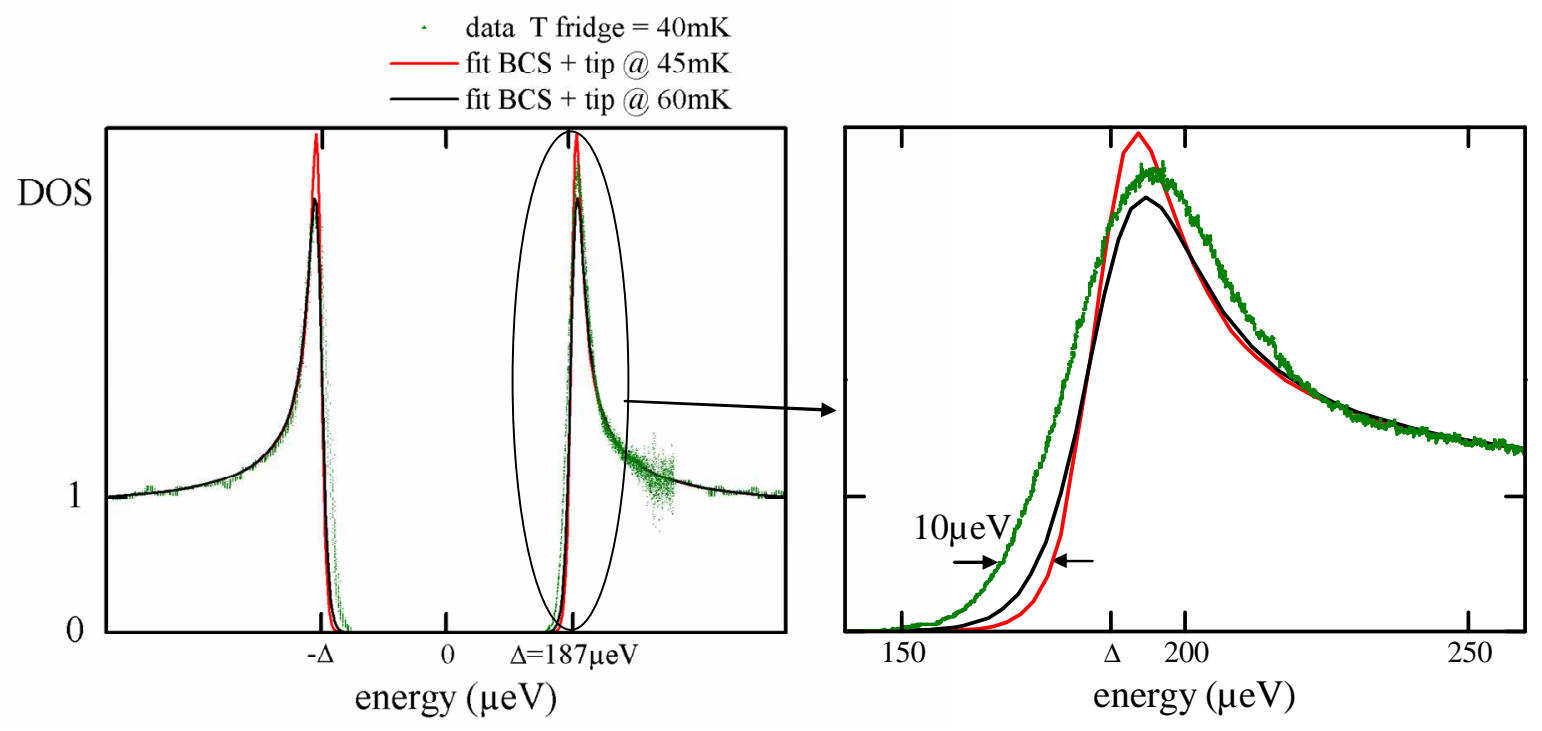

Fig. 1.5 First measurement of the tunneling density of states performed at low temperature ( $40 \mathrm{mK})$ on an aluminum film with a tungsten tip. The data (green points) are fitted using the BCS density of states with a superconducting gap $\Delta=187 \mu \mathrm{eV}$ for the sample, and an electronic temperature of $45 \mathrm{mK}$ (red curve) and $60 \mathrm{mK}$ (black curve) for the electron energy distribution in the tip, with no other adjustable parameter. The best fits are obtained for an effective temperature in the tip around $50 \mathrm{mK}$. The corresponding energy resolution for the density of states measurement is about $15 \mu \mathrm{eV}$. Right panel shows a zoom on the peak. The discrepancy between fit and data cannot be explained for a standard BCS-normal junction, even by inputting a higher effective temperature, or by introducing a depairing parameter; it is not presently understood. 


\subsection{An experiment on the proximity effect in $\mathrm{S}-\mathrm{N}-\mathrm{S}$ structures}

Spatially resolved tunneling spectroscopy is the ideal method to investigate the various kinds of proximity effects that develop at interfaces between materials having different electronic orderings. The superconducting proximity effect occurring at an interface between a superconducting material $(\mathrm{S})$ and a non-superconducting one $(\mathrm{N})$ is one good example. It manifests itself as the $\mathrm{N}$ side acquiring some superconducting properties, and a weakening of superconductivity on the $\mathrm{S}$ side. It can be described microscopically using Andreev-Saint James $([18,19])$ reflection processes in which an electron with a given spin coming from the $\mathrm{N}$ side onto the interface at a sub-gap energy is retro-reflected as a hole of opposite spin (the electron time-reversed particle), resulting in a Cooper pair being transferred to the superconductor's condensate (see Fig. 1.6). Proximity induced superconductivity is quite different from bulk superconductivity: for instance, the conductance of an $\mathrm{N}$ wire in contact at one end with an $\mathrm{S}$ electrode is unchanged at zero temperature despite the exclusion of the electric field in the $\mathrm{N}$ side close to the interface [20]; on the contrary, transport of Cooper pairs can develop in short enough SNS nanostructures, yielding a zero resistance state for the normal region $([21,22])$.

\section{Superconductor}

\section{Normal metal}

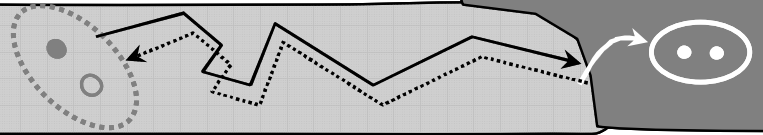

Fig. 1.6 At an interface between a superconductor and a normal metal, Andreev reflection processes take place. They account for the coupling between time-reversed states of quasiparticles propagating in the normal metal due to a pairing potential in the superconductor. Andreev reflection allows as well to describe supercurrent flow through a piece of normal metal connected at both ends to a superconductor.

Prior to this thesis work, superconducting proximity effect had been extensively investigated, both experimentally and theoretically. For reviews on this field see [23] and [24].

The theory for superconducting proximity effect is based on the de Gennes microscopic theory for inhomogeneous superconductivity which allows to treat consistently a space- 
dependent mean-field coupling between time-reversed electrons in an arbitrary structure [25]. Eilenberger showed how these equations could be more conveniently handled under a quasiclassical form, in which one focuses only on the variations of the (quasiclassical) Green functions of the electron-hole pairs at the scale of the so-called superconducting coherence length $\xi=\hbar v_{F} / \Delta$, much larger than the Fermi wavelength $\lambda_{F}[26]$. Here, $v_{F}$ denotes the Fermi velocity and $\Delta$ the superconducting gap of the bulk superconductor ${ }^{1}$. When one considers the generally relevant experimental case of diffusive conductors having a mean free path $\ell \ll \xi$, one can introduce further approximations for the behavior of the quasiclassical Green functions leading to the Usadel equations which are much easier to handle than Eilenberger's [27]. The Usadel equations are the diffusion differential equations for the Green functions in heterogeneous superconductors. Most of the theoretical results on proximity superconducting structures have been obtained within this framework ${ }^{2}$.

Predicting the behavior of a diffusive proximity effect structure amounts to solving the Usadel differential equations for the given sample geometry. The outcome of these equations is directly the space dependent field of Green functions. In particular, the real part of the retarded Green function is nothing else than the LDOS in the structure. Thus, measuring the LDOS in a superconducting proximity structure with our microscope is a means of directly and thoroughly testing the basic predictions of the quasiclassical theory of superconductivity. Within this theoretical framework, several generic features of the diffusive NS structures are known. In particular, it predicts that in an $\mathrm{N}$ wire of finite length $\mathrm{L}$ in good contact at one end with a superconducting electrode, a reduced superconducting gap with no available quasiparticle states, the so-called minigap, develops in the LDOS with a uniform value everywhere in the wire. This minigap is related to the characteristic time for diffusion $\tau_{D}=L^{2} / D$ through the whole wire, or, equivalently, is proportional to the Thouless energy $\hbar / \tau_{D}$, where $D$ is the diffusion constant. Note however that while the minigap is expected to be uniform, the detailed shape of the full DOS is space dependent. In the case where the wire is connected at both ends to superconducting electrodes, the LDOS is predicted to be a $2 \pi$ periodic function of the superconducting phase difference $\delta$ between the two superconductors, with the minigap being maximum at $\delta=0$ and suppressed at $\delta=\pi$.

\footnotetext{
${ }^{1}$ This definition for $\xi$ is valid in the ballistic regime. In the diffusive limit it becomes $\xi=\sqrt{\frac{\hbar D}{\Delta}}$.

${ }^{2}$ Some results on proximity effect can be derived within the scattering formalism [28] and using the random matrix theory for some classes of structures.
} 
Most experiments performed up to now on superconducting proximity effect consisted in transport measurements, for which comparison with theory implies integration over position, in contrast to LDOS measurement. Still, in some experiments the tunneling LDOS in NS proximity structures was measured [30], and STMs were already used for this on entirely conducting samples $([31,32])$. These measurements were generally found in good agreement with the predictions. However, none of these previous experiments investigated the phase dependence of the LDOS in a superconducting proximity structure.

With our instrument, it was possible to investigate the position and phase dependences of the LDOS in a superconducting proximity structure. The obtained results were assessed with the corresponding predictions of the Usadel equations.

In order to perform this test, we measured the LDOS along silver wires connected on both sides to a U-shaped superconducting Aluminum electrode. This way, by applying a magnetic flux $\Phi$ through the loop (see Fig. 1.7) on can control the phase difference $\delta$ across the wire according to:

$$
\delta=2 \pi \frac{\Phi}{\Phi_{0}}, \text { with } \Phi_{0}=\frac{h}{2 e} \text { the superconducting flux quantum. }
$$

Additionally, a set of normal wires of different lengths were fabricated on the same chip under the same conditions, thus keeping all other parameters (e.g. diffusion constant, N-S interfaces transparency, layer thickness etc) constant.

Our instrument is convenient to test such a geometric dependence, because it can easily probe multiple devices on the same chip, whereas in a transport measurement, this would imply connecting 2 to 4 times as many wires as devices to test, which rapidly becomes impractical.

The LDOS was measured at different positions in a normal wire of $300 \mathrm{~nm}$ (see Fig. 1.7). We found the presence of a minigap independent of position both on the normal and the superconducting sides, an essential feature predicted for proximity effect. In the normal region, (black, orange, and green curves in Fig. 1.7 b), a gap in the density of states of $\sim 50 \mu \mathrm{eV}$ is observed. Conversely, on the superconducting side, states are measured in the energy range between the minigap and the BCS gap (about $170 \mu \mathrm{eV}$ in our sample): states leak from the normal region into the superconductor, at energies below the gap, and above the minigap (compare blue and red curves). The observation of the proximity effect in both the $S$ and the $\mathrm{N}$ sides exemplifies the interest of our apparatus. 

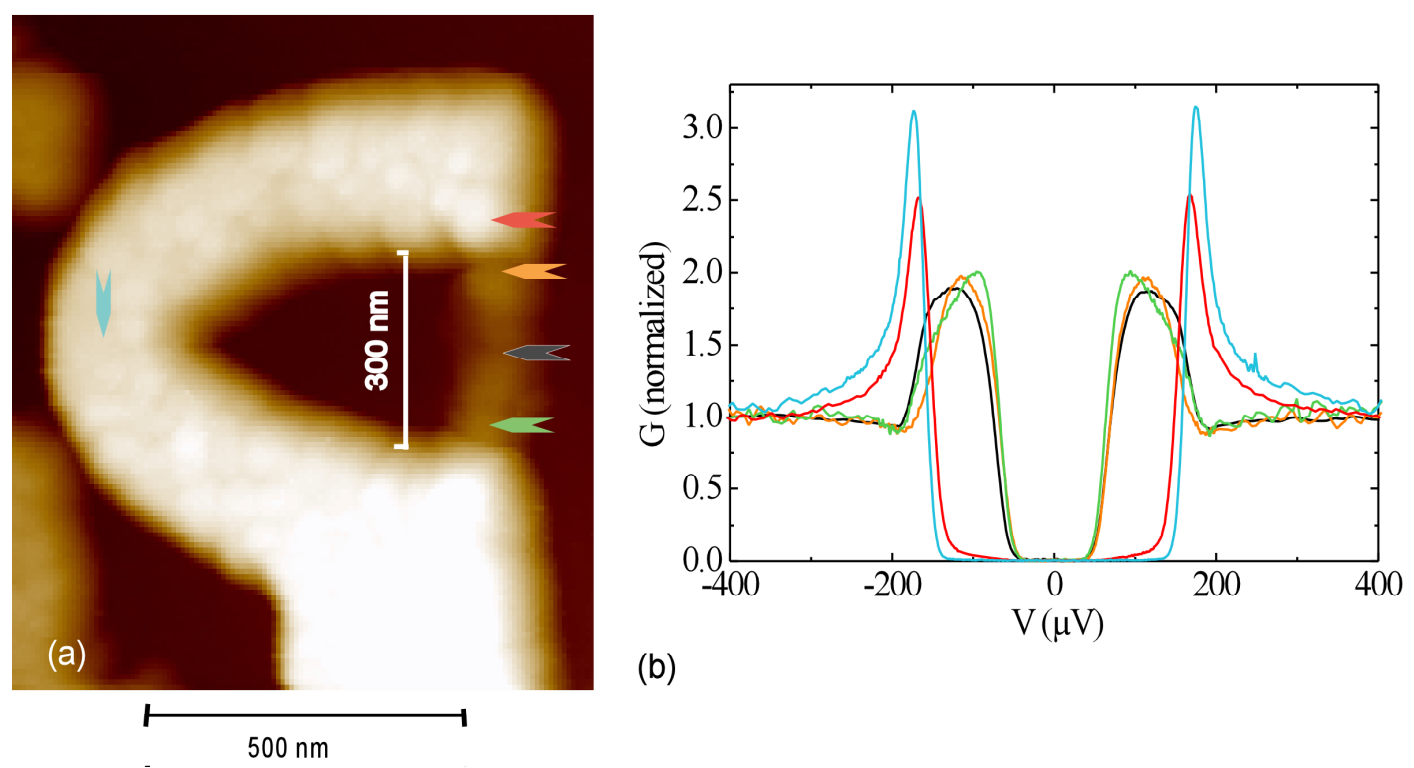

(b)

Fig. 1.7 (a) AFM image taken at $30 \mathrm{mK}$ of a hybrid Normal-Superconductor structure. The U-shaped electrode is made of $60 \mathrm{~nm}$ thick, $200 \mathrm{~nm}$ wide aluminum, and the silver wire inserted in the $U$ is $30 \mathrm{~nm}$ thick, $50 \mathrm{~nm}$ wide, and $300 \mathrm{~nm}$ long. The structure was fabricated on an insulating $\mathrm{Si} / \mathrm{SiO}_{2}$ substrate by standard e-beam lithography, and double-angle evaporation. (b) Density of states measured at various positions in the structure, with zero phase difference between both ends of the normal wire. The positions where the different curves of the graph were measured are indicated on the AFM image by arrows of the same color.

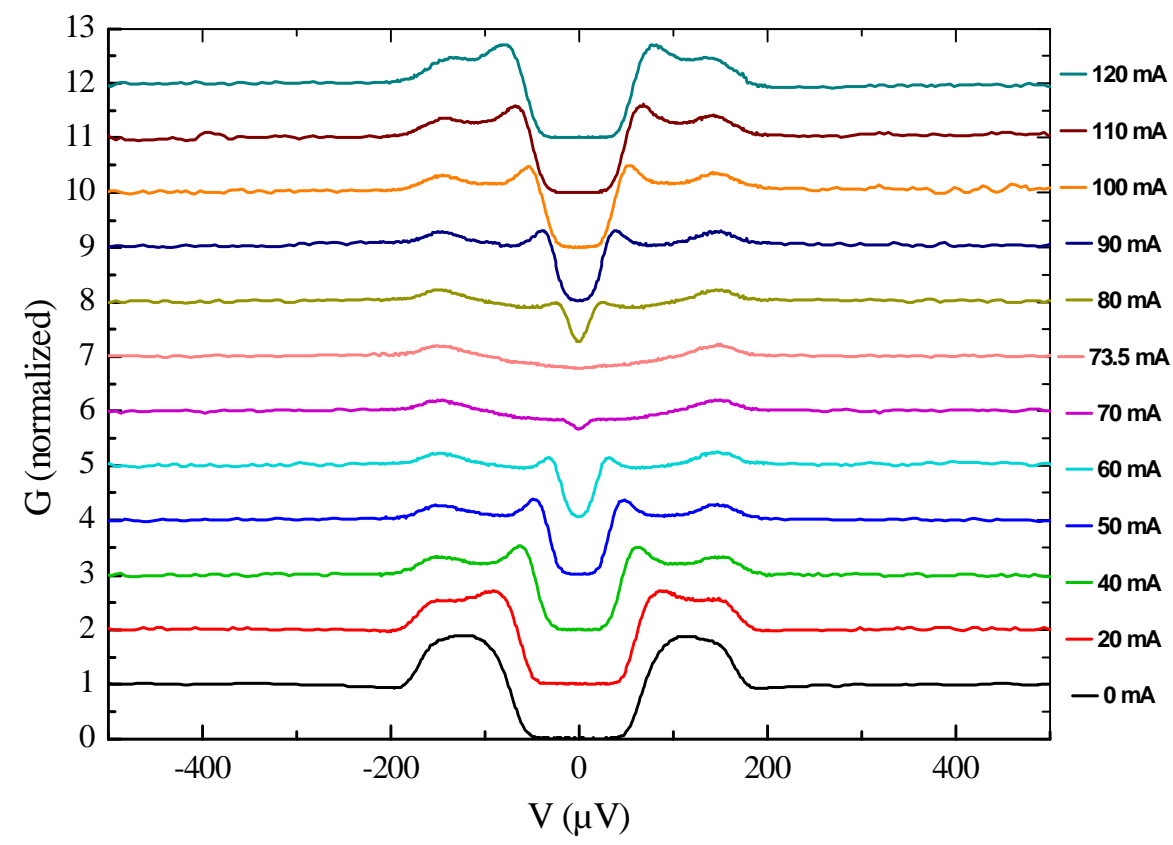

Fig. 1.8 Variations of the LDOS in the silver wire in presence of an applied magnetic field inducing a superconducting phase difference across the wire. Data are taken on the wire shown in Fig. 1.7, at the position labeled in black. Each curve is normalized to the high energy conductance value, and shifted vertically by 1 for clarity. This gives an overview of the closing of the minigap, when phase reaches $\pi$ (Icoil $=73,5 \mathrm{~mA}$ ) and of the overall variations of the DOS. Noticeably, a peak rises on the minigap edge and its position decreases simultaneously with the minigap. A rounder peak appears around $\Delta$, which does not depend on the phase difference. Such features were observed at all positions. 
The phase difference dependence of the LDOS, and of the minigap were also probed. The phase modulation of the LDOS is shown in Fig. 1.8 for the position colored in black on the wire of Fig. 1.7. We indeed clearly observed a periodic modulation of the minigap, with its disappearance at half-integer flux quantum in the loop.

Finally, we have probed the variation of the minigap as a function of the normal wire length. For all wires, the nominal transverse dimensions were the same (width: $50 \mathrm{~nm}$ wide, thickness: $30 \mathrm{~nm}$ thick). A summary of the results obtained at different positions in 4 wires ranging from $300 \mathrm{~nm}$ to $2.7 \mu \mathrm{m}$ is shown in Fig. 1.9.

When the wire is short enough, a minigap independent of position is present throughout the structure, with an energy gap depending on the length of the normal region, When the wire length is longer than the thermal length (equal to $1.8 \mu \mathrm{m}$ at $40 \mathrm{mK}$ with $\mathrm{D}=170 \mathrm{~cm}^{2} / \mathrm{s}$ ), no minigap is observed. This is similar to the case of an infinite normal wire.

These results are discussed in chapter 4 .

We have compared our measurements with the predictions of the Usadel equations. In doing this we have tried to render the essential features observed in the experiment, while keeping a minimum number of adjustable parameters and a numerical problem of reasonable size. We found it a good compromise to settle to a one-dimensional approximation of the SNS structure, taking into account reverse proximity effect, i.e. the modification of the superconducting order parameter close to the interface. As shown in Fig. 1.10, this modeling enables us to reproduce quite well the features observed in the experimental data. This agreement is obtained with only a few adjustable parameters. Indeed, the superconducting gap is known from a direct measurement on aluminum, the position in the wire is known from the AFM picture, and one can only vary the interface transparency (equal for all positions in a wire) and the diffusion constant (equal for all the different wires).

A detailed account for this comparison is given in chapter 4 . 

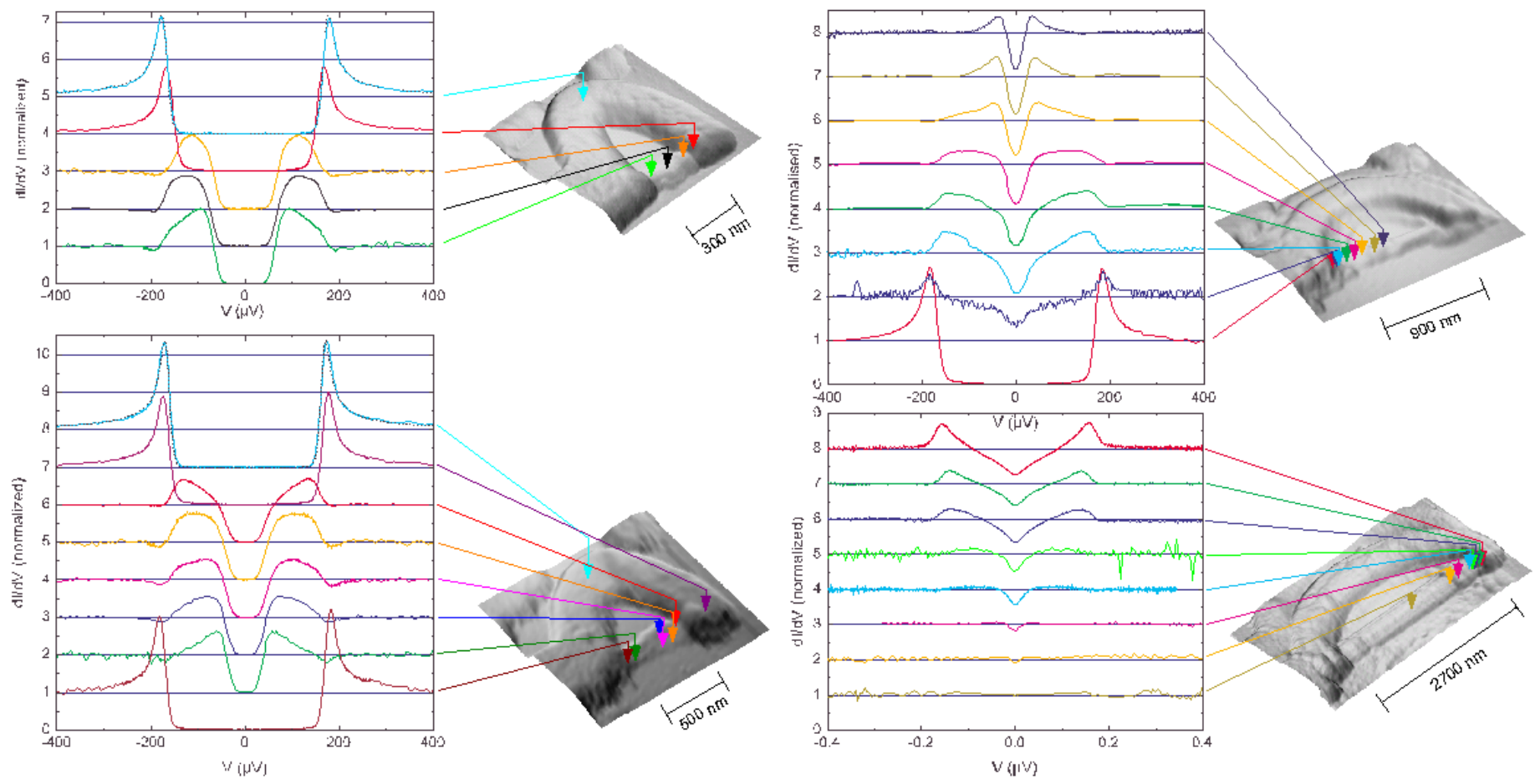

Fig. 1.9 On the same chip were fabricated SNS structures with normal wires of different lengths. Here we show a summary of the results obtained for 4 different structures. In each panel, an AFM picture taken at $40 \mathrm{mK}$ shows the structure on which the LDOS was measured. Each position is marked by a colored arrow corresponding to a curve of the same color. Data are normalized to their high energy conductance and shifted by one for clarity. Top-left: in the shortest $300 \mathrm{~nm}$ wire, the minigap is $\sim 50 \mu \mathrm{eV}$. Bottom-left: in a $500 \mathrm{~nm}$ long wire, the minigap is still clearly observed $(\sim 28 \mu \mathrm{eV})$. Top-right: In the $900 \mathrm{~nm}$ long wire, the predicted minigap of $9 \mu \mathrm{eV}$ is smaller than the energy resolution of $15 \mu \mathrm{eV}$ achieved with electrons at $50 \mathrm{mK}$, which explains why the measured density of states does not fall to zero. Bottom-right: Case of a long wire $(2.7 \mu \mathrm{m})$ : the DOS is modified by proximity effect only close to the interface.
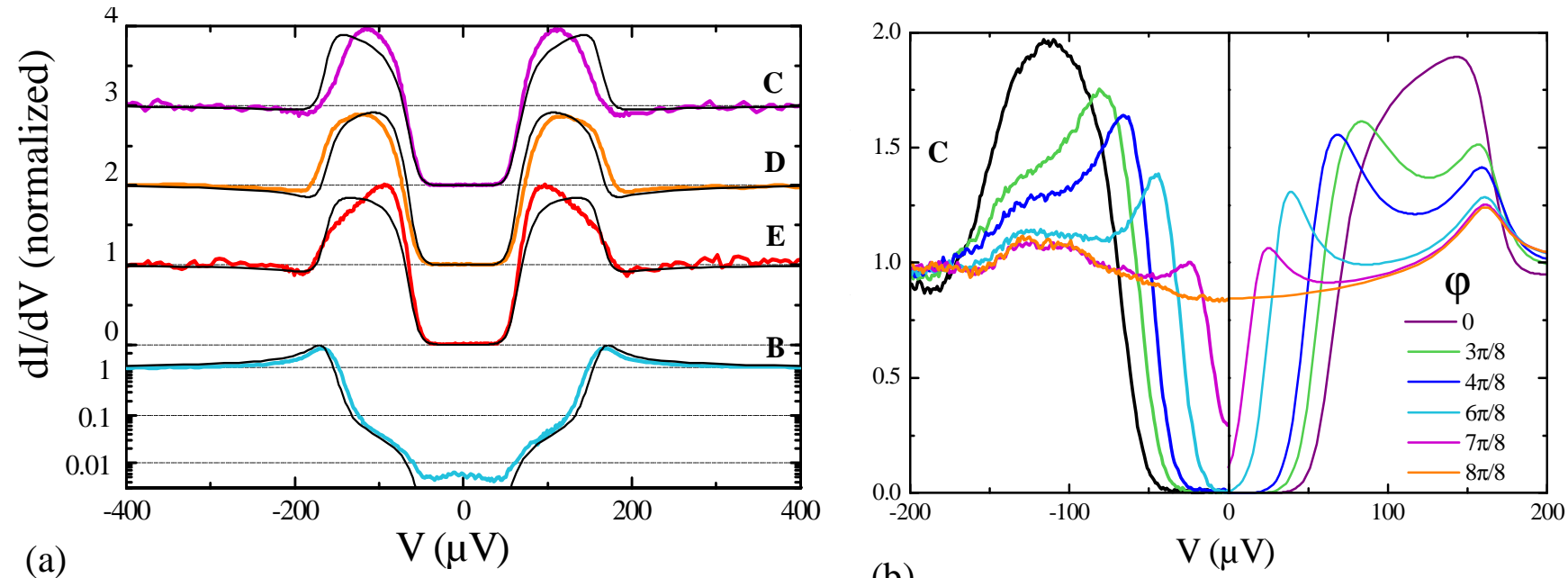

(b)

Fig. 1.10 (a) Comparison between measurements (color) and predictions (black lines) of the quasiclassical theory for the different positions already shown in Fig. 1.7. Data curves are plotted in the same color as in Fig. 1.7. (b) DOS at six different phases at the position labeled in orange on Fig. 1.7 (Left panel: data, right panel: theoretical predictions). The superconducting gap $(170 \mu \mathrm{eV})$, and the positions were measured, while the interfaces parameters, the effective temperature $(60 \mathrm{mK})$ as well as the diffusion constant $\left(170 \mathrm{~cm}^{2} / \mathrm{s}\right)$ were adjusted to obtain a good overall reproduction of all the data. 


\subsection{Perspectives}

The experiment on the superconducting proximity effect demonstrates the ability of our apparatus to probe the LDOS of nanostructures. This method is clearly more versatile than the fabrication of tunnel junctions at all the places where the DOS has to be measured. Thanks to the installed filtering and to the low temperatures, the unprecedented energy resolution of $\sim 15 \mu \mathrm{eV}$ for an STM gives access to many interesting phenomena in mesoscopic physics.

We outline here some possible experiments for which our apparatus would be best adapted. Some of them are already on the bench.

\subsubsection{Proximity effect in ballistic 1D systems}

We have probed the superconducting proximity effect in a diffusive $\mathrm{N}$ wire, which is described by the Usadel formalism. Another simple and poorly explored regime is the case of a ballistic 1D system. Possible N systems for this type of experiments are single wall carbon nanotubes (SWCNT), in which proximity superconductivity has already been demonstrated [33], or metallic wires carved from an epitaxially grown thin film of refractory metal such as tungsten (see 1.6.4). In either cases the proximity LDOS is expected to be different from the diffusive case. In particular, in the case of metallic SWCNT, where transport occurs through only two conduction channels, we ought to observe a discrete spectrum of Andreev Bound States.

The interest of single wall nanotubes is their ballistic behavior, but achieving good interface with a metal electrode is not easy. Metallic wires would provide much better NS interfaces, thus giving a better chance to observe proximity effect [34], but genuinely ballistic metallic wires have not been fabricated yet (except in 2DEGs).

\subsubsection{Proximity effect in a 2D system}

Bidimensional structures are also worth being investigated. Here, we think more specifically of graphene, which has attracted a lot of interest due to its very peculiar electronic properties. Even though transport in ideal graphene should be ballistic, the actually achieved mean-free paths in present experiments are still rather short, $10-100 \mathrm{~nm}$, due to impurity scattering.

A very interesting phenomenon was predicted by C. Beenakker to occur in graphene: Andreev reflection is expected to be specular, instead of a retro-reflection, provided the doping is low (close to the Dirac point) [35]. A possible geometry to probe this prediction is the electron 
focusing geometry developed at Stanford University in the group of D. Goldhaber-Gordon, which would allow to select electrons with a given incidence angle on the interface. Our goal would be to probe the electron-hole coupling at the specular reflection angle. However, this experiment would only be feasible if longer mean-free paths were achieved. We have already performed a preliminary experiment on a sample provided by D. Goldhaber-Gordon. The geometry of the graphene sheet was however not well defined, and we could not draw general conclusions from our preliminary measurements.

\subsubsection{Spin injection and relaxation in superconductors}

When a current flows between a ferromagnet and a BCS singlet superconductor, spinpolarized quasiparticles accumulate at the interface since Cooper pairs carry no spin. The recombination of these excess quasiparticles involves spin-flip processes, which were investigated long ago [36], but never satisfactorily explained. By measuring the local DOS, our instrument could bring some useful insight on these processes.

The investigation of the local DOS of the proximity induced spin-triplet superconductivity in $\mathrm{CrO}_{2}$ is also very interesting [37]. In particular, here again a spin-flip process is necessary at the interface between spin singlet and spin triplet superconductors.

In both cases, using a spin polarized tip would give access to the DOS for each spin state, and provide even more detailed information.

\subsubsection{Energy relaxation in quasi-ballistic ("Superdiffusive") structures}

In usual metallic thin films, electric transport is diffusive, due to electron scattering by impurities and crystalline defects. Recently, epitaxially grown metallic films of refractory metals (W, Mo...) in which bulk electron scattering is very small, were produced. In nanostructures carved in these films, electrons are mainly scattered by the boundaries of the conductors, and electric transport is dominated by the electrons in the lowest energy transverse sub-band, which are less scattered by the wire boundaries [38, 39]. In this so-called super-diffusive regime, electron-electron interactions are also expected to be modified with respect to the standard diffusive wires. In order to address this issue, we propose to determine the energy exchange rate between electrons in a super-diffusive wire. For this purpose, we propose to use a technique pioneered in the group 10 years ago in the case of diffuse wires [40]. In this technique one measures the out-of-equilibrium energy distribution function in a 
wire connected to two reservoirs with a potential difference V. This measurement gives access to the energy exchange rate between electrons. In a nut, the distribution function in the middle of the wire is the average of those in the reservoirs in the case of negligible energy exchange, whereas it is Fermi function with a local temperature $\mathrm{k}_{\mathrm{B}} \mathrm{T} \approx \frac{\sqrt{3}}{2 \pi} \mathrm{eV}$ in the case of strong energy exchange.

Probing the energy distribution function could provide useful insight on experiments in superdiffusive devices (see Fig. 1.11). Instead of a wire, more complex geometries can be thought of to exploit the challenging and interesting features of super-diffusive structures ${ }^{1}$.

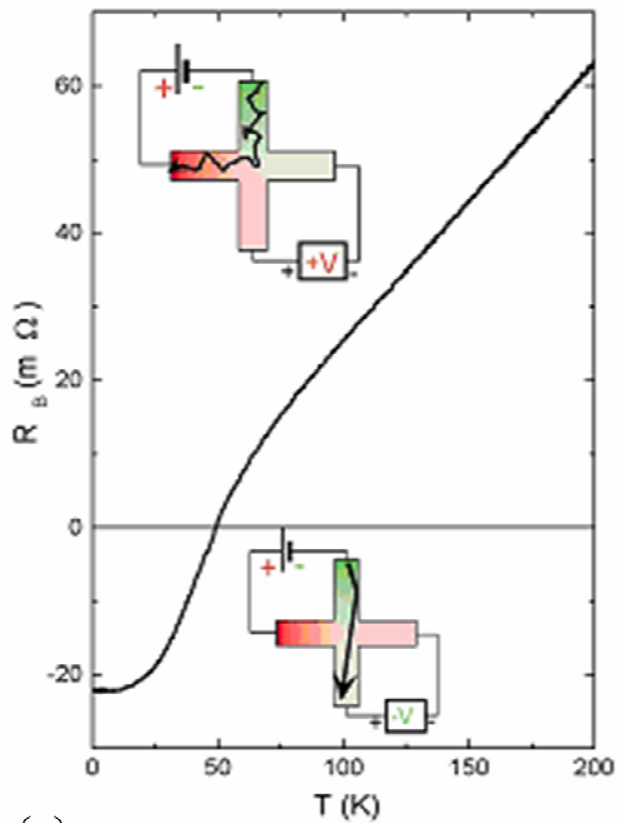

(a)
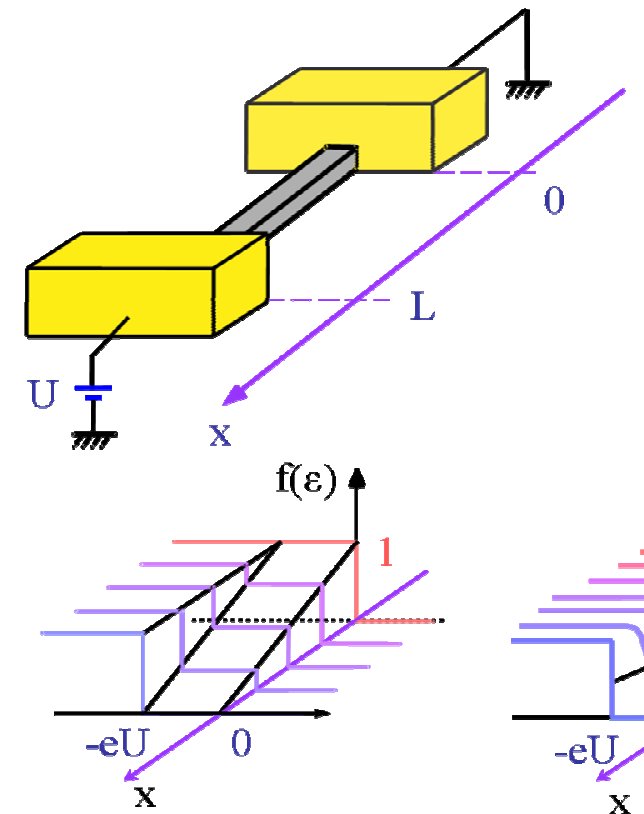

(b)

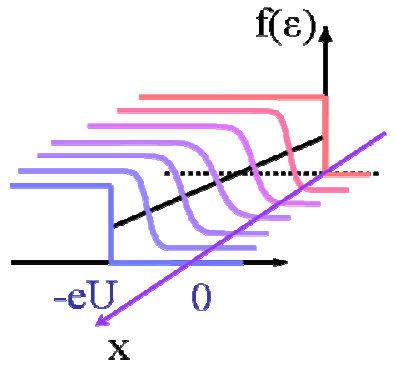

$\mathbf{X}$

Fig. 1.11 (a) A transport experiment has already been performed in the Quantronics group on epitaxial films of tungsten or molybdenum to show evidences of superdiffusive transport in such materials [38]. Below a certain temperature, phonon scattering becomes negligible and electrons motion is determined mostly by interface roughness. The measurement of the cross resistance in an epitaxial film shows a inversion of sign below $50 \mathrm{~K}$, indicating departure from the diffusive transport, as pictured in the insets. (b) An experiment designed to probe the energy exchange rate between electrons by measuring the distribution function $\mathrm{f}(\varepsilon)$ has already been performed on diffusive wires [40]. The case of weak electron interactions (left) and strong electron interactions (right) are pictured. The same experiment can be envisioned on superdiffusive wires. In both situations (a) and (b), using our microscope with a superconducting tip would allow us to probe in great detail the spatial and energy dependences of the distribution function.

\footnotetext{
${ }^{1}$ In super-diffusive systems, the relation between electric field and electrons motion is a tensor, which depends on geometry. Spatial variation of the distribution function $\mathrm{f}(\varepsilon)$ gives access to ballistic electronic paths, and the exact shape of $f(\varepsilon)$ depends on energy redistribution between electrons (see Fig. $1.11 \mathrm{~b}$ ), which is different for ballistic and for diffusive electrons.
} 
ChaPTer 2

DESIGN, FABRICATION

AND OPERATION OF

THE MICROSCOPE 
In this Chapter, I will describe the local probe microscope which was designed, assembled and operated during my thesis. This project aimed to build an instrument able to perform high resolution local tunneling spectroscopy at any point on a nanocircuit. This goal imposed a number of requirements which have driven the design of the microscope:

- High resolution tunneling spectroscopy requires low noise, low temperature operation. In turn, low temperature operation and implementation in a dilution refrigerator impose constraints on the choice of materials and actuators and on the construction of the microscope. This will be explained in $\S 2.1$ and $\S 2.2$, while low temperature instrumentation aspects will be addressed in the next chapter.

- Probing a single nanostructure necessitates aligning the tip on this particular nanostructure. Thus, the microscope must have accurate 3D displacement capability of the tip with respect to the sample. Coarse actuating will be discussed in $\S 2.3$.

- The nanostructures to be probed have insulating and conducting parts. As STMs unavoidably crash their tip on insulators, this requires a dual-mode probe. We have used a combined AFM-STM probe based on a quartz tuning fork which had already been used in several low temperature AFMs. This sensor will be presented in detail in $\$ 2.4$. 


\subsection{The microscope structure}

\subsubsection{General description}

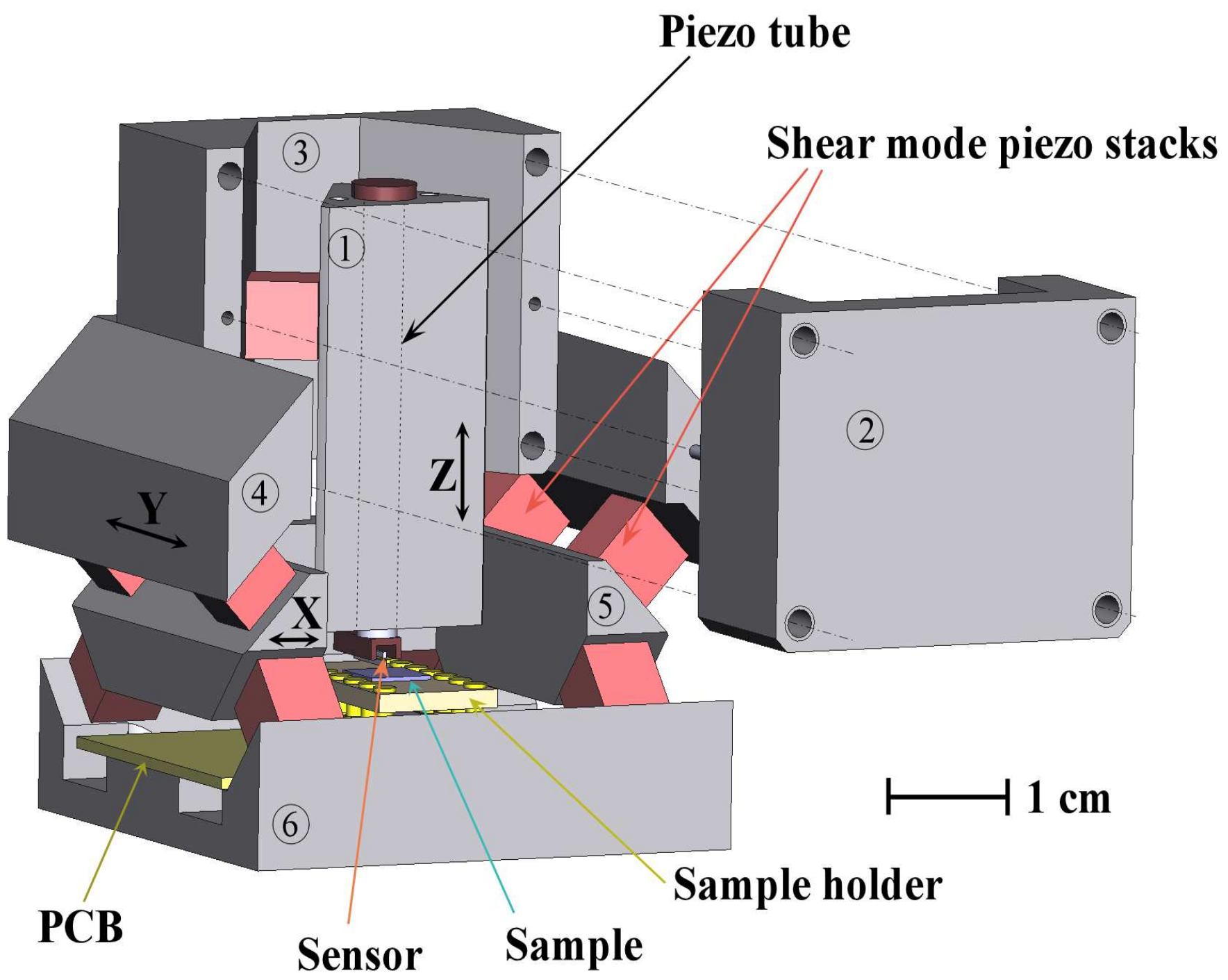

Fig. 2.1 CAD image of the microscope. Part $\mathrm{n}^{\circ} 2$ in front, separated for clarity, is normally aligned to part $\mathrm{n}^{\circ} 3$ by threaded rods (symbolized by dashed lines), and gently pressed against part $\mathrm{n}^{\circ} 1$ by springs. Mating prismatic shapes are used to guide sliding parts (along the indicated X, Y and Z axes) which provide coarse positioning of the sensor tip above a fixed sample on three independent orthogonal axes. The sliding parts are actuated by inertial stick-slip motion of four shear-mode piezo stacks for each axis. Fine scanning of the sample surface is done by a vertical piezoelectric tube, which performs 3-D positioning. The sample is fixed and connected onto a sample holder, which plugs into a socket soldered onto a printed circuit board (PCB). The base is thermally anchored to the $30 \mathrm{mK}$ plate of the inverted dilution fridge. 
Fig. 2.1 is an illustration of the overall structure of the microscope. It has a mobile tip above a fixed sample so that the sample can be electrically connected with fixed wires and thermally anchored to the mixing chamber of the refrigerator. The tip can be coarse-positioned above the sample using 3 independent orthogonal actuators indexed by capacitive sensors which will be discussed in $\$ 2.3 .1$ and $\$ 2.3 .2$. Fine scanning is performed by a piezoelectric scanning tube supporting the sensing system. This latter system consists of a metallic sensor tip mounted at one end of an arm of a miniature quartz tuning fork. This sensor will be described in \$2.4.2.

\subsubsection{Materials and construction}

\subsubsection{Material selection}

As in any complex assembly made for cryogenic use, we had to take great care of choosing materials with compatible contractions upon cooling down. This is particularly true for materials which are meant to be glued together. In this respect, the selection is primarily dictated by the use of available cryogenic piezoelectric actuators, all based on so-called PZT ceramics (See $\$ 2.2$ p. 45). Thus, we tried to use materials matching as closely as possible the contraction of theses PZT ceramics (See Table. 2.1).

Besides contractions, other criteria enter the material selection:

(i) It is important that bulk materials used at low temperature are good thermal conductors to thermalize well and rapidly.

(ii) The materials chosen for the microscope body should be preferably easily machined.

For both the above reasons, to fabricate the body of the microscope, we preferred using a metal such as Ta6V (a titanium alloy), which can be machined similarly to stainless steel, rather than machinable ceramics such as Macor® (used in STM bodies because of its matching to PZT contractions). Titanium has the further advantage, as compared to common metals, to be non magnetic which is mandatory for applications in a magnetic field or when using superconductors.

The fact that titanium is superconducting at low temperature (critical temperature of $390 \mathrm{mK}$ ) makes it a bad thermal conductor. This was cured by gold-plating the titanium parts with a few microns thick gold layer, to help their thermalization as much as possible (See §2.1.2.5). 


\begin{tabular}{|c|c|c|}
\hline Material & $\begin{array}{c}\text { linear CTE @ 320K } \\
\frac{\Delta l}{l}\left(\times 10^{-6} / \mathrm{K}\right)\end{array}$ & $\begin{array}{l}\text { integrated thermal contraction } \\
\left.\frac{\Delta l}{l}\right|_{\text {ave }} *(300 \mathrm{~K}-4 \mathrm{~K})\left(\times 10^{-3}\right)\end{array}$ \\
\hline Titanium Ta6V & 8.9 & 1.71 \\
\hline Copper & 16.4 & 3.26 \\
\hline Brass & 18.9 & 3.97 \\
\hline Stainless Steel, not used & 13 & 2.97 \\
\hline Tungsten & 4.4 & 1.1 \\
\hline PIC151® from Politec PI (the tube) & 6 & no published data ${ }^{6}$ \\
\hline PIC255®, Politec PI (the stacks) & 4 & no published data ${ }^{1}$ \\
\hline Al alloy & 24 & 4.19 \\
\hline Sintered alumina & 7.8 & $\sim 1$ \\
\hline Quartz & 8.1 & $\begin{array}{l}-0.01 \text { (silica) } \\
0.22 \text { (silicon) }\end{array}$ \\
\hline $\begin{array}{c}\text { Vespel® SP-22 (plastic) } \\
\text { (tube and tuning fork support) }\end{array}$ & 38 & 6.3 \\
\hline Stycast ${ }^{\circledR} 2850$ FT (epoxy) & 35 & 4.2 \\
\hline Stycast ${ }^{\circledR} 1266$ (epoxy), not used & & 11 \\
\hline Epotek® H20E, Politec PI (conductive epoxy) & 31 & no published data ${ }^{7}$ \\
\hline Macor ${ }^{\circledR}$ (ceramic), not used & 9.3 & 1.67 \\
\hline
\end{tabular}

Table. 2.1 Comparison of thermal contractions of the main materials used in the microscope assembly. Thermal contraction below $4 \mathrm{~K}$ is negligible.

\footnotetext{
${ }^{6}$ Matching of PZT ceramics and titanium thermal expansion down to cryogenic temperatures was brought to our knowledge by the UAM low temperature lab group.

${ }^{7}$ Epoxies can have thermal expansion coefficients up to 40 times higher than common metals.
} 


\subsubsection{Mechanical structure}

Local probe measurements, and, above all, tunneling spectroscopy, are very sensitive to vibrations. It is therefore crucial that a local probe microscope is built to be as much as possible immune to vibrations [41]. This means that vibrations reaching the base of the microscope should not change the tip-to-sample distance. This implies a very rigid structure with large eigenfrequencies.

In this respect, Ta6V is also a good choice: It is lighter and stiffer than other materials we could have chosen, so that the eigenfrequencies of the structure will be pushed up. We further tried to make it as small as possible, to minimize the mass and thus the cooling time.

\subsubsection{CAD Design}

I designed the structure of the microscope using "Solid Concept", a 3-D parametric CAD software. In this software, designing a fairly complex assembly such as this microscope is done quite simply by listing constraints (such as mating faces, dimensions, parallelism...) on the different parts. Changing any of these constraints automatically propagates and updates the design.

With such a tool, starting from preliminary ideas on how one would implement coarse motions, what would be a stiff and stable structure, what are the piezoelectric actuators dimensions and the required relative motions of the different parts, etc, one can rapidly end up with blueprints for machining the parts. Those blueprints are reproduced on Fig. 2.4. Each element is shown under the standard blueprint views, plus an additional isometric view for clarity.

In many respects, this design is not original, as it borrows many ideas to low-temperature STMs already successfully operated in other labs ${ }^{1}$. In particular, the coarse motion displacement motors based on stick-slip motion, shear mode piezo stacks and prismatic guiding, can be traced to the Pan design [42].

We also benefited from the knowledge of the low temperature laboratory of the Universidad Autónoma de Madrid [43] where such motors had been successfully operated prior to this work.

\footnotetext{
${ }^{1}$ Prior to this thesis no STM had been built in the laboratory.
} 

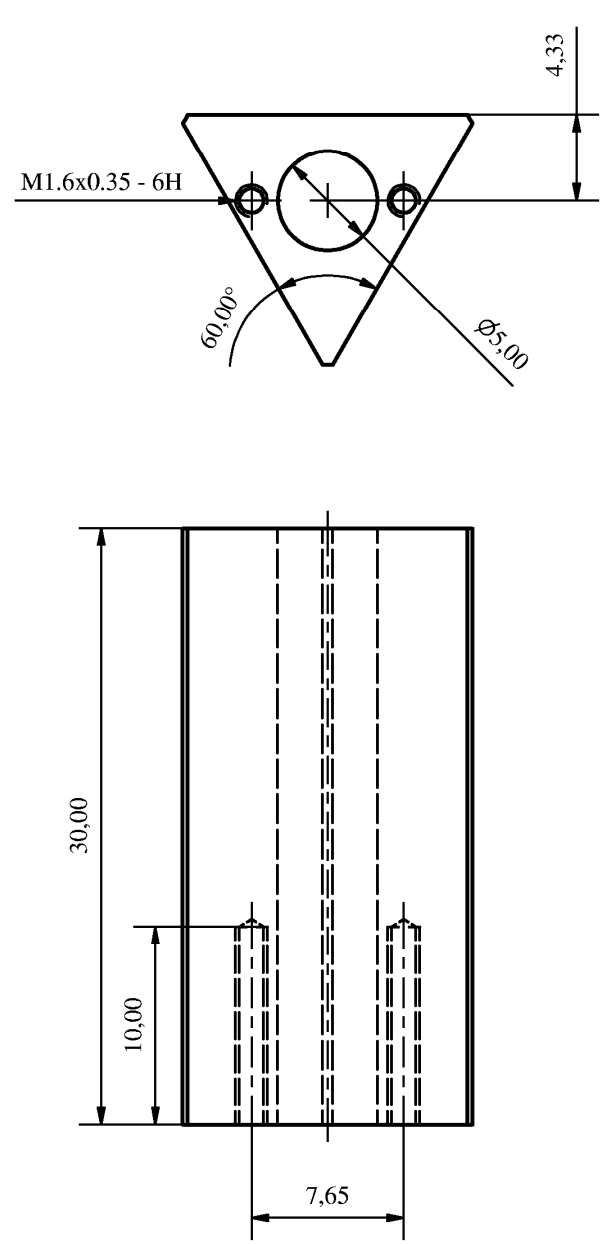

\begin{tabular}{|c|c|c|c|c|}
\hline Conçu par & \multicolumn{2}{|c|}{ CEA - QUANTRONICS GROUP } & Dat & $12 / 12 / 2004$ \\
\hline \multirow{2}{*}{ SCANNER CARRIAGE } & \multirow{2}{*}{ A4 } & \multirow{2}{*}{$\begin{array}{c}\text { Echelle } \\
3: 1\end{array}$} & Projet & AFM-STM \\
\hline & & & Mranere & Titanium (TA6V) \\
\hline
\end{tabular}
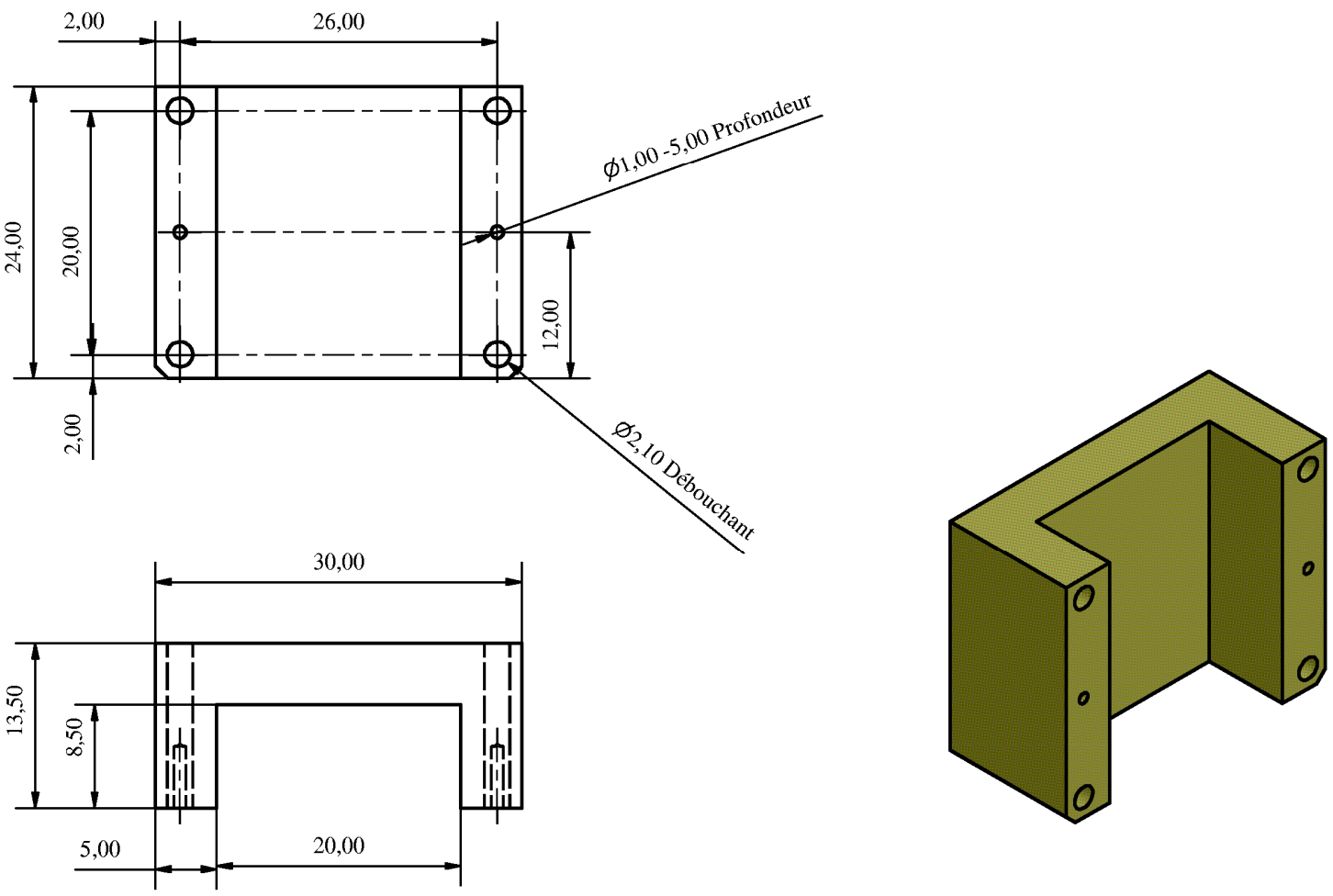

\begin{tabular}{|c|c|c|c|c|}
\hline \begin{tabular}{|l|} 
Conçu par \\
Hélène le Sueur
\end{tabular} & \multicolumn{2}{|c|}{ CEA - QUANTRONICS GROUP } & $\mathrm{Da}$ & $12 / 12 / 2004$ \\
\hline \multirow{2}{*}{$\mathrm{Z} 1$} & \multirow{2}{*}{$\mathrm{A} 4$} & \multirow{2}{*}{$\begin{array}{c}\text { Echelle } \\
2: 1\end{array}$} & Projet & AFM-STM \\
\hline & & & Matue & Titanium (TA6V) \\
\hline
\end{tabular}

Fig. 2.2 Blueprints of part 1 and 2 of Fig. 2.1 

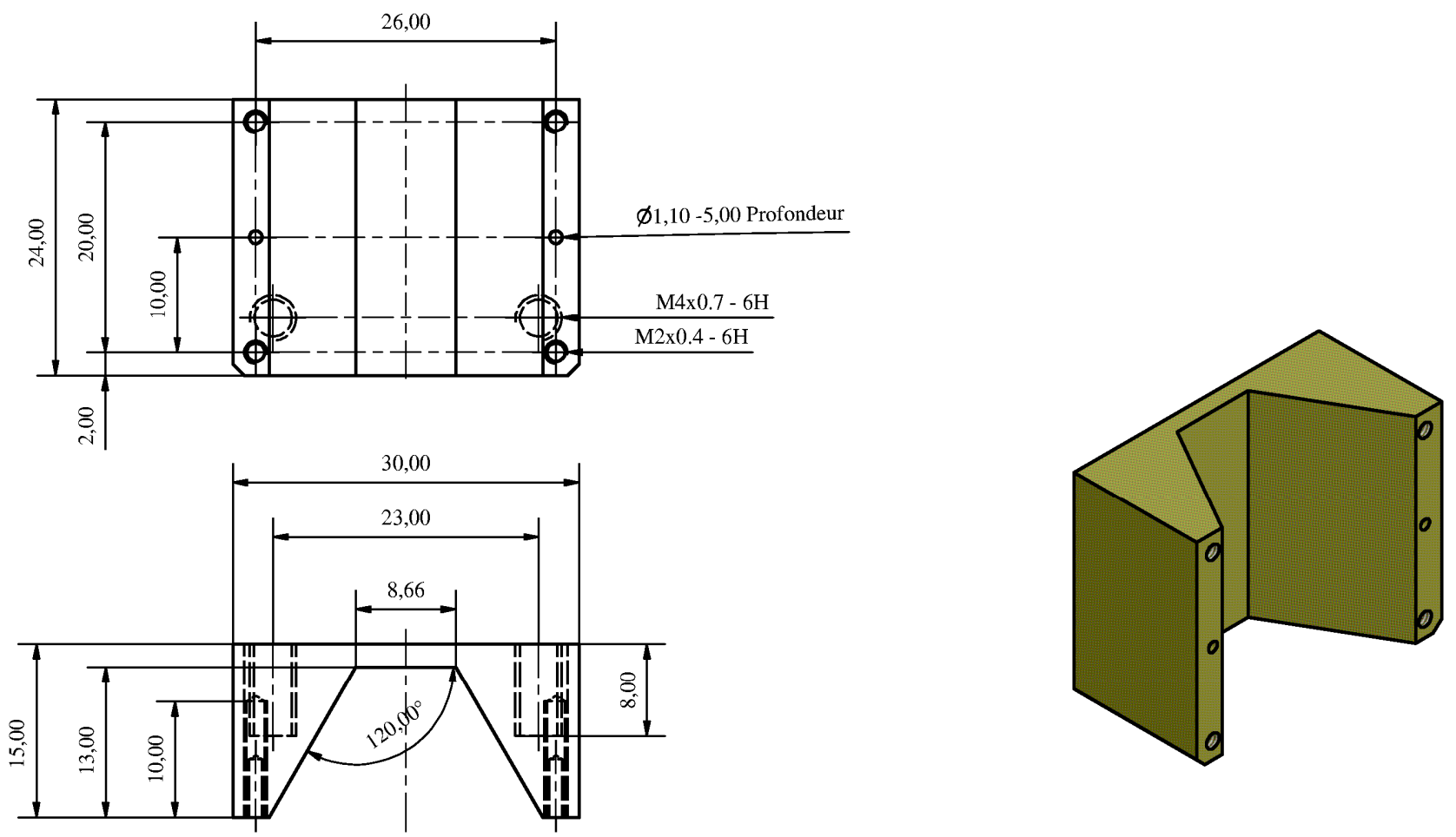

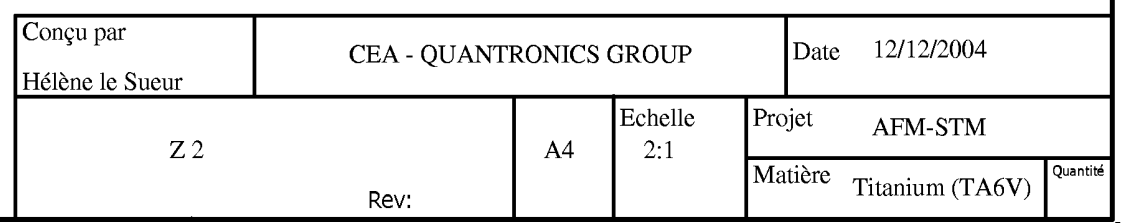
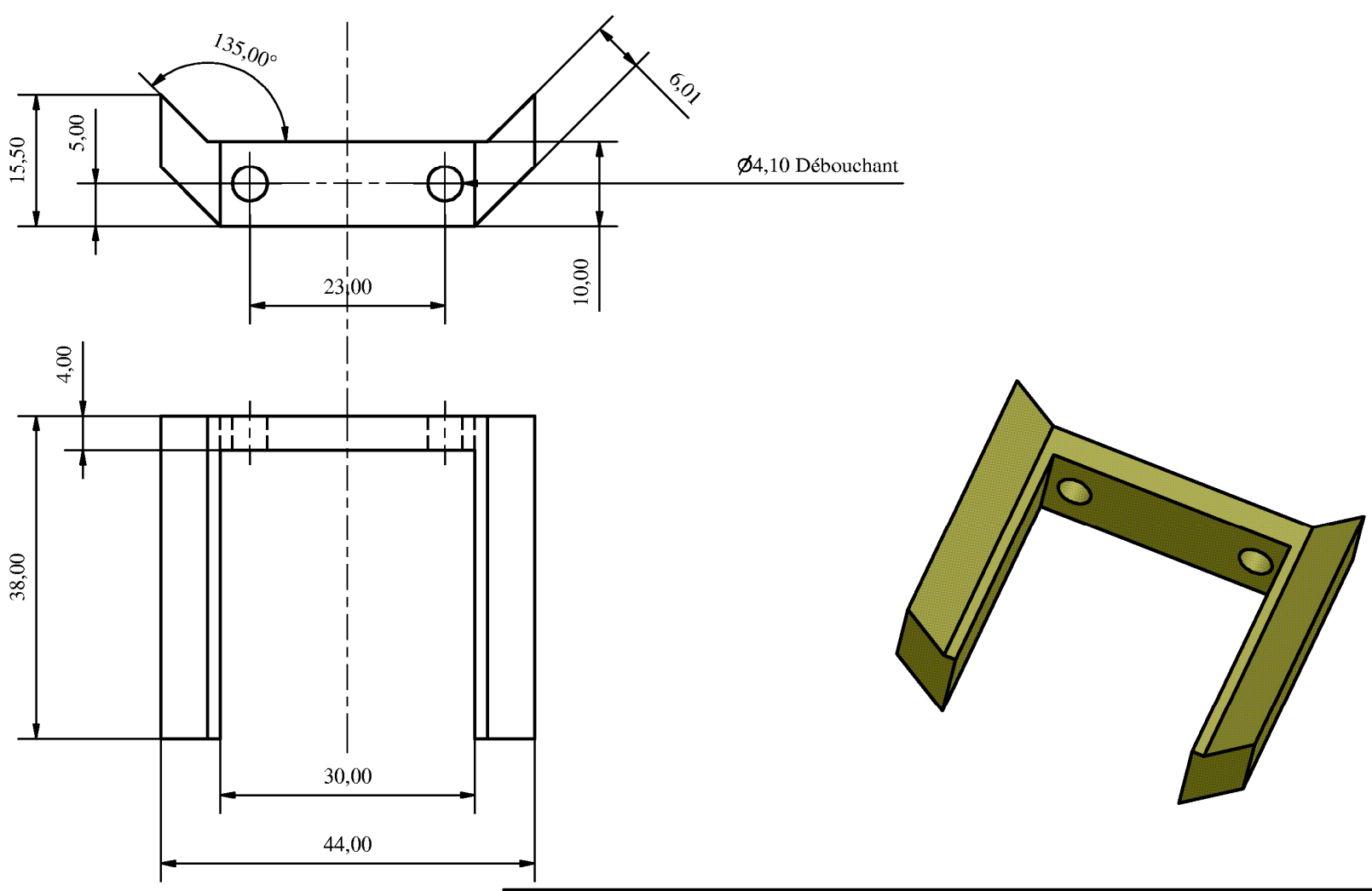

\begin{tabular}{|c|c|c|c|c|c|}
\hline \begin{tabular}{|l} 
Conçu par \\
Hélène le Sueur
\end{tabular} & \multicolumn{3}{|c|}{ CEA - QUANTRONICS GROUP } & Dat & $12 / 12 / 2004$ \\
\hline \multirow[t]{2}{*}{ (2) } & & \multirow{2}{*}{ A4 } & \multirow{2}{*}{$\begin{array}{c}\text { Echelle } \\
3: 2\end{array}$} & Projet & AFM-STM \\
\hline & & & & Matière & Titanium (TA6V) \\
\hline
\end{tabular}

Fig. 2.3 Blueprints of part 3 and 4 of Fig. 2.1 


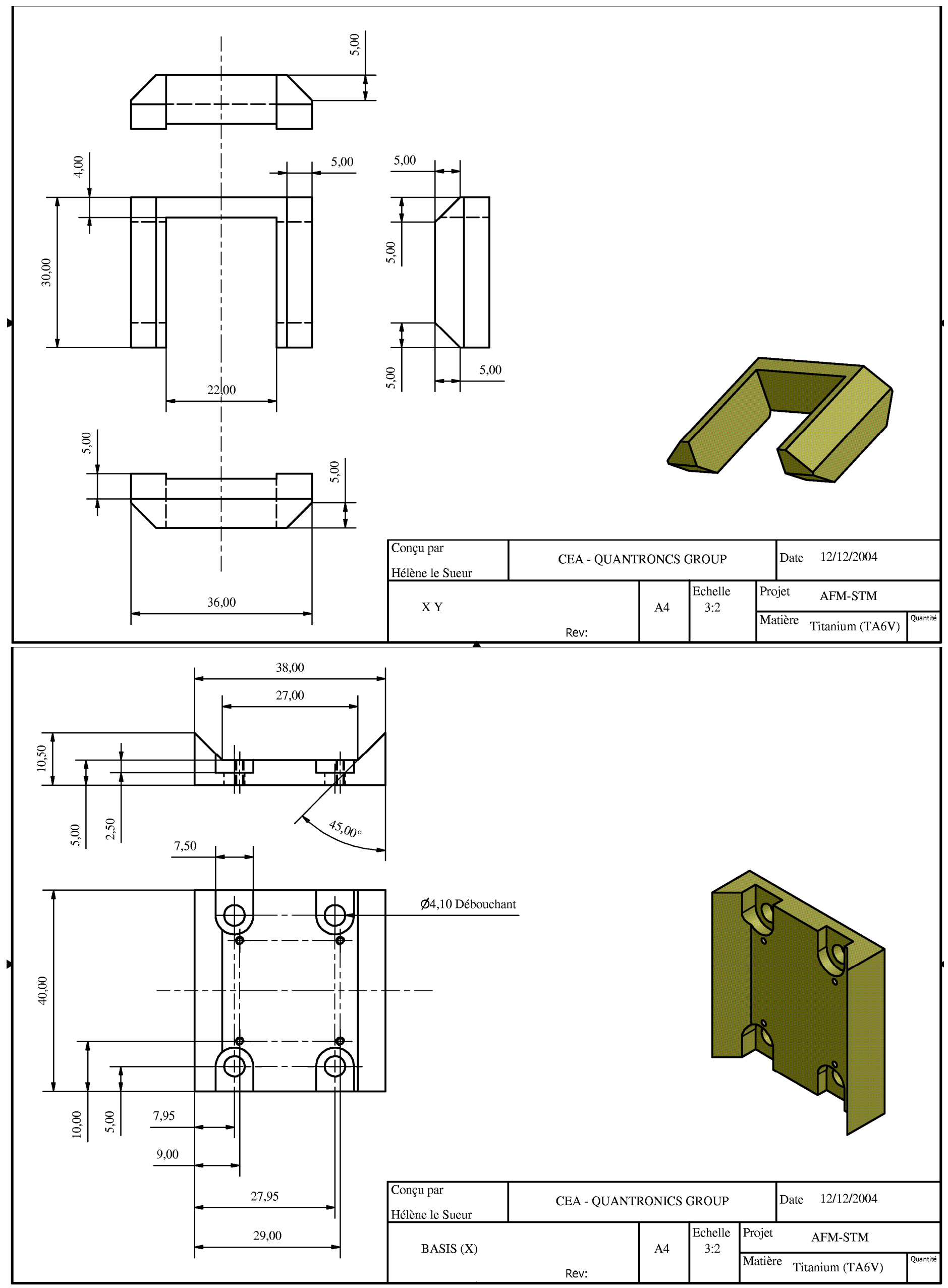

Fig. 2.4 Blueprints of part 5 and 6 of Fig. 2.1. 


\subsubsection{Assembly}

Epoxy Stycast ${ }^{\circledR} 2850$ FT is ubiquitously used by cryogenists, thanks to its thermal matching to copper. All joint parts which do not necessitate electrical bonding are glued together using Stycast ${ }^{\circledR}$. This concerns all piezoelectric stacks which are glued to the titanium parts, as well as the alumina plates, and the connectors of the microscope (see Fig. 1.4).

Electrically connected parts are bonded together by silver epoxy, Epotek® H20E or H20F from Politec PI. Since H20F is cured at a lower temperature than H20E, it is more appropriate for the assembly of the PZT piezo parts (see §2.2.2.3). However, it is softer, and more fragile. The AFM-STM probe is glued at the end of the piezoelectric tube using General Electric varnish, usually employed as an insulator on electronic circuits. The reason to use it here is that it is easily removable using isopropanol, and allows quick mount and un-mount of the probe sensor when necessary (for instance when the tuning fork is broken).

At the other end, the piezoelectric tube is glued with conductive epoxy to a tiny threaded titanium cap. When gluing the cap on the tube (see Fig. 2.5), one has to care about alignment of the axis of the cap with respect to the tube axis. This cap has two functions: first to connect the inner electrode of the tube, and second to hold the tube onto its support. The piezo tube is screwed on a machined Vespel® part (non conductive polymer, see Table. 2.1), which is itself screwed on the prismatic $\mathrm{Z}$ titanium part. Therefore the tube can be easily removed and replaced when needed (for instance to repolarize it (see 2.2.3.1). The electrical connections of the electrodes of the tube are done using conducting epoxy as shown in Fig. 2.5.

This holding of the piezo tube is made as rigid as a direct gluing of the tube in the titanium part would be, except that it can be conveniently replaced. 


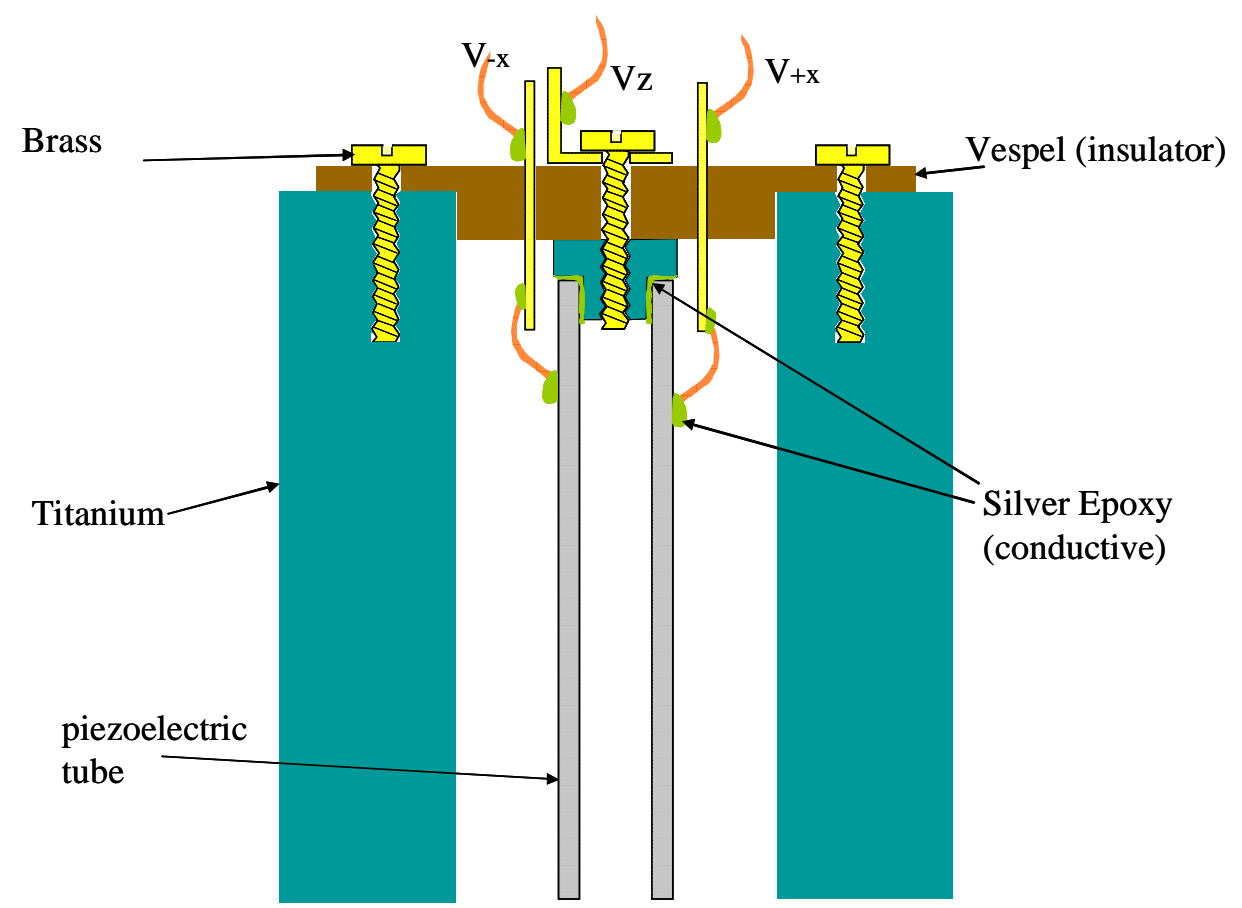

Fig. 2.5 Schematic view of the mounting of the tube in the titanium $Z$ prism (part 1 of Fig. 2.1). The external electrodes (see $\$ 2.2 .2 .3 . b \mathrm{p} 48$ ) of the tube are connected by silver epoxy to throughputs, and the central electrode is glued to a threaded titanium cap.

\subsubsection{Plating of titanium}

For better thermalization, the Ti parts of the microscope are covered with a thick gold and copper layer by local electrochemical deposition. The thickness is of the order of $10 \mu \mathrm{m}$, but is not uniform, due to the technique employed.

In this electrochemical technique, adhesion of the deposited metal onto titanium is normally poor due to bad sticking onto the uniform $\mathrm{TiO} 2$ layer covering titanium.

To improve this, we first perform a local anodization of titanium with a $\left\{\mathrm{HClO}_{4}+\mathrm{CH}_{3} \mathrm{COOH}\right\}$ solution at $-30 \mathrm{~V}$ potential. In this step we grow porous titanium oxide, which yields better adhesion of the metallic layer.

We then deposit alternatively gold and copper layers. These layers are deposited from cyanide-complexed gold and $\mathrm{CuSO}_{4}$ solutions at respective potentials of $+12 \mathrm{~V}$ and $+6 \mathrm{~V}$, with a carbon electrode. 


\subsection{Piezoelectric actuators}

Piezoelectric materials are the standard actuators used in local probe microscopy. They allow controlling electrically very small displacements, down to subatomic levels. In the following, I first review the basics of these actuators. Then, I detail how we use them in our microscope

- for accurately scanning the tip over the sample,

- for performing coarse positioning over the sample,

- in the AFM sensor.

\subsubsection{The piezoelectric effect}

Piezoelectricity was first predicted and explained by the Curie brothers in 1880. It is the property of certain crystals to generate charge when stressed. This effect is due to the intrinsic polarization of the crystalline unit cell (e.g. pyroelectric materials), and more generally to a non-centro-symmetric charge distribution.

When a crystalline cell with non uniform charge distribution is mechanically deformed, the geometric centers of positive and negative charges move by a different amount resulting in electric polarization. Conversely, the application of an electrical field results in different motions of the charges geometric centers in the cell, that finally induce macroscopic deformation of the material, either shear or longitudinal.

The converse piezoelectric effect was mathematically deduced by Lippmann in 1881 and was immediately confirmed by the Curie brothers, proving the complete reversibility of electroelasto-mechanical strains.

\subsubsection{General mathematical description of piezoelectricity}

In a microscopic (local) view, strain $(S)$ develops within any material, due to an applied stress $(T)$, and the link between those quantities is the mechanical compliance ${ }^{1}(s)$. Mechanical compliance is a local tensor (6x6), when describing continuous media with a large number of degrees of freedom (DOF) ${ }^{2}$.

Identically, from the electrical point of view, the electric displacement $(D)$ is related to the electric field $(E)$ by the permittivity $(\varepsilon)$, where $D$ an $E$ are 3-dimensional vectors, and $\varepsilon$ is a rank 3 diagonal matrix.

\footnotetext{
${ }^{1}$ Compliance is homogeneous to the inverse of the Young modulus.

${ }^{2}$ It can be reduced to a global tensor or even to a scalar when the material has symmetries, or is homogeneous.
} 
In a piezoelectric material, all the above mechanical and electrical quantities are coupled. The piezoelectric effect can be described by the so-called strain-charge coupled tensorial equations:

$$
\left\{\begin{array}{l}
S=[s] T+{ }^{\mathrm{t}}[d] E \\
D=[d] T+[\varepsilon] E
\end{array}\right.
$$

where $[d]$ is the piezoelectric coupling matrix $(3 \times 6)$ ( ${ }^{t}$ stands for transposition of a matrix).

Polarization and strain are therefore related by the coefficients of the $[d]$ matrix, depending on the orientation of the crystalline cells relative to the electric field. The coefficient $d_{\mathrm{ij}}$ of the [d] matrix represents the induced strain (relative length change) along axis $\mathrm{j}$ with $\mathrm{j} \in\{1,6\}$, per unit of electric field applied in direction i: numbers from 1 to 3 identify the three directions in the crystal cell referential, and 4 to 6 label the shear modes ${ }^{1}$. Usually, the $3^{\text {rd }}$ direction is taken as the polarization direction.

\subsubsection{Practical equations for piezoelectric devices}

Piezoelectric devices are objects made of piezoelectric materials. Their shape is cut relative to the polarization axis of the material and they are covered with metallic electrodes to apply an electric field to the material or to collect the piezoelectric charges, whether it is used in reverse or direct piezoelectric mode.

The tensorial relation (2.1) is a local microscopic description of the piezoelectric effect. By integration over the spatial coordinates, taking into account the shape of the electrodes, one establishes a link between the charge $q$ on the electrodes (from $\iint D$ ), the voltage $U$ applied $\left(\int E\right)$, the displacement $z$ of the object $\left(\int S\right)$, and the force $F$ applied $\left(\iint T\right)$, of the same form as (2.1), but with macroscopic parameters.

For example, in the case of a one dimensional actuator (i.e. designed to move an extremity in a single given direction), this yields a linear relationship between electrical and mechanical quantities:

$$
\left(\begin{array}{l}
z \\
q
\end{array}\right)=\left(\begin{array}{cc}
k^{-1} & s \\
s & c
\end{array}\right)\left(\begin{array}{l}
F \\
U
\end{array}\right),
$$

where $z$ is the motion of the extremity along the proper axis, $F$ the force applied at that point in the relevant direction, $q$ the charge collected by the electrodes, $U$ the voltage across the

\footnotetext{
${ }^{1}$ We use here the Voigt notation, where $S$ and $\mathrm{T}$ appear as 6D vectors instead of rank 2 tensors, and $[d]$ as a rank 2 instead of a rank 4 tensor.
} 
electrodes, $s$ the unloaded (i.e. with $F=0$ ) deflection of the actuator under unit applied voltage, $c$ the capacitance between electrodes (charge collected per unit voltage when $F=0$ ) and $k$ the actuator spring constant (deflection per unit force when $U=0$ ).

Energy conservation arguments further imply that the determinant of the above matrix is zero, and that its coefficients are linked by

$$
s^{2}=\frac{c}{k}
$$

This in turn implies that under all circumstances there is proportionality between $z$ and $q$ in piezoelectric materials:

$$
\begin{aligned}
& \alpha=\frac{q}{z}, \\
& \text { with } \alpha=k s=\frac{c}{s}
\end{aligned}
$$

Most devices are thus characterized by giving the ratio $\alpha$, the so-called piezoelectric coupling coefficient (in $\mathrm{C} / \mathrm{m}$ ), and their capacitance.

\subsubsection{Practical piezoelectric materials}

In the following I present the materials we have used in the microscope, quartz and the socalled PZT ceramics.

\begin{tabular}{|c|c|c|}
\hline \multirow{2}{*}{ Material } & \multicolumn{2}{|c|}{$\begin{array}{c}\text { Piezoelectric coupling } \\
\left(10^{-12} \mathrm{C} / \mathrm{m}\right) \text { or }\left(10^{-12} \mathrm{~N} / \mathrm{V}\right)\end{array}$} \\
\cline { 2 - 3 } & Room Temperature & Low Temperature (4K) \\
\hline Quartz & $d_{\mathrm{ij}} \sim 1$ & $\sim$ unchanged \\
\hline PZT & $d_{\mathrm{ij}} \sim 200-550$ & reduced by a factor 5 to 10 \\
\hline
\end{tabular}

Table. 2.2 piezoelectric coupling constants $d_{\mathrm{ij}}$ of the piezoelectric materials used in the microscope.

\subsubsection{Quartz crystal}

Crystalline quartz in its most abundant form $(\alpha)$ exhibits spontaneous piezoelectricity. Quartz can be produced in high quality monocrystals with excellent mechanical properties. This enables to fabricate extremely stable, high quality factor, electromechanical resonators based on the piezoelectric effect. Such resonators are ubiquitously used as time bases in electronic systems. The AFM sensor of our microscope uses such a resonator (see §2.4). 
For practical applications other than resonators, such as actuators, transducers, etc., quartz (or other natural piezoelectric material) is not very attractive because of its low intrinsic coupling constant. Quartz is however free from hysteresis and drift problems found in synthetic materials (see below).

\subsubsection{PZT piezoelectric material}

\subsubsection{3.a Description}

In order to improve the applicability of piezoelectric devices in particular in the domain of actuators, material research came up with ceramics having much larger coupling coefficients. The most widely used of these is a mix oxide of lead, zirconium, and titanium, the so-called PZT. It has a composition $\mathrm{Pb}\left(\mathrm{Zr}_{\mathrm{x}} \mathrm{Ti}_{1-\mathrm{x}}\right) \mathrm{O}_{3}$ with $\mathrm{x} \approx 0.52$, and can achieve strains of up to $0.2 \%^{1}$. At this particular composition, crystal is at a morphotropic phase ${ }^{2}$ boundary which greatly enhances its piezoelectric properties.

The crystalline structure of PZT is a perovskite. The unit cell consists of a small tetravalent metal ion, $(\mathrm{Ti}$ or $\mathrm{Zr})$ in a lattice of larger metal ions $(\mathrm{Pb}, \mathrm{Ba})$ and $\mathrm{O}^{2-}$ ions. Below the Curie temperature of the crystal, the cell acquires spontaneous anisotropy, and presents a permanent dipolar moment. The orientation of this moment can adopt several orientations, and can be changed by applying a sufficiently large electric field (it is thus a ferroelectric material). The adjacent cells in a crystal all have the same polarization orientation; a grain therefore presents a permanent macroscopic polarization.

\subsubsection{3.b PZT ceramics: poling and depoling}

For most applications ${ }^{3}$ the material consists of a ceramic made of small sintered grains. As fabricated, all the grains are randomly oriented, so that the ceramic element has no global dipolar moment, and no net piezoelectric effect.

\footnotetext{
${ }^{1}$ In view of the enormous potential for applications this is an active research field and since 2000 several "giant piezoelectric effect" materials such as PMN-PT, PZN-PT with coupling constants one order of magnitude larger than PZT have been obtained.

2 A phase transition between two crystallographic arrangements occurs when changing the stoechiometric composition (in PZT materials, it is a transition between rhombohedral and tetragonal phases).

${ }^{3} \mathrm{PZT}$ is also used in monocrystalline thin films in infrared imaging applications, for its pyroelectric properties.
} 
In order to make them usable, a net macroscopic piezoelectric effect is obtained in these ceramics by poling (i.e. polarizing) them. The poling process consists in using the ferroelectric property of the material: under a sufficiently large electric field (typically in the $\mathrm{MV} / \mathrm{m}$ range at room temperature), the microscopic individual crystalline cell dipoles can be permanently rotated. This process is often assisted thermally as the ferroelectric coercive field vanishes at the Curie temperature, which typically ranges from 150 to $350^{\circ} \mathrm{C}$ depending on the type of PZT. After this process, the grains that were earlier randomly oriented are globally aligned along the direction of the poling field, and a permanent polarization remains.

However, care must be exerted with these ceramics, as they may be "depoled" by applying a too large reverse electric field or by raising temperature above the Curie temperature.

\subsubsection{3.c PZT actuators shapes and functioning}

One can construct a variety of piezoelectric actuators which can be classified as follows:

\section{- "normal" piezoelectric mode.}

In these devices, the poling direction coincides with the subsequently applied field, and the resulting local deformation consists in a $d_{33}$ elongation (respectively compression) along the applied field, accompanied by a transverse $\left(d_{31}, d_{32}\right)$ contraction (resp. expansion) when the field is applied in (resp. opposite to) the direction of the poling field (see Fig. 2.6).
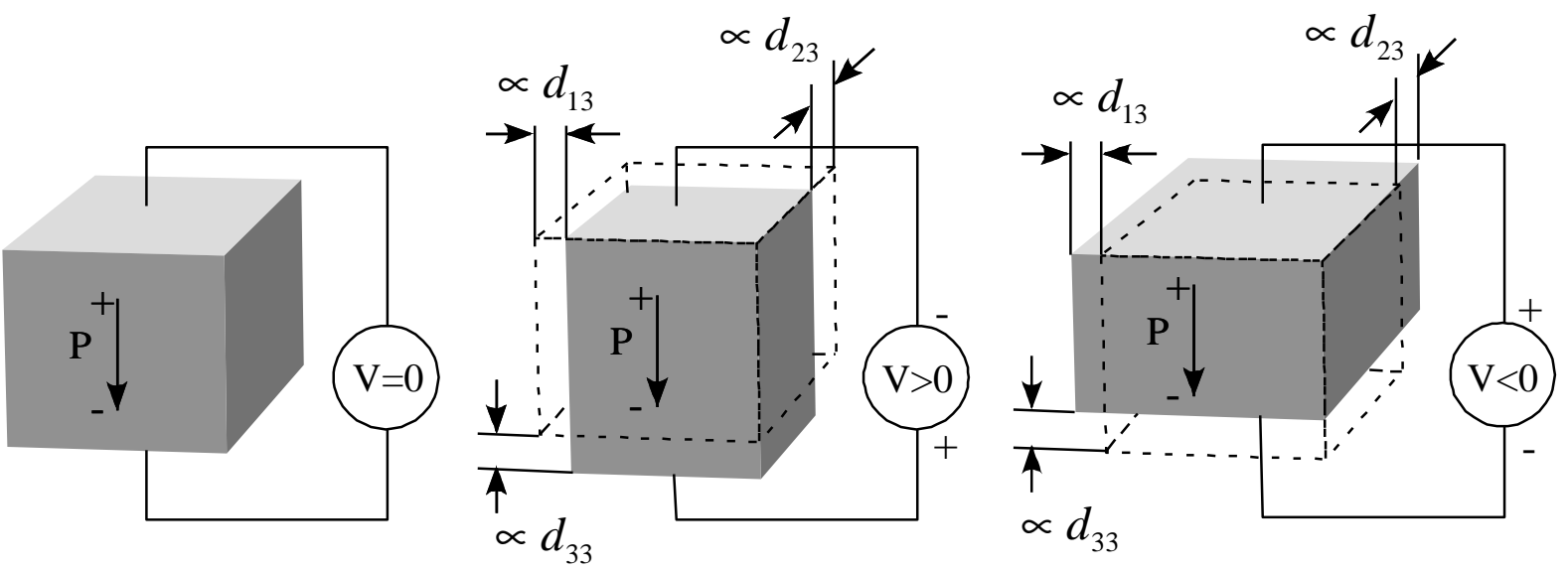

Fig. 2.6 Deformation of a piezoelectric material when an electric field is applied parallel to the polarization direction.

\section{- "shear" piezoelectric mode.}

In this kind of devices, the applied field is perpendicular to the poling field (indexes $\mathrm{i}=1$ or 2): a unit field applied in direction 1 (resp 2) yields a shear deformation of the material in the poling direction (see Fig. 2.7) proportional to $d_{15}$ (resp. $d_{24}$ ). The particularity 
of these devices is that poling was done prior to cutting the ceramic and plating it with electrodes parallel to the poling field. Consequently, if accidentally depoled, the device cannot be repoled in-situ and must be replaced.
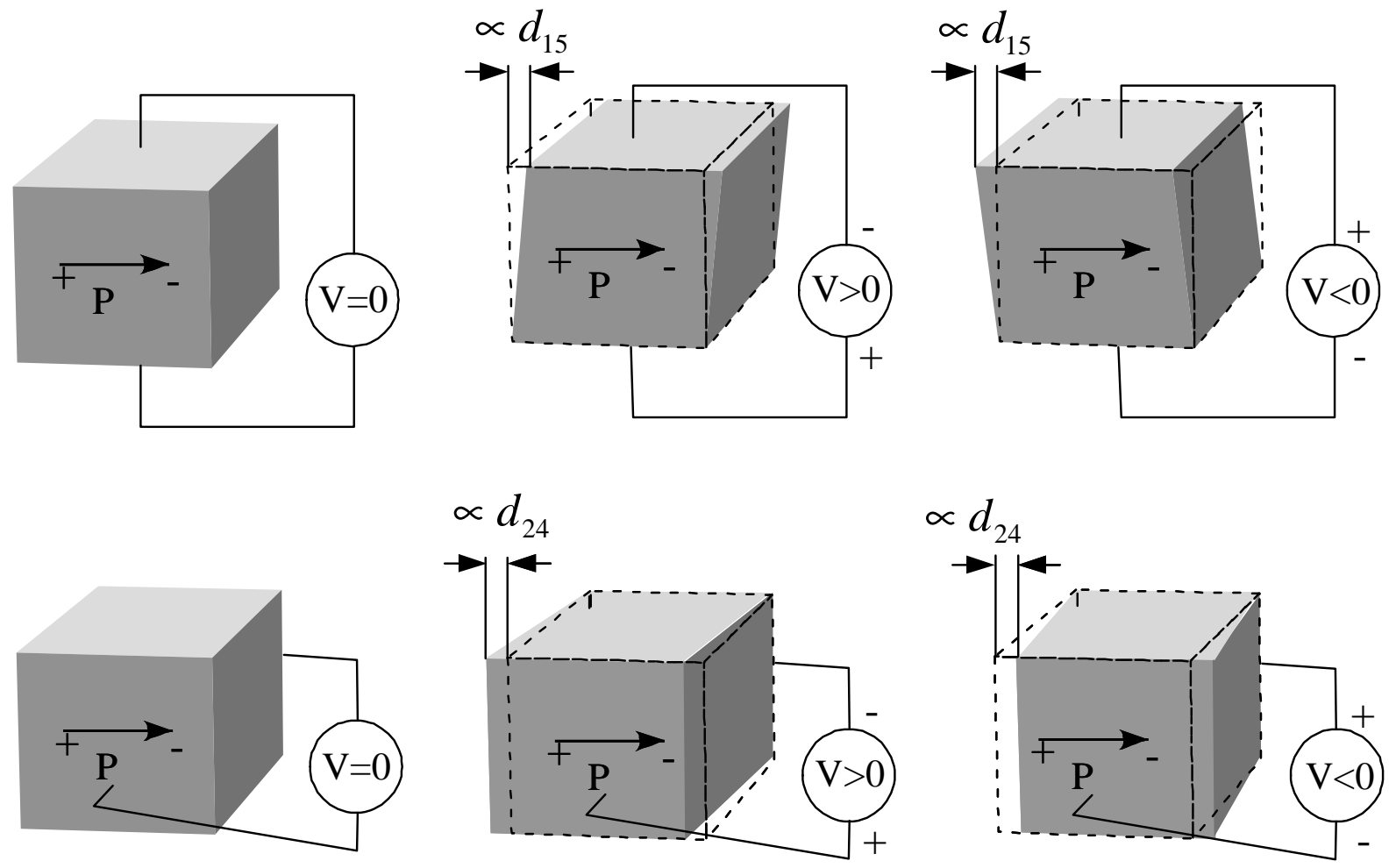

Fig. 2.7 Deformation of piezoelectric material when an electric field is applied perpendicular to the polarization direction: a torque is applied to crystalline cells which result in shear deformation.

\section{- Normal and shear stacked devices.}

Voltages can be easily produced and controlled up to a few $100 \mathrm{~V}$. For these voltages, the magnitude of the piezo coefficient $\left(d_{33}, d_{15}, \ldots\right)$ limits achievable displacements by an individual slab of material to the $\mathrm{nm}$ range. In order to increase these displacements, one resorts to stacking several slabs, so that their individual displacements add ${ }^{1}$. To do so, one stacks slabs with alternate polarization together with intermediate electrodes (see Fig. 2.8). These stacks form the most powerful piezoelectric devices, with displacements up to several hundred $\mu \mathrm{m}$, able to carry heavy loads. Stacks are usually made of many thin layers (to maximize the electric field, and therefore, the strain), which translates in devices having large capacitances. Also, large voltages must be handled with care in these devices, as one is more likely to exceed the breakdown field or the depoling field in thin layers.

\footnotetext{
${ }^{1}$ Large deflexions can be obtained in tubes, unimorph and bimorph structures without resorting to stacking, but then the available actuating force is small.
} 


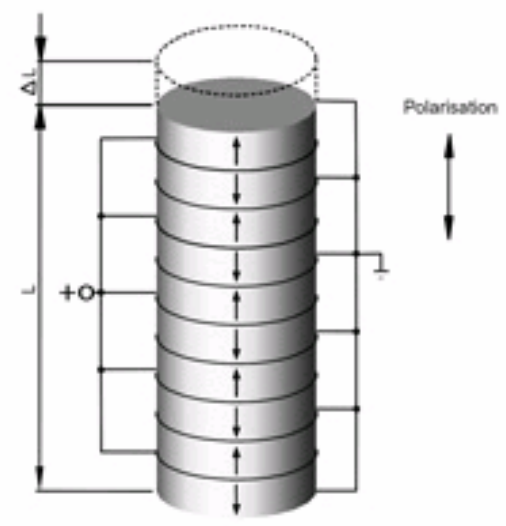

Fig. 2.8 Piezoelectric sheets can be stacked to increase their achievable displacement. Adjacent sheets have opposite polarizations, to share the same common potential. For a given applied voltage, the displacement is enhanced by a factor equal to the number of sheets in the stack. Stacks can be made either of normal or shear mode piezoelectric sheets.

\section{- Piezo-tube devices.}

The poling process of a ceramic allows orienting the polarization of the material in any convenient spatial distribution. Most notably, in tubes, one can easily obtain a radial polarization (Fig. 2.9).
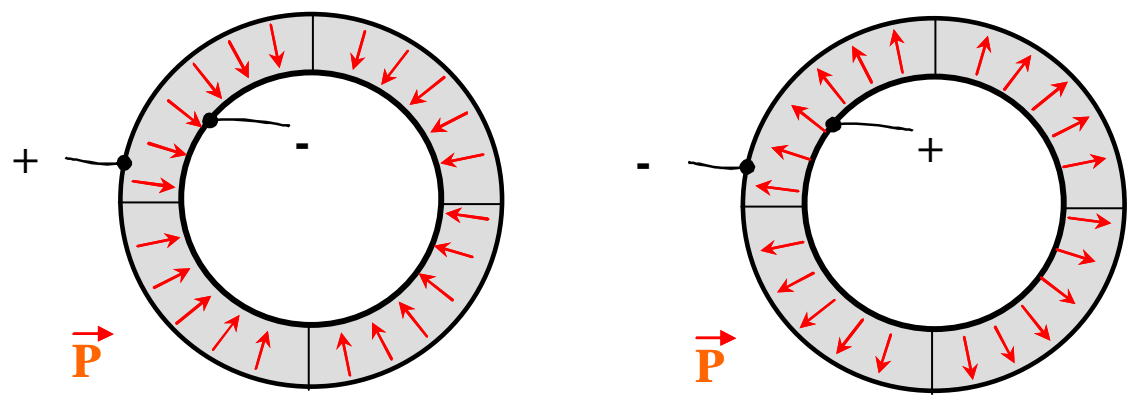

\section{polarization}

Fig. 2.9 Radial polarization of the tube is performed by setting all outer electrodes at the same potential with respect to the inner electrode. The polarization can be done indifferently setting the center electrode at the lowest bias or at the highest. This will result in opposite directions of polarization, and reverse scanning behaviours.

With such tubes, split electrodes allow to move the tube extremity in all 3 directions (see Fig. 2.10). The deflection of the piezoelectric tube works on the principle of a bi-metal: one can apply to two opposite electrodes an electric field in opposite radial direction, by applying them opposite voltages with respect to center electrode as depicted on Fig. 2.10. This results on one side in a dilatation of the wall, meanwhile the other side experiences a contraction. As a result of volume conservation, the whole tube bends in the direction where the radial dilatation has occurred, like in a bi-metal. 

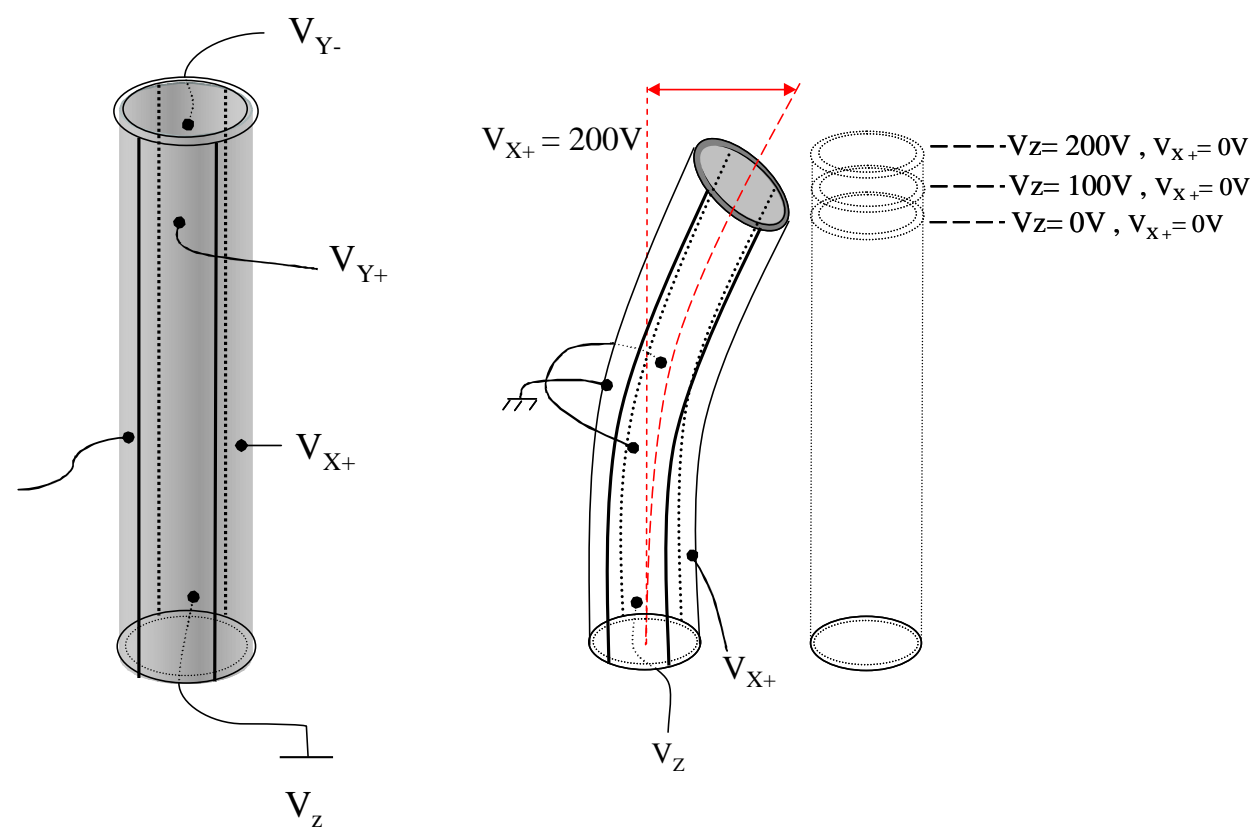

Fig. 2.10 Principle of piezoelectric tube X-Y deflection. In such tube, one uses the $d_{31}$ piezoelectric coefficient, that is, the polarization being along the electric field. When the field is applied uniformly between inner and outer electrodes, the wall thickness increases (decreases) and the tube globally retracts (extends). If the outer (or inner) electrode is cut into different pieces polarized independently, one can deflect selectively in a given $\mathrm{X}-\mathrm{Y}$ direction.

Lateral displacement at the tube end is proportional to the length of the tube and to elongation (contraction), and inversely proportional to the diameter. Elongation (contraction) is itself proportional to length, and inversely proportional to the wall thickness: for maximal displacement one has to choose the longest, smallest and thinnest tube. The drawback of this choice is to lower the resonant frequency of the structure (see discussion above).

Piezotubes are the most commonly used scanners for local probe microscopes.

\subsubsection{3.d Imperfections of PZT ceramics: drift and hysteresis}

Since the macroscopic piezoelectric effect of PZT ceramic devices results from the addition of microscopic contributions, it is subject to the motion of screening charges in the material and to thermodynamic fluctuations. As a result, the material has large drift and hysteresis (typically a sizeable fraction of the total range), as well as noise and energy losses.

In practice, no bi-univocal relationship exists between the position of an actuator and the voltage applied to it: an actual position depends on the history before reaching this point, and, for a fixed applied voltage, the position slowly relaxes (drifts) towards an equilibrium position. These imperfections are particularly pronounced when performing finite frequency motion of the actuator: the amplitude rapidly decreases with frequency and one must take care to avoid having too much internal dissipation which could lead to damaging the device (overheating the glue in stacks, for instance), or partly depoling it. 
As well, the piezoelectric coupling depends on temperature, and this also can cause drifts in position if the temperature changes.

Finally, PZT ceramics are prone to ageing, i.e. they are gradually depolarized, even in absence of a large reverse electric field.

These effects limit SPMs applications, as they hamper the stability of the local probe, the reproducibility of the tip positioning, etc. Modern SPMs overcome these problems by using linearized closed-loop scanners, where the piezo position is constantly monitored and corrected.

Note that these imperfections are not present in quartz actuators.

\subsubsection{3.e PZT ceramics at low temperature}

Fortunately, the above imperfections of PZT ceramics are cured when operating them at low temperature, and PZT actuators become nearly ideal with low losses and hardly any hysteresis and drift at all. This is probably due to the freezing of most mobile screening charges. The ageing is reduced as well, because spurious depolarization occurs at a lower rate.

The counterpart for this improvement in stability is that the piezoelectric coupling constant is considerably reduced, typically by a factor 5 to 10 . Note that contrary to PZT, the Quartz coupling constant very weakly depends on temperature (see Table. 2.2).

Overall, PZT ceramics are very convenient actuators for cryogenic applications: they provide high energy efficiency (thus low spurious heating), and they are very simple to use, with only electrical connections to implement. In practice, they are the most used actuators at low temperature.

In our microscope, coarse motion and fine scanning are made with PZT ceramics parts.

\subsubsection{The piezoelectric tube}

We use a 4 quadrants piezoelectric tube from Politec PI model PT130.94, with an inner diameter of $2.2 \mathrm{~mm}$, an outer diameter of $3.2 \mathrm{~mm}$, a length of $30 \mathrm{~mm}$, yielding at $300 \mathrm{~K} \mathrm{a}$ longitudinal displacement of $2.1 \mu \mathrm{m} / 100 \mathrm{~V}$, and a lateral displacement of $14 \mu \mathrm{m} / 100 \mathrm{~V}$. The maximal voltage one can apply in the direction reverse to polarization is $200 \mathrm{~V}$. The capacitance of each electrode is $2.4 \mathrm{nF}$.

Piezoelectric coupling of the tube is reduced at low temperature roughly by a factor 8 , giving a lateral motion of $1.8 \mu \mathrm{m} / 100 \mathrm{~V}$, and a vertical motion of $150 \mathrm{~nm} / 100 \mathrm{~V}$. The maximum scan range we can reach during our experiments is $\pm 300 \mathrm{~nm}$ vertically, and $\pm 4 \mu \mathrm{m}$ horizontally. 


\subsubsection{Poling procedure}

Due to ageing, or accidental depoling, it is necessary to repolarize the tube, to recover a full piezoelectric coupling. In our case, ageing occurs at low rates of a few percent per year, because we work with the tube only at very low temperatures.

Poling can be done as explained before by applying a very high electric field, however we found it safer and more reliable to heat the ceramic above its Curie temperature to fully release the constraint on dipole orientation, and cool it down while applying a substantial electric field. Curie temperature of our tube is around $250^{\circ} \mathrm{C}$, so we heated it around $350^{\circ} \mathrm{C}$, and the field we used was a little higher than the depoling field, i.e. $510^{5} \mathrm{~V} / \mathrm{m}$.

By monitoring the capacitance of the electrodes before and after the polarization procedure, one notices an increase by $\sim 20 \%$ (from $2.4 \mathrm{nF}$ up to $\sim 3 \mathrm{nF}$ ): indeed, after polarization, all charges have a maximal displacement in the field direction, enhancing the signal measured with the capacitance meter for a given applied voltage amplitude.

Right after polarization, the piezoelectric cell is highly hysteretic, and one has to "cycle" it by applying sinusoidal excitations on the full range of deflection. After a few hours of cautious cycling (not exceeding the depoling field etc), the piezoelectric cells relax and one gets a more predictable and less hysteretic behaviour.

\subsubsection{Calibration at high and low temperatures}

After poling or repoling, a calibration of the tube motion can be done by imaging patterns of known dimensions to determine the effective displacement per volt in the three directions. For this purpose, we use a design grid whose patterns consist in pads of known height and lateral dimensions engraved in the sample. Details of the fabrication are presented in chapter 3.

The scanner controller is a commercial controller model PicoPlus ${ }^{\mathrm{TM}}$ from Molecular Imaging. This controller comes with the software "Picoscan" that includes a calibration script. Other features are included in the software like spectroscopy functions, that record inputs/outputs of the controller versus one another (I(V), I(z), etc...).

The procedure is the following: calibration factors are set to unity (say $1 \mathrm{~nm} / \mathrm{V}$ ) and hysteresis corrections are set to zero. The grid is scanned once, yielding an image which is a priori distorted if performed at room temperature. Dimensions of the grid patterns known from the fabrication are input in the imaging software which deduces axis coefficients, and hysteresis corrections. A subsequent second-order polynomial fit permits to linearize the tube motion. As an illustration, images taken both before and after calibration are presented in Fig. 2.11. 

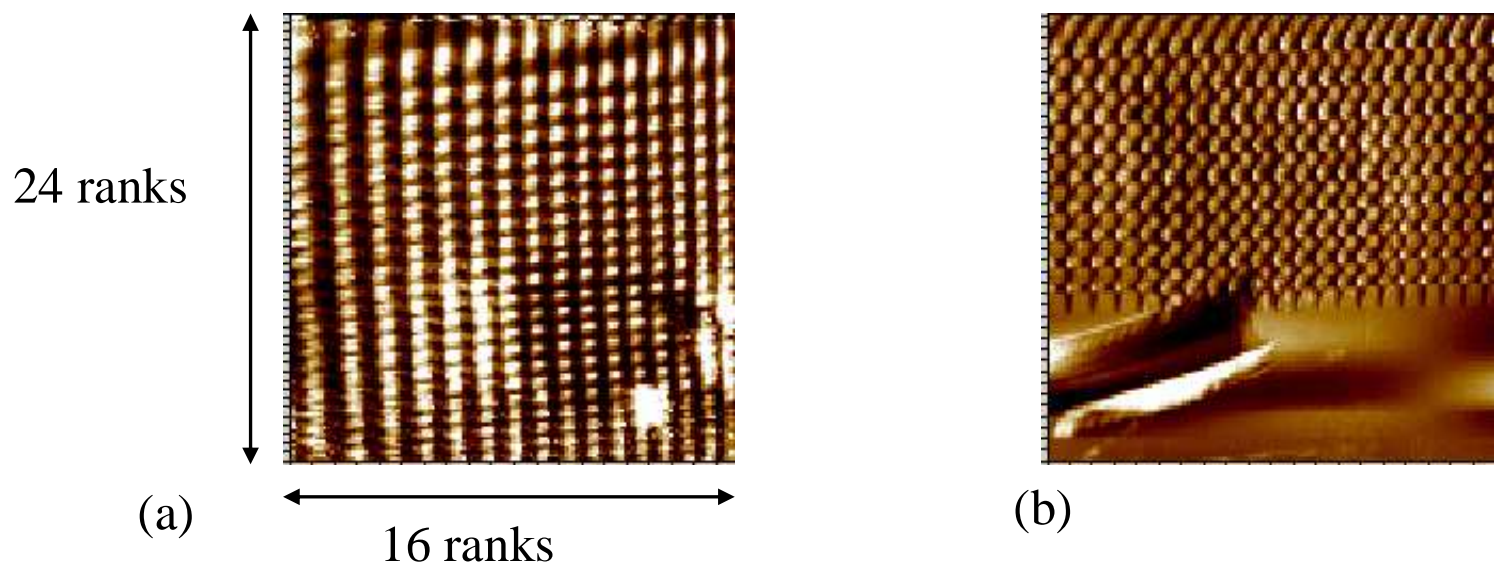

(b)

Fig. 2.11 Images shown are scanned from left to right (fast axis), and from top to bottom (slow axis). (a) before the calibration. (b) once the tube is calibrated (at another position, on the edge of the grid). On the first image (a), one can see the tube is deflected in a highly non linear way: the tube motion follows a second order polynomial profile. The fact that one sees more ranks of patterns on the slow axis is symptomatic of the frequency dependence of the piezoelectric coupling.

The calibration is given as three parameters: the linear deflection in $\mathrm{nm} / \mathrm{V}$, the non linear deflection in $\mathrm{nm} / \mathrm{V}^{2}$, and the percentage of over scan needed to correct hysteresis. Calibration coefficients at ambient temperature are the following:

$$
\begin{aligned}
& \text { fast axis: } 129.9 \mathrm{~nm} / \mathrm{V}, 0.392 \mathrm{~nm} / \mathrm{V}^{2} \text {, } \\
& \text { slow axis: } 133.8 \mathrm{~nm} / \mathrm{V}, 0.19 \mathrm{~nm} / \mathrm{V}^{2} \text {, } \\
& \text { for both axis, the hysteresis is } \sim 10 \% \text {, }
\end{aligned}
$$

$$
\text { Z: } 21.5 \mathrm{~nm} / \mathrm{V} \text {. }
$$

However the built-in calibration procedure is not satisfying, at least for room temperature use. As said earlier, the piezoelectric efficiency is highly frequency dependent. Since images are recorded line by line, one axis is used at a higher frequency than the other. This results in different calibration factors for each axis. As a consequence, the recorded parameters cannot be used for instance for a rotated image acquisition. Ideally, calibration should be done at different scanning speeds, and recalled whenever needed.

As already mentioned, this problem is solved at low temperatures: there is basically no difference between $\mathrm{X}$ and $\mathrm{Y}$, the second order term is negligible (one always remain in linear regime), and there is no hysteresis. The coefficients are then:

$$
\underline{\mathrm{X} \text { and } \mathrm{Y}}: 18.5 \mathrm{~nm} / \mathrm{V}, 0.0002 \mathrm{~nm} / \mathrm{V}^{2} \text {, and } \underline{\mathrm{Z}}: 1.5 \mathrm{~nm} / \mathrm{V} \text {. }
$$




\subsection{Coarse positioning and indexing}

The coarse positioning system of the tip relative to the sample, briefly mentioned previously, is now explained in more details.

\subsubsection{Design \& principle}

Each axis of the coarse positioning system is independent, and they are all similar. A given axis is schematically figured in Fig. 2.12. It is composed of a fixed part comporting a $\mathrm{V}$-shaped groove, on the side of which are glued four shear mode piezo stacks. A mobile $\mathrm{V}$-shaped part comes resting on these four stands, and slides along the groove. The contact surfaces are alumina plates (not shown on Fig. 2.12); one is glued on top of the piezo stacks, and the other one onto the moving part. For the $\mathrm{X}$ and $\mathrm{Y}$ axes, the fixed and moving parts are maintained in contact by gravity, whereas for the $\mathrm{Z}$ axis, the sliding part is gently pressed onto the piezo stacks by soft, adjustable springs.

\subsubsection{Stick-slip motion}

The shear mode piezo stack generates the motion along one axis using the stick-slip mechanism [44] described in Fig. 2.12: during a slow voltage ramp, the deformation of the piezo stacks drives the mobile part by static friction between the alumina plates. Then, in a very fast reverse voltage ramp, the piezo stacks are returned to their original position. During this fast motion, the friction force is not enough to overcome the inertia of the moving part which will essentially remain in place.

Electrically, this is equivalent to slowly charging the capacitance of the piezo stacks, and subsequently discharging it as fast as permitted by the driving circuit.

By repeating these cycles, arbitrary large displacement can be performed, limited only by the mechanical constraints (i.e. size of the moving parts...). In our design the accessible range is about $10 \mathrm{~mm}$ for both $\mathrm{X}$ and $\mathrm{Y}$ axes and $5 \mathrm{~mm}$ for the $\mathrm{Z}$ axis, which is comfortably large for the kind of samples we want to measure. We can even imagine putting two samples side by side in the microscope so that they are measured in the same cooling cycle. 


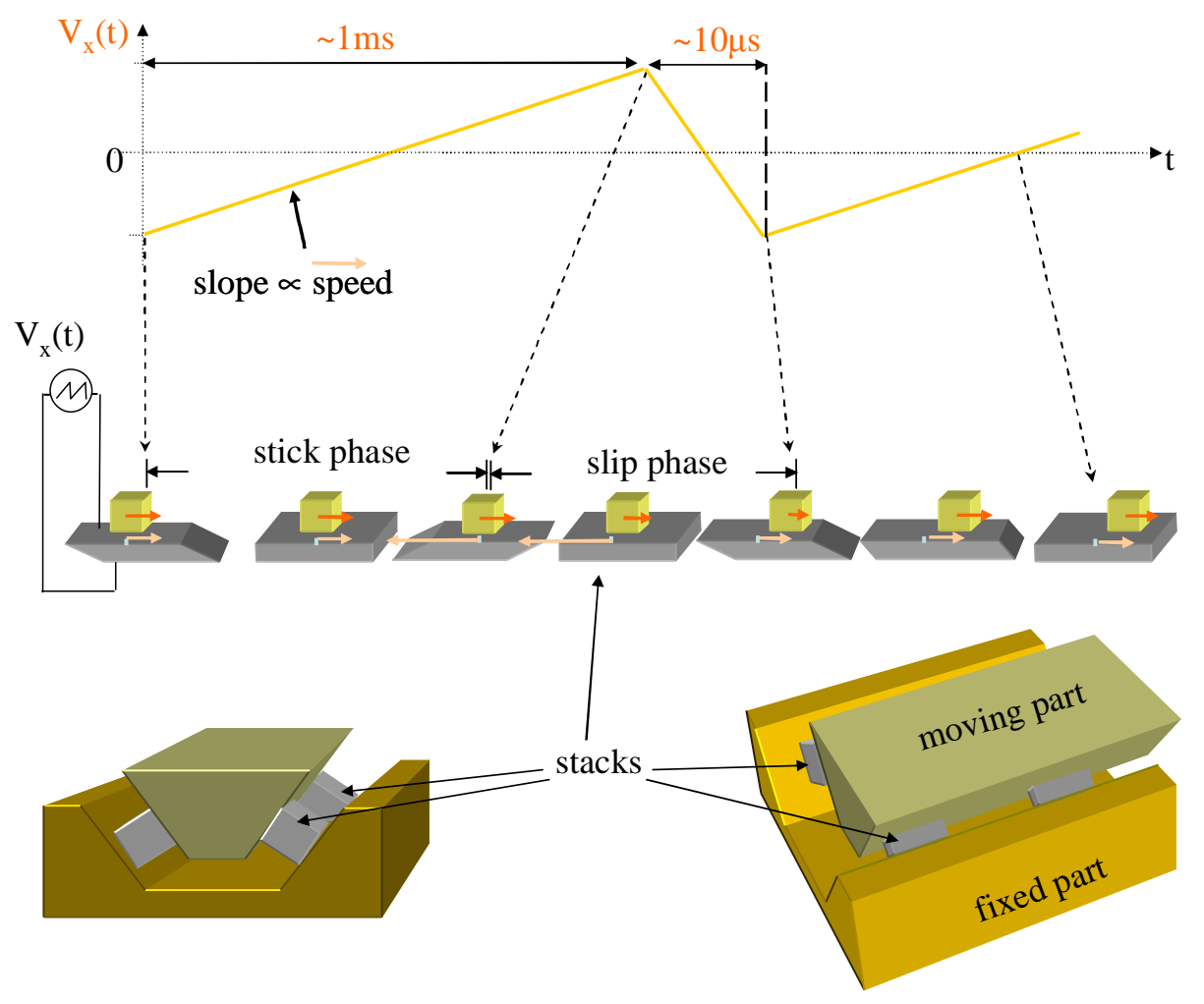

Fig. 2.12 Operation of the inertial stick-slip motors: there are three independent motors, one for each axis, X Y and Z. Each motor is composed of four shear mode PZT stacks of sheets glued by Stycast 2850 FT to one titanium part, say the "fixed part". All stacks are deflected simultaneously with a bipolar voltage. The inertial motion of the "moving part" is initiated by a sawtooth voltage pulse: during the slow slope, the part "sticks" to the stacks, while during the reverse fast slope it slips. Each step of $\pm 160 \mathrm{~V}$ at $30 \mathrm{mK}$ yields a net displacement of $\sim 200 \mathrm{~nm}$. The same displacement is obtained at room temperature using a $\pm 35 \mathrm{~V}$ ramp. Steps can be repeated up to $8 \mathrm{kHz}$ without any significant loss of displacement efficiency.

\subsubsection{Electrical requirements}

Each piezo stack (Politec PI model P-121.03) is composed of 6 ceramic sheets. Four stacks are used to motorize one axis, so that we have to charge 24 sheets, at the same time. Given the dimensions of the piezo sheets $\left(5 \times 5 \mathrm{~mm}^{2}\right.$ area, $0.5 \mathrm{~mm}$ thickness $)$, and the dielectric constant of PZT, the total capacitance per axis is of the order of $15 \mathrm{nF}$.

For a typical voltage ramp of $100 \mathrm{~V}$ and discharge time of about $10 \mu \mathrm{s}$, the driving source must be able to deliver a peak current of about $150 \mathrm{~mA}$ during those $10 \mu \mathrm{s}$. This is important to notice because high voltage sources often have relatively high output impedance. For instance high voltage sources commonly used to drive a piezo scanner cannot deliver such a large peak current. For performing inertial stick-slip motion one often needs to rely on dedicated electronics. For this purpose we use an MSCU® control unit from Omicron. 
Another factor which can limit the discharging current of the piezo stacks is the resistance in the wires and filters connecting the room-temperature source to the microscope in the refrigerator. In our case, the total wiring resistance for an axis, when the refrigerator is cold, is of the order of $600 \Omega$, giving an RC time constant of $10 \mu \mathrm{S}$. Anyhow, I checked that the efficiency of the motors at room temperature was not reduced by the introduction of a series resistance: they work properly with a bias resistance up to more than $1.4 \mathrm{k} \Omega$, without any measurable reduction of the average step size.

\subsubsection{Mechanical performance of the motors}

The behaviour of such an inertial stick-slip motor highly depends on the static and dynamic friction coefficients, which are hardly controlled. Thus, one has to find a trade -off between two opposite behaviors: on one limit, friction is too low, and the object slips even during the "stick phase", which limits the maximum frequency the motors can be driven at. On the opposite limit, friction is too high and the object sticks onto the ceramic during both alternations. Between those two limits, lies a usable dynamic range which is found more or less empirically.

The plates used are $0.254 \mathrm{~mm}$-thick polished sintered alumina plates. Their typical roughness is less than $80 \mathrm{~nm}$ RMS according to manufacturer. They are thick and rigid enough to remain very flat during assembly. Furthermore this material is extremely tough so that it would not wear when used repeatedly.

I found that the procedure chosen to assemble the constituting parts of a motor had a direct impact on its behaviour. The best performance and reliability were obtained when all elements were glued simultaneously to ensure that facing alumina plates are well resting one onto another.

On the other hand, one may fear that very flat plates perfectly facing each other may stick to each other upon cooling down, because of the freezing of adsorbed water on the surfaces.

We found that the "inertial stick-slip" motion works reliably at low temperature, even without special cleaning, or using any dry lubricant onto the alumina plates. This was a rather good surprise, since such actuators are notoriously tricky to operate at low temperature: they tend to stay blocked, or to operate erratically.

In practice, the good performance of the motors can be degraded by its surroundings. In particular, one must take care that all the wiring connecting the microscope, and the copper braids for thermalization, do not limit or hamper the displacements. 


\subsubsection{Inertial vs. non-inertial stick-slip motion}

In the driving mode we use, all four piezo stacks are driven at the same time and require a single driving line.

Several other implementations of stick-slip actuators in SPM rely on controlling individually each piezo stack [45], so that only one stack is sliding at a time. Of course such approach requires a more complex wiring and control process.

An intermediate approach, keeping a simple wiring, consists in dephasing the signal arriving on each stack, by making an RC cascade at low temperature [46]. In this latter approach, however, extra Joule power is dissipated at low temperature compared to our implementation.

\subsubsection{Position indexing}

When the microscope is operated at low temperature, we have no optical means to know whether the motors are moving properly, or to control their actual position. Because of their intrinsic irreproducibility, it is highly desirable to control that the motors move properly and even better to measure their position with sufficient accuracy to enable fast navigation on the samples. I now describe how such a position measurement is performed in our microscope.

\subsubsection{Design}

The benefit brought by a position indexing system should not be outweighed by a too large extra complexity in the setup. In particular, the mechanical assembly must be handy because the microscope should be easily mounted and dismounted, for instance to change the sample. Furthermore, the operation of the position indexing system must also be compatible with the low temperature environment and should be simple. These conditions forbid for instance the use of an optical interferometer.

We met these requirements with a rather simple solution, by incorporating a variable capacitor to each of the stick-slip actuators. These are planar variable capacitors consisting in gold electrodes deposited on the back side of the facing alumina plates sliding one onto another. The alumina plates therefore constitute the dielectric insulator of the capacitor.

The gold electrodes are $12 \mathrm{~mm}$-long, $6.5 \mathrm{~mm}$-wide rectangles on one side, and 12x8 $\mathrm{mm}$ on the other side. Neglecting edge effects, the maximum capacitance (when the electrodes fully face each other) is

$$
C_{\max } \approx \frac{\varepsilon_{0} \varepsilon_{r} W L}{2 t}=13.5 \mathrm{pF}
$$


where $W$ and $L$ are the length and width of the electrodes, $t=0.254 \mathrm{~mm}$ the thickness of one alumina plate, and $\varepsilon_{r}=9.9$ the dielectric constant of alumina.

\subsubsection{Resolution and accuracy of the capacitive position sensors}

To be useful, this position measurement should have a resolution at least equal to the range of the scanner, which is $8 \mu \mathrm{m}$ in our case. Thus, the resolution of the variable $\mathrm{X}$ and $\mathrm{Y}$ capacitances measurement should be better than $(8 \mu \mathrm{m} / 12 \mathrm{~mm}) C_{\max } \approx 6 \times 10^{-4} C_{\max } \approx 8 \mathrm{fF}$. These rather small capacitance values can still be easily measured using standard impedance bridges (so-called RLC meters).

In practice, however, measuring such capacitances requires some care, since the wiring capacitance presents a stray capacitance much larger than $\mathrm{C}_{\max }$, in parallel with the variable capacitor. This would then necessitate a much higher resolution in the capacitance measurements. In our setup, cross-talk in the electrical lines connecting the variable capacitors to the $\mathrm{C}$-meter were carefully considered and minimized by running the cables in different shields.

To circumvent the problem associated with measuring small capacitances, one can think of making capacitors with much smaller dielectric thickness. Actually, we deliberately chose to go the other way because it improves the accuracy of the position measurement, as I will now explain. In fact, in these variable planar capacitors, one has to worry about the thin air gap (vacuum gap at low temperature) between the two alumina plates. The gap thickness depends both on the roughness of the plates and on the mounting of the alumina plates. To be more explicit, in an imperfect setup, a displacement along the axis of the actuator is accompanied by another uncontrolled motion in the perpendicular direction. This changes the average air gap and the value of the capacitance by an amount not related to the actual displacement of the actuator we want to measure. Ideally, the design and the mounting of the system should be such that these parasitic capacitance changes are negligible. 


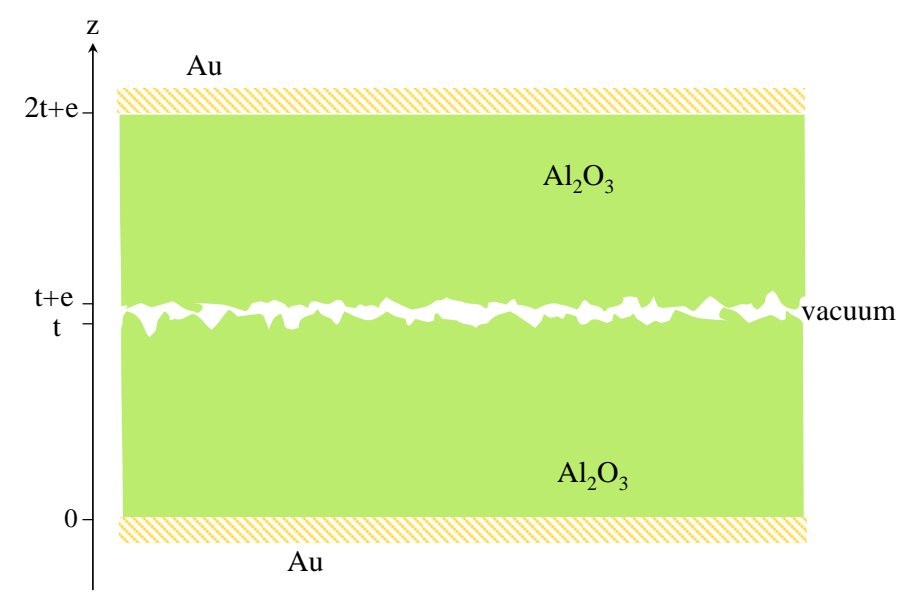

Fig. 2.13 Schematics of the 3-layer capacitance composed by two counterpart alumina plates and a gap of air in between.

Taking into account an average thickness $e$ of the air gap, the effective capacitance of the planar capacitor is:

$$
C=\varepsilon_{0} W L\left(\frac{2 t}{\varepsilon_{r}}+e\right)^{-1}
$$

If one assumes that $e$ takes more or less random values with typical distribution width $\delta e$, the relative change in the capacitance due to this randomness is:

$$
\frac{\delta C}{C}=\frac{\mathrm{d} \log C}{\mathrm{~d} e} \delta e
$$

Optimally, we should make this quantity smaller than the measurement resolution, so that it does not affect our determination of the position. Since it is difficult to control the amplitude $\delta e$, because it depends on how the system is assembled, it is safer to minimize $e / t$ right from the start.

It is this reasoning which led us to put the electrodes on the back side of these rather thick alumina plates. And this is why we are bound to measuring small capacitance values with the associated aforementioned difficulties.

\subsubsection{Practical implementation}

To increase the signal of interest on $\mathrm{X}$ and $\mathrm{Y}$ axis, we measure two capacitance elements, by tooling two neighbouring stacks up with gold plated alumina. The facing alumina plate, glued on the opposite titanium part, has thus two electrodes patterned on it (see Fig. 2.14). Care must be taken to assemble the setup so that the electrodes face each other correctly.

To mount these capacitances in the microscope, first of all, the two piezo stacks are glued with Stycast ${ }^{\circledR} 2850$ FT to their respective alumina plates. Then, all the three alumina plates are glued together with correct spacing using a glue which is soluble in acetone. This insures 
both planarity of the contact between the plates, and correct lateral positioning of the two small plates. After that, all the alumina plates are glued simultaneously onto their respective titanium parts. The removable glue is subsequently dissolved when the Stycast is set.

This procedure ensures as well that potential non planarity or misfit between the titanium parts is corrected.

In order to avoid measuring capacitance between the diagonally opposed electrodes, we set them at the same potential, and measure the sum of capacitance thus given. Fig. 2.14 shows how the motors are installed on one axis X or Y.

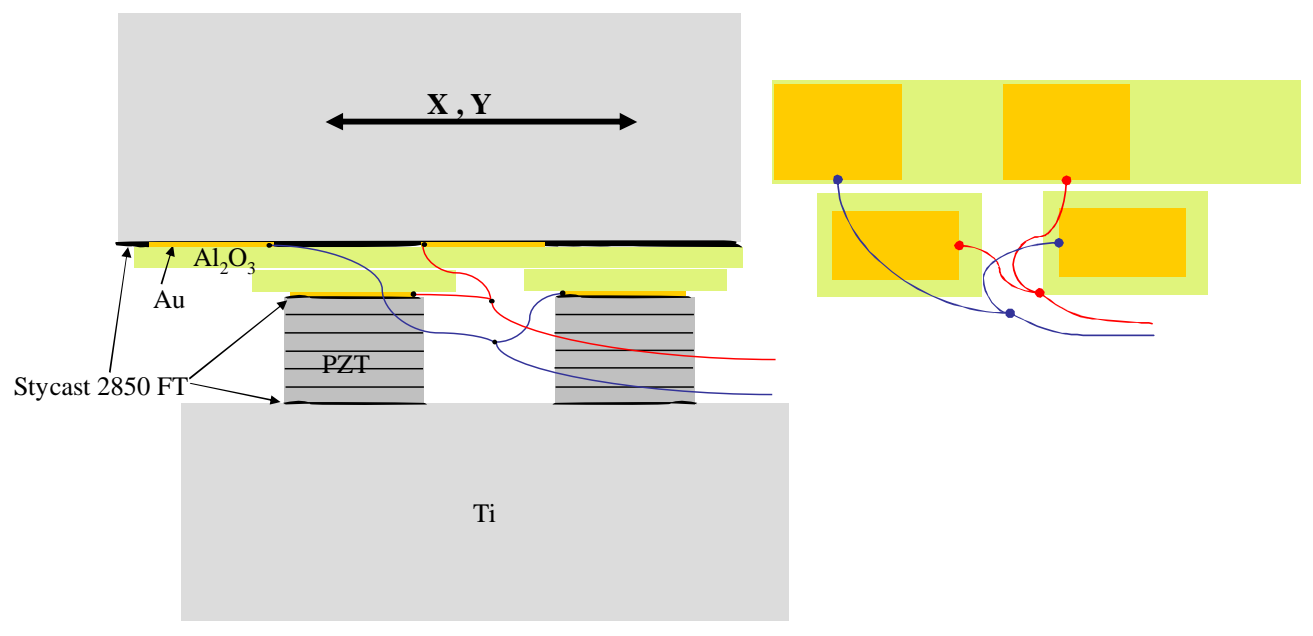

Fig. 2.14 Installation of the piezo stacks and of the gold plated alumina plates, and wiring of the capacitances. The schematic picture presented her stands for $\mathrm{X}$ and $\mathrm{Y}$ axis.

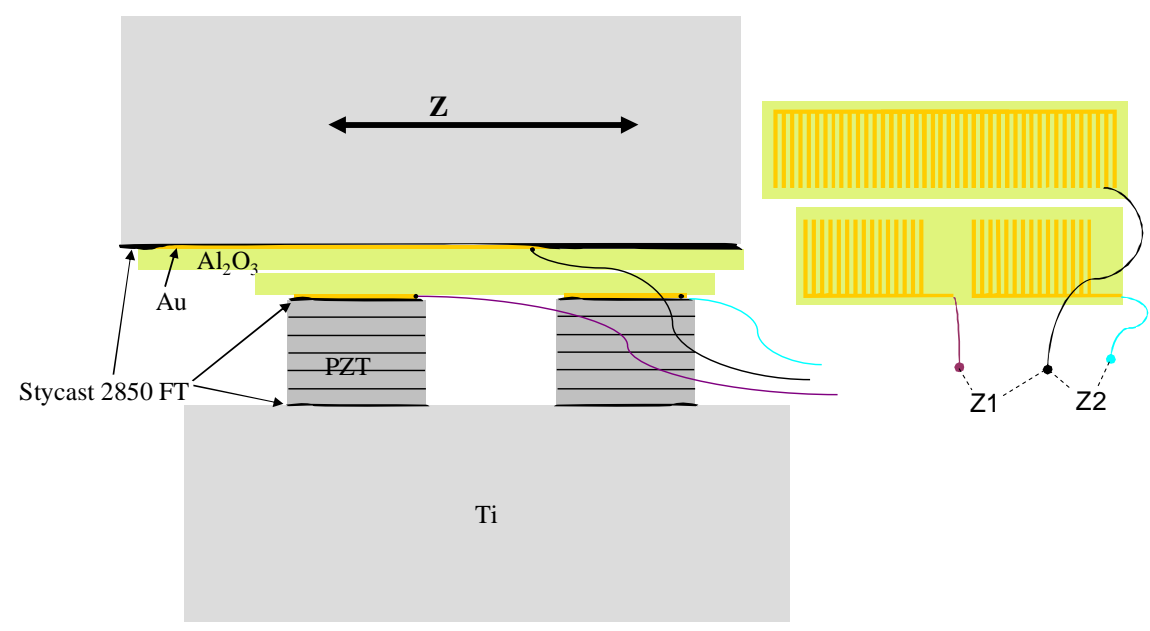

Fig. 2.15 Same installation as in Fig. 2.14 for the $\mathrm{Z}$ axis. The two piezo stacks are linked by the same alumina plate for reasons detailed in the text.

For $\mathrm{X}$ and $\mathrm{Y}$ axis indexing we have rectangular gold electrodes, which provide a linear variation as a function of displacement. For the $\mathrm{Z}$ motor, precision on position is not needed but instead, since the approach procedure involves small steps, we ideally need more sensitivity to displacements. Indeed, during the tip approach sequence, we typically perform 
displacements of $500 \mathrm{~nm}$ at a time, roughly equal to the maximal extension of the tube $(600 \mathrm{~nm})$, and it would be convenient to resolve these steps.

By using fingered capacitances as pictured in Fig. 2.15, instead of rectangles we tried to increase sensitivity. In this configuration, the capacitance variation is sinusoidal, instead of linear, and the absolute position is not known, unless periods are counted from the beginning of the course.

At first glance, the derivative of capacitance with respect to displacement is higher because one adds the signal from all fingers (increasing or decreasing at the same time). However, the edge effect limits the actual advantage of having a large number of parallel eletrodes, and one has to find a compromise: given the thickness of the plates, the optimum period (giving maximum contrast, together with fast enough oscillations) is $1.5 \mathrm{~mm}$.

In order to keep the same sensitivity on the whole period, we use two series of fingers, shifted by a quarter of a period. We read alternatively from one or the other quadrature. Two capacitances are thus measured on the $\mathrm{Z}$ axis.

These quadrature signals are summed with respectively $\mathrm{X}$ and $\mathrm{Y}$ signals, so that we only need 3 wires in all for capacitance measurements: one for $\mathrm{X}+\mathrm{Z} 1$, one for $\mathrm{Y}+\mathrm{Z} 2$, and one common wire. The first two are wired individually in coaxial lines, whereas the third one is part of a multi-wire line (see chapter 3).

Alumina plates were bought from Reinhard Microtech GmBh, already plated with a few nanometers of a tungsten/titanium adhesion layer and with $5 \mu \mathrm{m}$ of gold. Electrodes are patterned by optical lithography, and wet chemical etching. Gold is etched in a warm $\left(30^{\circ} \mathrm{C}\right)$ $\mathrm{KI}+\mathrm{I}_{2}$ agitated solution (in $\sim 1$ hour, no sonication), and $\mathrm{W} / \mathrm{Ti}$ is etched in a $\mathrm{H}_{2} \mathrm{O}_{2}$ solution.

\subsubsection{Testing actuators and sensors.}

The RCL meter used for capacitance measurements (LCR819 from INSTEK) displays a 5digit value and is specified for $20 \mathrm{fF}$ maximal precision when measuring a typical $40 \mathrm{pF}$ capacitance at $20 \mathrm{kHz}$ during $1 \mathrm{~s}$. For a $3 \mathrm{~s}$ long measurement of our variable capacitance at a fixed position, we obtain a typical accuracy of $5 \mathrm{fF}(\sim 5 \mu \mathrm{m})$ that sets the position resolution.

Given that, we could check the motors behaviour and calibrate the variations of the capacitance for a given displacement of the motors, at room temperature.

We present in Fig. 2.16 a record of the capacitance as a function of the number of steps performed, on both $\mathrm{X}$ and $\mathrm{Y}$ axis, on the whole range of displacement (i.e. $12 \mathrm{~mm}$ ), and on $\mathrm{Z}$ axis in a shorter range. 
This test was performed starting from one extremity of the course of $\mathrm{X}$ and $\mathrm{Y}$ motors, applying a given number of voltage ramps of $70 \mathrm{~V}$ amplitude at $200 \mathrm{~Hz}$, and measuring capacitance variations after each series of pulses.

Doing this, we cannot distinguish between two separate behaviours: the true variation of the capacitance with actual displacement, and the true displacement of the motors. The first is assumed perfectly linear, at least far from the edges, whereas the second is less predictable. We attribute the deviations from linear behaviour to bad functioning of the motors, except at the start and end points.

A check of the capacitance variations alone should consist in measuring separately the actual displacement with an interferometer for instance.

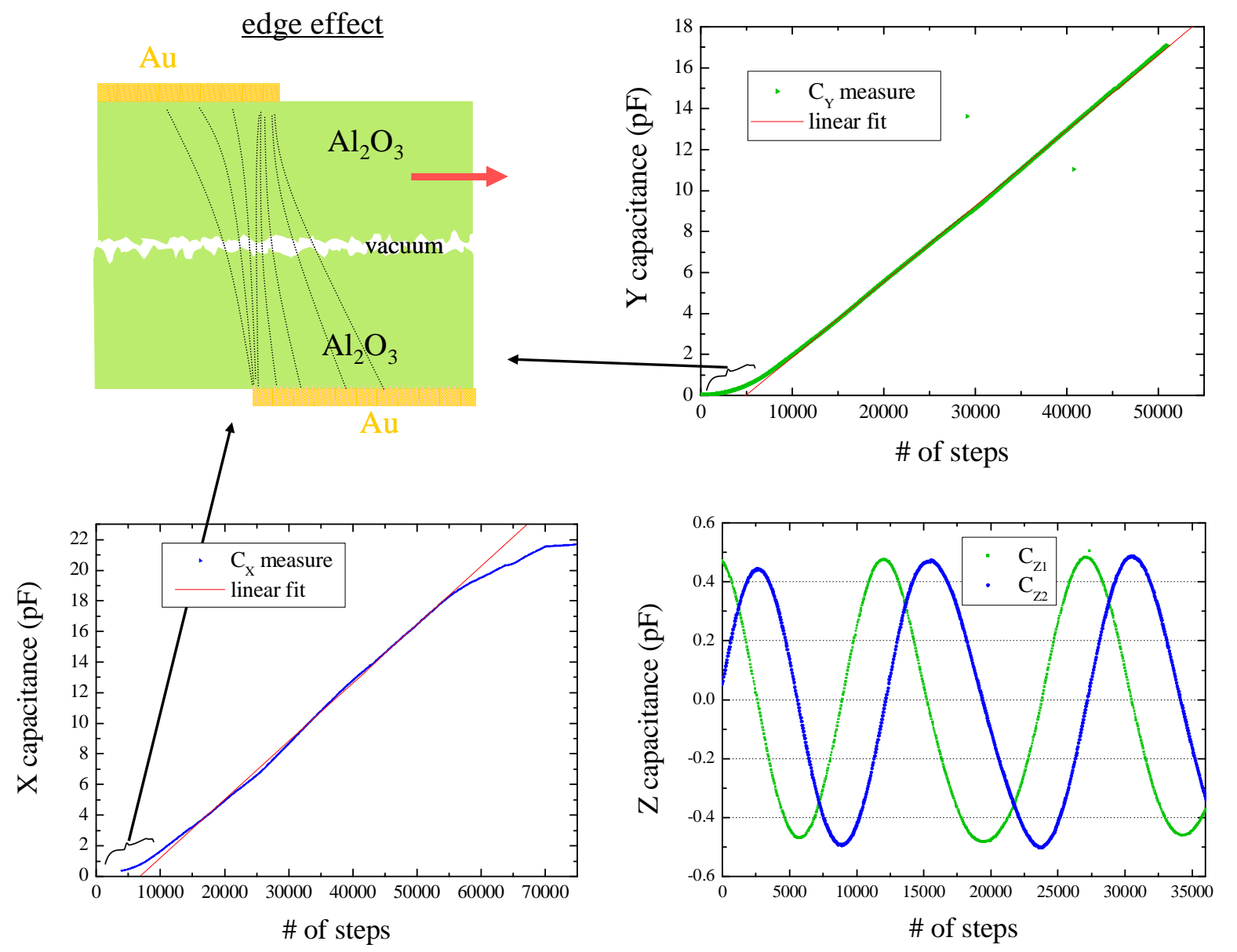

Fig. 2.16 Measurement at room temperature of the capacitances of the three axes when moving the motors by applying voltage ramps of $70 \mathrm{~V}$ amplitude at $200 \mathrm{~Hz}$. On the whole travel length, which is $12 \mathrm{~mm}$, the $\mathrm{X}$ and $\mathrm{Y}$ axis respond quasi linearly. At starting and ending points, when the electrodes do not cover each other, one observes edge effect. A linear fit of the central part allows to calibrate the capacitances at room temperature, assuming the linear range is $12 \mathrm{~mm}$. The $\mathrm{Z1}$ and Z2 signals are plotted on the right bottom side: a constant value has been subtracted to plot their variations around zero. The procedure has been done in both directions (up and down) and the curves are similar, except of course that the efficiency is lower by 20\% in the upwards direction. 
The linear fits of the $X$ and $Y$ motors give a value of $2 \mathrm{fF} / \mu \mathrm{m}$, nominal variation being $2.25 \mathrm{fF} / \mu \mathrm{m}$. The $\mathrm{Z}$ axis capacitance has a maximal variation of $2.5 \mathrm{fF} / \mu \mathrm{m}$. This value should be increased, for instance by using thinner and/or wider plates, because the current setup has proven not fully satisfactory. Indeed, the achieved resolution is a factor 4 to 5 higher than the small approach steps.

This $\mathrm{X}, \mathrm{Y}$ calibration was used at low temperature to position the tip over the sample, assuming constant dielectric and mechanical parameters of alumina upon cooling down.

Until now the motors have proven to work reliably at low temperature, by never being blocked for instance, and with relatively good reproducibility (though less than at room temperature). The well-behaved quasi-linear responses at room temperature, as well as the overall good functioning at low temperature validate our design of the microscope and the procedure for the assembly of the motors. 


\subsection{The atomic force sensor}

Binnig and co-workers implemented the first atomic force microscope in 1986 [47] in order to measure forces as small as $10^{-18} \mathrm{~N}$, with in mind applications in the field of nanotribology. They revisited the concept of the stylus profilometer (one could also evocate the phonograph), scaling it down to access inter-atomic interactions. They wanted to go beyond the native limitation of the STM, invented 5 years earlier (see chapter 1), which does not allow to probe a non-conducting material. In their original design, a soft lever to which was attached a sharp tip was probing the surface, and an STM was used to monitor the deflexion of the lever.

Since 1986, Atomic force microscopy has been developed in various domains. This field has brought great progress in surface science by allowing to access surface properties down to the atomic scale, involving various forces such as electrostatic, magnetic forces, force spectroscopy, tribology, etc. AFM has even been used as a lithography tool to manipulate and pattern nanostructures, and massively parallelized integrated AFMs were proposed as scheme to make read/write memory devices [48].

An atomic force microscope probe sensor is generically constituted of a lever to which is attached a tip. When approaching the tip from a surface, the short range forces affect the whole sensor, changing the lever static and dynamic properties. Surface imaging is done by scanning the tip above the sample and monitoring an appropriate sensor variable, such as its static deflexion or the amplitude of a resonant mode. In most cases, the dynamic range of the AFM is increased by implementing a feed-back mechanism which maintains the measured variable at a constant set point while scanning.

There are several ways of acquiring topographic images:

- Maintaining the tip in close contact with the surface all the time. This is the "contact mode", in which one detects the lever static deflection. In this mode, however, the tip generally wears quite rapidly, and there is a possibility that what is observed on the sample is due to the action of the tip.

Other modes are dynamic, with the tip performing an oscillatory motion. Dynamic AFM experiments are done by exciting the lever in a resonant mode. When the tip interacts with the surface, the lever resonance is modified. The dynamic modes file in two categories: 
- The tapping mode where the tip "hits" the surface at every point of the image. This mode yields the highest resolution, and much reduces the problem of surface and tip deterioration with respect to contact mode.

- The non-contact mode where the tip remains in the attractive field of the surface, and never reaches the repulsive part. The lateral resolution is degraded due to a larger tip-sample distance, but this allows a larger imaging speed.

\subsection{1 implementing AFM sensors at low temperature}

Among the many possible force detection methods, we need one suitable for operation in a dilution refrigerator. Despite their widespread development, atomic force microscopes have not been easily adapted to cryogeny, because of the difficulties to fit the force sensors to cryogenic environments.

\subsubsection{Optical sensors}

Indeed, the standard method used in commercial AFMs consists in measuring the deflection of the lever with a laser beam focused on its end (see Fig. 2.17), and reflected towards a 4 quadrants diode. In this approach, both the laser and the detector use semi-conductors which do not function at low temperatures since their charge carriers are frozen. It is still possible to use this technique in a low temperature AFM, by deporting the laser and diode outside of the fridge and using an optical fiber [49] to bring in and out the laser signal. Such a setup was successfully operated at $4 \mathrm{~K}$ [50] and down to $20 \mathrm{mK}$ [51]. Others have modified the photodiodes to work under vacuum and low temperatures [52], though not at temperatures below 4K. However the optical alignment remains a delicate issue in those setups, especially because differential thermal contractions of the materials employed cause spurious displacements of the laser beam upon cooling. 


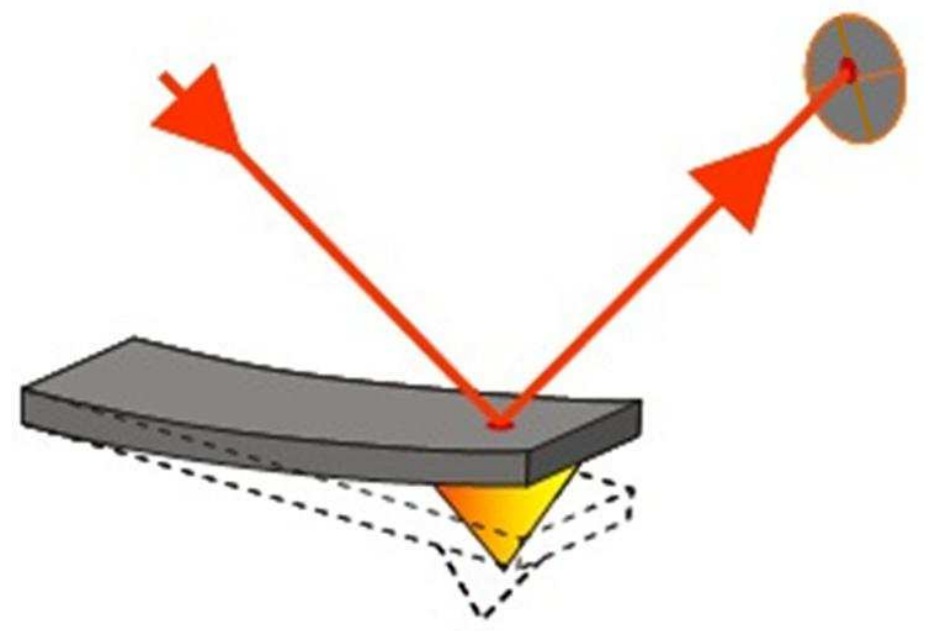

Fig. 2.17 Illustration of standard AFM deflection measurement. A laser beam is reflected on the end of the lever towards a 4 quadrants diode which measures the deflection of the lever either statically or dynamically.

Moreover, when operating at dilution fridge temperatures, the cooling power is very limited (of the order of $40 \mu \mathrm{W}$ at $100 \mathrm{mK}$ in our case), and the laser itself (with typical power in the $\mathrm{mW}$ range) would likely bring too much heat load to the fridge.

Finally, in low temperature experiments, the laser photons have energies much larger than that of thermal fluctuations and could cause unwanted excitations in the sample.

\subsubsection{Electrical sensors}

Given the above problems associated with optical detection, one quite naturally thinks of electrical detection.

Indeed, the use of piezoelectric and piezoresistive materials for local probe sensors was introduced just a couple of years after Binnig's original AFM publication. Those materials overcome the problems described above for low temperature experiments.

First came the piezoelectric quartz tuning fork ([53]) and then piezoresistive cantilevers $([54,55,56])$. In the latter, a deformation is converted into a resistance variation, which, with suitable design and operating conditions, can lead to detectable deflections as low as $0.01 \mathrm{~nm}$. However, given the intrinsic sensitivity and noise of these sensors, such performance with a reasonable bandwidth requires operating the sensor at large bias voltage, with a dissipated power in the $\mathrm{mW}$ range, which again is not suitable for very low temperature.

Thus, upon examination, the only remaining option for very low temperature AFM is the use of piezoelectric devices. In these devices, one naturally obtains low dissipation electromechanical oscillators, which are very simple to integrate in an electric circuit and to operate in a dilution refrigerator. 
Compared to optical and piezoresistive cantilevers, these sensors are only operated in dynamic mode. On the other hand, for this dynamic mode, piezoelectric sensors incorporate their own dithering actuator "for free", whereas the other types of sensors require a separate device to excite the resonator (a device which, ironically, is usually a piezoelectric element). Hence, regarding their implementation, piezoelectric sensors are the simplest possible AFM sensors.

In our microscope, the AFM sensor is a piezoelectric tuning fork which is intrinsically a high -Q resonator. This high -Q provides high sensitivity, which allows very low excitation amplitudes (of less than $1 \mathrm{~nm})$, with a minute dissipated power $(\sim \mathrm{pW})$.

\subsubsection{Quartz Tuning Fork (TF)}

The tuning fork was invented in 1711 by a trumpeter named John Shore, to ease tuning trumpets correctly. It is made of two parallel beams linked together by a common base (see Fig. 2.18 and Fig. 2.24).

\subsubsection{A high $Q$ harmonic oscillator}

The tuning fork is a harmonic oscillator with inherent high quality factor. The main resonant mode of the tuning fork is a symmetric mode with respect to the symmetry planes of the device (see Fig. 2.19).

This symmetric resonance is commonly used to provide the correct tune to musicians. The use of this peculiar fork shape instead of a single beam with the same resonant frequency is easy to understand: in the symmetric resonant mode, the motion of the arms is totally decoupled from the motion of the center of mass, and the frequency and the quality factor are then independent of the holder, but only depend on the damping within the material and surrounding air. By choosing stiff materials with low loss, the tone is accurate, lasts long and enables the musician to tune with a single bow on the tuning fork.

\subsubsection{Tuning forks for atomic force microscopes}

The first implementation of quartz tuning forks in local probe microscope was in a Scanning Near-field Acoustic Microscopy (SNAM) experiment by Günther et al. and dates back to 1988 ([53]). The growing use of tuning forks in different branches of local probe microscopy was initiated by the need to suppress light from laser-based detection and to ease experimental setups. Karrai and Grober for example expanded its use to Scanning Near-field Optical Microscopy (SNOM) in 1995 [57]. 

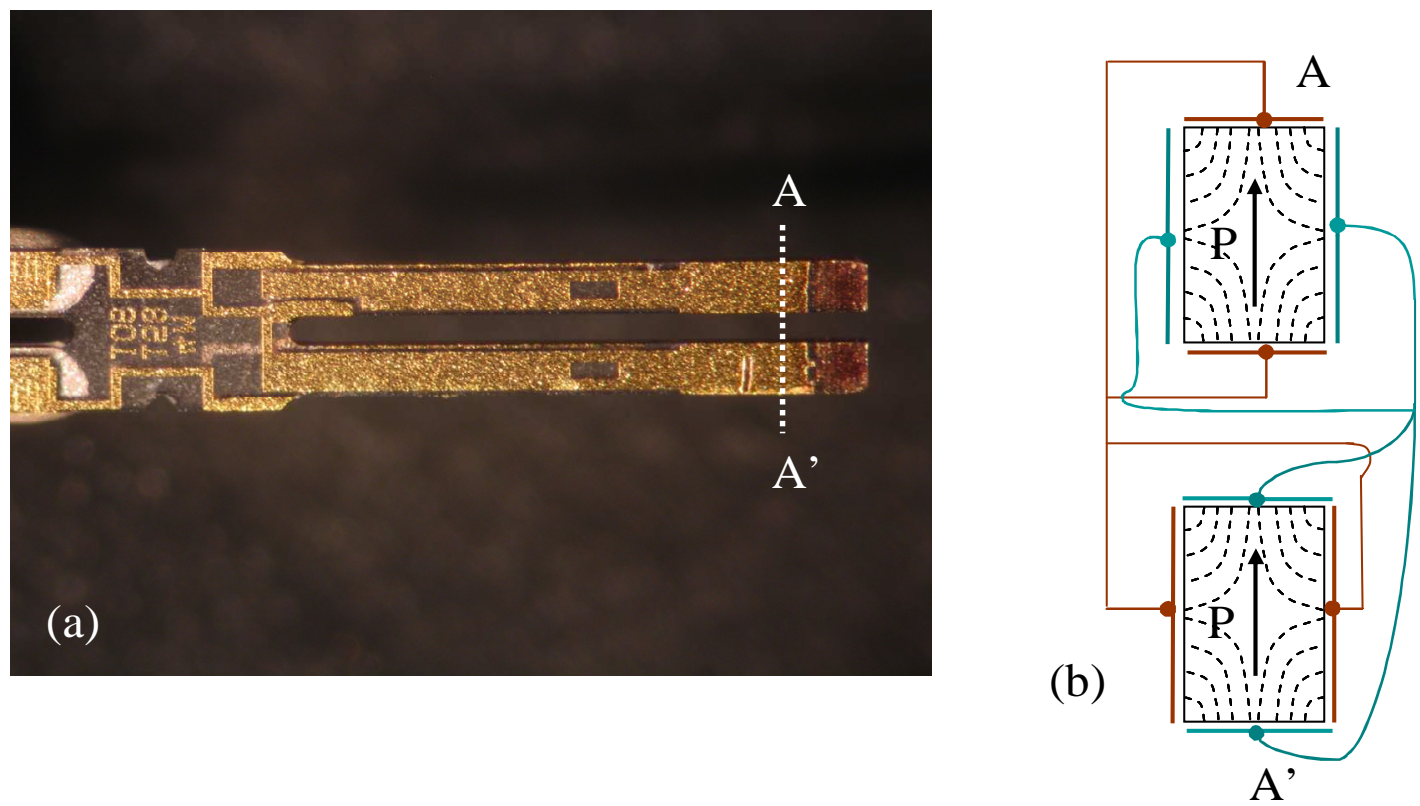

Fig. 2.18 (a) Photograph of a piezoelectric quartz tuning fork used in our AFM microscope. Gold electrodes are deposited on top of the quartz, in a special shape designed to excite selectively the symmetric mode (i.e. both arms move in phase opposition). (b) Schematic of a cross section A-A' of the tuning fork, showing the connections of the electrodes. $\mathrm{P}$ is the intrinsic polarization of the piezoelectric quartz. The dotted lines represent the field lines.

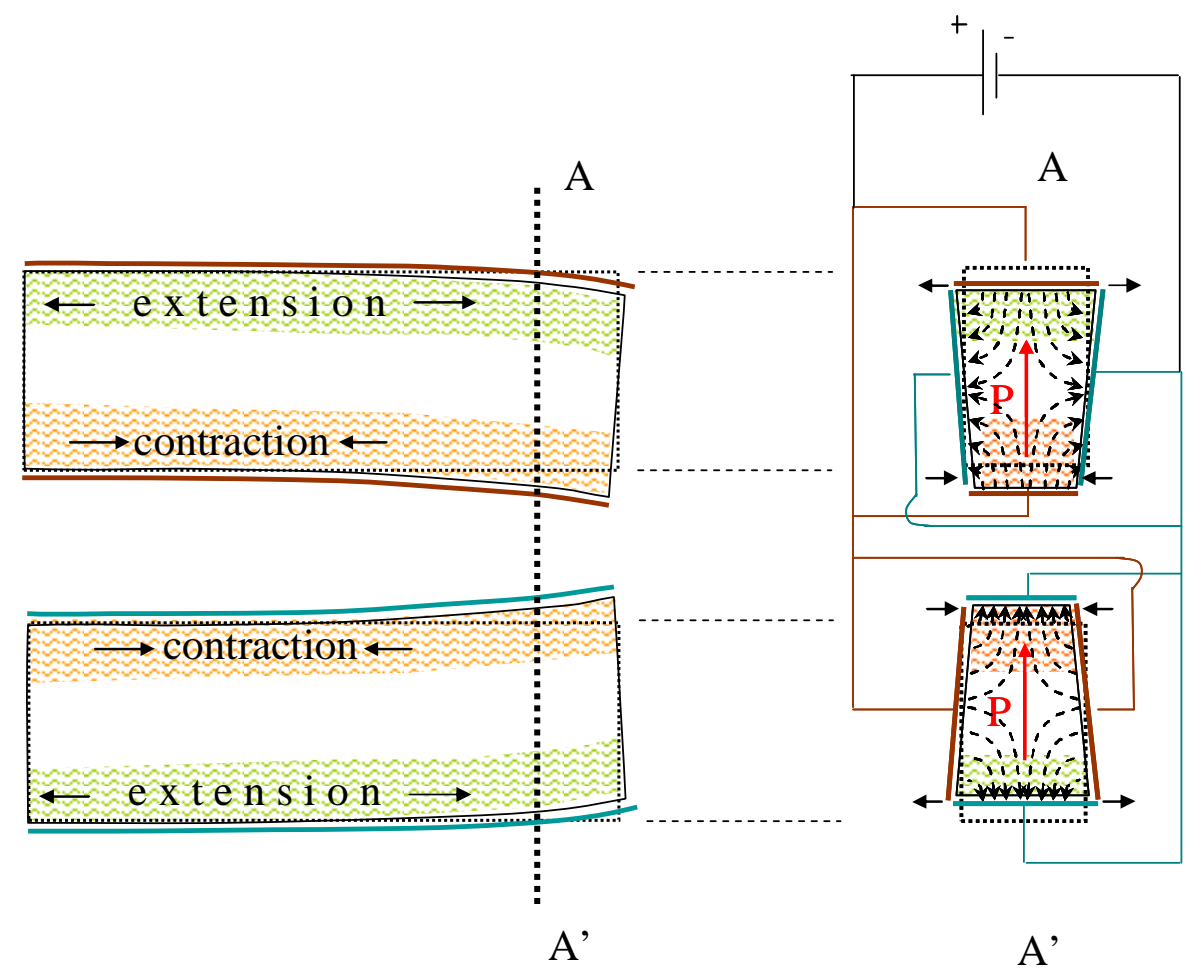

Fig. 2.19 The motion of the prongs can be explained as follows: when a potential difference is applied to the electrodes, opposite charges appear on facing electrodes, so that the arms deflect in opposite directions. To be more specific, when for instance polarization is opposite to electric field, dipoles experience a vertical contraction, and due to volume conservation, perpendicular directions experience a dilatation (see \$2.2.2.3.c). Moreover, if the upper region of the cross section experiences an extension, the lower part will experience a contraction since the electric field reverses in the arm, as depicted by the field lines. These stresses lead to a bending of the arm. The other arm being connected the opposite way simultaneously bends the opposite direction, yielding a symmetric motion. 
The Tuning Forks (TFs) used in AFM are standard parts mass-produced by the clockwork industry. They are made of monocrystalline piezoelectric quartz, a material with high stiffness and low loss. They are engineered [58] so that the symmetric mode frequency is accurately $32768 \mathrm{~Hz}\left(2^{15} \mathrm{~Hz}\right)$, designed to provide the $1 \mathrm{~s}$ time base of low power electric circuits (wristwatches for example) upon division by a simple shift register. Engineering also optimizes the stability of oscillation parameters versus temperature ${ }^{(1)}$, and the balance of the fork's arms (generally involving post-fabrication laser trimming).

To fabricate TFs, a quartz monocrystal is cut such that polarization is nearly ${ }^{1}$ aligned along the arms' thickness (see Fig. 2.18), and metallic electrodes are deposited on the arms in a way that only the symmetric mode can be electrically excited (see Fig. 2.18). Conversely, when a motion of the arms is initiated by an external force, only its symmetric part will be detected via the electrodes.

As fabricated, forks are sealed in a small can under rarefied atmosphere, so that their $Q$ is typically $10^{5}$ at room temperature. We have to remove this case for our application. We do this manually on a lathe, and we end with a bare tuning fork as shown in Fig. 2.18. We subsequently glue the fork on a stand, connect it, and then glue a tip on the end of the lower arm, as shown on Fig. 2.20.

\footnotetext{
${ }^{1}$ The so-called $+5^{\circ} \mathrm{X}$ cut of the quartz crystal, in which polarization is tilted by $5^{\circ}$ off the plane, is purposely chosen to cancel at first order the temperature dependence of the resonance frequency of the fork at room temperature.
} 


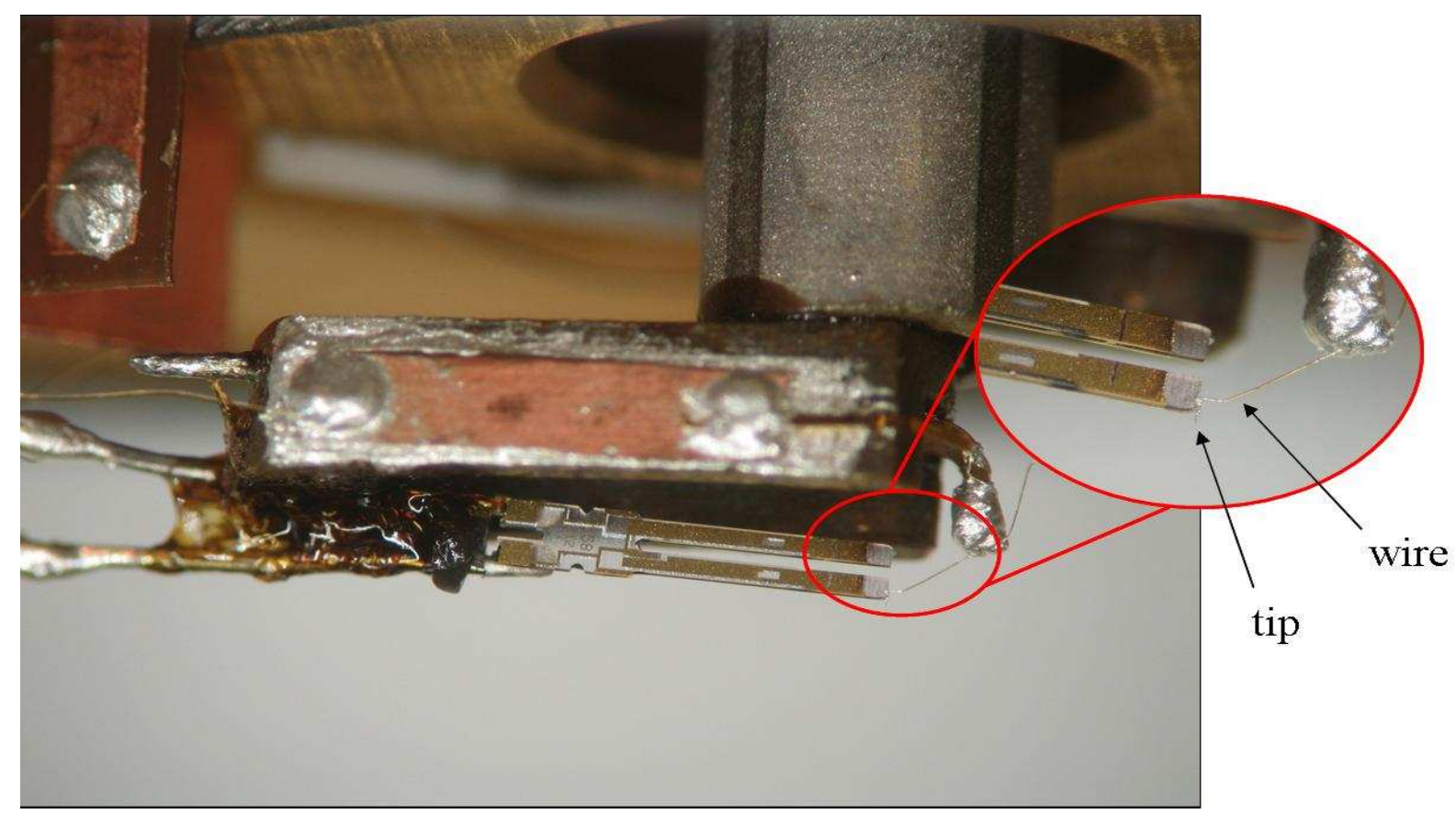

Fig. 2.20 Tuning fork fully mounted, hold at the end of the piezoelectric tube. The base of the tuning fork is glued onto the support, and the support itself is glued to the tube using GE varnish. The tip and the wire are glued with conductive silver epoxy Epotek H20E from Polytec PI. Both arms of the fork are free to move. Note the tuning fork is hold in a way that the tip is roughly aligned with the axis of the tube.

In our setup, we chose to connect the tip to the tunnel current amplifier through an additional wire, like in Refs.[59,60]. It would have been possible instead (and indeed much easier) to connect the tip to one of the electrodes of the tuning fork.

This brings benefits and raises issues:

- The tunneling current can be measured independently of the piezo current. This enables, to perform simultaneously STM and AFM measurements, at least in principle. If this separation is not done, tunneling current and piezo current mix and controlling the system becomes harder.

- This wire adds mass on one of the fork's arms. Doing so, it seems we unbalance the tuning fork more than if we only mount a tip. However, as we will see below, the wire also brings stiffness, which gives the opportunity to better preserve the fork's balance.

\subsubsection{Oscillating modes of the tuning fork}

A finite element modeling (FEM) performed on the geometry of our forks yields their eigenmodes of oscillation. Next figure shows the first six resonant modes, along with their frequencies. Out of these modes, only one (the symmetric mode, labeled 4) does not induce force or torque on the support. It will hence have the highest quality factor. 
By design, the geometry of the electrodes patterned on the tuning fork is such that for all modes except the symmetric mode (4), the electrical signal cancels. Thus, electrically, one can only induce and detect this mode.

(1)

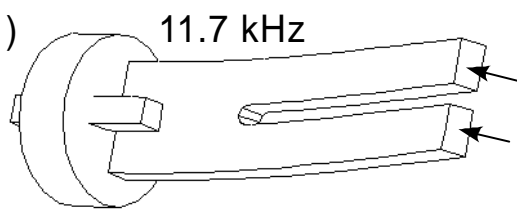

(3)

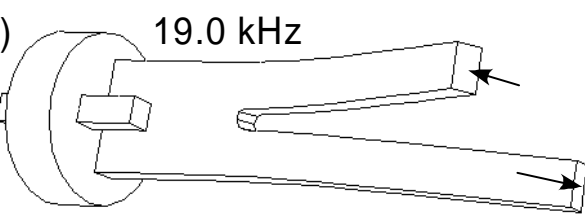

(5)

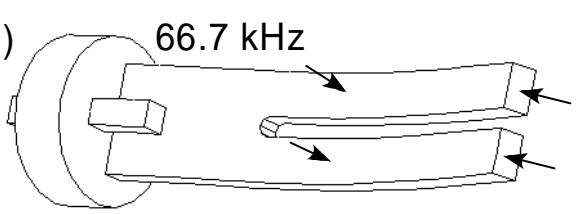

(2)

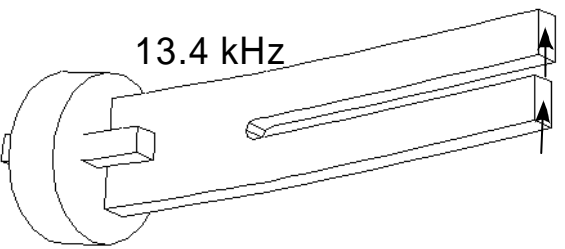

(4)

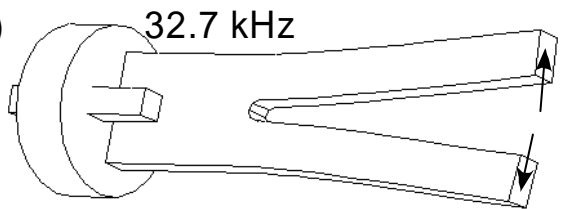

(6)

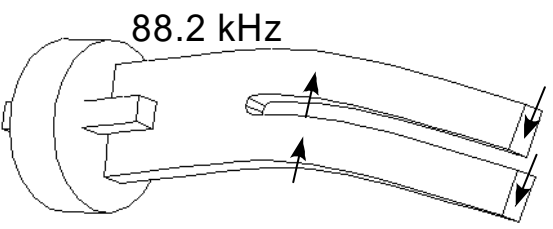

Fig. 2.21 First six resonant modes of the tuning fork. The frequencies are obtained by a finite element modeling. In these simulations the circumference of the cap is fixed. The three in-plane modes (24-6) can be described in a 3-Degrees of Freedom (DOF) oscillator model with three masses and three springs (see $\$ 2.4 .2 .6$ ).

\subsubsection{Basic analysis of a tuning fork: 1 Degree-of-Freedom harmonic oscillator model.}

Let's first assume for the time being that the tuning fork can be described as a mechanical harmonic oscillator with a single degree-of-freedom (DOF). This corresponds to practical cases where the tuning fork is mounted as indicated in Fig. 2.22, in which only a single beam of the fork moves. While it does not correspond to our setup, it has been actually used in some experiments (see e.g. [61]). 

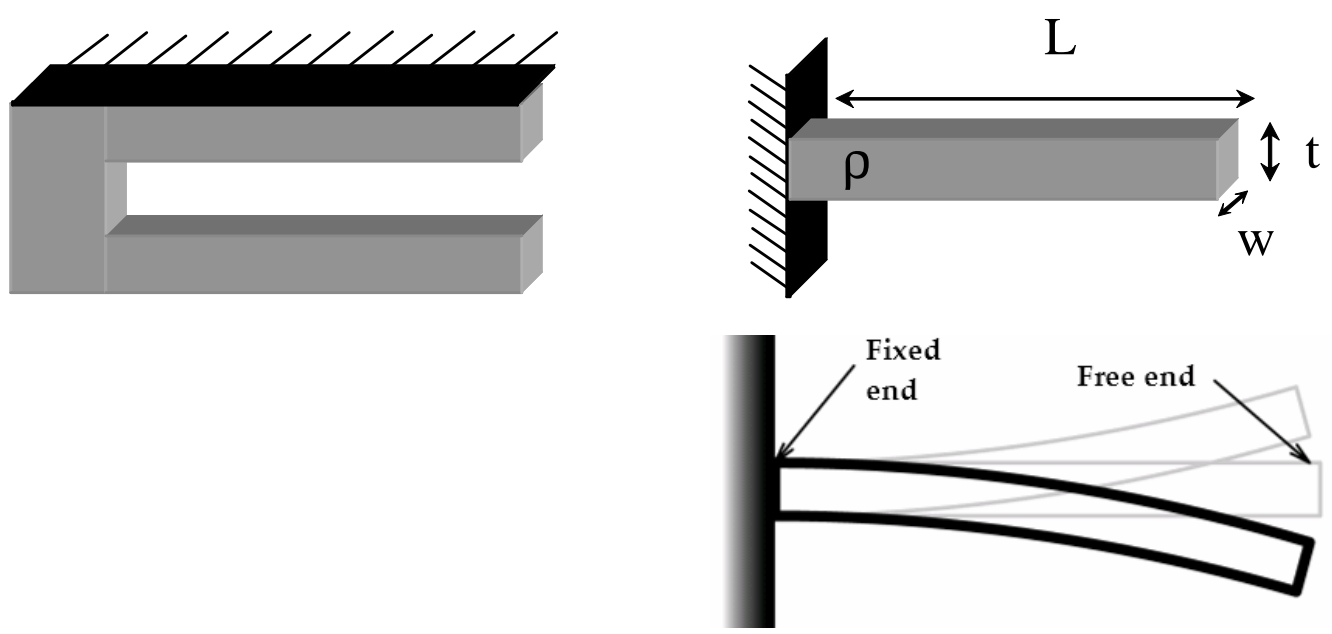

Fig. 2.22 An equivalent to the harmonic oscillator model with one DOF is represented here where the tuning fork is attached by one arm to ground. This yields the same result as a single beam attached at one end. Theory of elastic beams provides the correspondence between the geometrical parameters of the beam and the effective mass and spring constant of the corresponding 1-DOF model.

Even when the two arms are free to move, in the symmetric mode, the motion of one beam can be approximated by the mode of a fixed-free beam, neglecting the deformation in the base of the tuning fork.

We will thus first assume we can describe the tuning fork as such as single degree-of-freedom (1-DOF) harmonic oscillator. This, simple picture allows to obtain a good initial understanding of how the force sensor works.

\subsubsection{4.b Mechanical analysis}

In this framework, one can use the results from the theory of elasticity, to obtain the characteristics of the oscillator from the dimensions of the beams and quartz properties [62].

In this case, for a homogeneous parallelepiped, one can predict the stiffness of the beam as measured from its free extremity:

$$
k=\frac{Y t^{3} w}{4 L^{3}}
$$

and the frequency of the resonant modes:

$$
\omega_{i}=\sqrt{\frac{Y}{12 \rho}} \frac{t x_{i}^{2}}{L^{2}}
$$

where $\rho$ is the volumic mass and $Y$ the Young modulus of the material, $L, w$ and $t$ are respectively the beam length, width and thickness, and $x_{i}$ the roots of $\operatorname{Cosh} x \operatorname{Cos} x=-1$ :

$$
x_{i} \in\{1.8751,4.69409,7.85476,10.9955, \ldots\}
$$


The mode of interest to us is the first one. Note that these formula only approximately predict the properties of a tuning fork, as many details are not taken into account in this simple modeling: the anchoring of the beam is not absolutely fixed in an actual tuning fork (the base also deforms), the electrodes are not taken into account, and, finally, elastic properties of quartz are not fully isotropic.

As seen from its free extremity, when considering only the fundamental mode ${ }^{1}$, such a beam can be modeled as a simple 1-DOF mass and spring system, with the above calculated spring stiffness and an effective mass $m_{\text {eff }}=k / \omega_{1}^{2}$. This model is depicted in Fig. 2.23. Our forks have the following specifications: the arms are $2.4 \mathrm{~mm}$ long, $214 \mu \mathrm{m}$ thick and $130 \mu \mathrm{m}$ wide. Therefore, their spring constant is $k \sim 1800 \mathrm{~N} / \mathrm{m}$ (see Table. 2.4 for young modulus of quartz), and the effective mass is $m_{\text {eff }} \approx 43 \mu \mathrm{g}$, to be compared to the weight of one arm $m_{\text {arm }} \approx 178 \mu \mathrm{g}$.

\subsubsection{4.c Tip-sample interaction}

We now come to the point of analyzing how the tip-sample interaction modifies the fork's resonance, within the above 1-DOF model. We also take into account the piezoelectric coupling and further assume that the oscillator is driven by a low impedance source, so that voltage is imposed from the outside. Proportionality between position and charge mentioned in \$2.2.1.2, allows for two equivalent representations of the resonator: one mechanical, and one electrical. These representations are depicted in Fig. 2.23, and correspondences among parameters in both languages are given in Table. 2.3. In the absence of tip-sample interaction, the bare resonance pulsation is $\omega_{1}=\sqrt{k / m}=1 / \sqrt{L C}$. When the oscillator is subject to both a driving voltage and an interaction force, its dynamics obeys the mechanical equation:

$$
m \ddot{z}+g \dot{z}+\left(z+z_{0}\right) k=F_{\text {int }}\left(z+z_{0}\right)+a U_{\text {drive }}
$$

where only viscous damping force is assumed with coefficient $\gamma, U_{\text {drive }}$ is the driving voltage, $\alpha$ the piezo coupling constant, $F_{\text {int }}(z)$ the interaction force with the sample, $z_{0}$ the equilibrium position of the oscillator (i.e. the mean distance between tip and surface), and $z$ the deviation thereof. For small oscillation amplitude, $F_{\text {int }}$ can be expanded about $z_{0}$,

$$
F_{\text {int }}\left(z+z_{0}\right)=F_{\text {int }}\left(z_{0}\right)+\frac{\partial F_{\text {int }}}{\partial z}\left(z_{0}\right) z
$$

\footnotetext{
${ }^{1}$ The electrodes excite and detect only this fundamental mode, and the next bending mode is at a frequency $\sim 6.3$ time higher than the fundamental.
} 
which shows that the gradient of the interaction force can be treated as an additional spring constant $k_{\text {int }}=-\frac{\partial F_{\text {int }}}{\partial z}\left(z_{0}\right)$. Hence the modified resonance pulsation is

$$
\tilde{\omega}_{1}=\sqrt{\frac{k+k_{\mathrm{int}}}{m}}
$$

If we assume the interaction only weakly changes the bare resonance frequency, the shift of the resonance frequency is, at first order:

$$
\frac{\Delta \omega_{1}}{\omega_{1}}=\frac{k_{\text {int }}}{2 k}
$$

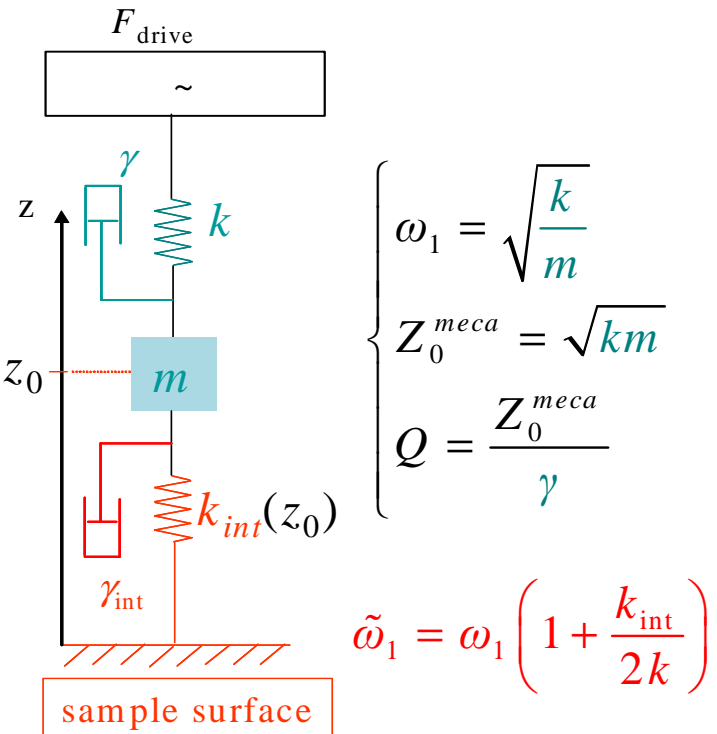

(a)

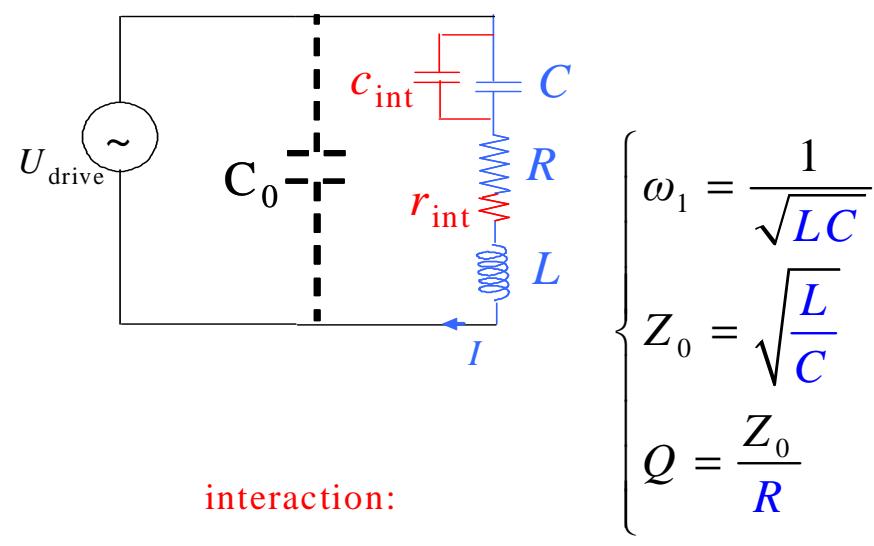

$c_{\text {int }} \leftrightarrow 1 / k_{\text {int }}$

$r_{\text {int }} \leftrightarrow \gamma_{\text {int }}$

(b)

Fig. 2.23 Tuning fork as an electro-mechanical oscillator (a) Schematics of the mechanical oscillator model derived in section 2.4.2.4.c (b) Schematics of the electrical model of a quartz tuning fork. As stressed in \$2.2.1.2 mechanical and electrical parameters are rigidly coupled through the piezoelectric relations. Only $\mathrm{C}_{0}$ does not have any mechanical interpretation: it represents the stray capacitance between the electrodes (i.e. the electrical coupling between the leads outside the piezoelectric, e.g. through air...).

The frequency shift thus senses only the interaction force gradients; it is insensitive to homogeneous field such as gravity, which only displaces the equilibrium point of the oscillator.

In the actual measurements we perform, and more generally when using piezoelectric resonators in electronic engineering, one does not measure the position of the oscillator, but rather the current flowing through the resonator

$$
I(t)=\dot{q}(t)=\alpha \dot{z}(t) .
$$

For an harmonic drive $U_{\omega}$, it is more convenient to work in Fourier transform, for which $I(\omega)$ obeys electrical counterpart of (2.2): 


$$
i L \omega I+R I+\frac{I}{i C \omega}=U_{\omega}+\frac{1}{a} F_{\mathrm{int}} .
$$

The electrical parameters of the resonator can be extracted by fitting the electrical resonance line shape (see $\$ 2.4 .2 .8 . \mathrm{d}$ p 88). From this and knowing the piezoelectric coupling coefficient $\alpha$, one can obtain all the mechanical parameters of the model, and in particular the amplitude of the oscillation, which is important for AFM measurements. The piezoelectric coupling coefficient $\alpha$ is most accurately obtained from an optical measurement of the resonance $[63,64]$. In the case of a single beam oscillator, Giessibl [61] also gave an analytical expression for $\alpha$, as a function of geometric parameters of the beam and of $Y$ (Young modulus) and $d_{31}$ (piezoelectric coefficient).

\begin{tabular}{|c|c|c|c|c|c|}
\hline \multicolumn{2}{|c|}{ Electrical } & \multicolumn{2}{|c|}{ Mechanical } & & \\
\hline parameter & $\begin{array}{c}\text { typical value } \\
{[4 \mathrm{~K}-300 \mathrm{~K}]}\end{array}$ & parameter & typical value & $\begin{array}{c}\text { ratio } \\
\text { Electrical } / \text { Mechanical } \\
\text { [unit] }\end{array}$ & $\begin{array}{c}\text { Energy } \\
\text { correspondence }\end{array}$ \\
\hline q (charge) & & $\mathrm{z}$ (position) & & $\alpha[\mathrm{C} / \mathrm{m}]$ & \\
\hline $\mathrm{U}$ & & $\mathrm{F}_{\mathrm{d}}$ & & $1 / \alpha[\mathrm{V} / \mathrm{N}]$ & \\
\hline $\mathrm{L}$ & $200 \mathrm{kH}$ & $\mathrm{m}$ & $43 \mu \mathrm{g}$ & $\frac{1}{\alpha^{2}}\left[\mathrm{NmA}^{-2} / \mathrm{kg}\right]$ & $\begin{array}{c}\frac{1}{2} \mathrm{mz}^{2} \equiv \frac{1}{2} \mathrm{lq}^{2} \\
\text { kinetic }\end{array}$ \\
\hline $\mathrm{C}$ & $0.13 \mathrm{fF}$ & $1 / \mathrm{k}$ & $5.510^{-4} \mathrm{mN}^{-1}$ & $\alpha^{2}\left[\mathrm{~F} / \mathrm{mN}^{-1}\right]$ & $\begin{array}{c}\frac{1}{2} \mathrm{kz}^{2} \equiv \frac{\mathrm{q}^{2}}{2 \mathrm{c}} \\
\text { potential }\end{array}$ \\
\hline $\mathrm{R}$ or $\mathrm{L} \omega_{0} / \mathrm{Q}$ & $\begin{array}{c}500 \mathrm{k} \Omega- \\
10 \mathrm{M} \Omega\end{array}$ & $\gamma$ or $\mathrm{m} \omega_{0} / \mathrm{Q}$ & $(200 \mu \mathrm{g} / \mathrm{s})$ & $\frac{1}{\alpha^{2}}$ & $\begin{array}{c}\gamma \dot{\mathrm{z}}^{2} \equiv \dot{\mathrm{q}}^{2} \\
\text { dissipation }\end{array}$ \\
\hline
\end{tabular}

Table. 2.3 Connection between electrical and mechanical models of the piezoelectric oscillator (see Fig. 2.22). The values correspond to beams found in most $32 \mathrm{kHz}$ tuning forks. The typical magnitude of the coupling constant is $\alpha \approx 1.35 \mu \mathrm{C} / \mathrm{m}$.

\subsubsection{Discussion of the 1-DOF model}

Implementing an AFM piezoelectric "force" measurement such as described above, where one arm of the tuning fork is fixed, is simple and easy to understand, but is not ideal. In particular, one looses the main benefit of using a tuning fork, namely that the oscillator is decoupled from its support. On the contrary, the motion of the beam couples with the whole 
structure of the microscope. In particular, when the tuning fork is mounted at the end of the piezo scanning tube, as in our setup, the stiffness of the tube depends on its deflection and extension, and thus changes while scanning an image. This change in stiffness induces a spurious change in the resonance frequency of the resonator, not related to the tip-to sample interaction, and appears as artifacts in the AFM image. It may also happen that the resonance of the beam mixes with other modes of the structure ${ }^{1}$.

To avoid such problems, we considered it was important to keep as much as possible the benefit of using a balanced resonator, naturally decoupled from its mechanical environment. In our setup, the tuning fork is held by its base, with its two arms free. In this case, however, the above 1-DOF model is not sufficient to establish the link between the frequency shift and the tip-sample interaction. There is actually a factor $1 / 2$ missing in relation (2.3) due to the fact there are 2 prongs instead of the one considered above (see below). Furthermore, as we necessarily break the built-in symmetry of the TF by gluing onto it the tip and its connecting wire, we need to understand how this changes its behavior. In particular we need to address the following questions:

- By which amount does this symmetry breaking couple the symmetric mode of the bare TF to its other modes?

-What is the oscillation amplitude of the tip in an asymmetric TF, and what is the link between the current flowing in the TF and the tip amplitude?

Such questions have not been investigated in depth in the past. The asymmetry was discussed in Ref [65] and [59], which describe the fork as two oscillating beams coupled by a spring. This analysis is however insufficient to answer all the above questions.

To address these issues, I present in the following a more complete model of the TF.

\subsubsection{Model of a tuning fork as three coupled masses and springs}

In the following we describe a simplified model developed during this thesis. In this model, the tuning fork as 3 masses coupled to each other and to ground by the means of 3 springs, each mass having only one degree of freedom (see Fig. 2.24). In this description, a central mass representing the base of the TF is coupled to the support through a spring $k_{0}$. In the ideal

\footnotetext{
${ }^{1}$ Standard AFM cantilevers should in principle have the same problems. However, their effective mass is about 1000 times lower than a tuning fork beam, so they comparatively couple much less to the rest of the structure. Also their Q factor is smaller which further reduces the visibility of such effects.
} 
symmetric case, this central mass would be at rest, and the TF would be decoupled from its support, as expected. However, when symmetry is broken, this central part has a finite motion and excites the rest of the microscope structure. This 3-DOF model allows to describe the first three in-plane modes of the TF (modes $n^{\circ} 2,4$ and 6 pictured in Fig. 2.21), and how they are modified, when symmetry is broken.

It is important to stress that, in our model, all the resonant modes of the connecting wire and the tip are not described, hence they are modelled as a 1DOF oscillator, rigidly linked to the fork.

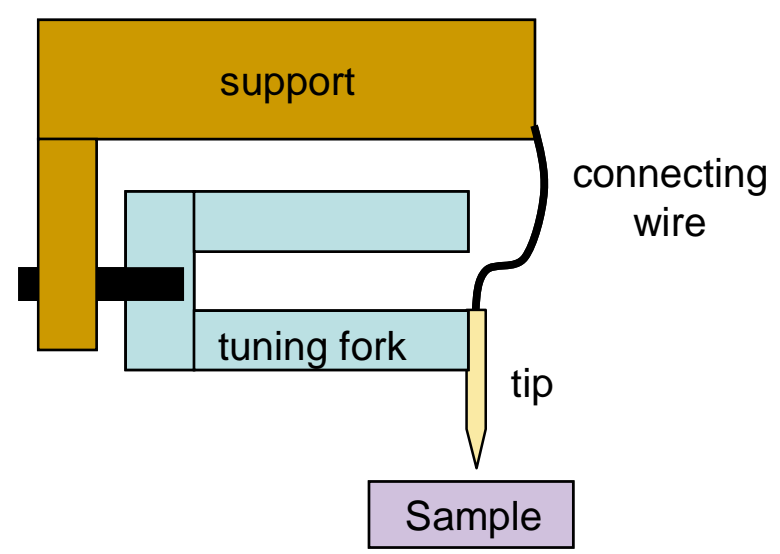

(a)

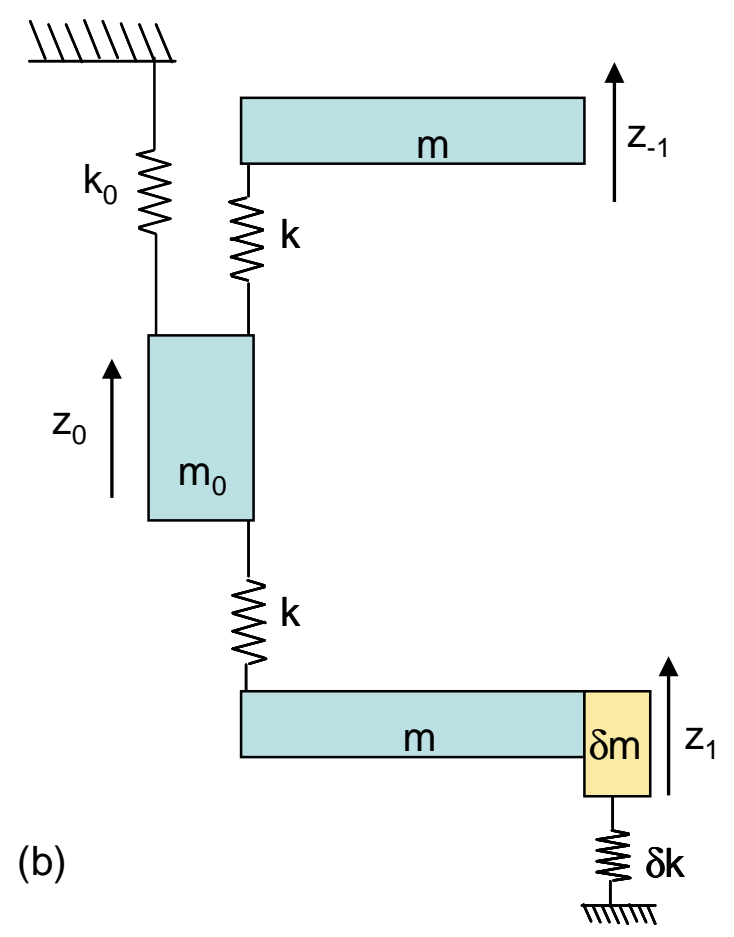

Fig. 2.24 (a) Schematics of a quartz tuning fork, with tip and wire for tunneling current. (b) Equivalent mechanical 3 DOF model. Both arms of the fork have the same effective mass $m$ and spring constant $\mathrm{k}$. The mass $\mathrm{m}_{0}$ is the effective mass of the TF's base, and $\mathrm{k}_{0}$ is the stiffness of its link of the fork to the support. The symmetry of the tuning fork is broken by the extra mass $\delta \mathrm{m}$ and extra stiffness $\delta \mathrm{k}$ due to the tip, wire and interaction with the surface.

\subsubsection{6.b Free dynamics of the 3-DOF model}

We can find the modes of the structure by solving the coupled equations of motion for the free dynamics of the three masses in a harmonic analysis. These equations can be conveniently written in a matrix form:

$$
K . Z=\omega^{2} M \cdot Z
$$

where $\omega$ is the pulsation of the mode,

$$
Z=\left(\begin{array}{c}
z_{-1} \\
z_{0} \\
z_{1}
\end{array}\right)
$$


is the position vector of the three masses,

$$
K=\left(\begin{array}{ccc}
k & -k & 0 \\
-k & 2 k+k_{0} & -k \\
0 & -k & k+\delta k
\end{array}\right)
$$

is the stiffness matrix coupling the displacements of the 3 masses, and

$$
M=\left(\begin{array}{ccc}
m & 0 & 0 \\
0 & m_{0} & 0 \\
0 & 0 & m+\delta m
\end{array}\right)
$$

is the diagonal matrix of effective masses. Equation (2.5) can be conveniently written as

$$
\Omega . Z=\omega^{2} Z,
$$

where

$$
\Omega=M^{-1} K=\left(\begin{array}{ccc}
\frac{k}{m} & -\frac{k}{m} & 0 \\
-\frac{k}{m_{0}} & \frac{2 k+k_{0}}{m_{0}} & -\frac{k}{m_{0}} \\
0 & -\frac{k}{m+\delta m} & \frac{k+\delta k}{m+\delta m}
\end{array}\right) .
$$

The modes of the structure are the eigenvectors of $\Omega$, and the resonant frequencies are the corresponding eigenvalues. Introducing the notations

$\omega_{1}=\sqrt{\frac{k}{m}}$ the pulsation of the unperturbed symmetric mode (or of an isolated arm),

$\gamma=\frac{k_{0}}{k}$ the ratio of stiffnesses in the tuning fork model,

$\beta=\frac{m}{m_{0}}$ the ratio of effective masses in the tuning fork,

$\mu=\frac{\delta m}{m}$ the relative change of mass of the lower arm, and

$\kappa=\frac{\delta k}{k}$ the relative change of stiffness for the lower arm,

the matrix $\Omega$ reads:

$$
\Omega=\omega_{1}^{2}\left(\begin{array}{ccc}
1 & -1 & 0 \\
-\beta & \beta(2+\gamma) & -\beta \\
0 & -\frac{1}{1+\mu} & \frac{1+\kappa}{1+\mu}
\end{array}\right) .
$$




\subsubsection{6.c Balanced tuning fork: the unperturbed modes}

Assuming first $\mu=\kappa=0$, one recovers the symmetric tuning fork. The eigenvectors then have the following components:

$$
\begin{aligned}
& Z_{1}=\left(\begin{array}{c}
1 \\
0 \\
-1
\end{array}\right) \text { with frequency } \omega_{1}, \\
& Z_{2}=\left(\begin{array}{c}
1 \\
1-x+y \\
1
\end{array}\right) \text { with frequency } \omega_{2}=\omega_{1} \sqrt{x-y}, \\
& Z_{3}=\left(\begin{array}{c}
1 \\
1-x-y \\
1
\end{array}\right) \text { with frequency } \omega_{3}=\omega_{1} \sqrt{x+y},
\end{aligned}
$$

where $x=\frac{1}{2}+\beta\left(1+\frac{1}{2} \gamma\right)$ and $y=\sqrt{x^{2}-\beta \gamma}$. These eigenmodes can be respectively identified with modes 4, 2 and 6 of Fig. 2.21. If the frequencies of these modes are known from experiment or from finite element calculations, one can deduce $\beta$ and $\gamma$, and finally extract all the values of our 3-DOF model, up to a global scaling factor. Fixing this global factor requires one more input value which can be, for instance, a static measurement (or Finite Element Model (FEM) calculation, or beam theory result) of the stiffness of an arm. For example, for the tuning fork we use, the ratios between resonance modes shown in Fig. 2.21 obtained from FEM yield $\beta=2.61, \gamma=0.468$, and $k=1800 \mathrm{~N} / \mathrm{m}$, completely determining the 3-DOF model.

\subsubsection{6.d Broken symmetry: a perturbative analysis}

In the case of interest to us where the symmetry of the tuning fork is broken by the presence of the tip, general analytical results can still be obtained with the above method. Unfortunately these analytical results involve general roots of a third degree polynomial and are so inconvenient to write and use, that, in practice, it is more efficient to perform numerical diagonalization.

The questions we want to address, however, do not require the knowledge of the general solution of the asymmetric case: we are only interested in weak asymmetry of the fork, and hence a perturbative approach at the lowest order in $\mu$ and $\kappa$ is sufficient.

Performing Taylor expansions on the general analytical solution to obtain the perturbative results is also impractical, because the general cubic roots prevent simplifying the expressions. Hence, we apply directly the perturbation theory, that relies only on the linearity 
of equations. We first perform a change of basis for the matrix $\Omega$ to the unperturbed eigenbasis

$$
\tilde{\Omega}(\mu, \kappa)=U_{0}^{-1} \Omega(\mu, \kappa) \cdot U_{0},
$$

where $U_{0}$ is the matrix diagonalizing the unperturbed system $\Omega(\mu=0, \kappa=0)$ :

$$
U_{0}^{-1} \cdot \Omega(0,0) \cdot U_{0}=\left(\begin{array}{ccc}
\omega_{1}^{2} & 0 & 0 \\
0 & \omega_{2}^{2} & 0 \\
0 & 0 & \omega_{3}^{2}
\end{array}\right) \equiv \tilde{\Omega}_{0}
$$

The matrix $U_{0}$ can be constructed using the above eigenvectors $Z_{\mathrm{i}}$, properly normalized. In the new basis, $\tilde{\Omega}(\mu, \kappa)$ can then be Taylor-expanded at first order in $\mu$ and $\kappa$ :

$$
\tilde{\Omega}(\mu, \kappa)=\tilde{\Omega}_{0}+P+O\left(\mu^{2}, \kappa^{2}, \mu \kappa\right),
$$

where $P$ is the perturbation, linear in both small parameters $\mu$ and $\kappa$ :

$$
P=\omega_{1}^{2}\left(\begin{array}{ccc}
\frac{\kappa-\mu}{2} & \frac{\kappa+(y-x) \mu}{\sqrt{4+2(1-x+y)^{2}}} & \frac{\kappa-(x+y) \mu}{\sqrt{4+2(-1+x+y)^{2}}} \\
\frac{(-1+x+y) \sqrt{2+(1-x+y)^{2}}(\kappa-\mu)}{4 y \sqrt{2}} & \frac{(-1+x+y)(\kappa+(y-x) \mu)}{4 y} & \frac{(-1+x+y) \sqrt{2+(1-x+y)^{2}}(\kappa-(x+y) \mu)}{4 y \sqrt{2+(-1+x+y)^{2}}} \\
\frac{(1-x+y) \sqrt{2+(-1+x+y)^{2}}(\kappa-\mu)}{4 y \sqrt{2}} & \frac{(1-x+y) \sqrt{2+(-1+x+y)^{2}}(\kappa+(y-x) \mu)}{4 y \sqrt{2+(1-x+y)^{2}}} & \frac{(1-x+y)(\kappa-(x+y) \mu)}{4 y}
\end{array}\right) .
$$

The changes in the eigenvectors and eigenvalues of the system in presence of the perturbation $P$ can be expressed simply in terms of the matrix elements of $P$. In particular, changes to the eigenvalues of the perturbed matrix are given by the diagonal of $\tilde{\Omega}_{0}+P$. Hence, due to the perturbation, the resonance frequency of the mode relevant for AFM (i.e. the symmetric mode) is shifted by

$$
\omega_{1}^{2} \rightarrow \omega_{1}^{2}\left(1-\frac{\mu}{2}+\frac{\kappa}{2}\right)
$$

which gives a relative frequency shift, at first order,

$$
\frac{\Delta \omega_{1}}{\omega_{1}} \simeq \frac{1}{4}\left(\frac{\delta k}{k}-\frac{\delta m}{m}\right) .
$$

Note that this result is a factor of two smaller than what is found in the single beam model (Eq. (2.3)).

The corresponding eigenvector is changed by the perturbation according to

$$
\bar{Z}_{1} \rightarrow \bar{Z}_{1}+\frac{P_{21}}{\omega_{1}^{2}-\omega_{2}^{2}} \bar{Z}_{2}+\frac{P_{31}}{\omega_{1}^{2}-\omega_{3}^{2}} \bar{Z}_{3},
$$


where the overbar denotes the properly normalized unperturbed eigenvectors (other modes are obtained by circular permutation on indices). All calculations performed, the amplitudes of the perturbed symmetric mode are changed according to

$$
\left(\begin{array}{c}
-1 \\
0 \\
1
\end{array}\right) \rightarrow\left(\begin{array}{c}
-1 \\
0 \\
1
\end{array}\right)+\frac{\Delta \omega_{1}}{\omega_{1}}\left(\begin{array}{c}
\frac{-1+\beta(2+\gamma)}{\beta} \\
2 \\
\frac{-1+\beta(2+\gamma)}{\beta}
\end{array}\right)
$$

\subsubsection{6.e Discussion}

From this calculation one can draw a number of important conclusions regarding the influence of the tip, the wire and the sample:

- All changes of the symmetric mode are proportional to its relative frequency change.

- The frequency shift due to loading the tuning fork with the tip's mass can be, in principle, exactly compensated by the extra stiffness brought by the connecting wire (as already noted in [59]). This compensation then maintains a perfectly symmetric tuning fork, completely decoupled from the rest of the structure.

- The amplitudes of deformation of the two arms in the symmetric eigenmode, relevant for AFM, are changed by opposite amounts. The current we measure is related to the sum of the deflections as indicated by eq. (2.4). Hence, the changes in the arms individual currents cancel, and for a given excitation amplitude one keeps a constant signal at resonance (see $\$ 2.4 .2 .8)$.

- The arms' amplitudes can be left unchanged when weakly breaking the symmetry of the tuning fork, provided $\frac{m_{0}}{m}=2+\frac{k_{0}}{k}$, even if the resonance frequency changes.

- When loading the tuning fork with the tip, for a given current flowing in the tuning fork, the change in the amplitude of the arm carrying the tip with respect to the unloaded tuning fork can be directly linked to the observed relative frequency shift between the two situations. This link is 
quantitative if the model parameters of the bare tuning fork are known (see above). This can be useful for calibrating the tip amplitude.

- The re-coupling of the resonator to the rest of the structure due to the motion of the tuning fork base (mass $m_{0}$ in the model) is also proportional to the relative frequency shift. Relative to the amplitude of the arms motion, the base amplitude is exactly twice the relative frequency shift and (surprisingly) independent of the stiffness $k_{0}$ of the mounting of the tuning fork.

In practice, using tips and connecting wires of very small diameters $(10-25 \mu \mathrm{m})$ with a minimum amount of glue, together with proper shaping of the connecting wire (found empirically), I could obtain quite consistently frequency shifts lower than $200 \mathrm{~Hz}$, i.e. a relative frequency change of the order or better than $0.5 \%$. Correspondingly, the amplitude of the tuning fork base is only of order $1 \%$ of the tip's amplitude. Such small amplitude can still excite resonances in the microscope structure, but given that the quality factors of typical structures are much lower than that of the tuning fork, it is unlikely that these resonances can cause problems during measurement.

The present results assume negligible damping, which is confirmed by experiment since the Q factor remains very high even when the tip interacts with the sample. A more complete description making use of imaginary spring constants could account for damping in the tuning fork, as well as non-conservative tip-sample interaction forces. Provided a model is known for the non-conservative interaction forces, this would give a more realistic evolution of the mode amplitudes, at the expense of introducing more parameters in the model.

\subsubsection{Estimating characteristics of the connecting wire for preserving the tuning fork balance}

From the above discussion, preserving the balance of the tuning fork requires that the added mass $\delta m$ and spring constant $\delta k$ are in the same ratio $\frac{\delta k}{\delta m}=\frac{k}{m}$ as that of the tuning fork's arms.

Our model turns into adding to the fork an "extra resonator" with ideally the same resonance frequency as the TF. However, this "extra resonator" is completely constrained by the TF 
since it shares the same $z_{1}$ DOF as the bottom arm. Hence, no mode mixing occurs, in contrast with the usual case of coupled oscillators bearing their own DOF.

Note however that our model is an approximation since the wire is a complex body having a lot more than 1 DOF. We thus pay special attention to constrain (i.e. plastically deform) the wire prior to adding it to the TF, so that its apex faces exactly the TF's end (position $z_{1}$ at rest).

Using the characteristics of the materials involved in the tip mounting, one can try to estimate the dimensions of the connecting wire so that its effective spring constant is in the correct range to perform the compensation.

For a well defined geometry, one can imagine to use FEM simulations. However, since one can hardly mount the tip and the wire in a perfectly controlled and reproducible manner, we found it simpler to have only estimates of the correct parameters ranges, and we subsequently relied on empirical observation to obtain reproducible low frequency shifts and high quality factors.

Simple estimates can be obtained by considering the masses added by the tip and glue, and treating the wire as a vibrating beam.

\begin{tabular}{|c|c|c|}
\hline material & $\mathrm{Y}\left(10^{10} \mathrm{~N} / \mathrm{m}^{2}\right)$ & $\rho\left(\mathrm{kg} / \mathrm{m}^{3}\right)$ \\
\hline Epotek H20E & 7.13 & 2550 \\
\hline tungsten & 40 & 19300 \\
\hline gold & 7.72 & 19320 \\
\hline quartz & 7.87 & 2659 \\
\hline
\end{tabular}

Table. 2.4 Mechanical parameters of typical materials used in our AFM/STM probe.

The tungsten tip's mass is estimated to be $0.9 \mu \mathrm{g}$, for $400 \mu \mathrm{m}$-length, $6 \mu \mathrm{m}$-radius. The glue's mass is estimated to be $0.7 \mu \mathrm{g}$ for a hemisphere of radius $50 \mu \mathrm{m}$. An upper value of added weight is of the order of $\delta m_{1}=2 \mu \mathrm{g}$.

To estimate the role of the connecting wire, we now assume it is a cylindrical rod, mounted in prolongation of the tuning fork's arm. For a clamped-free cylindrical beam ${ }^{1}$, the added

\footnotetext{
${ }^{1}$ In fact, the wire is clamped at both ends in a way which makes it difficult to perform calculations. Since we are looking only for orders of magnitude, we take here this simple approach, knowing that the numbers obtained for the stiffness will be underestimated.
} 
effective mass $m^{*}=\frac{k^{*}}{\omega^{* 2}}$ of the connecting wire is given by the ratio of its spring constant $k^{*}=\frac{3 \pi Y R^{4}}{4 L^{3}}$ and its eigenfrequency $\omega^{*}=\frac{3.52 R}{2 L^{2}} \sqrt{\frac{Y}{\rho}}, R$ being the wire radius, $Y$ the young modulus, $\rho$ the density of the material, $L$ the length of wire. Hence, the effective mass of this

$\operatorname{rod}$ is $m^{*}=0.76 \rho R^{2} L$. Solving $\frac{k^{*}}{\delta m_{1}+m^{*}}=\omega_{1}^{2}$, we find the necessary length of wire lies in a range of 100 to $300 \mu \mathrm{m}$. It is however very difficult to mount such a short wire. We instead mount a longer wire with a bend so that a $\sim 100 \mu \mathrm{m}$-long section is in the prolongation of the tuning fork beam and that it holds itself into that position before gluing it to the fork. By this procedure, we observed we could induce reproducible low perturbations on the fork's resonance, with frequency shifts of the order of $100 \mathrm{~Hz}(0.3 \%)$, and a quality factor of more than 6000 at room temperature and ambient pressure, instead of 8000 for the bare fork. The quality factor achieved by this method increases up to 150000 at low temperature. The results presented here are of course fork-dependant, and the forks employed in this work are among the lightest and smallest commercially available. That makes them more sensitive to force gradients since $k$ is proportional to the thickness, but they are also more sensitive to asymmetry, which results in damping associated to the motion of the TF's base (see $\$ 2.4 .2 .5$, p. 77).

\subsubsection{Measuring the oscillator parameters}

The first step in our AFM experiments is to set up the resonator for imaging, and first of all, we have to characterize its resonance, to ensure the fork is properly mounted.

The principle of the resonance measurement is the following: we impose an AC voltage bias at a known frequency, and measure the current flowing through the tuning fork at this frequency. This admittance measurement is done with a standard current amplifier (CurrentVoltage converter or CVC), and a lock-in synchronous detection. A complete schematic of the measurement is shown in Fig. 2.25.

Typical resonance curves recorded with this setup are shown in Fig. 2.26, for different stages of tooling up the tuning fork with a wire and a tip. 


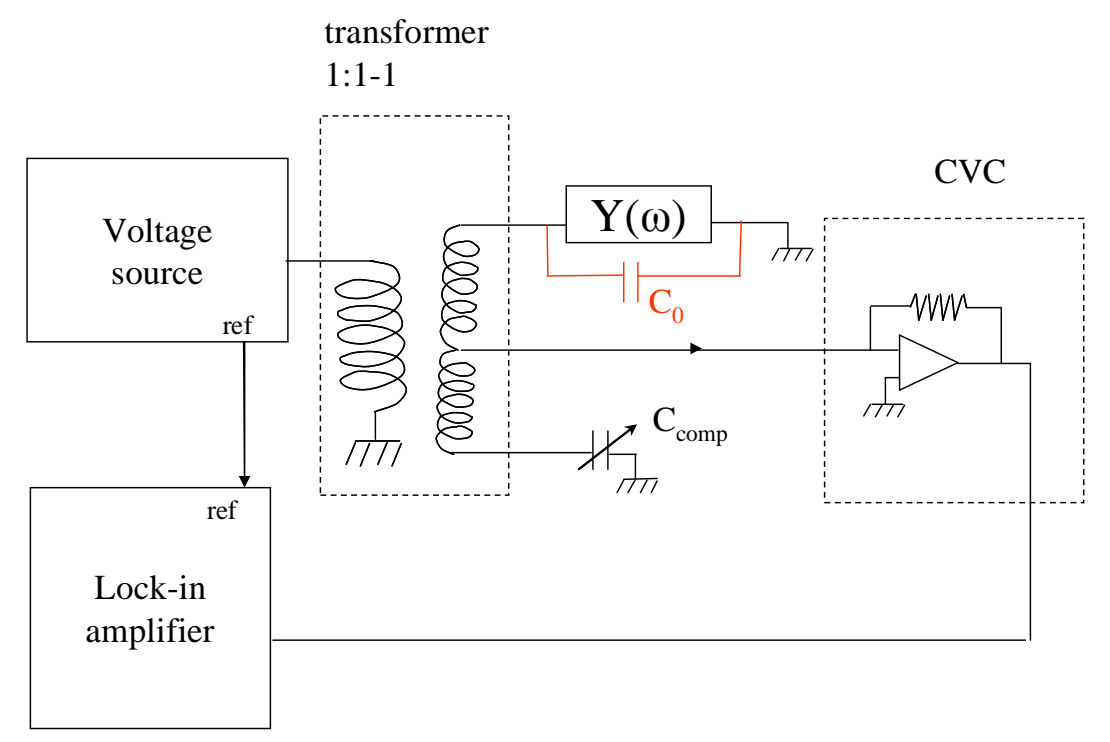

Fig. 2.25 Block diagram of the measurement setup. A voltage source excites the tuning fork (symbolized by an admittance $\mathrm{Y}(\omega)$ ). The current that flows through this admittance is amplified by a CVC, and fed to a lock-in. Current has a maximal amplitude at resonance, and is in-phase with the excitation voltage. With the lock-in, one can record in-phase and out of phase signals (or amplitude and phase), and by properly adjusting the phase of the incoming signal with respect to the reference, one can record a perfect Lorentzian resonance, such as shown in Fig. 2.27). The variable capacitance and the transformer allow to cancel the current flowing in the stray capacitance $\mathrm{C}_{0}$, and thus to extract the part of the signal corresponding to the resonance of the oscillator alone.

\subsubsection{8.b Resonance curves during the setup}

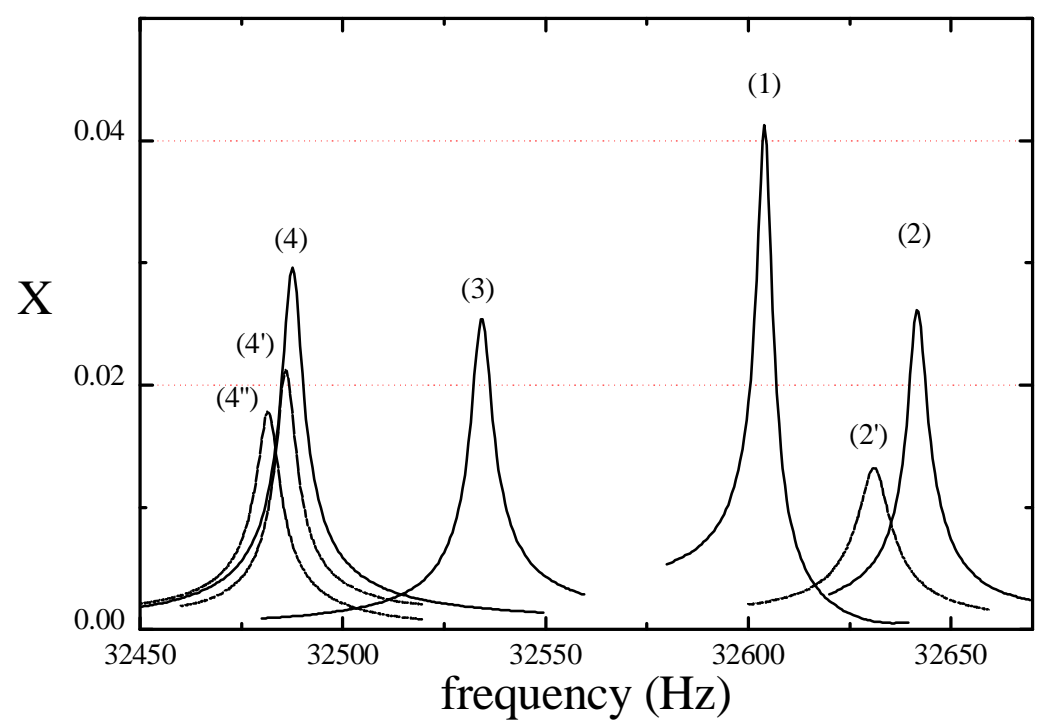

Fig. 2.26 Resonance curves measured in various mounting configurations of the tuning fork. The signal plotted is the in-phase lock-in signal measured with the setup described in Fig. 2.25. All data were taken at room temperature and ambient pressure. (1) is a bare tuning fork attached to its holder. (2) is taken after soldering at the end of one arm a $12 \mu \mathrm{m}$ diameter, $\sim 500 \mu \mathrm{m}$ long gold wire. (3) is taken after attaching the gold wire at its other end to the connection pad. (4) is taken after the tip was glued. The different curves associated with the same number are taken at different moments, by placing the wire in different positions. 


\subsubsection{8.c Capacitance compensation}

At resonance, the admittance of the resonator is real and maximal, however it is small compared to the imaginary admittance $i C_{0} \omega_{1}$ of the capacitance $C_{0} \sim 100 \mathrm{pF}$ of the coaxial wire connecting the tuning fork to room-temperature apparatus, in parallel with the resonator ${ }^{1}$. In order to properly measure only the current flowing through the resonator we use a bridge technique introduced by Grober et al. in Ref. [66]: a transformer and an adjustable capacitor are used to precisely compensate the current flowing in $C_{0}$ (see Fig. 2.25).

This compensation is important, because reliably tracking the tuning fork resonance in AFM imaging depends on having a change of sign of the imaginary part of the admittance at resonance (see §2.4.3). The procedure for this compensation is the following: we first take an uncompensated resonance curve of the tuning fork, in a frequency range large enough that we are sure it encompasses the resonance. Then we can determine the resonance frequency and set the generator to it. We then compensate the capacitance, trimming an adjustable capacitor down to the sub-pF level, canceling the out-of phase measurement of the lock-in at resonance frequency. This procedure can be iterated if necessary.

\subsubsection{8.d Extracting the resonator parameters}

To determine the resonator parameters we have set up a fitting procedure, which enables accurate extraction of all the parameters, among which the resonance frequency, the quality factor, and a measurement of the accuracy of the capacitance compensation. This also enables to accurately tune the phase of the PLL sensor (see below).

The procedure involves fitting simultaneously the in-phase and quadrature lock-in measurements of the resonance as a function of frequency $f$, and fitting them with the corresponding prediction for the RLC circuit.

The fitting function has the following mathematical expression for the in-phase expression:

$$
\begin{aligned}
X(f)=(o f f s e t & \left.+\frac{2 c \pi f_{1}^{3} Q f^{2}}{f_{1}^{4} Q^{2}+f_{1}^{2}\left(1-2 Q^{2}\right) f^{2}+Q^{2} f^{4}}\right) \cos \varphi \\
& -2 c \pi f\left(C_{\text {ratio }}+\frac{\left(f_{1}^{2} Q^{2}\left(f_{1}^{2}-f^{2}\right)\right)}{f_{1}^{4} Q^{2}+f_{1}^{2}\left(1-2 Q^{2}\right) f^{2}+Q^{2} f^{4}}\right) \sin \varphi
\end{aligned}
$$

The quadrature expression is the same, with $\varphi$ replaced by $\varphi+\pi / 2$. In these expressions, the fitting parameters are the central frequency $f_{1}$, the resonator capacitance $c$, the stray shunt

\footnotetext{
${ }^{1} C_{0}$ also incorporates a small stray capacitance contribution from the tuning fork itself.
} 
capacitance $C_{0}^{*}=C_{0}-C_{\text {comp }}=c^{*} C_{\text {ratio }}$ (which is zero when compensation is perfect, and which can be negative when over compensated), the $Q$ factor, the dephasing $\varphi$ due to cables and amplifiers, and a small offset that slightly improves the fit and can be seen as a leakage resistor in the setup in the range of 10-30 $\mathrm{M} \Omega$. The last two parameters arise from the setup and measurement technique, while the first four describe the system shown on Fig. 2.23.

When the lock-in phase is correctly set (i.e. when it compensates for dephasing in amplifier and cables), $\varphi=0$ and the $\cos \varphi$ term (resp. $\sin \varphi$ term) of equation (2.7) describes the real (resp. imaginary) part of the impedance of the tuning fork.

Such a fitting procedure yields a very high quality fit, and provides an accurate determination of the parameters. An example of fit is shown on Fig. 2.27.

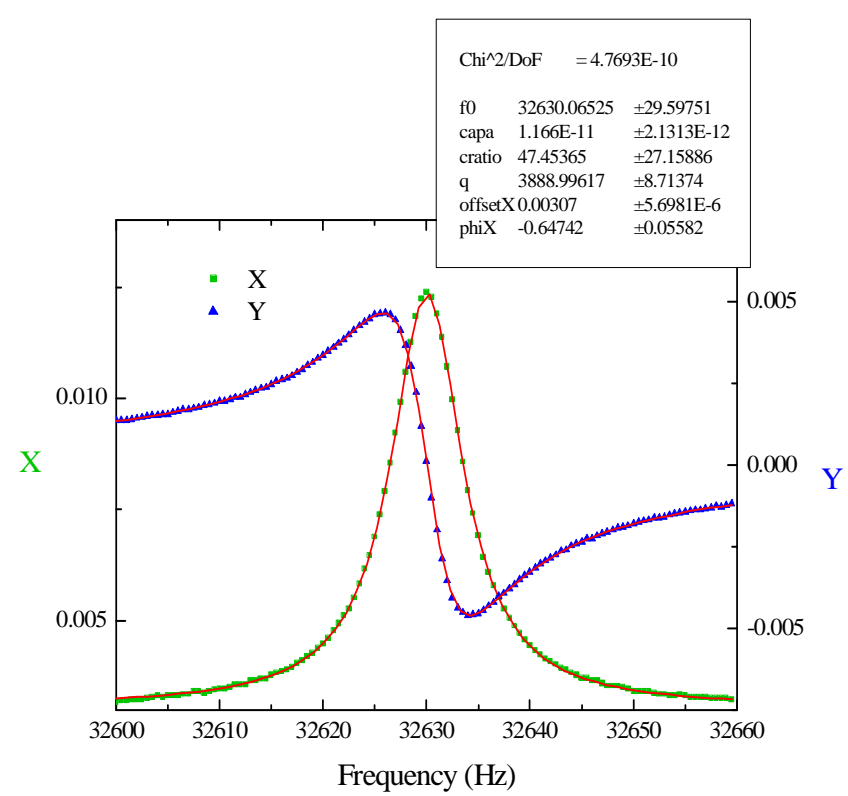

(a)

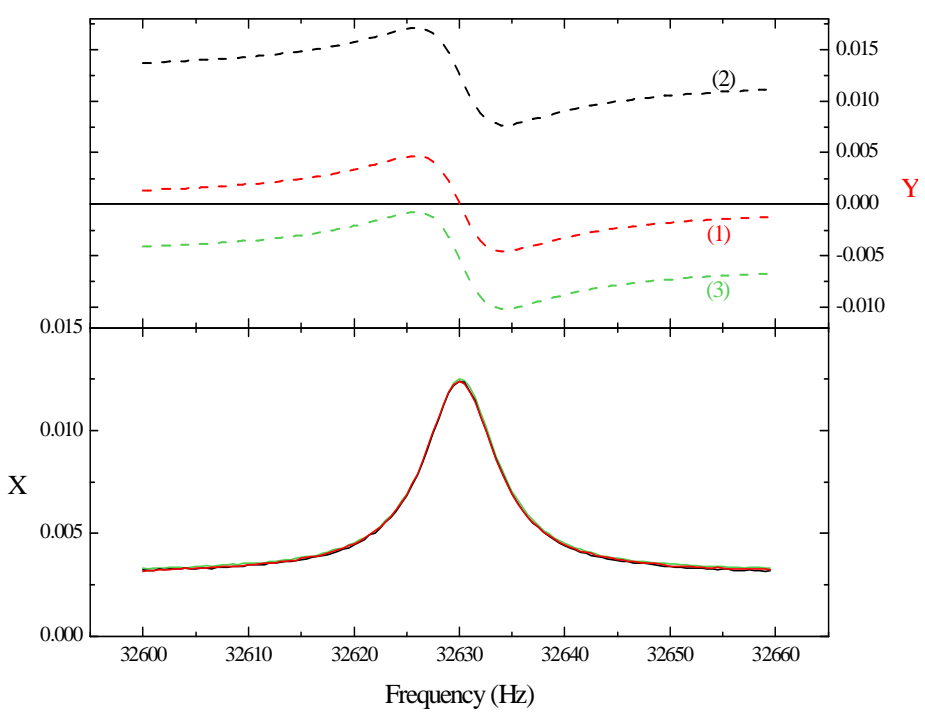

(b)

Fig. 2.27 Fit of the resonance curve and adjustment of the resonance parameters: (a) A resonance curve fitted with the model presented above. The resonance is shown in the in-phase (X) out-ofphase (Y) representation, with different scales. Taking into account all setup parameters, the impedance $\mathrm{Y}^{-1}(\omega)$ at resonance is $\sim 5 \mathrm{M} \Omega$. (b) Plot of resonance curves obtained with three different values of the compensation capacitance: (1) is obtained when the compensation is quasi perfect, (2) when the stray capacitance is under compensated, (3) when the stray capacitance is over compensated. For low quality factors, the compensation has to be accurate: curves shown here are obtained by a displacement of the order of $\sim 1 \mathrm{pF}$ of the adjustable capacitance.

\subsubsection{Force measurement}

I now discuss the nature of the interactions we typically detect in our experiments, and describe how we perform their measurement in AFM mode, using the tuning fork. 


\subsubsection{Typical forces on the atomic scale}

I consider here the general case of a metallic tip on a metallic or insulating sample. When the tip approaches the surface, atoms of the tip and of the surface interact. Detailed descriptions and measurements of these interactions can be found in several articles [64, 67, 68].

In the following, I assume there are no magnetostatic interactions so that only electromagnetic interactions between charges in the tip and the sample are to be considered, all other physical forces being neglected.

At infinitely large tip-sample distances, the tip does not interact with the surface, and the force is thus zero. The potential energy of the tip in the field of the sample is taken to be zero.

When lowering the tip, one encounters several attractive or repulsive forces which come into play at different scales [69].

If the surface is conductive, and if there is an electric potential difference between tip and sample, charges of opposite signs accumulate on facing electrodes. This leads to a "long range" attractive force between the two electrodes that depends on their shape, since the electrostatic potential energy writes $V_{\text {elec }}=1 / 2 C(z) U^{2}, U$ being the applied bias voltage, and $C$ the capacitance between the electrodes. We are mainly insensitive to this force since we normally keep a low bias voltage. However, varying the applied voltage would be a way to characterize the tip aspect ratio $[69,70]$ by measuring the capacitance (within e.g. a spherecone model, presented in ref [71]) or to perform scanning capacitance measurements [72].

An electrostatic force can also occur on a metallic tip above a charged insulating surface. I assume that the insulating parts of our samples are not charged, which seems to describe well the experimental observations.

In absence of a voltage applied to the tip and of a static charged sample, the dominating force at large tip-to-sample distance is the Van der Waals force. This is a net attractive force due to the polarizability of the materials. It is also a long-range tip-shape dependent force, with an approximate $\mathrm{d}^{-2}$ dependence, where $\mathrm{d}$ is the tip-to-sample distance.

At some point, the foremost atoms of the tip begin to experience a shorter range "chemical" interaction with the atoms of the surface. This can be seen as overlapping of orbitals at large distances and becomes eventually Coulomb repulsion when the atoms of the tip try to "enter" the sample. When both the tip and sample are metals, this interaction can be approximately described by the energy $E=-E_{B}(1+x) e^{-x}$, with $x=\left(d-d_{m}\right) / \lambda$, where $E_{B}$ is the binding 
energy, $d_{m}$ is the separation at $E=E_{B}$, and $\lambda$ is a scaling parameter that gives the range of the metallic interaction [64].

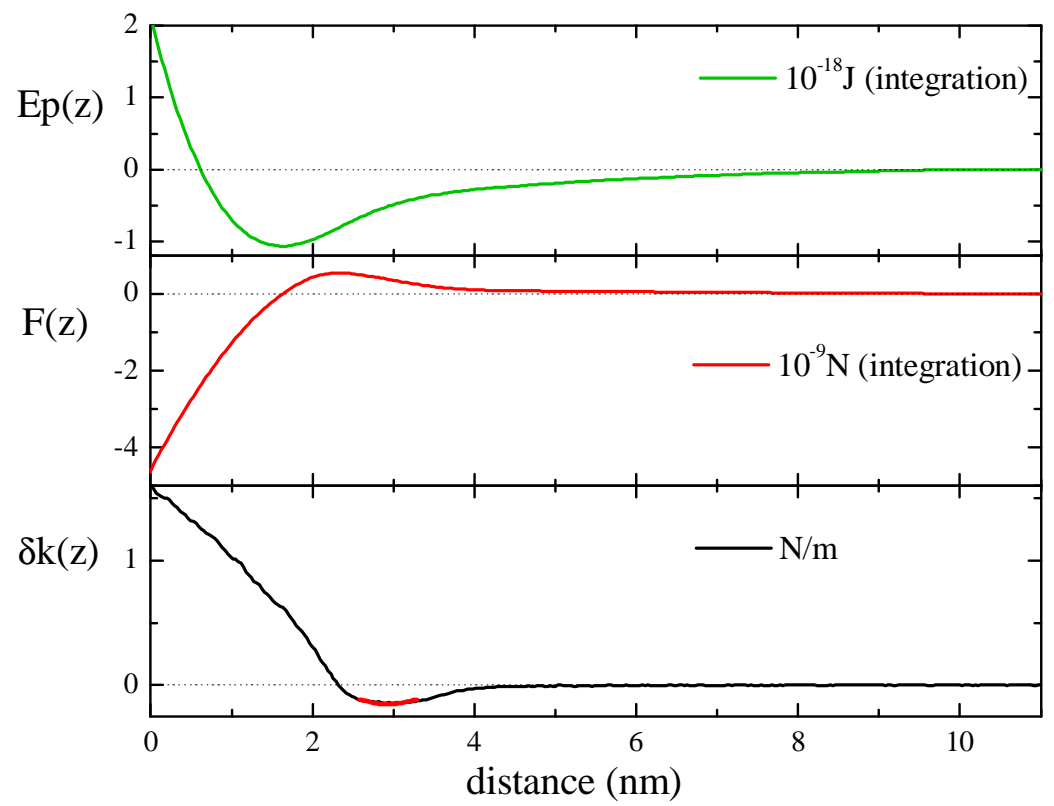

Fig. 2.28 From variations of the central frequency of the tuning fork with tip to sample distance, one can extract several pieces of information. The lower curve represents the actual variation of the spring constant $\delta k$, assuming a small perturbation on a spring of stiffness $k=1800 \mathrm{~N} / \mathrm{m}$ (see discussions above: 2.4.2.4.b and 2.4.2.6.d). From that curve were calculated the force and the potential by successive integration. Integration constants are chosen to cancel both quantities at large distances. Note this procedure is only qualitative because we do not account here for the finite amplitude of oscillation of the fork. The region highlighted on the $\delta \mathrm{k}$ plot is important when performing images with a feedback mechanism, as will be explained in 2.4.3.5.

The lower plot of Fig. 2.28 is a typical experimental curve recorded by either approaching towards or retracting the tip from the sample: approach and retract curve do not always superimpose due to tip sticking to the sample during the lift. From this data one can deduce the potential experienced by the tip close to the sample as well as the interaction force between tip and sample. The potential measured experimentally resembles the well-known Lennard-Jones potential describing interaction between two atoms.

A dip is usually present on the force gradient, even at 0 voltage bias. It depends on the tip shape: it happens there is no dip at all when the tip is sharp. When the tip gets rounder, the dip becomes deeper. This dip is due to the competition between an attractive Van der Waals force and a short-range repulsion.

\subsubsection{2 "Force" measurements: sensor stability and sensitivity}

As discussed in $\$ 2.4 .2 .2$, tracking the changes in the resonance frequency of a tuning fork excited with vanishing amplitude allows in principle to measure the force gradient felt by the tip at the sample surface. This simple principle however contains "built-in" limitations: 
- First of all, measuring the frequency of the resonator requires a finite vibration amplitude. During an oscillation, the tip-to-sample distance changes and hence, the actual frequency shift corresponds to an average force gradient. When the amplitude used is large compared to the scale of variation of the gradient at the surface, the features are smeared out. Notably, the measured dip in the frequency shift is smeared and reduced [75].

- A simple stability criterion of the tip-to-sample distance prevents to have the tip at rest, at distances $z$ where the force gradient is such that $\frac{d F(z)}{d z}<-k_{\text {eff }}$ where $F(z)$ is the total tip-to-sample force and $k_{\text {eff }}$ the effective spring constant of the tip holder. In these regions, the tip cannot be held at rest (neither with vanishing excitation) at a fixed distance from the sample and jumps to the nearest stable point. Using a sensor with high stiffness avoids such instability, which is mandatory for STM measurements. In this respect, the use of a tuning fork is much better than standard AFM cantilevers [77]; on most contacts it allows to measure the whole tip-to sample distance range, with a very low excitation amplitude, without the jump-to-contact usually observed with AFM cantilevers.

- Other stability criteria can be formulated in dynamic oscillation: since the force gradient $\delta k(z)$ is non constant in $z$ (anharmonic potential), there may be several stable oscillation modes at a given average distance, each mode having a different amplitude [68]. This results in the hysteretic behaviour (as a function of amplitude and frequency), encountered with soft standard AFM cantilevers. Non-conservative interaction forces can also induce such dynamic instabilities. However, when using a tuning fork, the interaction non-linearities weakly affect the dynamics of the oscillator $(\delta k(z) \ll k)$ which is mainly determined by the high spring constant and, as a consequence hysteretic behaviours are not observed.

- The ability of a resonator to measure frequency shifts is ultimately limited by the intrinsic thermal noise due to dissipative parts in the resonator. One can show $[73,74]$ that $\frac{\delta \omega_{\min }}{\omega_{1}}=\sqrt{\frac{2 k_{B} T B}{k A^{2} \omega_{1} Q}}$ where $k_{B}$ is the Boltzmann 
constant, $T$ the temperature of the dissipative part of the oscillator, $Q$ the quality factor, $k$ the spring constant, $A$ the mechanical amplitude of the oscillator, and $B$ the bandwidth of the frequency measurement. From this expression, and given that the frequency shift $\delta \omega_{1}$ of the oscillator in response to a force gradient $\delta k$ is $\delta \omega_{1}=\delta k \omega_{1} / 4 k$ (see 2.4.2.6.d), one can estimate the minimum detectable force gradient to be $\delta k_{\min } \approx \sqrt{\frac{4 k_{B} T B k}{\omega_{1} A^{2} Q}}$.

From the above analysis, one sees that the choice of a resonator is a balance between greater stiffness which gives better stability of operation and lower stiffness which gives better overall sensitivity. Quartz tuning forks being much stiffer than conventional cantilevers, they are very stable and convenient to use in AFM: they are not easily prone to jump-to-contact or hysteretic behavior upon a change of amplitude. Years of development on dynamic AFM have shown that $k$ values exceeding hundreds of $\mathrm{N} / \mathrm{m}$ provide adequate sensitivity and stability [75]. The tuning forks we mostly used had $k=1800 \mathrm{~N} / \mathrm{m}$, and are among the softest (i.e. thinnest) available. Those tuning fork typically yield a sensitivity to a force gradient: $\delta \omega_{1} / \delta k=\eta \approx 2.3 \mathrm{~Hz} /(\mathrm{N} / \mathrm{m})$, which is a standard value [74].

Furthermore, for a given sensitivity, lower excitation amplitudes can be achieved by using higher Q-factor or higher resonant frequencies. Since well balanced tuning forks can have high Q-factor (see discussion above), they can be very good AFM sensors, yielding atomic resolution [75].

In our setup, the above minimum detectable force gradient is not reached, by far, due to extra sources of noise introduced in the measurement of the sensor. Indeed, the above predicted ultimate frequency resolution we should be able to achieve with our tuning fork with, say, a $1 \mathrm{~nm}$ amplitude of the sensor and a $100 \mathrm{~Hz}$ (respectively, $1 \mathrm{kHz}$ ) bandwidth should be of the order of $0.15 \mathrm{mHz}$ (resp. $0.5 \mathrm{mHz}$ ) whereas the output noise of the PLL alone is already $2 \mathrm{mHz}$ rms (resp. $40 \mathrm{mHz} \mathrm{rms}$ ) when fed with a noiseless signal. In actual measurements of the resonator, the PLL frequency measurement accuracy is further degraded by the noise coming from the amplifier.

\subsubsection{3 "Force" measurements: detection schemes}

The easiest operation mode of a resonator is to drive it at a fixed amplitude and frequency, on the side of the resonance peak, and to measure its amplitude. This was the first mode used in dynamic AFM. In this mode, known as AM mode, when the tip-to-sample interaction changes 
in a step-like manner, the central frequency of the oscillator changes and its amplitude experiences a transient which damps exponentially with the characteristic time $\tau=2 Q / \omega_{1}$. For a $32 \mathrm{kHz}$ oscillator with a Q-factor of the order $10^{5}$, this transient, also known as the ringing-down (or up) of the oscillator amplitude associated with changing the energy of the oscillator, can last several seconds and makes it unusable as a practical AFM detector.

Still, high Q-factor oscillators can be used as AFM detectors by tracking the resonance frequency of the oscillator [76]. This frequency tracking mode is also called FM mode and can be implemented in two ways:

- In the first method, the oscillator is placed in a loop with an effective negative resistance around the resonance frequency (i.e. an amplifier with $\sim \pi$ phase shift) so that it enters self-oscillation.

- In the second method, the oscillator is inserted in a phase-locked loop (PLL) controller, such as described below. This latter method is somewhat more flexible than the first one in terms of controlling the resonance, and it has the advantage of directly providing a signal proportional to the frequency shift of the oscillator. This is the mode we used.

In either of these FM modes, when applying a step change in the interaction, there is no time penalty associated with changing the energy stored in the oscillator, like in the AM mode. In FM mode, one indeed tracks the change of the resonator parameters themselves that effectively occur at the sound velocity [77], rather than measuring the rate at which the oscillator absorbs (looses) energy.

In the self-oscillation mode the change in frequency is thus as fast as the resonance frequency itself.

In the PLL case, which is of interest to us, the response time is limited by the speed at which one can measure the changes in the phase of the oscillation [73, 74, 77]. Of course, this speed has a tradeoff with the stability of the frequency, and therefore with the sensitivity and the accuracy of the force measurement. The actual accuracy and speed of the AFM sensor then depends on details of the setup, and in particular on the signal-to-noise ratio in the measurement of the oscillator. In practice, with our setup which uses standard roomtemperature amplifiers and PLL electronics and reasonable excitation amplitude, it is possible to track the resonance frequency within a bandwidth in the $100-1000 \mathrm{~Hz}$ range, i.e. much faster than $\tau^{-1}=\omega_{1} / 2 Q \sim 0.2 \mathrm{~Hz}$, which allows reasonable scanning rates in AFM imaging, in the $0.1-1 \mathrm{~Hz}$ range. 


\subsubsection{Phased locked loop detection}

In the following, I describe more in details the operation of the tuning fork in FM mode with a PLL detector. The block diagram of the PLL is shown in Fig. 2.29.

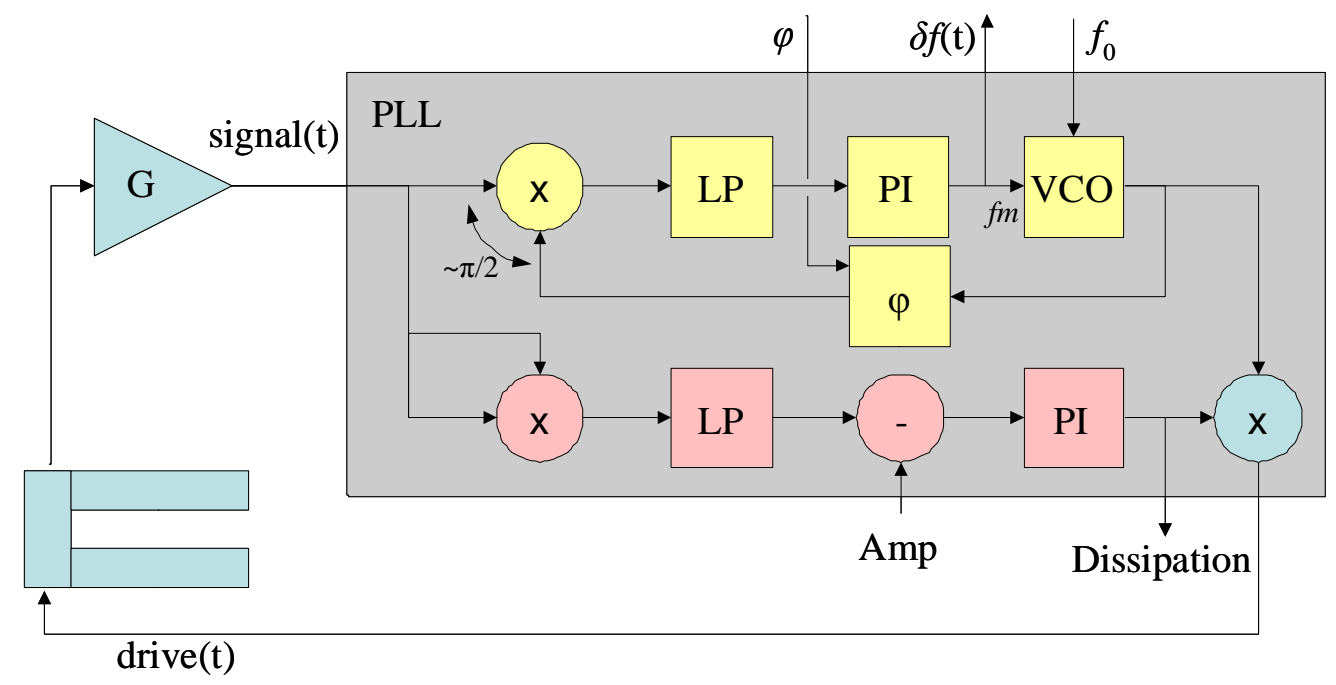

Fig. 2.29 Block diagram of a PLL detector (yellow path) with automatic amplitude control (pink path). VCO stands for Voltage Controlled Oscillator, PI for Proportional-Integral, LP for Low Pass, $\mathrm{x}$ for multiplier, $\Delta$ for difference, $\varphi$ for dephaser, $\mathrm{G}$ for amplifier. The input control signals for the PLL are the amplitude set point Amp, the central VCO frequency $f_{0}$ and the dephasing angle $\varphi$. The output monitoring signals are the frequency shift $\delta f(\mathrm{t})$ and the amplitude of the drive signal "Dissipation", which is proportional to the sum of the sensor dissipation and the dissipation due to non-conservative tip-sample interaction.

Its principle of operation is the following: the VCO is set with a central frequency $f_{0}$ close to the experimentally determined resonance by fitting the resonance curve. It delivers a sine oscillation at a frequency $f=f_{0}+\lambda f m$, where $f m$ is the voltage at the $\operatorname{VCO} f m$ input and $\lambda \mathrm{a}$ constant giving the frequency sensitivity of the VCO. This signal, multiplied by the amplitude control signal is sent to the sensor, amplified and comes back at the PLL input. There, it enters a phase comparator, composed of a multiplier and a low pass filter to remove the $2 f$ component. The PLL dephaser can be tuned to accommodate for dephasing in the amplifier. It is set such that it delivers a signal with a $\pi / 2$ phase lag signal with respect to the input signal right on resonance, so that the phase comparator signal is zero on resonance. This way, on resonance, the system is at a fixed stable point: the integrator output is constant and the frequency remains fixed. On the other hand, when the VCO frequency does not correspond to the sensor resonance, the response of the sensor is not in phase with the drive and the phase detector gives a non-zero phase shift, with a negative sign (resp. positive) if the drive frequency is above (resp. below) the resonance frequency. This phase shift is integrated by the PI resulting in a change in the drive frequency until the phase detector signal drops to zero, when resonance condition is met again. 
This phase-locking mechanism has a single (stable) operation point when the resonator output is such that the signal component in quadrature with respect to the drive goes through zero in the frequency range of the VCO. This condition is met only when the parallel stray capacitance is perfectly compensated (see Fig. 2.27 b). When the capacitance compensation is imperfect, there may be zero or two solutions. In the first case the system cannot phase lock on resonance and the VCO oscillates either at its maximum or minimum frequency. In the second case, one solution corresponds to an unstable situation, and the other to a metastable phase locking. In the latter case, operating an AFM is possible although risky, as the PLL can unlock when scanning over large steps on the sample.

Our PLL further controls the amplitude of the drive so that the output signal remains constant. The amplitude (in fact, the power) of the input signal is determined by squaring it and filtering to remove the $2 f$ component. This filtered value is compared to the setpoint and the difference is integrated to obtain the amplification factor to be applied to the drive. This way, the difference between the setpoint and the detected amplitude eventually drops to zero.

This implementation of a PLL actually consists in two parallel loops:

- A first loop that feeds a correct frequency to the tuning fork, to avoid detuning from the resonance. This one is the fastest (see 2.4.3.3).

- Another loop that compensates for energy losses either due to a detuning of the excitation, or to damping in the tip-sample interaction. Energy loss has a time constant $\tau=2 Q / \omega_{1}$, as discussed above. Amplitude regulation is essential to prevent from loosing signal when the $Q$-factor drops while scanning the surface. This loop should not be set to regulate at a rate higher than $\tau^{-1}$, to prevent amplitude oscillations.

We use a commercially available PLL system to control the tuning fork: the Nanosurf Easy PLL system. This system is partly analog, and partly digital, which is convenient to interface and control the PLL from within a program. However, the analog multipliers used in the system are noisy components which make the PLL behave less than optimally regarding the sensitivity and frequency stability. A well designed fully digital PLL should outperform it easily. 


\subsubsection{Feedback loop in imaging mode}

Once the PLL is locked, image in AFM mode is performed by closing the feedback loop which controls the average tip-to-sample distance. The Block diagram of this loop is shown in Fig. 2.30.

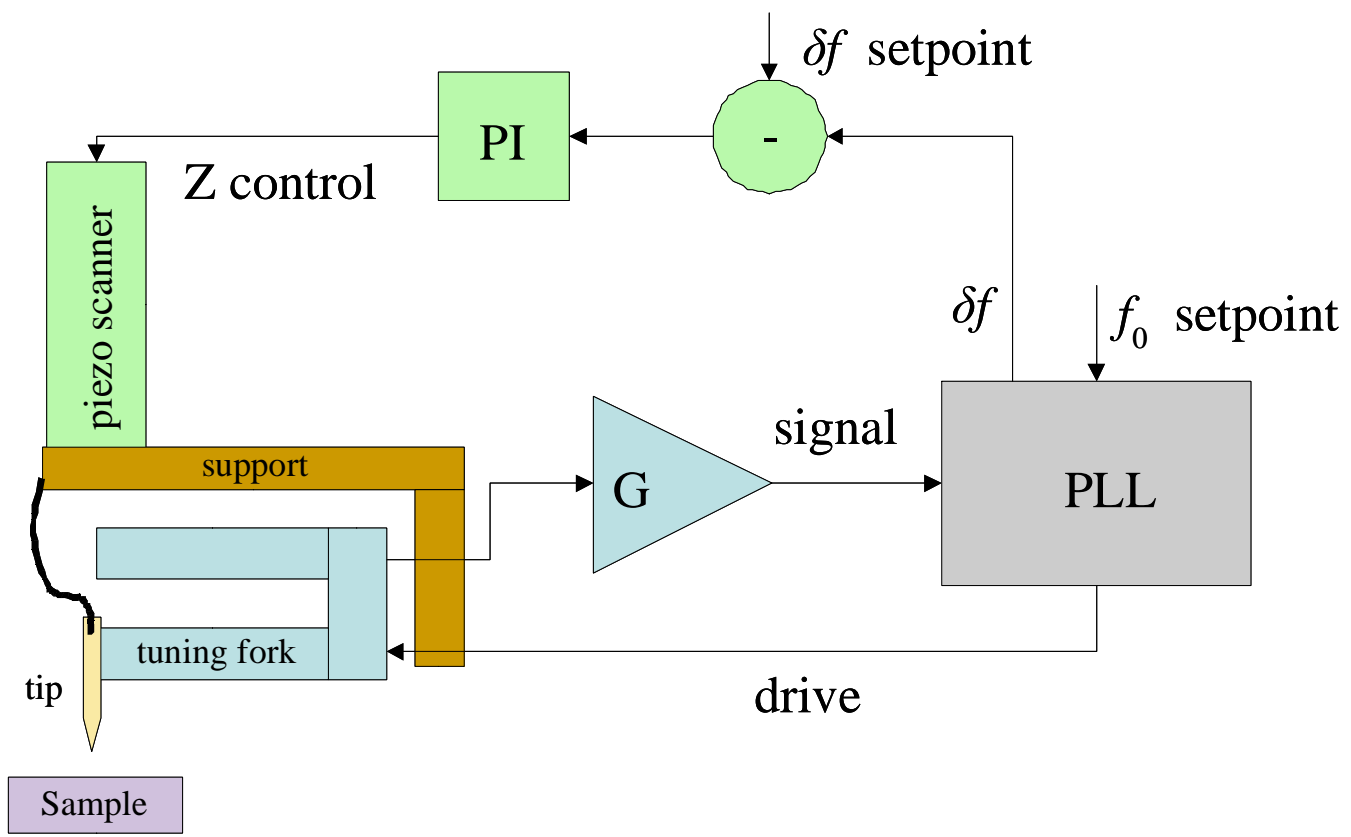

Fig. 2.30 The feedback loop operated for AFM imaging is composed of the following elements: the quartz tuning fork (TF) whose resonant frequency is a measure of the tip-sample distance, the PLL that both drives the TF at its resonant frequency and measures the error $\delta f$ in frequency signal, and a controller that adjust the piezo scanner voltage so as to keep a constant tip-sample interaction (Edistance). What is important to notice here is that we basically have two imbricate loops: one for the excitation at resonance of the fork, having typical response up to $1 \mathrm{kHz}$, and another one for the tip-sample distance adjustment, which has a time response of $\sim 1 \mathrm{~Hz}$. Speed limitation of the feedback on position comes from the noise on the $\delta f$ signal which imposes inserting a low pass filter $(120 \mathrm{~Hz})$. Given this limitation, an optimization of the scanning speed is done by choosing appropriate feedback parameters (see text).

When the tip-to-sample distance is varied, the resonance of the oscillator changes as shown in

Fig. 2.28. In regions of the curve where $\delta f$ varies with the distance, this signal can be used to maintain the distance at a fixed value. Given the shape of the curve, different imaging modes exist:

- The so-called "tapping mode" is operated closest to the sample surface in the negative slope region (of $\delta k(z)$ ), where coulomb repulsion dominates all other contributions. The feedback is then set in "negative" direction, meaning that it will react to an increase of gradient by retracting the tip. The tapping mode, though oscillating, operates similarly to the "contact mode" in non-dynamic AFM. 
- The non-contact mode is operated in the region of positive slope, where either electrostatic or Van der Waals force dominate. The feedback is then set to "positive" direction.

It is clear that the region in-between (emphasized by a red line on Fig. 2.28) is subject to ambiguous and slow behavior of the feedback loop. Moreover when imaging in non-contact mode, one has to be very careful in order not to trespass the cross-over region or the tip will crash. For this reason, it is better to image reasonably flat surfaces or at higher distances in this mode. When imaging is performed in contact mode, there is no unstable behavior. This is why we preferred this mode. In this mode, however, the cross-over region is responsible for a slower feedback response to an increase in tip-to sample distance (a "down" step on the sample surface) than for a decrease (up step). In order to compensate for this asymmetric behavior, we found convenient to use logarithmic feedback control, similar to what is used in STM mode [78].

To operate in tapping mode, we set a positive $\delta f$ setpoint, typically $1 \mathrm{~Hz}$. Alternatively, we could set the VCO center frequency below the free resonance frequency. The piezoelectric tube voltage is adjusted by the PI element of the controller to keep $\delta f$ at the setpoint. Hence, the sign of the feedback is set so that an increase in the resonant frequency yields a retraction of the piezotube.

While imaging, the tube is deflected laterally by the controller to scan the sample, at a typical rate of 1 line per 5 seconds $\left(\mathrm{v}_{\mathrm{x}} \sim 1.6 \mu \mathrm{m} / \mathrm{s}\right)$, while maintaining $\delta f$ at the setpoint. One line is $8 \mu \mathrm{m}$ wide for our tube, at $30 \mathrm{mK}$, and the number of lines (depending on the desired resolution) is typically 100 . Thus it takes about 8 '20 to record an $8 \times 8 \mu \mathrm{m}$ image.

\subsubsection{5.b Optimizing AFM imaging}

Good performance of AFM imaging requires tuning the gains of the PI elements in the feedback loops properly, for optimizing the desired characteristics (scanning speed, topographic resolution...). Several articles have addressed this point both experimentally and theoretically [59, 68, 79-81]. Theoretical analysis enables determining the feedback settings for optimal dynamic performance. These analyses however rarely take into account the adverse effect of noise on imaging and stability. In practice we determined "optimal" feedback parameters by tuning the step-response of the AFM loop of Fig. 2.30 to a small voltage step added to the piezo tube polarization $\mathrm{Vz}$ (in both directions), which simulates a topographic step. 


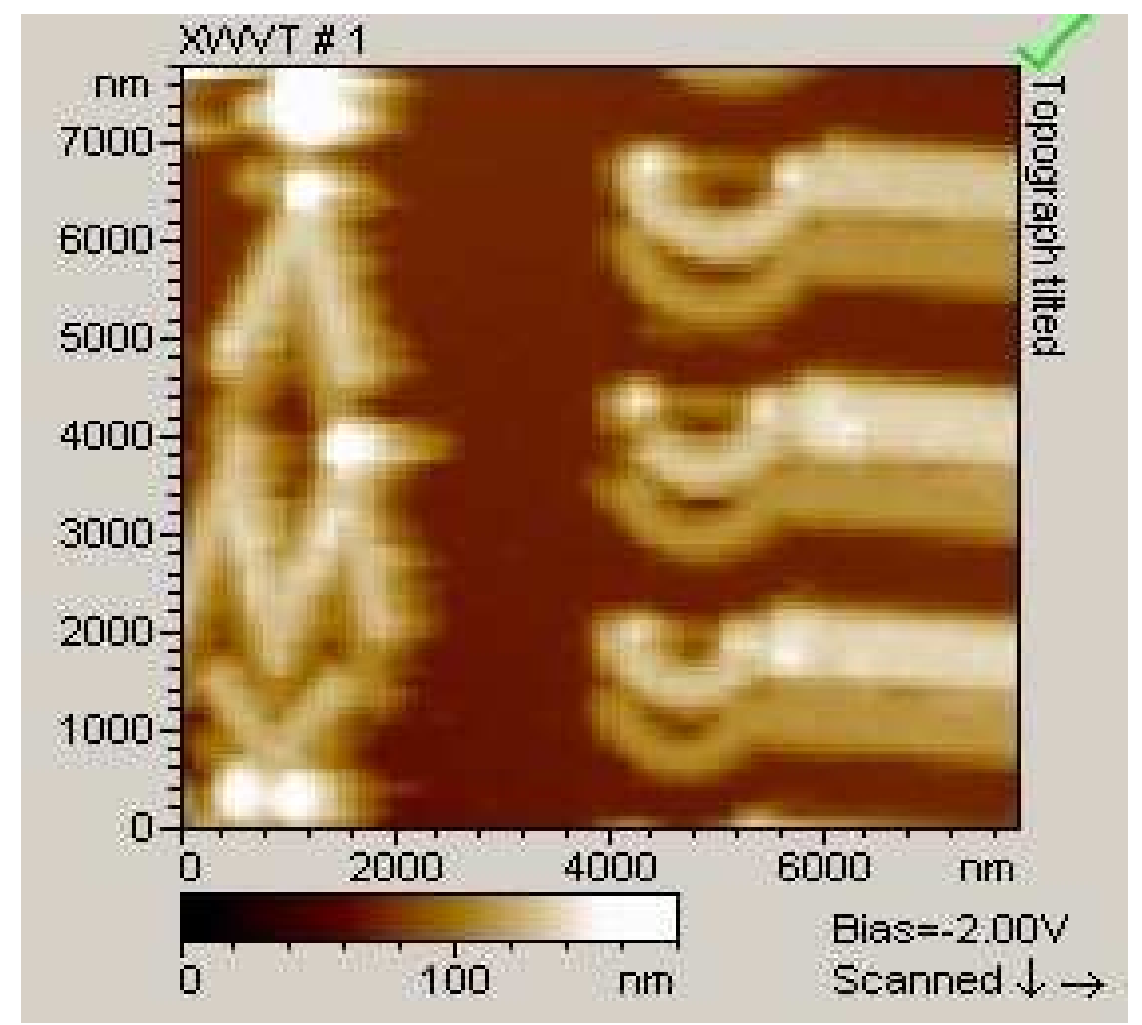

Fig. 2.31 Full range $\left(8 x 8 \mu \mathrm{m}^{2}\right)$ image of a sample studied during this thesis (see chapter 4$)$. Here, the resolution is limited by the number of recorded pixels, fixed to $64 \mathrm{pts} / \mathrm{lines}$. The maximum step height on this sample is $60 \mathrm{~nm}$. This image was scanned at $2 \mu \mathrm{m} / \mathrm{s}$. 
Chapter 3

\section{EXPERIMENTAL}

\section{TECHNIQUES}


In this Chapter, I will give an overview of the experimental techniques involved in the environment and operation of the AFM-STM built during this thesis work. I will give details regarding:

- the special type of dilution refrigerator we have used, and the problems encountered,

- the requirements on wiring and filtering for very low temperature experiments in general, and how we implemented them in the present case,

- data acquisition and experiment control,

- fabrication techniques for tips and sample. 


\subsection{A dilution refrigerator well adapted for a local probe microscope}

\subsubsection{Functioning of the inverted dilution refrigerator}

To cool our microscope down to the $50 \mathrm{mK}$ temperature range we use a special ${ }^{3} \mathrm{He}-{ }^{4} \mathrm{He}$ dilution refrigerator, designed by Alain Benoit at the CRTBT (Grenoble) and called Sionludi. It has an "inverted" geometry, in which the coldest plate is topmost (see Fig. 3.1), contrary to most dilution fridges. This is very convenient for an application like ours, which requires good accessibility to the experiment, to be able for instance to work comfortably on the microscope with binocular lenses. The microscope is simply laid (and screwed) on the Sionludi top plate. No additional setup is required to hold it, and it is easily removable. This is required because the mounting of the tip on the tuning fork, for instance, cannot be done in place: it requires removing part of the microscope to work on it on a workbench under binocular lenses (see Chapter 2).

Another specificity of the fridge is the absence of a helium bath, ensuring a steady stage at $\sim 4.2 \mathrm{~K}$ in ordinary dilution fridges (see Fig. 3.1 and Fig. 3.2). Instead, the cryogenic liquid is contained in a Helium dewar placed underneath the fridge, which one slightly pressurizes ( $\sim .1 \mathrm{~atm}$ ) to obtain a cooling $\mathrm{L}^{4} \mathrm{He}$ circulation inside a dedicated circuit of the fridge. Liquid reaches a box anchored to the " $4 \mathrm{~K}$ stage", evaporates in that box and circulates downwards into a continuous exchanger. The output goes directly to the helium recovery. Helium flow is regulated by a Bronkhorst EL-FLOW® digital mass flow controller. The flow is set large enough to provide a steady $4 \mathrm{~K}$ stage temperature, while keeping a reasonable helium consumption of about $12 \mathrm{~L}$ of liquid per day. The downward ${ }^{4} \mathrm{He}$ flow is used in permanent regime to thermalize the incoming cooling mixture, so the "main exchanger" is properly dimensioned to recover the enthalpy of the outgoing ${ }^{4} \mathrm{He}$. Temperature fluctuations on the $4 \mathrm{~K}$ stage can cause fluctuations of the mixing chamber temperature, either because the mixture is not properly cooled when it reaches the dilution stages, or due to radiations.

The Sionludi has a second separate circuit, containing the ${ }^{3} \mathrm{He}-{ }^{4} \mathrm{He}$ mixture with an approximate atomic ratio 30\%-70\%. Capillaries passing inside the $4 \mathrm{~K}$ exchanger bring the mixture up into the fridge. One of them is a low impedance bypass circuit that brings the mixture to the mixing chamber without passing through any impedance. This bypass circuit lets cold mixture gas circulate with a large flow for precooling the upper stages from room 
temperature down to $4 \mathrm{~K}$. Such a circuit is necessary in this design since, as a consequence of the absence of a Helium bath, one cannot use exchange gas in the vacuum can to cool down as in ordinary fridges. The other capillary brings mixture to the impedances at the input of dilution exchanger.

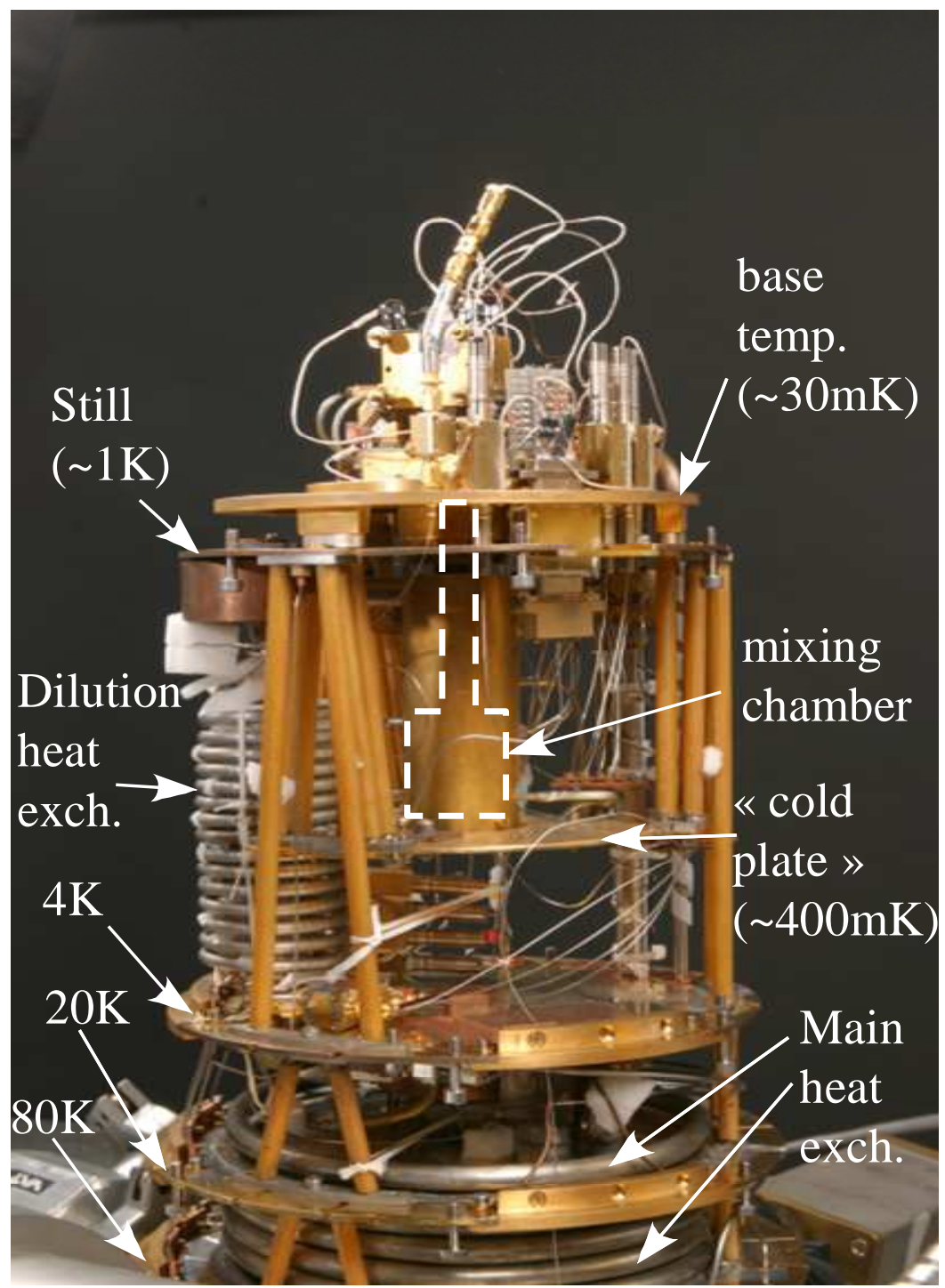

Fig. 3.1 Photograph of the Sionludi where the principal components are indicated. 


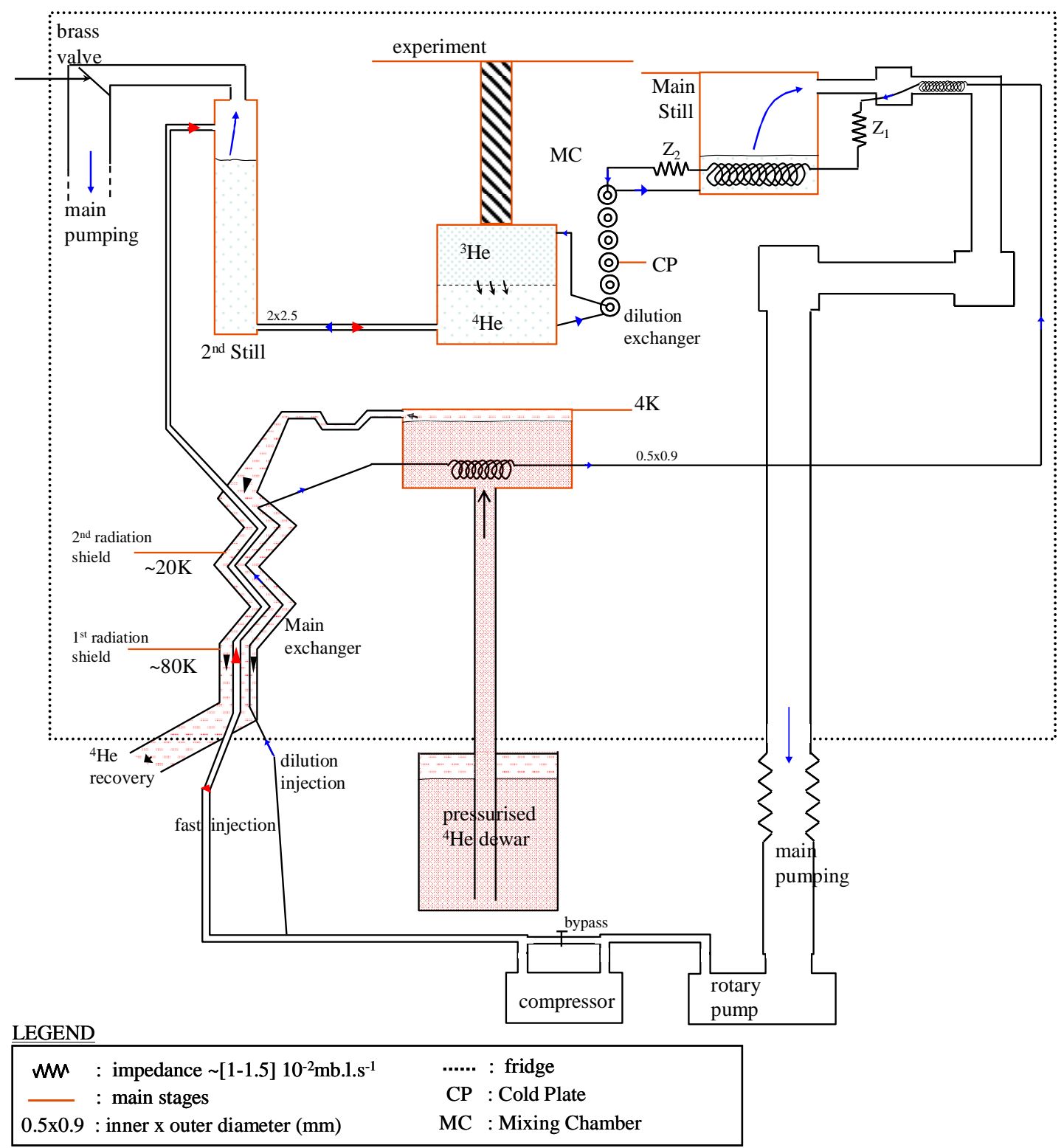

Fig. 3.2 Schematic of the principle of gas flows in the Sionludi (see text). The fridge's enclosing is shown by a dotted line, corresponding to the vacuum can represented on Fig. 3.3.

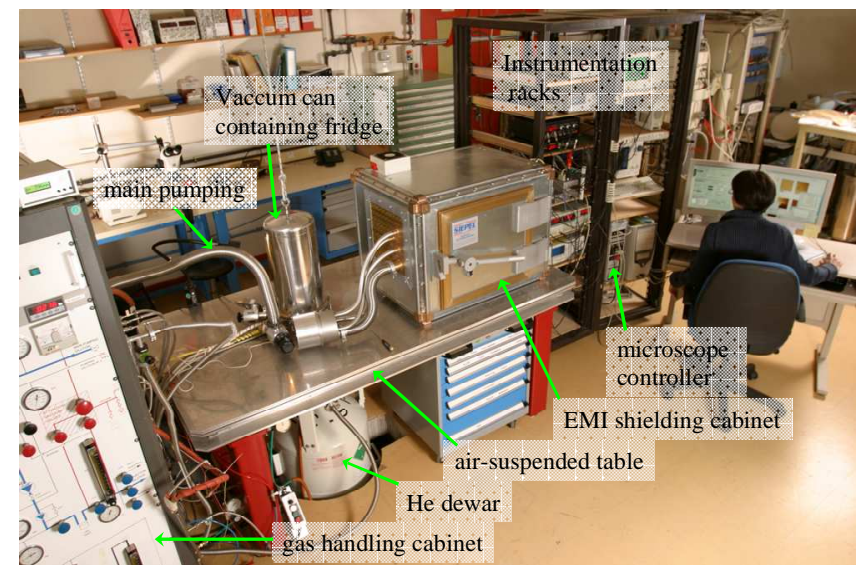

Fig. 3.3 Ensemble view of the lab. From left to right: the gas handling system, the vibration isolation table, on which is laid the fridge (enclosed in vacuum can here) and the helium Dewar attached under the table, the shielding cabinet linked to the fridge by metallic shields, and finally the electronic cabinets and the operator in front of the command computer. 
To reach such low temperatures, one must take great care to avoid thermal leaks through radiation and conduction. To limit them, our fridge is enclosed in a vacuum can and a set of copper thermal radiation shields arranged like Russian dolls and tightly screwed to stages of the refrigerator, to ensure their good thermalization. In this way, each stage receives radiations only from a stage close in temperature, and the inner stage (the coldest) is well separated from the outside room temperature radiations.

When this thesis work started the refrigerator was being completely refurbished at the CNRS Grenoble, because the main heat exchanger had become leaky due to defective materials, and the dilution heat exchanger had completely plugged. This reparation lasted much more than anticipated. During this phase I participated in testing and debugging the refrigerator on several occasions. Even after we recovered a working fridge in Saclay, we made some modifications, aiming at reducing liquid helium consumption and oscillatory instabilities in the $4 \mathrm{~K}$ stage temperature.

\subsection{2 (not so good) Vibrations in dilution refrigerator}

Vibrations are a major problem in STM experiments since the tunnel current varies exponentially with the tip to sample distance, with a characteristic scale of $\sim 0.1 \mathrm{~nm}$. Many setups try to compensate, or eliminate vibrations reaching the microscope, which introduce noise in the measurement. As already mentioned in Chap. 2, the general strategy for decoupling from external vibrations is to design a microscope that is as stiff and as light as possible to increase the resonance frequencies of the structure, and to embed it into a low-pass mechanical filtering system with a cut-off frequency as low as possible, typically <1 Hz. In our case, this system consists of an air-suspended vibration isolation table onto which the fridge is mounted (the Dewar being suspended below the table) to isolate the microscope from the vibrations of the building (see Fig. 3.3) ${ }^{1}$. However, this setup does not fully eliminate vibrations from the environment of the microscope, as explained below.

In usual dilution fridges, the incoming mixture is condensed at $\sim 100 \mathrm{mb}$ by thermal contact on a $1.5 \mathrm{~K}$ pumped liquid ${ }^{4} \mathrm{He}$ bath called the " $1 \mathrm{~K}$ pot". Another difference between Sionludi

\footnotetext{
${ }^{1}$ However the table does not protect well against acoustic vibrations transmitted by air. For instance, clapping hands induces vibrations which are easily detected with the STM. Efforts could be made to further enhance acoustic isolation.
} 
and more standard dilution fridges is the absence of a $1 \mathrm{~K}$ pot. The initial condensation of the mixture is thus operated on the $4 \mathrm{~K}$ stage, which means that it is necessary to pressurize above atmospheric pressure, using a compressor. Once the dilution is started, condensation is achieved by exchanging heat with cold gases in the main pumping line (see Fig. 3.2), and one should normally be able to switch off the compressor and work with an injection pressure in the 500-700 mb range which can be delivered by the rotary pump alone. In our Sionludi, however, this is not the case, and we can only work in a steady state with a pressure of the mixture injection line permanently above 1.5 bars, i.e. with the compressor permanently on. This generates some acoustic pressure and a substantial level of vibrations, both of which are a nuisance for the microscope operation. Even worse, the mixture flow itself becomes turbulent and generates a lot of vibrations, presumably when it flows through the impedances, at a stage that is tightly linked to the experiment plate.

We diagnosed this problem and somewhat measured the level of vibrations in the fridge by putting a microphone on the experiment plate, next to the microscope. The microphone is made of a piezoelectric multilayer stack PICMA® P-885.30 from PI glued vertically, at the end of which a $\sim 6 \mathrm{~g}$ copper block is attached ${ }^{1}$ (see Fig. 3.4). When vibrations arrive on the microphone, the copper block provides an "inertial force" proportional to its mass, which the stack converts to an electrical signal. This electric signal is amplified by a fixed-gain (x100) low noise amplifier LI75A by NF-Electronics and fed to the input audio line of the computer. Note that the setup is sensitive only to vertical vibrations of the experimental plate.

\footnotetext{
${ }^{1}$ The model we use has a bare structural resonant frequency of $90 \mathrm{kHz}$, and a stiffness of $68 \mathrm{~N} / \mu \mathrm{m}$. With the copper block, the resonance frequency is $17 \mathrm{kHz}$.
} 


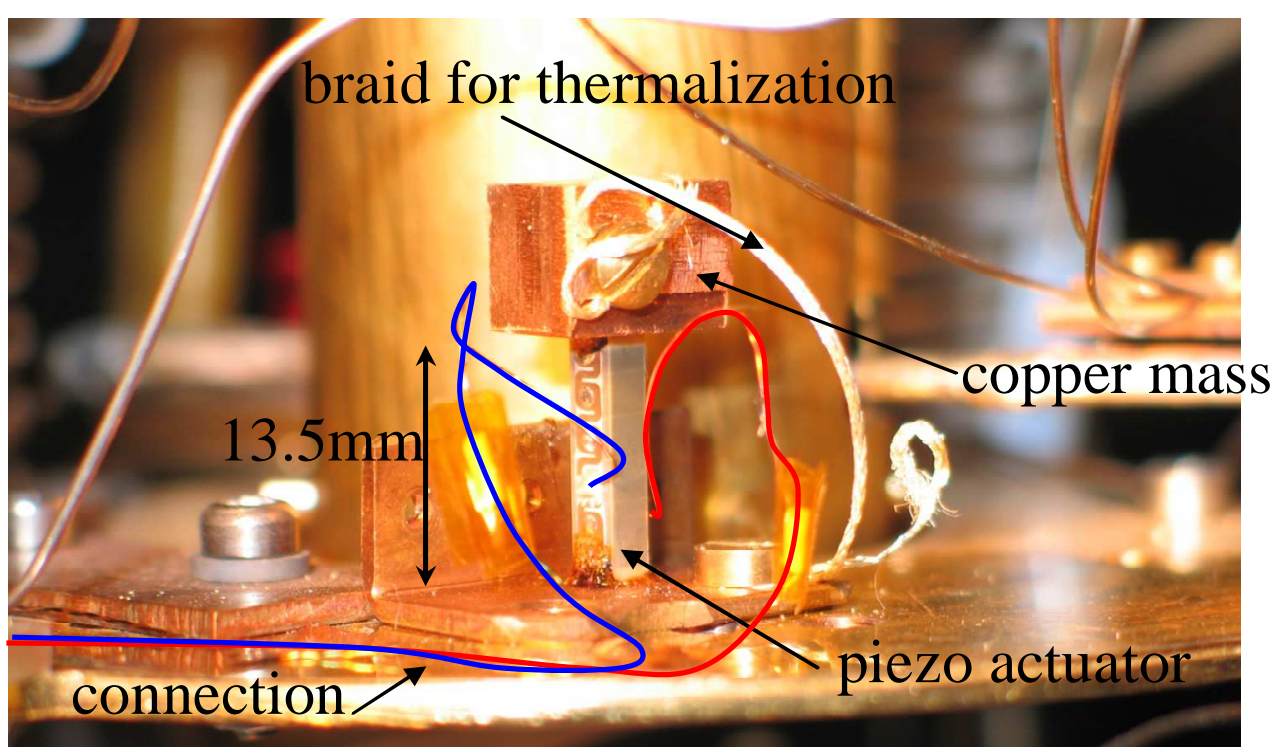

Fig. 3.4 Photography of the microphone used to detect vibrations inside the refrigerator. The electric signal produced by a stress (induced by vibrations of the plate) is collected on the electrodes of a piezoelectric actuator, amplified and fed to the audio input of the computer.

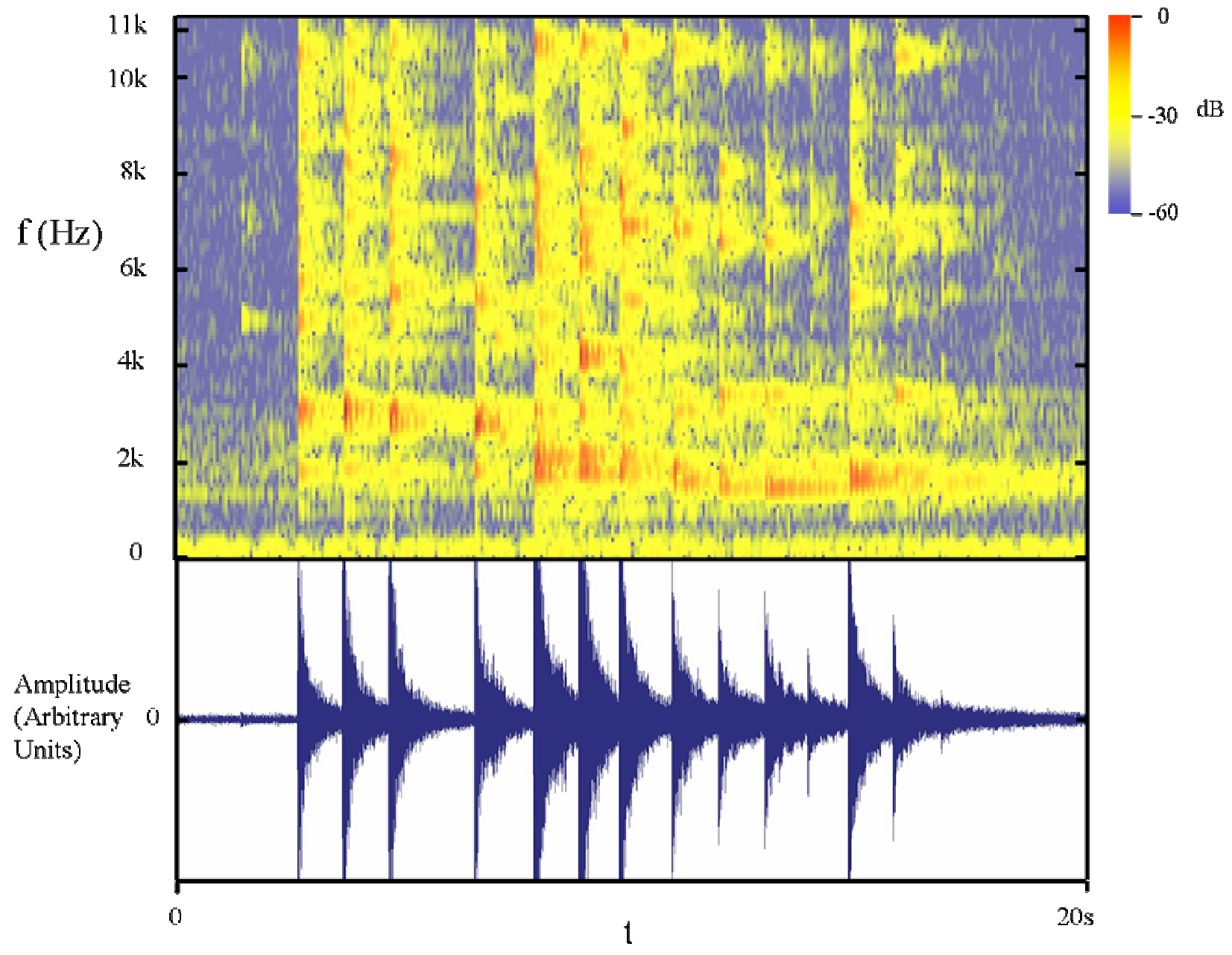

Fig. 3.5 Audio trace of vibrations of the fridge, recorded at the base temperature. Top: spectrum of the signal from $\sim \mathrm{DC}$ to $11 \mathrm{kHz}$. The color indicates the amplitude of the Fourier component relative to the maximum amplitude, in $\mathrm{dB}$. The signal spectrum presents the characteristic resonant frequencies of the fridge structure. Bottom: Corresponding wave signal, in arbitrary units. 
We have recorded several tracks in different circulation regimes of the refrigerator.

Sometimes it sounds more or less like a continuous turbulent flow, and sometimes it sounds more like metallic pings on pipes or bells (Fig. 3.5). We do not understand the differences observed between the various regimes. In all cases, the level of vibration is too high to allow stable spectroscopic measurements in true vacuum tunneling. It would even hinder performing STM imaging of a clean metallic surface. Note however that these vibrations do not seriously affect AFM mode operation.

We have checked that when the return line pressure is reduced by diverting the mixture momentarily, the fridge becomes indeed much quieter. There is no fundamental reason why our refrigerator could not operate with a lower return line pressure, like other Sionludis. We believe that the high return line pressure is due to some defective part, (likely a heat exchanger), and it will eventually be fixed, when the problem is precisely understood. This will certainly require a few trial-and-error runs involving modifications to the dilution circuit, and it is hardly predictable how long this can take. Since we were still able to perform stable tunneling spectroscopy in spite of the vibrations (see below), we decided this needed not be done during this thesis work.

\subsubsection{Living with vibrations}

In spite of this annoying level of vibrations, we have found a way to perform stable and accurate local electronic spectroscopy with our STM. Since our setup is too unstable to perform tunneling through a vacuum gap (as in normal STM operation), we instead put the tip in mechanical contact with the sample and tunnel through a thin oxide barrier covering the sample. This way, the distance between the two metals is well defined and remains fixed, in spite of the vibrations. In some sense, we mimic here the fabrication of planar tunnel junctions in situ, during the experiment.

Of course this requires the presence of an appropriate oxide layer on the sample. Such oxide spontaneously grows on aluminum films exposed to air. However, noble or semi-noble metals do not naturally form a good oxide layer appropriate for tunneling. In this case, one can still obtain a good tunnel barrier by covering the noble metal by a thin aluminum layer (i.e. a couple of nm thick) which will completely oxidize in air and form the barrier.

We can let the STM tip stand on this oxide layer and perform stable spectroscopy, with contacts lasting for hours without any change in transmission, in spite of the vibrations. 


\subsubsection{Possible improvements}

For increased immunity to vibrations, most local probe microscopes used at low temperature are suspended by springs $[82,83]$. We did not initially choose this option because of the coarse motion mechanism which changes the balance of the microscope when displaced, and because it complicates wiring and thermalizing the sample. If much better stability becomes necessary for some future experiment, this choice could be reconsidered. 


\subsection{Wiring a microscope for very low temperature experiments}

Connecting a $30 \mathrm{mK}$ experiment with room temperature equipment requires fulfilling a number of requirements which are detailed below.

\subsubsection{Thermal load}

The most basic requirement for the installation of a low temperature setup is to properly thermalize all the elements, without warming up the refrigerator. Wires need to be thermally anchored at each temperature stage, and one has to adjust the necessary length in between stages such that thermal flux that can be compensated by the available cooling power. As one goes down in temperature, the cooling power is reduced, but thermal conductivity of materials as well.

Estimating the thermal load brought by the wires (and optimizing their length) is done by using tables of temperature dependent thermal conductivity of typical materials used (steel, $\mathrm{Cu}-\mathrm{Ni}$, copper, manganin ${ }^{\circledR}$, superconducting wire in resistive matrix...) and by calculating the thermal flux as a function of their cross-section and temperature difference between stages.

Our fridge has a cooling power of $\sim 50 \mu \mathrm{W}$ at $100 \mathrm{mK}$, which means that if we feed a total power of $50 \mu \mathrm{W}$ down to the mixing chamber, its temperature will establish at $100 \mathrm{mK}$.

The steady state temperature of a dilution fridge is given by the balance of the cooling power $\dot{Q}=84 T^{2} \dot{n}$ ( $T$ is the temperature of the mixing chamber, the power is expressed in Watts and $\dot{n}$ is the ${ }^{3} \mathrm{He}$ throughput, in mole. $\mathrm{s}^{-1}$ ), and the heat load brought by:

- radiation from hotter parts of the fridge,

- conduction through the materials,

- convective heat brought by the incoming fluid, due to imperfect heat exchangers.

Our bare fridge had a base temperature of $\sim 20 \mathrm{mK}$. From the measured cooling power at $100 \mathrm{mK}$, one can then estimate that the residual heat loads listed above are of the order of $2 \mu \mathrm{W}$ in total. When installing the wiring for the experiment, this obviously increases the conduction heat load and the final base temperature will be more or less affected depending on how this additional load compares with the former figure. 
We installed a total of 50 shielded wires from ambient temperature down to the mixing chamber. They are divided as follows:

- a 15-wire line for the thermometry of the experiment,

- a 21-wire line for the control of the microscope,

- a twisted pair line for a small superconducting coil,

- 4 twisted pair cables

- and 4 coaxial lines for transport measurements on the sample or other purpose $^{1}$.

Most lines are home made with common cryogenic materials having low thermal conductivity. For the shielding, we employ stainless steel or $\mathrm{Cu}-\mathrm{Ni}$ tubes. Because of their cross-section, the thermal conductance of the shielding capillaries is the dominant contribution, so we diminished as much as we could their diameter and wall thickness: for instance, we fitted $2450 \mu \mathrm{m}$-diameter wires in a $0.6-0.7 \mathrm{~mm} \mathrm{CuNi}$ capillary to make the multi-wire line from $4 \mathrm{~K}$ to $30 \mathrm{mK}$.

Inner conductors are chosen depending on their use: the lines through which large currents are fedwere made of superconducting wires which do not dissipate, and are good thermal insulators. For current feeding of the coil we use $100 \mu \mathrm{m}$-diameter $\mathrm{Cu} / \mathrm{Mn}$ down to $4 \mathrm{~K}$ and $50 \mu \mathrm{m}$-diameter $\mathrm{Nb} / \mathrm{Ti}$ in $\mathrm{Cu} / \mathrm{Ni}$ matrix insulated wires from $4 \mathrm{~K}$ to $30 \mathrm{mK}$. Lines where high currents are not needed (DC transport, thermometers, tunnel and tuning fork measurement) are made of resistive material: for those we use either $70 \mu \mathrm{m}$-diameter insulated manganin all along, or commercial $0.5 \mathrm{~mm}$ diameter microcoax ${ }^{\circledR}$, made of stainless steel both for the inner conductor and the shielding. Finally for the (21 wires) control lines of the microscope we use $70 \mu \mathrm{m}$ manganin down to $4 \mathrm{~K}$ and then NbTi in CuNi matrix down to $30 \mathrm{mK}$; the latter choice is done to minimize the thermal load brought by this multi-wire line.

The multi-wires lines are assembled by pulling all the wires along with silicon glue into a tube of adjusted length and minimal diameter. Glue is used to improve the thermal contact between the inner conductors and the shielding, to ensure that conductors are well thermalized. It also prevents vibrations, which are sources of microphonic or triboelectric noise. Prior to inserting the wires, the inside of the tube is polished by passing through it a coiled tungsten or steel wire, to prevent wearing the wire insulation on the tube wall asperities which could cause shunts to ground.

\footnotetext{
${ }^{1}$ In addition to these wires going to the mixing chamber, other unshielded wires are installed for auxiliary thermometry and microphony (See previous section) purpose at higher temperatures.
} 
Before the installation of the wires, our fridge had a base temperature of $20 \mathrm{mK}$, and the whole wiring raised the temperature to $30 \mathrm{mK}$, corresponding to an additional load of $\sim 2.5 \mu \mathrm{W}$. Given the large number of lines installed, this is satisfactory.

\subsubsection{Low level signals and low noise requirements}

As explained above, all the lines reaching the $30 \mathrm{mK}$ plate are shielded. This is necessary to prevent electromagnetic noise from the outside (antennas, power supply, cell phones...) to couple to the experiment, which would prevent us from measuring low level signals. It is also integral part of the filtering strategy described below.

To reach a high signal-to-noise ratio, it is also necessary to avoid cross talk between some lines, in particular between low-level and high level signals. There are two kinds of wire geometry that can be used: when one wants to measure small voltages differential signals, it is necessary to minimize magnetic flux picking by twisting two wires all along. When one needs large bandwidths above $100 \mathrm{MHz}$, the coaxial geometry is the best solution.

Finally, in low-noise measurements, the grounding of the signals has to be carefully considered to avoid ground loops in which uncontrolled flux pickup from the lab ambient magnetic noise harshly affect measurements. Ground loops can generally be avoided by selecting a proper wiring topology with a unique, well localized, voltage reference point. In low temperature experiments, however, good grounding practice sometimes conflict with cable thermalization, and compromises have to be made. This topic is further discussed in the article on the tunnel current amplifier published in Review of Scientific Instruments 77, 123701 (2006), reproduced below (3.2.4.3).

\subsubsection{Filtering}

The last requirement for setting up properly the wiring is to filter the high frequency noise in each line. This is necessary to ensure that the experiments are performed in thermal equilibrium at the fridge temperature. During my thesis we have developed microfabricated filters to fulfil this requirement. We have published this work in Review of Scientific Instruments 77, 115102 (2006). Below I give detailed explanations regarding these filters which did not all fit in the article. The article itself is reproduced at the end of this section. 


\subsubsection{Necessity of filtering}

Black-body radiations emitted by dissipative elements can propagate inside the fridge, especially on the coaxial lines which are good wave guides. They are absorbed and re-emitted by electrons all along the lines. These radiations extend in a frequency range up to $h v \sim k_{B} T$, as stated by Planck's law, where T is the emitter's temperature.

When a photon emitted from a distribution at temperature $T_{1}$ is absorbed by a metal at a temperature $T_{2}$, an electron of this metal may go out of equilibrium with the distribution at $T_{2}$ if $T_{1}>T_{2}$. Its only way to relax is either to exchange energy with the surrounding electron bath, to reemit the radiation, or to give the extra energy to the phonons of the lattice. The latter process has a temperature-dependent rate. At room temperature, electron-phonon coupling is so strong that difference between electron temperature and phonon temperature is hardly ever measurable. But the coupling decreases as temperature decreases, so that at $\mathrm{T}<1 \mathrm{~K}$, electrons can be easily out of equilibrium with the phonons [84]. In particular, if one does not take great care to cut the black-body radiations from wires at $300 \mathrm{~K}$, it is unlikely that electrons in the lines ever get as cold as the refrigerator.

In order to perform high resolution tunneling spectroscopy with a normal probe (see chapter 1), it is important to take appropriate measures to ensure that electrons are in equilibrium at low temperature. In many other types of mesoscopic physics experiments a good filtering is also necessary to protect from unwanted photon assisted processes [85-89].

\subsubsection{Why filtering all the lines?}

One could think that by filtering properly the low-level signals connecting the sample, one would obtain the desired electronic temperature in the sample.

However, any unshielded cable acts as an antenna that absorbs from the environment and radiates on the experiment. It is therefore mandatory that all the lines entering the experimental environment are properly filtered and shielded all along so that they do not bear thermal radiations from $300 \mathrm{~K}$.

Most STM experiments performed in dilution refrigerators did not filter all the lines (or not sufficiently), including the controls of the microscope, which may partly explain why they could not reach the desired low effective electronic temperatures [90-92] (see Chap. 1 and below). 


\subsubsection{Propagation of thermal noise in networks}

When setting up an experiment that aims at performing low noise measurements at low temperatures, one needs to know the effective "electromagnetic temperature" of the lines connected to the sample. Assuming the instrumentation is ideal, the minimum noise reaching the sample is due to thermal noise propagating down the lines and emitted from all impedances connected in the setup, from $300 \mathrm{~K}$ to the base temperature. The question for the experimentalist is to know whether the noise density reaching the sample can be expressed as a thermal noise at base temperature, i.e. as emitted by an impedance at a temperature close to the fridge's.

In the following, I present a general treatment of this problem, and derive useful formulas to evaluate the effectiveness of a filtering setup.

The first goal is to find which amount of the noise produced by an impedance in the system is radiated on the sample. One basically uses Thevenin's theorem.

As seen from any two connections of the sample, the wiring connecting it to the outside can be modeled by an arbitrary network of impedances like the one described on Fig. 3.6.

We assume here that the network is sufficiently subdivided that each impedance $Z_{i}$ of the network is at equilibrium at its local temperature $T_{i}$. The non-symmetrised thermal noise spectral density, distinguishing emission $(\omega>0)$ and absorption $(\omega<0)$, across impedance $Z_{i}$ is given by Planck's law:

$$
S_{V_{i}}(\omega)=\frac{2}{\pi} \frac{\hbar \omega \operatorname{Re} Z_{i}(\omega)}{e^{\frac{\hbar \omega}{k_{B} T_{i}}}-1}
$$

One can obtain the contribution to the noise generated at the sample port due to each source in the following way (see Fig. 3.6 and Fig. 3.7 for a pictorial description): 


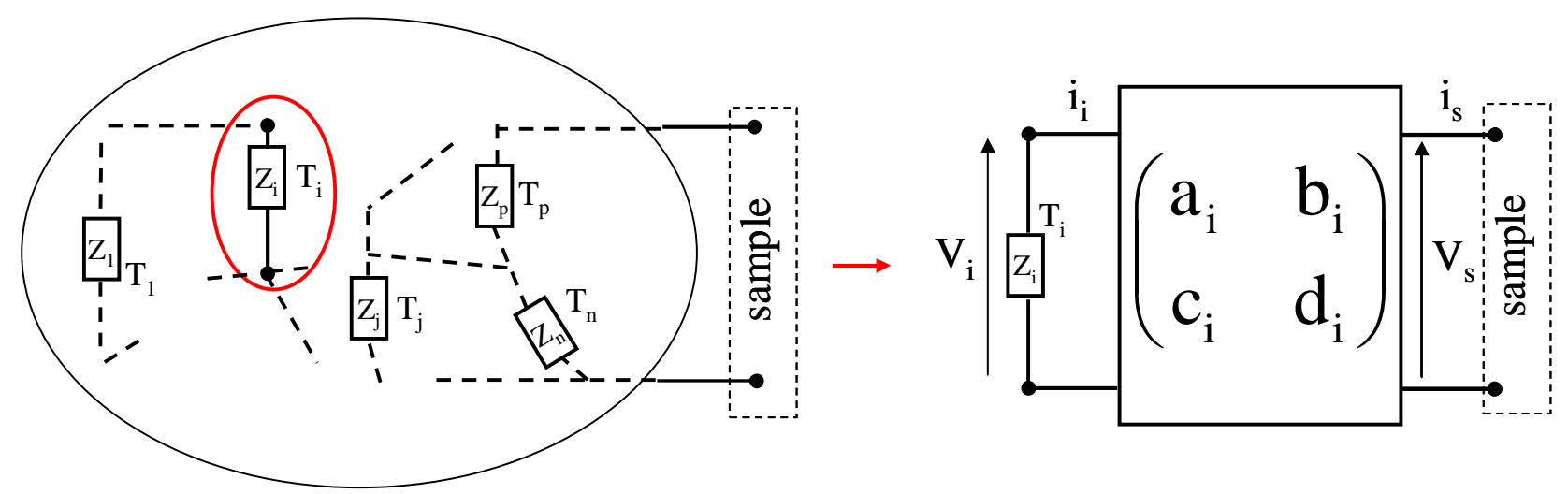

Fig. 3.6 As seen from the sample, the wiring is modeled by a network of impedances at different temperatures. To know the thermal noise spectrum seen by the sample, one may evaluate the contribution to noise at the sample from one particular impedance $Z_{i}$, and then sum all contributions from all $Z_{\mathrm{i}}$ s. For each $Z_{\mathrm{i}}$, one can reduce the rest of the network to an equivalent transfer matrix.

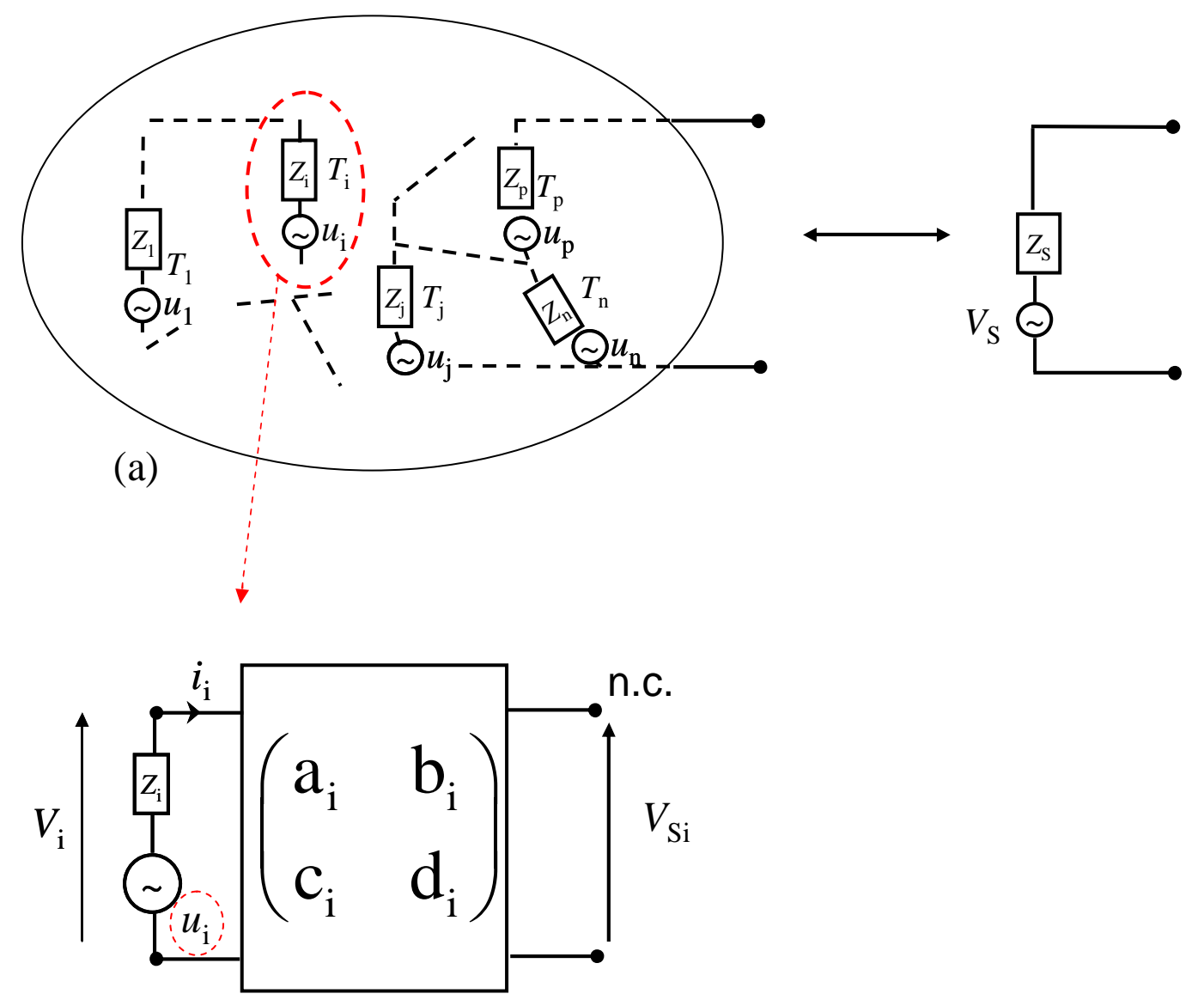

(b)

Fig. 3.7 Any impedance of the network can be modeled as a noiseless element in series with a voltage noise source $u_{\mathrm{i}}$ having a power spectrum given by (3.1). Each noise source $u_{\mathrm{i}}$ contributes to the output with an attenuation $a_{\mathrm{i}}$ that has to be determined. (a) Thevenin theorem tells us that the network viewed from the sample port is equivalent to $Z_{S}$ in series with a voltage source $V_{S}$. The relation between $Z_{\mathrm{S}}$ and the $Z_{\mathrm{i}} \mathrm{s}$ is known from the network theory (see text). (b) To know the relation between $V_{S}$ and the $u_{i} \mathrm{~s}$, one switches off all the noise sources except one $\left(u_{\mathrm{i}}\right)$, and calculates its voltage transfer at the sample port $V_{s_{i}} / u_{i}$. 
An individual impedance $Z_{i}$ is singled out from the rest of the network. The network in between $Z_{i}$ and the sample is linear and can be modelled by its $\mathrm{M}$ matrix. The sample load is not included in this picture. Following the conventions of Fig. 3.6, transfer equations between input port (indices i corresponding to $Z_{i}$ ) and output port (indices s for Sample) quantities write

$$
\left\{\begin{array}{l}
V_{i}=a_{i} V_{S}+b_{i} i_{S} \\
i_{i}=c_{i} V_{S}+d_{i} i_{S}
\end{array}\right.
$$

The total equivalent impedance $Z_{S}$ seen from output is defined by the ratio $-V_{S} / i_{S}$. From (3.2) one gets

$$
Z_{S}=\frac{b_{i}+Z_{i} d_{i}}{a_{i}+Z_{i} c_{i}}
$$

Thermal noise generated by $Z_{i}$ can be represented by a random voltage source $u_{i}$ placed in series with $Z_{i}$. Let's estimate the contributions of $u_{i}$ to the output voltage, with no load connected (see Fig. 3.7). Since $V_{i}=u_{i}-Z_{i} i_{i}$, one has ${ }^{1}$

$$
\alpha_{i}=\frac{V_{S}}{u_{i}}=\frac{1}{a_{i}+c_{i} Z_{i}}
$$

From the observation that

$$
\frac{\partial Z_{S}}{\partial Z_{i}}=\frac{1}{\left(a_{i}+c_{i} Z_{i}\right)^{2}}=\alpha_{i}^{2},
$$

one sees that the transfer coefficient for $u_{i}$ is readily obtained from the network's output impedance function, as seen from the sample. Using the superposition theorem, the voltage $V_{S}$ at output port, due to all voltage noise sources $u_{i}$ in each $\operatorname{arm} Z_{i}$ is:

$$
V_{S}=\sum_{i} \alpha_{i} u_{i}
$$

The power spectral density radiated at the sample port by source $i$ writes:

$$
S_{V S_{i}}(\omega)=\left|\alpha_{i}\right|^{2} S_{u_{i}}(\omega)
$$

with $\left|\alpha_{i}\right|$ the voltage gain calculated above, and $\left|\alpha_{i}\right|^{2}$ the power gain from source $i$ to the output.

\footnotetext{
${ }^{1}$ Since a reciprocal network verifies $\left|a_{i} d_{i}-b_{i} c_{i}\right|=1$, there exists a reversed similar relation between currents: $\frac{i_{i}}{i_{s}}=\alpha_{i}$.
} 
All noise sources are uncorrelated so their contributions add quadratically at the output (powers add):

$$
S_{V s}(\omega)=\sum_{i}\left|\alpha_{i}\right|^{2} S_{u_{i}}(\omega)=\sum_{i}\left|\frac{\partial Z_{S}}{\partial Z_{i}}\right| S_{u_{i}}(\omega)
$$

If we first imagine the whole network to be at an homogeneous temperature $T_{0}$, the noise at the sample is a perfect thermal noise at temperature $T_{0}, S_{V_{S}}(\omega)$ given by

$$
S_{V_{S}}(\omega)=\frac{2}{\pi} \frac{\hbar \omega \operatorname{Re} Z_{S}(\omega)}{e^{\frac{\hbar \omega}{k_{B} T_{0}}}-1}
$$

using (2.2), (3.7) and (3.8), and simplifying the Bose factors, one then obtains the following relation of general validity ${ }^{1}$ :

$$
\operatorname{Re}\left(Z_{S}\right)=\sum_{i}\left|\frac{\partial Z_{S}}{\partial Z_{i}}\right| \operatorname{Re}\left(Z_{i}\right)
$$

However, in the general situation when the network is not at a single temperature, the noise does not have the simple (3.8) form with a fixed temperature. Instead, to easily quantify the thermal character of noise at the output, we express $S_{V}(\omega)$ as in (3.8), but with an effective frequency-dependent temperature $T_{\text {eff }}(\omega)$ :

$$
\begin{aligned}
S_{V_{S}}(\omega) & =\frac{2}{\pi} \frac{\operatorname{Re} Z_{S}(\omega) \hbar \omega}{e^{\frac{\hbar \omega}{k_{B} T_{e f f}(\omega)}}-1} \\
& =\frac{2}{\pi} \frac{\hbar \omega}{\frac{\hbar \omega}{e^{\frac{k_{B} T_{e f f}}{(}(\omega)}}-1} \sum_{i}\left|\frac{\partial Z_{S}}{\partial Z_{i}}\right| \operatorname{Re} Z_{i}(\omega) .
\end{aligned}
$$

Making use of (3.7) and inverting the latter relation, we get the effective temperature $T_{\text {eff }}(\omega)$ of electrons at the output port:

\footnotetext{
${ }^{1}$ A similar relation exists linking not only the real parts of $Z_{S}$ and all the $Z_{i}: Z_{s}=\sum_{i} \frac{\partial Z_{s}}{\partial Z_{i}} Z_{i}$. This latter relation is an expression of Euler's relation for the impedance $Z_{s}$. Euler's relation applies to homogeneous functions of class $k$, defined by $f\left(t x_{1}, \ldots, t x_{n}\right)=t^{k} f\left(x_{1}, \ldots, x_{n}\right)$. Any impedance is thus a homogeneous function of class 1 . This general result for networks is not directly useful for our situation but it is nonetheless remarkable.
} 


$$
T_{e f f}(\omega)=\frac{\hbar \omega}{k_{B}}\left(\ln \left(1+\frac{\sum_{i}\left|\frac{\partial Z_{S}}{\partial Z_{i}}(\omega)\right| \operatorname{Re} Z_{i}(\omega)}{\sum_{i}\left|\frac{\partial Z_{S}}{\partial Z_{i}}(\omega)\right| \frac{\operatorname{Re} Z_{i}(\omega)}{e^{\frac{\hbar \omega}{k_{B} T_{i}}}-1}}\right)\right)^{-1}
$$

As could be expected, this noise is in general not equivalent to the noise of a single impedance thermalized at an intermediate temperature.

To sum up, knowing only the $Z_{i}$, their individual temperatures, and the topology of the network, one can calculate the output impedance $Z_{s}$ as a function of the $Z_{i}$, and subsequently using (3.1) and (3.7) the exact total output noise density as seen from the sample. With (3.11), one can finally check the efficiency of the filtering setup at each frequency. In a real setup the question is: how far is $T_{\text {eff }}(\omega)$ from the temperature of the refrigerator?

We made use of these results, combined with the calculated characteristics of our filters to predict their performance for thermalizing the electric lines (See below, section 3.2.3.8).

\subsubsection{Filtering technique}

Filtering the lines and thermalizing electrons is done using dissipative low pass filters, which attenuate the electromagnetic radiations received from hotter parts above their cutoff frequency, and reemit a thermal spectrum at their own temperature. Room temperature blackbody radiation extends up to frequencies $v \sim\left(k_{B} / h\right) 300 \mathrm{~K}=6 \mathrm{THz}$. In order to properly thermalize photons at $30 \mathrm{mK}$ the filters have to cut the spectrum above $v \sim\left(k_{B} / h\right) 30 \mathrm{mK}=600 \mathrm{MHz}$, and all the way up to $6 \mathrm{THz}$ with very high attenuation. In particular, there must be no "hole" in the stop-band. Thus, one has to be particularly careful when designing the filter to avoid transmission resonances. This is why we always prefer dissipative rather than reflective ones, which unavoidably present complicated resonances above their cutoff frequencies.

The filters usually consist of distributed RC lines providing better high frequency attenuation than a cascade of discrete $\mathrm{R}$ and $\mathrm{C}$ elements with the same total values. Indeed, for a cascade of discrete RC elements, the attenuation slope above the cut-off is given by the number of poles. In a distributed line, the attenuation starts more slowly but its slope keeps increasing 
with frequency. Furthermore, at very high frequency (typically a few GHz), discrete elements like surface mounted resistors get shunted by their parasitic parallel capacitance and attenuation decreases.

Diverse methods have been successfully applied to fabricate such devices: resistive coaxial cables [87, 88], and powder filters based essentially on the skin effect in small metallic grains [89].

During this thesis work, we have redesigned micro-fabricated meander filters that had been developed in the Quantronics group in 1994 [86]. The new design has an improved geometry to get a better attenuation, in particular to avoid TE and TM transmission resonances [93]. It also involves new materials, fabrication process and packaging with the goal of getting more robust filters than those of the old version, which were fabricated on silicon substrates, and for which the insulator was $\mathrm{SiN}$ which cracked after a few thermal cycles and provoked shunts to ground. The design of our distributed RC lines is shown in Fig. 3.8.
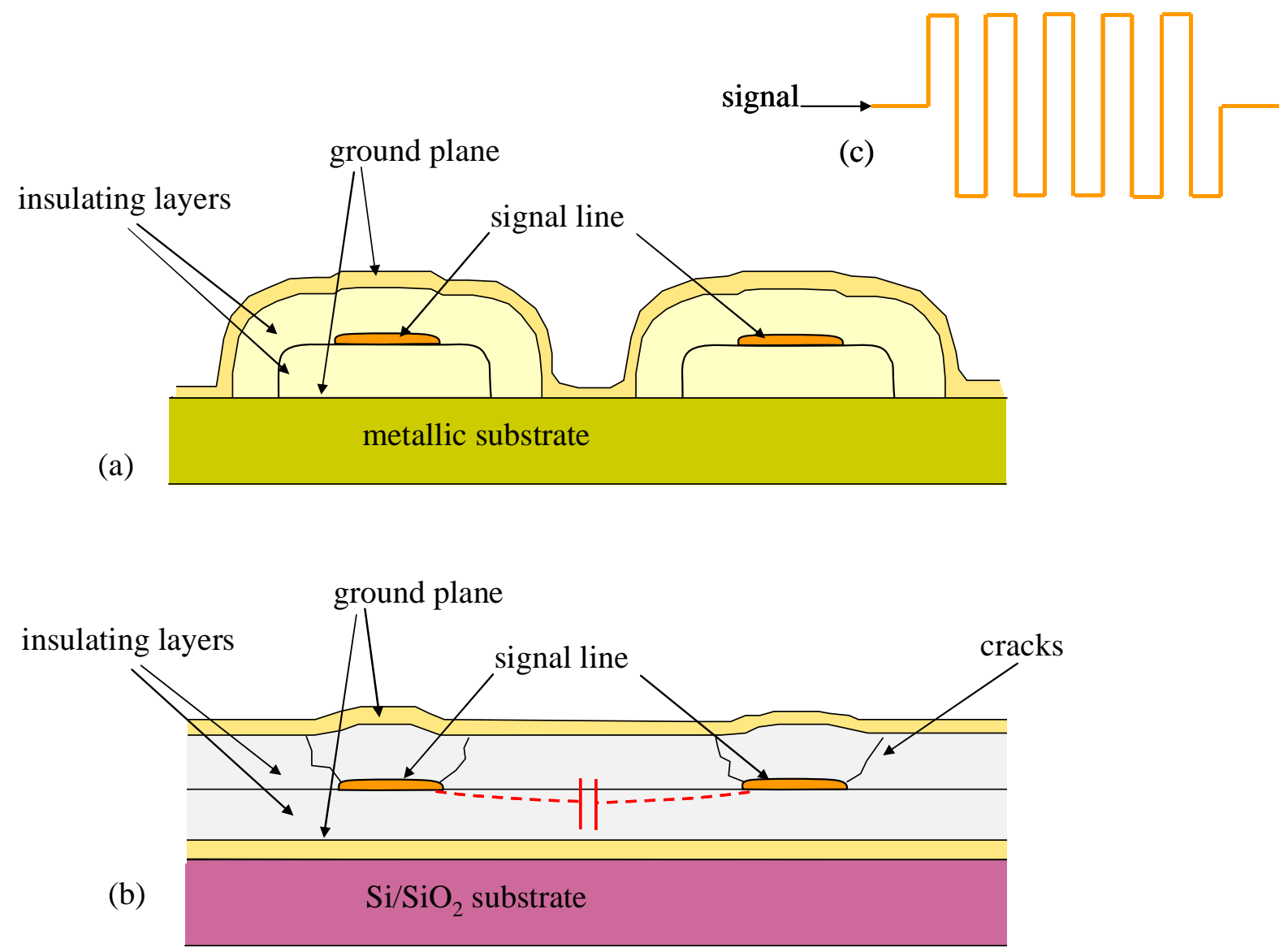

Fig. 3.8 Cross section view of the micro-fabricated filters. For readability, height and width are not to scale. (a) New design. (b) Former design. (c) Schematic top view. The new design is equivalent to an on-chip coaxial resistive line. The line is meandering on the chip to increase the available total length, to reach a sufficient attenuation with a single chip. The resistance and capacitance are distributed along the line, like in the old design, but no cross-talk can happen between two parts of the line since the line is fully encapsulated by ground. 


\subsubsection{Fabrication}

We have chosen the various materials by paying special attention to differential thermal contractions, which, in the old version of the filters, were responsible for failure either by shunt to ground or open circuit after a couple of thermal cycles. Filters are mounted in metallic shielding boxes made of brass, and we chose a brass substrate for the meanders accordingly.

As can be seen in Fig. 3.8, the meander is completely enclosed by ground, in a "coaxial" geometry. To make this geometry requires 4 fabrication steps, to pattern each layer: the first insulating layer, the meander, the second insulating layer, and the top ground plane. All patterns are done by lithography of photo-resists using a UV mask aligner MJB 3 UV 400 IR from Karl Suss.

All fabrication parameters are given in the article Rev. Sci. Instrum. 77, 115102 (2006) reproduced below. Here I just add schematic views of the process, and detailed comments.

\section{Insulating layers (Fig. 3.9)}

The insulating layers consist of Cyclotene ${ }^{\circledR} 402440$ BysbenzoCycloButene (BCB), a photosensitive polymer from Dow Chemicals. Their thickness can be adjusted at will from a few hundreds nanometers up to a few microns, by properly choosing the viscosity and the spinning velocity of the resist. This is useful for filters since, depending on the desired application, this tuneable thickness allows to vary capacitance to ground without changing the filter layout. In our case, we had an additional constraint on the dielectric thickness since the filters need to withstand the high voltages necessary for the piezoelectric actuators. To withstand $400 \mathrm{~V}$ with $\mathrm{BCB}$ (nominal electric field breakdown of $300 \mathrm{~V} / \mu \mathrm{m}$ ), we need at least $1.3 \mu \mathrm{m}$. This sets a lower limit to thickness, keeping in mind that sharp defects will enhance the field and may provoke breakdowns at lower voltages. To be on the safe side, we planned twice that thickness, i.e. between 2.5 and $3 \mu \mathrm{m}$. The first step of the process is schematically pictured in Fig. 3.9. 


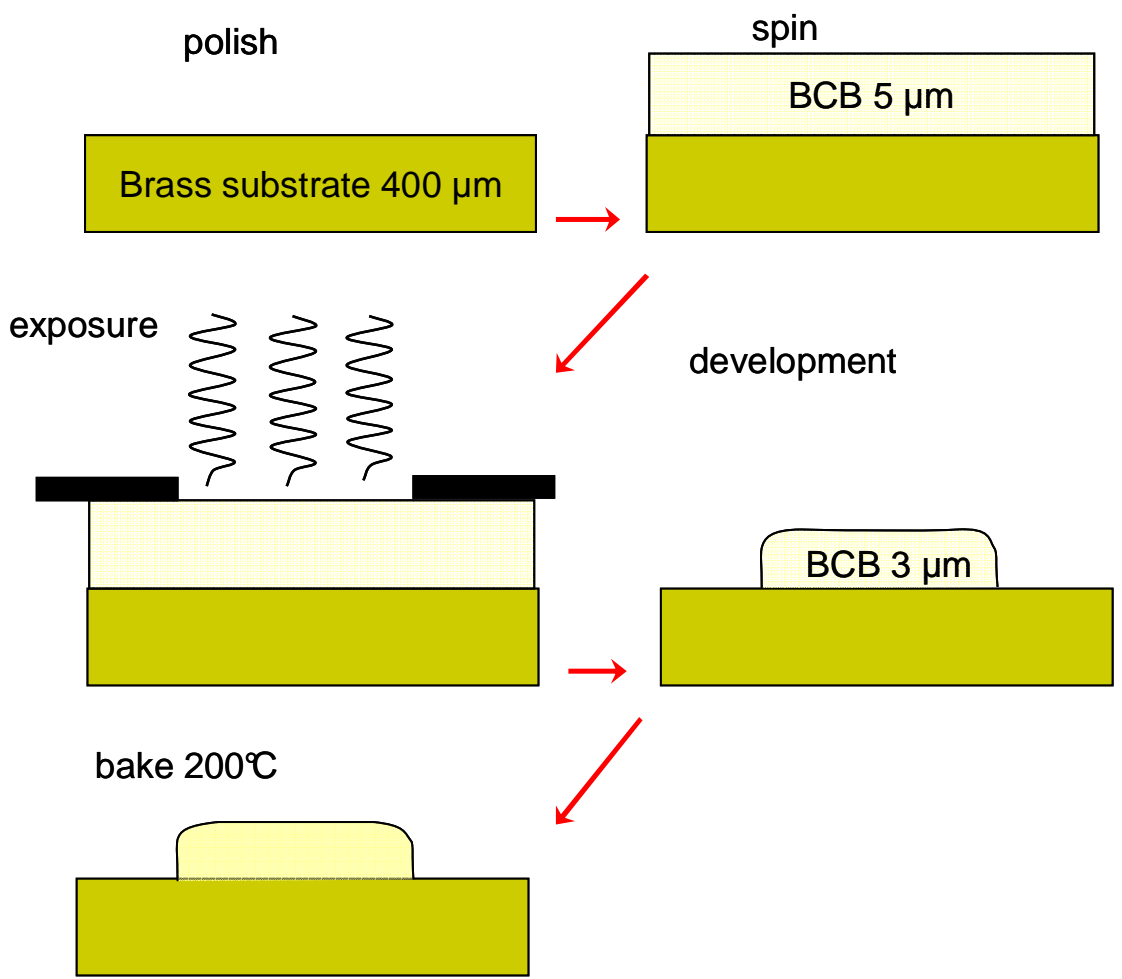

Fig. 3.9 Fabrication of the filters: step $^{\circ} 1$, insulating layer. A BCB layer of $5 \mu \mathrm{m}$ is spinned onto a polished brass substrate, to yield a final thickness of $3 \mu \mathrm{m}$. It is then UV exposed through a mask. $\mathrm{BCB}$ cross-linking is initiated where it is exposed (negative resin). After development, a $200^{\circ} \mathrm{C}$ 1 hour cure is done in vacuum oven to yield $\sim 75 \%$ cross-linking of the BCB. This step allows $\mathrm{BCB}$ to endure the following fabrication steps while enhancing subsequent layers adhesion onto the $\mathrm{BCB}$, in comparison to a fully cured $\mathrm{BCB}(98 \%)$.

\section{Meander (Fig. 3.10)}

\section{- Design}

The control lines used for inertial piezoelectric coarse motion of the microscope must allow fast $(\sim \mu \mathrm{s})$ charging of capacitances $(\sim 10 \mathrm{nF})$. This imposes an upper limit of the order of $\sim 100 \Omega$ for the series resistance of the filters. Given this resistance, and the dielectric thickness already mentioned, the geometry of the filter was chosen to provide a suitable cutoff frequency. After considering several designs, we found that the layout shown in Fig. 3.11, was satisfactory: it presents parts with a small cross-section, intended to procure series resistance, and pads with a large cross-section providing with capacitance to ground. It is thus an intermediate design between a uniform cross-section distributed RC line and a cascade of discrete $\mathrm{RC}$ elements. For the same total $\mathrm{R}$ and $\mathrm{C}$, it even provides a steeper cut-off than the uniform cross-section line, due to reflections at impedance (cross-section) discontinuities.

Besides electromagnetic propagation, another important consideration in the design of the meander is the total volume of resistive material, because large volumes are needed to thermalize well electrons at the lowest temperature, due to weak electron-phonon coupling. 
For a given layout, the volume of the resistive material can be changed by using materials with different resistivities.

- Fabrication

We fabricated the meander with a 50\% atomic content copper-gold alloy.

The reason to choose an alloy is to introduce as much disorder as possible so that the ratio of residual resistance (RRR) at $4 \mathrm{~K}$ would be as close as possible to 1 , in order to have rather constant filtering performances over the temperature range.

The alloy was deposited by co-evaporation in a Joule evaporator. Since gold and copper evaporate at different rates, we prepared each time a fresh load that was fully evaporated, so that reproducible properties were achieved. In a fixed geometry, one can tune the total resistance to the design target by varying the layer thickness. We obtained 75-90 $\Omega$ total resistance by evaporating a full load of $0.4 \mathrm{~g}$ of copper and $1.2 \mathrm{~g}$ of gold, at an initial rate $\sim 1 \mathrm{~nm} / \mathrm{s}$, yielding a total thickness of about $350 \mathrm{~nm}$.

A $5 \mathrm{~nm}$-thick Ti layer was deposited as an adhesion layer for the alloy on BCB. However, the adhesion remains poor, and the final yield is of the order of $50 \%$, in good part because of pealing of the meander which causes open circuits and/or shorts to ground. To improve adhesion, a process is described in the BCB technical data. It consists in a short time ion milling with $1 \mathrm{keV}$ argon ions prior to deposition, in order to create carbon radicals that would make chemical links to titanium. We have not tried this process since we do not have the available ion gun in our joule evaporator, but this will hopefully solve most adhesion problems encountered in the process.

We have tested an alternative solution to solve these adhesion issues, consisting in plasma etching the surface of $\mathrm{BCB}$ with a mixture of $\mathrm{O}_{2}$ and $\mathrm{SF}_{6}$ in a Reactive Ion Etching machine just before introducing the sample in the evaporator. This has improved slightly the results but progress needs to be done to improve the yield.

Fabrication step $\mathrm{n}^{\circ} 2$ is explained in Fig. 3.10. It consists in patterning a mask in a photosensitive resist soluble in acetone through which one evaporates the meander. The mask is then removed leaving only the patterns of the meander on top of the BCB. To obtain a neat lift-off, we harden the resist skin (the first $\sim 100 \mathrm{~nm}$ ) in chlorobenzene prior to development, to make an overhang. It is also possible to spin a true bilayer, using LOR 30B as a first layer (see 3.3.2.2). 


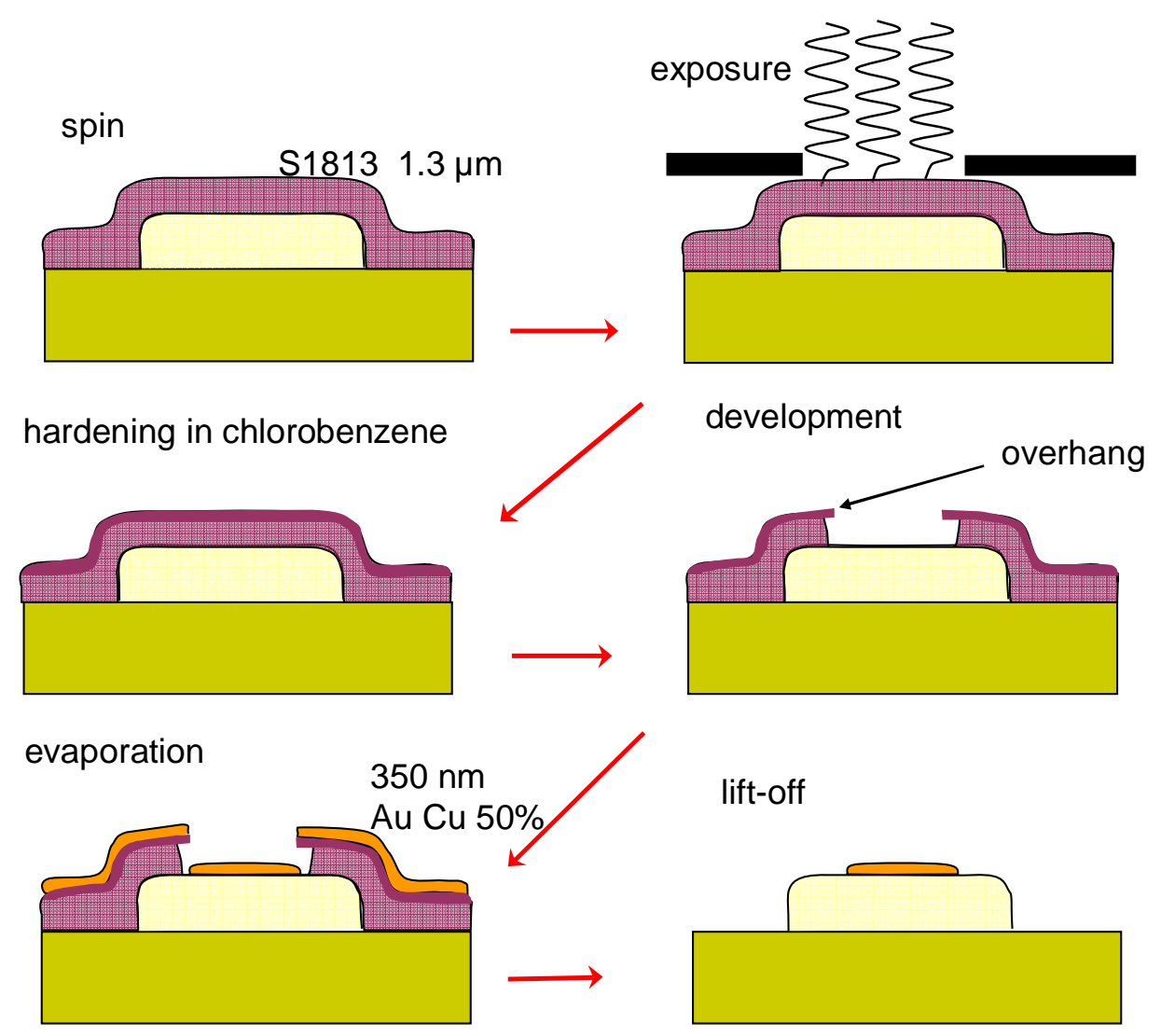

Fig. 3.10 Fabrication of the filters: step $n^{\circ} 2$, meander deposition. For masking the alloy evaporation we use positive photosensitive resist soluble in acetone. The surface of the resist is hardened in chlorobenzene to make a "crust" that does not dissolve during development. This yields an overhang which eases the lift-off. Otherwise, since the flanks of the resist are smooth the metal layer can be continuous, and lift may result in uprising of the edges, and sometimes complete removal of the circuit, since adhesion is poor.

\section{Ground plane and shielding (Fig. 3.11)}

The final ground plane is made of a $1 \mu \mathrm{m}$-thick gold layer. This film is made thick enough to be a perfect reflector in the whole relevant frequency range, hence providing good isolation of adjacent arms of the meander.

Filters are batch processed on $75 \mathrm{~mm}$ diameter wafers; they are subsequently cut into chips of $8 \mathrm{~mm}$ per $4 \mathrm{~mm}$, and mounted in the boxes as depicted in Fig. 3.11. The chips are contacted to the connectors by $100 \mu \mathrm{m}$-diameter insulated copper wires. These wires are glued to the chip bonding pad with conductive silver epoxy Epotek H20E from Politec PI, and soldered to the connector. The empty space around the chip is filled with H20E, to shield as well as possible the input from the output.

We chose this one because it is of convenient use, with a reasonable polymerisation temperature $\left(\sim 100^{\circ} \mathrm{C}\right)$, and a long life (up to one day) at room temperature once the ingredients are mixed. However, its thermal contraction is higher than that of brass. To our 
knowledge, there is no conductive epoxy whose thermal contraction is matched to brass (or copper). Despite this we observed only a few percent failures after about ten brutal cycles in liquid nitrogen. We do not have yet much statistics on the ageing of these filters, but they have proved quite reliable until now, over the first year of experiment (4 out of 76 failed at the first cooling, and none since then).

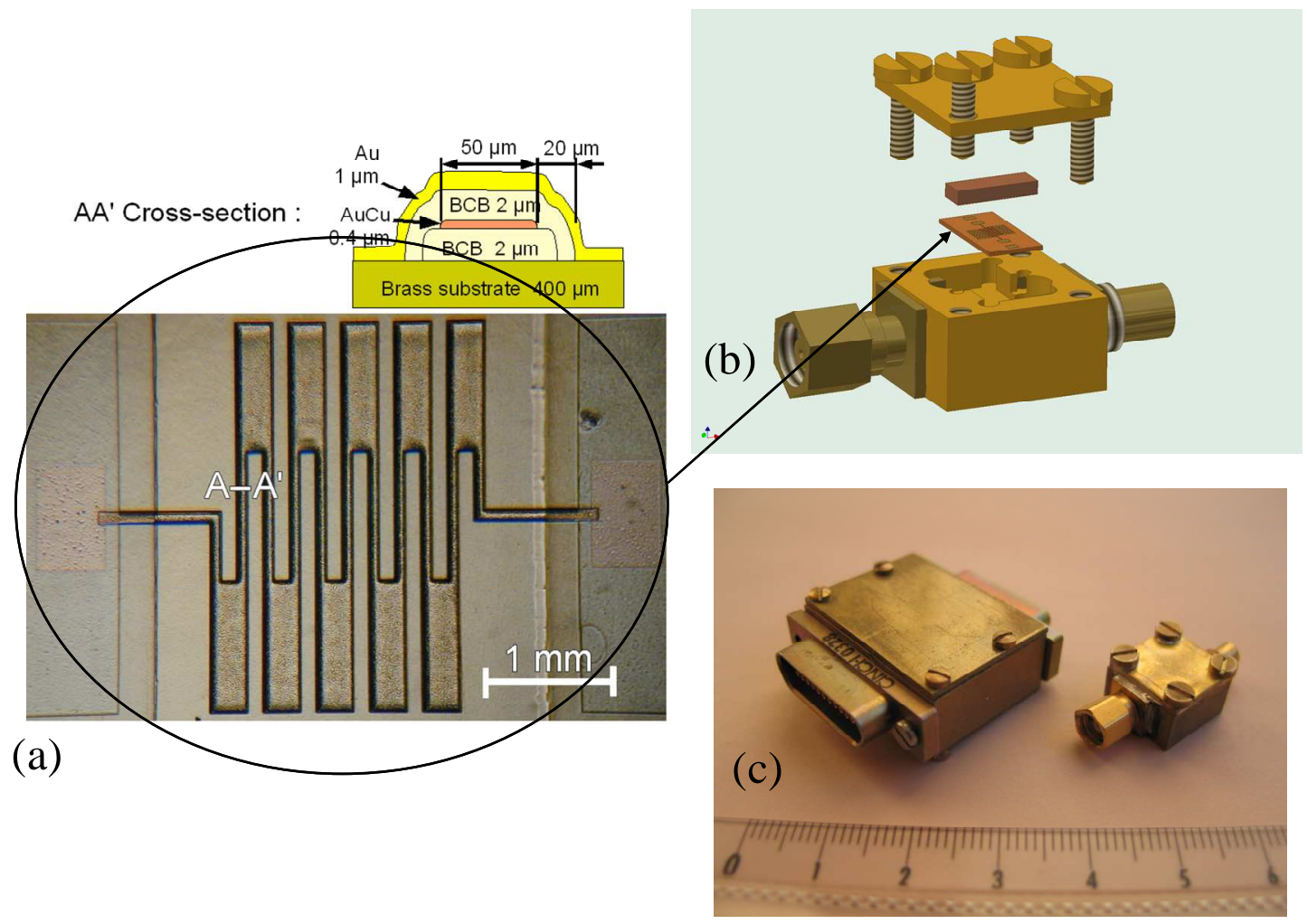

Fig. 3.11 (a) Photo and cross section view of a filter. The connecting pads are laid on a layer of BCB. There are two pads in series (second not shown on the photograph) to insert, if desired, a CMS component like a ferrite. (b) Scheme of the mounting: the chip is installed in a tightly adjusted housing. A brass part comes above the filter: It is electrically contacted to the ground plane and shields the input from the output. To avoid microwave leaks, electrical continuity is ensured by filling the vacuum intervals with silver epoxy. Finally the box cover is glued with silver epoxy as well. (c) The smallest boxes are $12 \times 10 \times 8 \mathrm{~mm}^{3}$ containing one filter connected with SMC connectors. The largest box is $34.5 \times 30.1 \times 13 \mathrm{~mm}^{3}$ containing 28 filters out of which 25 are used. Its connectors are micro-SubD from Cinch. We also use $13 \times 22 \times 8 \mathrm{~mm}^{3}$ boxes containing 2 filters connected by miniature triaxial Fischer series 101 connectors. All the boxes have a male connector on one side and a female on the other so they can be easily inserted or removed from the lines.

By design, the filters are not matched to $50 \Omega$, and the connection by bonding pads provides as well a poor impedance matching. If necessary, efforts could be made to perform for instance high frequency experiments in the $100 \mathrm{MHz}-1 \mathrm{GHz}$ range, by making use of appropriate $50 \Omega$ microstrip design and launcher connectors. 


\subsubsection{Electromagnetic simulations of the filters.}
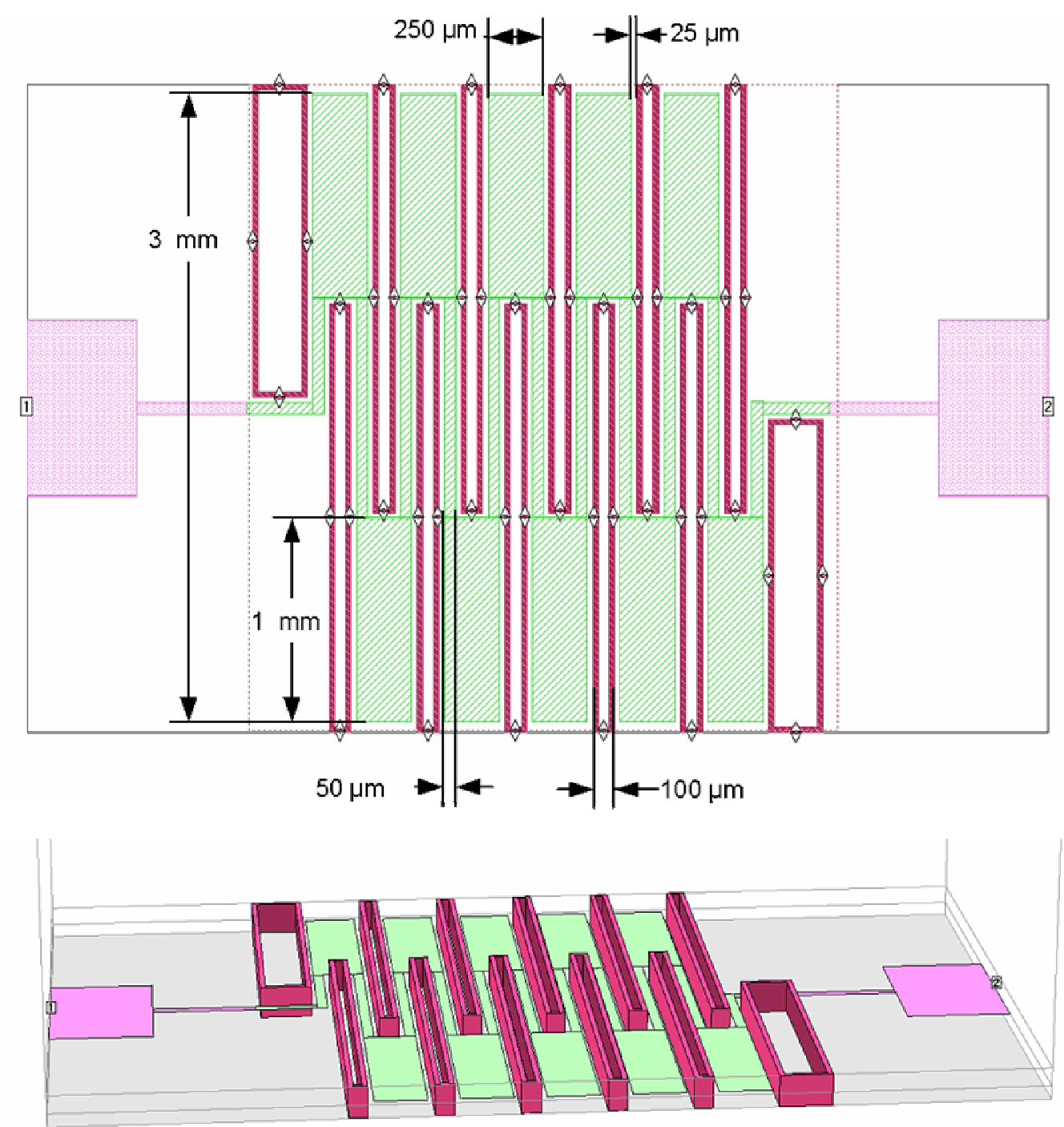

Fig. 3.12 The picture shows the design file used for electromagnetic simulation with Sonnet ${ }^{\circledR}$ software. The green areas represent the resistive meander and the red rectangles are vias connecting top and bottom ground planes (not shown) to isolate adjacent arms. In the bottom part, a $3 \mathrm{D}$ view is shown, with a $40 \mathrm{x}$ magnification in vertical scale.

Electromagnetic simulations were carried out to check the design and performance of the filters. For this purpose we have mostly used Sonnet, a 2.5D simulator well adapted for planar circuits. In these simulations, we used the exact filter layout shown in Fig. 3.12. This technique relies on finite elements subdivision of the structure. As the frequency increases, the size of elements is reduced too so that in a planar EM simulation software as we use, in worse cases, the size of the memory requirement scales as $\mathrm{f}_{\max }{ }^{2}$, and the computation time as $\mathrm{f}_{\max }{ }^{6}$. As a result, these simulations are rapidly computationally demanding and intensive. Indeed, above $100 \mathrm{GHz}$ it became impossible to run the full simulation of a filter on our PCs 
within a reasonable time (because memory swapping occurred). Moreover the accuracy of the simulation is challenged even before one runs out of memory, since already at $12 \mathrm{GHz}$ the noise floor of the calculation is reached due to the very high attenuation of the filters around $\sim-150 \mathrm{~dB}$.

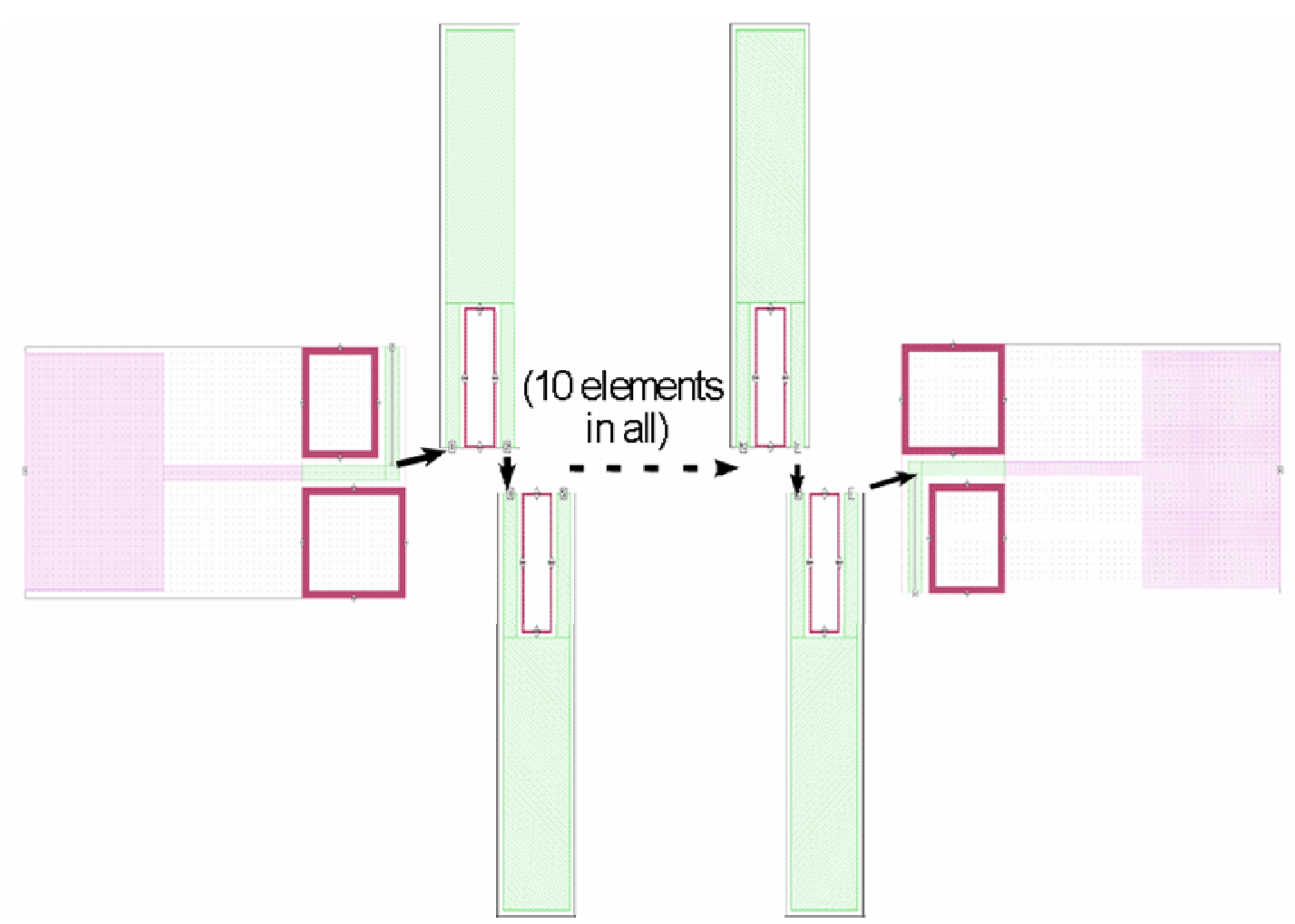

Fig. 3.13 In order to reduce the computational size of the simulation, to be able to reach very high frequencies, we have subdivided the filter in smaller independent parts and cascaded the resulting $\mathrm{S}$ matrices as depicted in this figure.

To extend the simulation at very high frequencies and still obtain meaningful results, we have subdivided the simulation in smaller parts whose $\mathrm{S}$ matrices are subsequently cascaded to obtain the full behaviour of the filter. These subdivisions require less memory and time to be simulated and, since their attenuation is smaller, there is no problem with accuracy anymore. The subdivision we made is shown in Fig. 3.13. We have checked that, in the range where both the complete simulation and the subdivided simulation are possible, they give the same result. In this way we were able to perform simulations up to $6 \mathrm{THz}$. We have checked that the hypotheses on which the simulation is based (namely that the propagation effects in the films thicknesses are not important) are still valid at such high frequencies. In particular, we have used a quasi-optical program (MODE, from Lumerical Inc.) to check whether TE or TM waveguide modes could propagate in the narrow cross sections of the filters (in contrast to the TEM coaxial mode we are simulating with Sonnet), and we found that no such mode can 
propagate below $\sim 20 \mathrm{THz}$, and even then, they propagate with very large losses (data not shown).

Using the exact geometric and material (dielectric constant) parameters, and the measured total DC resistance of the meander to get the material resistivity, the simulations give the results shown on Fig. 3.15, with no adjustable parameter.

Of course, when predicting attenuations in the hundreds of $\mathrm{dBs}$, one must be very careful as there is absolutely no way to check such predictions. One can even be sceptical because leaks in connectors, for instance, can easily produce much larger transmissions.

We have compared the predicted performance of the present filter design with that of the former generation [86] where the adjacent arms were not isolated. To do this, we have simply removed the vias connecting top and bottom ground plane in Fig. 3.12. When doing so, we see that the attenuation of the filters saturates around $-90 \mathrm{~dB}$ above $5 \mathrm{GHz}$, and shows transmission peaks around $50 \mathrm{GHz}$, due to TE modes. Such simulations cannot be performed above $100 \mathrm{GHz}$, because here we cannot subdivide the network into independent smaller pieces.

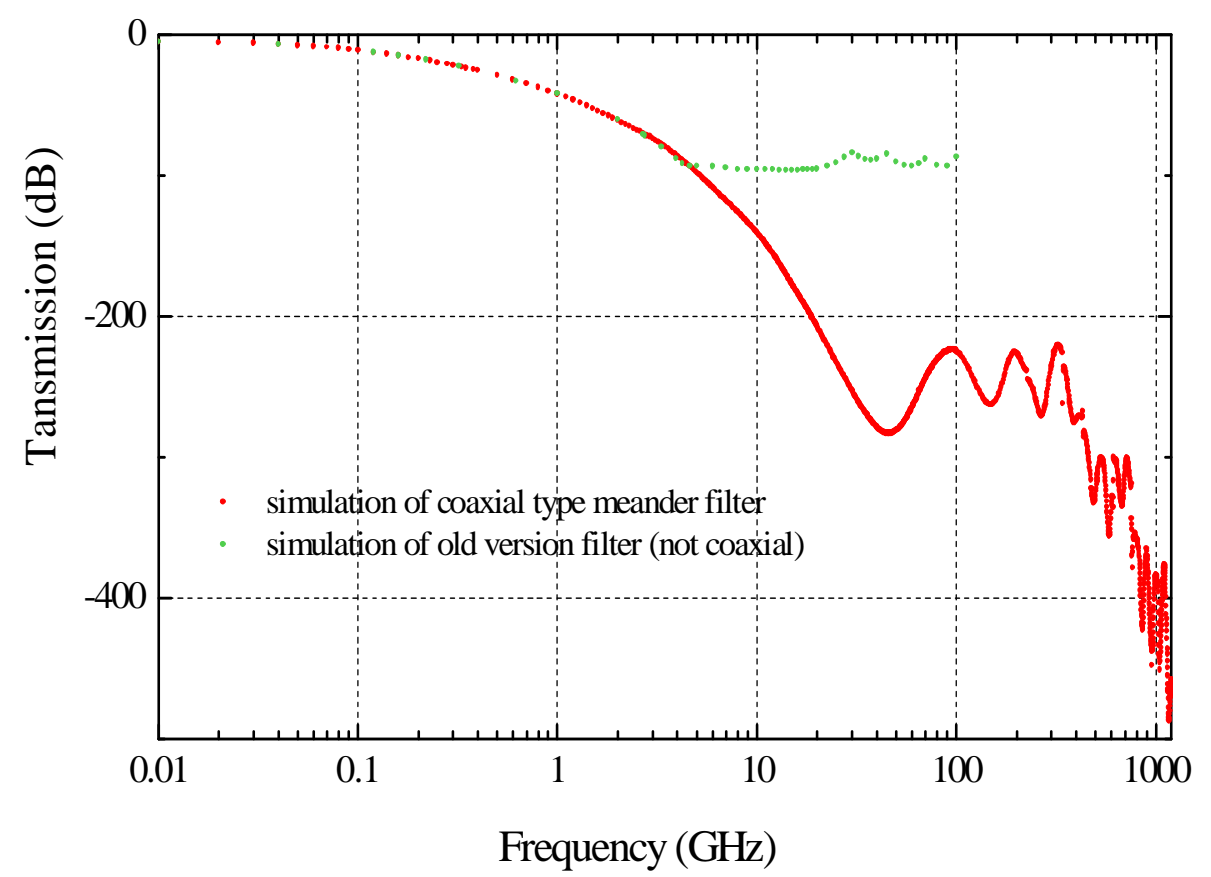

Fig. 3.14 Simulation of the filters using Sonnet, up to very high frequencies $(1.2 \mathrm{THz})$ in order to check their theoretical efficiency. The transmission curve obtained with the actual geometry of filters is plotted in red. The transmission of the former design filters, where the adjacent arms are not isolated is plotted in green. This shows the theoretical expectation is far better for the coaxial type filter, since no transverse electric or magnetic mode can be transmitted directly from input to output in this version, whereas with no encapsulation, it is easy to find transmission resonances, even at about ten $\mathrm{GHz}$. 


\subsubsection{Filter attenuation}

Figure (Fig. 3.15) shows transmission measurement from $40 \mathrm{MHz}$ to $65 \mathrm{GHz}$ of one of the filters (connected with coaxial SMC connectors). These data are taken at room temperature, using a $65 \mathrm{GHz}$ vector network analyzer (Anritsu 37297D).

From DC to $4 \mathrm{GHz}$, the measured filter attenuation follows very closely what is expected from simulations (see \$3.2.3.6). Above $4 \mathrm{GHz}$, we do not measure any transmission above the noise floor of the analyzer, which suggests that no sharp resonances are present. The finite attenuation at low frequency comes from the dc resistance of the filter which makes a divider with the $50 \Omega$ port of the analyzer.

SMC connectors are specified to pass signals from DC up to $12 \mathrm{GHz}$. They introduce $3 \mathrm{~dB}$ reflection at $12 \mathrm{GHz}$. The measurement was done with SMA to SMC transitions and thus does not give access to the filter attenuation alone, but rather to the full assembly including the adapters. The noise floor for ambient temperature measurements was around -100 dB. It was worse for $4 \mathrm{~K}$ measurements, due to losses in cables. In principle, it would be possible to reach a $\sim 20 \mathrm{~dB}$ lower noise floor by using a large bandwidth amplifier, but we had none with an appropriate bandwidth.

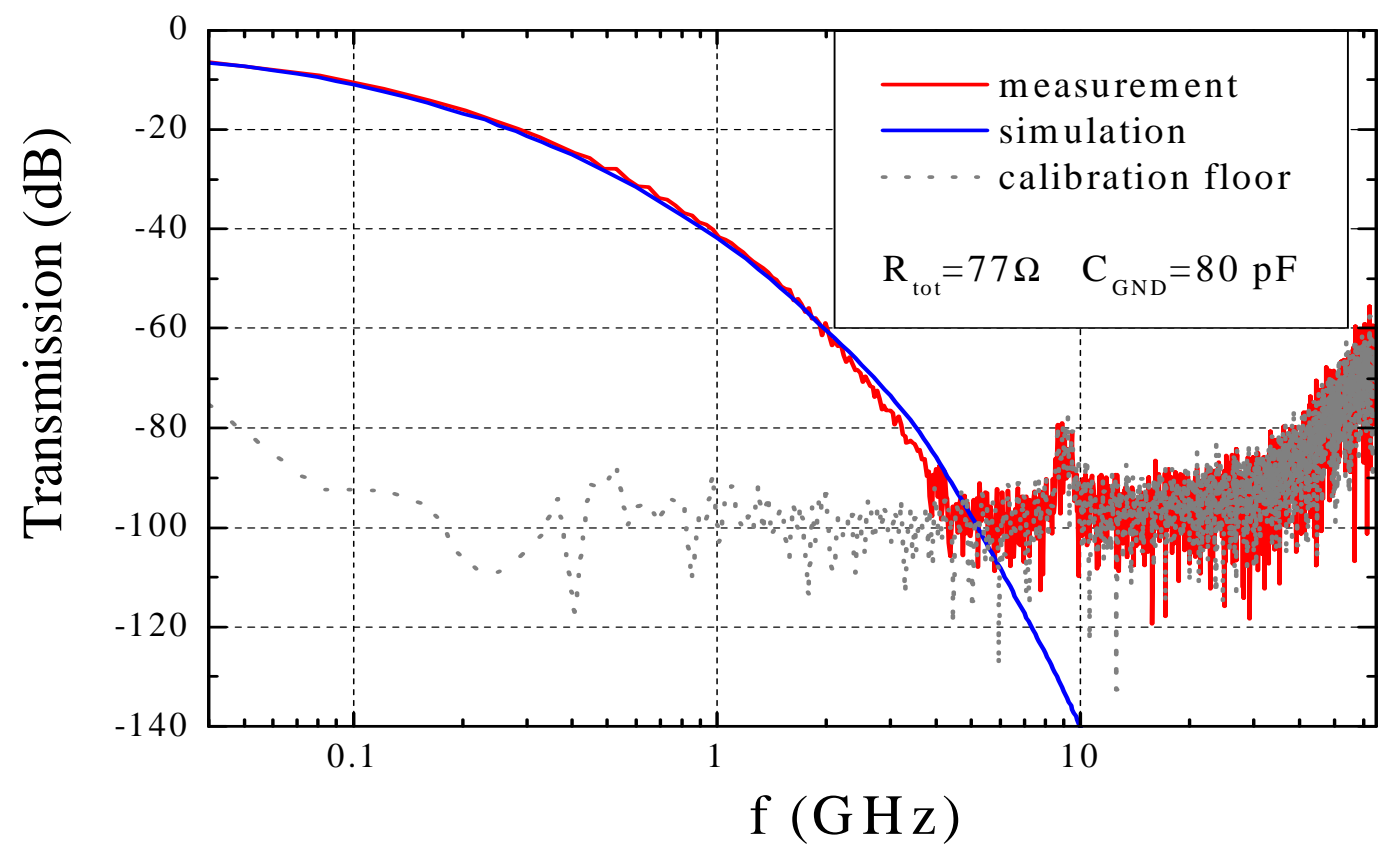

Fig. 3.15 Transmission of a coaxial meander low pass filter, mounted in a shielded case, and connected with SMC connectors. Data (red curve) is taken at room temperature with a Anritsu 37297D $65 \mathrm{GHz}$ network analyzer. On the same plot we show the noise floor of the network analyzer (grey dots). These data are to be compared to the simulations done under Sonnet electromagnetic simulation software (blue curve). Around $5 \mathrm{GHz}$, the curve crosses the noise floor, but the prediction states that it should continue to go down. The peak in the data around $9 \mathrm{GHz}$ is a calibration artifact (also present on the calibration floor). The small discrepancy between simulation and data between 2 and $6 \mathrm{GHz}$ is probably due to the connection to the filter which introduces reflection not included in the simulation with direct $50 \Omega$ ports connection. 


\subsubsection{Analysing the full setup}

We chose to install 2 sets of filters for each line; one is anchored at $4 \mathrm{~K}$ and the other at $30 \mathrm{mK}$. First, in order to have an idea whether this filtering setup is appropriate, one can plot the expected spectrum of a black body radiation emitted at $300 \mathrm{~K}$ and filtered by the setup. The resulting spectrum is plotted in Fig. 3.16.

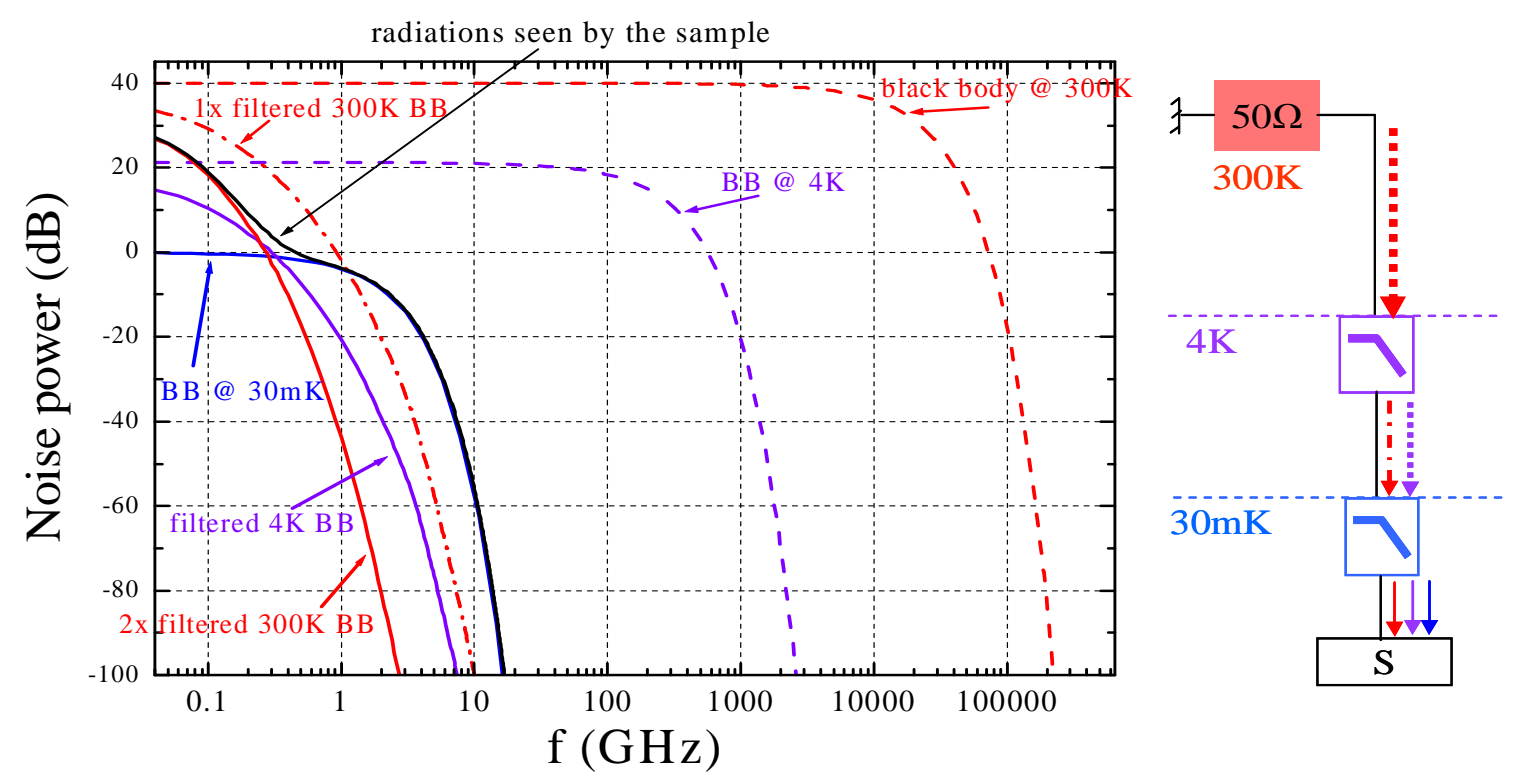

Fig. 3.16 Graphical analysis of the thermal noise filtering. One can calculate the thermal noise power spectrum received by the sample at the end of a filtered line, using the simulation results. The setup envisioned here consists of a $50 \Omega$ resistor at $300 \mathrm{~K}$ and a line that includes two stages of filters: one at $4 \mathrm{~K}$, and one at $30 \mathrm{mK}$. To obtain the total noise at $30 \mathrm{mK}$, one has to sum up the contributions from all impedances, with the corresponding attenuation provided by the filters. Contributions are plotted at different stages of filtering: full lines correspond to the effective radiations seen by the sample, which consist of the $300 \mathrm{~K}$ black body (BB) spectrum attenuated twice, the $4 \mathrm{~K} \mathrm{BB}$ spectrum filtered once, and the $30 \mathrm{mK} \mathrm{BB}$ spectrum. The dot-dashed curve shows the $300 \mathrm{~K}$ BB spectrum filtered once. Dashed curves show the $300 \mathrm{~K}$ and $4 \mathrm{~K}$ BB spectrums not filtered. For clarity, all curves are referenced to the noise power spectrum $\mathrm{Sv}(0) @ 30 \mathrm{mK}$ (cf equation (2.2)). From this picture one can grasp the very large attenuation needed to filter the $300 \mathrm{~K}$ radiation for proper $30 \mathrm{mK}$ thermalization.

Sonnet simulations also provide the $\Pi$ or $\mathrm{T}$ impedance model of the filter at all simulated frequencies. We can use this output, together with the analysis performed in 3.2.3.3, to calculate the effective frequency dependent radiation temperature as seen from the experiment, given by (3.11). The outcome of this calculation is shown in Fig. 3.17. In this calculation, the role of the lossy coaxial line connecting the filters is neglected, for simplicity ${ }^{1}$.

\footnotetext{
${ }^{1}$ It is in principle possible to include these lossy lines in the noise analysis of the network. To do this, one has to take into account the temperature gradient (which has to be calculated), and to subdivide the line into several sections assumed at thermal equilibrium. However, since these lines have a higher cut-off frequency than the microfabricated filters, the full analysis would not change much the results presented here.
} 
From this result, one sees that the effective photon temperature is equal to the fridge temperature from $1 \mathrm{GHz}$ to $50 \mathrm{GHz}$. At low frequencies, radiations are out of equilibrium, but are not too dangerous since they correspond to photons already present in the spectrum of a dissipative element placed at $30 \mathrm{mK}$, with a higher level though (equivalent to that of a high impedance). This could be solved by increasing attenuation in that range, using discrete elements as mentioned in the paper. At higher frequency, the effective temperature of the noise reaching the sample is also out of equilibrium, but since $h v \gg k_{B} T_{\text {eff }}$ the corresponding photon flux density is negligibly small. To fix ideas, the flux is plotted on Fig. 3.17, assuming that the sample is a $50 \Omega$ resistor. The number of photons reaching the sample can be found by integrating this curve over the bandwidth of the setup; the integral from $10 \mathrm{MHz}$ to $6 \mathrm{THz}$ corresponds to one photon every $10^{16} \mathrm{~s}$.

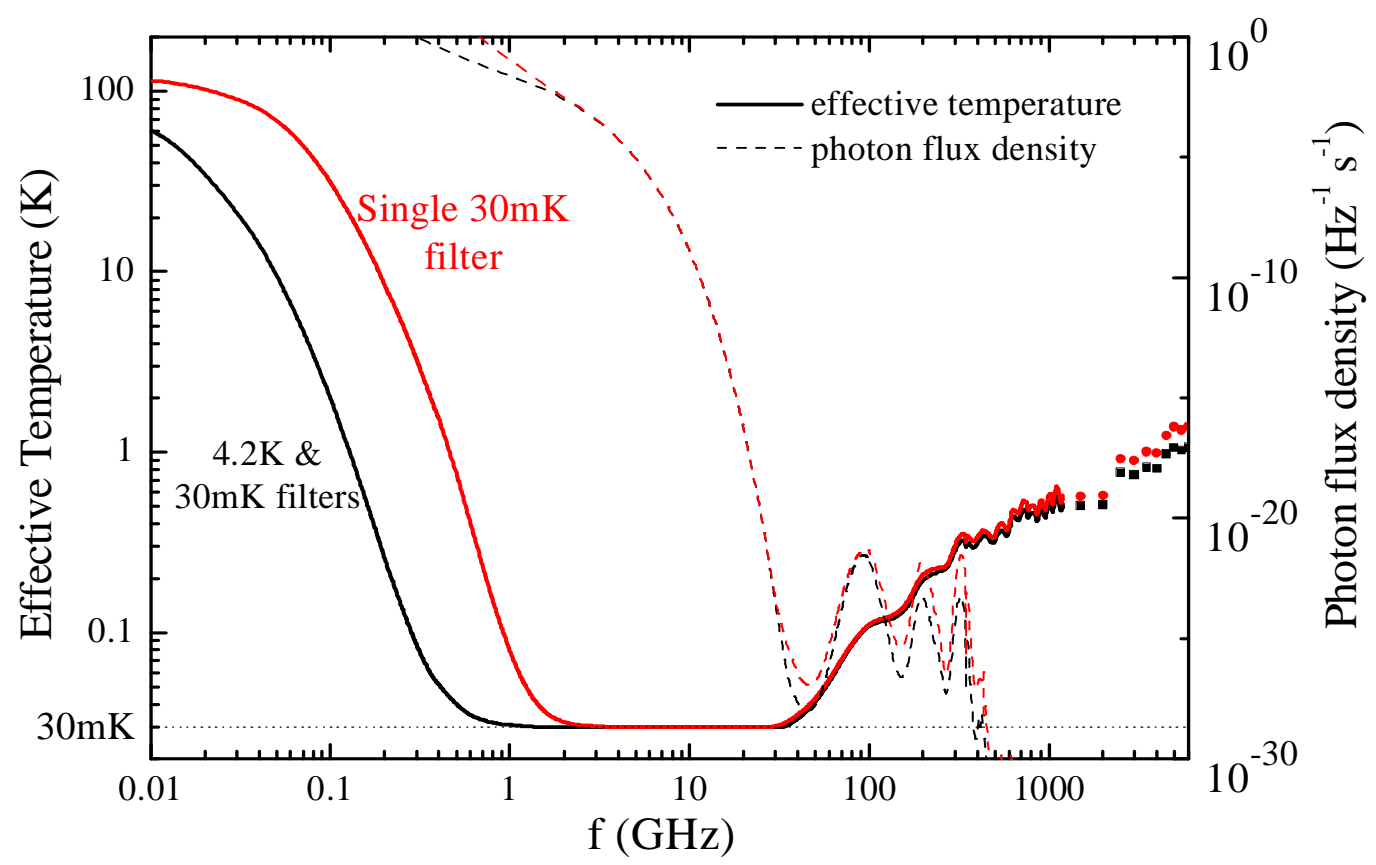

Fig. 3.17 Solid lines: effective temperature of the radiations emitted by elements at $300 \mathrm{~K}$ with a single stage of filter at $30 \mathrm{~m} \mathrm{~K}$ (red curve), and after two stages, one at $4 \mathrm{~K}$, the other at $30 \mathrm{mK}$ (black curve). The effective temperature is calculated using the expressions of the network theory given in 3.2.3.3 and the results of the numerical simulations. Dashed lines: the photon flux density, given by the noise power spectrum absorbed by a load impedance of $50 \Omega$ at $30 \mathrm{mK}$ (the sample), divided by the energy of one photon $h v$. This tells us for instance that one photon reaches the sample every $10^{16} \mathrm{~s}$ in the total energy interval shown here.

These results give us confidence that the electrons of the sample can be brought to equilibrium with the phonon distribution at $30 \mathrm{mK}$. As already mentioned, no microwave network measurements can check in detail these predictions. The only possible check comes from real experiments: for instance tunneling spectroscopy, or josephson junction switching probability measurement. 


\subsubsection{Experimental validation of the filtering setup}

To demonstrate the effect of filtering the lines, we compared conductance curves of an N-S tunnel contact obtained in the two different configurations: first using the bare lines with no micro-fabricated filter installed, second with all the micro-fabricated filters installed. The data obtained are plotted in Fig. 3.18. Data are taken in each case on a $25 \mathrm{~nm}$-thick aluminum film, with a tungsten tip, at a fridge temperature around $40 \mathrm{mK}$.
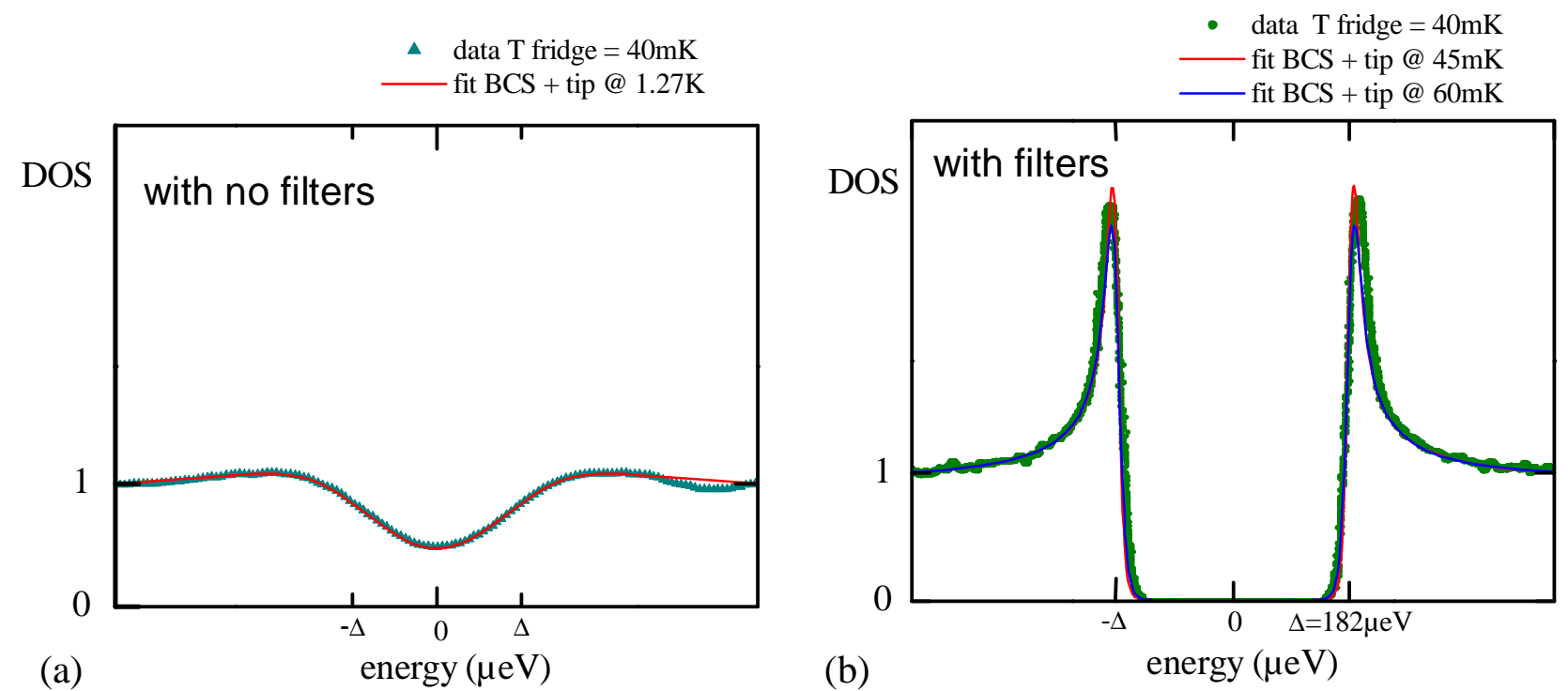

Fig. 3.18 Comparison of tunneling density of states measurements of a superconductor with a normal tip. (a) with no microfabricated filters installed, (b) with the filters. In the first case, the measured density of states is convoluted with a Fermi distribution at $1.27 \mathrm{~K}$, indicating that the electrons are very poorly thermalized. In the second case, the measured density of states can be fitted using an electronic temperature in the tip of about $50 \mathrm{mK}$.

These measurements completely validate the filtering setup, since electrons of the tip are thermalized to a temperature very close to the fridge's when all lines are filtered. The effective temperature obtained in our experiments is the best electronic temperature achieved with an STM until now: our setup enhances by a factor $\sim 3$ results obtained in former experiments, and allows to envision several experiments where a high resolution is needed.

\subsubsection{Article reprint: microfabricated filters}

The article published in Rev. Sci. Instrum. 77, 115102 (2006) is reproduced hereafter. 


\title{
Microfabricated electromagnetic filters for millikelvin experiments
}

\author{
Hélène le Sueur and Philippe Joyez \\ Service de Physique de l'Etat Condensé (CNRS URA 2464), CEA Saclay, 91191 Gif sur Yvette, Cedex, France \\ (Received 26 July 2006; accepted 30 September 2006; published online 16 November 2006)
}

\begin{abstract}
In this article we report on the design, fabrication and tests of micro-fabricated broadband filters suitable for proper electromagnetic thermalization of electrical lines connected to sensitive quantum electronics experiments performed at dilution fridge temperatures. Compared to previous such miniature filters, the new design improves on performance and reliability. These filters can be packed in space-saving cases with either single or multi-contact connectors. Measured performance in the accessible range compares well to simulations. We use these simulations to discuss the effectiveness of these filters for electromagnetic thermalization at $30 \mathrm{mK}$. [DOI: $10.1063 / 1.2370744]$
\end{abstract}

\section{INTRODUCTION}

A number of quantum electrical experiments need to be operated at millikelvin temperatures for optimum performance. One can think for instance of solid-state qubits or of tunnel spectroscopy experiments (in which energy resolution is directly proportional to the temperature). If cooling a sample down to the $\mathrm{mK}$ range is rather easy, ensuring that an experiment is performed at full thermal equilibrium is not. The reason is well known : leads connecting the sample to room temperature apparatus feed in electromagnetic noise which may directly couple with the device under test (e.g. in the case of a qubit) and/or heat up the electrons of a small device above the temperature of the crystalline network. Indeed, many experimental results in the field of low temperature quantum electronics can only be interpreted with an electronic temperature greater than that of the refrigerator. The noise generated by room temperature instruments is at the minimum the Johnson thermal noise (the electrical equivalent of Planck's black body radiation) extending in frequency up to $300 \mathrm{~K} \cdot \mathrm{k}_{\mathrm{B}} / \mathrm{h} \sim 6 \mathrm{THz}$, in the infrared frequency range. One thus needs to insert in the leads connected to the sample filters able to absorb this very wide band noise and to reemit a thermal spectrum at the experiment temperature which corresponds to a bandwidth of a fraction of $1 \mathrm{Ghz}$. This problem has been investigated theoretically, and the stringent requirements this imposes on noise attenuation have been evaluated for operating Coulomb blockade devices [1,2].

Unfortunately, there exists no ready-made commercial filters with such wide stop-band and sufficient attenuation. This is why in the last 25 years many custom filter designs have been proposed in order to effectively thermalize experiments at $\mathrm{mK}$ temperatures. Most solutions rely on the use of shielded lines (usually coaxial lines) and shielded distributed RC filters. These filters being dissipative, they must themselves be placed at the lowest temperature so that their own thermal noise is at equilibrium. A popular solution (easy to make and use) is to combine both the line and the filter by using a lossy coax such as Thermocoax $[3,4]$. One drawback with this approach is that the length required for a low enough cutoff frequency is often of the order of meters, which is impractical to install in many dilution fridges where space is often scarce. Moreover, when such a line is running between two or more different temperatures, estimating the resulting output noise temperature is not easy. Other popular filters are powder filters based on skin effect in metallic grains [5]. These are very effective filters, but once again they tend to be rather large and their performance is not reliably predictable at design time.

Some ten years ago, in the need for space-saving filters for Coulomb blockade experiments, one of us was involved in the design and fabrication of miniature filters, as reported in Ref. [2]. These filters were based on microfabricated chips made by optical lithography which consisted of a resistive meander line sandwiched between two insulating layers and ground planes, implementing a distributed RC filter. These filters had a predictable performance in the $0-20 \mathrm{GHz}$ range which could be tailored at design time. They had several weaknesses, though : a limited and poorly predictable very high frequency attenuation due do shortcomings in the filter chip (coupling between adjacent meander lines) and casing designs, and reliability issues upon ageing and thermal cycling attributed to differential contraction of the various materials involved in the filter assembly. Critically reviewing these problems, we came up with a new design of microfabricated filters which corrects all these problems. We now consistently obtain much better stop-band attenuation together with other desirable characteristics : less DC resistance, less total capacitance, better ageing and high-voltage capability. In this article we describe this design, along with the fabrication, packing and performance of the corresponding filters.

\section{DESIGN}

When designing a filter such as we need one can imagine that very high frequency electromagnetic waves (hereafter microwaves for short, but extending in fact up to infrared) will sneak into the least metallic hole, slot or crack and propagate through vacuum (or dielectric) much like a fluid would : If there is a dielectric path between input and output, microwaves will find their way. Thus, designing an efficient filter is much like making the device leak-tight to fluids. The key step towards electromagnetic "hermeticity" in our new design is the use of a brass substrate for the microfabricated filter chip in place of the more standard 


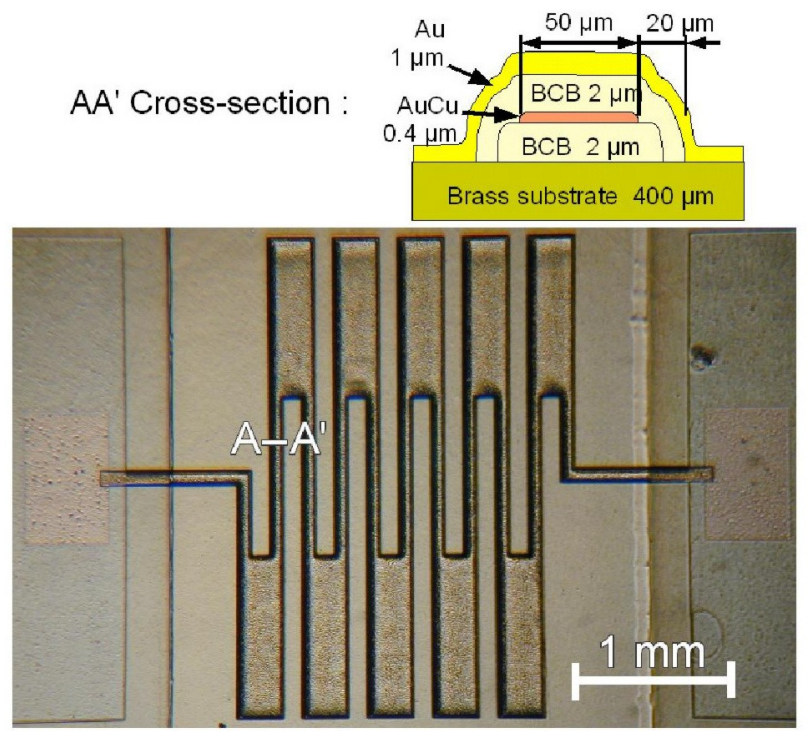

Fig 1. Picture of a microfabricated filter chip, and cross-section of a meander arm with materials and respective thickness (note different vertical and horizontal scales).

silicon substrate used in the old design. This way, not only we suppress the dielectric substrate which is difficult to seal perfectly and which behaves as a wave guide, but we also suppress differential thermal contraction of the filter chip with respect to the brass case which ensures that no cracks will appear upon thermal cycling. The design of the filter chip itself is shown in Fig. 1. It consists of a resistive meander line connecting the input pad to the output pad. The meander line alternates narrow parts and large pads. This provides a steeper cut-off than a meander with uniform width having the same total resistance and capacitance, due to reflections on impedance discontinuities. The meander line is completely surrounded by an insulating material and a ground sheath, which provides decoupling of adjacent arms of the meander at high frequency. Apart from the different sections, it is like a miniature distributed resistive coaxial cable. Its behavior is thus somewhat similar to that of Thermocoax-type filters, but its waveguide modes are rejected at higher frequencies due to the smaller cross-sections of the dielectric.

\section{FABRICATION}

Fabrication starts from 0.4 mm-thick brass sheet out of which we cut $75 \mathrm{~mm}$ diameter wafers. Each wafer is mechanically polished down to a $1 / 4 \mu \mathrm{m}$ grain size to yield a fair optical polish. Then we deposit the bottom insulating layer using spin coating. This layer consists of Cyclotene 4024 bisbenzocyclobutene-based (BCB) negative photoresist from Dow Chemicals. For our requirements we chose a spin speed of $6000 \mathrm{rpm}$, thus obtaining a resist thickness of $2.5 \mu \mathrm{m}$. By varying the dilution of the photoresist or/and the spin speed we can tune this thickness reliably down to $500 \mathrm{~nm}$, and change the filter capacitance accordingly. The resist is then patterned using UV photolithography. The wafer is subsequently immersion-developed and submitted to a moderate cure at $210{ }^{\circ} \mathrm{C}$ under inert atmosphere, to convert about $75 \%$ of the resist into the final polymer. This hardens the layer, which can then handle further microfabrication steps, and enhances subsequent layers adhesion. In this step, the BCB looses $\sim 10 \%$ thickness. Then, following the manufacturer recommendation, the wafer is submitted to an $\mathrm{SF}_{6} / \mathrm{O}_{2}$ Reactive Ion Etching plasma to eliminated resist residues and enhance subsequent layer adhesion on BCB. This plasma etching further reduces the resist thickness by about $100 \mathrm{~nm}$. Next, we spin on a $1.3 \mu \mathrm{m}$ thick layer of Shipley S1813 positive photoresist. The meander pattern is then exposed, aligned onto the previous insulating layer. Prior to develop, the wafer is immersed $30 \mathrm{~s}$ at $30^{\circ} \mathrm{C}$ in dichlorobenzene to obtain after development an overhanging resist profile needed for a good lift-off. The substrate is then placed in a Joule evaporator and we deposit a $5 \mathrm{~nm}$-thick titanium adhesion layer followed by a 100 $400 \mathrm{~nm}$ thick copper-gold alloy at 50\% atomic content with a resistivity of $\sim 1.3 \times 10^{-7} \Omega \mathrm{m}$, to form the resistive meander. The thickness of the layer is chosen to obtain the desired total resistance of the meander and filter characteristics. As copper and gold evaporate at different rates, we prepare a fresh load at each pump down and evaporate it completely to obtain a reproducible alloy. After deposition the resist is lift-off in acetone. In a third lithographic step, we pattern a second BCB layer to terminate encapsulation of the meander in the central part of the chip while leaving uncovered the substrate between arms of the meander. This second $\mathrm{BCB}$ layer differs from the first in that the connecting pads of the meander are not covered and it is $10 \mu \mathrm{m}$ wider than the first layer in the rest of the structure (see figure 1). The $\mathrm{BCB}$ is finally hard cured 1 hour at $250{ }^{\circ} \mathrm{C}$ under inert atmosphere to achieve $98 \%$ polymer conversion and bring it to nominal electrical and mechanical characteristics. In the last lithographic step, another layer of Shipley S1813 is used to evaporate a $\sim 1 \mu \mathrm{m}$-thick gold sheath on top of the meander to terminate encapsulation and electrical shielding of the meander. Finally, the brass wafer is diced into $3 \times 8 \mathrm{~mm}$ chips for individual filters. For multi-line filters, several adjacent filters are left attached together.

\section{ASSEMBLY}

Filter chips are then mounted in cases. In Fig. 2 we show the design of a case for a single filter equipped with soft-soldered SMC connectors. The goal is to mount the filter chip in between two perfectly separated chambers. The two chambers are defined by a small metallic barrier running across the meander. Perfect electromagnetic separation of the chambers is reached by filling all machining gaps with silver epoxy. In each chamber we establish a connection from the connector pin to the filter pad using thin insulated wire and silver epoxy. Finally, we close the cover applying here also silver epoxy to make it electromagnetically leak-tight. Following the same design, other cases with different connectors can be made. In particular, double filters for shielded twisted pair lines are assembled in cases fitted with miniature triaxial connectors (Fischer series 101), and up to 25 filters can be packed in a $34.5 \times 30.1 \times 13 \mathrm{~mm}$ case equipped with two 25 contacts shielded micro-subD connectors.

\section{TESTING}




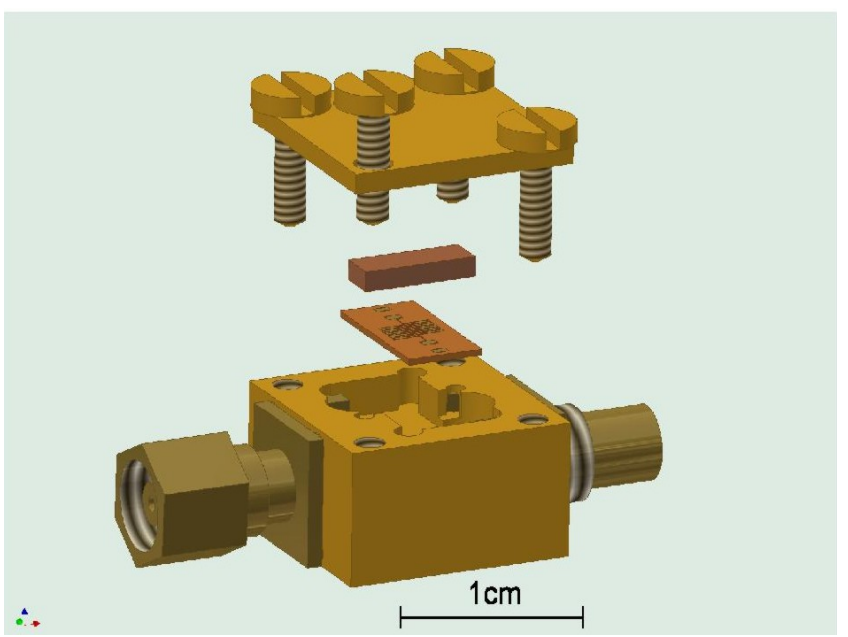

Fig 2. Exploded view of a single filter case equipped with SMC coaxial connectors. Connections between connector pins and filter pads are made with thin insulated wire and silver epoxy (not shown). All machining gaps and joints are filled with silver epoxy to achieve perfect electromagnetic separation between input and output connector chambers. Screws can be used to thermally anchor the case.

Fig. 3 shows the measured attenuation of a single filter equipped with SMC coaxial connectors. These measurements were performed using a $65 \mathrm{GHz}$ two $50 \Omega$ ports vector network analyser (Anritsu 37297D). The $S_{21}$ forward transmission data show that the filter has a steep cut-off reaching an attenuation greater than the $\sim-100 \mathrm{~dB}$ noise floor of the analyser at $4.5 \mathrm{GHz}$, and remains below the noise floor up to $65 \mathrm{GHz}$. Attenuation measurements carried out at $4.2 \mathrm{~K}$ (data not shown) show little change in the response of the filter, essentially due to a $\sim 20 \%$ reduction of the resistivity of the alloy we use. However, due to losses in the cables running form the network analyser into the liquid helium dewar, the sensitivity of this low temperature measurement is much lower than that of

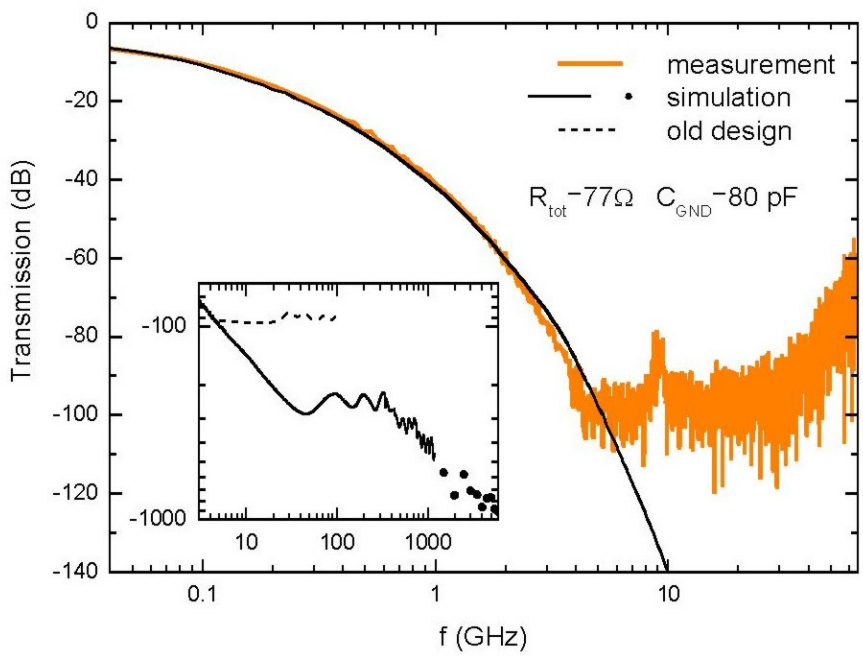

Fig 3. In the main panel, transmission of an assembled filter having a dc resistance of $77 \Omega$ and capacitance to ground of $80 \mathrm{pF}$. The data were measured using a microwave network analyser up to $65 \mathrm{GHz}$. Above $4.5 \mathrm{GHz}$ the noise floor of the analyser is reached (the peak at $9 \mathrm{GHz}$ is also noise and is due to a calibration artefact). We also plot the results of a microwave simulation with no adjustable parameters (see text). In the inset, results of the microwave simulation at higher frequency and predictions for the transmission of the same filter if the meander arms were not screened, as in Ref [2]. the room temperature measurements shown in Fig. 3, and thus it is less suited to test for proper attenuation performance. Repeated thermal cycling of the filter showed no measurable change in properties. Since one application for these filters in our laboratory will be to filter high voltage lines connected to piezo elements in a low temperature scanning probe microscope, we further submit all assembled filters to a $30 \mathrm{~s} 400 \mathrm{~V}$ test between the meander line and ground. A significant fraction of the filters do not pass this test, developing a permanent short to ground. According to the specifications, the BCB break down field is greater than $300 \mathrm{~V} / \mu \mathrm{m}$. Given the $\mathrm{BCB}$ thicknesses used, $400 \mathrm{~V}$ should not exceed the breakdown voltage of the dielectric. We think the lower breakdown voltage observed in many filters is due to the field enhancement occurring on sharp defects. The overall yield after assembly and high voltage test is of the order of $50 \%$, which could likely be improved by a better control of process parameters and cleanliness. Note that given their rather low resistance, these filters are acceptable on lines driving $\sim 10 \mathrm{nF}$ piezo elements used for inertial stick-slip motion, where a large instantaneous current is needed.

\section{SIMULATIONS}

We now compare the measurements shown in Fig. 3 to the expected behaviour of this filter. For this we have modelled the full filter chip using a 2.5-D planar electromagnetic simulator (Sonnet). Given the feature sizes and aspect ratios used, approximations made in such a simulation are valid throughout the whole frequency range we consider [6]. The data input in the geometrical model were the nominal dimensions of the meander line and the measured thickness of both BCB layers $(1.6 \mu \mathrm{m}$ for the bottom layer and $2.2 \mu \mathrm{m}$ for the top layer) obtained using a thin film profile meter. The sheathing of the meander is modelled using vias between metallic layers above and below the dielectric layers. Electrical data for the BCB are taken from the manufacturer : $\varepsilon_{\mathrm{r}}=2.5$, the relative dielectric constant and a loss tangent of $2 \square 10^{-3}$. These inputs yield a $81 \mathrm{pF}$ capacitance to ground of the filter at low frequency, in remarkable agreement with the $80 \mathrm{pF}$ measured value. The conductivity of the meander is adjusted to reproduce the $77 \Omega \mathrm{dc}$ resistance of the filter. Given these inputs, the calculated forward transmission also shown in Fig. 3 is in very good agreement with the measurements.

The small discrepancy showing up above $2 \mathrm{GHz}$ might be due to the fact that we do not take into account the circuit outside the filter chip. For instance, the small wires connecting the chip to the connectors introduce some inductance in series with the filter which increases attenuation as frequency rises. The connectors also introduce losses in the transmission above $10 \mathrm{GHz}$. These simulations also show the superiority of the present design compared to the former one [2]: if we remove isolation between adjacent arms of the meander, the filter transmission remains between $-85 \mathrm{~dB}$ and $-95 \mathrm{~dB}$ in the 5 to $100 \mathrm{GHz}$ range.

Given the good agreement obtained, one can wish to push this simulation up to higher frequencies to get insight on the behaviour of the filter in ranges where it would be 
extremely difficult to measure. However, it turns out that the full chip simulation remains at the simulator noise floor (between -150 and $-170 \mathrm{~dB}$, depending on the frequency [6]) above $12 \mathrm{GHz}$, and furthermore becomes too large to be performed on an average workstation for frequencies larger than $\sim 100 \mathrm{GHz}$. In order to go beyond these limitations one needs to recourse to subdividing the filter into smaller parts and to cascading the resulting $\mathrm{S}$ matrices. Note that this assumes that the subdivided parts are independent otherwise than through their connecting ports (i.e. no coupling through the sheathing, which is what one expects from a bulk metallic shield). Doing so, we can push the simulation up to $6 \mathrm{THz}$ without excessive complications (See inset of Fig. 3). We find that above $50 \mathrm{GHz}$ oscillating features appears with a $95 \mathrm{GHz}$ period, corresponding to standing waves in the $1 \mathrm{~mm}$-long features of the meanders, and a second one at $\sim 400 \mathrm{GHz}$ corresponding to a standing wave in the width of a large pad in the meander. To sketch the filter behaviour, we can say that after the initial cut-off, the transmission curve forms a quasi-plateau between roughly 20 and $400 \mathrm{GHz}$ with a maximum transmission of $\sim-220 \mathrm{~dB}$ and subsequently falls rapidly again with an overall $\exp (-\sqrt{f})$ dependency, due to skin effect losses [7]. Note that these simulations do not take into account a number of effects which might be important at very high frequency such as increase of the loss tangent of the dielectric, surface roughness of the conductors and which likely would further increase filter attenuation.

\section{DISCUSSION}

Based on these simulations, we can evaluate the effectiveness of these filters for experiment thermalization. To do so, we calculate the effective temperature seen by a device connected to a line comprising a $50 \Omega$ source at $300 \mathrm{~K}$ and either a single filter anchored at $30 \mathrm{mK}$ or two filters respectively placed at $4.2 \mathrm{~K}$ and $30 \mathrm{mK}$. This frequency-dependent effective temperature is the temperature that an impedance equal to the output impedance of the line should have to have the same noise level than that reaching the device connected to the line at $30 \mathrm{mK}$ at a given frequency. The equivalent noise source at the output of the line can be evaluated exactly taking into account the contributions coming from the various parts of the line at different temperatures with the corresponding attenuation provided by the setup [8]. The result is displayed in Fig. 4 and shows that by using two filters, effective $30 \mathrm{mK}$ thermalization is reached in the $1-30 \mathrm{GHz}$ range [9]. At higher frequencies, the photon flux at the output of the line is larger than that of a $30 \mathrm{mK}$ impedance but remains in absolute so low that it's probability to spoil an actual experiment is negligible. On the lower end of the spectrum, the effective temperature is also above equilibrium but corresponds to photons present in the thermal spectrum at $30 \mathrm{mK}$. Further reduction of noise in this part of the spectrum may be desired, in particular to protect the experiment against electromagnetic interferences (EMI) in the laboratory ambient. In this frequency range, this can be done using carefully assembled discrete elements. In this goal, the connecting pad of the filter chip were made to accommodate 0805-size

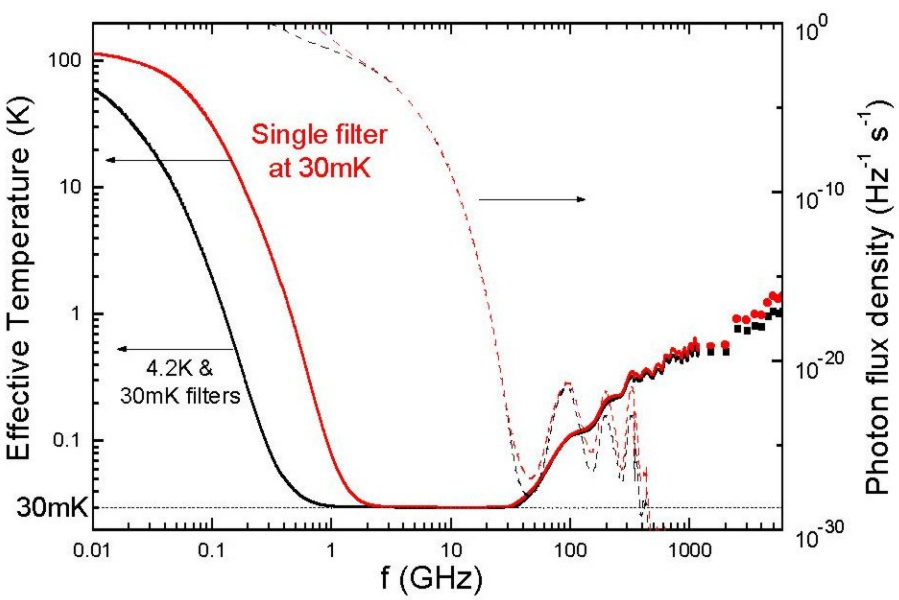

Fig 4. Calculated effective temperature (full lines, left axis) seen by a device connected to a line comprising a $50 \Omega$ source at $300 \mathrm{~K}$ and either a single filter (red curves) anchored at $30 \mathrm{mK}$ or two filters (black curves) respectively placed at $4.2 \mathrm{~K}$ and $30 \mathrm{mK}$ and photon flux density (dashed lines, right axis) at the output of this line on a $50 \Omega$ load.

surface mounted components such as readily available lossy inductors sold for EMI filtering with good attenuation in the $0.1-1 \mathrm{GHz}$ range.

At low temperature, Joule effect in such a resistive filter can easily heat the electrons of the filter out of equilibrium, due to the poor electron-phonon coupling [10]. Therefore, care should be taken no to drive excessive currents through these filters. For a given allowed relative increase $\eta$ of the electronic temperature, we can evaluate at lowest order the maximum dc current allowed to flow in the filter as $i_{\max }=\sqrt{5 \sigma V T_{p h}^{5}(\eta-1) / R}$ where $\sigma \sim 1 \mathrm{nW} / \mu \mathrm{m}^{3} / \mathrm{K}^{5}$ is the coupling constant, $V$ is the volume of the resistive material volume where electron-phonon coupling takes place, $T_{p h}$ is the phonon temperature and $R$ the resistance of the filter. To fix ideas, if one takes the whole volume of resistive material of the filter measured above, $V=1.510^{6} \mu^{3}$ and a $5 \%$ increase in the electron temperature $(\eta=1.05)$ above $T_{p h}=30 \mathrm{mK}$, one gets a maximum allowed dc current $i_{\max }=0.34 \mu \mathrm{A}$.

\section{POSSIBLE IMPROVEMENTS}

As mentioned above, the resistivity of the copper-gold alloy we use depends on temperature. This alloy was chosen mainly for its ease of use in our Joule evaporator as it yields a relatively low-stress film. This alloy could be replaced by another type of resistive alloy less dependent on temperature, provided maybe another deposition technique was used. Possible choices could then be Ni-Cr, $\mathrm{Cu}-\mathrm{Ni}$ or $\mathrm{Cu}-\mathrm{Mn}$.

\section{CONCLUSION}

In conclusion, we have developed and fabricated new miniature filters for proper thermalization of electrical experiments performed in dilution fridges. In the range where they can be measured, these filters behave as predicted. Calculations show that using two such filters on each line should permit to effectively thermalize experiments at $30 \mathrm{mK}$ above $1 \mathrm{GHz}$. 


\section{ACKNOWLEDGEMENTS}

We gratefully thank P.F. Orfila, P. Senat, D. Vion and D. Esteve for their help and support throughout this

[1] John M. Martinis and M. Nahum, Phys. Rev. B 48, 1831618319 (1993)

[2] D. Vion, P. F. Orfila, P. Joyez, D. Esteve, and M. H. Devoret, J. Appl. Phys, 77, 2519 (1995).

[3] A. B. Zorin, Rev. Sci. Instrum., 66, 4296 (1995).

[4] D. C. Glattli, P. Jacques, A. Kumar, P. Pari, and L. Saminadayar, J. Appl. Phys, 81, 7350 (1997).

[5] J. M. Martinis, M. H. Devoret, and J. Clarke, Phys. Rev. B, 35, 4682 (1987).

[6] From modal analysis in the narrowest part of the meander we conclude a $2.5 \mathrm{D}$ solver remains appropriate up to $\sim 20 \mathrm{THz}$, when TE modes start to propagate in the waveguide (but with high losses). development, and N. Feltin, B. Steck and P. Bertet for their interest and involvement in this project. We acknowledge useful discussions with H. Courtois and D.C. Glattli. This work was supported in part by the French Research Ministry project AC Nano 2003 NR110.

[7] J. C. Rautio, in Proc. European Microwave Conf. Dig., Munich, Germany, 2003, pp. 463-466. http://www.sonnetusa.com/support/downloads/publications/li mits_euromic_oct03.pdf

[8] D. Pozar, Microwave Engineering, Addison Wesley.

[9] P. Lafarge, p.xxx, Ph. D thesis, Université Paris VI, 1993.

[10] Note that this effective temperature prediction relies on calculated attenuations of several hundred $\mathrm{dBs}$. In practice, leaks in the connectors of the filters or any other part of the system could much reduce effective attenuation.

[11] C. Wellstood, C. Urbina, and John Clarke, Phys. Rev. B 49, 5942-5955 (1994) 


\subsubsection{Tunneling spectroscopy}

\subsubsection{Tunneling spectroscopy measurements}

The first step in local spectroscopy is to identify where the measurement will take place in the circuit under investigation. This is done visually, on an AFM topographic image (see Chap. 2). Once a satisfying image is taken, the tip is retracted, the tuning fork is stopped, and the microscope is switched to STM (current) feedback mode.

Then, we choose the position at which we want to perform spectroscopy on the AFM image, by just clicking at the desired location on the image. In response, the control software deflects the piezoelectric tube at the designated point of the image, by applying the appropriate static voltages to the tube's outer electrodes. Afterwards, we only change the tube elongation by varying the inner electrode's potential, to close or open the contact at will, so that the tip remains at the same position over the sample.

When first closing the contact, we apply a constant bias voltage between the tip and the sample, large enough to ensure that a current will flow when the desired contact transparency is reached (if a superconductor is probed, the bias voltage should not be in the gap of the superconductor). We typically used a few $\mathrm{mV}$ for the experiments performed so far on aluminum. Then we tune the contact transparency by extending or retracting the tube to reach contact resistances in the range from the hundreds of $\mathrm{k} \Omega$ to a few $\mathrm{M} \Omega$. This can either be done manually, or by setting a current setpoint and putting the STM in closed loop. These resistance values are used because they are well suited for tunneling spectroscopy $\left(\mathrm{R}>>\mathrm{h} / 2 \mathrm{e}^{2} \approx 13 \mathrm{k} \Omega\right.$ ) while still providing currents which can be measured with a good signalto-noise ratio. Indeed, the tunneling spectroscopy method presented in Chapter 1 is based on the assumption that the tunnel coupling of the states in both electrodes is only a weak perturbation of these states, and that tunneling can be treated at the lowest order in perturbation theory. This is the case only if the normal state tunnel resistance of each conduction channel in the contact is much larger than $h / 2 \mathrm{e}^{2} \approx 13 \mathrm{k} \Omega$. On clean surfaces the contact resistance can be made much lower if desired but such measurements cannot be interpreted simply as spectroscopy measurements (see chapter 1).

Once a stable contact established, we perform spectroscopic measurements which consist in measuring the differential conductance of the tunnel contact. This can be done in two ways: 
(i) the first one is to record $I(V)$ curves, and perform numerical differentiation with respect to voltage. Better results can be obtained by averaging several such curves to reduce the noise.

(ii) The second method is to measure directly the derivative $d I(V) / d V$ using a lock-in technique: a small sinusoidal signal is added to the DC voltage and the corresponding AC response current is measured synchronously. In such a measurement, the energy resolution is given by $\sim \sqrt{V_{A C}^{2}+V_{\text {Noise }}^{2}}$, where $V_{\text {Noise }}$ is the equivalent total RMS noise including both instrumental contributions and intrinsic thermal width of the Fermi distribution in the tip (See reprint in section 3.2.4.3, page 143), and $V_{A C}$ is the AC drive amplitude. Hence, to preserve the resolution it is best to work at low AC drive, but it reduces the signal to be measured. In our experiments $V_{\text {Noise }}$ was a couple of $\mu \mathrm{V}$ (See reprint). We normally operated with $V_{A C} \in[1-3] \mu \mathrm{V}$, which means that the typical magnitude of the AC current to be measured was of the order of $1 \mathrm{pA}$, and much less in the gap of a superconductor.

The main advantage of this synchronous detection with respect to the previous one is that it picks only noise in a narrow band around the frequency of the modulation, resulting generally in a much better signal to noise ratio. In particular one can avoid this way the 1/f noise which cannot be averaged with the first method. The lock-in frequency is optimally chosen where the signal-to-noise ratio at the output of the tunnel current amplifier is optimum. Given the amplitude of the signal measured, it was important to optimize this point, as well as the tunnel current amplifier sensitivity (See reprint, in 3.2.4.3).

A high quality $d I / d V$ spectrum with very low noise required long lock-in averaging time for each voltage point, and was typically taken over a couple of minutes. This makes those measurements very sensitive to mechanical drift or sudden reconfiguration in the contact transmissions. Fortunately, as already mentioned, by operating in contact on a thin oxide layer we could often measure reproducible spectra on timescales of a few hours.

When for some reason a good stability cannot be reached, it is often worth to use the first method, which, however also requires some cautions: when successive $I(V)$ characteristics deviate too much from each other, averaging them makes no real sense. So it is more a question of how to accept or reject the data, and it can become more subjective in the end, even though objective rules have been tentatively formulated [94]. 
In the following I discuss the data acquisition setup and more specific details regarding the current measurement.

\subsubsection{Data acquisition setup}

A schematics of the setup is shown in Fig. 3.19

The DC voltage source is a programmable DC source 7651 from Yokogawa. It allows to voltage bias from $-30 \mathrm{~V}$ to $30 \mathrm{~V}$, with 5 digits precision, down to $100 \mathrm{nV}$ increments using the appropriate range.

The lock-in is a Stanford Research SR830 DSP digital lock-in amplifier. It has internal AC source, providing AC excitation, from $4 \mathrm{mV}$ to $5 \mathrm{~V}$ and from $1 \mathrm{mHz}$ to $102 \mathrm{KHz}$.

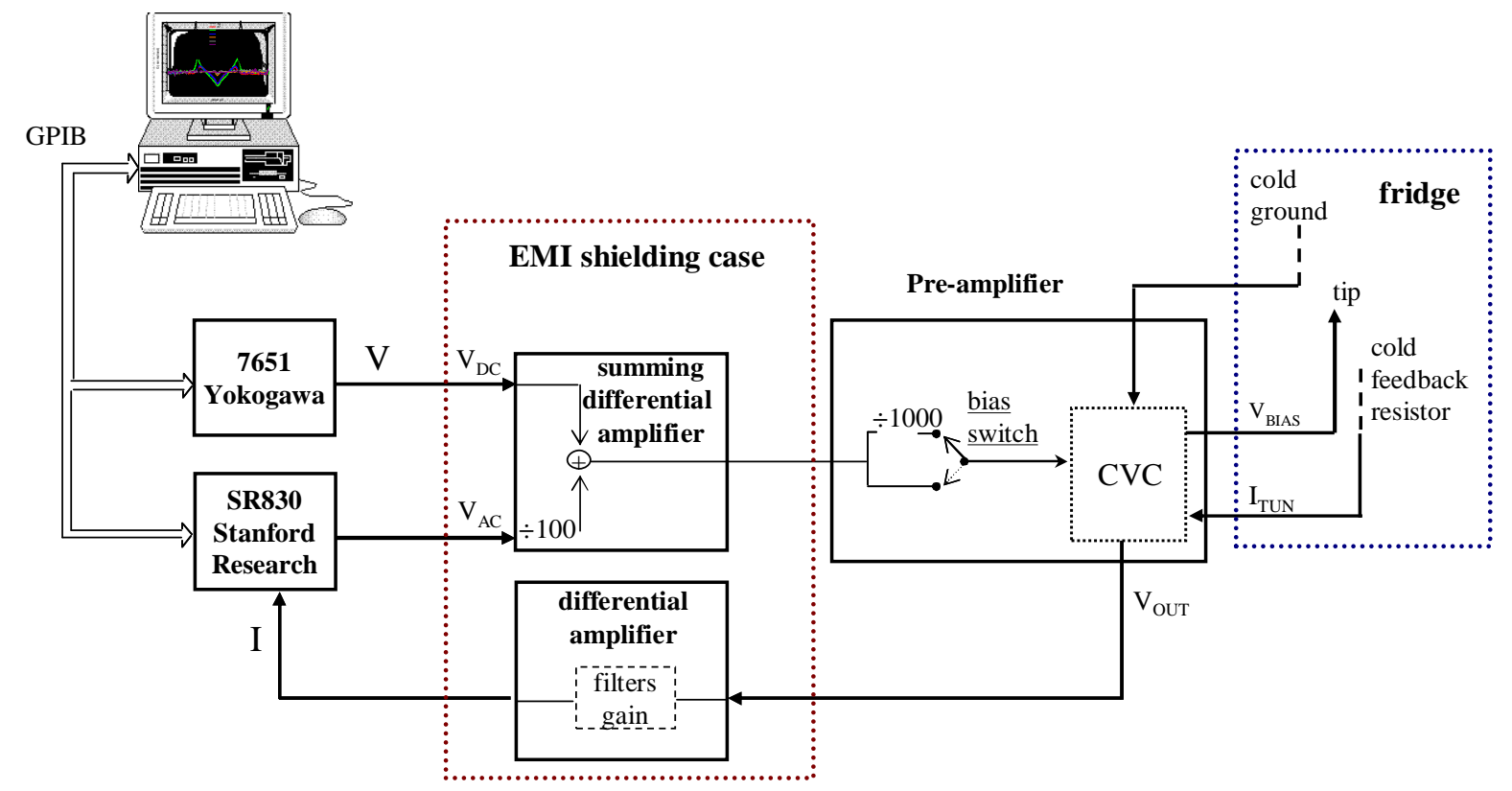

Fig. 3.19 Block diagram of the density of states measurement setup. Via the GPIB bus, a computer controls a DC source and a lock-in amplifier to make DC voltage ramps of desired speed and step size, and record the differential conductance $d I / d V(V)$ of the contact. Voltage bias is applied to the tip and the current flow is pre-amplified by a custom current to voltage converter (CVC) we have developed (see \$3.2.4.3, p 143). The input and output connections to the preamplifier are made through a home made instrumentation amplifier, that decouples the experiment ground from the rest of the electronics. On the output it provides, if desired, another stage of amplification and filtering, with adjustable parameters. Its output is fed through a differential connection to the input A-B of the lock-in.

Both the AC excitation delivered by the lock-in source and the DC voltage enter a battery powered differential low noise summing amplifier to decouple the experiment ground from the rest of the electronics ( $\mathrm{PC}$ etc.). Additionally this differential amplifier divides the AC signal by a factor 100, and adds it to the DC bias. This composite signal is then sent to the current preamplifier to be applied as the tip bias. Optionally, the preamplifier can further divide again this signal by a factor of 1000, when low level bias are required, as when 
measuring the superconducting gap of aluminium. The summing differential amplifier is situated in a so-called "Electro-Magnetic Interference" (EMI) shielding case. This shield also contains all the batteries that power the pre-amplifier and the differential input and output amplifiers. The pre-amplifier is mounted right at the output connector of the refrigerator, to avoid for extra noise and stray capacitance (see Fig. 3.20). It contains the heart of the current amplification system, and was carefully optimized to deliver minimal back-action noise on the tunnel contact, high current sensitivity and large bandwidth. A complete description of the pre-amplifier and its performances was done in an article, which is reproduced below (see reprint, 3.2.4.3), so I will not discuss this part further here.

The output signal of the pre-amplifier is again amplified by a home-made low noise differential amplifier. The gain of this stage can be chosen from 1 to 1000, and filters can be applied from DC to $100 \mathrm{kHz}$, either low, high or band pass. This amplifier is also battery powered and situated in the EMI shielding case.

The signal coming out of the differential amplifier is fed to the input of the lock-in, used in differential mode (A-B) to avoid ground loops. Input parameters of the lock-in were typically: AC coupled, normal reserve, $300 \mathrm{~ms}$ integration time.

In our setup, the tip is polarized, though one could imagine polarizing the sample instead, while setting the tip to ground. However, this choice was made to allow independent sample transport measurements, requiring sample biasing. 


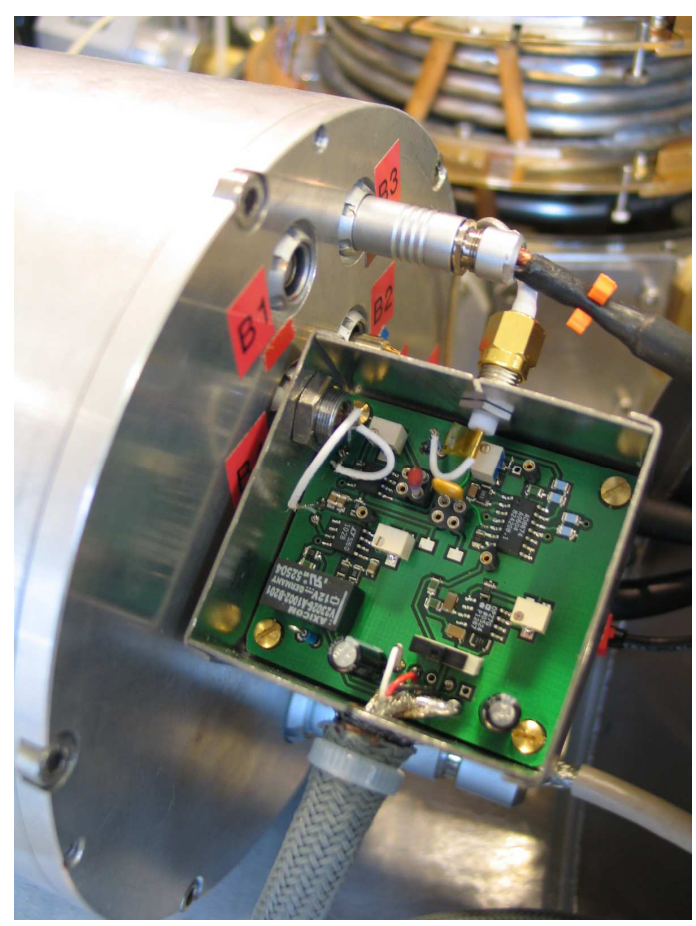

Fig. 3.20 Picture of the tunnel current pre-amplifier, with cover and shield removed. It is plugged right at the output of the refrigerator.

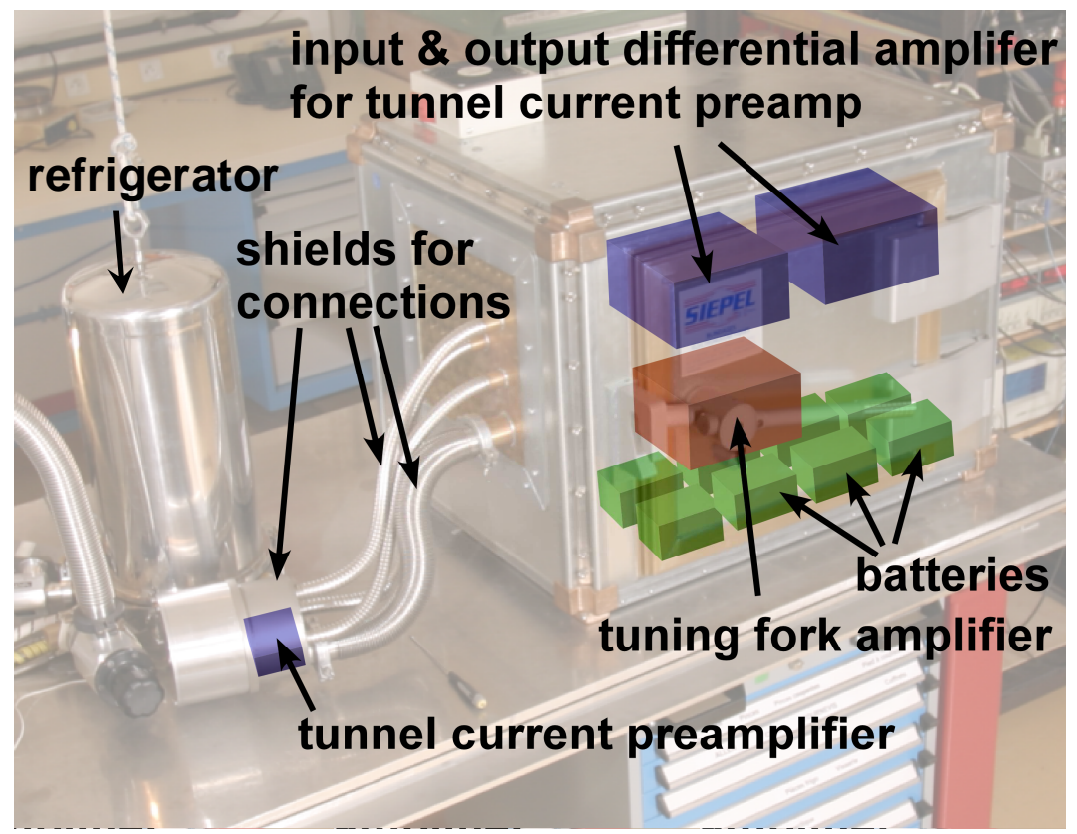

Fig. 3.21 Details for the installation of the low noise electronics 


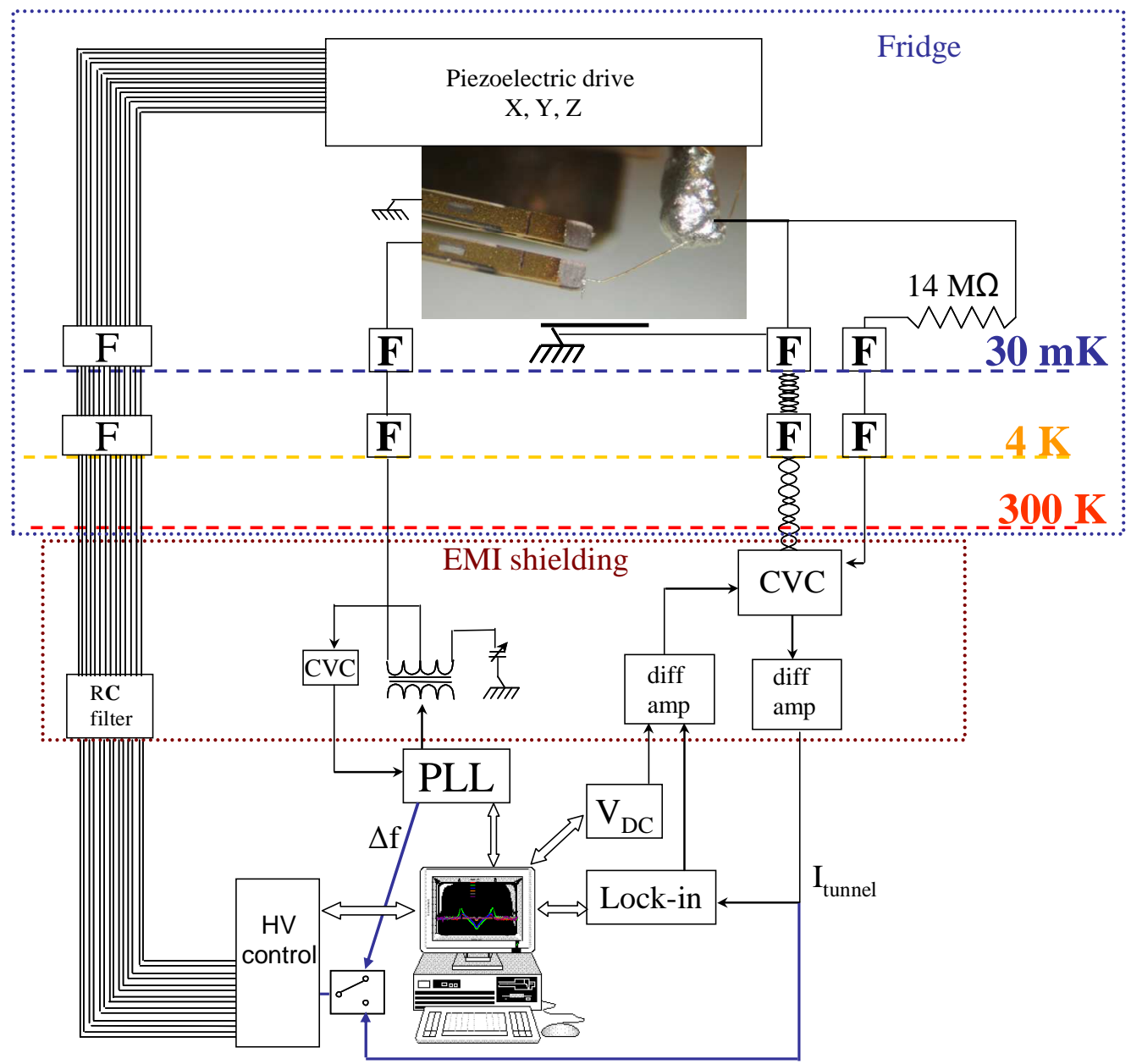

Fig. 3.22 Block diagram of the setup of the AFM-STM microscope in the dilution refrigerator. A computer controls all the electronics through buses. The tuning fork is connected to the PLL which gives the information of the frequency shift to the feedback system. The tip is electrically connected to our home-made amplifier, which gives information on the tunnel current. The feedback can be set to control either frequency or current, depending on the desired operating mode. The high voltage electronic drives the piezoelectric tube for precise motion of the tip. All quantities can be recorded by the computer. Symbols F represent the microfabricated filters; an additional low pass filter stage consisting of discrete RC elements is set at room temperature on the high voltage lines. All setups pictured here were already presented in the previous sections (see chapter 2 for the AFM control).

As already mentioned, the EMI shielding case contains all low level signals amplifiers, and their batteries. It is laid on the vibration isolation table, and is a through-route to all connections to the fridge. High level signals entering the cabinet, like high voltages for piezo elements go through feed-through low-pass filters. All connections between this cabinet and the refrigerator are completely shielded with flexible metallic $\mu$-metal EMI shielding tube, as can be seen in Fig. 3.21.

Data are acquired by a personal computer that commands the different apparatus through the available I/O ports (GPIB and RS232). These instruments are interfaced by a program I have 
developed using Testpoint ${ }^{\circledR}$ from National Instruments, that performs the simultaneous operations necessary for a good control over the microscope, and data acquisition.

Many modules are used to control the microscope:

-Coordination of stick-slip actuators \& capacitive position sensors

-Tuning fork control: resonance measurements, PLL control.

-Interfacing with the standard Molecular Imaging microscope front-end program, for controlling approach and withdraw of tip, changing between AFM and STM mode...

-Interfacing with remote refrigerator temperature monitor.

For $d I / d V$ measurements, the program sets the instrument parameters and subsequently controls the DC voltage sweep and data acquisition. The DC voltage sweep is done by changing the Yokogawa source output stepwise. After each step, the program waits five times the integration constant of the lock-in and then reads the lock-in measurement over the GPIB bus before performing the next DC voltage increment.

\subsubsection{Article reprint : Tunnel current pre-amplifier}

The article published in Review of Scientific Instruments, 77, 123701, (2006), is reproduced below. 


\title{
Room-temperature tunnel current amplifier and experimental setup for high resolution electronic spectroscopy in millikelvin STM experiments
}

Hélène le Sueur and Philippe Joyez

Service de Physique de l'Etat Condensé (CNRS URA 2464), CEA Saclay, 91191 Gif sur Yvette, Cedex, France (Received 26 July 2006; accepted 26 October 2006; published online 1 December 2006)

\begin{abstract}
The spectroscopic resolution of tunneling measurements performed with a scanning tunneling microscope is ultimately limited by the temperature at which the experiment is performed. To take advantage of the potential high spectroscopic resolution associated with operating an STM in a dilution refrigerator we have designed a room temperature tunnel current amplifier having very small back-action on the tunnel contact and allowing to nearly reach the predicted energy resolution. This design is a modification of the standard op-amp based tip-biasing current-voltage converter which implements differential voltage sensing and whose back action on the tip voltage is only $\sim 2 \mu \mathrm{V}$ rms for a $14 \mathrm{MV} / \mathrm{A}$ transimpedance and $22 \mathrm{kHz}$ bandwidth. [DOI: 10.1063/1.2400024]
\end{abstract}

\section{INTRODUCTION}

Scanning tunneling microscopes (STM) operated in spectroscopy mode provides valuable local information on the energy dependence of electronic mechanisms in the sample. For this purpose, low temperature STMs are in particular widely used to investigate recently discovered superconductors, or proximity effects. In this tunneling spectroscopy technique, when one of the electrodes is nonsuperconducting, the energy resolution is ultimately limited by the temperature at which the experiment is carried out. Indeed, the most precise results in this field are obtained using STMs in dilution refrigerators [1-7] with which one can commonly cool experiments in the $10-100 \mathrm{mK}$ range. However, in these experiments the energy resolution usually achieved is in practice significantly less than the thermal fundamental limit. There is thus a need for better instrumentation allowing to fully exploit the potential of these low temperature measurements.

In this article we discuss the required characteristics of the experimental setup in order to achieve optimal spectroscopic resolution and we present a room temperature amplifier optimised to have a back-action noise on the tunnel contact low enough that the energy resolution is close to the temperature limited value in an experiment carried out at $40 \mathrm{mK}$.

\section{TUNNEL SPECTROSCOPY BASICS}

For a perfect voltage bias between two electrodes made of good metals, the tunnel current can be expressed as

$$
\begin{aligned}
I(V)=\frac{4 \pi e|\tau|^{2}}{\hbar} \int & \left(n_{L}(\epsilon-e V)-n_{R}(\epsilon)\right) \rho_{L}(\epsilon-e V) \\
& \times \rho_{R}(\epsilon) d \epsilon
\end{aligned}
$$

where $n_{L, R}(\varepsilon)$ are the occupancies of the electronic states at energy $\varepsilon$ in the left or right electrode, $\rho_{L, R}(\varepsilon)$ are the densities of states in the electrodes and $\tau$ is the tunneling Hamiltonian matrix element between the two electrodes, assumed here to be independent of energy. When one knows the density of states and the occupation of the energy levels in one of the electrodes, the measurement of the $I-V$ characteristic of the contacts allows to extract information on the electronic state of the second electrode. For instance, if the left electrode is a normal metal in equilibrium at zero temperature, $\rho_{L}$ can be assumed nearly constant near the fermi level and $n_{L}$ is a step function at the fermi energy. Then, the derivative $\frac{d I(V)}{d V}$, gives a direct measurement of $\rho_{R}(\mathrm{eV})$. If the temperature $T$ is finite, the step function becomes a Fermi function, i.e. a rounded step function. In this case, the differential conductance $\frac{d I(V)}{d V}$ is given by the convolution product $\rho_{R} * f_{L}^{\prime}$ :

$$
\frac{d I(V)}{d V} \propto \int_{-\infty}^{\infty} \rho_{R}(\varepsilon) f_{L}^{\prime}(e V-\varepsilon) d \varepsilon
$$

where $f^{\prime}{ }_{L}$ is the derivative of the Fermi function $f_{L}$ of the left electrode which is a bell-shaped function having a width $k T$. Such a convolution product smears out the details in $\rho_{R}$, limiting the energy resolution to $\sim k T$.

In real life experiments the bias voltage cannot be perfectly fixed, however. It has fluctuations characterized by a distribution function $P(V)$ and what is actually measured is the average value [8]

$\left\langle\frac{d I}{d V}\right\rangle(\bar{V})=\int_{-\infty}^{\infty} \frac{d I(V)}{d V} P(V) d V$

where $\bar{V}=\int V P(V) d V$ is the average voltage. By inserting (1) into (2) and permuting integration orders, Equ. (2) can rewritten under the same form as (1) with the convolution product $P * f_{L}^{\prime}$ in place of $f_{L}^{\prime}$. Thus, not surprisingly, noise will further blur the resolution and appear under most circumstances as an excessive temperature. If $P(V)$ is Gaussian with an rms width $V_{n}$, the effective temperature can be evaluated as $T_{e f f}=\sqrt{T^{2}+3\left(e V_{n} / k \pi\right)^{2}}$ [9]. 


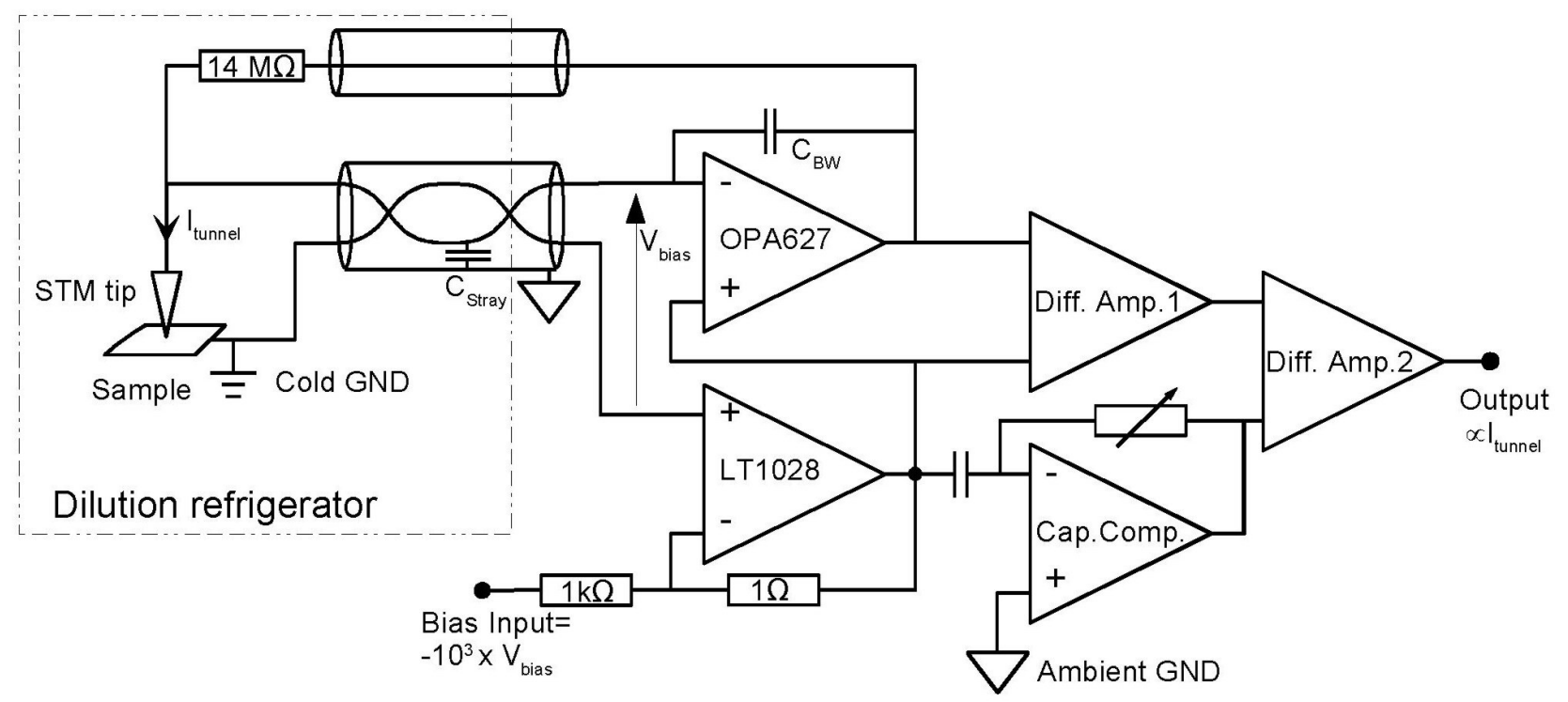

Fig1. Simplified schematics of the amplifier and connection to the tunnel contact. The tip and sample « cold ground » are connected to the amplifier through a shielded twisted pair with filters (not shown). The « cold ground » signal is sensed by an LT1028 ultra low voltage noise op-amp and used to offset the desired tip bias voltage. This sum is then used as the voltage reference of the current voltage converter. The first difference amplifier (Diff. Amp. 1) is made using high precision resistors (0.01\%) to accurately subtract the bias voltage from the output of the CVC. The two other circuits (Diff. Amp. 2 and Cap. Comp.) compensate the ac currents flowing to ground in the stray capacitance $\mathrm{C}_{\text {Stray }} \sim 200 \mathrm{pF}$. For best current sensitivity, the $14 \mathrm{M} \Omega$ feed-back resistor of the CVC is placed at low temperature and connected through a coaxial cable. Bandwidth of the CVC is adjusted to $22 \mathrm{kHz}$ to limit noise peaking by the use of $\mathrm{C}_{\mathrm{BW}} \sim 1 \mathrm{pF}$.

\section{EXPERIMENTAL CONSIDERATIONS}

Following the above discussion, in order to reach the highest level of spectroscopic resolution in a tunneling spectroscopy experiment it appears necessary to perform the experiment at the lowest possible temperature, which in practice means in dilution refrigerator. It also appears equally important that the electromagnetic noise reaching the tunnel contact through the connecting wires has both a distribution and a spectrum compatible with the energy resolution sought. To reach such a low noise level in an experiment one must act at three levels. At the first level, the wires reaching the tunnel contact must be properly thermalized, so that the contact really "sees" an electromagnetic temperature equal to that of the refrigerator. This is achieved by careful shielding and filtering of the cables connected to the tunnel contact so that thermal radiation coming from room temperature apparatus is removed [10]. At the second level, the wiring of the experiment must follow well established low-noise techniques to avoid stray pick-up of electromagnetic signals in the laboratory ambient (mains, radio signals...) by the wires connected to the tunnel contact. In particular, if not using a magnetic shield enclosing the whole experimental setup, one has to select a proper circuit topology and grounding strategy to avoid noise due to inductive coupling in so-called ground loops [11]. At the third level, the intrinsic voltage noise of any instrument connected to the tunnel contact must be considered. Contrarily to the output noise of voltage generators which can usually be reduced to appropriate levels by using simple voltage dividers, the input noise of amplifiers is generally fully present on the tunnel contact in the bandwidth of the setup and can be seen as a back-action on the tunnel contact. Thus, in order to reach the ultimate energy resolution, one must use an amplifier with rms input voltage noise $V_{n}$ such that $V_{n} \ll k T / e$. As experiments are performed at lower temperatures, this puts more stringent requirements on the amplifier noise characteristics. Yet, in the low-temperature STM literature, this back-action is seldom considered and the current sensitivity (i.e. output voltage noise density divided by the transimpedance ) is often the only figure of merit given to characterize the measurement chain.

\section{IMPLEMENTATION}

In our installation of an STM in a dilution refrigerator we have followed systematically the above considerations. The electromagnetic thermalization of all the wiring connected to the STM is achieved using shielded lines and custom microfabricated low-pass filters described in Ref. [10]. Besides this, we realized that having a very good control of the effective bias voltage applied to the tunnel contact is almost impossible when one uses a standard current-voltage converter (CVC) because such a circuit does not follow the good practice of having a single voltage reference point in a low signal level circuit. Namely, the voltage reference ("ground") of the CVC usually sitting at room temperature is physically separated from the electrode of the tunnel contact which should be precisely at the same potential. This makes the system prone to ground-loop noise problems. To overcome this problem we designed a modified CVC which allows differential sensing of the voltage on the tunnel contact. A simplified schematics of this amplifier in shown in Fig. 1.

In this design, the tip and sample « cold ground » are 


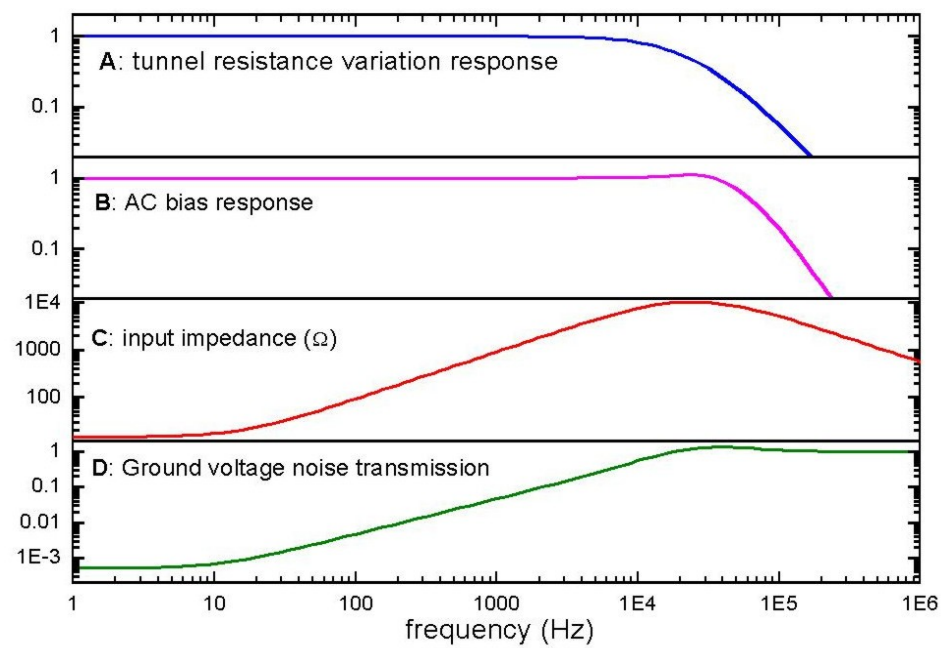

Fig 2. Results of Spice simulations. Panel A: normalized linear ac response at the output of the amplifier in response to a small variation of the tunnel resistance, as a function of frequency. The normalization value is $1.4 \mu \mathrm{V}$ per $\%$ variation of a $1 \mathrm{M} \Omega$ tunnel resistance under a dc bias of 10 $\mu \mathrm{V}$. Panel B: normalized linear ac response at the output of the amplifier in response to ac bias. The normalization value is $14 \mu \mathrm{V}$ per $\mu \mathrm{V}$ ac bias on a $1 \mathrm{M} \Omega$ tunnel resistance. Panel $\mathrm{C}$ : Input impedance of the CVC as seen from the tunnel contact. Panel D: Assuming an ac voltage difference between hot and cold grounds, fraction of which still present in the tunnel contact bias.

connected to the amplifier through a shielded twisted pair. The « cold ground » signal is sensed by an LT1028 ultra-

low voltage noise op-amp and added to the desired tip bias voltage. This sum is then used as the voltage reference of the CVC. This design allows to cancel tip voltage fluctuations due to magnetic noise pick-up from the lab environment at low frequency which cannot be easily shielded. The current-voltage converter is made with an OPA627 which offers a good combination of current and voltage noises and bandwidth. Given the $\sim 200 \mathrm{pF}$ total capacitance to ground of the tunnel current line in the twisted pair cable, the $14 \mathrm{M} \Omega$ feed-back resistor and a $\sim 1 \mathrm{pF}$ capacitor which limits output noise peaking at cut-off frequency [12], the OPA627 delivers a $22 \mathrm{kHz}$ bandwidth, sufficient for STM imaging. The noise coming from the LT1028 is also limited by reducing its bandwidth using a $100 \mathrm{nF}$ over-compensation capacitor and RC low-pass filter (neither shown). The operational amplifiers used in the rest of the circuit are low noise op-amps (OP27 or similar) which add negligible noise to the signal.

The amplifier is constructed using surface mounted components and fits easily on a 45 x $50 \mathrm{~mm}$ printed circuit board. The circuit board is mounted in a small metallic box which plugs directly into the socket of the twisted pair cable at the output of the refrigerator to avoid adding further cable length and stray capacitance. The amplifier is battery-powered and ground loops with other instruments are strictly avoided by using differential instrumentation amplifiers both in front of the bias input and following the output of the amplifier. The batteries and the instrumentation amplifiers are placed in an EMI shielding cabinet and a continuous metallic shield between the refrigerator and the cabinet completely encloses the amplifier and its connections to the batteries and the instrumentation amplifiers.

\section{AMPLIFIER PERFORMANCE}

We used Spice [13] simulations extensively during the design stage to optimize the level of noise of our amplifier and its dynamic behavior. Spice simulations offer a rapid and simple way to evaluate several design options and particularly regarding the choice of the op-amps to use, as most manufacturers provide Spice models for their op-amps and many of these models include noise characteristics. In Fig. 2 we show the predicted frequency dependences for key properties of our amplifier. The ac response to changes in the tunnel contact resistance and in the bias voltage are flat up to a $-3 \mathrm{~dB}$ bandwidth of $22 \mathrm{kHz}$ and $30 \mathrm{kHz}$ respectively. The input impedance of the amplifier as seen from the tunnel contact is $14 \Omega$ at very low frequency and then rises linearly with frequency up to a maximum of $10 \mathrm{k} \Omega$ at the cut-off frequency of the amplifier. The CVC thus delivers a good voltage bias at all frequencies for a tunnel contact in standard tunneling conditions (tunnel resistance $\gg h / e^{2} \approx 26 \mathrm{k} \Omega$ ). We have also investigated the efficiency of the differential voltage sensing scheme implemented in the amplifier at different frequencies. For this we simulated an ac voltage difference between the cold ground and the room-temperature ground and looked how much of this voltage was present on the tunnel contact bias (Fig. 2D). This shows that this setup can efficiently suppress low frequency noises due to currents flowing in the ground, and thus, provided the twisted pair wiring avoids picking up flux, it should be effective against pickup of mains power and its harmonics.

In order to make accurate noise calculations, we need to properly take into account the noise due to the dissipative wires and filters sitting at different temperatures in our setup. Even though Spice normally has a single temperature for all elements in the model, it is fairly easy to make a model for resistors at different temperatures for this purpose [14]. What the noise calculations show is that the input voltage noise of the amplifier, i.e. the back-action of the amplifier on the tunnel contact, is essentially determined by the input voltage noise of the CVC (OPA627) and resistor noise in the twisted pair and its filters [15]. The input noise of the amplifier does not depend on the input current noise of the CVC, nor on the Johnson noise in the feed-back resistor which both only appear in the output noise of the OPA627 owing to the low input impedance of the CVC 


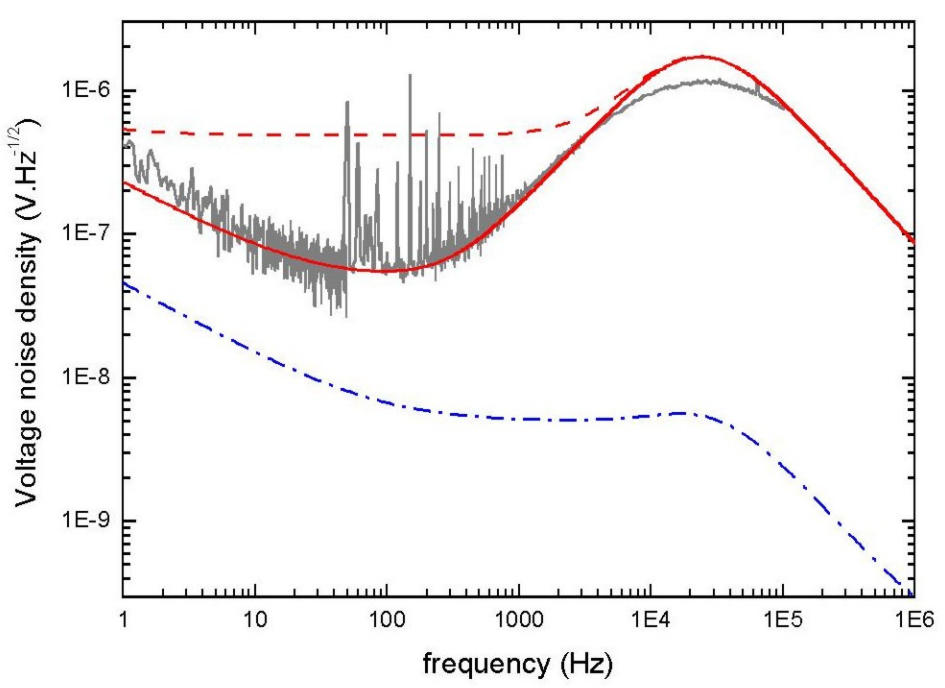

Fig 3. Noise performance of the amplifier. Dash-dotted curve : Predicted input noise voltage density on the tunnel contact. The input noise is independent of the feed-back resistor temperature in this range. The integrated noise gives $\sim 2 \mu \mathrm{V}$ rms. Thick top curves : predicted output noise with the feed-back resistor at room temperature (dashed line) or at low temperature (solid line). Thin gray line: measured output noise while experiment was at low temperature. No adjustable parameters are used. The discrepancy above $10 \mathrm{kHz}$ is attributed to an additional stray feedback capacitor on the OPA627 in parallel with the $C_{\mathrm{BW}}=1 \mathrm{pF}$ installed capacitor (see Fig. 1).

provided by feed-back loop. It does not depend neither on the LT1028 voltage sensor because its noise at the non inverting input of the OPA627 is negligible compared to the intrinsic voltage noise of the OPA627. For this setup, Spice simulations predict that when running the experiment at $30 \mathrm{mK}$, the amplifier and wiring make a $\sim 2.0 \mu \mathrm{V} \mathrm{rms}$ back action noise on the tip bias voltage. The smearing of the spectroscopic resolution due to this voltage noise has the same rms width than a $13 \mathrm{mK}$ Fermi function, and its broadening of a $30 \mathrm{mK}$ fermi function is similar to a $2.6 \mathrm{mK}$ increase in temperature. Since this noise level is essentially due to the input voltage noise of the OPA627, it could be reduced by narrowing the bandwidth or by substituting it by an op-amp with less voltage noise. For this purpose one could use for instance an AD745, but its greater current noise would adversely affect the output noise of the CVC, i.e. its current sensitivity. As stated above, the output noise also depends on the Johnson noise of the feed-back resistor of the CVC. In fact, for the large resistor value we use, at room temperature this is the dominant output noise contribution at low frequency. Consequently, the sensitivity of the amplifier can be significantly improved by reducing the temperature of this resistor [16], as shown in Fig. 3. Using one extra coaxial line in the refrigerator, we placed the feed-back resistor at the temperature of the microscope (see Fig.1) but it could also be placed at $4 \mathrm{~K}$ with no change in performance. This, together with the twisted pair cable, makes a total of three lines for the tunnel contact bias and measurement. In this configuration, the output noise of the amplifier is peaked around the cutoff frequency of the amplifier, typical of an optimized CVC [12]. At low frequency, it rises due to $1 / f$ noise. In between, around $100 \mathrm{~Hz}$, the output noise has a flat minimum corresponding to a current sensitivity of $4 \mathrm{fA} / \mathrm{Hz}^{1 / 2}$. This minimum is the most favorable frequency range for lock-in measurements of differential conductance since it provides the highest signal-to-noise ratio for the weak ac current one needs to measure when using an ac bias voltage well below $k T / e$ which is necessary to preserve the spectral resolution.

\section{EXPERIMENTAL RESULT}

In Figure 4, we show a differential tunnel conductance measured with this amplifier. In this experiment the STM was equipped with a tungsten tip and the sample was a $25 \mathrm{~nm}$-thick evaporated aluminium film deposited on an oxidized silicon substrate. The data were recorded at a temperature of $\sim 40 \mathrm{mK}$, well below the superconducting transition temperature of the aluminium film, but above that

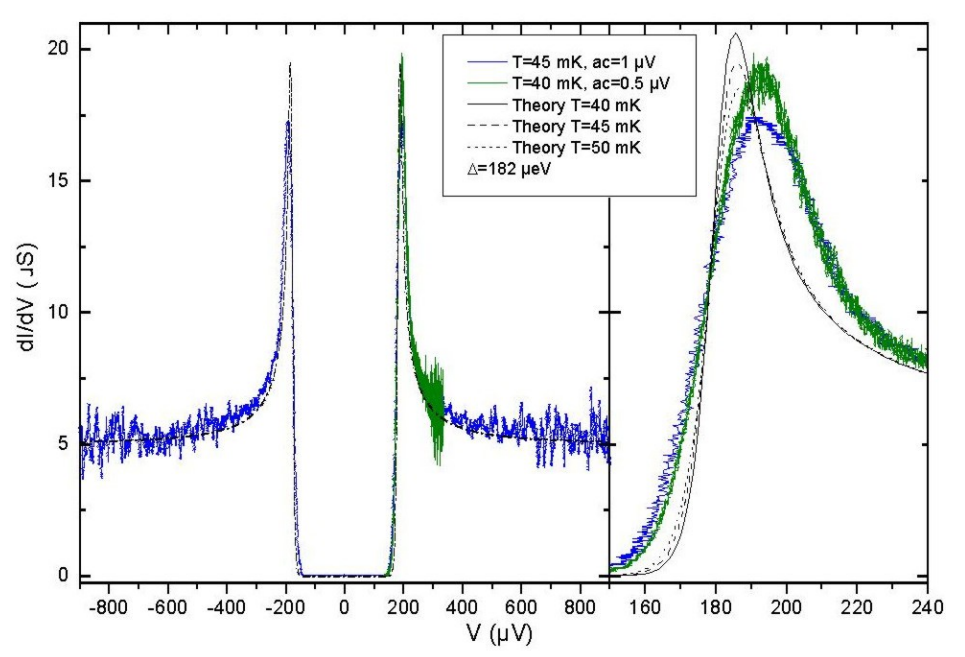

Fig 4. Experimental differential conductance taken on a 25 $\mathrm{nm}$ thick aluminium film with a tungsten tip for two different temperatures and ac excitations, and comparison with the prediction for Normal-Superconductor tunnel spectrum at different temperatures, with a gap $\Delta=182 \mu \mathrm{eV}$. On the right panel, a close-up on the right hand side peak. In spite of the unexplained discrepancy, the aspect ratio of the peak and its dependence on ac amplitude and/or temperature in this range indicate that the effective temperature of the measurement is close to the refrigerator temperature and proves that the back-action of our amplifier is not larger than expected from the simulations. 
of tungsten $(15 \mathrm{mK})$. In this case, theory predicts that the differential conductance should be simply proportional to a convolution product of the BCS density of states of the film with the derivative of the fermi function of the normal state tip. The only adjustable parameters in this curve are the superconducting gap of aluminium [17], the asymptotic value of the conductance at large voltage and the temperature of the tip. Fitting the data with theory should thus give access to the effective temperature of the tip, including all noise contributions. In our case, for a reason not presently understood, a precise fitting of the data within this model is not possible. Nevertheless, the peak height in the differential conductance of such normal metalsuperconductor contact gives a good indication of the effective temperature of the normal metal. Based only on the height of the peak, the effective temperature would be around $45 \mathrm{mK}$ for a measurement performed at $40 \mathrm{mK}$. The fact that the peak height still varies when temperature and ac excitation are lowered confirms such an estimate and it also proves that the amplifier input noise is close to the predicted value. Thus, the spectroscopic resolution of this

[1] L. Crétinon, A. K. Gupta, H. Sellier, F. Lefloch, M. Fauré, M. Fauré, A. Buzdin and H. Courtois, Phys. Rev. B 72, 024511 (2005)

[2] M. Crespo, H. Suderow, S. Vieira, S. Bud'ko, and P. C. Canfield, Phys. Rev. Lett. 96, 027003 (2006)

[3] B. Sacépé, C. Chapelier, C. Marcenat, J. Kačmarčik, T. Klein, M. Bernard, and E. Bustarret Phys. Rev. Lett. 96, 097006 (2006)

[4] T. Hanaguri, C. Lupien, Y. Kohsaka, D.-H. Lee, M. Azuma, M. Takano, H. Takagi \& J. C. Davis, Nature 430, 1001 (2004)

[5] M. D. Upward, L. P. Kouwenhoven, A. F. Morpurgo, N. Kikugawa, Z. Q. Mao, and Y. Maeno, Phys. Rev. B 65, $220512(2002)$

[6] E. Ishikawa, R. Shoda, N. Matsuura, M. Ono, T. Eguchi, A. Sakai, M. Kubota and Y. Hasegawa, e-J. Surf. Sci. Nanotech. Vol. 2, p.151 (2004)

[7] H. Kambara, T. Matsui, Y. Niimi H. Fukuyama, Jpn. J. Appl. Phys. 45, 1909 (2006)

[8] This result is valid if the measurement time is long compared to the typical timescales of voltage noise variations, $t$ hemselves slow compared to the tunneling process. This is generally the case when performing lock-in measurements of $t$ he differential conductance. More detailed analysis is needed for peculiar noise sources, like slow telegraphic noise or for very high frequency noise for which photon assisted tunneling may appear.

[9] In tunnel spectroscopy experiments where the tip and the sample are both superconductors well below their transition measurement is very close to the ultimate thermal limit and a factor of $\sim 4$ better than the best previously published resolution obtained with an STM.

\section{CONCLUSION}

In conclusion, we have discussed the requirements for a high resolution, low temperature tunneling spectroscopy setup. We have shown how to fulfill this requirements by designing a new low noise current voltage converter which implements differential voltage sensing. Measurements performed with this amplifier in a thoroughly filtered setup show that we indeed reach a spectroscopic resolution only limited by the fundamental thermal noise.

\section{ACKNOWLEDGEMENTS}

The authors gratefully acknowledge discussions with G. Rubio-Bollinger and $\mathrm{H}$. Courtois and useful comments of C. Urbina. This work was supported in part by the French Research Ministry project AC Nano 2003 NR110.

temperature, the ultimate resolution is fixed by the width of the singularities in the density of states, and not by the temperature. However, the actual energy resolution is still affected in the same way by the bias voltage noise, and the amplifier we present here will also help improving these measurements.

[10] H. le Sueur and P. Joyez, Preprint.

[11] H.W. Ott, Noise Reduction Techniques in Electronic Systems, 2nd Edition, John Wiley and sons, ISBN: 0-47185068-3

[12] Texas Instrument application note SBOA055A available at http://focus.ti.com/lit/an/sboa055a/sboa055a.pdf

[13] Spice circuit simulation software. See SPICE entry at www.wikipedia.org, for instance.

[14] In SPICE, a resistor at lower temperature can be simulated by

a smaller resistor in series with a "noiseless resistor" made with a behavioral voltage source with a voltage proportional to the current in the small resistor. Some c omercial versions of Spice can natively handle elements at different temperatures.

[15] Each line has a total resistance of $\sim 300 \Omega$ coming from two $80 \Omega$ filters sitting at $4 \mathrm{~K}$ and $30 \mathrm{mK}$, the rest being in the wires themselves, mostly between $4 \mathrm{~K}$ and $30 \mathrm{mK}$. The total capacitance of each line to ground is $\sim 200 \mathrm{pF}$.

[16] N. Moussy, H. Courtois and B. Pannetier, Rev. Sci. Instrum., 72, $128(2001)$

[17] in Al thin films the actual gap depends on the impurity content of the film and on its thickness. 


\subsection{Fabrication techniques}

\subsubsection{Tip fabrication}

The preparation of the tip is essential to obtain at the same time a good spatial resolution using the Atomic Force sensor, and good tunneling results.

The first point is obvious: lateral resolution achieved with a force sensor depends on the tip shape and the sample local topography. Ideally, the "perfect tip" should have, at large scale a high aspect ratio, and at small scale, an apex with a small radius. However, tips that are too thin are very fragile and should be avoided in low temperature experiments where there is no way to interchange the tip.

The second point deals with tunneling spectroscopy: we need a well characterized metallic tip, with a known density of states around Fermi level. This requirement is worth mentioning, as most metals tend to spontaneously oxidize in air, and some of these oxides (mixed valence oxides of $\mathrm{Nb}$, for instance) may have "exotic" electronic properties. Also, obviously, the oxide should not be so thick as to prevent tunneling at all. In most cases it is preferable to clean the tip from its oxide prior to attempt tunneling.

Materials commonly used for STM tips are thus preferably stiff, not too reactive metals, either normal or superconducting depending on the application, and easily machined to form high aspect ratio tips. Those are typically Tungsten, Gold and Iridium or Platinum / Iridium alloys for normal tips, and Niobium for superconducting ones.

\subsubsection{Electrochemical etching}

The simplest yet effective way to fabricate tips consists in cutting a wire with a blade. This works for STM images on rather flat sample typically used in STM surface studies, where tips with high aspect ratios are not needed: there is an atom of the tip which is closest to the surface, and tunneling occurs from this atom. Such tips are not suited for our needs, as there is little hope that they would have the desired high aspect ratio shape needed for AFM. The need for sharp, high quality tips has fostered strong research effort during the past years (for reviews on the subject see [95-97]), and a large number of methods are now employed in the field of microscopy to fabricate local probe tips.

Among the possibilities to fabricate AFM-STM tips, electrochemical etching is one of the simplest and most reproducible method. Its main drawback is the sequential processing of 
tips, contrary to parallel procedures where many tips are processed together out of the same wafer, which has long been done for e.g. silicon AFM Probes [98]. In contrast, electrochemical etching is "craftsmanship": each tip is fabricated individually out of a thin wire of the desired metal. The wire is cut to desired length and dipped into an adequate electrolyte. Either DC or AC voltage polarization is applied to the setup until the wire is sufficiently etched to display a sharp shape.

We usually process tips in small batches. After fabrication, each tip is subsequently observed in a Scanning Electron Microscope (SEM) to determine which tips of the batch are the best suited for the experiments, taking into account both fragility (i.e. aspect ratio), and sharpness (i.e. apex radius).

The setup for electrochemical etching of tips is described in Fig. 3.23. The principle is to etch the metal of the wire (oxidize it) and benefit from the differential mobility of ions in the solution to get a differential etch rate, and the desired final shape. Indeed, near the surface of the solution, ions are less mobile than in the "bulk", due to confinement. Consequently the wire will be preferentially etched in the bulk of the solution, and with a decreasing effectiveness as reaching the surface. In tungsten there is an additional effect enhancing the latter, called the necking phenomenon [99] that is responsible for such accentuated concave profiles most valued by AFM users.

This setup has originated from a very well known setup for etching STM tips, actually derived from the first makings of field emission electron microscopy (FEEM) and field ion microscopy (FIM) tips ([100-103]) and modified by Kulawik et al.[104] to provide easy control over the end of the chemical reaction. Indeed, in an electrochemical etching process it is mandatory to turn the reaction off when the optimal sharpness is reached. If the reaction is prolonged after this optimal point is reached, the tip only gets blunter, and one might not get the desirable overall shape either [105]. The key advantage of the specific setup shown in Fig. 3.23 is to automatically (and simply) stop the chemical etching when the tip is wellformed, and the apex is sufficiently thin for the wire to break under its own weight. 


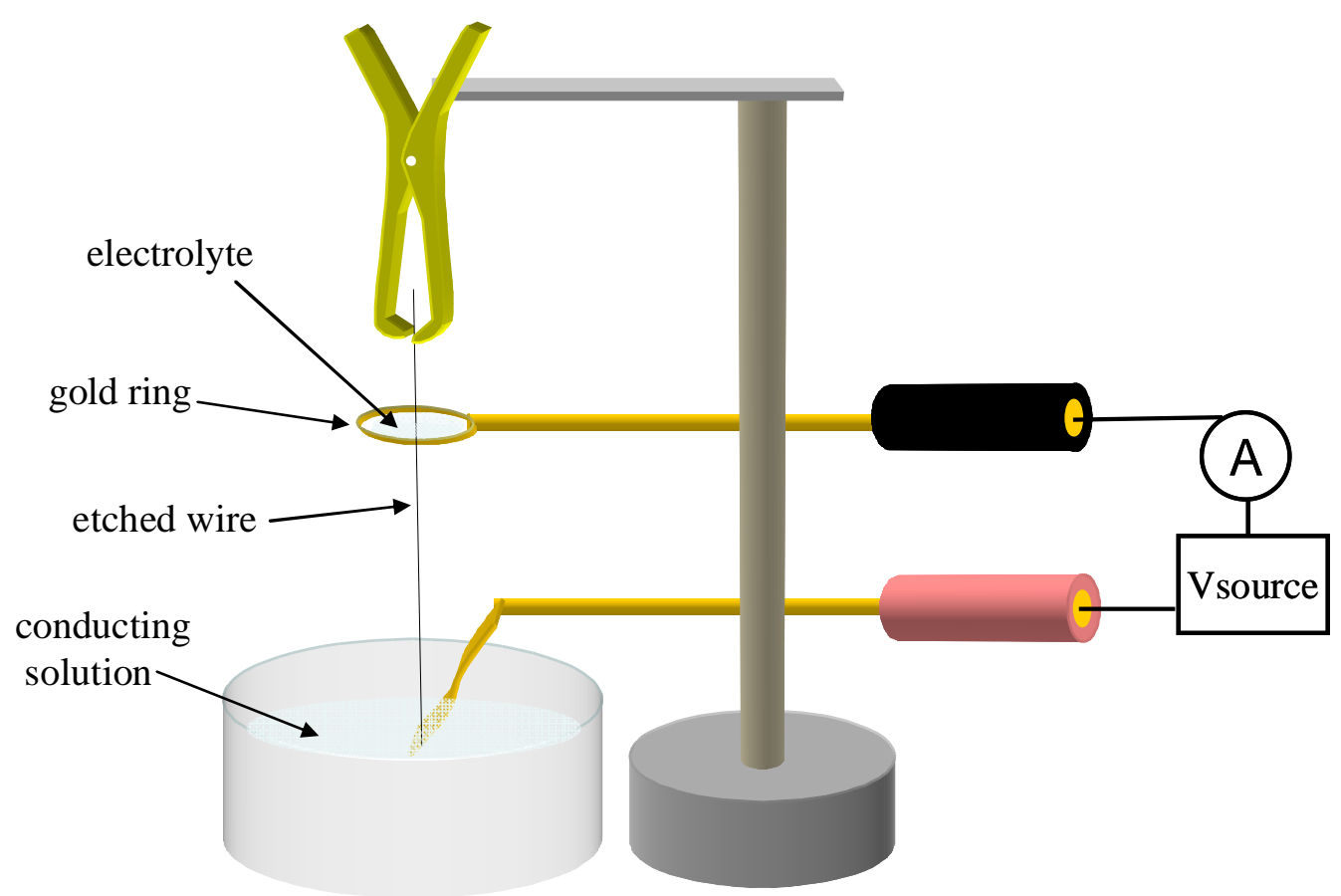

Fig. 3.23 Setup used for electrochemical etching of tips. The two gold electrodes soaked with a different solution. The wire to be etched links electrically the two electrodes: at one end (the anode) the wire is oxidized, at the other end (the cathode), water is reduced. Here the wire is oxidized on the top gold ring, in which the etchant (electrolyte) is held by capillary forces; charges produced by oxidation flow to the bottom electrode passing by the wire, and into the conducting solution. When the wire is completely oxidized at the anode, it breaks under its own weight, thus opening the circuit, and falls in the bottom beaker, where one can collect it. The upper part can make good tips as well (see Fig. 3.24).

\subsubsection{Tungsten tips}

Tungsten is the most widely used material to form electrochemically etched STM tips for several reasons:

- High stiffness:

It is among the stiffest materials with a Young modulus of $4.10^{11} \mathrm{~N} / \mathrm{m}^{2}$, about 7 times more than quartz for instance (cf Chap. 2). This confers it an appropriate behaviour for controlling contacts of atomic size: for instance tungsten apex are not prone to the so-called "jump to contact" usual for gold and other ductile materials. Indeed the dynamics of the jump is governed by a competition between bonding forces and elastic forces in the material [106]. In tungsten, the stiffness is high enough to prevent from a jump to contact in a Mechanically Controlled Break Junction (MCBJ) [107] experiment.

In our case tips made of tungsten are interesting because they are more robust than tips of other materials suitable for STM, and, if short enough their (elastic) resonance modes do not interfere with the use of the tuning fork. 
- Electrical properties of tungsten:

Tungsten is a non-magnetic normal metal with good conductivity $\left(\sim 1.8 .10^{7} \Omega^{-1} \mathrm{~m}^{-1}, \sim 4\right.$ times less than gold) down to very low temperatures, and becomes superconducting with a variable Tc from $\sim 15 \mathrm{mK}$ for the crystalline form alpha up to $3.5 \mathrm{~K}$ for the beta form. Experimental studies on the diverse crystalline forms of tungsten have shown that the critical temperature can be tuned at will by varying the proportions of those phases in a polycrystalline system ([108-110]).

We have tried several diameters, starting with a pure $90 \mu \mathrm{m}$-diameter drawn wire from an old stock, a $25 \mu \mathrm{m}$-diameter pure $(99,95 \%)$ and a $10 \mu \mathrm{m}$-diameter gold-coated $(\sim 1 \mu \mathrm{m})$ both bought from Goodfellow®. All the types tried showed normal densities of states at $30 \mathrm{mK}$.

- Drawbacks:

The main drawback of tungsten for STM applications is the formation of an oxide layer which hampers correct tuning of the contacts conductance. In STM applications this requires in-situ cleaning of the tip before making measurements. Most in-situ cleaning techniques require very high temperatures or intense electric fields [111].

Another technique consists in covering the tip with a thin inert gold layer, after removing the oxide by ion-milling for instance (see below)

- Simple etching procedure:

A key advantage of Tungsten is the ease with which it can be electrochemically etched: it is very simply oxidized in a basic aqueous solution like $\mathrm{KOH}, \mathrm{NaOH}$, at low chemical potentials, and its most stable oxide $\mathrm{WO}_{4}{ }^{2-}$ forms a soluble complex with $\mathrm{OH}^{-}$, easy to remove by dipping the wire in deionised water. The basic oxydo-reduction reactions involved in the etching process are [99]:

Cathode:

$$
6 \mathrm{H}_{2} \mathrm{O}+6 \mathrm{e}^{-} \rightarrow 3 \mathrm{H}_{2}^{(\mathrm{g})}+6 \mathrm{OH}^{-}
$$

Anode:

$$
\mathrm{W}^{(\mathrm{s})}+8 \mathrm{OH}^{-} \rightarrow \mathrm{WO}_{4}^{2-}+4 \mathrm{H}_{2} \mathrm{O}+6 \mathrm{e}^{-}
$$

Actually, a correct description would involve the creation of intermediate chemical species like tungsten trioxide $\mathrm{WO}_{3}$, which is not soluble. Furthermore, the reaction at the anode involves the formation of bubbles, which is not mentioned in the reaction above. The formation of an oxide layer during electrochemical etching is discussed in several papers $[99,112,113]$, though none of them really concludes on the dependency of its thickness on 
the applied external parameters like the bias voltage, current density, $\mathrm{OH}^{-}$concentration etc. It is usually a few nanometers thick, and has to be subsequently removed using either of the techniques described in literature [111].

We have tried to improve several aspects of the fabrication. First of all, the curvature radius as well as the aspect ratio are thought to depend a lot on the thickness of the meniscus in which the reaction takes place [99]. This parameter is controlled by the surface tension of the electrolyte, and that is the reason why one generally adds to the etchant (in our case $\mathrm{KOH}$ 4M) a significant proportion (20\%) of ethanol, acetone [114], or isopropanol which lowers surface tension. We use a setup in which a drop of electrolyte is held in a gold ring, which also requires in itself a low surface tension. Reviewing those points, we decided to incorporate soap in the solution. In that manner, the electrolyte film held in the gold ring can be almost as thin as desired.

In our application, the need to connect the tip electrically and to unbalance the tuning fork as little as we could (see chapter 2), made us opt for a very thin $10 \mu \mathrm{m}$-diameter wire, and several millimetres long, to ensure electrical continuity from the tip to the big gold pad (see tuning fork setup). Thus, because of its thinness, it is prone to be stuck by capillary forces in the electrolyte film, instead of falling. We thus attach at the bottom end a small copper chip, which smoothly stretches the wire, and helps it falling when the reaction is over.

The wire we use is $\sim 1 \mu \mathrm{m}$ gold coated to prevent the tungsten from oxidizing. The presence of gold does not prevent the reaction to take place.

After etching, tips have to be cleaned in deionised water using ultra sound to remove some chemical reaction by-products. Some of them are not easily removed $[112,115,116]$ so a subsequent step of ion milling mechanical cleaning can be necessary [113]. After having done several experiments using non ion-milled tips, we decided it was preferable to process this second step, to avoid in-situ tip cleaning, which are not really controlled, and result in tip blunting, and/or damage to the sample.

The ion-milling consists in exposing the tips $\sim 30 \mathrm{~min}$ to a $1 \mathrm{keV} \mathrm{Ar}$ ions beam. We have verified, according to [113], that the tip radius further decreases when submitted to ion milling, but we have not experienced double tips forming or other non desirable effects due to crystalline structure of tungsten. However, we are not able to draw ferm conclusions based on our few tests since we had no tool to investigate the crystalline structure. A subsequent gold layer of $10 \mathrm{~nm}$ is deposited in-situ after ion-milling, to protect tungsten from oxidation, and hopefully enhance the tunneling behaviour. 
Parameters of tungsten tip etching processes are gathered in the following figure together with illustration of the results obtained. We quite reproducibly obtain $\sim 10 \mathrm{~nm}$ radius of curvature directly after etching, and an etching length of $\sim 400 \%$ of the original diameter. As already noted, the $10 \mu \mathrm{m}$ diameter wire is more difficult to etch using this setup due to its lightness.
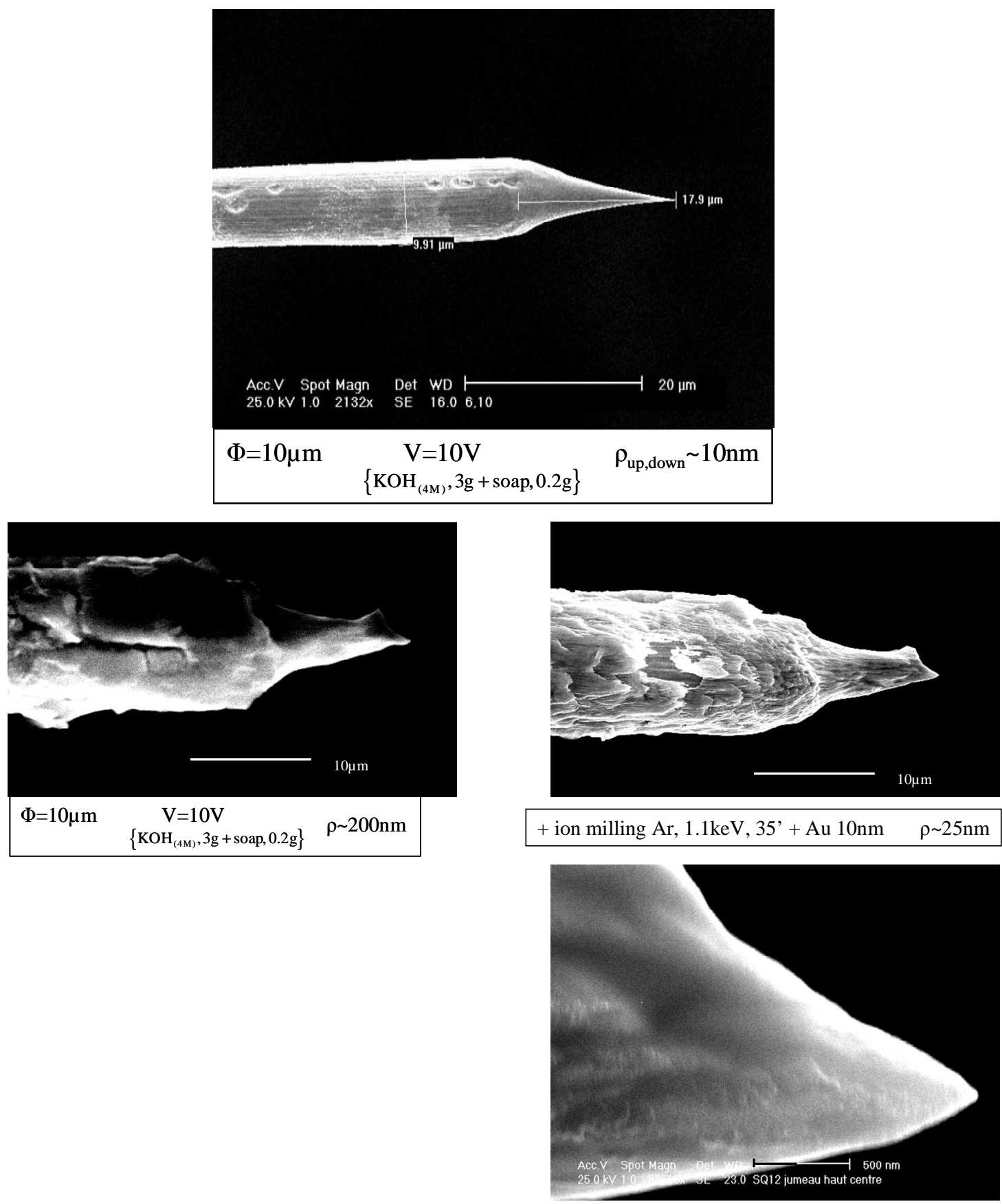


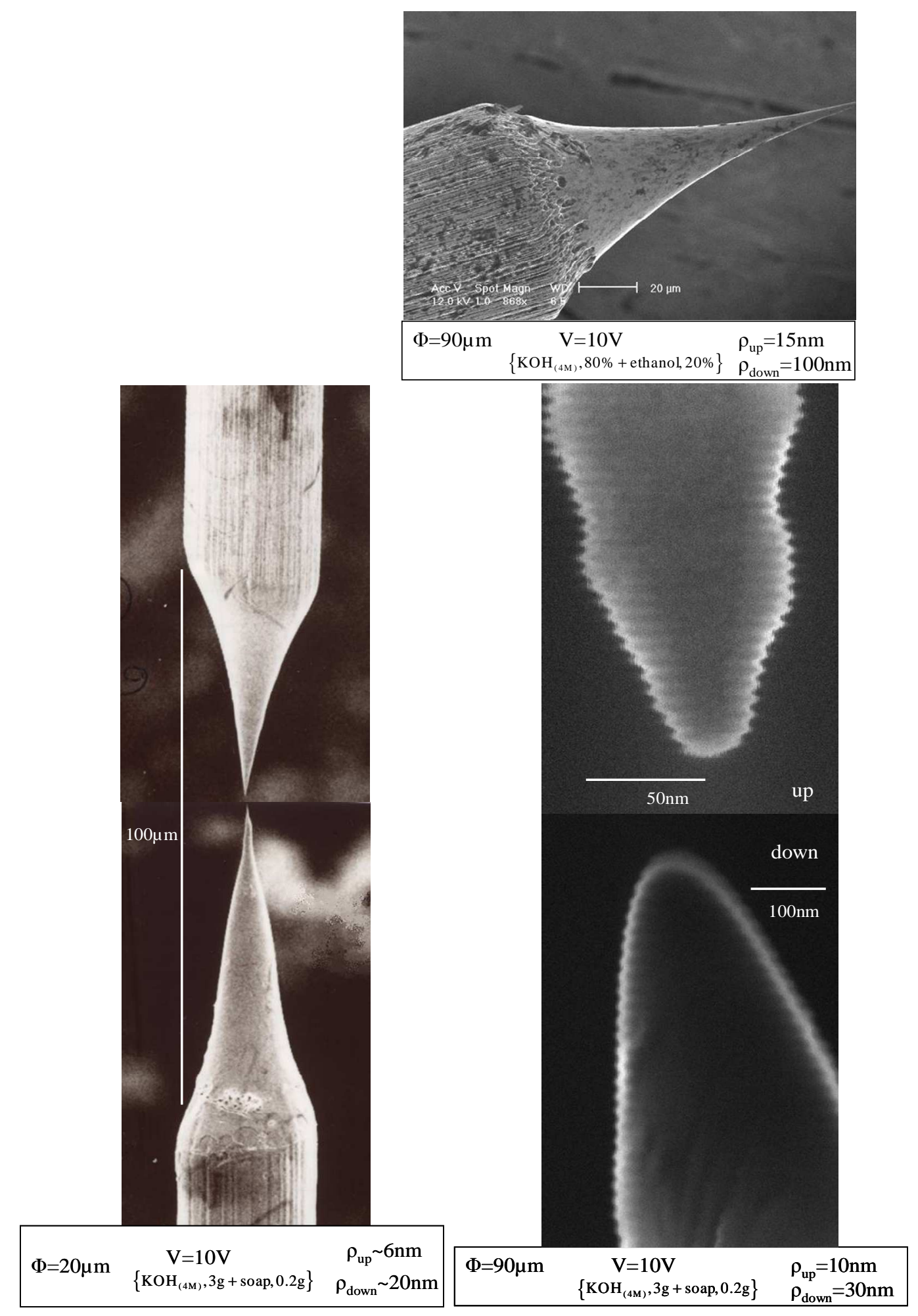

Fig. 3.24 Parameters used in this thesis work for the fabrication of tungsten tips, and the corresponding results photographed in a scanning electron microscope (SEM). The measured curvature radius of the apex is noted $\rho$, with a subscript up for the upper part and a subscript down for lower part of the etched wire. The best tip profiles (see text) were obtained using a $90 \mu \mathrm{m}$ diameter wire. We could reproducibly obtain good tip apex with the $20 \mu \mathrm{m}$ diameter wire, though the profile was less predictable. The $10 \mu \mathrm{m}$ diameter wire gives the worst results, both concerning shape and curvature, because we have to attach some mass to it, and this is not much reproducible from one batch to another. Wigles observed on the greatest magnifications are due to mechanical noise in the SEM, enhanced by the fact that wires are $\sim 1 \mathrm{~mm}$ long. 


\subsubsection{Niobium tips}

We have not used so far superconducting tips for energy distribution function measurements. However we have tried making some either by electrochemical etching or by RIE. Niobium reacts mainly with Fluor based chemicals, so a wet etching would imply Hydrofluorhydric acid (HF), and a dry etching $\mathrm{SF}_{6}$ or $\mathrm{CF}_{4}$. It is also possible to attack niobium using a mix of Nitric, sulphuric and acetic acid.

A major problem with niobium, also encountered in thin films fabrication is the formation of badly conductive oxides. These oxides may change the superconducting gap amplitude, provoke losses in resonators or quasiparticles traps in single electron devices. These oxides are of course also annoying for STM use, and need to be removed before tip use.

\subsubsection{In-situ tip cleaning}

Our experiment is very different from high vacuum setups, in which all devices are fabricated under extreme cleanness conditions, and never exposed to air before being measured.

Indeed, the first time we want to form a tunnel contact, it is necessary to remove the oxide layer formed on the tip, which is too thick to tunnel through. Moreover, some parts of the sample might present thick enough native oxide layers and/or ice, to prevent tunneling as well. We found this in particular on bulk aluminum, where the native oxide is of a few nanometers $(\sim 5-10 \mathrm{~nm})$.

To do in-situ cleaning, we apply a relatively high voltage to the tip $(\sim+5$ to $+10 \mathrm{~V})$, and extend the piezoelectric tube manually (i.e. not in feedback mode) at low speed towards the surface until a current flows. Then we quickly go back to lower bias voltage ( a few $\mathrm{mV}$ ) and try to tune the conductance and get stable contacts. After performing such "cleaning", the conductance is higher than before at the same $\mathrm{Z}$ position, indicating that oxide layers have been removed either from the tip or from the sample.

The mechanism likely to be involved in such cleaning is electron sputtering: a bias voltage of up to $10 \mathrm{~V}$ is applied over distances of the order of $1 \mathrm{~nm}$. The resulting field of $10^{10} \mathrm{~V} / \mathrm{m}$ is therefore intense. Electrons are thus much accelerated and can leave the metal more easily [111]. This may allow some contaminants to be sputtered from the tip, or from the sample, depending on the polarization.

If the sample cleaning is too long, one might remove completely the oxide layer on the sample so that a stable contact is not any longer possible at the same place. 


\subsubsection{Tip damages and tip reshaping}

Depending on the nature of the local oxide layer and of the tip apex, it may be necessary to press the tip down on the sample to obtain the desired tunnel conductance for tunneling spectroscopy.

How hard one needs to press can be estimated by noting the difference in $\mathrm{Z}$ position of the piezoelectric tube between the two modes AFM and STM at the same (X,Y) position. Knowing the amplitude of excitation of the tuning fork (see chapter 2), one can roughly deduce the actual tip position and determine whether it is harshly pushed in the sample or not $^{1}$.

Under such stresses, the tip may bend or deform, becoming blunter. This can in principle be diagnosed by checking that the feedback in AFM mode is the same before and after the contact has been established. Also, after the tip has been laid on the structure to make tunnel contacts it is likely to be contaminated with some material unintentionally collected from the surface (ice, oxide...). After having established a few contacts, we often observe that the AFM imaging resolution is much worse than initially.

Since changing a rounded tip requires a complete thermal cycling for the refrigerator, we have investigated possibilities to re-sharpen the tip, by using the procedure described in [117] (also applied in [118]) and consisting of pulling a small metallic neck out of a metallic electrode on the sample. Using this method, we were able to more or less restore sharp tip apex from badly rounded tips. The procedure is not fully reproducible, for example when the tip is bent, but it has had a good yield until now.

\footnotetext{
${ }^{1}$ This is only an estimation since the position of the tube recorded in the AFM mode is not the average position of the tip, but depends on the tip-sample potential shape, and on the amplitude of oscillation of the tuning fork (see Chap. 2). Therefore, one cannot simply subtract the fork amplitude to deduce the rest position.
} 
In practice, the following reshaping procedure was done with the tungsten tip over a silver electrode:

- The tube is extended roughly a few nanometers above the surface (found from the force mode feedback position), and the feedback is turned off.

- The feedback gains are set to very low values, so that the maximum tip velocity obtained is around $\sim 0.5 \mathrm{~nm} / \mathrm{s}$.

- Current setpoint is set to $80 \mathrm{nA}$, which is the maximum signal one can measure with the cold feedback resistor. The setup of the amplifier allows us to plug a different resistance at room temperature. By switching to lower resistance, one could reach higher currents, but we found the actual current flow convenient.

- Tip voltage bias is set to $\sim-10 \mathrm{~V}$.

- Feedback is turned ON. The tube extends towards the sample until it reaches the setpoint value, and the feedback then regulates current.

- Then voltage bias is set to lower absolute values $\sim-4 \mathrm{~V}$. The tube further extends towards the sample.

- Some back and forth movements of low amplitude $(\sim 2 \mathrm{~nm})$ are done slowly with the tip to enhance sticking of the metal onto the tip.

- Current setpoint is now reduced to $\sim 2 \mathrm{nA}$, so the tube retracts, very slowly. Since the feedback is slow, the actual current remains at the maximum value ( $~ 80 \mathrm{nA})$ during a few nanometers while the tip is rising, indicating that the tip-to sample contact is submitted to deformation (preferably plastic). Current now diminishes by steps, corresponding to new configurations in the tip shape.

- The current setpoint has to be increased once more, to repeat the procedure.

One stops the procedure when the current remains high while retracting over large distances, approximately equal to the thickness of the metallic layer: this is roughly the best one can hope to do. Of course this depends on mechanical properties of the material in which one dips the tip: a ductile, low melting temperature material is preferable. Fig. 3.25 shows the mechanism of tip reshaping.

Capillary forces exerted by plunging the tip into the electrode may not alone be responsible for tip sharpening: the same typical applied voltages have been reported elsewhere [119] to 
provoke tip sharpening while keeping the tip away from the surface. Atomic migration under a high electric field is likely to be involved in such procedure. It may also be part of the process of in-situ tip cleaning described above, which amounts to reorganizing the tip apex.

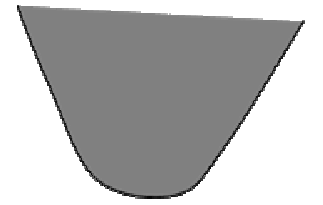

(a)
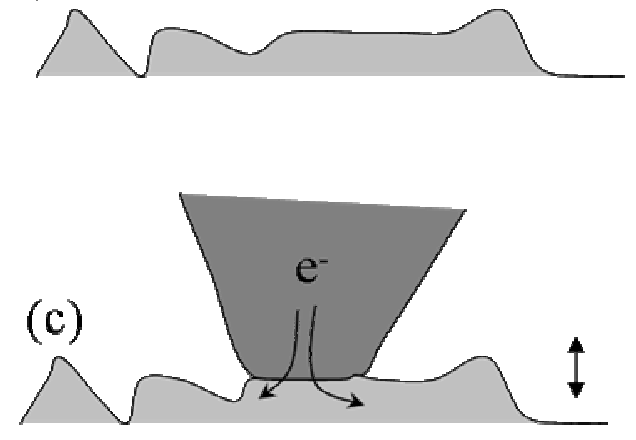

(e)

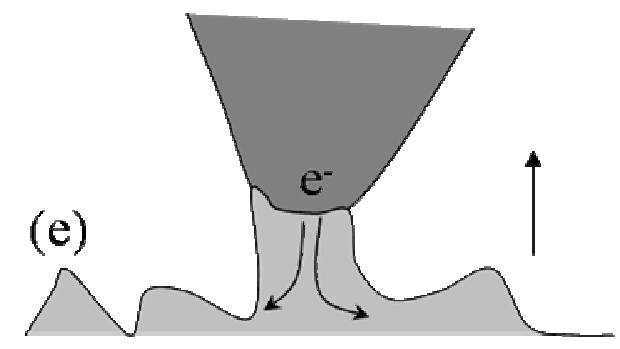

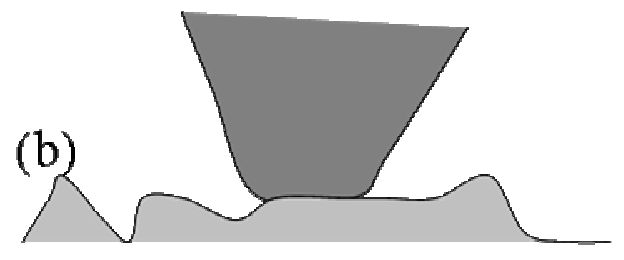
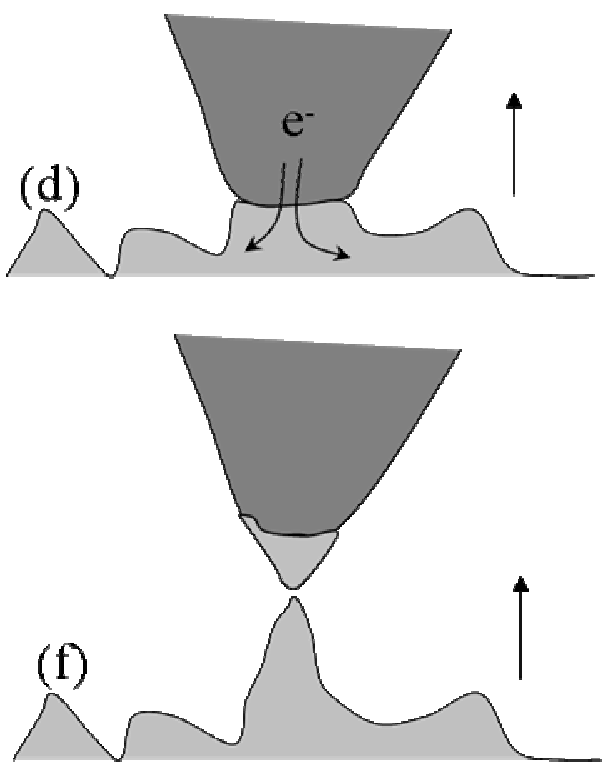

Fig. 3.25 When one plunges the tip in a ductile material, adhesive forces may overcome elastic deformation of the material, so that it can stick to the tip. If the two materials can melt, sticking can be enhanced by flowing a current meanwhile dipping the tip. When the tip is retracted, a neck forms. This neck should ideally form as late as possible during the retraction. The neck goes thinner as the tip moves back and finally breaks leaving a fine tip apex.

Finally, the type of metal used to reform the tip has to be compatible with the spectroscopic measurement carried out. That is to say if the tip is normal, one should not use superconducting metal...

In Fig. 3.26 we show an example of how the image quality can be greatly enhanced after performing such a reshaping procedure. The same region of a sample is shown before and after a tip was reformed. The maximum height of the sample is $\sim 60 \mathrm{~nm}$, and the tip has been reshaped in a $30 \mathrm{~nm}$ silver thin film.

The edge of the $60 \mathrm{~nm}$ features are well distinguished, indicating the procedure was successful over expectancies: indeed, this implies the tip had been refined over a length at 
least equal to $60 \mathrm{~nm}$. On other trials to reshape the tip, the features walls were not as distinct as on Fig. 3.26, though small features could be distinguished on the flat parts.

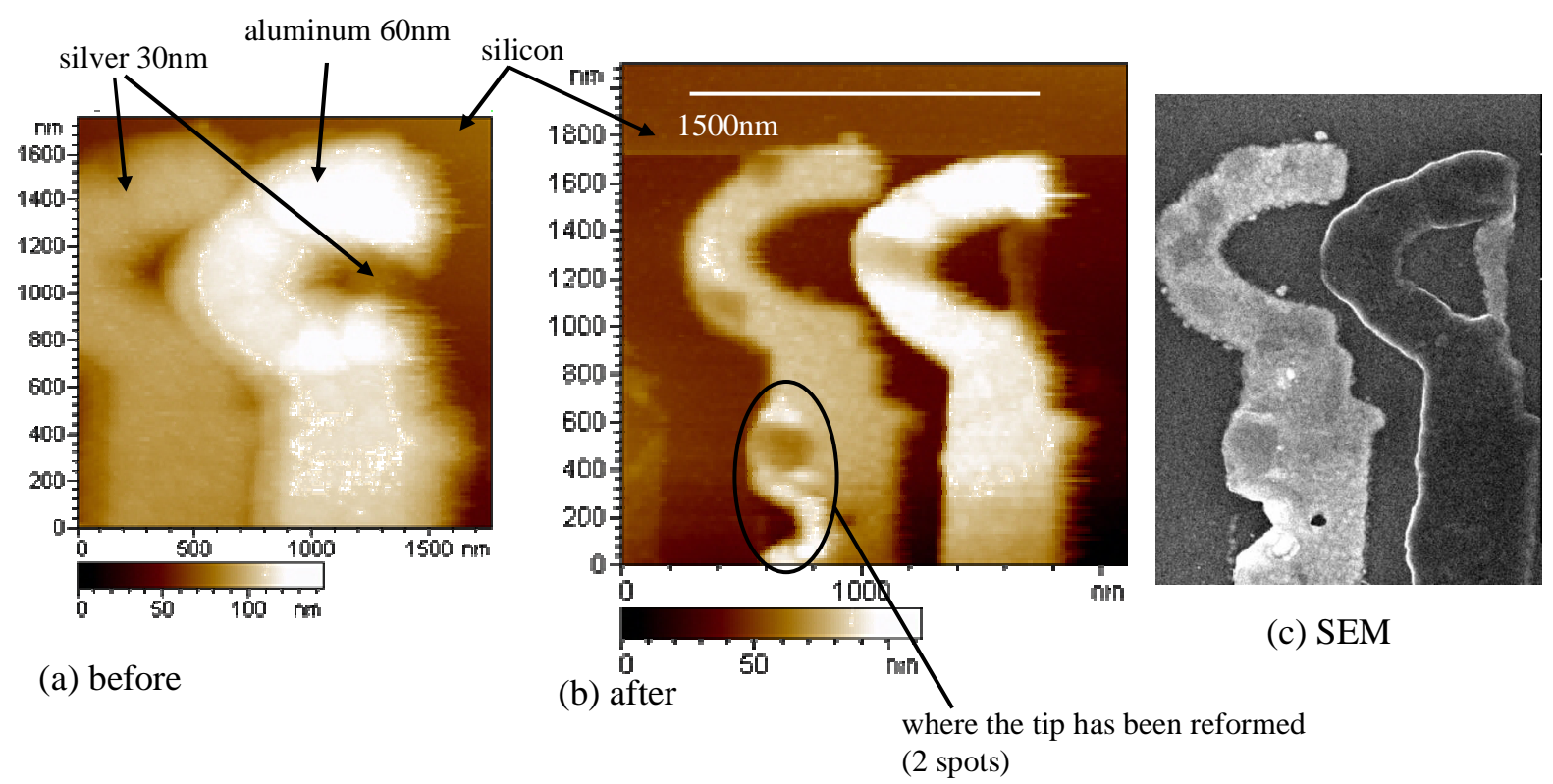

Fig. 3.26 (a), (b), Two images taken with the microscope in AFM mode at $40 \mathrm{mK}$, on approximately the same region of the sample, comprising a silver layer of $30 \mathrm{~nm}$, and one of aluminium of $60 \mathrm{~nm}$ as indicated on the images. The left image (a) is taken after the tip had been damaged by laying on the sample to make tunnel contacts through the oxide layer (see 3.1.2, p105). The right one (b) is taken just after having reshaped the tip in the silver film located on the left. (c) Scanning Electron Microscope image taken after the experiment was finished.

\subsubsection{Sample preparation}

Samples were prepared using standard lithography techniques: e-beam or UV patterning of sensitive resists, dry or wet etching, joule or electron gun evaporation for the deposition of thin films... I describe here the different processes used during this work.

\subsubsection{Position encoding grid}

As briefly mentioned in the introduction chapter, our samples were designed so that it is possible to find easily a particular nanostructure of a few microns wide in the middle of a sample of a few millimetres. Since our imaging range with the piezoelectric tube at low temperatures does not exceed $8 \mu \mathrm{m}$, this would require way too much efforts in trials-errors, if no means of locating the tip with respect to the sample were included.

The microscope possesses its own relative position meter, owing to the capacitive measurement (see chapter 2), but it does not give access to the absolute position over the sample. 
This drawback has several origins:

- The sample is glued by hand on the sample holder, hence not reproducibly,

- The tip does not always have the same length, neither does the sample have the same thickness,

- Finally, stray capacitances are sensitive to the detailed arrangement of the cables, humidity levels, etc, which change from one run to another.

All these typically change the capacitance measurement by $\sim 0.1 \mathrm{pF}$ from one run to the other, which corresponds to a fictitious displacement of $100 \mu \mathrm{m}$.

One then has to tackle the problem differently, and encode directly on the sample a coordinate system relative to e.g. the center, which can be seen as a local map of the sample.

Since the full range of our scanner is $8 \mu \mathrm{m}$, we pattern a coordinate code every $16 \mu \mathrm{m}$, such that wherever the scanner lands on the sample surface, the first image contains at least a part of a code. After having deciphered the position, using the motors combined to capacitance measurements, the desired nanostructure can be reached in only a couple of images and displacements. The whole procedure of finding a nanostructure thus takes generally less than $1 / 2$ hour.

The binary code is presented in Fig. 3.27. Each axis $\mathrm{X}$ and $\mathrm{Y}$ is encoded on 8 bits spread over two lines. The first two lines encode the $\mathrm{X}$ position, the last two the $\mathrm{Y}$ position. The first line left bit of each axis is the sign bit, and the other 7 bits stand for powers of 2 . The full code tells the position from the center in the form of a multiple of $16 \mu \mathrm{m}$.

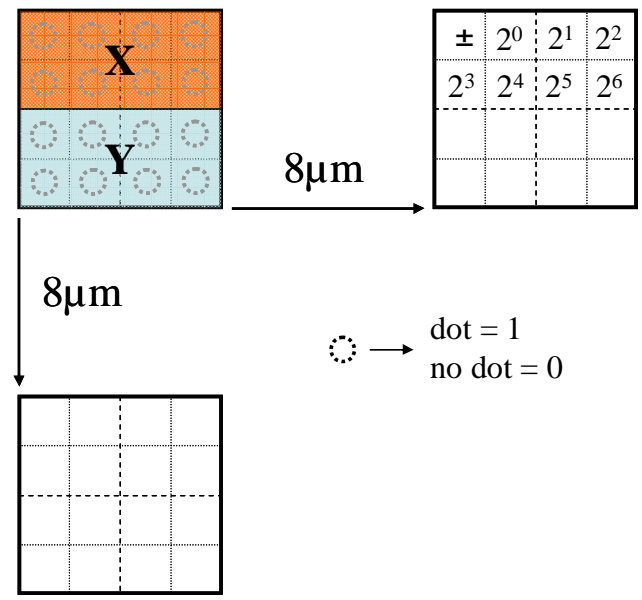

Fig. 3.27 Binary code patterned all over the surface of our samples to ease the location of the interesting structure. This is the local map of the sample. 
It is encoded as bumps or dips in the sample surface, much like the Braille alphabet for blinds. This technique of patterning the sample with recognizable shapes is standard in near-field microscopy (as opposition to far field, where the area of interest can be accessed at a glance). The true innovation in the form presented here, is that information is everywhere so that it is impossible to miss it, and it is unique so that there is no ambiguity. This lightens very much the efforts to reach the interesting point, as compared to other techniques, and it is not too difficult to design. Note however that the design of the encoding grid must not disturb the fabrication and the functioning of the structures to be studied. For this reason, we leave intact a square of $100 \mu \mathrm{m}$ side at the center of the sample on which we fabricate the finest patterns. Within this area, there are usually enough recognizable objects with small designs (connecting pads etc...) so that if the tip lands there, it is not difficult to locate.

Fabrication of the encoding grids was done usually by optical lithography, prior to fabrication of structures on the sample. This way, it can be wafer processed, using photolithography masks.

- One starts from a standard $\mathrm{Si} / \mathrm{SiO}_{2}$ substrate, on which one spins Shipley® S1805 photoresist at $4000 \mathrm{rpm}$ during 45". The resist is then dried 1'30 at $110^{\circ} \mathrm{C}$, and cooled down at ambient temperature. The thickness yield of the film is about $500 \mathrm{~nm}$.

Resist should not be thicker than the minimal pattern size which is $1 \mu \mathrm{m}$, because of light diffraction by the edges of the mask. A $1 \mu \mathrm{m}$ resolution is commonly achievable with most photosensitive resists and UV mask aligners, but requires some care: in particular the contact between the mask and the wafer has to be very tight.

- The resist is exposed at $100 \mathrm{~mJ} / \mathrm{cm}^{2}$ using a MJB3 UV Aligner from Karl Süss $(\lambda=365 \mathrm{~nm})$, with hard contact using a vacuum chuck. The exposure dose is $\sim 10 \%$ lower than recommended, to avoid enlarging of the patterns.

- The resist is developed 35" in MF319 Shipley® developer, ODI rinsed and dried.

The substrate is subsequently engraved with reactive ion etching using fluor based chemicals adequate for $\mathrm{Si}$.

- We used a flow of SF6 at $28 \mathrm{cc} / \mathrm{min}$, in a chamber pressure of $10 \mu \mathrm{bar}$. RF power is set to $50 \mathrm{~W}$, which results in ions being accelerated at $170 \mathrm{~V}$. These parameters yield an anisotropic etching, at a rate of $\sim 1 \mathrm{~nm} / \mathrm{s}$. 
We generally etched $\sim 70 \mathrm{~nm}$ down in the substrate to provide clear contrast on AFM images.

Fig. 3.28 shows the aspect of samples with an encoding grid.

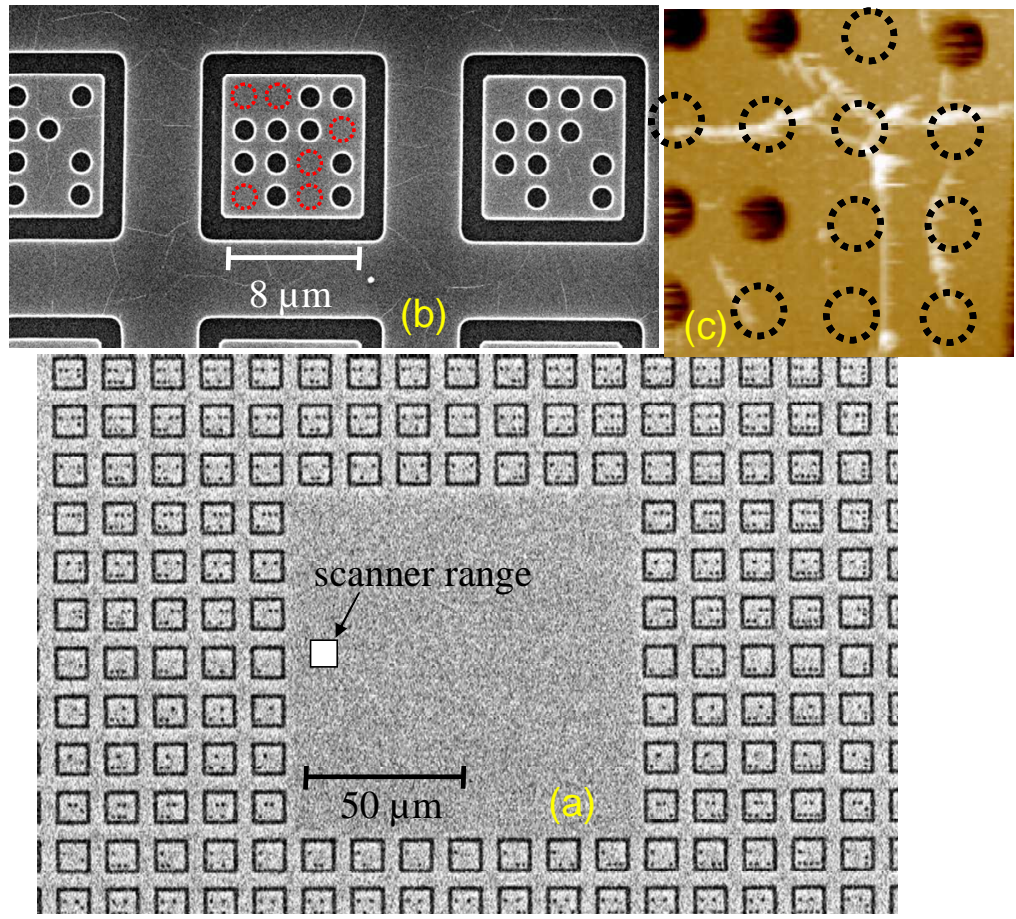

Fig. 3.28 (a) large range SEM image of the sample once the grid is engraved. The center is bare to let some room for fine structures. Large pads can be deposited without problem over the grid: one can still see the code, and the pads are continuous. (b) fine SEM zoom on three consecutive patterns. The dots represent a one, and the absence of dot (marked by red dashed circles) stands for a zero. (c) AFM image taken just after the landing; the position with respect to the center is known immediately from the code: $(1+4) \times 16 \mu \mathrm{m}$ to the left $\left(1^{\text {st }}\right.$ bit on) in $\mathrm{X}$ and $(1+8) \times 16 \mu \mathrm{m}$ to the bottom ( $1^{\text {st }}$ bit on $)$ in $\mathrm{Y}$.

With this engraving recipe, the codes appear as dips in the topography of the sample.

It is also possible to evaporate material through the resist mask, which would result in bumps on the topography. This technique is applied for instance when the grid cannot be patterned before the fabrication of structures.

\subsubsection{Multiple angle evaporation}

Metallic circuits we measure generally consist in multiple metallic layers of various shapes, which are deposited one after another. These layers can be deposited by metal evaporation through a mask of resist, or they can be etched out of uniform layers.

Whenever possible, it is convenient to evaporate all the different metallic layers under the same vacuum, using a unique resist mask.

This is true for several reasons: first, when small structures are patterned, it is technically challenging, and time-consuming to carry out several lithography steps aligned on each other. 
Second, when one needs to control the contact transparency between two layers, it is better to evaporate them in a controlled environment, without breaking the vacuum. It is possible though to circumvent the latter problem by machining the surface of an air (water) contaminated sample using argon ion beam milling (see 3.3.1.2).

Suspended masks are used to evaporate multiple metallic layers of different shapes without having to break the vacuum, as depicted on Fig. 3.29. They are formed with a bilayer of resist, either photosensitive or electro-sensitive, depending on the size of patterns required. The top layer has less solubility in the developer than the bottom layer so that one can form an undercut in the mask. The top layer should also be stiff enough so that it does not collapse when the bottom is evacuated.

Finally, the height of the bilayer and the angle under which one evaporates define the displacement between the patterns.

For electron beam lithography, we use a bilayer fabricated in the following way:

- spin of MMA 8.5 MAA EL10 from Micro Chem at $1500 \mathrm{rpm}$ 60",

- bake on the hot plate for 2' at $170^{\circ} \mathrm{C}$,

- wait for thermalization at ambient temperature,

- go through the procedure from step 1 to 3 once again, to increase, if needed, the thickness of MMA (caution: one has to start the spin as soon as the resist is dispensed on the wafer to avoid re-dissolution of the first layer)

- $\quad$ spin PMMA A3 from Microchem® at 5000 rpm for 60"

- bake $20^{\prime}$ at $170^{\circ} \mathrm{C}$

These yield a bilayer thickness of $\sim 1 \mu \mathrm{m}$ MMA, and $70 \mathrm{~nm}$ PMMA on top. MMA is more sensitive than PMMA to electron exposure so it is possible to expose the MMA with low doses without breaking the PMMA layer. This is basically how one can do undercut, and overhang masks as shown in Fig. 3.29.

For optical lithography, we use LOR30B from Micro Chem as the under layer, and S1800 from Shipley® as the top layer. LOR30B is not an optically sensitive resist, it can be made very thick and it does not disolve into S18 developer. All this makes fabrication less tricky than using MMA/PMMA bilayers. 

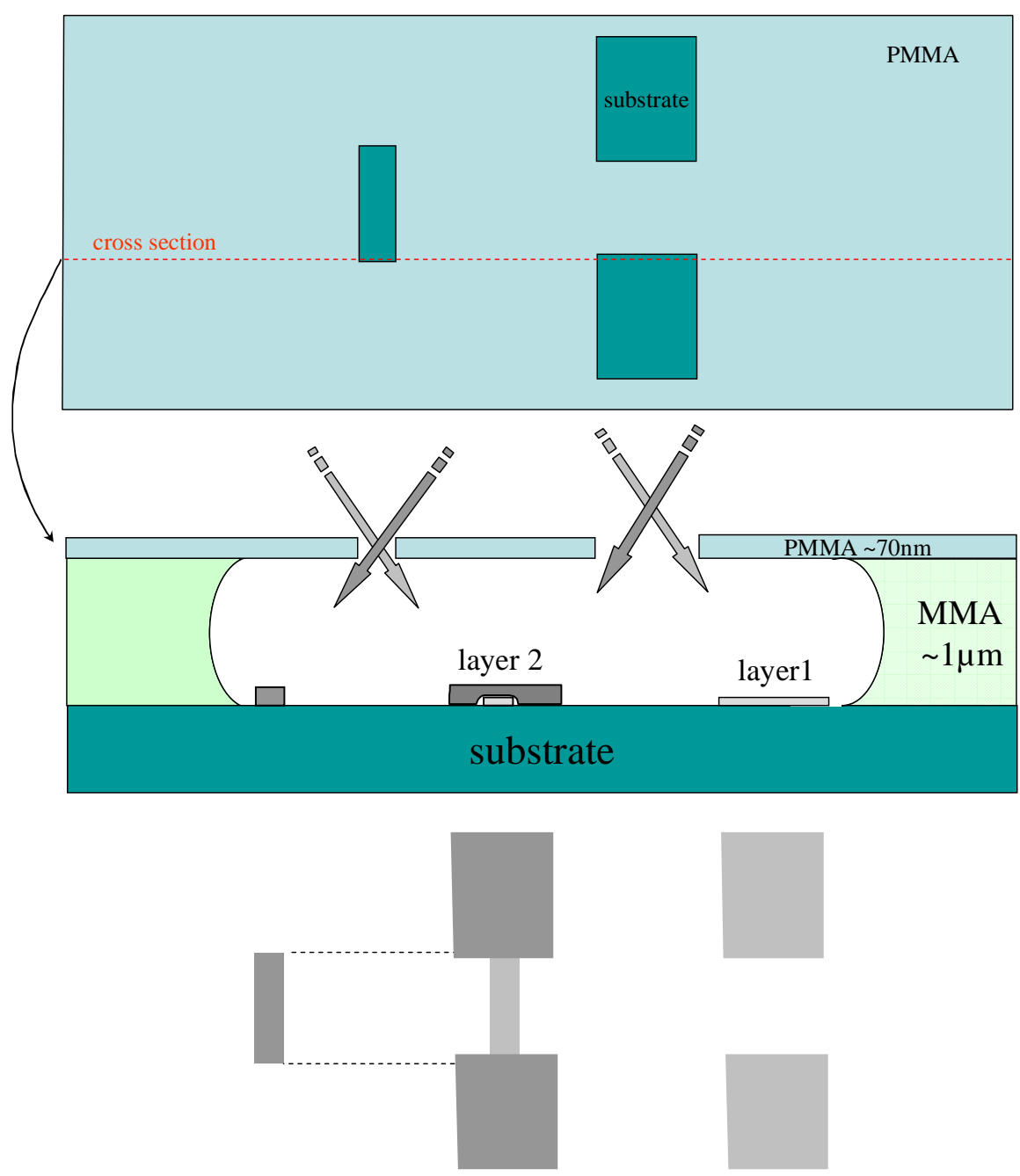

Fig. 3.29 The suspended mask is formed by a a first thick layer of resist, and a thin top layer of another resist that is less soluble or less sensitive. One can form circuits with two different metallic layers as pictured on the scheme, by simply evaporating materials through the same mask under different angles. 
Chapter 4

$\underline{\text { MESOSCOPIC }}$

SUPERCONDUCTIVITY 


\subsection{Introduction to proximity effect}

The superconducting order parameter is a subtle quantity whose space and time variations present a great richness of behaviours. It describes the pairing correlations between electrons with opposite spins occupying time-reversed orbital states. The pairing potential that drives the superconducting order only occurs in superconducting materials, and originates in BCS superconductors from the electron-phonon coupling [14].

The interest of mesoscopic superconductivity is to combine materials with different properties, and to probe the superconductivity on all the relevant scales.

Proximity superconductivity occurs when a non-superconducting $(\mathrm{N})$ material is placed in contact with a superconducting $(\mathrm{S})$ one. Pairing correlations leak from the $\mathrm{S}$ side into the $\mathrm{N}$, and the order parameter of the $S$ is reduced close to the interface. Some superconducting properties are transferred onto the $\mathrm{N}$ side: the electric field is excluded close to the interface, diamagnetism occurs [120], the density of states is modified and may sometimes acquire a true gap [121, 122], and a supercurrent may flow across an SNS structure [123]. However, the resistance of an $\mathrm{N}$ wire connected on one end to an $\mathrm{S}$ electrode is equal to its normal state value at zero temperature, despite a reduction at intermediate temperatures [124]. Proximity superconductivity is thus not simply a reduced superconductivity, and its investigation, started in the years 1960s, is not yet completed.

A key element in our understanding of proximity effect is the Andreev reflection process [125]: when an electron incoming from the N side impinges an NS interface with an energy smaller than the superconducting gap energy, it is not simply transmitted to the $\mathrm{S}$ side, but is retro-reflected as a hole, with an extra pair transferred to the superconductor. We will exploit this very physical point of view whenever possible.

An SNS structure in which an $\mathrm{N}$ wire is connected at both ends to $\mathrm{S}$ electrodes is a model structure for probing proximity superconductivity. Up to now, most experiments measured only the maximum supercurrent in such structures, and the theoretical predictions have not been fully probed. Our aim was to exploit the space and energy resolution of our AFM/STM machine to investigate proximity superconductivity through the local DOS in a model SNS structure in which the phase difference is controlled externally. 


\subsection{Theoretical description of Proximity Effect}

\subsubsection{Inhomogeneous superconductivity}

The investigation of proximity superconductivity was initiated by the Russian school around Bogolubov [126- 128], and by the Orsay group led by de Gennes [129]. From the theoretical point of view, proximity superconductivity is part of the wider field of inhomogeneous superconductivity. In metallic structures containing superconducting materials, the quantum states of quasiparticles obey the celebrated Bogolubov-de Gennes equations [130]. A solution for the treatment of inhomogeneous superconductivity was derived by Gor'kov [131] using quantum field theory methods for calculating electronic Green functions. This formalism was further simplified by Eilenberger [132] using the disorder averaged Green function technique. In the case of diffusive conductors in which the electronic mean free path is short, Usadel [133] derived a set of equations sufficiently simple to be handled. Other important situations have also been addressed. In particular, the transport properties of fully coherent proximity effect structures were calculated by Beenaker [134] in terms of their scattering matrix, which allowed to make predictions using the Random Matrix Theory (RMT).

\subsubsection{The Bogolubov - de Gennes equations}

The formation of pairs is described in the BCS theory by the exchange of virtual phonons [14], but it can be equivalently formulated as an interaction between time-reversed conjugates, without specifying the nature of the coupling. In this point of view, the electron and hole quasiparticles of the normal metal are coupled by the pairing Hamiltonian. Thus, the eigenstates of the system no longer correspond to electron or hole-like quasiparticles, but to a superposition of them. The two corresponding amplitudes $u(\boldsymbol{r})$ and $v(\boldsymbol{r})$ obey the Bogolubov-de Gennes equations [130]:

$$
\left(\begin{array}{cc}
H_{e} & \Delta \\
\Delta^{*} & -H_{e}
\end{array}\right)\left(\begin{array}{l}
u(\boldsymbol{r}) \\
v(\boldsymbol{r})
\end{array}\right)=\varepsilon\left(\begin{array}{l}
u(\boldsymbol{r}) \\
v(\boldsymbol{r})
\end{array}\right)
$$

where $H_{e}$ is the usual electronic Hamiltonian containing the kinetic and potential terms, and $\Delta(\boldsymbol{r})$ is the pair potential, to be determined self-consistently. A way to solve a system is to determine all eigenstates of Eq. (4.1), and to fill the states up to the Fermi level. In most circumstances, this strategy is however too difficult to handle. 


\subsubsection{Theoretical description of the proximity effect in diffusive systems at equilibrium}

The Green function formalism does not require to determine the solutions of the Bogolubovde Gennes equations. Here, the formalism is outlined, and the main results pertaining to diffusive conductors are presented.

Symbols employed in this part follow the following rules: $U$ is a scalar, $\boldsymbol{U}$ is a vector, $\hat{U}$ is a $2 \times 2$ matrix, $\langle\ldots\rangle_{n}$ is an average, $[\ldots, \ldots]$ a commutator, and $\{. . . .$.$\} an anti-commutator.$

\subsubsection{Electronic Green functions}

Different types of electronic Green functions are defined. The retarded one write:

$$
G^{R}\left(\boldsymbol{r}, t \mid \boldsymbol{r}^{\prime}, t^{\prime}\right)=-i \theta\left(t-t^{\prime}\right)\left\langle\psi_{H}\left|\left\{\psi_{\sigma}(\boldsymbol{r}, t), \psi_{\sigma}^{+}\left(\boldsymbol{r}^{\prime}, t^{\prime}\right)\right\}\right| \psi_{H}\right\rangle
$$

where $\left|\psi_{H}\right\rangle$ is the ground state stationary wave function of the system, $\theta(t)$ the Heaviside function, and $\psi_{\sigma}(\boldsymbol{r})\left(\right.$ resp. $\left.\psi_{\sigma}^{+}(\boldsymbol{r})\right)$ is the annihilation (resp. creation) operator of a fermionic quasiparticle with spin $\sigma$ at position $\boldsymbol{r}$. The advanced Green function writes:

$$
G^{A}\left(\boldsymbol{r}, t \mid \boldsymbol{r}^{\prime}, t^{\prime}\right)=i \theta\left(t^{\prime}-t\right)\left\langle\psi_{H}\left|\left\{\psi_{\sigma}(\boldsymbol{r}, t), \psi_{\sigma}^{+}\left(\boldsymbol{r}^{\prime}, t^{\prime}\right)\right\}\right| \psi_{H}\right\rangle
$$

The retarded Green function contains the full information on equilibrium properties.

\subsubsection{Green functions in the Nambu space}

Due to electron-hole coupling, superconductors are described by a richer set of field correlators, represented by a $2 \times 2$ matrix in the so-called Nambu space:

$$
\hat{M}\left(\boldsymbol{r}, t \mid \boldsymbol{r}^{\prime}, t^{\prime}\right)=\left\langle\left(\begin{array}{cc}
\left\{\psi_{\uparrow}(\boldsymbol{r}, t), \psi_{\uparrow}^{+}\left(\boldsymbol{r}^{\prime}, t^{\prime}\right)\right\} & \left\{\psi_{\uparrow}(\boldsymbol{r}, t), \psi_{\downarrow}\left(\boldsymbol{r}^{\prime}, t^{\prime}\right)\right\} \\
-\left\{\psi_{\downarrow}^{+}(\boldsymbol{r}, t), \psi_{\uparrow}^{+}\left(\boldsymbol{r}^{\prime}, t^{\prime}\right)\right\} & -\left\{\psi_{\downarrow}^{+}(\boldsymbol{r}, t), \psi_{\downarrow}\left(\boldsymbol{r}^{\prime}, t^{\prime}\right)\right\}
\end{array}\right)\right\rangle_{0},
$$

where diagonal elements $\left\langle\left\{\psi_{\sigma}, \psi_{\sigma}^{+}\right\}\right\rangle_{0}$ represent the conventional occupation numbers or "normal" correlations, while anti-diagonal elements measure the so-called "anomalous" pair correlations, $\left\langle\left\{\psi_{\downarrow}, \psi_{\uparrow}\right\}\right\rangle_{0}$ or the amplitude for creating or annihilating a pair from the system, \langle\rangle$_{0}$ being a statistical average over the state of the system. Since in the following we only discuss equilibrium situations, we limit the discussion to the retarded Green function:

$$
\hat{G}^{R}\left(\boldsymbol{r}, t \mid \boldsymbol{r}^{\prime}, t^{\prime}\right)=-i \theta\left(t-t^{\prime}\right) \hat{M}\left(\boldsymbol{r}, t \mid \boldsymbol{r}^{\prime}, t^{\prime}\right)
$$




\subsubsection{Quasiclassical Green functions in the dirty limit - Usadel equation}

The above Green functions obey the microscopic Gor'kov equations [131]. They oscillate with the difference $\boldsymbol{r}-\boldsymbol{r}^{\prime}$ at the scale of the Fermi wavelength, which is much shorter than characteristics lengthscales occurring in superconducting devices, such as $\hbar v_{F} / \Delta$, the coherence length in a clean superconductor. By integrating over the relative coordinate one obtains the quasiclassical Green functions, which follow the Eilenberger equations [132, 135]. Usadel [133] showed that a further simplification can be carried out when considering diffusive conductors, in which case one needs only considering isotropic Green functions of the type

$$
\hat{G}_{D i s .}^{R}\left(\boldsymbol{r}, t-t^{\prime}\right)=-i \theta\left(t-t^{\prime}\right)\left\langle\hat{M}\left(\boldsymbol{r}, t \mid \boldsymbol{r}, t^{\prime}\right)\right\rangle_{D i s .}
$$

where \langle\rangle$_{\text {Dis. }}$ denotes disorder and statistical averaging over the state of the system. By taking the Fourier transform $\mathcal{F}$ over the time coordinate one obtains the position-energy retarded Green function in the diffusive case ${ }^{1}$

$$
\hat{R}(\boldsymbol{r}, E)=\mathcal{F}\left(\hat{G}_{D i s .}^{R}\left(\boldsymbol{r}, t-t^{\prime}\right)\right)
$$

$\hat{R}$ obeys the Usadel equation :

$$
\hbar D \hat{\nabla}_{r}\left(\hat{R} \hat{\nabla}_{r} \hat{R}\right)=\left[-i \hat{H}_{0}+\hbar \Gamma_{s f} \hat{\sigma}_{z} \hat{R} \hat{\sigma}_{z}, \hat{R}\right]
$$

where $D$ is the diffusion constant of the material, $\Gamma_{s f}$ is the spin-flip scattering rate and

$$
\hat{H}_{0}=\left(\begin{array}{cc}
E & i \Delta(\boldsymbol{r}) \\
i \Delta^{*}(\boldsymbol{r}) & -E
\end{array}\right),
$$

where $E$ is the energy referenced to the Fermi level $\left(E_{\mathrm{F}}=0\right)$ and $\Delta(\boldsymbol{r})$ is the mean-field effective pair potential of the BCS theory, which a priori depends on space in a proximity system. We discuss it further below.

\subsubsection{Properties of the Green function elements - Physical quantities}

The $\hat{R}$ matrix can be written as

\footnotetext{
${ }^{1}$ The advanced Green function can be expressed as $\hat{A}=-\hat{\sigma}_{z} \hat{R}^{\dagger} \hat{\sigma}_{z}$, with $\hat{\sigma}_{z}$ the third Pauli matrix.
} 


$$
\hat{R}(\boldsymbol{r}, E)=\left(\begin{array}{cc}
G & F \\
F^{\dagger} & -G
\end{array}\right),
$$

where $F$, the anomalous Green function, and $G$, the normal Green function, are complexvalued functions of space and energy, and $\dagger$ denotes time-reversal. The Green function is traceless $(\operatorname{Tr} \hat{R}=0)$ and normalized $\left(\hat{R}^{2}=\hat{I}\right)$, so that

$$
G^{2}+F F^{\dagger}=1 \text {. }
$$

Expressed in terms of $F$ and $G$, the Usadel equation becomes:

$$
\begin{aligned}
& \hbar D\left(G \nabla_{r}^{2} F-F \nabla_{r}^{2} G\right)=-2 i E F-2 \Delta G+4 \hbar \Gamma_{s f} G F \\
& F\left(\hbar D \nabla_{r}^{2} F^{\dagger}+2 \Delta^{*}\right)=F^{\dagger}\left(\hbar D \nabla_{r}^{2} F+2 \Delta\right)
\end{aligned}
$$

All the equilibrium physical quantities can be obtained from these Green functions. For instance,

- The quasiparticle density of states is given by

$$
\rho(\boldsymbol{r}, \varepsilon)=\rho_{N} \operatorname{Re} G(\boldsymbol{r}, \varepsilon),
$$

where $\rho_{N}$ is the normal state density at the Fermi level.

- The supercurrent writes

$$
\mathbf{J}=\frac{\sigma_{N}}{2 e} \int_{0}^{\hbar w_{D}} \mathrm{~d} E \tanh \left(\frac{E}{2 k_{B} T}\right) \operatorname{Re}\left(F \nabla F^{\dagger}-F^{\dagger} \nabla F\right)
$$

where $\sigma_{N}=\rho_{N} e^{2} D$ is the normal state conductivity of the electrode

- The superconducting pair potential (also known as order parameter) is

$$
\Delta(\boldsymbol{r})=\frac{\rho_{N} V(\boldsymbol{r})}{2 i} \int_{0}^{\hbar \omega_{D}} \mathrm{~d} E \tanh \left(\frac{E}{2 k_{B} T}\right)\left(F(\boldsymbol{r}, E)-\left(F^{\dagger}(\boldsymbol{r}, E)\right)^{*}\right)
$$

where $V(\boldsymbol{r})$ is the phonon-mediated interaction potential, which is zero in normal metals and finite in superconductors [136].

The last equation (4.6) explains why solving the Usadel equations in proximity effect problems is generally hard. Indeed, solving (4.4) for $F$ and $G$ is "only" a non-local problem in space which can be treated energy by energy. Equation (4.6) however transforms the problem into a much more complicated energy-integral problem, since the solutions for $F$ and $G$ at a given energy then also depend on the solutions at all other energies! 
One can express the pair potential as the product

$$
\Delta(\boldsymbol{r})=\rho_{N} V(\boldsymbol{r}) \mathcal{P}(\boldsymbol{r})
$$

of space-dependent material properties with the pair amplitude function $\mathcal{P}(\boldsymbol{r})$. This distinction between the pair potential and the pair amplitude is important: in NS heterostructures for instance, the discontinuity of $V(\boldsymbol{r})$ at interfaces will show up in $\Delta(\boldsymbol{r})$, resulting in $\Delta(\boldsymbol{r})=0$ in normal metal regions, whereas $\mathcal{P}(\boldsymbol{r})$ is generally non-zero even in the normal metal regions and varies smoothly across clean interfaces (for an illustration see below, Fig. 4.10). The pair amplitude function can itself be expressed as the integral of an energy-resolved pair amplitude function $p(E, \boldsymbol{r})$ :

$$
\mathcal{P}(\boldsymbol{r})=\int_{0}^{\hbar \omega_{D}} \mathrm{~d} E p(E, \boldsymbol{r}),
$$

where

$$
p(E, \boldsymbol{r})=\tanh \left(\frac{E}{2 k_{B} T}\right)\left(F(\boldsymbol{r}, E)-\left(F^{\dagger}(\boldsymbol{r}, E)\right)^{*}\right) .
$$

\subsubsection{Boundary conditions}

Deep inside reservoir electrodes at equilibrium, in the absence of supercurrent flow, the gradient terms in (4.4) vanishes and the Green functions assume their bulk values. In a normal metal $\hat{R}=\hat{\sigma}_{z}$. In a superconducting electrode

$$
\hat{R}_{S}=\frac{\operatorname{Sign}(E+|\Delta|)}{\sqrt{E^{2}-|\Delta|^{2}}}\left(\begin{array}{cc}
E & i \Delta \\
i \Delta^{*} & -E
\end{array}\right) .
$$

At an interface between two metals, one needs in general to take into account scattering due to an imperfect interface. The (dis)continuity of the Green functions in this case has been worked out in Refs.[137-140]. Introducing the spectral current density

$$
\hat{j}(\boldsymbol{r}, E)=\sigma_{N} \hat{R} \nabla \hat{R},
$$

the Green functions (dis)continuity relations can be expressed as a spectral current conservation equation across the interface

$$
\int_{\mathrm{S}} \mathrm{d} \boldsymbol{S}_{12} \cdot \hat{\vec{j}}_{1}=\hat{I}_{12}=-\int_{\mathrm{S}} \mathrm{d} \boldsymbol{S}_{21} \cdot \hat{\boldsymbol{j}}_{2}
$$

where the subscripts 1,2 denote the side of the interface, $\mathrm{d} \boldsymbol{S}_{\mathrm{ij}}$ an elementary surface element pointing from side $\mathrm{i}$ to side $\mathrm{j}$, and with the current 


$$
\hat{I}_{12}=G_{0} \sum_{n} \frac{T_{n}\left[\hat{R}_{1}, \hat{R}_{2}\right]}{4+T_{n}\left(\frac{1}{2} \operatorname{Tr}\left\{\hat{R}_{1}, \hat{R}_{2}\right\}-2\right)} .
$$

In the latter expression, $G_{0}=2 e^{2} / h$ is the conductance quantum and the $T_{n}$ are the transmissions of the channels across the interface, considered as a scatterer for electrons, $\grave{a} l a$ Landauer.

Following Nazarov [139], we re-write

$$
\hat{I}_{12}=\left[\hat{R}_{1}, \hat{R}_{2}\right] \frac{G_{N}}{4} Z\left(\frac{1}{4} \operatorname{Tr}\left\{\hat{R}_{1}, \hat{R}_{2}\right\}\right)
$$

where $G_{N}=G_{0} \sum_{n} T_{n}$ is the normal state conductance of the interface and $Z(u)$ is a function characterizing the distribution of the interface channel transmissions:

$$
Z(u)=\frac{G_{0}}{G_{N}} \sum_{n} \frac{2 T_{n}}{2+T_{n}(u-1)}
$$

which is such that for any interface $Z(u=1)=1$.

For various known probability distribution functions $P(T)$ of transmissions across the interface, $Z(u)$ can be evaluated as

$$
Z(u)=\frac{\int_{0}^{1} \frac{2 T P(T)}{2+T(u-1)} \mathrm{d} T}{\int_{0}^{1} T P(T) \mathrm{d} T}=\left\{\begin{array}{l}
\text { uniform } \rightarrow \frac{2}{2+t(u-1)} \\
\text { tunnel } \rightarrow 1 \\
\text { ballistic } \rightarrow \frac{2}{1+u} \\
\text { dirty } \rightarrow \sqrt{\frac{2}{1+u}} \\
\text { diffusive } \rightarrow \frac{\arccos u}{\sqrt{1-u^{2}}}
\end{array}\right.
$$

Above we have considered several distributions for the $T_{n}$

- Uniform stands for an interface having all channels with identical transmissions $T_{n}=t$.

- Tunnel and ballistic are sub-cases of the above with $t \ll 1$ and, $t=1$ respectively.

- The "dirty" case assumes the universal Schep \& Bauer channel transmission distribution valid for disordered interfaces [141] 


$$
P(T)=\frac{2 G_{N}}{\pi G_{0}} \frac{1}{T^{3 / 2} \sqrt{1-T}}
$$

- Finally, the "diffusive" case assumes the Dorokhov distribution [142] found in diffusive conductors.

$$
P(T)=\frac{G_{N}}{G_{0}} \frac{1}{T \sqrt{1-T}}
$$

Note however that the latter case is given just for information: it cannot correspond to a physically realistic interface since the Dorokhov distribution is valid only for transport through a thick diffusive medium.

\subsubsection{Parameterization of the Green functions}

The retarded Green function matrix is a $2 \times 2$ matrix with the following constraints:

$$
\begin{aligned}
& \operatorname{Tr} \hat{R}=0 \\
& \hat{R}^{2}=\hat{I} \Leftrightarrow G^{2}+F F^{\dagger}=1
\end{aligned}
$$

Hence, the four elements of the matrix are not independent; only two independent parameters need to be specified to construct the matrix, with several possible choices. In the following I present two common parameterizations of the retarded Green function.

\subsubsection{1 $\theta, \varphi$ parameterization}

The most widely used parameterization in the literature is the $\theta-\varphi$ parameterization:

$$
\widehat{R}(\boldsymbol{r}, E)=\left(\begin{array}{cc}
\cos \theta & \sin \theta e^{i \varphi} \\
\sin \theta e^{-i \varphi} & -\cos \theta
\end{array}\right),
$$

where $\theta(\boldsymbol{r}, E)$ and $\varphi(\boldsymbol{r}, E)$ are generally complex functions. This parameterization provides an intuitive geometrical representation of the Green function at the Fermi level, when both $\theta$ and $\varphi$ are real angles, allowing picturing the state of the system on a sphere (see Fig. 4.1):

$\theta$ is the "pairing angle", with $\theta=0$ for a normal metal at the pole of the sphere and $\theta=\pi / 2$ for a BCS superconductor at the equator.

$\varphi$ is the phase of the superconductor, corresponding to the "longitude".

In this representation, the density of states of excitations is given by $\rho(E=0)=\operatorname{Re} \cos \theta$, corresponding to the projection of the point on the vertical axis of the sphere. 


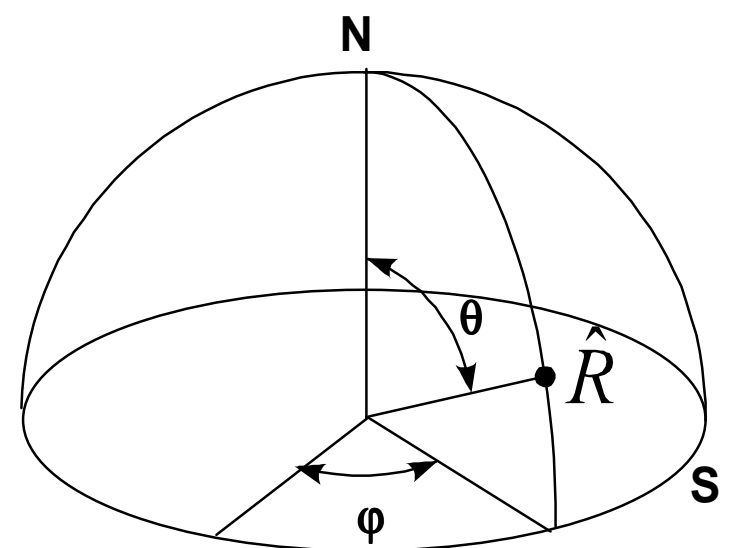

Fig. 4.1 Geometrical representation of the state of a proximity system at zero energy in the $\theta, \varphi$ parameterization.

In absence of a magnetic field, $\theta$ and $\varphi$ satisfy the coupled second order differential equations:

$$
\begin{gathered}
4 i E \sin \theta+2 \cos \theta\left(e^{-i \varphi} \Delta+e^{i \varphi} \Delta^{*}\right)+2 \hbar D \theta^{\prime \prime}=\sin 2 \theta\left(4 \hbar \Gamma_{s f}+\hbar D\left(\varphi^{\prime}\right)^{2}\right) \\
2 \cos \theta \theta^{\prime} \varphi^{\prime}+\sin \theta \varphi^{\prime \prime}=i \frac{e^{-i \varphi} \Delta-e^{i \varphi} \Delta^{*}}{\hbar D}
\end{gathered}
$$

derived from the Usadel equations (4.4), where the symbol prime denotes the spatial derivative.

\subsubsection{1.b Nazarov's Andreev circuit theory}

Based on this geometrical interpretation, Nazarov has described an elegant mechanical analogue way of solving the Usadel equations at the Fermi energy in arbitrary proximity effect networks [137]: he showed that the state of a proximity system can be found by solving the mechanical equilibrium of a set of springs on the surface of the sphere, where the springs represent the links of the network with strengths proportional to their conductance.

As a simple example, one can determine the Fermi level density of states of a system schemed on Fig. 4.2, representing a T-shaped normal metal connecting a normal reservoir and two superconducting reservoirs to which a phase difference is applied. One can guess that, if short enough, the normal wire connecting the two superconductors may sustain supercurrent, though since it is attached to a normal reservoir, its density of states will be finite at the Fermi energy. We are in the presence of what is called a "gapless" superconductor. 

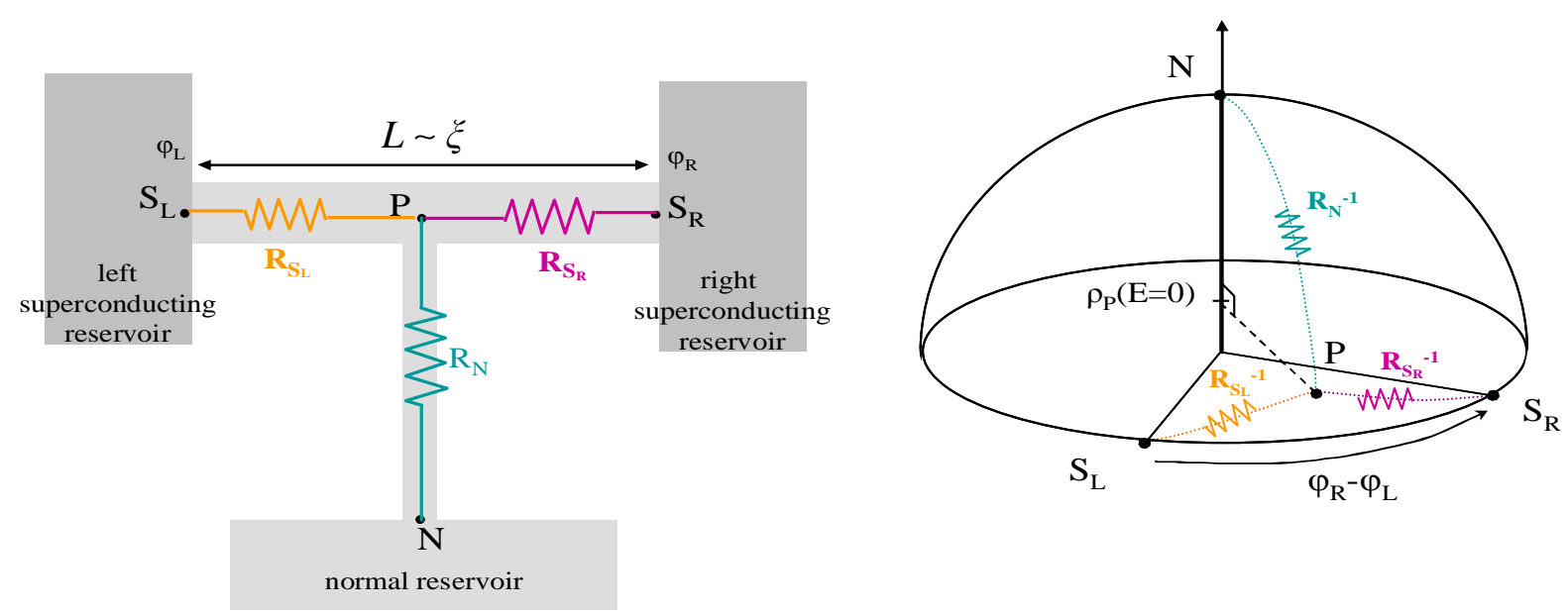

Fig. 4.2 Left: Sketch of an SNS system where the normal region is gapless, but can sustain a supercurrent. Right: circuit representation on the unit sphere: one finds a finite density of states at zero energy.

\subsubsection{Ricatti parameterization}

An alternate parameterization scheme of the Green function is the Ricatti parameterization [143, 144, 146, 149]:

$$
\hat{R}=\frac{1}{1+\tilde{\gamma} \gamma}\left(\begin{array}{cc}
1-\tilde{\gamma \gamma} & 2 \gamma \\
2 \tilde{\gamma} & \tilde{\gamma} \gamma-1
\end{array}\right),
$$

where $\gamma$ and $\tilde{\gamma}$ are also complex functions of $\boldsymbol{r}$ and $E$.

The Usadel equations (4.4) expressed in terms of the $\gamma$ and $\tilde{\gamma}$ functions are:

$$
\left\{\begin{array}{l}
2 i E \tilde{\gamma}-\Delta \tilde{\gamma}^{2}+\Delta^{*}+4 \hbar \Gamma_{s f} \tilde{\gamma} \frac{(\gamma \tilde{\gamma}-1)}{1+\gamma \tilde{\gamma}}+\hbar D\left(-\frac{2 \gamma}{1+\gamma \tilde{\gamma}}\left(\tilde{\gamma}^{\prime}\right)^{2}+\tilde{\gamma}^{\prime \prime}\right)=0 \\
2 i E \gamma-\Delta^{*} \gamma^{2}+\Delta+4 \hbar \Gamma_{s f} \gamma \frac{(\gamma \tilde{\gamma}-1)}{1+\gamma \tilde{\gamma}}+\hbar D\left(-\frac{2 \tilde{\gamma}}{1+\gamma \tilde{\gamma}}\left(\gamma^{\prime}\right)^{2}+\gamma^{\prime \prime}\right)=0
\end{array}\right.
$$

the prime still representing the spatial derivative. One notes here the symmetry between the two equations, which yields the general relation:

$$
\tilde{\gamma}(E)=\gamma(-E)^{*}
$$

related to the electron-hole (or time reversal) symmetry. In general, this symmetry does not simplify the task of solving the system, except in situations where $\Delta$ can be taken real throughout the system (i.e. no phase difference is present). In this case, $\gamma=\tilde{\gamma}$ and has an energy-even real part and an odd imaginary part, and the Usadel equations reduce to a single differential equation. 


\subsubsection{2.a Advantages of this parameterization}

Compared to the $\theta-\varphi$ parameterization, the two parameter functions are here treated on the same footing, and they are only algebraically combined instead of through transcendental functions. As a consequence, Equations (4.15) are somewhat more easily handled numerically than (4.13).

On a more physical ground, the Ricatti parameters are deeply related to the Andreev equations [125] themselves derived from the Bogolubov - de Gennes equations (4.1). This link is particularly obvious if one considers the clean case, in which the impurity scattering is neglected [143] (i.e. the Eilenberger equations in the clean limit). In the diffusive case, this formal link is still visible in the boundary conditions for $\gamma$ and $\tilde{\gamma}$, deep in the superconducting reservoirs (see below).

\subsubsection{2.b Ricatti parameters in reservoirs}

Deep into reservoirs, the gradient terms in (4.15) vanish. For a normal reservoir the solution is then trivial: $\gamma=\tilde{\gamma}=0$. For a BCS reservoir with a pair potential $\Delta e^{i \varphi}$ (with $\Delta$ real positive), assuming there is no pair-breaking $\left(\Gamma_{s f}=0\right), \gamma$ and $\tilde{\gamma}$ verify

$$
\left\{\begin{array}{l}
2 i E \tilde{\gamma}=\Delta e^{i \varphi} \tilde{\gamma}^{2}-\Delta e^{-i \varphi} \\
2 i E \gamma=\Delta e^{-i \varphi} \gamma^{2}-\Delta e^{i \varphi},
\end{array}\right.
$$

whose solutions are given by either of the following equivalent expressions ${ }^{1}$

$$
\begin{aligned}
\gamma(E)=\gamma_{S}(E) & =\exp \left(i \varphi+i \frac{\pi}{2}-i \operatorname{Sign}(\Delta-E) \operatorname{Arccos} \frac{E}{\Delta}\right) \\
& =i e^{i \varphi}\left(\frac{E+\sqrt{E^{2}-\Delta^{2}}}{\Delta}\right)^{-\operatorname{Sign}(\Delta+E)} \\
& =e^{i \varphi}\left(\frac{i E-\operatorname{Sign}(\Delta+E) \sqrt{\Delta^{2}-E^{2}}}{\Delta}\right)
\end{aligned}
$$

Expressions for $\tilde{\gamma}$ are the same with $\varphi$ replaced by $-\varphi$. The physical solution is retained by the argument that correlations must tend to zero for high energies. These expressions coincide with the Andreev reflection amplitudes [125] and are plotted in Fig. 4.3.

\footnotetext{
${ }^{1}$ Here, the principal determination of square roots of negative number are always taken: $\sqrt{-|x|}=+i \sqrt{|x|}$.
} 


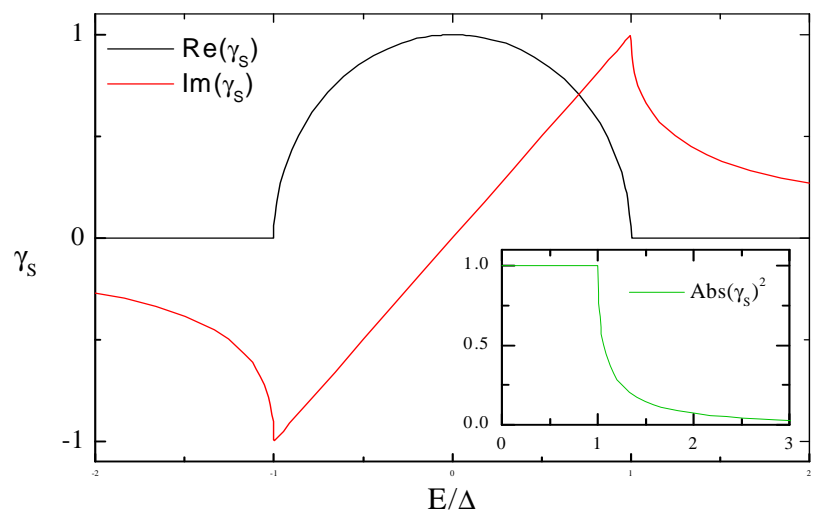

Fig. 4.3 Ricatti parameter $\gamma(\mathrm{E})$ of the retarded Green function in bulk superconductor with real pairing potential $(\varphi=0)$, as a function of energy. In this case, $\tilde{\gamma}=\gamma$. These functions coincide with the Andreev reflection amplitude at a perfect N-S interface. In the inset, the modulus square of $\gamma$ is plotted as a function of energy. It coincides with the Andreev reflection probability which is unity below the gap of the superconductor.

\subsubsection{2.c (Dis-)Continuity of Ricatti parameters at interfaces}

Expressing Green functions in terms of the Ricatti parameters, Equations (4.11)-(4.12) transform to

$$
\begin{aligned}
& \gamma_{1}{ }^{\prime}=-\frac{G_{N}}{A_{1} \sigma_{1}} \frac{\left(\gamma_{1}-\gamma_{2}\right)\left(1+\gamma_{1} \tilde{\gamma}_{2}\right)}{\left(1+\gamma_{2} \tilde{\gamma}_{2}\right)} Z\left(1-\frac{2\left(\gamma_{1}-\gamma_{2}\right)\left(\tilde{\gamma}_{1}-\tilde{\gamma}_{2}\right)}{\left(1+\gamma_{1} \tilde{\gamma}_{1}\right)\left(1+\gamma_{2} \tilde{\gamma}_{2}\right)}\right) \\
& \tilde{\gamma}_{1}{ }^{\prime}=-\frac{G_{N}}{A_{1} \sigma_{1}} \frac{\left(\tilde{\gamma}_{1}-\tilde{\gamma}_{2}\right)\left(1+\tilde{\gamma}_{1} \gamma_{2}\right)}{\left(1+\gamma_{2} \tilde{\gamma}_{2}\right)} Z\left(1-\frac{2\left(\gamma_{1}-\gamma_{2}\right)\left(\tilde{\gamma}_{1}-\tilde{\gamma}_{2}\right)}{\left(1+\gamma_{1} \tilde{\gamma}_{1}\right)\left(1+\gamma_{2} \tilde{\gamma}_{2}\right)}\right)
\end{aligned}
$$

where $G_{N}$ denotes the conductance of the interface, $A_{1}$ the area of the conductor on side 1 of the interface ${ }^{1}$, and $\sigma_{1}$ the normal state conductivity of electrode 1 . The above expressions are symmetric by exchanging tilde and non-tilde terms, and similar terms are obtained on side 2 , by permuting indices 1 and 2 , and changing sign (see (4.11)). These equations link the derivative of the Ricatti parameters on either side of the interface to the jump of the parameters across the interface.

\subsubsection{Spectral quantities in the Ricatti parameterization}

Following from (4.5), the normalized density of states writes

$$
\rho(\boldsymbol{r}, E)=\rho_{N} \operatorname{Re}\left(\frac{1-\gamma \tilde{\gamma}}{1+\gamma \tilde{\gamma}}\right)
$$

\footnotetext{
${ }^{1}$ In the 1-D approximation discussed below, the difference between cross sections of the electrodes on both sides of a contact can be accounted for by the term $A_{\mathrm{i}}$.
} 
the order parameter is expressed as the integral over energy of the pair amplitude:

$$
\Delta(\boldsymbol{r})=-i \rho_{N} V(r) \int_{0}^{\hbar \omega_{D}} \tanh \left(\frac{E}{2 k_{B} T}\right)\left(\frac{\gamma}{1+\gamma \tilde{\gamma}}-\left(\frac{\tilde{\gamma}}{1+\gamma \tilde{\gamma}}\right)^{*}\right) \mathrm{d} E
$$

\subsubsection{A word on the non-equilibrium theory}

In non-equilibrium situations the retarded and advanced Green functions need to be supplemented with the Keldysh Green function which contains the electron and hole distribution functions. In this non-equilibrium formalism the equations are very similar to those presented here. In spite of this formal similarity, solving numerically the nonequilibrium equations [144] can be much more complicated than solving the equilibrium situation, which is already not so easy, as will be explained in the next section.. 


\subsection{Solving the Usadel equations numerically}

As explained above, for a given diffusive proximity effect structure at equilibrium, all physical properties can be obtained from the field of the retarded Green function, which is the solution of the Usadel equation in the whole structure, with the appropriate boundary conditions. However, since the Usadel equations are coupled non-linear differential equations, it is not possible to obtain analytical solutions (except in rare limit cases [145]). Hence, one needs to recourse to some numerical method to solve these equations. Many fields in physics (hydrodynamics, plasmas...) face problems of similar type and generic Finite Element engines have been developed to solve for multi-dimensional non-linear differential equations. One could in principle use these tools for proximity effect too, as they allow for arbitrary shapes and easily make use of distributed computing architectures.

\subsubsection{Reduction of the problem dimensionality}

However, up to now, all numerical work on Usadel equations was done using specifically written code applicable only to idealized reduced dimensionality problems, mostly $1-\mathrm{D}^{1}$. This approach allows reducing the number of geometrical parameters, maintaining better control on the numerical process and having much shorter resolution times. This approach is particularly well suited to model large translation-invariant systems like planar junctions made of different stacked materials. It is also applicable for small systems having a typical "wire" geometry with transverse dimensions small enough to assume the Green functions are homogeneous in any cross-section of the wire, while varying along the wire. In practice, this 1-D approximation is a good assumption for diffusive wires if the diameter of the wire is small compared to the diffusive coherence length $\xi=\sqrt{\hbar D / \Delta}$, where $D$ is the diffusion constant of the material and $\Delta$ the relevant order parameter in the structure. For proximity structures made with Aluminum as the superconductor, and standard diffusive metals, $\xi$ is of the order of a hundreds of $\mathrm{nm}$. Hence, one can easily fabricate a quasi 1-D structure using ebeam lithography.

We have used the 1-D approximation of the Usadel equations for modelling the SNS experiment we have performed and which is presented in $\S 4.6$. In the following we assume and discuss only this case.

\footnotetext{
${ }^{1}$ Notable exceptions are 2D resolutions in [146] for the diffusive limit and [147] in the ballistic case.
} 


\subsubsection{Specifying the 1-D problem}

From now on we consider the resolution of the SNS problem shown in Fig. 4.4, in the 1-D approximation. We thus have to solve the Usadel equations to obtain the set of functions $\gamma(x, E), \tilde{\gamma}(x, E), \Delta(x)$ where $x$ is the coordinate along the system and these functions obey (4.15) in the bulk of the electrodes and (4.18) at the interfaces. Far enough in the superconducting electrodes we assume the superconductor reaches the bulk Green functions. Hence at the left and right boundaries $x= \pm\left(L / 2+L_{S}\right)$ where is $L_{S}$ taken large enough, we impose the bulk Green functions (4.17) as boundary conditions, with the appropriate phase difference. Assuming an exponential relaxation of the superconductor towards its bulk Green function, $L_{S} \sim 5 \xi$ should make a negligible difference with an infinitely long system. Note that the problem is not necessarily symmetric due to the possibility of specifying different interfaces.
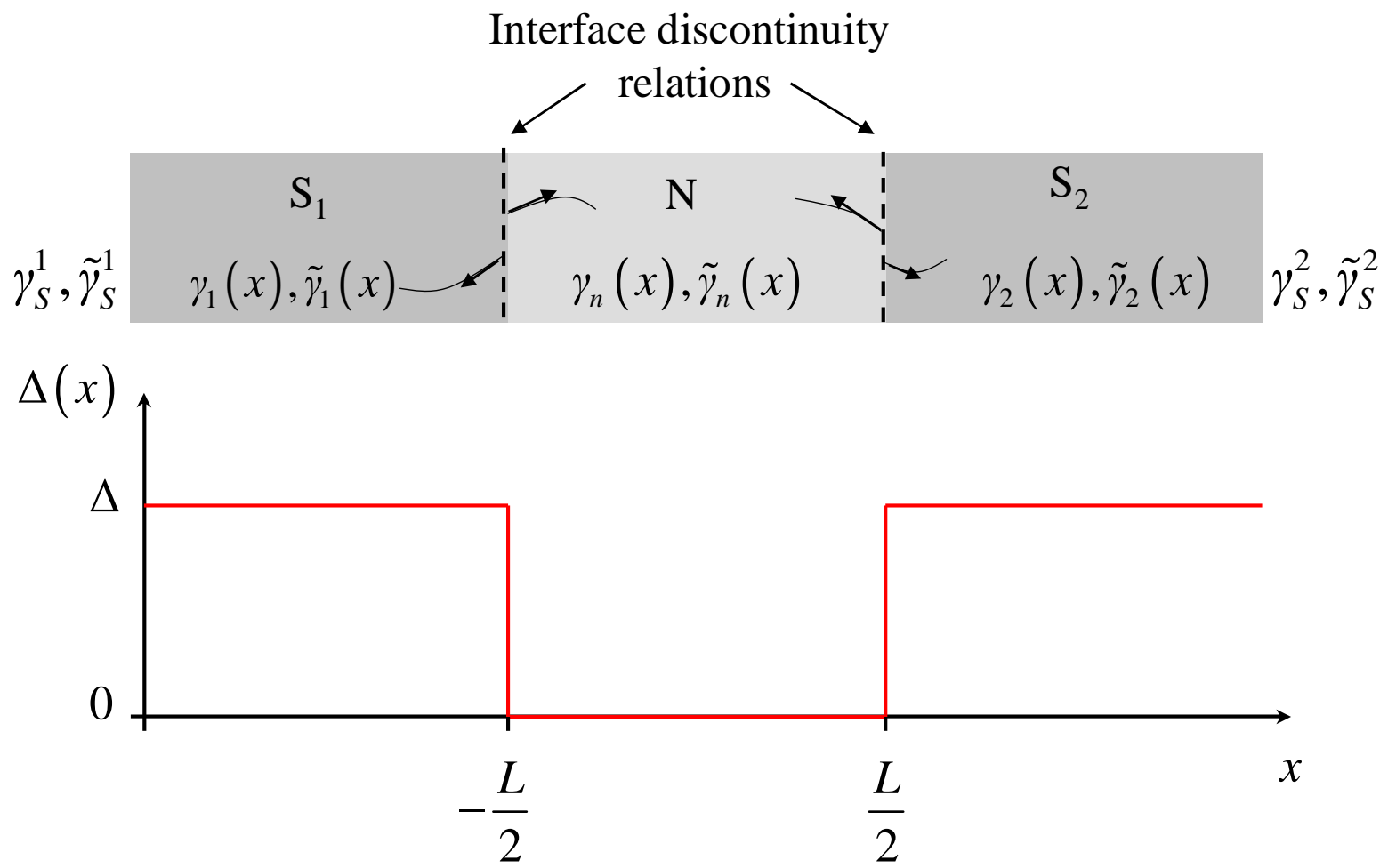

Fig. 4.4 1-D SNS system to be solved using Usadel equations with the Ricatti parameters. In each electrode we must solve for a pair of coupled energy and space dependent functions, plus the self consistency equation giving the order parameter. At the left and right of the system we assume the electrodes recover the state of the bulk BCS superconductor, with possibly a phase difference between them, while at the imperfect NS interfaces, a discontinuity relation links the derivatives of the functions on both sides with the jump in the functions. The initial profile of the order parameter is plotted in the bottom part: the modulus of the order parameter is assumed constant in the superconductors and zero in the normal part. 


\subsubsection{Solving strategy - self-consistency}

As a starting point to numerical computation, it is necessary to impose a profile for the order parameter $\Delta(x)$. The simplest starting assumption is to take $\Delta(x)$ as a step function, assuming constant values inside the superconductors and zero inside $\mathrm{N}$, for the phononmediated attraction doesn't exist in normal metals: $V_{N}=0$ (See Fig. 4.4).

From this point, corresponding $\gamma(x, E)$ and $\tilde{\gamma}(x, E)$ are then found by solving (4.15) and (4.18) energy by energy in a large enough energy range (from $E=0$ to many times $\Delta$ ) so that we can subsequently compute an updated profile of the order parameter using (4.20). The procedure is then iterated until consistency is reached. An example showing the convergence of such iterative calculation is shown below, in section 4.4.2.2, Fig. 4.10.

In inhomogeneous phase situations, we will assume a weak supercurrent density in the superconductors so that the phase drop occurs only across the normal wire. Otherwise, the boundary conditions we consider for the extremities are not suited. Note further that selfconsistent magnetic effects may need to be taken into account if the supercurrent is large. In the present treatment we neglect the effect of the magnetic field, except maybe through a spin-flip scattering rate $[146,148]$.

\subsubsection{Implementation details}

The interface boundary conditions introduce point-like discontinuities in $\gamma(x), \tilde{\gamma}(x)$ at $x= \pm L / 2$. Such discontinuities cannot be handled by standard numerical integration algorithms which assume smooth functions. It is better to split $\gamma$ and $\tilde{\gamma}$ into distinct functions having different domains:

$$
\gamma(x), \tilde{\gamma}(x) \rightarrow\left\{\begin{array}{llc}
\gamma_{1}(x), \tilde{\gamma}_{1}(x) & \text { for } & x<-L / 2, \\
\gamma_{n}(x), \tilde{\gamma}_{n}(x) & \text { for } & -L / 2<x<L / 2, \\
\gamma_{2}(x), \tilde{\gamma}_{2}(x) & \text { for } & x>L / 2,
\end{array}\right.
$$

so that these functions are smooth and have boundary conditions only at their ends. These functions need to be solved simultaneously even though they are now on distinct intervals. In order to solve them in a single differential integration, and not sequentially, we apply a trick which consists in "folding" them onto a single interval, as schematized in Fig. 4.5. We thus need to solve a set of 6 second-order differential equation (DE), on a single interval. These 
equations are coupled 2 by 2 (by sets of $\gamma, \tilde{\gamma}$ ) along the interval and re-coupled altogether at the extremities (by the boundary conditions).

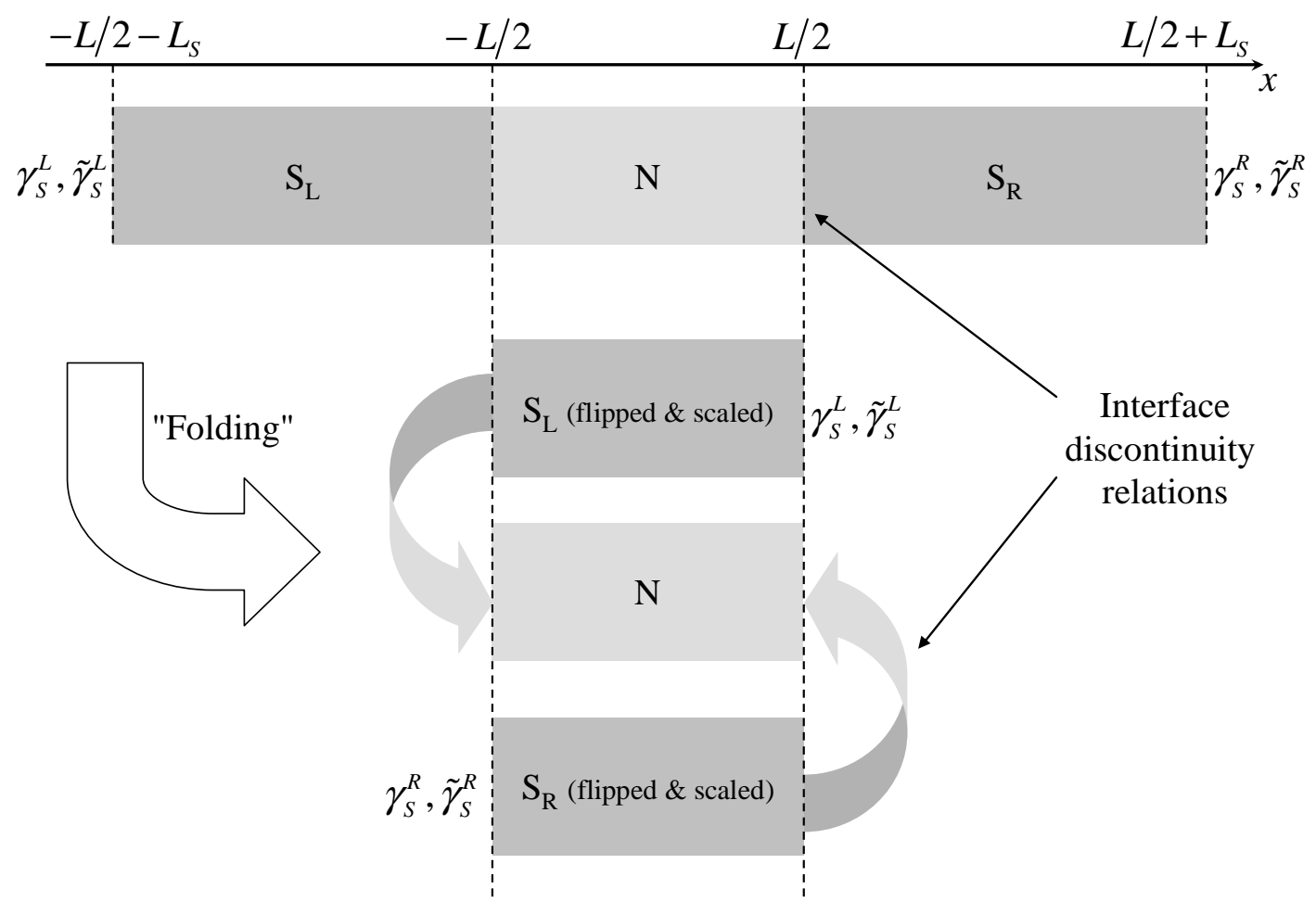

Fig. 4.5 To numerically solve the equation for boundary conditions in the SNS system, it is convenient to fold the system as described, so that all boundary conditions are brought at the extremities of a single interval, a standard situation for which algorithms exist.

The Usadel equations to solve for each electrode are non linear differential equations (NLDE) with boundary conditions given across an interval. There are no efficient algorithms for solving this kind of NLDE; they all basically work by trial and error, trying to find the initial value of the derivatives on one side of the interval which allow meeting the boundary conditions of the function on the opposite side. These so-called "shooting" algorithms may converge badly, for instance when the DE has an exponential sensitivity to its initial conditions. In contrast, for linear DE, deterministic algorithms exist to solve such boundary condition problems. We can thus use such an algorithm by linearizing the Usadel equation. To do so, we express each of the above 6 Ricatti parameters as $\gamma(x, E)=\gamma_{0}(x, E)+\delta \gamma(x, E)$ where $\gamma_{0}(x, E)$ is a trial function and $\delta \gamma(x, E)$ is a corrective term (assumed small) to be determined. By performing a first-order expansion in $\delta \gamma(x, E)$, one obtains a linear DE for $\delta \gamma(x, E):$ 


$$
\begin{aligned}
& \Delta+2 i E \gamma_{0}-\gamma_{0}^{2} \Delta^{*}+\hbar D\left(\gamma_{0}^{\prime \prime}-2 \frac{\tilde{\gamma}_{0} \gamma_{0}^{2}}{1+\tilde{\gamma}_{0} \gamma_{0}}\right)+\left(2 i E-2 \gamma_{0} \Delta^{*}+2 \hbar D \frac{\tilde{\gamma}_{0}^{2} \gamma_{0}^{\prime 2}}{\left(1+\tilde{\gamma}_{0} \gamma_{0}\right)^{2}}\right) \delta \gamma, \\
& -4 \hbar D \frac{\tilde{\gamma}_{0} \gamma_{0}^{\prime}}{1+\tilde{\gamma}_{0} \gamma_{0}} \delta \gamma^{\prime}+\hbar D \delta \gamma^{\prime \prime}=0
\end{aligned}
$$

(written here for $\Gamma_{s f}=0$, for simplicity) which can be solved efficiently, yielding a calculated first-order correcting function. If the computed correcting function is indeed found small everywhere, it is added to $\gamma_{0}$, while if it is large at some places, only a small fraction of it is added to $\gamma_{0}$ to obtain a new function closer to the real solution. The process is iterated until sufficient convergence is reached. Finally, each second order DE is transformed to two coupled first order equations for numerical resolution.

We have first implemented such a numerical resolution using Mathematica, which allows flexible and compact programming of complex problems and immediate visualization of the results. In particular, in numerical integrations of DE problems one does not need to spend much effort about numerical details such as the discretization of the equations. This implementation worked, but was too slow to realistically perform self-consistent gap profile calculations, or to explore many interface parameters when trying to reproduce the experimental data presented in the second part of this chapter.

With the help of Juan Carlos Cuevas (D.F.T.M.C. Universidad Autónoma de Madrid), and based on one of his programs which solved the SNS problem in N with rigid S boundary conditions, we implemented this calculation in FORTRAN code. This code makes use of a very efficient algorithm from Numerical Recipes, known as the "relaxation method". The algorithm is based on computing a large matrix linking finite differences in $\delta \gamma$ and inverting it with a highly optimized routine. Filling the matrix coefficients necessitates $\sim 800$ lines of algebraic FORTRAN code. Since producing such a large piece of code error-free (or even debugging it) is clearly beyond possibilities of mere humans, we generated this FORTRAN code automatically. Starting from the Usadel equations, all the required algebraic manipulations were carried out using Mathematica. Finally the FORTRAN syntax itself was output using the FortranForm function. For nearly interactive use, we still call the FORTRAN program from within Mathematica and visualize its results with Mathematica. Parameters and results are passed through text files. The resulting FORTRAN code is about two orders of magnitude faster than the corresponding (perhaps not fully optimized) Mathematica program. Using the FORTRAN code, Green functions of an SNS structure at a hundred points in each electrode (i.e. 300 in all) can be calculated for 1500 values of the 
energy in $\sim 60 \mathrm{~s}$ on a regular PC. Self-consistent gap calculation, if needed, requires further iterating this whole calculation, usually less than 10 times. Note that if gap self-consistency is not necessary, one can much reduce the number of energies to sample while still obtaining accurate Density of States (DOS) profiles in the structure in only about $\sim 10$ s. Hence, a selfconsistent DOS calculation can be 60 times longer than its non-self consistent counterpart.

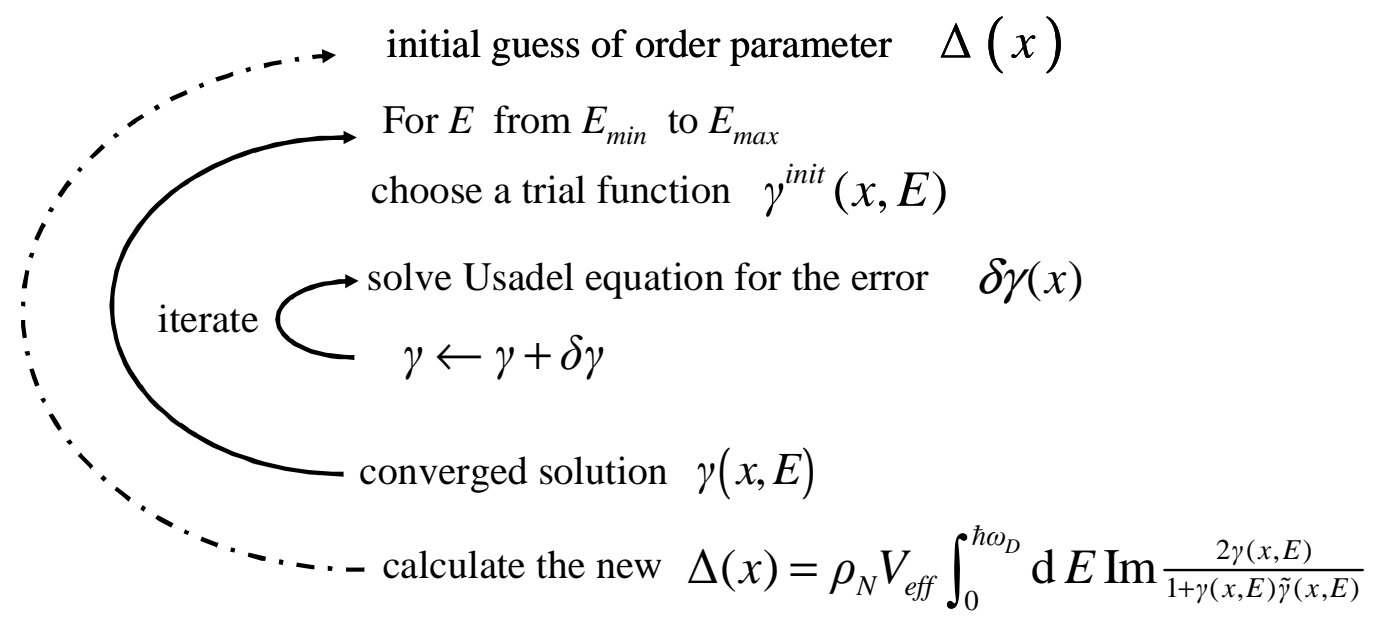

Fig. 4.6 Steps for the numerical resolution of the Usadel equations. First a profile of order parameter is guessed. Then for each energy, we first guess an initial value for the $\gamma$ functions, and use a linearized Usadel equation to compute a corrective term which we add to the guess function. This step is repeated until convergence is reached, then the next energy is evaluated. Once the correlations are found at all positions and energies, one can deduce the corresponding profile of the order parameter and iterate the procedure, if a full self-consistent calculation is desired. 


\subsection{General results on proximity effect in SNS structures}

In this section, I present the known proximity effect features encountered in SNS structures. To illustrate these features, I show results obtained from the numerical resolution of the Usadel equation presented in the previous section.

\subsection{1 "Minigap" in the DOS}

Fig. 4.7 shows the normalized density of states (DOS) for the simple case of a 1D SNS structure with $L=2 \xi$ ( $\xi$ is the superconducting coherence length), and perfect interfaces (i.e. no discontinuity of the Green functions at S-N interfaces).

Far away from the normal metal, the superconducting BCS DOS is recovered, as expected (it is the imposed boundary condition, after all).

The most striking feature in this DOS is the presence of an energy band smaller than the original gap of the bulk superconductor with exactly zero DOS. This so-called minigap is present throughout the structure (even in the $S$ electrodes), with a uniform value. The minigap is a salient generic feature occurring in any proximity effect structure with finite-size normal metal parts [121], not only in 1D SNS structures.

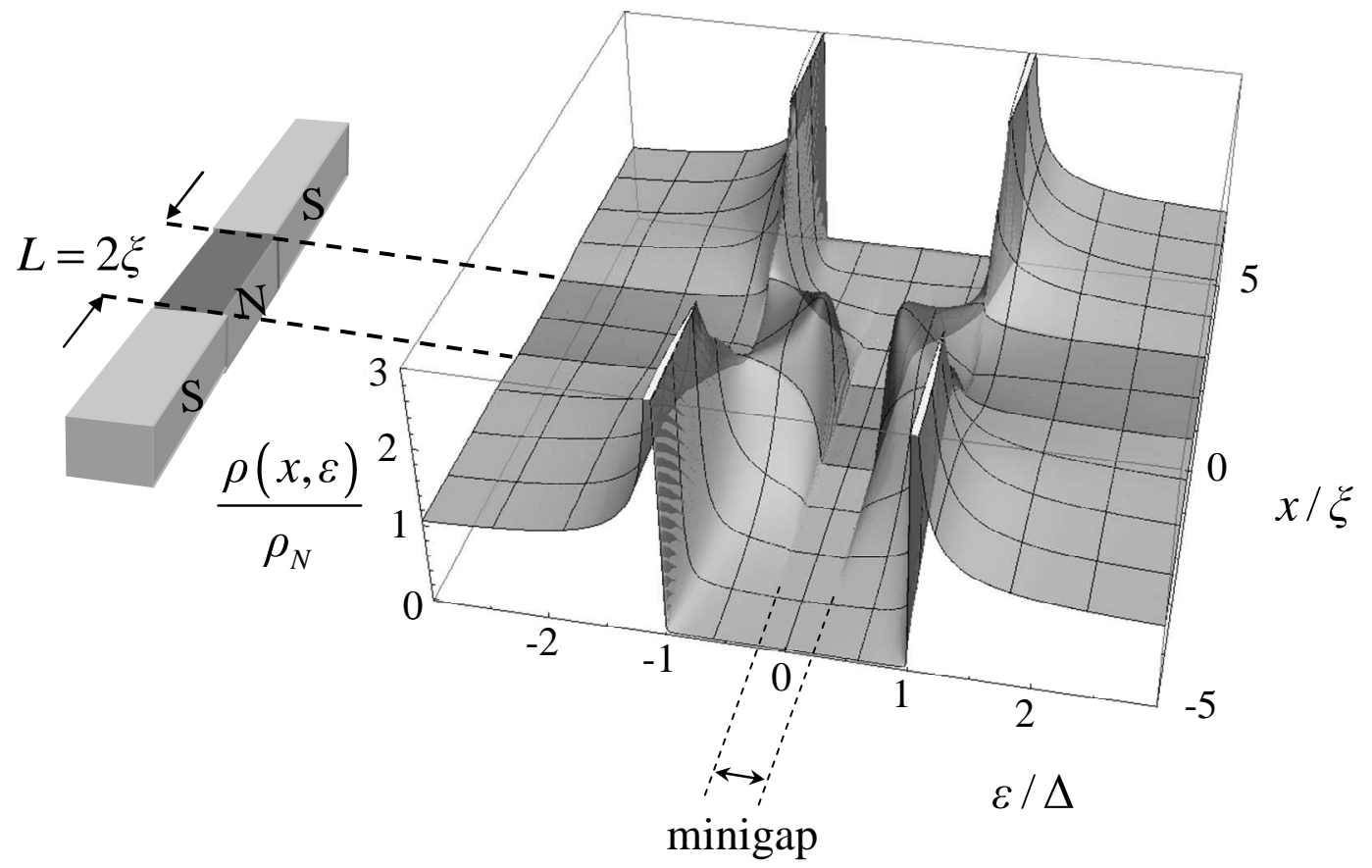

Fig. 4.7 Normalized Density of States (DOS) predicted by the numerical resolution of the Usadel equation in an S-N-S geometry with perfect interfaces, zero phase difference and uniform crosssections in $\mathrm{S}$ and $\mathrm{N}$ electrodes. In this image and in the following, the part of the 3-D graph corresponding to the $\mathrm{N}$ electrode is made darker than the rest. 


\subsubsection{Inverse proximity effect - Pairing amplitude and pairing potential}

From Fig. 4.7 it is clear that the DOS is also modified inside the superconductor. This modification can be seen as the evanescent part of the wavefunction of normal electrons undergoing Andreev reflection at the N-S interface. The evanescent wave has a characteristic decay scale of $\xi$. A consequence of this modification of the DOS is a change in the energy resolved pair amplitude Eq. (4.9) which is shown in Fig. 4.8, at zero phase difference across the structure, and in Fig. 4.9, for a $\pi$ phase difference.

\section{zero phase difference}

DOS

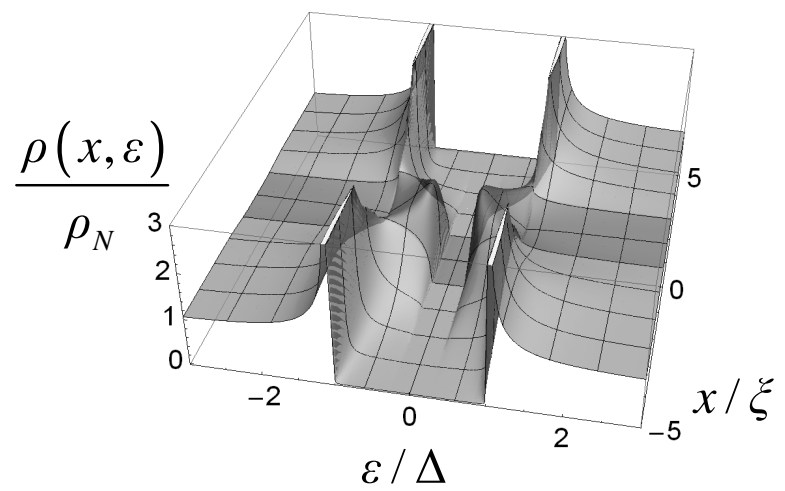

energy-resolved pair amplitude

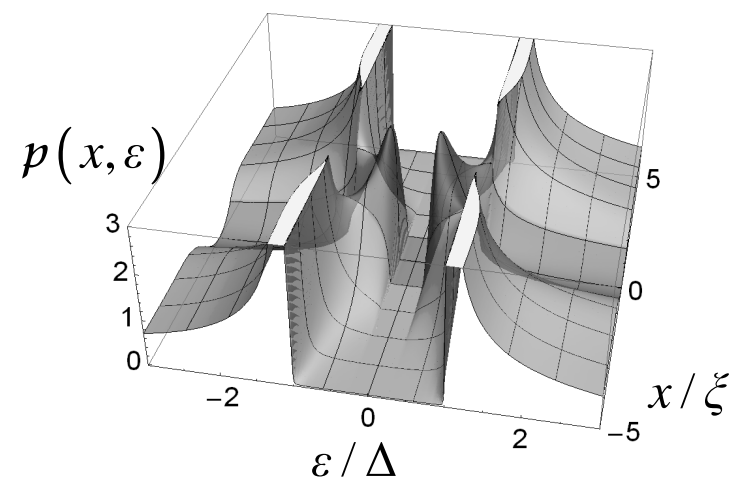

Fig. 4.8 For the same system ideal model system as in the previous figure at zero phase difference, we compare here side by side and at the same scale, the Density of States (DOS) and the energyresolved pair potential (Eq. (4.9), at zero temperature).

\section{$\pi$ phase difference}

\section{DOS}

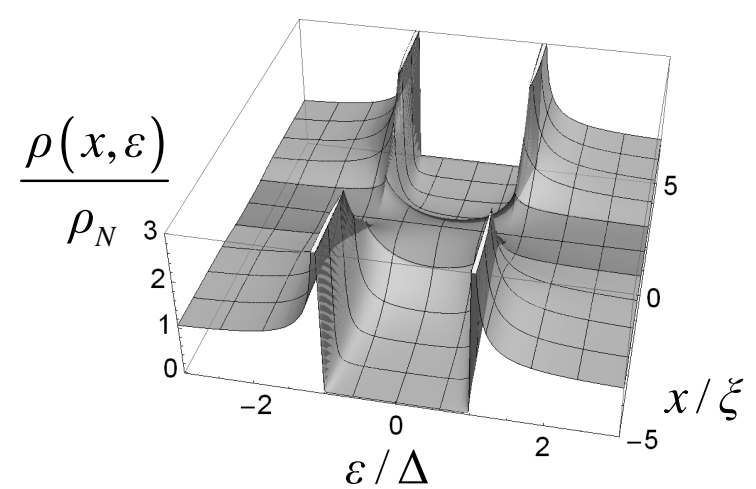

energy-resolved pair amplitude

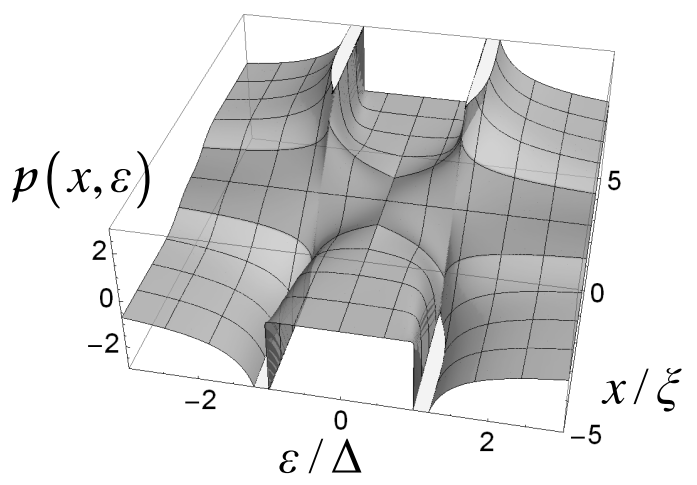

Fig. 4.9 Same figure as Fig. 4.8, for $\pi$ phase difference. 
Both of these phenomena occurring on the superconductor side of the contact are known as the "inverse" proximity effect.

We see that the amplitude of the energy-resolved pair amplitude function bears many resemblances with the DOS: it displays the same "minigap" feature, and peaks at the same positions. However, it falls to zero at high energy where the DOS goes to 1 . In the case of the $\pi$ phase difference, there is no minigap (or its width is zero). Here the pair potential changes sign across the structure (corresponding to the $\mathrm{e}^{\mathrm{i} \pi}$ term of the phase shift), and exactly in the middle of this symmetric structure, one recovers a normal state metal (i.e. constant DOS and zero pair amplitude).

\subsubsection{Self-consistency}

The pair potential is obtained according to Eq. (4.7)-(4.9), by integrating $p(E, \boldsymbol{r})$ to obtain the integrated pair amplitude. As previously explained (see §4.3.3), this calculation involves iterating the numerical resolution of the Usadel equations to reach self-consistency. Fig. 4.10, shows the iterated calculation of the pair potential in the structure shown in Fig. 4.7 to Fig. 4.10. One observes the reduction of the pair potential in the $\mathrm{S}$ electrodes on a length scale of the order of $\xi$, a manifestation of the inverse proximity effect. In the system we consider here, with high transparency interfaces and equal $\mathrm{S}$ and $\mathrm{N}$ area, the inverse proximity effect is particularly important, which makes reaching full self-consistency difficult. Here, it can be considered reached after 10 iterations. In many cases when quantitative predictions are not needed, the first iteration already qualitatively predicts correctly the behaviour of the system. This can be seen for instance in Fig. 4.11, where we compare the self-consistent value of the minigap to the non-self-consistent value obtained at first iteration assuming a step like potential, and the result of a calculation not taking into account inverse proximity effect (i.e. assuming rigid bulk superconducting Green functions up to the interface) [144]. In this figure we can also check the accuracy of our numerical results by comparing the predicted minigap in SNS system with clean interfaces as a function of the $\mathrm{N}$ length, to previously published results. We more or less recover the results of Belzig et al. [143], but the observed discrepancy is presently not understood ${ }^{1}$.

\footnotetext{
${ }^{1}$ A careful examination of our numerical results shows that the calculated solutions for symmetrical structures are slightly asymmetric (See e.g. Fig. 4.10), for a reason not yet elucidated. This could explain the discrepancy.
} 

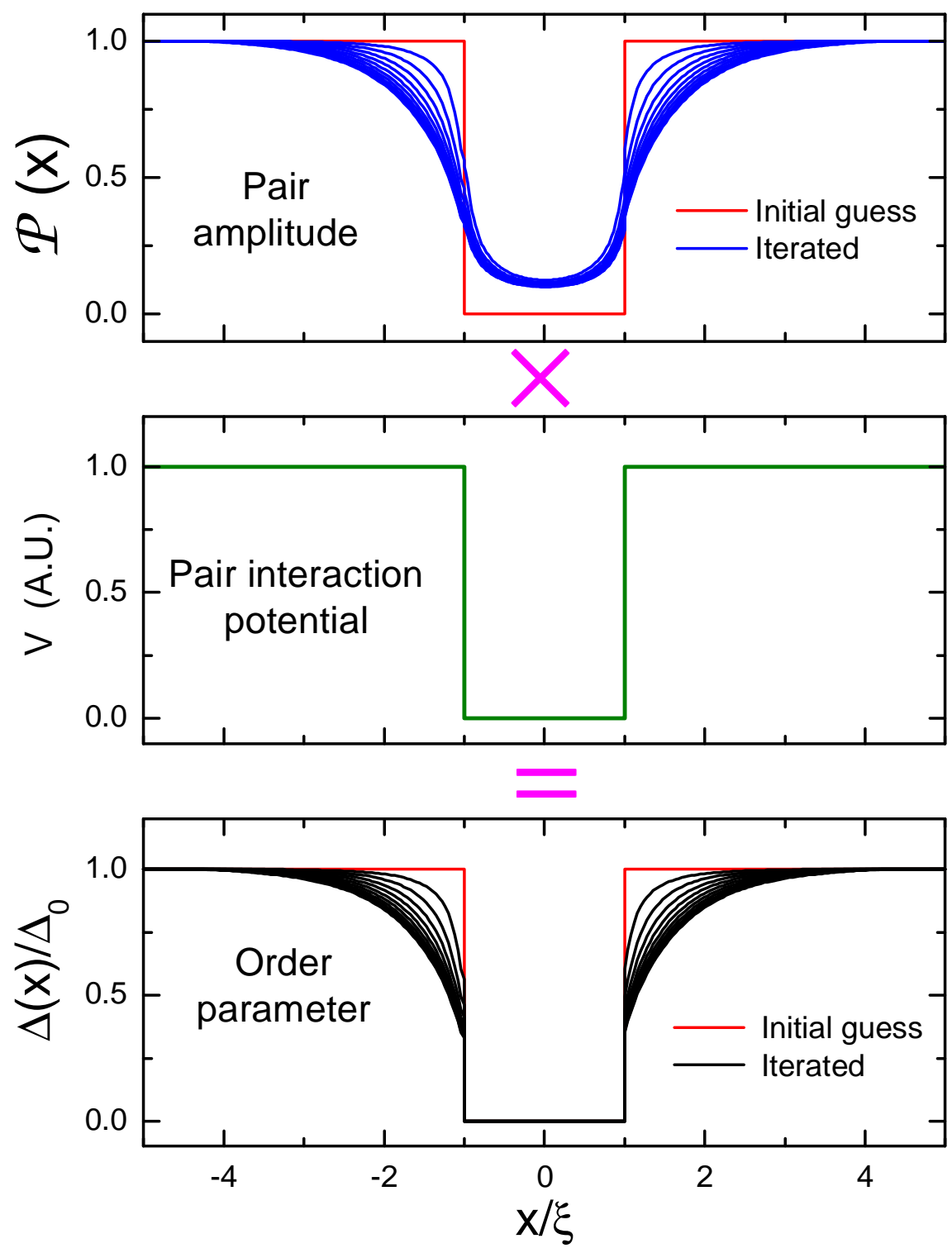

Fig. 4.10 Iterated pair amplitude and order parameter profiles along the S-N-S structure. The curves in the bottom panel are obtained by multiplying the curves in the top panel by the pair interaction potential depicted in the middle panel. Hence, inside the normal metal the pair amplitude is non zero, while the order parameter is zero due to the absence of pairing interaction. The pair potential is reduced in the superconducting electrodes, due to the inverse proximity effect. One sees clearly that the typical length scale is of the order of $\xi$. 


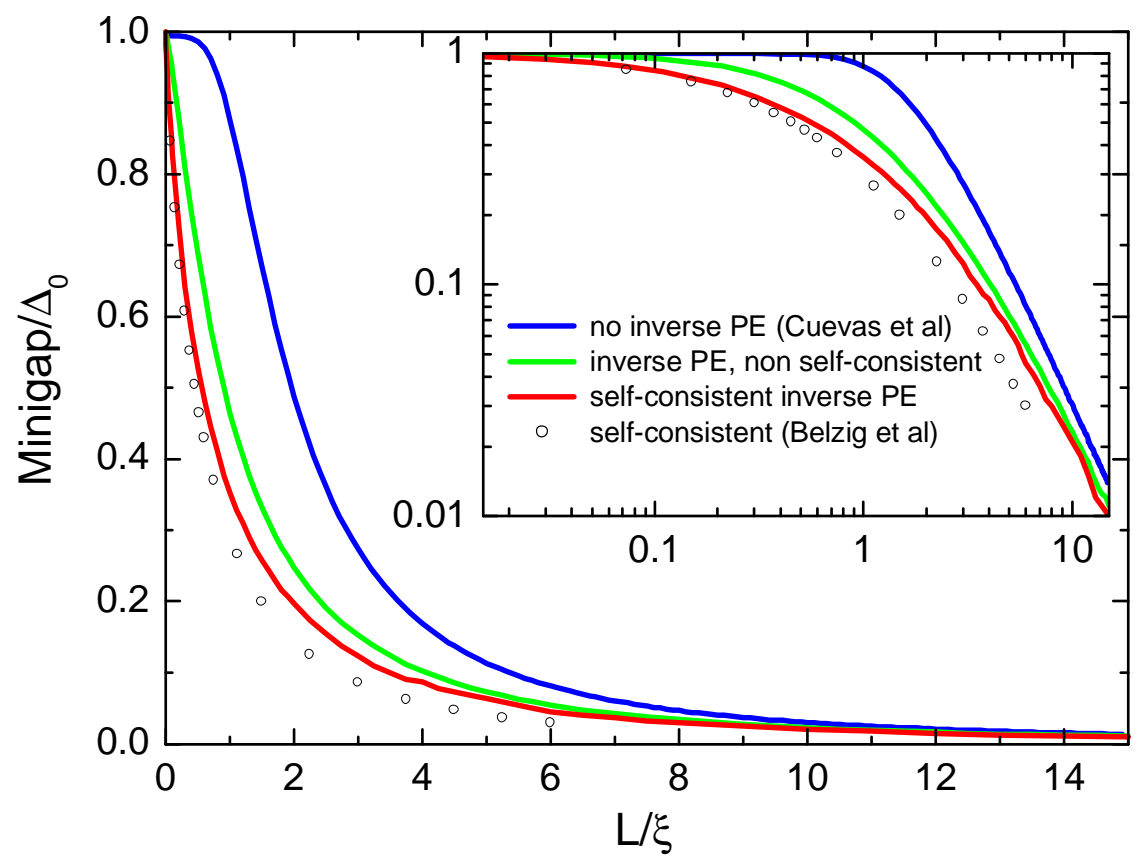

Fig. 4.11 Predicted values of the minigap in SNS structures with clean interfaces, as a function of L, the normal metal length. Green and red curves are the results of our calculation, taking into account the inverse proximity effect. Green is the non-self-consistent obtained at first iteration, and red is the converged result at iteration 10. The blue curve is taken from Fig. 1 in [144] and the open dots, from Fig. 3 in [143]. In the inset, the same data are plotted in a log-log scale, showing an asymptotic $\mathrm{L}^{-2}$ dependency of the minigap at large $\mathrm{L}$.

\subsubsection{Role of interfaces}

Imperfect interfaces reduce the strength of the proximity effect: the minigap is reduced, and on the $\mathrm{S}$ side, the inverse proximity effect, with the associated reduction of the pair potential is weaker (See Fig. 4.12). This can be easily understood in terms of scattering : at a dirty interface, an electron coming from the normal metal has, in addition to the Andreev reflection process, a finite probability to experience a normal reflection, which does not induce pair correlations in the $\mathrm{N}$ part. For a detailed investigation of the role of the interface parameters see Ref. [149].

Even with perfect interfaces, the 1D model can still show discontinuities in the derivatives of the Green functions at interfaces if the electrodes do not have the same cross-section (while sill staying in the 1D approximation). In this case, if the superconductor is much larger than the normal metal, the inverse proximity effect is also reduced (See Fig. 4.13). 

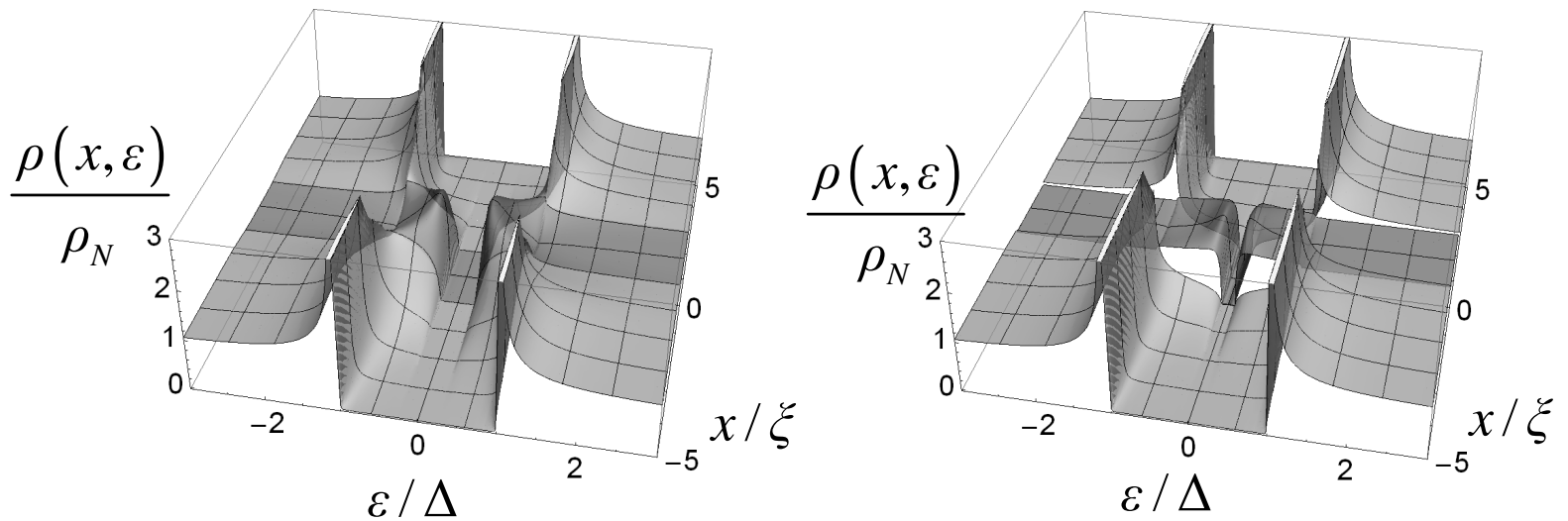

Fig. 4.12 Comparison of the DOS in the same ideal SNS structure with perfect interfaces as in Fig. 4.7 - Fig. 4.8 (left) and same structure with resistive interfaces (right), at zero phase difference. In this latter plot we assume the interfaces contribute to the total normal state resistance by the same amount than the wire.

\section{uniform cross-section}

\section{larger S cross-section}
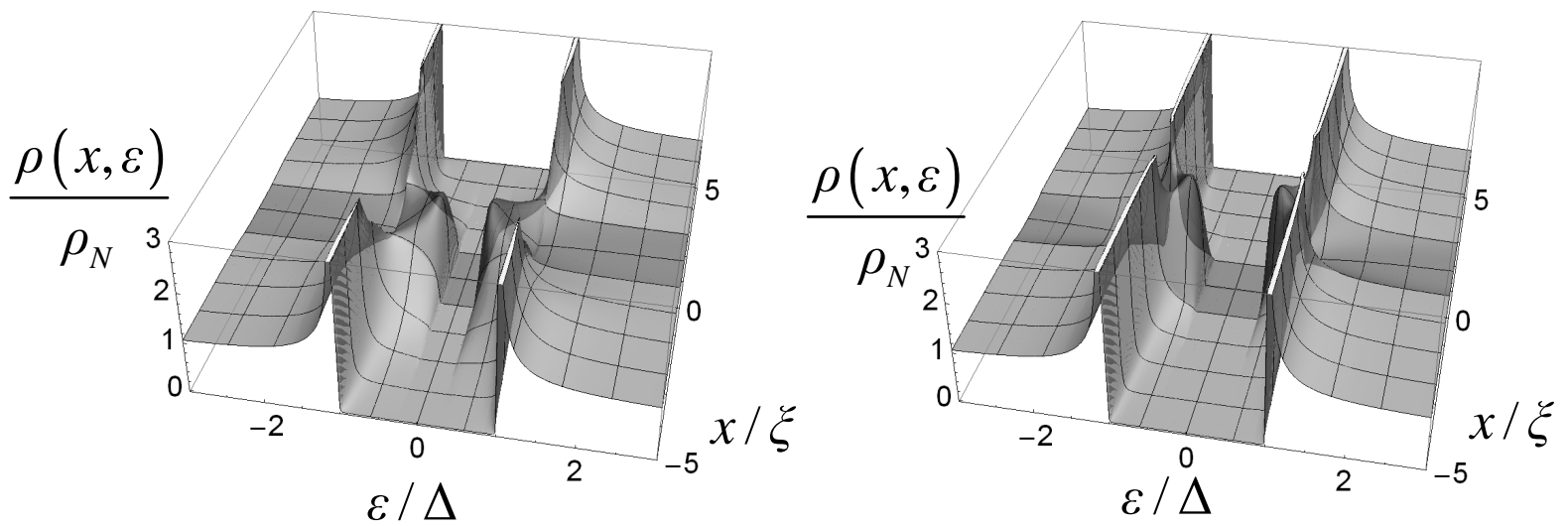

Fig. 4.13 Comparison of the DOS in SNS structures with perfect interfaces but different cross sections in $\mathrm{S}$ and $\mathrm{N}$ parts. On the left, $\mathrm{N}$ and $\mathrm{S}$ are of equal cross-section (Fig. 4.7 - Fig. 4.8), while on the right, the $\mathrm{S}$ cross section is ten times larger than the $\mathrm{N}$ cross sections. In this case, the superconductor appears more "rigid": the inverse proximity effect is reduced, and the minigap increased.

\subsubsection{Dependence of the minigap on $\mathrm{N}$ size}

Fig. 4.11, shows the dependence of the minigap in a 1D SNS structure with clean interfaces, as a function of the length $L$ of the $\mathrm{N}$ part. In the $\log$-log inset, one sees that at large $L$, the minigap varies as $L^{-2}$. This dependence can be traced to the diffusive character of the problem: an electron in the $\mathrm{N}$ part has a characteristic dwell time $\tau=L^{2} / D$, which defines the Thouless energy scale $E_{T}=\hbar / \tau=\hbar D / L^{2}$, frequently encountered in mesoscopic systems. The 
proportionality noted above shows that the minigap $E_{g}$ is proportional to the Thouless energy when $E_{g} \ll \Delta[143]$. The precise proportionality factor depends on the details of the system, and, in particular, on the interfaces, as shown in the previous section.

\subsubsection{Phase modulation of the proximity effect}

The originality of the equilibrium SNS system is that one can impose a fixed superconducting phase difference $\delta$ across the structure. Experimentally, this can be achieved by closing the system in a loop and threading a flux in the loop (see below) or by driving a supercurrent though the system. This phase difference affects the DOS of the whole structure in a periodic manner [150]. We show the evolution of the DOS of an SNS structure with $\delta$ in Fig. 4.14. When the phase is varied, the minigap evolves roughly as $|\cos \delta|$, pinching off linearly at $\delta=\pi \bmod 2 \pi$.

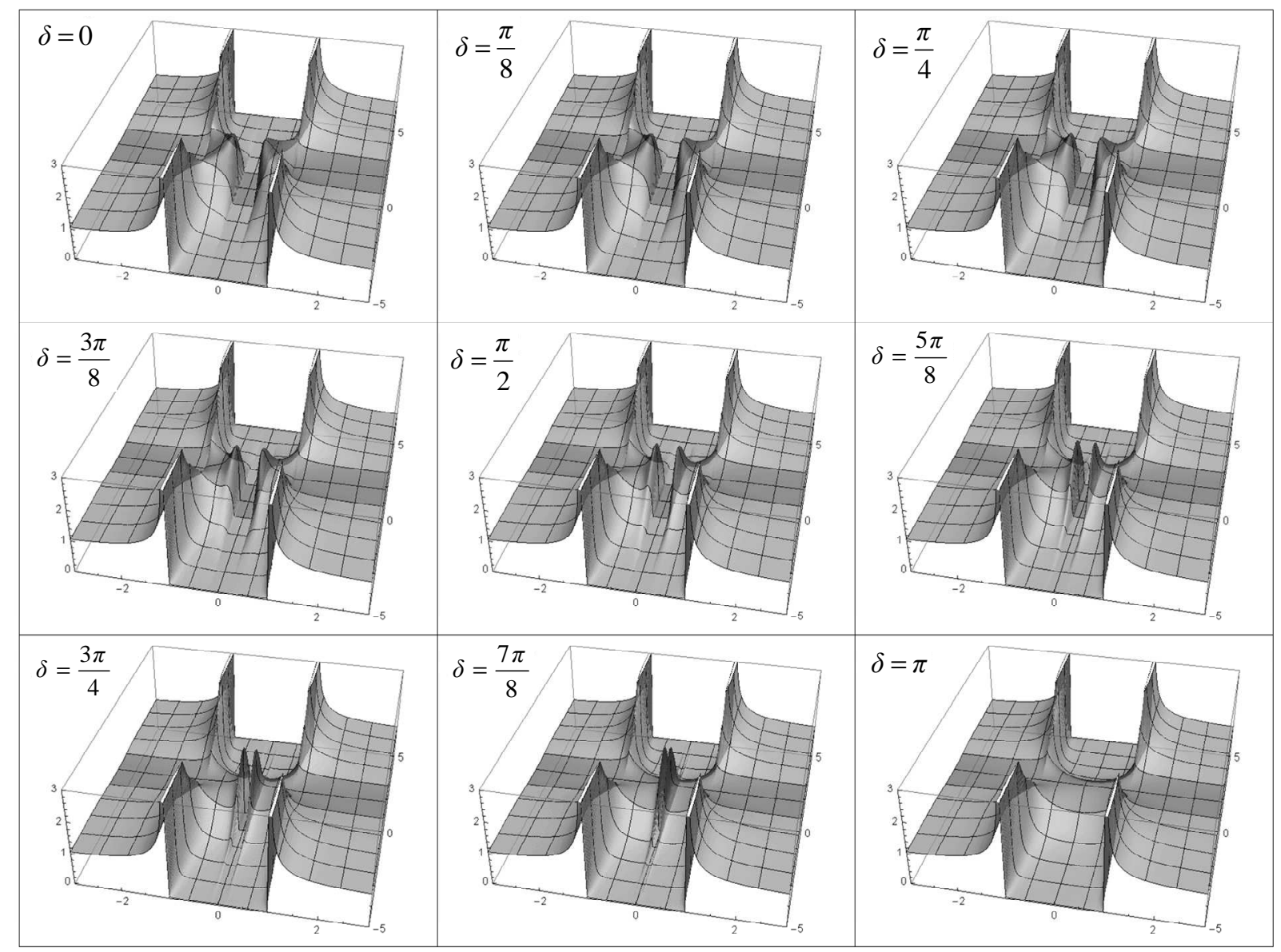

Fig. 4.14 Evolution of the DOS of an SNS structure (same as in Fig. 4.7 - Fig. 4.9 ) for a phase difference between the superconductors varying between 0 and $\pi$ (the behavior is even, $2 \pi$ periodic in phase difference). 


\subsection{SNS systems viewed as scattering structures}

Pioneered by Landauer, the description of quantum coherent structures in terms of scatterers for electronic conduction channels has proved a powerful way of understanding mesoscopic devices. In particular, its formal simplicity often allows intuitive insights on the behaviour of the systems. For proximity effect structures, it is a complementary tool for the Green functions approach which gives its predictions only through the "black box" of numerical resolution of the Usadel equations.

The scattering approach of SNS structures is well explained by Beenakker in Ref. [134]. In this approach one combines the ordinary electronic scattering matrix $s$ of the $\mathrm{N}$ part with the Andreev scattering matrix $a(\varepsilon, \varphi)$ describing the NS interfaces and which couples electron and hole motions in the $\mathrm{N}$ part of the structure. This matrix $a(\varepsilon, \varphi)$ depends on both the energy and the phase of the superconductor and can be expressed with the $\gamma_{S}$ given in (4.17). Working out the scattering matrix of the whole structure, one finds resonant conditions at well defined energies $|\varepsilon|<\Delta$, given by the roots of

$$
\operatorname{Det}\left[1-a\left(\varepsilon,-\varphi_{1}\right)^{*} s(\varepsilon) a\left(\varepsilon, \varphi_{2}\right) s(-\varepsilon)^{*}\right]=0
$$

where 1 is the unity matrix [134]. These resonances correspond to quasiparticle states for which after an Andreev reflection at each extremity the particle interferes in phase with itself (Fig. 4.15), giving rise to a localized state inside the N part, called an Andreev Bound State (ABS). Such a structure can be viewed as a Fabry-Perot resonator made with phaseconjugation mirrors, and the ABS are the modes of the resonator.

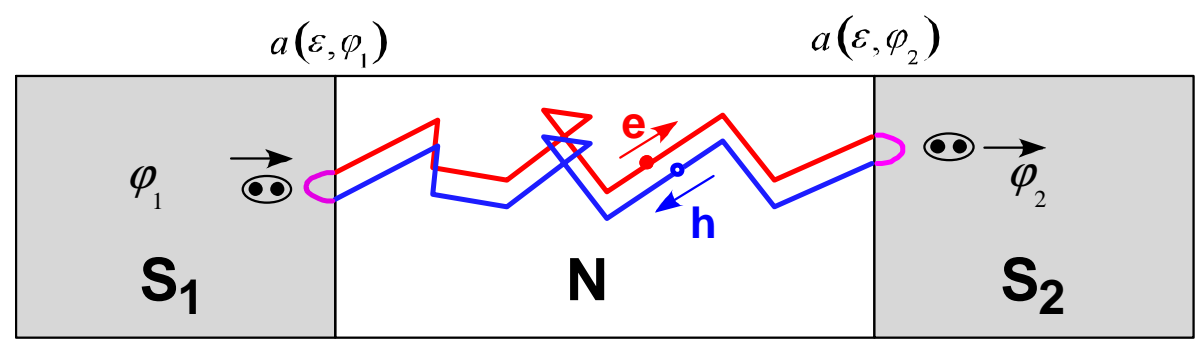

Fig. 4.15 Sketch explaining the formation of Andreev bound states in an SNS structure. At well defined energies, when following a trajectory such as described in this figure, the particle can interfere constructively, giving rise to a localized state with the particle confined mostly inside the normal metal.

All the properties of the system can be obtained from the global scattering matrix, provided the statistical properties of the constituents are known (and, in particular, their energy dependence) [151]. This is where this approach merges with the field of Random Matrix 
Theory [152]. In particular one can predict the DOS of the structure, which, at energies below $\Delta$, is the distribution of the roots of (4.21).

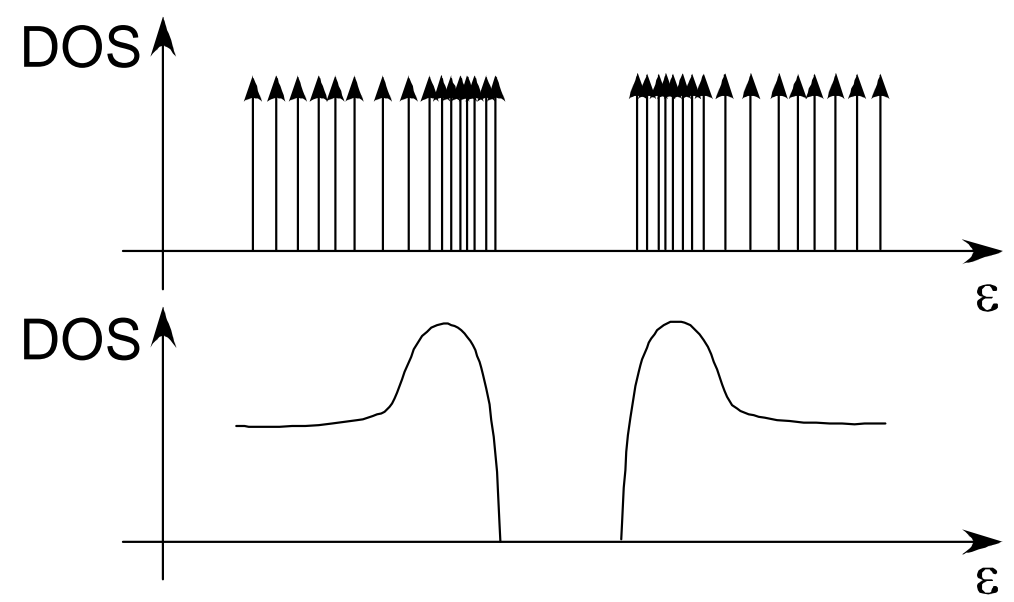

Fig. 4.16 Sketch showing the link between a discrete collection of Andreev bound states (top) obtained in the scattering formalism, and the DOS obtained from the Green functions formalism.

This approach can be simply and completely worked out in the case of short SNS structures $(L \ll \xi)$, for which the energy dependence of the $\mathrm{N}$ part scattering matrix can be neglected and assuming "rigid" BCS superconducting reservoirs (i.e. assuming no inverse proximity effect). The Andreev bound states are then completely determined and one predicts their energies to be [134]:

$$
\varepsilon_{i}^{ \pm}= \pm \Delta \sqrt{1-\tau_{i} \sin ^{2}\left(\frac{\delta}{2}\right)},
$$

where $\delta=\varphi_{2}-\varphi_{1}$ is the phase difference across the structure and $\left\{\tau_{1}, \ldots, \tau_{i}, \ldots, \tau_{n}\right\}$ is the set of eigenvalues of $s^{\dagger} s$, i.e. the normal state transmission probabilities of the electronic channels in the N part. The ABS come in pairs, symmetric about the Fermi energy, hence the \pm sign.

In this latter short contact limit with rigid BCS reservoirs, the fact that the pair interaction potential is zero or finite does not change the properties of the system (see e.g. blue curve in Fig. 4.11), and hence, this description applies as well to fully superconducting systems with a short constriction. This scattering approach of mesoscopic superconductivity has indeed been thoroughly tested in atomic contact experiments, in which the number of channels is small and their individual transmissions can be determined [153-157], and spectroscopic measurements of transitions among ABS using microwave irradiation are now being attempted. 


\subsection{An STM experiment on the proximity effect}

Although the proximity effect in various structures has been investigated for many years [158], no experiment could investigate it in detail. Many predictions were found in agreement with the theoretical predictions, though a comprehensive comparison is still lacking. The main results obtained are:

- The tunneling density of states was measured at a few distances away from a NS interface using a few fixed tunneling probes [122].

- The maximum supercurrent of an SNS structure was determined [159].

In order to probe the proximity effect in greater detail, we have fabricated a series of SNS structures of various sizes and measured the local tunneling DOS at many different positions in the structure. Our aim was in particular to investigate the dependence with the length of the $\mathrm{N}$ part, and the dependence of the proximity effect with the phase difference between the two $\mathrm{S}$ electrodes. Here, an important goal was to probe and measure the minigap in the local DOS predicted to occur for short enough $\mathrm{N}$ wires.

Last but not least, this experiment provided us with a real test-bed for the experimental system.

\subsubsection{Sample geometry}

Our aim is to investigate quasi-1D S-N-S structures in which the phase difference can be controlled externally. For this purpose, we have inserted an $\mathrm{N}$ wire in a superconducting loop, which allows to control this phase difference by an applied magnetic field. On the same sample, we designed many wires with different lengths covering all the different regimes of the proximity effect (\$4.4.4): the long junction regime equivalent to two independent S-(semiinfinite $\mathrm{N}$ wire) systems and in which the local DOS does not vary with the phase difference, and the short junction regime in which proximity superconductivity develops all along the $\mathrm{N}$ wire and in which the local DOS varies with the phase difference as explained in $\$ 4.4 .5$. The samples fabricated using electron beam lithography are shown in Fig. 4.17. 

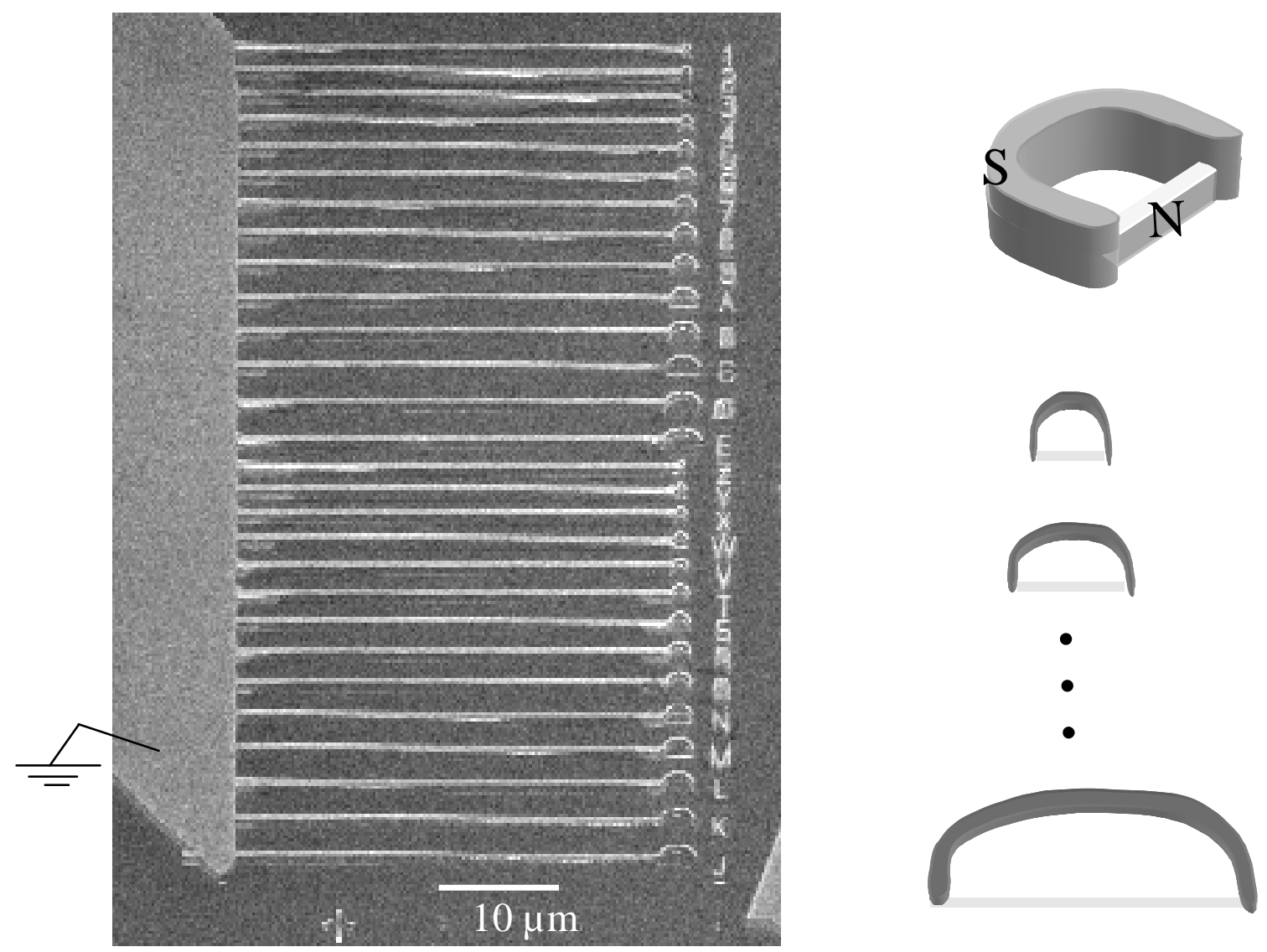

Fig. 4.17 Our samples consist of an array of SNS structures with a loop geometry, the normal wire being inserted in a superconducting loop. The normal length, ranges from $300 \mathrm{~nm}$ to $3 \mu \mathrm{m}$. The labels (number or letters) allow to identify the structure using AFM imaging. Leads connect all the structures to a common ground, necessary to sink the tip current in tunnel spectroscopy measurements.

\subsubsection{Implementation of the experiment}

\subsubsection{Achieving a good phase bias}

We discuss in this section the conditions for a suitable control of the phase difference across the $\mathrm{N}$ wire. The flux of the magnetic field determines the integral of the phase gradient around the loop. We designed our experiment so that the phase difference across the $\mathrm{N}$ wire is given by the flux applied externally. Two correcting effects have to be considered:

- A part of the integral arises from the $\mathrm{S}$ part of the wire due to its non-zero inductance.

- The applied magnetic flux is modified by the screening current that develops in the loop. 


\subsubsection{1.a Kinetic inductance correction}

At a given flux, the ratio between the phase difference accumulated along the $\mathrm{S}$ wire and along the $\mathrm{N}$ wire, $\delta^{(S)} / \delta^{(N)}$, is also the ratio of their kinetic inductances, if their geometric inductance is relatively small. The kinetic inductance of an S wire writes [160]:

$$
\mathcal{L}_{K}^{(S)}=\frac{1}{\pi} R_{N}^{(S)} \frac{\hbar}{\Delta}
$$

where $R_{N}^{(S)}$ is the normal state resistance of the $(\mathrm{S})$ wire. Similarly, the kinetic inductance of the $\mathrm{N}$ wire subject to proximity effect takes, up to a numerical factor ${ }^{1}$, a similar expression:

$$
\mathcal{L}_{K}^{(N)} \propto R_{N}^{(N)} \frac{\hbar}{\varepsilon_{G}}
$$

with $\varepsilon_{G}$ the minigap energy. One thus obtains the relation:

$$
\frac{\delta^{(S)}}{\delta^{(N)}} \approx \frac{\varepsilon_{G}}{\Delta} \frac{R_{N}^{(S)}}{R_{N}^{(N)}}
$$

This ratio is small for all our samples. It decreases from about $10^{-1}$ in the shortest wire down to $10^{-3}$ in the longest one.

\subsubsection{1.b Circulating currents correction}

The geometric self-inductance $\mathcal{L}_{G}$ of the loop brings a correction to the externally applied flux, due to the circulating currents (see Fig. 4.18). Since those currents depend themselves on the phase bias, this gives rise to a self-consistent equation for the true phase bias

$$
\delta=\frac{2 \pi}{\Phi_{0}}\left(\Phi_{e x t}-\mathcal{L}_{G} I(\delta)\right),
$$

whose solutions are a reduced phase $\delta$ for flux $\Phi_{\text {ext }}<\Phi_{0}$ and an enhanced phase above $[161,162]$.

When the product $\mathcal{L}_{G} i_{C}$ of the inductance and the critical current of the weak link is not small compared to the flux quantum, this causes hysteretic behaviour and a jump of phase bias around $\pi$ (see Fig. 4.18) [163].

However if the geometrical inductance term of Eq. (4.22) can be neglected, this effect does not show up. The geometrical inductance is at first order $\mathcal{L}_{G} \propto \mu_{0} l$ with $l$ the effective loop

\footnotetext{
${ }^{1}$ From [148] and [159], one can evaluate the kinetic inductance of a diffusive normal wire subject to proximity superconductivity, assuming $\varepsilon_{G} \sim 3.1 \varepsilon_{T}[143]$.
} 
radius (for the short wires the loop is almost circular, whereas for the long wires, the shape is ellipsoidal - see Fig. 4.19). For all wires, $\mathcal{L}_{G}$ is lower than $\mathcal{L}_{K}^{(N)}$ (see 4.6.2.1.a).

Since the critical current of the $\mathrm{N}$ part is $i_{C} \propto \varepsilon_{T} /\left(e R_{N}^{(N)}\right)$ [159], the maximum phase correction is $\delta_{G} \approx 2 \pi \mu_{0} l \varepsilon_{T} /\left(\Phi_{0} e R_{N}^{(N)}\right)$. This correction is negligible for all samples, including the shortest $\mathrm{N}$ wires. Numerical estimates are in the following ranges:

$$
\begin{gathered}
\mathcal{L}_{G} \in[1-2] \mathrm{pH}, \\
\mathcal{L}_{K}^{(S)} \in[3-9] \mathrm{pH}, \\
\mathcal{L}_{K}^{(N)} \in[25-5300] \mathrm{pH}, \\
\varepsilon_{T} \in[1.5-90] \mu \mathrm{eV}, \\
i_{C} \in[0.04-17.5] \mu \mathrm{A},
\end{gathered}
$$

yielding in the worst case (the smallest loop) a geometrical flux $\left(\mathcal{L}_{\mathrm{G}} i_{C}\right)$ around $1 \%$ of $\Phi_{0}$.
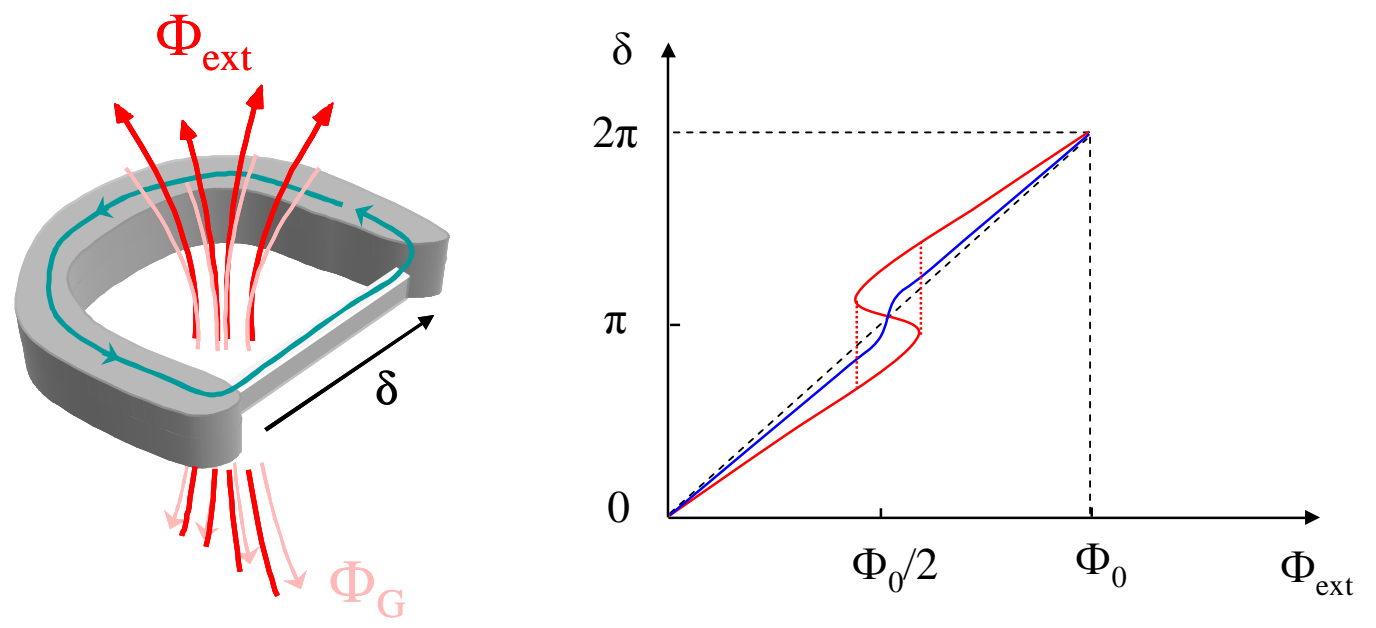

Fig. 4.18 Currents in the wire contribute to the total flux through the loop, which affects the phase difference across the $\mathrm{N}$ wire. In our experiment, this correction is small since the geometric inductance is small compared to the total kinetic inductance of the loop. The phase difference accumulated along the $\mathrm{S}$ part is also small compared to that along the $\mathrm{N}$ part because its kinetic inductance is smaller. Right: red curve: when the geometric inductance of the loop is not negligible the S-N-S junction exhibits an hysteretic behaviour, and there is a jump of phase around $\pi$; blue curve: in our experiment, the geometric inductance is negligible, and we can achieve phase biases continuously from 0 to $\pi$.

\subsubsection{Sample fabrication}

We have fabricated SNS structures made of aluminum and silver. Aluminum is a weak-coupling superconductor well described by the BCS theory, and silver a metal with 
good electronic coherence properties due to the rarity of magnetic impurities in the sources ${ }^{1}$. Furthermore, the Al-Ag interface can have a good transparency, which is essential to obtain a sizeable proximity effect. The interface quality is indeed of great importance for the propagation of electronic correlations into the normal metal (see $\$ 4.4 .3 \mathrm{p} \mathrm{190)}$ ).

For that reason, one has either to fabricate the sample by evaporating both layers without breaking the vacuum, or to mill the surface of the first layer before depositing the second one. Both techniques are described in Chapter 3. We have opted for the first one, using shadowmask deposition at two angles, with a bilayer of resist forming a suspended mask.

This method faces the difficulty that two distinct electrodes (the normal and the superconducting ones) have to be deposited, with only two connections, and no overlap at any other place. This imposes both small structures and large deposition angles through a suspended mask with a large height. To put numbers, we employed $\pm 17^{\circ}$ deposition angles on $\mathrm{a} \sim 1 \mu \mathrm{m} / 70 \mathrm{~nm}$ thick MAA / PMMA bilayer, which yields a $\sim 600 \mathrm{~nm}$ lateral displacement sufficient to avoid overlap between the patterns. The loops are half ellipses, with one radius defined by half the normal length from $150 \mathrm{~nm}$ to $1.5 \mu \mathrm{m}$, the other radius being at most $1 \mu \mathrm{m}$ for the largest and $0.6 \mu \mathrm{m}$ for the smallest. This method also faces the challenge to deposit thin and narrow wires (nominal width of $50 \mathrm{~nm}$ ) at large angles. Indeed, the deformation of the suspended mask due to stresses results in wires wider than expected (up to $70 \mathrm{~nm}$ ), and with a poorly defined shape.

Avoiding overlaps between $\mathrm{N}$ and $\mathrm{S}$ in another place than the wire edges ensures that the superconducting region remains genuine, which allows considering a bulk superconductor away from the interface, and investigating the inverse proximity effect in the superconductor.

\subsubsection{Overview of the measured SNS structures}

Some of the structures of Fig. 4.17 which we measured during the experiment are shown below with their dimensions. Those photographs were taken in a SEM after the experiment was completed.

\footnotetext{
${ }^{1} \mathrm{Fe}$ is often the dominant impurity in $\mathrm{Au}$, and copper oxide is magnetic.
} 

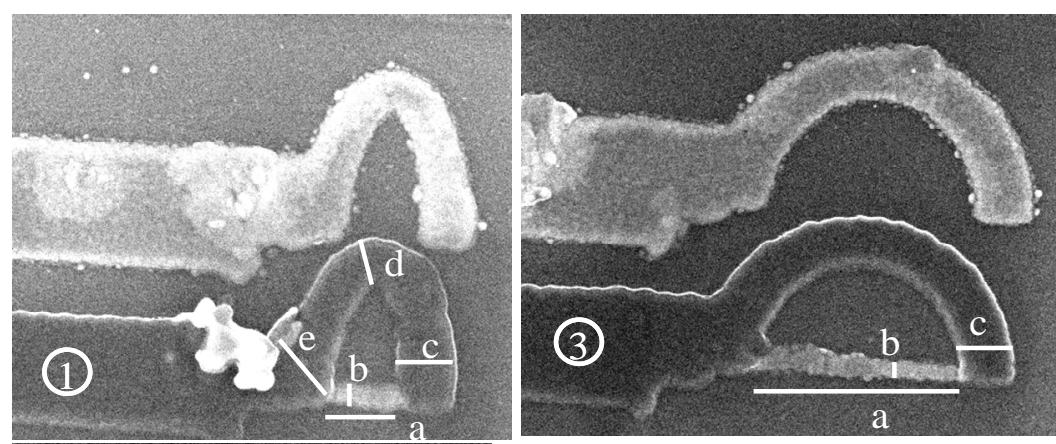

\begin{tabular}{|c|c|c|c|c|c|}
\hline & & (1) & (2) & (3) & (4) \\
\hline \multirow{2}{*}{$L_{N}$} & nom. & 400 & 600 & 1000 & 2800 \\
\cline { 2 - 6 } & meas & 405 & 575 & 975 & 2810 \\
\hline \multirow{3}{*}{ a } & nom. & 300 & 500 & 900 & 2700 \\
\cline { 2 - 6 } & meas & 307 & 430 & 870 & 2550 \\
\hline \multicolumn{2}{|c|}{ b } & 59 & 65 & 61 & 64 \\
\hline \multicolumn{2}{|c|}{$\mathrm{c}$} & 220 & 275 & 240 & 220 \\
\hline \multicolumn{2}{|c|}{$\mathrm{d}$} & 175 & 300 & & \\
\hline \multicolumn{2}{|c|}{$\mathrm{e}$} & 300 & 360 & & \\
\hline
\end{tabular}
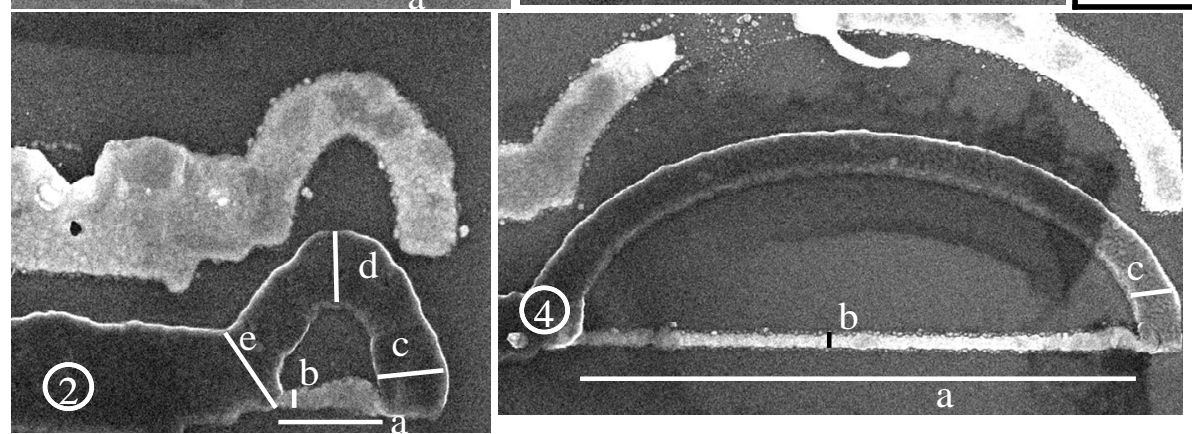

Table: dimensions (nm)

Fig. 4.19 Scanning electron micrographs of the S-N-S proximity structures. The darker material is aluminum, and the lighter is silver. Silver (normal) wires of different lengths are connected across a loop of aluminum (superconductor). The total length $L_{N}$ of each wire (indicated on the table) was measured from the maxima of the overlap regions, with an AFM. Both nominal and measured length are mentioned. Other dimensions were measured with the SEM; in particular the "free" length (a) of the wires, difficult to obtain with the AFM due to tip size effects. The wires were designed to be $50 \mathrm{~nm}$ wide but they come out slightly wider. Marks on the different structures come from the tip reshaping procedures (see chap 3), done to recover a good tip shape in situ. From place to place, the normal wire is enlarged, possibly due to mask deformation.

\subsubsection{What do we measure?}

During this experiment we have first used our AFM to locate the systems, and image them. Once a satisfying image was obtained, we turned off the AFM mode (see chap 2), positioned our tip above the desired place, formed a tunneling contact between the tip and the sample with a resistance in the $100 \mathrm{k}-1 \mathrm{M} \Omega$ range, and recorded the conductance of the contact as a function of voltage, flux through the loop....

We first present the complete set of data taken on the four systems shown in Fig. 4.19, and we draw the main conclusions that can be inferred from them. The longest wire (\#4) in which the proximity effects developed at both ends do not interfere in the middle of the wire is described first; we then present the strong interference regime that occurs in the short wires \#1 and \#2; the intermediate regime found in wire \#3 is then presented. 
Throughout the whole data presented in this chapter, the positions at which the spectra were taken are indicated on an AFM image, with arrows of different colours labelling the corresponding curves. All plots presenting the position dependence follow this format. All spectra are normalized to the high voltage conductance value, for the ease of comparison, and usually offset by integers for clarity.

\subsubsection{Density of states in a long SNS structure}

The first measurements I will present here were done in the longest wire of length $L_{N}=2.8 \mu \mathrm{m}$ labelled 4 in Fig. 4.19. The density of states measured at various positions in the normal wire \#4, and at zero magnetic flux, is shown in Fig. 4.20. The DOS differs from its normal state value only close to the NS interface, and no effect is observed in the center part of the wire. At $30 \mathrm{mK}$, the thermal length $L_{T}=\sqrt{\hbar D / k_{B} T}$ is $\sim 2 \mu \mathrm{m}$ for $D=170 \mathrm{~cm}^{2} / \mathrm{s}$, roughly equal to the $\mathrm{N}$ wire length. In this regime, one expects a small reduction of the density of states in the central part of the wire. Interface imperfections can further reduce the proximity effect.

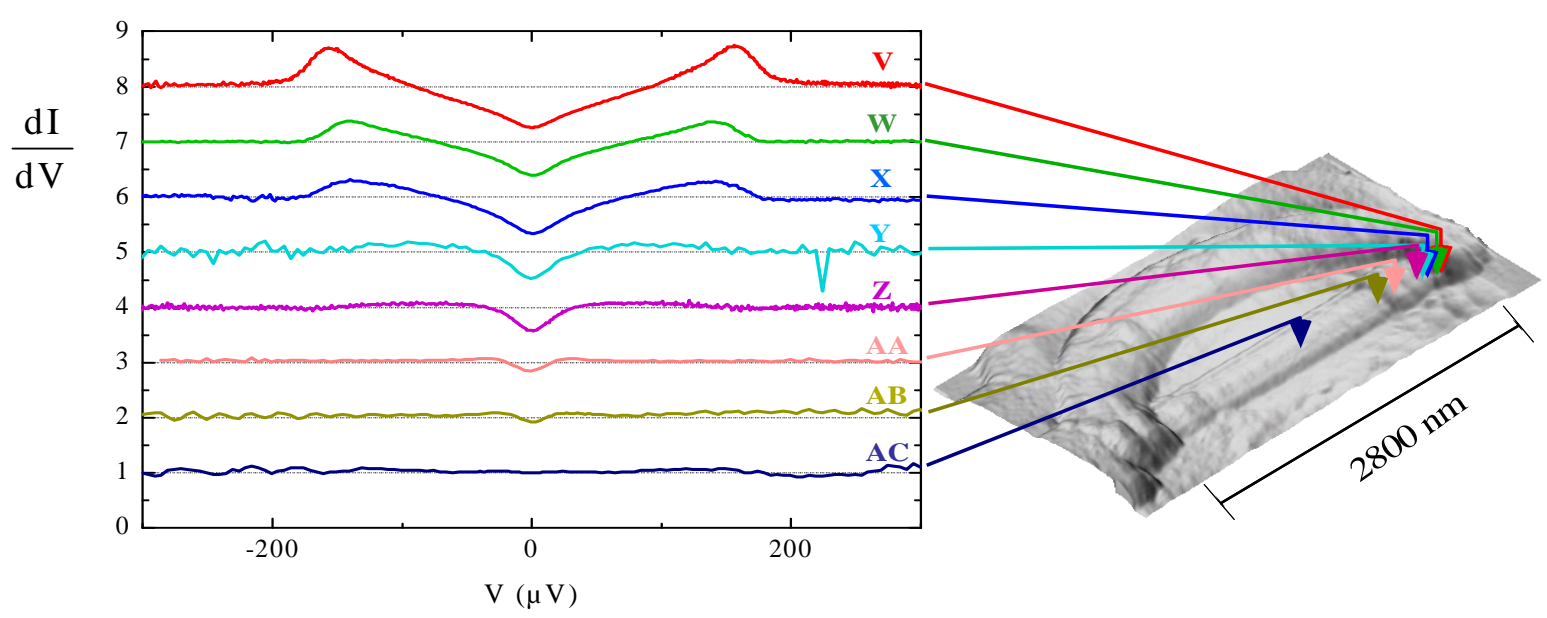

Fig. 4.20 In a normal wire of total length $L_{N}=2800 \mathrm{~nm}$ the density of states exhibits strong modification close to the interface with the superconductor, and recovers the shape of a normal metal density of states (i.e. a flat one) as one goes away from the superconductor. On the right is shown the AFM picture of the structure, on which are indicated the various contact points. On the left are plotted the normalized conductance data taken at each contact point. Curves are offset by integers for clarity.

The proximity effect decays with the distance to the interface at all energies. At the Fermi level, proximity effect has the longest extension in the normal region. In Fig. 4.21 is plotted the evolution of the DOS at the Fermi level as a function of distance from the interface. We 
find that the recovery to the normal DOS is close to an exponential, with a characteristic length of $240 \mathrm{~nm}$.

In absence of proximity effect in the central part of the wire, no effect of the phase difference is expected. This will have to be checked in the next experiment since it was not done during the present one. When these data were taken, we could not drive enough current in the coil, and this wire was not measured again once we had solved the coil issue.

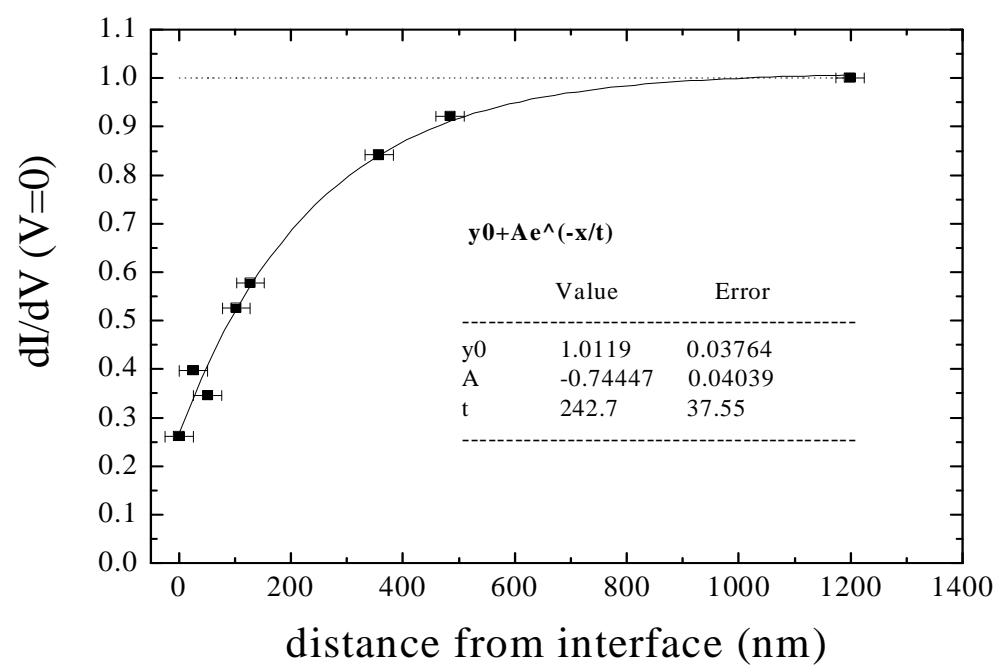

Fig. 4.21 Normalized conductance at the Fermi level as a function of distance to the interface. The density of states recovers progressively its normal state value, with a characteristic distance of $240 \mathrm{~nm}$. The positions are indicated with an error bar of $50 \mathrm{~nm}$ (see $\$ 4.6 .7)$.

\subsubsection{Density of states in a short SNS structure}

\subsubsection{1 position dependence}

We consider here wires with total length $400 \mathrm{~nm}$ (\#1) and $600 \mathrm{~nm}(\# 2)$, and with an overlap region of $\sim 50 \mathrm{~nm}$ for $\# 1$ and $\sim 70 \mathrm{~nm}$ for \#2 at each end (see discussion in $§ 4.6 .7$ ).

Local DOS data are presented for the two wires in Fig. 4.22 and Fig. 4.23. In each structure the density of states was measured at various positions in the normal wire and in the superconducting electrode.

- Wire \#1 (length: $400 \mathrm{~nm}$ )

One observes on those plots the presence of a true energy gap independent of position in the $\mathrm{N}$ wire: this is the predicted minigap. On the superconducting side, and close to the interface, one notices the existence of states between the minigap and the superconducting gap. This phenomenon, called the inverse superconducting proximity effect (see 4.4.2), had not been observed before. 
These measurements of inverse proximity effect are technically challenging, because the DOS is small, which requires a high current sensitivity (see chapter 3 ). The measured currents were of the order of $20 \mathrm{fA}$ in the minigap region, and $100 \mathrm{fA}$ in between the minigap and the gap, with a noise of $\sim 5$ fA. Data B shown on a logarithmic scale in Fig. 4.22 allows to estimate the measurement noise: an apparent conductance of $\sim 710^{-3} \mu \mathrm{S}$ is measured in the minigap with an $\mathrm{AC}$ excitation of $3 \mu \mathrm{V}$, and the peak to peak noise is $210^{-3} \mu \mathrm{S}$. We take the value of $710^{-3} \mu \mathrm{S}$ to be the offset of our measurement in that case: this was the typical value that we read when the tip was away from the sample, which can be seen as a leak resistor in the setup. 

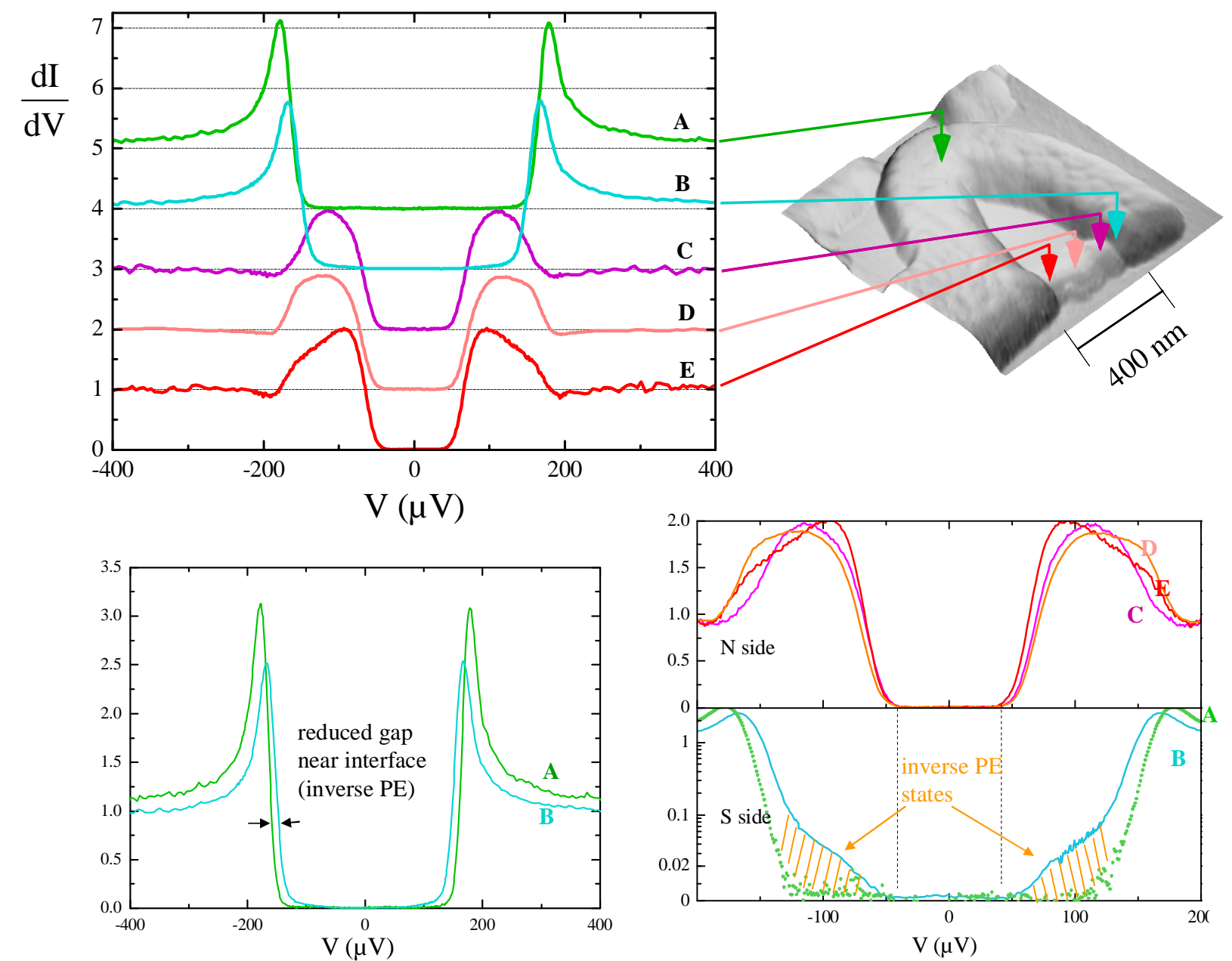

Fig. 4.22 Local DOS in the shortest wire measured, with total length $400 \mathrm{~nm}$ and "free" length around $300 \mathrm{~nm}$. The spectra are recorded at various points in the structure, both in the normal and the superconducting regions. Data are plotted on the graph (upper left), and the corresponding positions are shown on the AFM image (upper right). In the normal wire, we show three contact positions labeled C, D and E. Tunneling data at those points exhibit a true minigap around the Fermi level, with a width independent of position. The density of states varies nevertheless strongly with position, as shown in the lower-right graph. The tunnel spectroscopy has also been recorded in the superconducting region at positions labeled $\mathrm{A}$ and $\mathrm{B}$, A being far from the normal region, and $\mathrm{B}$ close to it. Data taken at these points are shown on the lower-left graph: the density of states at position B is non-zero below the gap energy. To make it clear, data at position A and B are also shown using a logarithmic scale, on the lower-right graph. Unfortunately, data were noisier at pos. A due to less averaging, and they are spoiled by a high noise level, compared to pos. B. A non-zero DOS is observed below the superconducting gap, but not below an energy close to the minigap energy, observed in curves C, D, E and B. 
- Wire \#2 (length: $600 \mathrm{~nm}$ )

Similar observations were performed in this slightly longer wire. The main difference is a smaller minigap energy, as expected.

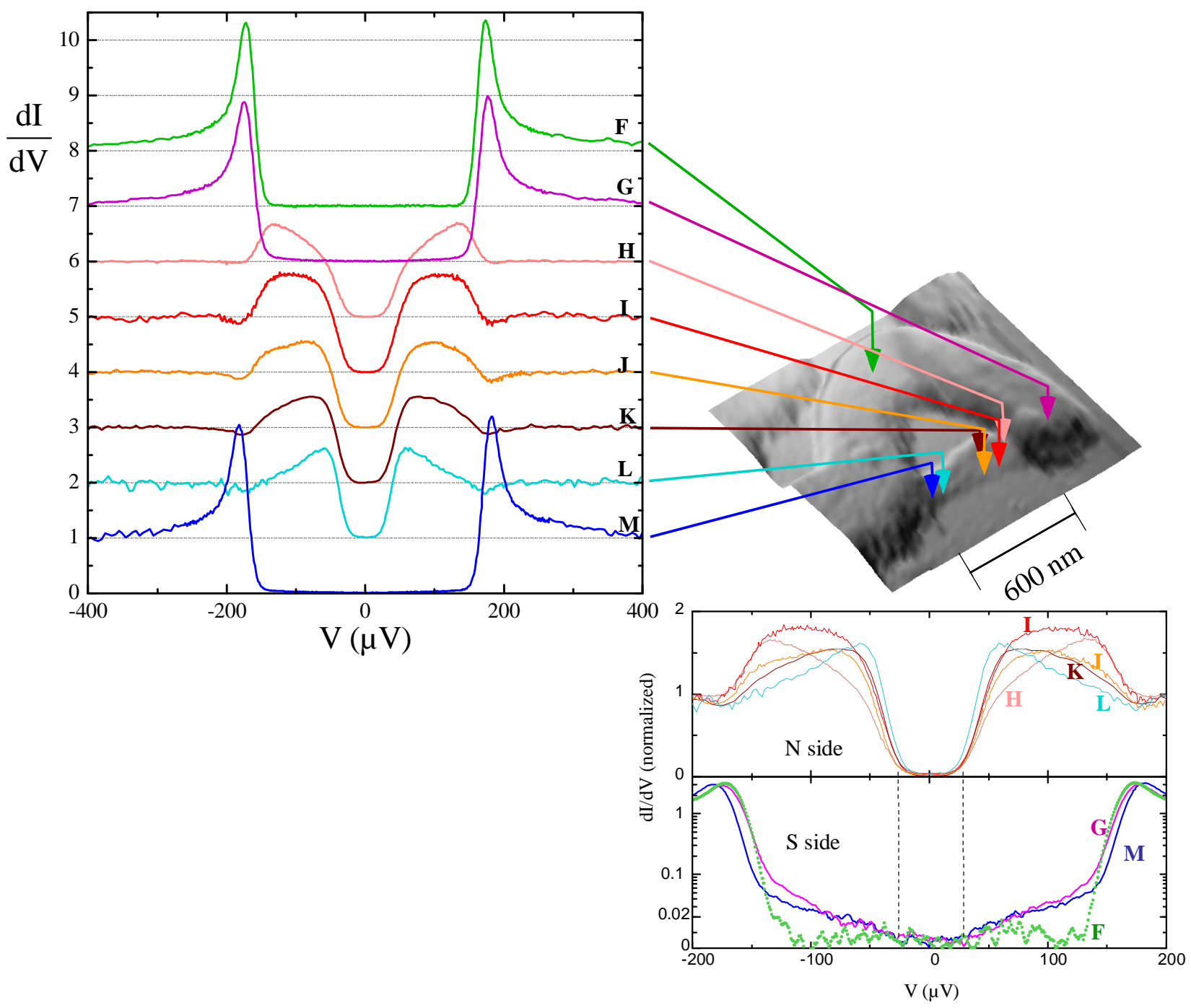

Fig. 4.23 Local DOS in the wire \#2 $(600 \mathrm{~nm})$. A minigap is observed again, smaller than in wire \#1. The graphs have the same formatting than in Fig. 4.22. The spectra on the S side that were not taken with enough sensitivity to display them in logarithmic plot. Positions in S close to the interface $(G, M)$ still show a characteristic energy which coincides with the minigap, above which a non-zero DOS can be seen (contrary to what happens in the bulk at pos. F). As in Fig. 4.22 positions $A$ and $B, F$ should exhibit a larger gap than $G$ and $M$. The variations of superconducting gap between these different positions may arise from thickness variations of the aluminum wire (the thinner, the higher the gap). Even though M seems closer to the normal wire than $\mathrm{G}$ on the image, the DOS at G is not less affected by inverse PE than the DOS M (see bottom-right panel). In fact, $\mathrm{G}$ is on top of the N-S overlap (see topography). Adjustements of the data (see \$4.6.7.2) showed that the interface near $\mathrm{G}$ is cleaner than the other interface, which explains why the gap is more reduced at G. 


\subsubsection{2 phase dependence}

At each position presented on Fig. 4.22 and Fig. 4.23, the phase was modulated by applying a magnetic flux. Fig. 4.24 shows how the minigap closes as the phase goes through $\pi$, as well as the appearance of features in the DOS shape.

Noticeably, a peak emerges on the minigap edge, similar to the BCS coherence peak in a true superconductor. This is what one would naively expect in a superconductor with an order parameter equal to the minigap. Since the minigap varies with the phase difference, the peak is displaced and its shape evolves with the phase difference. 


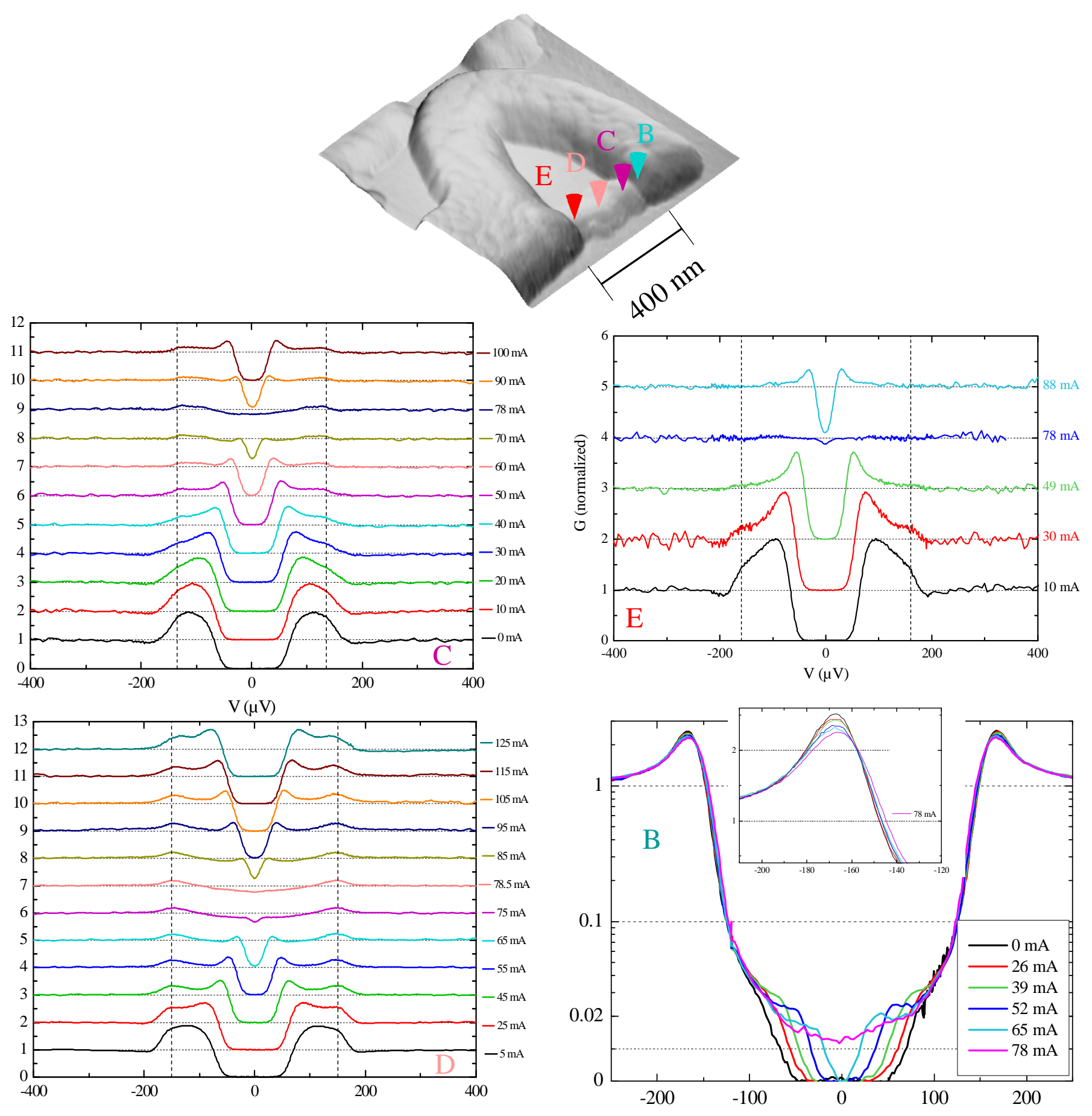

Fig. 4.24 Normalized conductance for different values of current in the coil; each graph represents a different positions in wire \#1, labeled by the same letters as in Fig. 4.22. For clarity, curves are displaced by integers on all graphs except for the position B in the superconductor where data are shown on a logarithmic scale, for there is only a small amount of states that are modified by inverse proximity effect. 

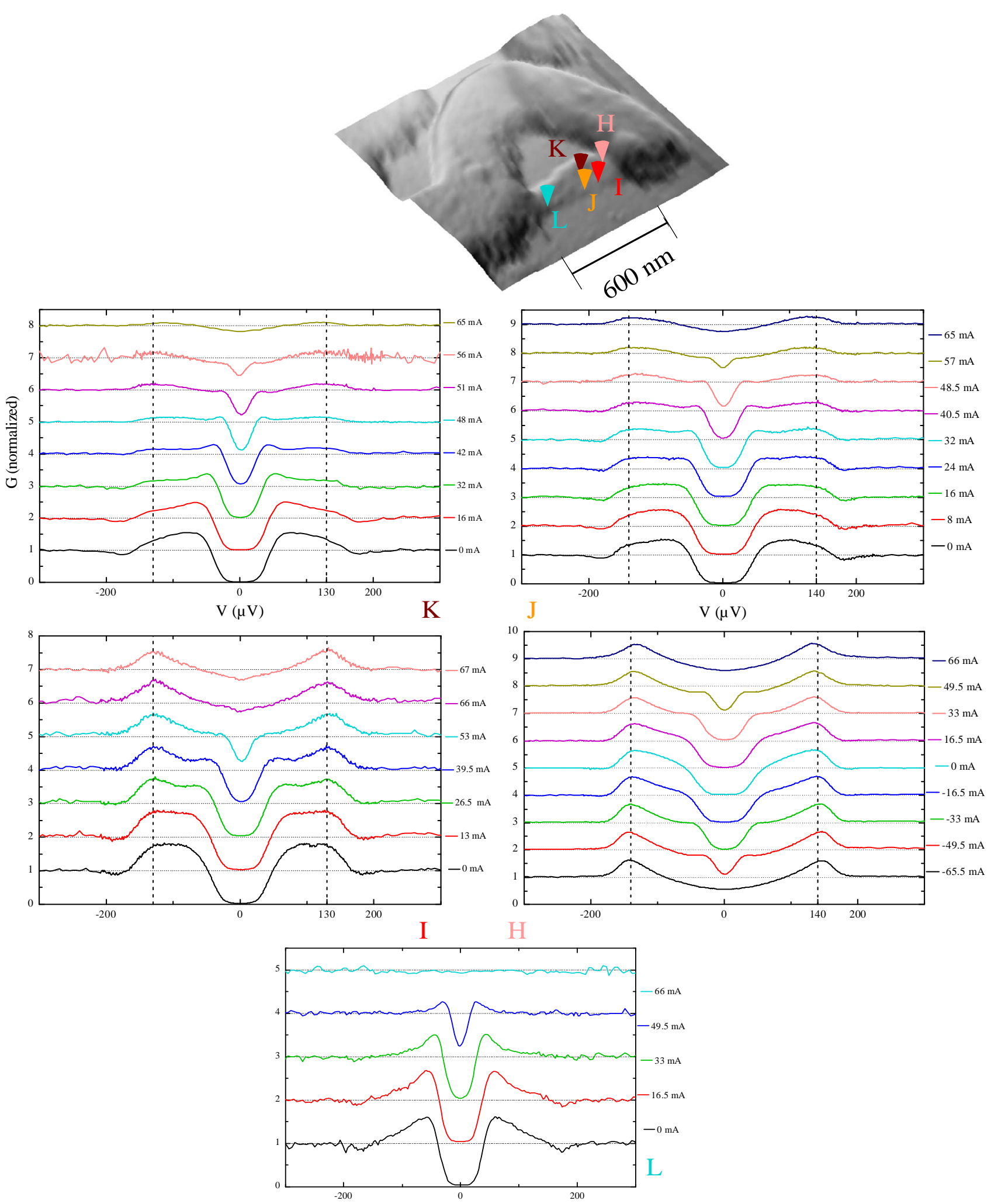

Fig. 4.25 Same as in Fig. 4.24 for wire \#2. Positions G and M on the superconducting side were not measured as a function of phase bias. 


\subsubsection{2.b Phase calibration}

In order to obtain the coil current to phase conversion, we determine the flux which corresponds to a $\pi$ phase difference. We find $78 \pm 1 \mathrm{~mA}$ in wire \#1, and $65 \pm 1 \mathrm{~mA}$ in wire \#2. All data plotted as a function of the phase assume those values calibration values.

\section{phase difference}

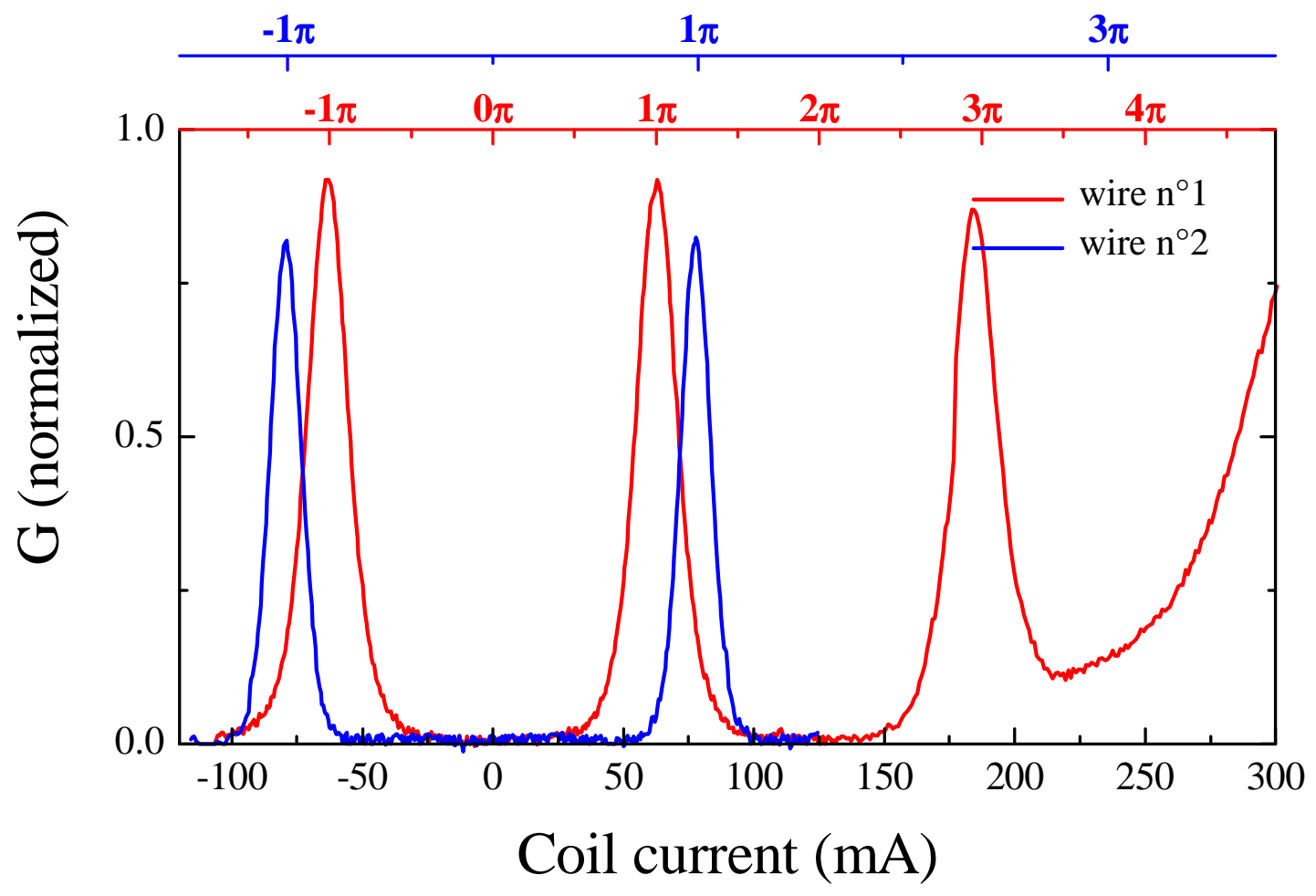

Fig. 4.26 Normalized conductance at the Fermi level, as a function of coil current, at one position in the two wires presented in this section. Since the size of the loop is not constant, one has to apply a higher magnetic field to thread one flux quantum through the loop, so the period is not a constant function of coil current. The $\mathrm{X}$ axis is shown either in direct coil current values (bottom axis) or in phase value (top axis), assuming the aforementioned values of the current for a $\pi$ phase bias. When the coil current is too high, one can observe depairing in the superconductor, resulting in an increase in the Fermi level density of states. This is observed above $180 \mathrm{~mA}$ at the position presented here in wire \#2. The data were not taken in wire \#2 for I $>125 \mathrm{~mA}$.

\subsubsection{2.c minigap as a function of phase}

The criterion employed to determine the amplitude of the minigap should rigorously detect the value of polarization at which the density of states begins to be non zero, with a threshold as small as the accuracy in the DOS measurement. However, due to finite energy resolution of the density of states measurement (see Chap. 3), this criterion yields a zero gap value over a whole phase range around $\pi$. To go beyond this limitation, and show data on the whole range of phase bias, we define the minigap amplitude to be the position of the maximum of the 
derivative of each curve, obtained numerically from the data. Gap determinations obtained with this procedure are shown in Fig. 4.27.

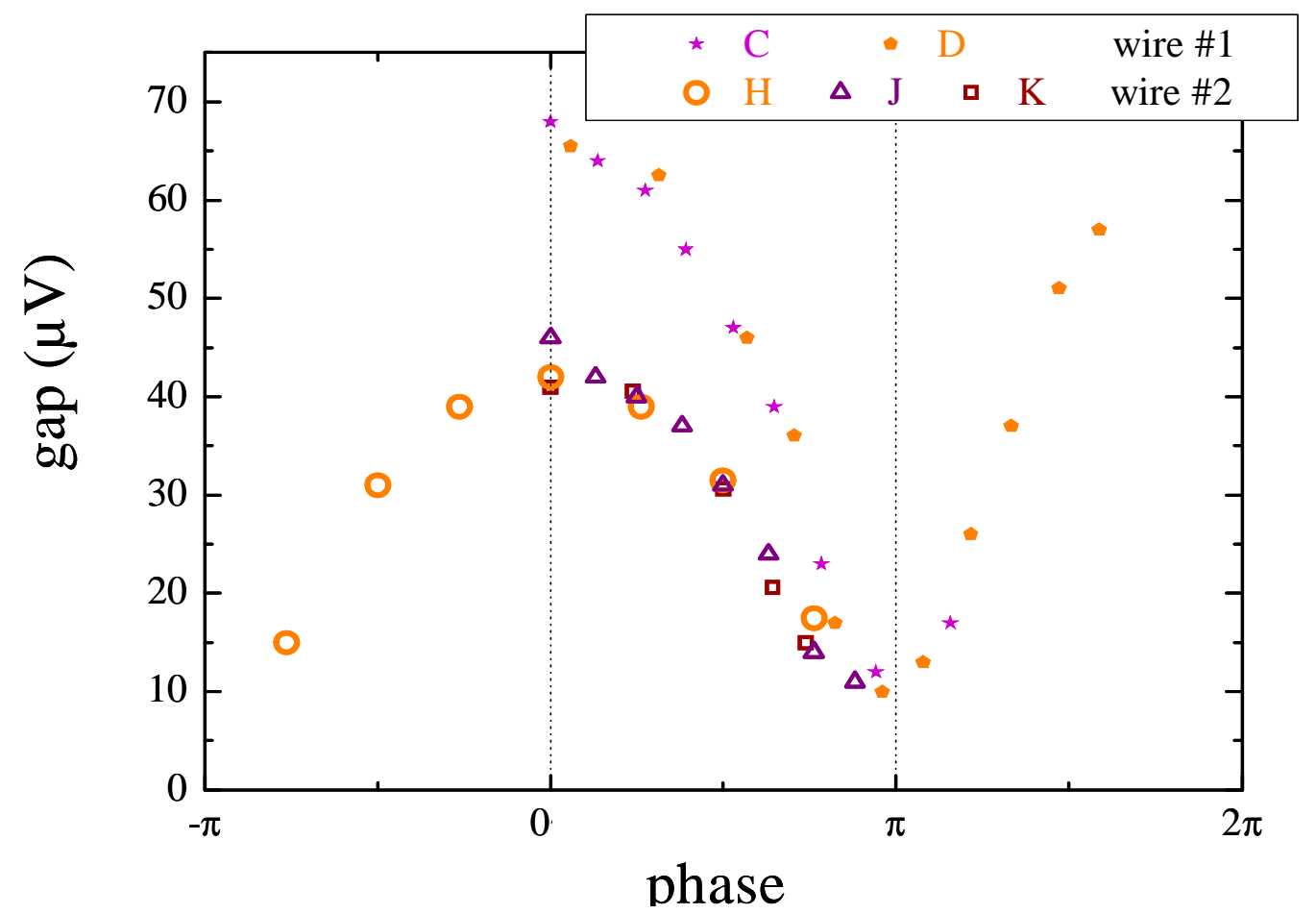

Fig. 4.27 In wires \#1 and \#2 the gap amplitude has been plotted as a function of phase bias. One sees a diminution of the gap amplitude as the phase increases towards $\pi$, and an increase after $\pi$. Considering all the data, one can see this behavior is symmetric around 0 and $\pi$. For each wire, data are shown for various positions, and the coincidence between data show that the minigap amplitude is independent on position.

\subsubsection{Is the Al electrode affected by the applied field?}

The phase modulation data that we have presented require to apply magnetic fields not small compared to the critical field of the Aluminum films. Our current to field calibration, derived from the flux calibration using the loop area, is 0.65 Gauss $/ \mathrm{mA}$. The field applied in the phase modulation experiments, up to about 50 Gauss, is not small compared to the critical field of $\mathrm{Al}$ wires. The depairing due to the field is characterized by the depairing energy [148]

$$
\Gamma(B)=\frac{D}{6 \hbar} e^{2} B^{2} w^{2},
$$

with $B$ the magnetic field and $w$ the width of the superconductor.

The predictions of this model were found in quantitative agreement with experiments performed on Al wires with similar widths [148, 164]. 
The DOS measurements taken in the Al electrode at two positions close to the NS interface show an effect of the magnetic field over the coil-current range $(70 \mathrm{~mA})$ explored in phase modulation experiments. These data, presented in Fig. 4.28, point out that the depairing effect is larger in the region close to the current-sinking wire (see Fig. 4.17) with a larger width of $500 \mathrm{~nm}$, than on the narrower opposite side, where the depairing is smaller. Fits with the Skalski model [165] are consistent with the estimated width variations.

Note however that the simple wire model yielding (4.23) is not rigorous for our structure whose geometry varies on the length-scale of the wire width. These results also indicate that a symmetric design of the $\mathrm{S}$ electrode at the two ends of the $\mathrm{N}$ wire would have been more appropriate.

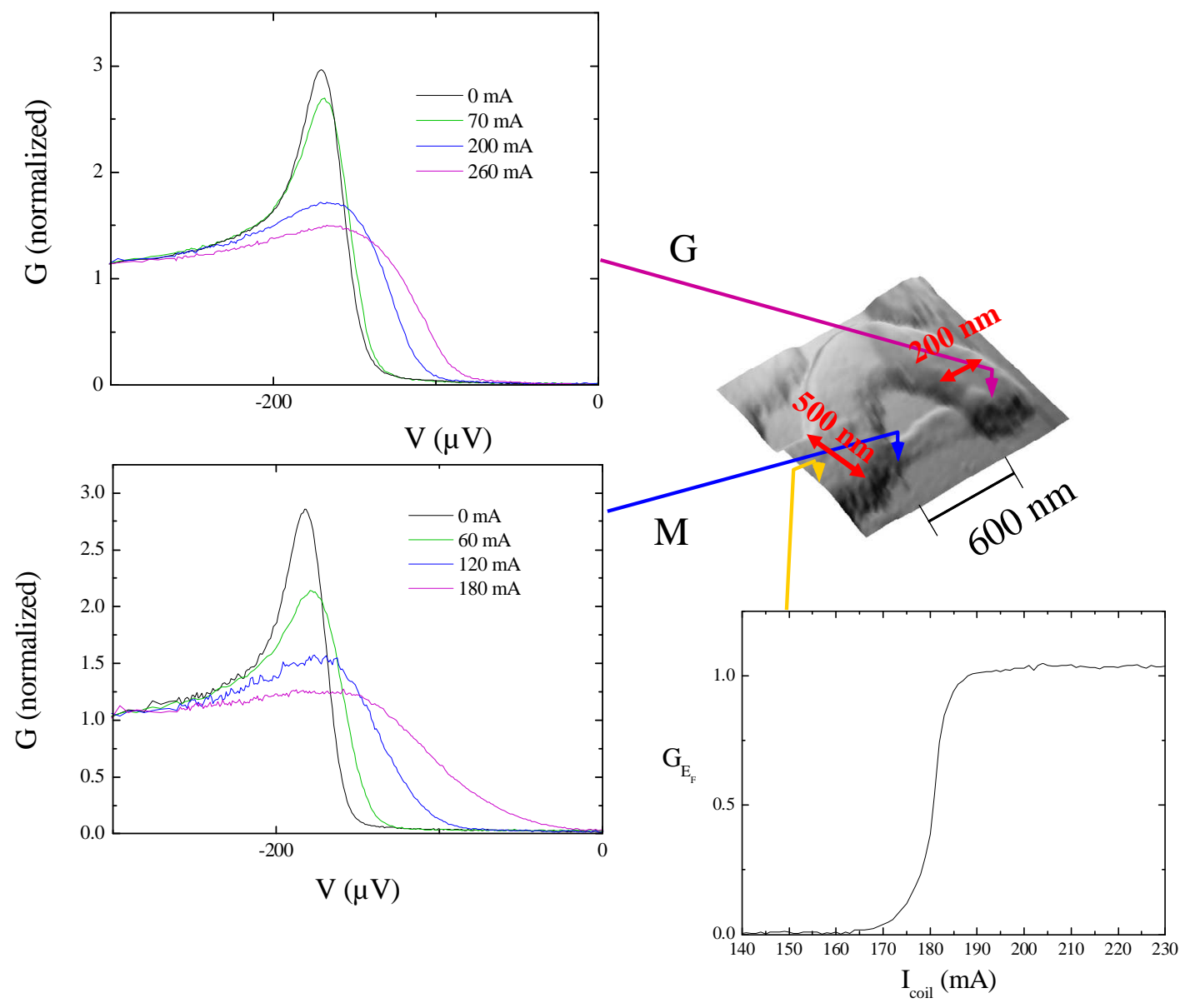

Fig. 4.28 Left: Local DOS at two positions in the Al S electrode, for different coil currents $(0.65 \mathrm{G} / \mathrm{mA})$. The gap reduction is slightly more important at position $\mathrm{M}$ than at position $\mathrm{G}$ because of a larger depairing energy in $\mathrm{M}$, due to a wider electrode. Bottom right: Variations of the zero energy DOS with the coil-curren, in the bulk of the sinking wire. The critical field here is about 120 Gauss, for a wire of $500 \mathrm{~nm}$-width. 


\subsubsection{Density of states in a medium-size SNS structure}

\subsubsection{Intermediate regime}

When the length of the normal wire is increased, superconducting correlations are expected to be present throughout the structure, till it exceeds the thermal length. From the experimental point of view, the picture is however less simple because, when the minigap becomes too small, the resolution achieved with the tunneling method (see chapter 1 and 3) does not allow to observe a zero DOS inside the minigap, but only a dip. Note that this was already observed in the phase dependence of the minigap around $\pi$.

In the $1 \mu \mathrm{m}$-long wire \#3 the minigap, estimated by extrapolating the values obtained on the other wires, is $9 \mu \mathrm{V}$. Given the $10 \mu \mathrm{V}$ resolution of our microscope, we indeed cannot expect to observe a true gap.

Data taken in wire \#3 are plotted on Fig. 4.29. Close to the interface, the curves distinguish by pronounced features just below $\Delta$ on the $\mathrm{N}$ side, and by a peak at $E=\Delta$ on the $\mathrm{S}$ side. As one goes towards the middle of the wire, features appear at lower energy: states aggregate at the minigap edge. 


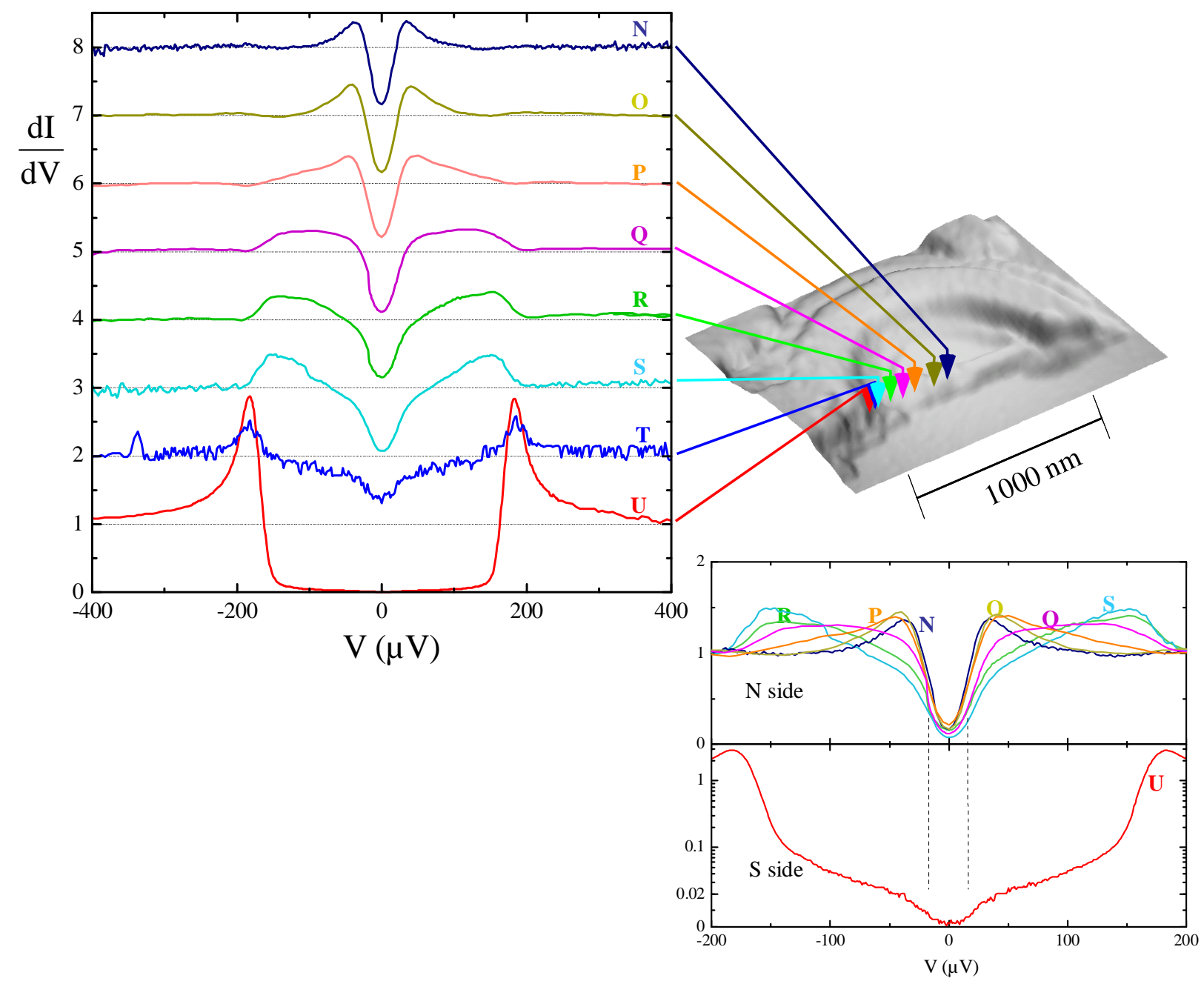

Fig. 4.29 Left: Local DOS in the $1 \mu \mathrm{m}$ long wire \#3. Spectra were taken at positions very close to the $\mathrm{N} \mathrm{S}$ interface, as shown at $\mathrm{U}$ on the $\mathrm{S}$ side, and $\mathrm{S}, \mathrm{T}$ on the $\mathrm{N}$ side. The spectrum evolves from a near-BCS spectrum at position U (see Fig. 4.7), to a gapless spectrum at position $\mathrm{T}$ still on the $\mathrm{S}$ side. Other positions display some of the features shown in Fig. 4.22 and Fig. 4.23. An estimated minigap can be obtained from the width of the conductance dip (dashed lines). Position $\mathrm{T}$ is noisy partly due to mechanical instabilities of this contact, and partly to the very low conductance of the contact: we did not achieve more than $\sim 0.04 \mu \mathrm{S}$ at this position and could never again observe this kind of data.

\subsubsection{Comparison with theory}

We now compare the above results to the predictions of the quasiclassical theory presented above, using the the technique described in $\S 4.3$.

\subsubsection{Parameters entering the theory}

(i) For the superconducting aluminum electrode, we only need to know the value of the gap of aluminum $\Delta$. This value is obtained from a separate DOS measurement in the bulk, i.e. "far away" from the normal region and at zero magnetic field. As discussed in chapters 1 and 3, the conductance is the density of states convolved with the electronic energy distribution 
function in the tip. From the fits, one obtains both the temperature of electrons in the tip, and the gap of the superconductor. This value of $\Delta$ should be constant on all the samples, but actually, the apparent gap near low resistance interfaces needs to be reduced by a few percent, to take into account inverse proximity effect which is not calculated self-consistently here, for simplicity (cf. Fig. 4.22).

(ii) For the $\mathrm{N}$ wire, we mainly need to know the diffusion constant $D$. It is assumed to be the same for all wires, fabricated in the same deposition step. The diffusion constant in the $\mathrm{S}$ electrode also enters the theory, but we noticed that the predictions are weakly sensitive to this value, due to interface parameters (see point $(i v)$ ), so we took the same value as for silver.

(iii) Geometrical parameters and positioning accuracy: The 1-D approximation used in the numerical calculations requires making some simplifying assumptions on the geometry and defining effective lengths (See Fig. 4.30). In particular, the effective length $\ell$ of the wire is taken intermediate between the "free" length $a$ and the total length $L_{\mathrm{N}}$. Even in the absence of positional uncertainty relative to the sample image, the position of the tip in the model also needs to be redefined: in the model, what is relevant is the distance to a sharp interface, whereas in the experiment, the position of the tip is not at a well defined distance from the interface. Furthermore, the tip-to-interface distance $x_{\text {eff }}$ entering the 1-D model is different from the distance $x$ to the apparent interface in the AFM image. In the crudest approximation, we consider, in the $\mathrm{N}$ wire,

$$
x_{\mathrm{eff}}=x+\frac{\ell-a}{2} .
$$

We stress here that this is not to be taken as an exact mapping: such a mapping does not exist. On the $\mathrm{S}$ side also, a similar redefinition is required, since the $\mathrm{Al}$ thickness has to be taken into account.

Once this mapping done, one expects the discrepancies between the 1-D model and the experiment to be in relative most important for the shortest wires and close to the interfaces. Additionally, the experimental tip positioning accuracy, mainly limited by tip deformations, is estimated at $\pm 25 \mathrm{~nm}$ in worst cases. Hence, small adjustments of the positions were allowed for a few curves in order to obtain a predicted DOS in better agreement. 

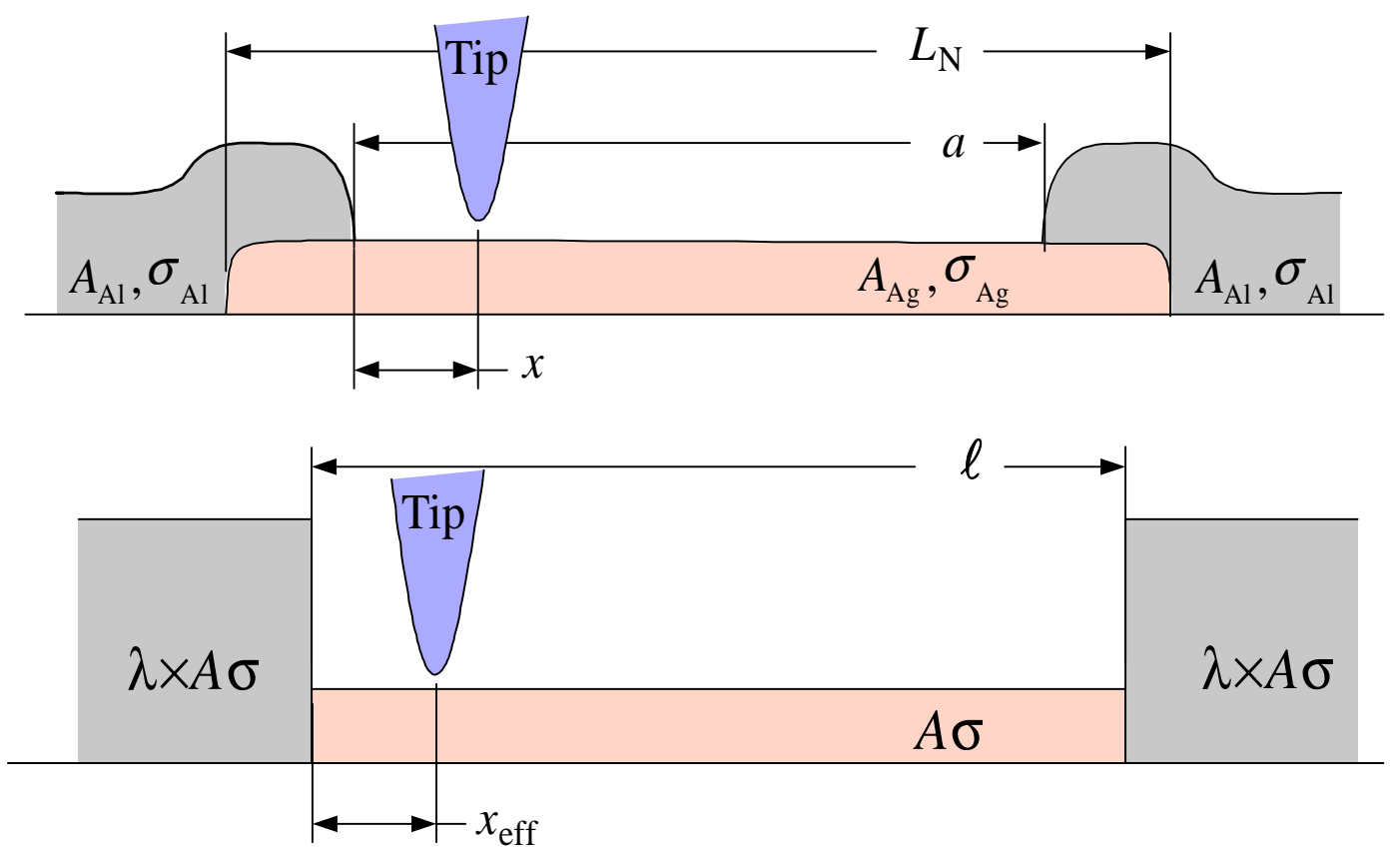

Fig. 4.30 Top: relevant geometric and material parameters to describe the overlap junctions in the SNS structures. $A_{\mathrm{Ag}, \mathrm{Al}}$, are the nominal cross sections of the structure. Bottom: in the 1-D model, we introduce effective parameters discussed in the text.

(iv) As discussed in the theory section, the interface transparency is an important parameter, which, experimentally, is not well controlled. In the model we have to provide the transmission distribution $P(T)$ of the conduction channels of the interface (see $\$ 4.2 .3 .5$, p. 172). While attempting to reproduce the experimental measurements, we found the depression just above $\Delta$ found in some data could only be reproduced by assuming the channels transmission is close to unity. For simplicity, and to reduce the number of parameters, we assumed all transmitted channels are ballistic channels $(\tau=1)$. This is consistent with the fabrication procedure where both metal are deposited in the same vacuum, thereby ensuring interface relatively good cleanness. Doing so, we are left only with the number of interface channels as a free parameter. This also is reasonable since the effective overlap between the electrodes can vary due to the dispersion in the fabrication process, which is clearly seen on the scanning electron micrographs of the structures (Fig. 4.19). Moreover, as already mentioned, the cross-section area differs from one side to the other side of the interface. Thus, some channels belonging to the superconductor are not coupled to the normal region, which weakens the inverse proximity effect in the superconductor. For convenience in the dimensionless numerical equation, for each interface we rather specify the resistance ratio $r=R_{N} / R_{\xi}$, where $R_{N}=G_{N}^{-1}=\left(N G_{0}\right)^{-1}$ is the normal state resistance of the contact and $\mathcal{N}$ the number of transmitted channels, and 


$$
R_{\xi}=\frac{\xi}{A \sigma_{N}}=\frac{\xi}{A \rho_{N} e^{2} D}
$$

is the resistance of a length $\xi$ of the $\mathrm{N}$ wire, $A$ being its cross-section, and $\rho_{\mathrm{N}}$, the density of states.

Finally, in the parameters describing the interfaces in (4.18) enters the product of the cross section with the conductivity of the material. Since we assume (ii) $D$ is the same in both materials, and knowing the density of states of $\mathrm{Al}$ at the Fermi energy, $\rho_{\mathrm{Al}}=2.15 \times 10^{47} \mathrm{~J}^{-1} \mathrm{~m}^{-3}$ and of $\mathrm{Ag}, \rho_{A g}=1.03 \times 10^{47} \mathrm{~J}^{-1} \mathrm{~m}^{-3}$, we expect a conductivity about twice as large in Al. According to the nominal geometrical parameters $A_{\mathrm{Al}}=8 A_{\mathrm{Ag}}$. Hence, in the calculation we take the product $A_{\mathrm{Al}} \sigma_{\mathrm{Al}}=\lambda A_{\mathrm{Ag}} \sigma_{\mathrm{Ag}}$, with $\lambda=16$.

\subsubsection{Comparison with theoretical predictions}

\subsubsection{2.a Determination of the parameters}

Determining appropriate model parameters was done in an trial and error process in order to obtain a good overall rendering of the data. First, the diffusion constant is fixed and then the left and right interface resistance ratios $r_{\mathrm{L}, \mathrm{R}}$ are adjusted for each wire in order to try reproducing the width of the minigap, and, if possible, the individual shapes of the curves. In spite of the apparent large parameter space, by playing with the numbers one rapidly acquires the feeling of the direction in which to adjust the various parameters.

Indeed, initially the parameters space was even much larger since we were also adjusting the average transmission of the interface channels (and even trying other distributions), before realizing that the optimum was consistently found only for high transmissions, at which point we decided to freeze this parameter to unity for all interfaces.

In the following table are summarized the set of parameters we have converged to. 


\begin{tabular}{|r|c|c|c|c|c|}
\hline & wire \#1 & wire \#2 & wire \#3 & wire \#4 \\
\hline Effective length $\ell(\mathrm{nm})$ & 350 & 550 & 950 & 2750 \\
\hline Diffusion constant $D\left(\mathrm{~cm}^{2} \mathrm{~s}^{-1}\right)$ & \multicolumn{4}{|c|}{170} \\
\hline Gap energy $(\mu \mathrm{eV})$ & 165 & $170 ; 173$ & 175 & 170 \\
\hline \multirow{2}{*}{ Left interface } & average $\tau$ & \multicolumn{4}{|c|}{1} \\
\cline { 2 - 6 } & $r_{\mathrm{L}}$ ratio & 0.7 & 4.0 & 0.5 & 1.0 \\
\hline \multirow{2}{*}{ Right interface } & average $\tau$ & & \multicolumn{4}{|c|}{1} \\
\cline { 2 - 6 } & $r_{\mathrm{R}}$ ratio & 0.5 & 0.05 & 2.0 & 0.6 \\
\hline
\end{tabular}

Table. 4.1 Sample parameters used for the calculated results presented in this section. In wire \#2, the gap energy differs between the two superconducting electrodes: the first given value corresponds to the left one.

One can check whether these parameters are reasonable. From the diffusion constant, one can calculate $\xi=250 \mathrm{~nm}$, and $R_{\xi}=3.7 \Omega$, corresponding to a resistance per square $R_{\square}=0.7 \Omega$ quite reasonable for this kind of films. From the nominal cross-section of the wires $\left(30 \times 50 \mathrm{~nm}^{2}\right)$, and the silver Fermi wavelength $\lambda_{\mathrm{F}}=5.2410^{-10} \mathrm{~m}$, one arrives to a number of $\mathcal{N} 22000$ channels in the wire corresponding to a minimum interface resistance $R_{\min }=\left(\mathcal{N} G_{0}\right)^{-1}=0.6 \Omega$. Hence the interface resistance ratio could be as low as $R_{\min } / R_{\xi}=0.015$. The values obtained are globally larger, indicating either a deviation from clean interfaces, or a variation in the number of transmitted channels from the simple estimation above. Since we obtain a better agreement in the fits by assuming ballistic channels rather than a disordered interface, we conclude that the actual overlap between aluminium and silver is responsible for the observed variations. This is checked by having a look at AFM images, where the overlap can be roughly evaluated. This method is not really sensitive, and only allows checking whether interface parameters are reasonable or not, especially for wire \#2 where there is a huge variation between left and right interfaces. In next figure are plotted the cross sections measured parallel to the silver wire on AFM images, for both wire \#1 and wire \#2. On this figure, one can observe that $(i)$ on wire \#1 the overlap is quite equivalent for both interfaces, wich is consistent with the values $r_{L}$ and $r_{R}$ for this wire, and (ii) on the contrary, in wire \#2, the right interface overlap is clearly observable whereas the left one does not appear at all, which once again backs up the fitting values $r_{L}$ and $r_{R}$. On wire \#3 and \#4, the left and rigth overlaps look similar (data not shown).

These observations allow us to infer that the number of transmitted channels is not limited by the wire cross section, but rather by the overlaps. 

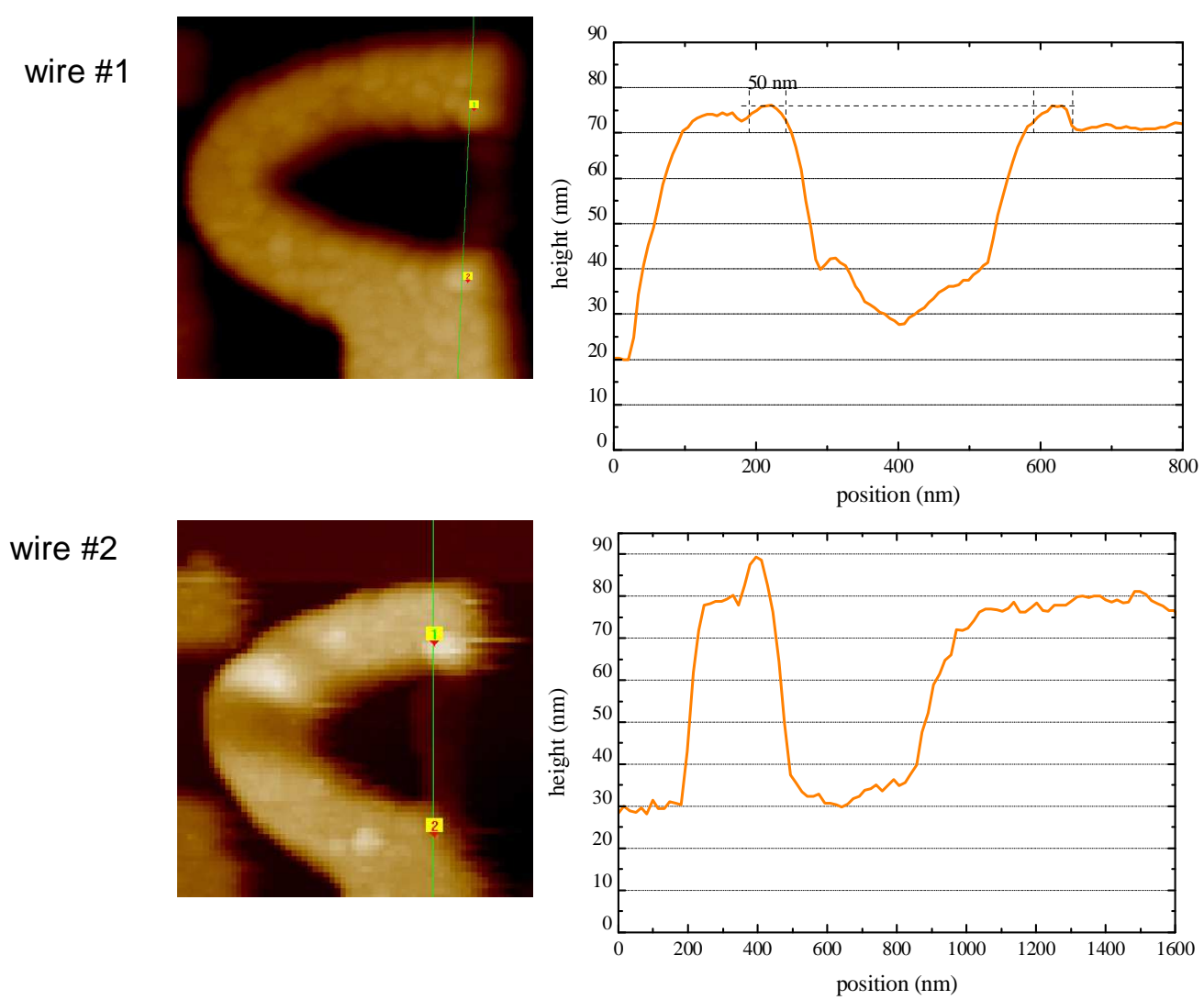

Fig. 4.31 Cross-section views of wire \#1 and wire \#2. Left panel: AFM images, on which are indicated by a straight line the locations where were taken the height data. Right panel: height data along the line. One can see that on wire \#1 the overlap is balanced between left and right interfaces. On the contrary, on wire \#2 one can see the overlaps are really asymmetric.

Finally, regarding the value of the gap used for each wire (to avoid doing self-consistent calculations), we see that indeed these gap values correlate somewhat with the quality of the interfaces we consider: lower gap values are found for wires with clean interfaces. A finer analysis would have required taking two gaps to take into account the asymmetry of the contacts. Indeed, careful examination of the curves $\mathrm{G}$ and $\mathrm{M}$ in Fig. 4.23 shows that the gap is larger on the "left" side of the wire (M curve) than on the "right" side (G curve), corresponding to the interface resistances we find. Since by construction the model cannot capture all details of the experiment, we thought it was a bit pointless trying to refine further this particular aspect. In principle, though, as explained in the theory part, such details on the order parameter should be captured by performing self-consistent calculations, which are only a matter of patience.

Using the above parameters, the calculated DOS profiles at $\delta=0$ for the four wires are shown in Fig. 4.32. 

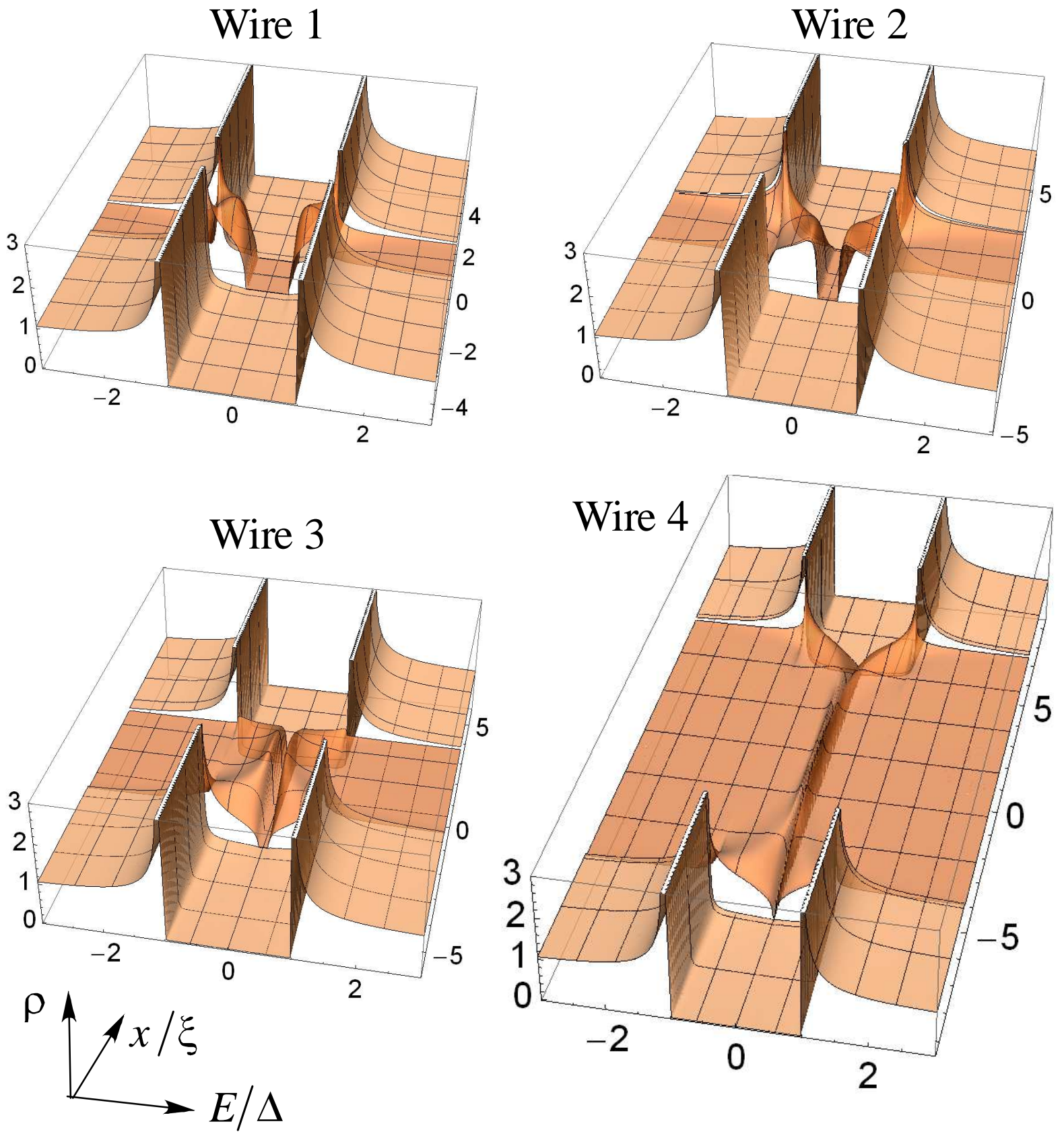

Fig. 4.32 Calculated DOS profiles at $\delta=0$ for the four wires. The interface parameters are shown in Table. 4.1 and have been chosen to obtain the best overall fit at all the measured positions, presented hereafter.

\subsubsection{2.b Position dependence}

The theoretical predictions for the conductance, obtained by convolving the DOS with a thermal rounding function taken at $60 \mathrm{mK}$, are plotted in Fig. 4.33 together with the experimental data for the 4 wires measured. All positions are labeled with the same letters as in Fig. 4.22, Fig. 4.23 and Fig. 4.29. 
Most features of the conductance curves are quantitatively accounted for, except at a few positions such as $\mathrm{C}$ and $\mathrm{E}$ in the shortest wire \#1. Here, we attribute the discrepancy to the failure of the 1D model, which is corroborated by the better agreement obtained for the longer wires. Note that some small position adjustments, within the estimated position accuracy, have been made for fitting some curves.

The dip predicted at zero voltage at positions $\mathrm{AA}, \mathrm{AB}, \mathrm{AC}$ is not observed experimentally. A possible explanation for this effect is the presence of a small depairing process in the $\mathrm{N}$ wire. More calculations are needed to test this hypothesis.

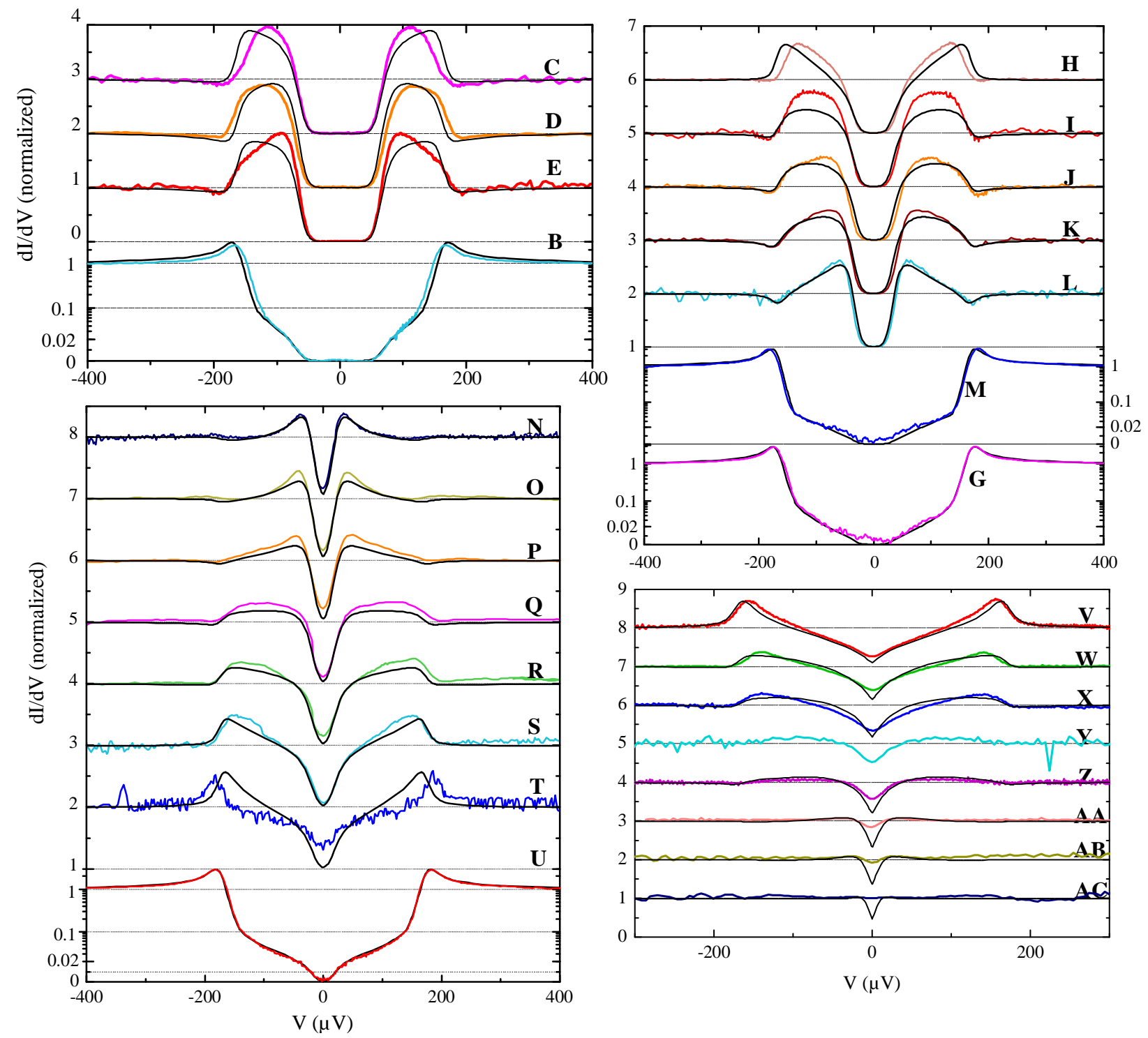

Fig. 4.33 Comparison of data with theory at zero phase bias for all wires (top left: \#1, top-right: \#2, bottom-left: \#3, bottom-right: \#4) presented in sections 4.6.4 to 4.6.6. Each curve is labeled by a letter corresponding to the position labels. 


\subsubsection{2.c Phase dependence}

We have then compared the data with the predicted phase variations for the conductance. Data and predictions are compared in Fig. 4.34 for two positions on the two sides of the NS interface in wire \#1.

The important result demonstrated here is that the minigap is observed unambiguously on the $\mathrm{S}$ side: the minigap measured in the $\mathrm{N}$ part and the associated feature observed on the $\mathrm{S}$ side have the same phase evolution.

The presence of the same energy scale (the minigap) thoughout the SNS structure is an important characteristic of the influence of the finite $\mathrm{N}$ electrode on the $\mathrm{S}$ side, which is well reproduced by the theoretical predictions.

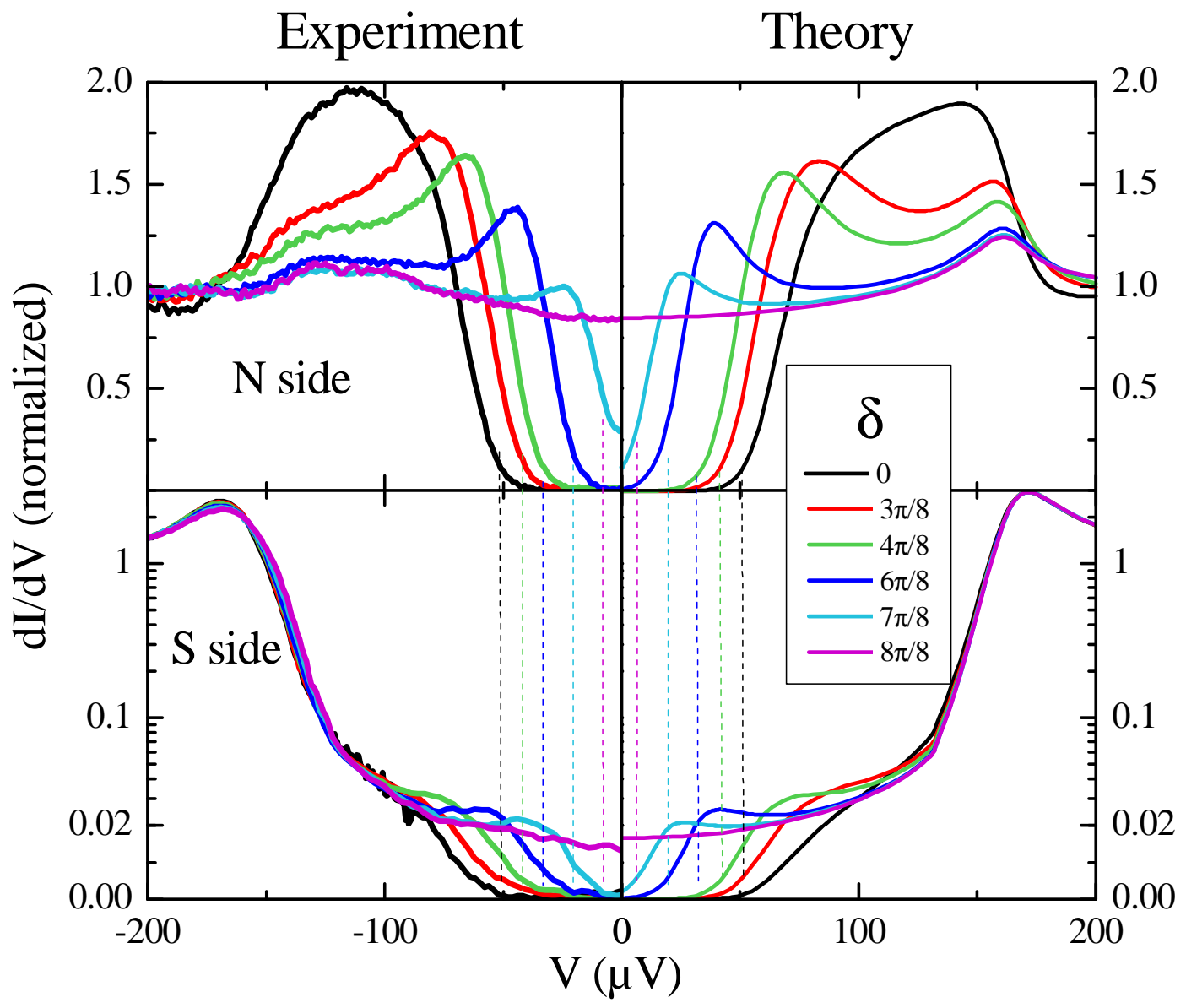

Fig. 4.34 Comparison between tunneling conductance data and predictions at two positions on the two sides of the NS interface in wire \#1. Experimental data (left) and theoretical predictions (right) at position $\mathrm{C}$ on the normal side (top), and at position $\mathrm{B}$ on the $\mathrm{S}$ side (bottom). The minigap in the $\mathrm{N}$ part and its associated feature in the $\mathrm{S}$ side follow the same phase variations. 


\subsubsection{2.d Length dependence}

The different S-N-S structures were fabricated altogether on the same chip. In these conditions, one can hope to get the most similar interface parameters and metal properties. Although interfaces are not identical (see Table. 4.1), the dependence of the minigap with the $\mathrm{N}$ wire length could be approximately checked during the experiment.

Tunneling spectra are shown in Fig. 4.35 in the central positions of the four wires measured. The measured minigaps are in agreement with the calculated ones. On Fig. 4.35 are also shown theoretical curves presented in a paper from J.C. Cuevas et al. [144].

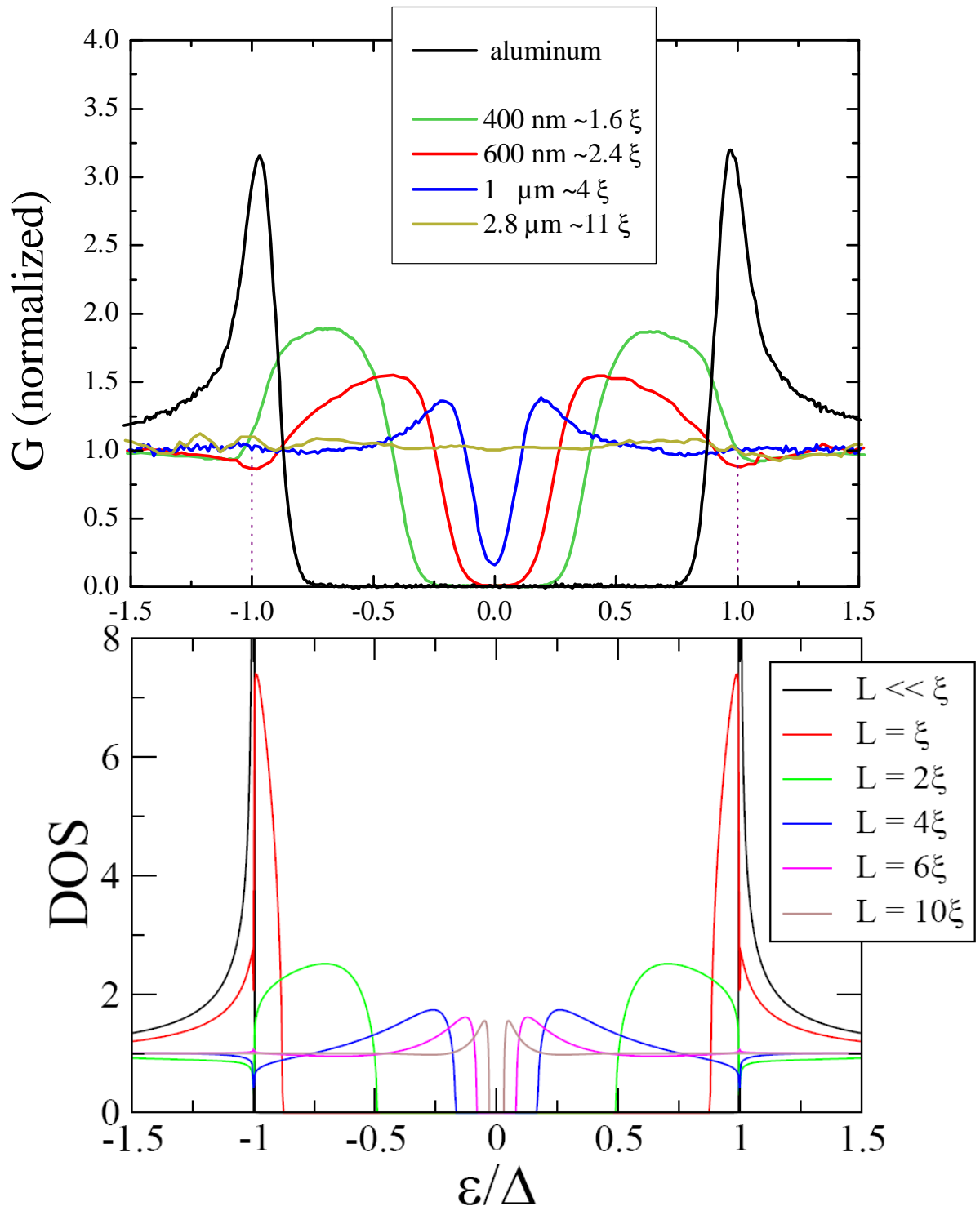

Fig. 4.35 Top: tunneling spectra in the middle of four wires (\#1-4) with different lengths, expressed in units of $\xi$. Bottom: theoretical predictions for the DOS in the middle of the $\mathrm{N}$ wire for different lengths, assuming perfect interfaces, and neglecting inverse PE. This figure is reproduced from FIG. 1 of Ref. [144]. The dependence of the measured minigap is not easily comparable to theoretical predictions because the interface parameters vary from one wire to another. However, our measurements already qualitatively resemble the predictions. 


\subsubsection{Summary of the fitting parameters}

\begin{tabular}{|c|c|c|c|c|c|}
\hline & Position & $\begin{array}{c}x \\
(\mathrm{~nm})\end{array}$ & $x / a$ & $\begin{array}{c}x_{\text {eff }} \\
(\mathrm{nm})\end{array}$ & $\begin{array}{c}\delta x_{\mathrm{eff}} \\
(\mathrm{nm})\end{array}$ \\
\hline \multirow{4}{*}{ Wire 1} & $\mathbf{E}$ & 41 & $13 \%$ & 62 & \\
\hline & D & 154 & $50 \%$ & 175 & \\
\hline & $\mathrm{C}$ & 266 & $87 \%$ & 287 & \\
\hline & B & 338 & $110 \%$ & 420 & \\
\hline \multirow{7}{*}{ Wire 2} & M & 0 & $0 \%$ & -66 & \\
\hline & $\mathbf{L}$ & 67 & $16 \%$ & 127 & \\
\hline & $\mathbf{K}$ & 255 & $59 \%$ & 315 & \\
\hline & $\mathbf{J}$ & 304 & $71 \%$ & 364 & -31 \\
\hline & I & 324 & $75 \%$ & 384 & -40 \\
\hline & $\mathbf{H}$ & 421 & $98 \%$ & 485 & -73 \\
\hline & $\mathbf{G}$ & 516 & $120 \%$ & 660 & 110 \\
\hline \multirow{8}{*}{ Wire 3} & $\mathbf{U}$ & -19 & $-2 \%$ & -1 & \\
\hline & $\mathbf{T}$ & 26 & $3 \%$ & 66 & \\
\hline & $\mathbf{S}$ & 52 & $6 \%$ & 92 & \\
\hline & $\mathbf{R}$ & 131 & $15 \%$ & 171 & -21 \\
\hline & $\mathbf{Q}$ & 191 & $22 \%$ & 231 & -44 \\
\hline & $\mathbf{P}$ & 261 & $30 \%$ & 301 & \\
\hline & $\mathbf{O}$ & 348 & $40 \%$ & 388 & \\
\hline & $\mathbf{N}$ & 435 & $50 \%$ & 475 & \\
\hline \multirow{7}{*}{ Wire 4} & $\mathrm{AC}$ & 1352 & $53 \%$ & 1402 & \\
\hline & $\mathbf{A B}$ & 2066 & $81 \%$ & 2116 & \\
\hline & $\mathbf{A A}$ & 2193 & $86 \%$ & 2243 & \\
\hline & $\mathbf{Z}$ & 2346 & $92 \%$ & 2498 & -102 \\
\hline & $\mathbf{X}$ & 2499 & $98 \%$ & 2549 & \\
\hline & $\mathbf{W}$ & 2547 & $100 \%$ & 2677 & -89 \\
\hline & $\mathbf{V}$ & 2576 & $101 \%$ & 2597 & \\
\hline
\end{tabular}

Table. 4.2 Parameters used for the fits presented in Fig. 4.33 \& Fig. 4.34. $x$ is the effective apparent position of the tip from the AFM image, while $x_{\text {eff }}$ is the effective position using the crude mapping discussed in §4.6.7.1. The last column are shifts which were applied to the listed effective positions to improve the fits. The most important corrections were needed in wire 2 , in which the geometry of the wire was not nominal (See micrograph in Fig. 4.19) 


\subsubsection{Experimental limitations}

Some experimental limitations were already discussed at the beginning of the experimental section. We discuss here several aspects of the experimental realization of importance for the comparison wit theory.

\subsubsection{4.a Positioning accuracy}

AFM pictures were usually taken just before the acquisition of the spectra. Whenever it was necessary to take more extra image(s), they were superimposed with the reference image, in order to show all points on the same image, and be able to compare the position of the different contacts. Although this superimposition procedure is accurately done on visual appreciation, it induces a small inaccuracy in the absolute position of the tunneling contacts measured, because the imaging resolution generally changes from image to image. Note however that an extra uncertainty on the tip position arises from tip deformations during the establishment of a tunneling contact with sufficiently large conductance (see chapter 3 ). The overall positioning accuracy is estimated at $\pm 25 \mathrm{~nm}$.

For this reason, some conductance curves may be better fitted by theoretical predictions at slightly different positions.

\subsubsection{4.b Energy resolution}

A standard issue in STM spectroscopy is the energy resolution linked to the electronic temperature in the tip, when using a normal tip, and to the external electromagnetic noise. The broadening of the DOS by these effects hinders the observation of too small minigaps, as discussed in $\$ 4.6 .4$, p. 201.

To reproduce the data, we convolved the calculated LDOS with a thermal broadening function at an effective temperature of $65 \mathrm{mK}$, inferred from tunneling experiments on the $\mathrm{S}$ electrode.

We could, in principle, take the problem the other way around, and deconvolve the experimental data from this broadening to allow a more accurate determination of the minigap. However, we did not try this procedure because it does not work well on noisy data, with non-monotonic shapes. 


\subsubsection{Model limitations}

Here are presented some points that could be enhanced in the theoretical treatment used to reproduce the data.

\subsubsection{5.a Depairing}

At low energies, on the LDOS of wire \#4 (Fig. 4.33), and far in the normal region, one predicts a dip in the density of states at low energy which is not observed.

The thermal broadening introduced in the calculation, which accounts well for the other data, and in particular for the rounding of the DOS at the minigap edges, does not account well here for the data at small energies.

A possible explanation for this discrepancy is the existence of a small depairing process in the $\mathrm{N}$ wire.

This can be handled theoretically in the quasiclassical formalism, by setting a non zero spinflip rate term in the Usadel equations (4.15).

However, the source of silver was specified with a very high purity (1 ppm impurity), which does not favour this hypothesis.

\subsubsection{5.b Self consistent treatment of the proximity effect}

As mentioned above, the order parameter in the S part close to the NS interface is reduced. This implies in return a reduction of the induced proximity effect. An iterative calculation has to be performed until the process converges (see \$4.4.2.2 p. 188) and yields a stable order parameter profile (see Fig. 4.10). Note that this inverse proximity effect is small because the superconducting electrode has more channels than the narrow normal wire.

\subsubsection{5.c 1-D model of the NS interface}

In our 1-D model, the geometry of the NS interface is not taken into account. In our samples, the superconducting electrode is deposited on top of the $\mathrm{N}$ wire, over a $50 \mathrm{~nm}$ nominal overlap length, comparable to the width of the $\mathrm{N}$ wire. Since this overlap length is smaller than the coherence length, $L_{\text {overlap }}<<\xi$, a 1-D model is roughly justified, but may yield quantitative differences. Note however that the collapse of the suspended mask, observed sometimes, may have resulted in a larger overlap. In any case, the precise position of the N-S interface is not known with a better accuracy than the overlap length, comparable to the positioning accuracy. 
A simplified analysis of a 3-D system, nevertheless capturing some of changes brought by the 3-D geometry [166], yielded a modified profile of the order parameter, with two steps instead of a single one as shown in Fig. 4.36. This prediction could not be checked in our experiments. The full self-consistent solution of a 3-D model, and the detailed investigation of an N-S overlap junction, constitutes a real challenge in the field.

\begin{tabular}{|c|c|c|}
\hline $\mathrm{S}$ & $\mathrm{d}_{\mathrm{S}}$ & \\
\hline & $\mathrm{d}_{\mathrm{N}}$ & $\mathrm{N}$ \\
\hline
\end{tabular}

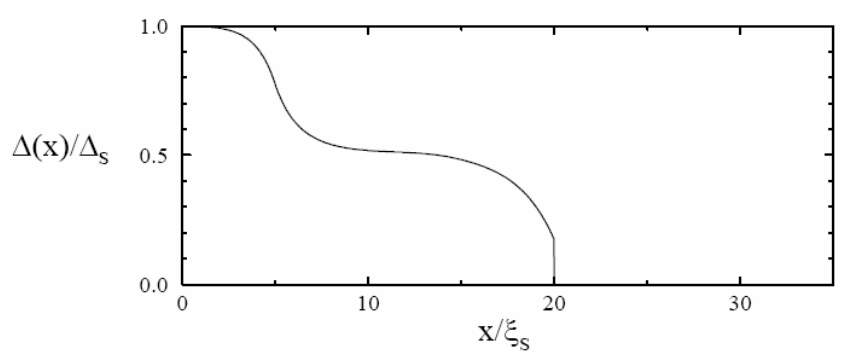

Fig. 4.36 Model of the overlap junction considered in [166]. A two step profile is predicted for the order parameter.

\subsubsection{5.d Transverse extension, sample geometry}

In our case, another, and probably more critical, assumption is the 2-D character of the electrodes.

The 1-D model applies when the transverse dimensions are much smaller than $\xi$. In our situation, $\xi=\sqrt{\hbar D / \Delta} \approx 250 \mathrm{~nm}$ for the assumed value $D=170 \mathrm{~cm}^{2} / \mathrm{s}$. The diffusion constant in aluminum was not measured in the samples, but other experiments have given values in the range $D=100-300 \mathrm{~cm}^{2} / \mathrm{s}$. The width of the aluminum wire is thus not small compared to the coherence length, and lateral inhomogeneities have to be expected in the contact area.

A more symmetric design between the wide current-sinking side and the other one would also avoid a dissymmetry in the boundary conditions for the superconducting gap. Indeed, when a magnetic field is applied for varying the phase difference, the width dependent depairing affects both sides differently: the gap on the wider current-sinking side is more depressed than on the other side (see Fig. 4.17).

\subsubsection{Article reprint: proximity effect in S-N-S structures}

We reproduce below an article published in Physical Review Letters 100, 197002 (2008). 


\title{
Phase controlled superconducting proximity effect probed by tunneling spectroscopy
}

\author{
H. le Sueur, P. Joyez, H. Pothier, C. Urbina, and D. Esteve \\ Quantronics group, Service de Physique de l'Etat Condensé (CNRS URA 2464), CEA-Saclay, 91191 Gif-sur-Yvette, France
}

(Dated: April 15, 2008)

\begin{abstract}
Using a dual-mode STM-AFM microscope operating below $50 \mathrm{mK}$ we measured the Local Density of States (LDoS) along small normal wires connected at both ends to superconductors with different phases. We observe that a uniform minigap can develop in the whole normal wire and in the superconductors near the interfaces. The minigap depends periodically on the phase difference. The quasiclassical theory of superconductivity applied to a simplified 1D model geometry accounts well for the data.
\end{abstract}

PACS numbers: 74.45.+c, 07.79.-v, 74.78.Na, 73.20.At

The proximity effect at the interface between a normal metal (N) and a superconductor (S) consists in the weakening of electron pair correlations on the $\mathrm{S}$ side and in their appearance on the $\mathrm{N}$ side, which can then acquire superconductor-like properties (for a review, see [1]). In the case of diffusive metals, the quasiclassical theory of superconductivity [2] provides a powerful framework to describe this proximity effect. It predicts that if the $\mathrm{N}$ region is smaller than the electron phase coherence length, it then behaves as a genuine superconductor, i.e. it can carry a supercurrent and there is an energy range $[-\delta,+\delta]$ around the Fermi energy in which there are no available states for quasiparticles. As $\delta$ is smaller than the gap $\Delta$ of the bulk superconductor, it has been dubbed the "minigap" [2]. A remarkable feature is that this minigap is the same everywhere in the structure, i.e. both in the $\mathrm{N}$ and in the $\mathrm{S}$ electrodes. In particular, this implies a non-zero Local Density of States (LDoS) in the superconductor between $\delta$ and $\Delta$. This additional density of states, which can be seen as the evanescent tail of normal electrons undergoing Andreev reflection [1] at the NS interface, dies exponentially when moving into the superconductor. These predictions are illustrated in Fig. 1a for the case of a one dimensional SNS structure in which $\delta$ is furthermore predicted to depend periodically on the phase difference $\varphi$ between the order parameters of the two superconductors [3] and vanishes at $\varphi=\pi \bmod 2 \pi$, as shown in Fig. 1b. Although spectral properties of proximity structures have been partially probed in spatial measurements of the LDoS [4-8] and in transport measurements (refs. in [1] and [9-12]), neither the existence of a uniform minigap nor its phase modulation have been reported. In this Letter we present high resolution measurements of the phase and space dependence of the LDoS on both sides of the interfaces of SNS structures, and compare them to the theoretical predictions.

In a Scanning Tunneling Microscope (STM), the dependence of the tunneling current $I$ on the tip-to-sample voltage $V$ directly probes the electronic states available at energy $\mathrm{eV}$ in the electrode just underneath the sharp tip. Variations of the LDoS over small distances can therefore be accessed taking advantage of the high spatial

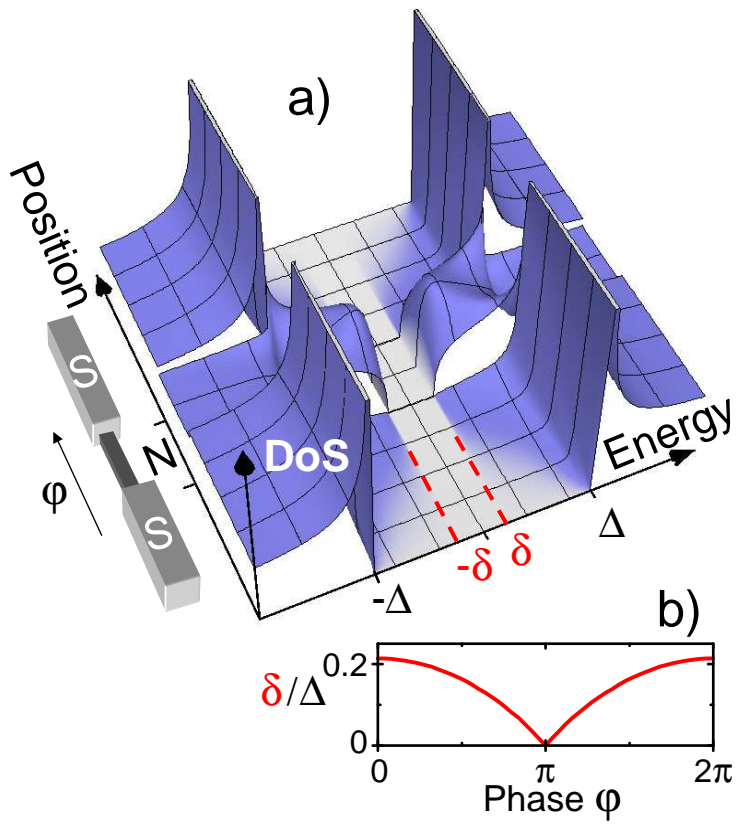

FIG. 1: (color online) (a) LDoS in a 1-D SNS structure, obtained by solving the Usadel equations of the quasiclassical theory of superconductivity in diffusive metals, taking into account scattering at interfaces and geometrical parameters [15]. $\Delta$ is the gap of the superconductor. A uniform minigap $\delta$, defining a range of energy $2 \delta$ in which the LDOS is exactly zero, appears across the normal region and in the $\mathrm{S}$ electrodes. In the latter, the finite conductance between $\delta$ and $\Delta$ decreases exponentially away from the interfaces, and the BCS DoS is eventually recovered. The color scale was chosen to enhance the visibility of the minigap. (b) Predicted $2 \pi$-periodic phase modulation of $\delta$.

resolution of the STM. In a measurement with a normal metal tip, the differential conductance $d I / d V(V)$ at small energies is simply proportional to the LDoS of the electrode, broadened by an instrumental function which is ideally the derivative of the Fermi distribution function of the electrons in the tip at temperature $T_{e}$ [13]. This sets a limit to the energy resolution of such tunneling spectroscopy at about $3.5 k_{B} T_{e}$, which can be nevertheless small enough to resolve the features due to the prox- 

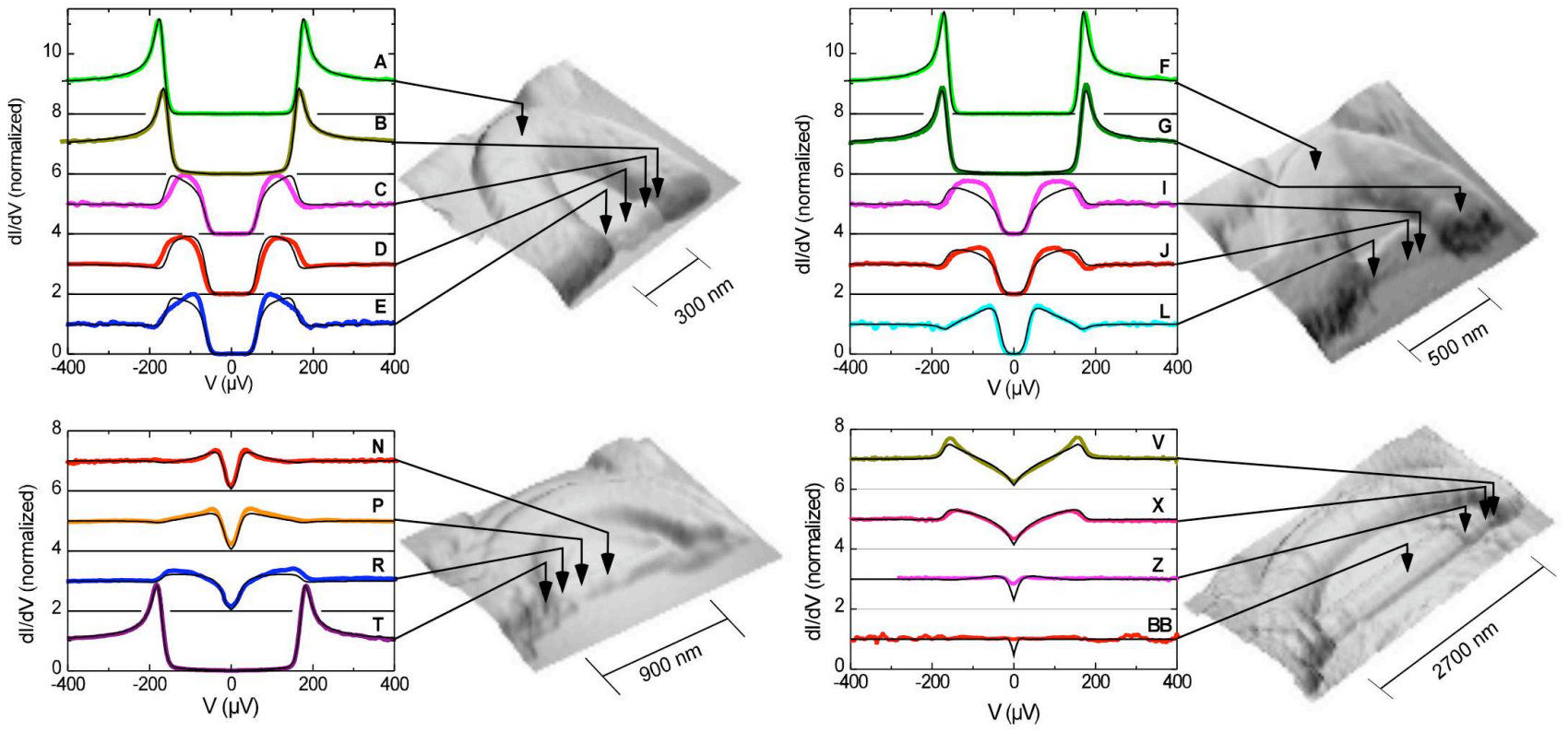

FIG. 2: (color online) Measured (thick grayed lines) differential conductance taken for zero flux through the loop at various positions (indicated by the arrows and labeled by capital letters) of the four SNS structures shown by the AFM images (taken at $35 \mathrm{mK}$ ). The length of the silver wire is respectively (a) $300 \mathrm{~nm}$, (b) $500 \mathrm{~nm}$, (c) $900 \mathrm{~nm}$, (d) $2700 \mathrm{~nm}$. The curves are normalized to 1 at large voltage and are shifted by integer numbers for clarity. The thin black solid lines correspond to the model based on the quasiclassical theory of superconductivity introduced in the text and described more in depth in Ref. [15].

imity effect, provided the electrons in the tip are cooled well below the temperature $\delta / k_{B}$.

In order to perform such measurements on nanocircuits, which contain large insulating areas on which STMs cannot be operated, we have designed and built a cryogenic dual mode STM-AFM (Atomic Force Microscope) $[14,15]$, operating with a single metallic tip. This local probe sensor consists of an electrochemically sharpened tungsten wire [16] glued on one prong of a miniature piezoelectric quartz tuning fork. The latter is a high quality factor electromechanical resonator, which here serves as the AFM transducer [17]. Other dual mode instruments are being developed and used elsewhere [18, 19]. The AFM mode is used to acquire detailed topographic images of the samples which later on allow for accurate positioning of the tip for STM spectroscopy. The microscope is mounted in a table-top dilution refrigerator with a base temperature of $\sim 35 \mathrm{mK}$. By itself this is not sufficient to ensure a low electronic temperature $T_{e}$ and therefore a high energy resolution: it is of critical importance to also shield and filter all the electrical lines needed to operate the microscope, and to measure the tunnel current with a low back-action amplifier. For these purposes, we developed microfabricated cryogenic filters [20] and a custom low-noise, differential-sensing current amplifier [21]. After cooling down, the tip is moved on the sample substrate towards the structures of interest, using a 3-axes capacitance-indexed coarse-positioning sys- tem based on stick-slip motion. During this stage, the microscope is operated in AFM mode, and recognition of superficial patterns etched in the substrate is used to locate the structures. Once a satisfactory AFM image is recorded spectroscopy measurements are performed in STM mode at several positions along the mapped structure.

AFM pictures of four of the SNS structures that were measured are shown in Fig. 2. They consist of a loop formed with a nominally $60 \mathrm{~nm}$-thick, $200 \mathrm{~nm}$-wide Ushaped superconducting aluminum $(\mathrm{Al})$ electrode and a nominally $50 \mathrm{~nm}$-wide and $30 \mathrm{~nm}$-thick normal silver (Ag) wire. The Al film is designed to overlap the $\mathrm{Ag}$ wire at both ends over nominally $50 \mathrm{~nm}$. Several structures were fabricated simultaneously on the same insulating substrate chip with Ag wire lengths ranging from 300 $\mathrm{nm}$ to $3 \mu \mathrm{m}$ (for details on the fabrication see $[14,15]$ ). The aspect ratio of the structures was chosen such that a magnetic flux $\Phi$ threading the loop imposes a phase difference $\varphi \simeq 2 \pi \Phi / \Phi_{0}$ across the silver wire [22], with $\Phi_{0}=h / 2 e$ the flux quantum. To sink the tunnel current when performing spectroscopy, the $\mathrm{Al}$ electrode is connected to ground on one side. The visible part of the Ag wires is covered by a fully oxidized $2 \mathrm{~nm}$-thick aluminum film. By putting the tip in mechanical contact with this oxide layer, one can obtain nearly drift-free tunnel contacts with conductance of the order of $1 \mu \mathrm{S}$. This allows to take reproducible, high resolution spectra, by measur- 
ing the differential conductance as a function of the DC tip-sample bias voltage, using a lock-in amplifier with typically a $1 \mu \mathrm{V}$ AC excitation.

When the tip is placed on top of an $\mathrm{Al}$ electrode far away from any contact to the Ag, the differential conductance (Fig. 2a curve A, and Fig. 2b curve F) displays the well known behavior of a Bardeen-CooperSchrieffer (BCS) superconductor, with an energy gap and its characteristic singularity at the gap edge. These curves are well fitted with a BCS DoS, with an energy gap $\Delta=170 \mu \mathrm{eV}$ for $\mathrm{Al}$, and an effective electronic temperature $T_{e}=65 \mathrm{mK}$. This is higher than the temperature of the refrigerator during the measurements $(35 \mathrm{mK})$, probably because of a still insufficient reduction of the electrical noise. The corresponding energy resolution of $20 \mu \mathrm{eV}$ is nevertheless, to our knowledge, the best obtained so far in STM spectroscopy.

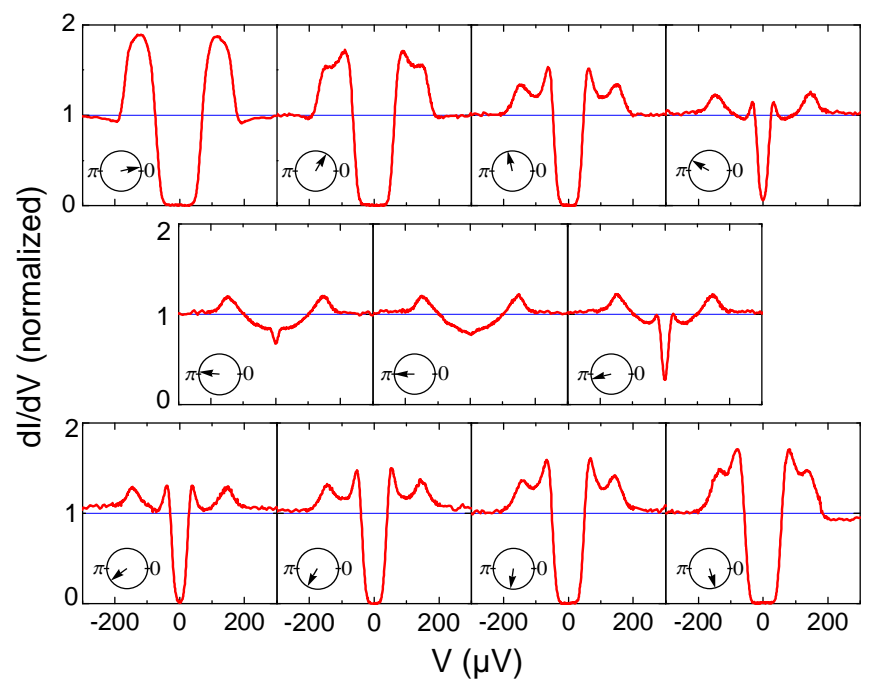

FIG. 3: Differential conductance $d I / d V(V)$ versus voltage measured in the middle of the $300 \mathrm{~nm}$-long Ag wire (position $\mathrm{D}$ ) for different values of the phase difference across it as shown by the phase-clocks $(\varphi / \pi=0.06,0.32,0.57,0.83 ; 0.96$, $1.00,1.08 ; 1.21,1.34,1.46,1.59)$.

In Fig. 2 we also show the LDoS measured at zero flux for several positions along each of the four normal wires. Both in the 300 and in the $500 \mathrm{~nm}$-long Ag wire, the differential conductance vanishes around zero voltage, on an energy range independent of position, which is the signature of a minigap in the DoS. The minigap is larger for the shorter wire. In the $900 \mathrm{~nm}$-long wire, the conductance is strongly reduced on a smaller energy range around zero, but remains always finite. Finally, in the middle of the $2.7 \mu \mathrm{m}$-long wire, $d I / d V(V)$ is nearly constant, i.e. unaffected by the proximity effect. Yet, in this long wire, the LDoS is strongly affected near the ends of the wire. When the tip is placed on the $\mathrm{Al}$ near a contact to the Ag wire, a small finite conductance is observed at subgap voltages (see e.g. curve $\mathrm{G}$ in Fig. $2 \mathrm{~b}$, curve $\mathrm{T}$

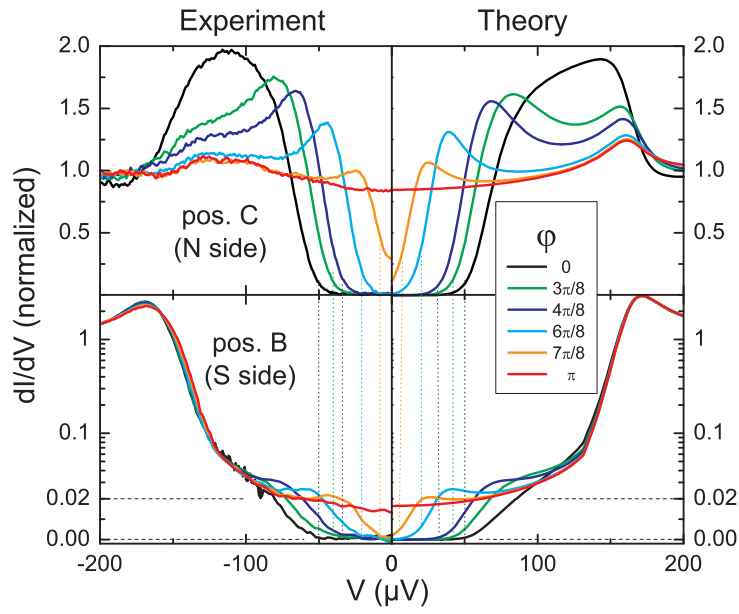

FIG. 4: (color online) Differential conductance versus voltage, for different values of the phase difference $\varphi$ across the $300 \mathrm{~nm}-$ long Ag wire. Left quadrants : measured; Right quadrants : calculated. Top : on the $\mathrm{N}$ side (position $\mathrm{C}$ ); Bottom : on the $\mathrm{S}$ side, close to the interface (position B - Note that the scale is linear up to 0.02 and logarithmic above, to magnify the variation for small subgap conductance). This shows that the minigap is also present on the superconductor side with the same value as on the $\mathrm{N}$ side. Dashed lines are guides to the eye.

in Fig. 2c and curve B in Fig. 2a and the corresponding close-up in the bottom part of Fig. 4). In structures displaying a finite minigap in the $\mathrm{N}$ wire we find that the same minigap is also present on the $\mathrm{S}$ side, despite very different overall shapes of the LDoS. Correlatively, the order parameter is slightly reduced close to the interface as shown by a shift in the position of the peak in the density of states $(160 \mu \mathrm{V}$ at position $\mathrm{B}$ vs. $170 \mu \mathrm{V}$ at position A in Fig. 2a). Farther from the normal metal (positions $\mathrm{A}$ and $\mathrm{F}$ in Fig. 2), the subgap LDoS vanishes.

The variations with the phase difference $\varphi$ of the LDoS in the middle of the shortest wire (position D) are shown in Fig. 3. As $\varphi$ is increased, $d I / d V(V)$ gradually deforms and $\delta$ disminishes. Around $\varphi=\pi$, the minigap vanishes, and the LDoS approaches that of the normal state: superconductivity is then maximally frustrated in the wire. This behavior is $\Phi_{0}$-periodic with the applied flux [15], which provides the phase calibration for each structure. The left quadrants of Fig. 4 show the measured variations of the subgap conductance on both the $\mathrm{S}$ and $\mathrm{N}$ side (positions $\mathrm{B}$ and $\mathrm{C}$ respectively), establishing further that for all values of $\varphi$, the minigap is the same in both the $S$ and $\mathrm{N}$ part of the structure. A similar phase modulation is observed in the 500 and 900 nm-long wires.

The above measurements can be compared with the predictions of the quasiclassical theory of superconduc- 
tivity in diffusive metals. This theory is based on the evaluation of the quasiclassical retarded Green function which, for a superconductor, is a $2 \times 2$ matrix of disorder-averaged correlation functions in electron-hole (or Nambu) space. This matrix depends on position and energy and obeys a diffusion equation, known as the Usadel equation, with boundary conditions at the interfaces [23]. Finding the LDoS in our structures requires, in principle, to specify their full 3-D geometry (including the interfaces and their properties), a complex task which, as far as we know, has never been done. For simplicity, we restrict here to solving a crude onedimensional SNS model involving geometrical and material parameters common to all structures (as they are fabricated simultaneously), and per-structure parameters characterizing the NS interfaces [15]. Due to the finite extent of the overlap interfaces of $\mathrm{N}$ and $\mathrm{S}$ in the samples, one cannot define exactly the tip-to-interface distance. Hence, for simplicity, in the model the effective position of the tip along the effective $\mathrm{N}$ wire length is crudely mapped to the apparent position of the tip on the AFM image, with an offset roughly equal to half the overlap length (see [15] for further details). Finally, in order to account for the effective temperature of the tip, we compare the measurements with the outcome of this model convolved with the derivative of Fermi function at $65 \mathrm{mK}$. In spite of the simplifying assumptions of the model, an overall agreement is obtained for the phase, space, wire length and energy dependence of all the conductance curves, as shown in Figures 2 and 4 . Deviations are most pronounced in the shortest wire at positions near the wire ends (e.g. positions $\mathrm{C}$ and $\mathrm{E}$ ), for which the 1D approximation neglecting the finite extent of the overlap junction geometry is relatively more important. Also, a narrow dip in the conductance predicted near $V=0$ at the middle of the $2.7 \mu \mathrm{m}$-long wire is not observed (see Fig. 2d), which may point to some uncontrolled depairing mechanism not taken into account in the model.

In conclusion, we have measured with unprecedented energy resolution the spatial and phase dependence of the superconducting proximity effect in a series of diffusive structures. In particular, we have shown that an identical phase-dependent minigap is present in both the $\mathrm{N}$ and $\mathrm{S}$ electrodes. This works complements our understanding of the proximity effect and supports its description by the quasiclassical theory. These findings are a first illustration of what can be achieved with this low temperature AFM-STM. There is a large variety of hybrid nanocircuits, which combine for example magnetic materials, semiconductors or even molecules such as carbon nanotubes, that should also give rise to a number of interesting short range "proximity effects" at interfaces. In addition, the capabilities of this new instrument can be extended in various ways. For instance, a superconducting tip would allow probing the superconducting condensate [24] or the electronic distribution functions in nanocircuits driven out-of-equilibrium, providing insight on local electron dynamics in the samples [25]. One could also use spin-polarized tips [26] to resolve spin order, or use the sensor for local gating or electrostatic force measurements [27] on the circuit. This combined AFM-STM cryogenic microscope is thus a versatile tool that opens broad perspectives in mesoscopic physics.

We gratefully acknowledge technical support by P.-F. Orfila and P. Senat and help from the whole Quantronics group. We are also indebted to N. Agraït, H. Bouchiat, C. Chapelier, H. Courtois, S. Guéron, T. Heikkilä, G. Rubio-Bollinger, E. Scheer, H. Suderow and Y. de Wilde for useful exchanges. We particularly thank J.C. Cuevas for guidance on theory and numerics. We acknowledge support by ANR-07-Blan-0240-01 and AC Nano 2003 NR110.

[1] B. Pannetier and H. Courtois, J. Low Temp. Phys. 118, 599 (2000).

[2] W. Belzig et al., Superlatt. and Microstruct. 25, 1251 (1999).

[3] F. Zhou et al., J. Low Temp. Phys. 110, 841 (1998).

[4] S. Guéron et al., Phys. Rev. Lett. 77, 3025 (1996).

[5] M. Vinet, C. Chapelier, and F. Lefloch, Phys. Rev. B 63, 165420 (2001).

[6] N. Moussy, H. Courtois, and B. Pannetier, Europhys. Lett. 55, 861 (2001).

[7] A.K. Gupta et al., Phys. Rev. B 69, 104514 (2004).

[8] W. Escoffier et al., Phys. Rev. Lett. 93, 217005 (2004).

[9] P. Dubos et al., Phys. Rev. Lett. 87, 206801 (2001).

[10] V.T. Petrashov et al., Phys. Rev. Lett. 95, 147001 (2005).

[11] M. S. Crosser et al., Phys. Rev. Lett. 96, 167004 (2006).

[12] L. Angers et al., arXiv:0708.0205 (2007).

[13] O. Fischer et al., Rev. Mod. Phys. 79, 353 (2007).

[14] H. le Sueur, Ph.D. Thesis (in English), Université Paris 6, 2007. Available online at http://tel.archivesouvertes.fr/tel-00261434/en/

[15] See EPAPS Document No. E-PRLTAO-??-????? for additional data, as well as experimental and modeling details (http://www.aip.org/pubservs/epaps.html).

[16] M. Kulawik et al., Rev. Sci. Instrum. 74, 1027 (2003).

[17] K. Karrai and R. D. Grober, Appl. Phys. Lett. 66, 1842 (1995).

[18] J. Senzier, P.S. Luo, and H. Courtois, Appl. Phys. Lett. 90, 043114 (2007).

[19] R. H. M. Smit et al., Rev. Sci. Instrum. 78, 113705 (2007).

[20] H. le Sueur and P. Joyez, Rev. Sci. Instrum. 77, 115102 (2006).

[21] H. le Sueur and P. Joyez, Rev. Sci. Instrum. 77, 123701 (2006)

[22] Within correcting factors of the order $\left(L_{S}+L_{G}\right) / L_{N}$, where $L_{N}$ and $L_{S}$ are the kinetic inductance of respectively the normal and the superconducting wires and $L_{G}$ is the geometrical inductance of the complete loop. For our samples these corrections are always smaller than $10 \%$. See $[14,15]$ for details. 
[23] Yu.V. Nazarov, Superlatt. and Microstruct. 25, 1221 (1999).

[24] Th. Proslier et al., Europhys. Lett. 73, 962 (2006).

[25] H. Pothier et al., Phys. Rev. Lett. 79, 3490 (1997).

[26] M. Bode and R. Wiesendanger, in Magnetic Microscopy of Nanostructures, H. Hopster and H. P. Oepen, Eds, Springer (Berlin, Heidelberg) (2005).

[27] M. T. Woodside and P. L. McEuen, Science 296, 1098 (2002). 
This animation should play automatically when using Adobe Reader 6 or higher. If it does not play, please use the animation_s1.avi file.

(attempting to play animation_s1.avi)

Animation S1. This animation shows the evolution of the DoS plotted in Figure 1 of the Letter when the superconducting phase difference across the structure is varied. This shows the periodic behavior of the LDoS and the vanishing of the minigap around $\varphi=\pi$. Each division along the position axis corresponds to a coherence length $\xi$. 


\subsection{Conclusions}

There is an overall excellent agreement between the conductance data taken at various positions in SNS structures with different lengths and the predictions based on the theory of inhomogeneous superconductivity in diffusive conductors, using a 1-D model, and supplemented with tunneling theory.

Many aspects of the superconducting proximity effect in a diffusive system were observed. Noticeably the normal length dependence of the minigap $\varepsilon_{g}$, as well as its modulation with the phase bias, were observed and found in quantitative agreement with the predictions. The inverse proximity effect, i.e. the existence of a sizeable DOS in the S electrode in the energy range $\left[\varepsilon_{g}-\Delta\right]$ was investigated for the first time. Remaining small discrepancies with the predictions can be attributed to a combination of experimental problems, and of an insufficient modelling of the complex sample 3-D geometry.

From the experimental point of view, a more detailed investigation of the N-S interface region would allow to probe the validity of the 1-D model, and of more advanced 3-D calculations. Solving Usadel equations self-consistently in a full 3-D geometry with finite element methods, and boundary conditions (see $\$ 4.2 .3 .5, \mathrm{p} 172$ ) is worth only if an appropriate pavement of the system allows to limit the amount of calculations to be performed.

\subsubsection{Further experiments}

Some interesting experiments follow from those presented in this thesis.

\subsubsection{Out of equilibrium proximity effect}

A full derivation of the out-of-equilibrium proximity effect in diffusive S-N-S structures was performed recently [144]. The $I(V)$ characteristic and filling factors of a long SNS junction were predicted, as a function of the normal wire length.

The investigation of this well defined non-equilibrium situation is important because even its transport properties, i.e. the $I(V)$ characteristic, has not been probed yet. We propose to perform a combined investigation of the local DOS and of transport properties. Note that the present theory does not take into account the inverse proximity effect, which was shown to be important in our experiments. In particular, we wonder how the subgap density of states is modified in out-of-equilibrium situations. 


\subsubsection{Gapless superconductivity}

Proximity SNS structures in which the N electrode is also connected to a normal reservoir have a zero minigap, but can still carry a supercurrent. This gapless superconductivity still deserves more detailed investigations (see $\$ 4.2 .4 .1 . b$, Fig. 4.2). 


\section{REFERENCES}

[1] S.J. Tans, A. Verschueren, and C. Dekker, "Room-temperature transistor based on a single carbon nanotube”, Nature 393, 49 (1998).

[2] E.B. Myers et al., "Current-Induced Switching of Domains in Magnetic Multilayer Devices", Science 285, 867 (1999).

[3] D. Goldhaber-Gordon, Hadas Shtrikman, D. Mahalu, D. Abusch-Magder, U. Meirav and M. A. Kastner, "Kondo effect in a single-electron transistor”, Nature 391, 156 (1998).

[4] D.W. Pohl, W. Denk, and M. Lanz, "Optical stethoscopy: Image recording with resolution lambda/20”, Appl. Phys. Lett. 44, 651 (1984).

[5] A.V. Bragas and O.E. Martínez, "Field-enhanced scanning optical microscope", Optics Letters 25, 631 (2000).

[6] F.-R.F. Fan, and A.J. Bard, "Photoassisted scanning tunneling microscopy and tunneling spectroscopy of n-type tungsten diselenide (n-WSe[2]) single crystals", J. Phys. Chem. 97, 1431 (1993).

[7] I. Giaever, “Energy gap in superconductors measured by electron tunneling”, Phys. Rev. Lett. 5, 147 (1960); I. Giaever, “Electron tunneling between two superconductors”, Phys. Rev. Lett. 5, 147 (1960); I. Giaever, “Electron tunneling and superconductivity”, Rev. Mod. Phys 46, 245 (1974).

[8] G. Binnig, H. Rohrer, Ch. Gerber, and E. Weibel, “Tunneling through a controllable vacuum gap”, Appl. Phys. Lett. 40, 178 (1981).

[9] For recent a review on tunneling spectroscopy, see O. Fischer, M. Kugler, I. MaggioAprile, C. Berthod, C. Renner, "Scanning tunneling spectroscopy of high-temperature superconductors”, Rev. Mod. Phys. 79, 353 (2007).

[10] J. Bardeen, “Tunneling from a Many-Particle Point of View”, Phys. Rev. Lett. 6, 57 (1960).

[11] J. Bardeen, “Tunneling Into Superconductors”, Phys. Rev. Lett. 9, 147 (1962).

[12] M.H. Cohen, L.M. Falicov and J.C. Philips, "Superconductive Tunneling”, Phys. Rev. Lett. 8, 316 (1962).

[13] J. Tersoff and D.R. Hamann, "Theory and Application for the Scanning Tunneling Microscope”, Phys. Rev. Lett. 50, 1998 (1983); J. Tersoff and D.R. Hamann, “Theory of the scanning tunneling microscope”, Phys. Rev. B 31, 805 (1985). 
[14] J. Bardeen, L.N. Cooper and J.R. Shrieffer, “Theory of Superconductivity”, Phys. Rev. 108, 1175 (1957).

[15] A. Anthore, F. Pierre, H. Pothier, and D. Esteve, "Magnetic-Field-Dependent Quasiparticle Energy Relaxation in Mesoscopic Wires”, Phys. Rev. Lett. 90, 076806 (2003).

[16] M.F. Crommie, C.P. Lutz, D.M. Eigler, "Confinement of electrons to quantum corrals on a metal surface”, Science 262, 218 (1993).

[17] For a review see H.J. Güntherodt and R. Wiesendanger, "Scanning Tunneling Microscopy”, volume I (Springer-Verlag) (1994).

[18] A.F. Andreev, "The thermal conductivity of the intermediate state in superconductors”, Zh. Eksp. Teor. Fiz. 46, 1823 (1964) [Sov. Phys. JETP, 19, 1228].

[19] P. de Gennes, and D. Saint-James, "Elementary excitations in the vicinity of a normal metal-superconducting metal contact”, Phys. Lett. 4, 151 (1963).

[20] F. Zhou, B. Spivak, and A. Zyuzin, "Coherence effects in a normal-metal-insulatorsuperconductor junction”, Phys. Rev. B 52, 4467 (1995).

[21] H. Courtois, Ph. Gandit, and B. Pannetier, "Proximity-induced superconductivity in a narrow metallic wire”, Phys. Rev. B 52, 1162 (1995).

[22] F. Zhou, B. Spivak, "Resistance of superconductor-normal-metal-superconductor (SNS) junctions”, JETP Letters 65, 369 (1997) [arXiv:cond-mat/9604185].

[23] W. Belzig, F.K. Wilhelm, C. Bruder, G. Schon, and A.D. Zaikin, "Quasiclassical Green's function approach to mesoscopic superconductivity”, Superlattice and Microstructures 25, $\mathrm{n}^{\circ}$ 5/6 (1999) [arXiv:cond-mat/9812297].

[24] B. Pannetier and H. Courtois, "Andreev Reflection and Proximity effect", J. Low Temp. Phys. 118, 599 (2000) [arXiv:cond-mat/9912024].

[25] P.G. de Gennes, “Superconductivity of Metals and Alloys”, Benjamin, New York (1966).

[26] G. Eilenberger, "Transformation of Gorkov's equation for type II superconductors into transport-like equations”, Z. Phys. 214, 195 (1968).

[27] K. Usadel, “Generalized diffusion equation for superconducting alloys”, Phys. Rev. Lett. 25, 507 (1970).

[28] C. Beenakker, "Universal limit of critical-current fluctuations in mesoscopic Josephson junctions”, Phys. Rev. Lett. 67, 3836 (1991) [arXiv:cond-mat/0406127]. 
[29] J.A. Melsen, P.W. Brouwer, K.M. Frahm, and C.W.J. Beenakker, "Superconductorproximity effect in chaotic and integrable billiards”, Physica Scripta T69, 223 (1997) [arXiv:cond-mat/9607036].

[30] S. Guéron, H. Pothier, N.O. Birge, D. Esteve, and M.H. Devoret, "Superconducting Proximity Effect Probed on a Mesoscopic Length Scale”, Phys. Rev. Lett. 77, 3025 (1996).

[31] M. Vinet, C. Chapelier and F. Lefloch, "Spatially resolved spectroscopy on a superconducting proximity structure”, Phys. Rev. B 63, 165420 (2001) [arXiv:cond-mat/0004340].

[32] N. Moussy, H. Courtois and B. Pannetier, "Local spectroscopy of a proximity superconductor at very low temperature”, Europhysics Letters 55, 861 (2001) [arXiv:cond-mat/0106299].

[33] A.Yu. Kasumov, R. Deblock, M. Kociak, B. Reulet, H. Bouchiat, I.I. Khodos, Yu.B. Gorbatov, V.T. Volkov, C. Journet, and M. Burghard, "Supercurrents Through SingleWalled Carbon Nanotubes”, Science 284, 1508 (1999).

[34] G.E. Blonder, M. Tinkham, and T.M. Klapwijk, "Transition from metallic to tunneling regime in superconducting microstrictions: excess current, charge imbalance, and supercurrent conversion”, Phys. Rev. B 25, 4515 (1982).

[35] C.W.J. Beenaker, "Specular Andreev reflection in graphene”, Phys. Rev. Lett. 97, 067007 (2006) [arXiv:cond-mat/0604594].

[36] B. Leridon, J. Lesueur, and M. Aprili, "Spin-bottleneck due to spin-charge separation in a superconductor”, Phys. Rev. B 72, 180505 (2005).

[37] R.S. Keizer, S.T.B. Goennenwein, T.M. Klapwijk, G. Miao, G. Xiao and A. Gupta, “A spin triplet supercurrent through the half-metallic ferromagnet CrO2”, Nature 439, 825 (2006).

[38] G.M. Mikhailov, A.V. Chernykh, J.C. Maan, J.G.S. Lok, A.K. Geim, D. Esteve, P. Joyez, "The edge and bulk electron state dominated magnetotransport in multi-terminal single-crystalline refractory metal nanostructure”, Nanotechnology 11, 379 (2000).

[39] G.M. Mikhailov, I.V. Malikov, A.V. Chernykh, L.A. Fomin, P. Joyez, H. Pothier, D. Esteve, E. Olsson, "Signatures of ballistic transport in the magnetoresistance of nanostructures made of single-crystalline refractory metals", Nanotechnology 13, 226 (2002).

[40] H. Pothier, S. Guéron, N.O. Birge, D. Esteve, and M.H. Devoret, "Energy distribution of quasiparticles in mesoscopic wires”, Phys. Rev. Lett. 79, 3490 (1997). 
[41] Y. Kuk and P.J. Silverman, “Scanning tunneling microscope instrumentation”, Rev. Sci. Instrum. 60, 165 (1989).

[42] S.H. Pan, E.W. Hudson, and J.C. Davis, "3He refrigerator based very low temperature scanning tunneling microscope”, Rev. Sci. Instrum. 70, 1459 (1999).

[43] J.G. Rodrigo, H. Suderow, S. Vieira, E. Bascones, and F. Guinea, "Superconducting nanostructures fabricated with the scanning tunneling microscope”, J. Phys. Condens. Matter 16, 1151 (2004).

[44] D.W. Pohl, “Dynamic piezoelectric translation devices”, Rev. Sci. Instrum. 58, 54 (1987).

[45] Maud Vinet, "Etude par STM de nanostructures supraconductrices par proximité”, $\mathrm{PhD}$ thesis, Université Joseph Fourier, Grenoble (2001).

[46] G. Rubio Bollinger, private communication.

[47] G. Binnig, Ch. Gerber, and C.F. quake, "Atomic Force Microscope", Phys. Rev. Lett. 56, 930 (1986).

[48] IBM, "Millipede project”, http://www.zurich.ibm.com/news/05/millipede.html .

[49] D. Rugar, H.J. Mamin, R. Erlandsson, J.E. Stern, and B.D. Terris, "Force microscope using a fiber-optic displacement sensor", Rev. Sci. Instrum. 59, 2337 (1988).

[50] R. Euler, U. Memmert, and U. Hartmann, "Fiber interferometer-based variable temperature scanning force microscope", Rev. Sci. Instrum. 68, 1776 (1997).

[51] D.V. Pelekhov, J.B. Becker, and G. Nunes Jr, "Atomic force microscope for operation in high magnetic fields at millikelvin temperatures”, Rev. Sci. Instrum. 70, 114 (1999).

[52] A. Radenović, E. Bystrenová, L. Libioulle, M. Taborelli, J.A. DeRose, and G. Dietler, “A low-temperature ultrahigh vacuum atomic force microscope for biological applications”, Rev. Sci. Instrum., 74, 1022 (2003).

[53] P. Günther, U.Ch. Fischer, and K. Dransfeld, "Scanning near-field acoustic microscopy”, Appl.Phys. B: Laser and Optics 48, 89 (1988).

[54] M. Tortonese, H. Yamada, R.C. Barrett, CF Quate, and E. Ginzton, “Atomic Force microscopy using a piezoresistive cantilever", IEEE conference on Solid-State Sensors and Actuators p448-453, San-Francisco, USA, (1991).

[55] M. Tortonese, R.C. Barrett, and C.F. Quate, "Atomic resolution with an atomic force microscope using piezoresistive detection”, Appl. Phys. Lett. 62, 834 (1993). 
[56] R.E. Thomson, "Low-temperature atomic force microscope using piezoresistive cantilevers”, Rev. Sci. Instrum. 70, 3369, (1999).

[57] K. Karrai, and R.D. Grober, "Piezoelectric tip-sample distance control for near field optical microscopes”, Appl. Phys. Lett. 66, 1842, (1995).

[58] Fortiming Corporation, "Basic technology of Quartz crystal resonator", http://www.4timing.com/techcrystal.htm, M-tron ${ }^{\mathrm{TM}}$ engineering notes, p100.

[59] T. Ihn, T. Vančura, A. Baumgartner, P. Studerus, K. Ensslin, “Operating a phase locked loop controlling a high-Q tuning fork sensor for scanning force microscopy”, arXiv:cond-mat/0112415, (2001).

[60] J. Rychen, T. Ihn, P. Studerus, A. Herrmann, and K. Ensslin, “A low-temperature dynamic mode scanning force microscope operating in high magnetic fields", Rev. Sci. Instrum. 70, 2765 (1999) [arXiv:cond-mat/9901023].

[61] F. Giessibl, "Atomic resolution on Si(111)-(7x7) by noncontact atomic force microscopy with a force sensor based on a quartz tuning fork”, Appl. Phys. Lett. 76, 1470 (2000).

[62] Khaled Karrai, "Lecture notes on shear and friction force detection with quartz tuning fork”, Ecole thématique du CNRS on near field optics, La Londe les Maures, France, (march 2000).

[63] J. Rychen, T. Ihn, P. Studerus, A. Herrmann, and K. Ensslin, H.J. Hug, P.J.A. van Schendel, and H.J. Güntherodt, "Operation characteristics of piezoelectric quartz tuning forks in high magnetic fields at liquid helium temperatures”, Rev. Sci. Instrum. 71, 1695 (2000).

[64] G. Rubio-Bollinger, P. Joyez, N.Agrait, "Metallic adhesion in atomic-size junctions”, Phys. Rev. Lett. 93, 116803 (2004).

[65] Nanonis GmbH, "Piezoelectric Quartz Tuning Forks for Scanning Probe Microscopy”, (2003).

[66] R.D. Grober, J. Acimovic, J. Schuck, D. Hessman, P. Kindelmann, J. Hespanha, A.S. Morse, K. Karrai, I. Tiemann, S. Manus, "Fundamental limit to force detection using quartz tuning fork”, Rev. Sci. Instrum., 71, 2776, (2000).

[67] F. Giessibl, "Forces and frequency shifts in atomic-resolution dynamic-force microscopy”, Phys. Rev. B 56, 16010 (1997).

[68] J. Polesel, "Modeling an Experiments in Dynamic Force Microscopy in Ultra High Vacuum”, PhD Thesis, Université Paul Sabatier, Toulouse III (2005). http://tel.archivesouvertes.fr/tel-00009666 
[69] B. Gottsmann and H. Fuchs, "Dynamic Force Spectroscopy of Conservative and Dissipative Forces in an Al-Au(111) Tip-Sample System”, Phys. Rev. Lett. 86, 2597 (2001).

[70] S. Belaidi, P. Girard and G. Leveque, "Electrostatic forces acting on the tip in atomic force microscopy: modelization and comparison with analytic expressions”, J. Appl. Phys. 81, 1023 (1997).

[71] S. Hudlet, M. Saint Jean, C. Guthmann, and J. Berger, "Evaluation of the capacitive force between an atomic force microscopy tip and a metallic surface”, Eur. Phys. J. B 2, 5 (1998).

[72] R.P. Stomp, “Dissipative and Electrostatic Force Spectroscopy of InAs Quantum Dots by Non-contact Atomic Force Microscopy”, Ph.D. Thesis, McGill University, Montreal (2005).

[73] T.R. Albrecht, P. Grütter, D. Horne, and D. Rugar, “Frequency modulation detection using High-Q cantilevers for enhanced force microscope sensitivity”, J. Appl. Phys. 69, 668-673, (1991).

[74] J. Rychen, T. Ihn, P. Studerus, A. Herrmann, and K. Ensslin, H.J. Hug, P.J.A. van Schendel, and H.J. Güntherodt, "Force-distance studies with piezoelectric tuning forks below 4.2K”, Appl. Surf. Sci. 157, 290 (2000).

[75] F. J. Giessibl, “Advances in atomic force microscopy”, Rev. Mod. Phys. 75, 949 (2003).

[76] T.R. Albrecht, P. Grütter, D. Horne, and D. Rugar, “Frequency modulation detection using high-Q cantilevers for enhanced force microscope sensitivity”, J. Appl. Phys. 69, 668 (1991).

[77] H. Edwards, L. Taylor, W. Duncan, and A.J. Melmed, "Fast, high-resolution atomic force microscopy using a quartz tuning fork as actuator and sensor”, J. Appl. Phys. 82, 980, (1997).

[78] F.Giessibl, M. Reichling, "Investigating atomic details of the CaF ${ }_{2}(111)$ surface with a qPlus sensor”, Nanotechnology 16, 118 (2005) [arXiv:cond-mat/04124430].

[79] G. Couturier, R. Boisgard, L. Nony, and J.P. Aimé, "Noncontact atomic force microscopy: stability criterion and dynamical responses of the shift of frequency and damping signal”, Rev. Sci. Instrum. 74, 2726 (2003).

[80] U. Dürig, H.R. Steinauer, and N. Blanc, "Dynamic force microscopy by means of the phase-controlled oscillator method”, J. Appl. Phys. 82, 3641 (1997). 
[81] Ch. Loppacher, M. Bammerlin, F. Battiston, M. Guggisberg, D. Müller, H.R. Hidber, R. Lüthi, E. Meyer, H.J. Güntherodt, "Fast digital electronics for application in dynamic force microscopy using high-Q cantilevers”, Appl. Phys. A 66, 215 (1998).

[82] S. Park and F. Quate, "Scanning tunneling microscope”, Rev. Sci. Instrum. 58, 2010 (1987).

[83] S. Park and F. Quate, "Theories of the feedback and vibration isolation for the scanning tunneling microscope”, Rev. Sci. Instrum. 58, 2004 (1987).

[84] F.C. Wellstood, C. Urbina, and J. Clarke, "Hot-electron effects in metals", Phys. Rev. B 49, 5942 (1994).

[85] J.M. Martinis and M. Nahum, "Effect of environmental noise on the accuracy of Coulomb-blockade devices”, Phys. Rev. B 48, 18316 (1993).

[86] D. Vion, P.F. Orfila, P. Joyez, D. Esteve, and M.H. Devoret, "Miniature electrical filters for single electron devices”, J. Appl. Phys. 77, 2519 (1995).

[87] A.B. Zorin, "The thermocoax cable as the microwave frequency filter for single electron circuits”, Rev. Sci. Instrum. 66, 4296 (1995).

[88] D.C. Glattli, P. Jacques, A. Kumar, P. Pari, and L. Saminadayar, "A noise detection scheme with $10 \mathrm{mK}$ noise temperature resolution for semiconductor single electron tunneling devices”, J. Appl. Phys. 81, 7350 (1997).

[89] J.M. Martinis, M.H. Devoret, and J. Clarke, "Experimental test for the quantum behaviour of a macroscopic degree of freedom: The phase difference across a Josephson junction”, Phys. Rev. B 35, 4682 (1987).

[90] B. Sacépé, C. Chapelier, C. Marcenat, J. Kaćmarćik, T. Klein, M. Bernard, and E. Bustarret, "Tunneling spectroscopy and vortex imaging in boron-doped diamond", Phys. Rev. Lett. 96, 097006 (2006) (cond-mat/0510541).

[91] J. Senzier, P.S. Luo, H.Courtois, "Combined scanning force microscopy and scanning tunneling spectroscopy of an electronic nano-circuit at very low temperature”, Appl. Phys. Lett. 90, 043114 (2007).

[92] H. Suderow, M. Crespo, P. Martinez-Samper, J.G. Rodrigo, G. Rubio-Bollinger, S. Vieira, N. Luchier, J.P. Brison, P.C. Canfield, "Scanning tunneling microscopy and spectroscopy at very low temperatures”, Physica C 369, 106 (2002).

[93] H. Courtois, O. Buisson, J. Chaussy, and B. Pannetier, "Miniature low-temperature high-frequency filters for single electronics”, Rev. Sci. Instrum. 66, 3465 (1995). 
[94] W. Escoffier, "Quantum coherence in superconductor - normal metal systems studied by scanning tunnelling microscopy and spectroscopy”, $\mathrm{PhD}$ thesis, $\mathrm{p} 30$, Université Joseph Fourier, Grenoble (2004). http://tel.archives-ouvertes.fr/tel-00007327

[95] A.J. Melmed, “Art and science and other aspects of making sharp tips”, J. Vac. Sci. Technol. B 9, 601 (1991).

[96] R. Wiesendanger, "Scanning Probe Microscopy and Spectroscopy: Methods and Applications”, Cambridge University Press (1994).

[97] D. Bonnell, "Scanning Probe Microscopy and Spectroscopy: Theory, Techniques, and Applications”, Wiley-VCH, 2nd edition (2001).

[98] T.R. Albrecht, S. Akamine, T.E. Carver, and C.F. Quate, "Microfabrication of cantilever styli for the atomic force microscope”, J. Vac. Sci. Technol. A 8, 3386 (1990).

[99] J.P. Ibe, P.P. Bey Jr., S.L. Brandow, R.A. Brizzolara, N.A. Burnham, D.P. DiLella, K.P. Lee, C.R.K. Marrian, and R.J. Colton., "On the electrochemical etching of tips for scanning tunneling microscopy”, J. Vac. Sci. Technol. A 8, 3570 (1990).

[100] E.W. Muller, “Beobachtungen über die Feldemission und die Kathodenzerstäubung an thoriertem Wolfram”, Z. Phys. 106, 132 (1937).

[101] E.W. Muller and T.T. Tsong, "Field Ion Microscopy_Principles and Applications" p. 187-189, Elsevier, New York (1969).

[102] P.J. Bryant, H.S. Kim, Y.C. Zheng, and R. Yang, "Technique for shaping scanning tunneling microscope tips”, Rev. Sci. Instrum. 58, 1115 (1987).

[103] M. Klein and G. Schwitzgebel, “An improved lamellae drop-off technique for sharp tip preparation in scanning tunneling microscopy”, Rev. Sci. Instrum. 68, 3099 (1997).

[104] M. Kulawik, M. Nowicki, G. Thielsch, L. Cramer, H.P. Rust, H.J. Freund, T.P. Pearl and P.S. Weiss, “A double lamellae dropoff etching procedure for tungsten tips attached to tuning fork atomic force microscopy/scanning tunneling microscopy sensors”, Rev. Sci. Instrum. 74, 1027 (2003).

[105] R. Nicolaides et al., “Scanning tunneling microscope tip structures”, J. Vac. Sci. Technol. A 6, 445 (1988).

[106] P.A. Taylor, J.S. Nelson, and B.W. Dodson, "Adhesion between atomically flat metallic surfaces”, Phys. Rev. B 44, 5834 (1991). 
[107] A. Halbritter, Sz. Csonka and G. Mihály, E. Jurdik, O.Yu. Kolesnychenko, O.I. Shklyarevskii, S. Speller and H. van Kempen, "Transition from tunneling to direct contact in tungsten nanojunctions”, Phys. Rev. B 68, 035417 (2003) [arXiv:condmat/0304389].

[108] W.L. Bond, A.S. Cooper, K. Andres, G.W. Hull, T.H. Geballe, and B.T. Matthias, "Superconductivity in films of $\beta$ tungsten and other transition metals", Phys. Rev. Lett. 15, 260 (1965).

[109] S. Basavaiah and S. R. Pollack, "Superconductivity in evaporated tungsten films" Appl. Phys. Lett. 12, 259 (1968).

[110] A.E. Lita, D. Rosenberg, S. Nam, A.J. Miller, D. Balzar, L.M. Kaatz, R.E. Schwall, "Tuning of tungsten thin film superconducting transition temperature for fabrication of photon number resolving detectors”, IEEE Transactions on Applied Superconductivity 15, 3528 (2005).

[111] A.S. Lucier, "Preparation and Characterization of Tungsten Tips Suitable for Molecular Electronics Studies”, PhD Thesis, McGill University, Montreal (2004).

[112] J. Garnaes, F. Kragh, K.A. Mørch, and A.R. Thölén, "Transmission electron microscopy of scanning tunneling tips”, J. Vac. Sci. Technol. A 8, 441 (1990).

[113] D.K. Biegelsen, F.A. Ponce, J.C. Tramontana and S.M. Koch, "Ion milled tips for scanning tunneling microscopy”, Appl. Phys. Lett. 50, 696, (1987).

[114] L. Libioulle, Y. Houbion, J.M. Gilles, "Very sharp platinum tips for scanning tunneling microscopy”, Rev. Sci. Instrum. 66, 97 (1995).

[115] A. Cricenti, E. Paparazzo, M.A. Scarselli, L. Moretto and S. Selci, "Preparation and characterization of tungsten tips for scanning tunneling microscopy”, Rev. Sci. Instrum. 65, 1558 (1994).

[116] R. Zhang and D. G. Ivey, "Preparation of sharp polycrystalline tungsten tips for scanning tunneling microscopy imaging”, J. Vac. Sci. Technol. B 14, 1 (1996).

[117] J.G. Rodrigo, H. Suderow, S. Vieira, E. Bascones, F. Guinea, "Superconducting nanostructures fabricated with the STM”, arXiv:cond-mat/0410500 (2004).

[118] A.E. Gildemeister, T. Ihn, M. Sigrist, K. Ensslin, D.C. Driscoll and A.C. Gossard, "In Situ Treatment of a Scanning Gate Microscopy Tip”, arXiv:cond-mat/0703605 (2007).

[119] J. Wintterlin, J. Wiechers, H. Brune, T. Gritsch, H. Höfer, and R. J. Behm, “AtomicResolution Imaging of Close-Packed Metal Surfaces by Scanning Tunneling Microscopy”, Phys. Rev. Lett. 62, 59 (1989). 
[120] Y. Oda and H. Nagano, “Meissner effect in Cu of thick Cu clad Nb", Solid State Commun. 35, 631 (1980).

[121] W.L. McMillan, “Tunneling Model of the Superconducting Proximity Effect”, Phys. Rev. 175, 537 (1968).

[122] S. Guéron, H. Pothier, N.O. Birge, D. Esteve, and M.H. Devoret, "Superconducting Proximity Effect Probed on a Mesoscopic Length Scale”, Phys. Rev. Lett. 77, 3025 (1996).

[123] H. Courtois, Ph. Gandit, and B. Pannetier, "Proximity-induced superconductivity in a narrow metallic wire”, Phys. Rev. B 52, 1162 (1995).

[124] F. Zhou, B. Spivak, "Resistance of superconductor-normal-metal-superconductor (SNS) junctions”, JETP Letters 65, 369 (1997) [arXiv:cond-mat/9604185].

[125] A.F. Andreev, "The thermal conductivity of the intermediate state in superconductors”, Zh. Eksp. Teor. Fiz. 46, 1823 (1964) [Sov. Phys. JETP 19, 1228].

[126] N.N. Bogoliubov, “A New Method of Superconductivity I”, Zh. Eksp. Teor. Fiz., 34, 58-73 (1958) [Sov. Phys. JETP 741 (1958)].

[127] N.N. Bogoliubov, V.V. Tolmachev, and D.V. Shirkov, “A New Method of Superconductivity”, Academy of Sciences of USSR, Moscow, (1958).

[128] N.N. Bogoliubov, Uspekhi Fiz. Nauk 67, 549 (1959) [Soviet Phys. Uspekhi 67, 236 (1959)].

[129] G. Deutcher and P.G. de Gennes, "Superconductivity”, edited by R.D. Parks, Marcel Dekker, New York p1005 (1969).

[130] P.G. de Gennes, "Superconductivity of metals and allloys", W.A. Benjamin, New York (1966), Addison-Wesley Chapter 4, 5.

[131] L.P. Gor'kov, Zh. Eksp. Teor. Fiz. 36, 1918 (1959); 37, 1407 (1959) [Sov. Phys. JETP 9, 1364 (1959); 10, 998 (1960)].

[132] G. Eilenberger, “Transformation of Gorkov's equation for type II superconductors into transport-like equations”, Z. Phys. 214, 195 (1968).

[133] K. Usadel, “Generalized diffusion equation for superconducting alloys”, Phys. Rev. Lett. 25, 507 (1970).

[134] C.W.J. Beenakker, "Universal limit of critical-current fluctuations in mesoscopic Josephson junctions”, Phys. Rev. Lett. 67, 3836 (1991) [arXiv:cond-mat/0406127].

[135] G. Eilenberger, "General approximation method for the free energy functional of superconducting alloys”, Z. Phys. 190, 142 (1966). 
[136] P.G. de Gennes, "Impurity and boundary effects in superconductors", Rev. Mod. Phys. 36, 225 (1964).

[137] Y.V. Nazarov, “Circuit theory of Andreev conductance”, Phys. Rev. Lett. 10, 1420 (1994).

[138] M.Y. Kuprianov and V.F. Lukichev, "Influence of boundary transparency on the critical current of “dirty” SS’S structures”, Sov. Phys. JETP 67, 1163 (1988).

[139] Y.V. Nazarov, "Novel circuit theory of Andreev reflection”, arXiv:cond-mat/981155 (1998).

[140] T.T. Heikkilä, J. Särkkä and F.K. Wilhelm, "Supercurrent-carrying density of states in diffusive mesoscopic Josephson weak links”, Phys. Rev. B 66, 184513 (2002).

[141] K.M. Schep and G.E.W. Bauer, "Universality of Transport through Dirty Interfaces”, Phys. Rev. Lett. 78, 3015 (1997).

[142] O.N. Dorokhov, "Transmission coefficient and the localization length of an electron in N bound disorder”, JETP Lett. 36, 318 (1982).

[143] For a review on the quasiclassical approach see: W. Belzig, F.K. Wilhelm, C. Bruder, G. Schon, and A.D. Zaikin, “Quasiclassical Green's function approach to mesoscopic superconductivity”, Superlattice and Microstructures 25, n 5/6 (1999) [arXiv:cond-mat/9812297].

[144] J.C. Cuevas, J. Hammer, J. Kopu, J.K. Viljas, M. Eschrig., “Proximity Effect and Multiple Andreev Reflections in Diffusive SNS Junctions”, Phys. Rev. B 73, 184505 (2006) [arXiv:cond-mat/0507247].

[145] D.A. Ivanov, R. von Roten, and G. Blatter, "Minigap in a long disordered SNS junction: Analytical results”, Phys. Rev. B 66, 052507 (2002).

[146] J.C. Cuevas and F.S. Bergeret, "Magnetic Interference Patterns and Abrikosov Vortices in Diffusive SNS junctions", (preprint).

[147] V. Barzykin and A.M. Zagoskin, "Coherent transport and nonlocality in mesoscopic SNS junctions: anomalous magnetic interference patterns”, Superlat. and Microstruct. 25, 797 (1999) [arXiv:cond-mat/9805104].

[148] A. Anthore, "Decoherence mechanisms in mesoscopic conductors", $\mathrm{PhD}$ Thesis, Université Paris 6, CEA - Saclay (2003). http://tel.archives-ouvertes.fr/tel-00003518

[149] J.C. Hammer, J.C. Cuevas, F.S. Bergeret, W. Belzig, "Density of states and supercurrent in diffusive SNS junctions: role of non-ideal interfaces and spin-flip scattering”, arXiv:cond-mat/07042358. 
[150] F. Zhou, P. Charlat, B. Spivak, B. Pannetier, "Density of States in SuperconductorNormal Metal-Superconductor Junctions” J. Low Temp. Phys. 110, 841 (1998).

[151] C.W.J. Beenakker, “Andreev Billiard”, Lect. Notes Phys. 667, 131 (2005) [arXiv:cond-mat/0406018].

[152] For a review on the use of the scattering formalism for quantum objects see: C.W.J. Beenakker, "Random-matrix theory of quantum transport”, Rev. Mod. Phys. 69, 731 (1997).

[153] N. Agrait, A. Levy-Yeyati, J.M. van Ruitenbeek "Quantum properties of atomicsized conductors" Phys. Reps. 81, 377 (2003).

[154] M.F. Goffman, R. Cron, A. Levy Yeyati, P. Joyez, M.H. Devoret, D. Esteve, C. Urbina, "Supercurrent in atomic point contacts and Andreev states", Phys. Rev. Lett. 85170 (2000).

[155] M.L. Della Rocca, M. Chauvin, B. Huard, H. Pothier, D. Esteve and C. Urbina, "Measurement of the current-phase relation of superconducting atomic contacts", to appear in Phys. Rev. Lett.

[156] R. Cron, "Atomic contacts: a test bed for mesoscopic physics", $\mathrm{PhD}$ Thesis, Université Paris 6, CEA-Saclay (2001). http://tel.archives-ouvertes.fr/tel-00001329

[157] J.J. Riquelme, L. de la Vega, A. Levy Yeyati, N. Agrait, A. Martin-Rodero, G. Rubio-Bollinger "Distribution of conduction channels in nanoscale contacts: Evolution towards the diffusive limit”, Europhys. Lett., 70, 663 (2005) [arXiv:cond-mat/0506017].

[158] For a review, see B. Pannetier and H. Courtois, "Andreev reflection and proximity effect”, J. Low Temp. Phys. 118, 599 (2000) [arXiv:cond-mat/9912024].

[159] P. Dubos, H. Courtois, B. Pannetier, F.K. Wilhelm, A.D. Zaikin, and G. Schön, “Josephson critical current in a long mesoscopic S-N-S junction”, Phys. Rev. B 63, 064502 (2001).

[160] M. Tinkham, "Introduction to Superconductivity”, McGraw-Hill, New York, 1975.

[161] A. Barone and G. Paternò, "Physics and Applications of the Josephson Effect", Wiley-Interscience, p359 (1982).

[162] W. Belzig, R. Shaikhaidarov, V.V. Petrashov, and Yu.V. Nazarov, "Negative magnetoresistance in Andreev interferometers”, Phys. Rev. B 66, 220505 (2002). 
[163] V. Antonov, V. T. Petrashov and P. Delsing, "Phase sensitive phenomena in Andreev interferometer”, Physica C: Superconductivity 352, 173 (2001); B.J. van Wees, S.G. den Hartog, and A.F. Morpurgo, "Comment on "Phase Controlled Conductance of Mesoscopic Structures with Superconducting Mirrors"”, Phys. Rev. Lett. 76, 1402 (1996).

[164] A. Anthore, H Pothier, and D. Esteve, "Density of States in a Superconductor Carrying a Supercurrent”, Phys. Rev. Lett. 90, 127001 (2003).

[165] S. Skalski, O. Betbeder-Matibet, and P.R. Weiss, "Properties of Superconducting Alloys Containing Paramagnetic Impurities”, Phys. Rev. 136, 1500 (1964).

[166] W. Belzig, "Magnetic and spectral properties of superconducting proximitysystems”, PhD Thesis, Universität Karlsruhe (TH), Karlsruhe (1999). 